NISSUNA UMANA INVESTIGAZIONE SI PUO DIMANDARE VERA SCIENZIA S'ESSA NON PASSA PER LE MATEMATICHE DIMOSTRAZIONI

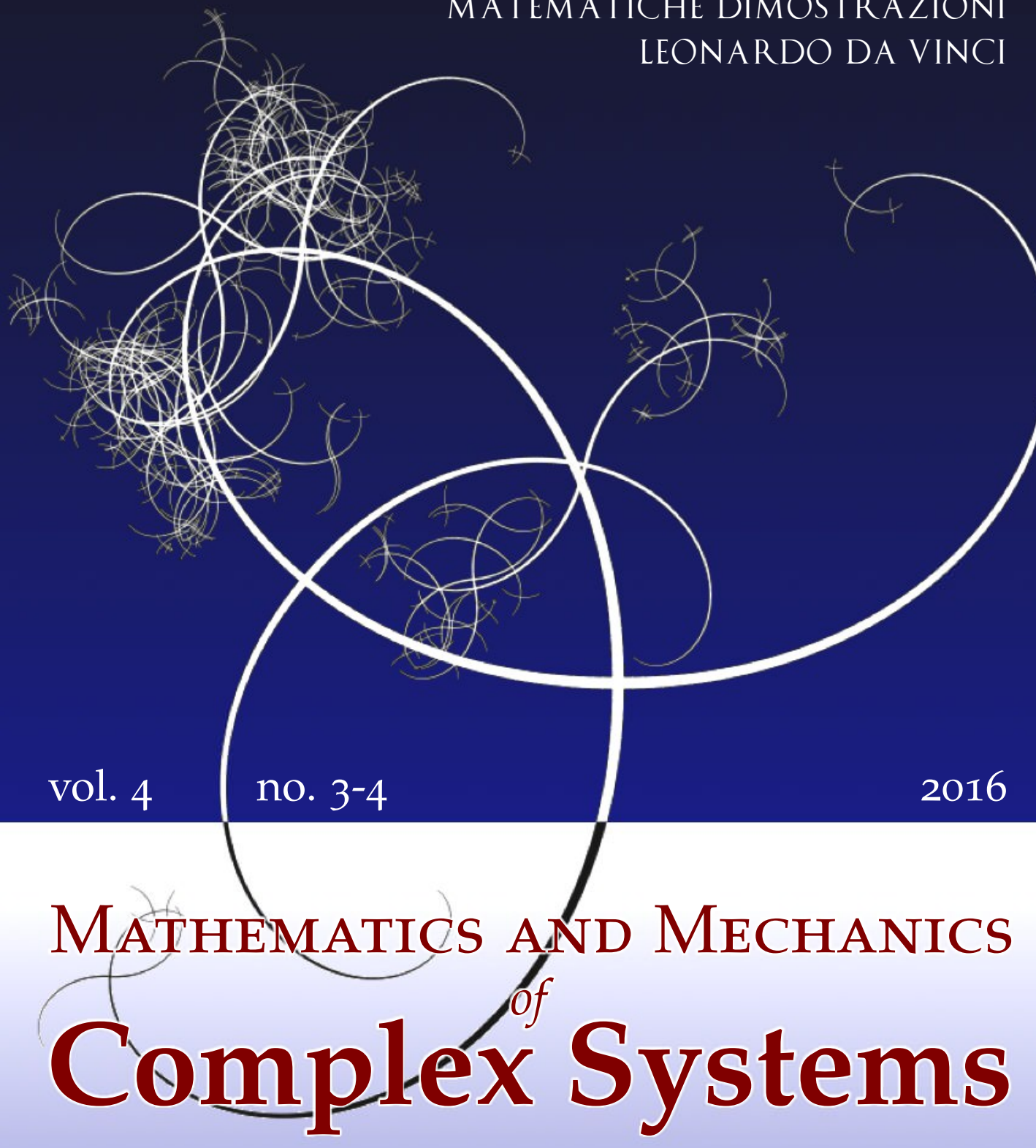

Special issue in honor of

Lucio Russo 
EDITORIAL BOARD

ANTONIO CARCATERRA

ERIC A. CARLEN

FRANCESCO DELL'ISOLA

RAFFAELE ESPOSITO

ALBERT FANNJIANG

Gilles A. FranCFORT

Pierangelo MARCATI

JEAN-JACQUES MARIGO

PETER A. MARKOWICH

MARTIN OSTOJA-STARZEWSKI

PIERRE SEPPECHER

DAVID J. STEIGMANN

PAUl STEINMANN

PierRe M. SuQueT

MANAGING EDITORS

MICOL AMAR

CORRADO LATTANZIO

ANGELA MADEO

MARTIN OSTOJA-STARZEWSKI

ADVISORY BOARD

ADNAN AKAY

Holm AltenBaCH

MICOL AMAR

HARM ASKES

TEODOR ATANACKOVIĆ

VICTOR BERDICHEVSKY

GUY BOUCHITTÉ

ANDREA BRAIDES

ROBERTO CAMASSA

MAURO CARFORE

ERIC DARVE

FELIX DARVE

ANNA DE MASI

GianPiEtro DEL Piero

EMMANUELE Di BENEDETTO

BERNOLD FIEDLER

IRENE M. GAMBA

DAVID Y. GAO

SERGEY GAVRILYUK

TIMOTHY J. HEALEY

DOMINIQUE JEULIN

ROGER E. KHAYAT

CORRADO LATTANZIO

ROBERT P. LIPTON

ANGELO LUONGO

ANGELA MADEO

JUAN J. MANFREDI

CARLO MARCHIORO

GÉRARD A. MAUGIN

ROBERTO NATALINI PATRIZIO NEFF

ANDREY PIATNITSKI

ERRICO PRESUTTI

MARIO PULVIRENTI

LUCIO RUSSO

Miguel A. F. SANJUAN

PATRICK SElVADURAI

ALEXANDER P. SEYRANIAN

MIROSLAV ŠILHAVÝ

GUIDO SWEERS

ANTOINETTE TORDESILLAS

LEV TRUSKINOVSKY

JUAN J. L. VELÁZQUEZ VINCENZO VESPRI ANGELO VULPIANI msp.org/memocs

Università di Roma "La Sapienza", Italia

Rutgers University, USA

(CO-CHAIR) Università di Roma "La Sapienza", Italia

(TREASURER) Università dell'Aquila, Italia

University of California at Davis, USA

(CO-CHAIR) Université Paris-Nord, France

Università dell'Aquila, Italy

École Polytechnique, France

DAMTP Cambridge, UK, and University of Vienna, Austria

(CHAIR MANAGING EDITOR) Univ. of Illinois at Urbana-Champaign, USA

Université du Sud Toulon-Var, France

University of California at Berkeley, USA

Universität Erlangen-Nürnberg, Germany

LMA CNRS Marseille, France

Università di Roma "La Sapienza", Italia

Università dell'Aquila, Italy

Université de Lyon-INSA (Institut National des Sciences Appliquées), France

(CHAIR MANAGING EDITOR) Univ. of Illinois at Urbana-Champaign, USA

Carnegie Mellon University, USA, and Bilkent University, Turkey

Otto-von-Guericke-Universität Magdeburg, Germany

Università di Roma "La Sapienza", Italia

University of Sheffield, UK

University of Novi Sad, Serbia

Wayne State University, USA

Université du Sud Toulon-Var, France

Università di Roma Tor Vergata, Italia

University of North Carolina at Chapel Hill, USA

Università di Pavia, Italia

Stanford University, USA

Institut Polytechnique de Grenoble, France

Università dell'Aquila, Italia

Università di Ferrara and International Research Center MEMOCS, Italia

Vanderbilt University, USA

Freie Universität Berlin, Germany

University of Texas at Austin, USA

Federation University and Australian National University, Australia

Université Aix-Marseille, France

Cornell University, USA

École des Mines, France

University of Western Ontario, Canada

Università dell' Aquila, Italy

Louisiana State University, USA

Università dell'Aquila, Italia

Université de Lyon-INSA (Institut National des Sciences Appliquées), France University of Pittsburgh, USA

Università di Roma "La Sapienza”, Italia

Université Paris VI, France

Istituto per le Applicazioni del Calcolo "M. Picone", Italy

Universität Duisburg-Essen, Germany

Narvik University College, Norway, Russia

Università di Roma Tor Vergata, Italy

Università di Roma "La Sapienza”, Italia

Università di Roma “Tor Vergata”, Italia

Universidad Rey Juan Carlos, Madrid, Spain

McGill University, Canada

Moscow State Lomonosov University, Russia

Academy of Sciences of the Czech Republic

Universität zu Köln, Germany

University of Melbourne, Australia

École Polytechnique, France

Bonn University, Germany

Università di Firenze, Italia

Università di Roma La Sapienza, Italia

MEMOCS (ISSN 2325-3444 electronic, 2326-7186 printed) is a journal of the International Research Center for the Mathematics and Mechanics of Complex Systems at the Università dell'Aquila, Italy.

Cover image: "Tangle” by $\odot$ John Horigan; produced using the Context Free program (contextfreeart.org).

PUBLISHED BY

7 mathematical sciences publishers

nonprofit scientific publishing

http://msp.org/

(C) 2016 Mathematical Sciences Publishers 


\section{LUCIO RUSSO: A MULTIFACETED LIFE}

Lucio Russo was born in Venice, Italy, on 22 November 1944. He attended high school at the Liceo Ginnasio Giambattista Vico in Naples; in the same city he then studied at the Università degli Studi Federico II, from which he graduated cum laude in 1969 with a thesis on quantum diffusion (Laurea in physics). In 1970-72 he held a research fellowship at the Istituto di Fisica Teorica of

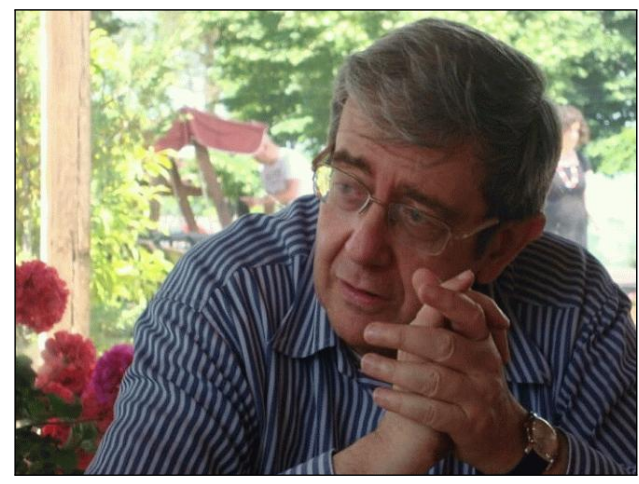
Naples and in 1973-78 he was adjunct professor (professore incaricato) at the University of Naples. In 1977-80 he was assistant professor of Rational Mechanics at the University of Modena, becoming full professor there in 1980. In 1982 he spent a research period at the Institut des Hautes Études Scientifiques (Bures-surYvette, France), and in 1982-83 he was visiting professor at Princeton University (USA). From 1984 until his retirement in 2015 he was full professor at University Tor Vergata of Rome. In 1999-2000 and 2000-2001 he obtained a secondment at the Accademia Nazionale dei Lincei. In 2010 he was awarded the International Prize "Tullio Levi-Civita" for the Mathematical and Mechanical Sciences.

During his professorship he taught, among other courses, Probability, General Physics, Rational Mechanics, Mathematical Methods for Physics, Mathematical Physics, Partial Differential Equations, Numerical Analysis, Real Analysis, History of Science, History of Mathematics.

His research activity covered several topics, from statistical mechanics (Gibbs measures of the Ising model) to probability theory (percolation theory, finite systems of random variables), reconstruction of images and recognition of shapes, chaotic nonlinear transformations (Hénon map) and history of science. Among his books, The Forgotten Revolution (Springer, 2004), Segmenti e bastoncini (Feltrinelli, 1998), Flussi e riflussi (Feltrinelli, 2003), Ingegni minuti (Feltrinelli, 2010; with E. Santoni), L'America dimenticata (Mondadori Università, 2013), and Stelle, atomi e velieri (Mondadori Università, 2015).

His results in technical directions, as well as his contributions in history of science, have been characterized by remarkable ingenuity and freshness, answering some important questions and asking interesting new ones. 
We, editors of this special issue, dedicate it to Lucio in friendship and admiration, on behalf of the whole Editorial Board of MEMOCS.

RAFFAELE ESPOSITO

M\&MOCS, Università degli Studi dell' Aquila

FRANCESCO DELL'ISOLA

Università di Roma "La Sapienza" 


\title{
THE WORK OF LUCIO RUSSO ON PERCOLATION
}

\author{
GEOFFREY R. GRIMMETT \\ Dedicated in friendship to Lucio Russo
}

\begin{abstract}
The contributions of Lucio Russo to the mathematics of percolation and disordered systems are outlined. The context of his work is explained, and its ongoing impact on current work is described and amplified.
\end{abstract}

\section{A personal appreciation}

Prior to his mid-career move to the history of science in the early 1990 s, Lucio Russo enjoyed a very successful and influential career in the theory of probability and disordered systems, in particular of percolation and the Ising model. His ideas have shaped these significant fields of science, and his name will always be associated with a number of fundamental techniques of enduring importance.

The author of this memoir is proud to have known Lucio in those days, and to have profited from his work, ideas, and company. He hopes that this brief account of some of Lucio's results will stand as testament to the beauty and impact of his ideas.

\section{Scientific summary}

Lucio Russo has worked principally on the mathematics of percolation, that is, of the existence (or not) of infinite connected clusters within a disordered spatial network. The principal model in this field is the so called percolation model, introduced to mathematicians by Broadbent and Hammersley in 1957, [14]. Consider, for definiteness, the hypercubic lattice $\mathbb{Z}^{d}$ with $d \geq 2$, and let $p \in[0,1]$. We declare each edge to be open with probability $p$ and closed otherwise, and different edges receive independent states. The main questions are centred around the existence (or not) of an infinite open component in $\mathbb{Z}^{d}$. It turns out that there exists a critical probability $p_{\mathrm{c}}=p_{\mathrm{c}}(d)$ such that no infinite open cluster exists when $p<p_{\mathrm{c}}$, and there exists a unique such cluster when $p>p_{\mathrm{c}}$. (It is not still known which of these two occurs when $p=p_{\mathrm{c}}$ for general $d$, specifically when $3 \leq d \leq 10$. See [20].)

\section{Communicated by Raffaele Esposito.}

MSC2010: 60K35, 82B20.

Keywords: percolation, Ising model, Russo's formula, RSW inequality, box crossing, approximate zero-one law, influence, sharp threshold. 
Let $C$ be the open cluster of $\mathbb{Z}^{d}$ containing the origin. Two functions that play important roles in the theory are the percolation probability $\theta$ and the mean cluster size $\chi$ given by

$$
\theta(p)=P_{p}(|C|=\infty), \quad \chi(p)=E_{p}|C|,
$$

where $P_{p}$ and $E_{p}$ are the appropriate product measure and expectation. The above model is the bond percolation model; the site percolation model is defined similarly, with sites being open/closed. A fairly recent account of percolation may be found in [25].

The question was raised in 1960 (by Harris, [31]) of whether or not $p_{\mathrm{c}}(2)=\frac{1}{2}$, and the search for a rigorous proof attracted a number of fine mathematicians into the field, including Lucio. Several important partial results were proved, culminating in 1980 with Kesten's complete proof that $p_{\mathrm{c}}(2)=\frac{1}{2}$, [37]. The interest of the community then migrated towards the case $d \geq 3$, before returning firmly to $d=2$ with the 2001 proof by Smirnov, [44; 45], of Cardy's formula.

Lucio contributed a number of fundamental techniques to percolation theory during the period 1978-1988, and the main purpose of the current paper is to describe these and to explore their significance. We mention Russo's formula, the Russo-Seymour-Welsh (RSW) inequalities, his study of percolation surfaces in three dimensions, and of the uniqueness of the infinite open cluster, and finally Russo's approximate zero-one law. Russo's formula and RSW theory have proved of especially lasting value in, for example, recent developments concerning conformal invariance for critical percolation.

In Section 8, we mention some of Lucio's results concerning percolation of $+/-$ spins in the two-dimensional Ising model. It was quite a novelty in the 1970s to use percolation as a tool to understand long-range order in the Ising model. Indeed, Lucio's work on the percolation model was motivated in part by his search for rigorous results in statistical mechanics. His approach to the Ising model has been valuable in two dimensions. In more general situations, the correct geometrical model has been recognised since to be the random-cluster model of Fortuin and Kasteleyn (see [26]).

This short account is confined to Lucio's contributions to percolation, and does not touch on his work lying closer to ergodic theory and dynamical systems, namely [R2; R6; R8; R11], and neither does it refer to the paper [R7]. A comprehensive list of Lucio's mathematical publications, taken from MathSciNet, may be found at the end of this paper.

Results from Lucio's work will be described here using 'modern' notation. No serious attempt is made to include comprehensive citations of the related work of others. 


\section{Russo's formula}

Let $\Omega=\{0,1\}^{E}$ where $E$ is finite, and let $P_{p}$ be product measure on the partially ordered set $\Omega$ with density $p \in[0,1]$. An event $A \subseteq \Omega$ is called increasing if:

$$
\omega \in A, \omega \leq \omega^{\prime} \Rightarrow \omega^{\prime} \in A .
$$

Let $\omega \in \Omega$. An element $e \in E$ is called pivotal for an increasing event $A$ if $\omega_{e} \notin A$ and $\omega^{e} \in A$, where $\omega^{e}$ and $\omega_{e}$ are obtained from $\omega$ by varying the state of the edge $e$ thus:

$$
\omega_{e}(f)=\left\{\begin{array}{ll}
0 & \text { if } f=e, \\
\omega(f) & \text { if } f \neq e,
\end{array} \quad \omega^{e}(f)= \begin{cases}1 & \text { if } f=e, \\
\omega(f) & \text { if } f \neq e .\end{cases}\right.
$$

In other words, $e$ is said to be pivotal for $A$ if the occurrence of $A$ depends on the state of $e$.

Theorem 3.1 (Russo's formula, [R14]). Let A be an increasing event. We have

$$
\frac{d}{d p} P_{p}(A)=\sum_{e \in E} P_{p}(e \text { is pivotal for } A) .
$$

Similar techniques are encountered independently in related fields. For example, Russo's formula is essentially equation (4.4) of Barlow and Proschan's book [7, p. 212] on reliability theory. Such a formula appeared also in the work of Margulis, [39], in the Russian literature. A characteristic of Lucio's work is the geometric context of the formula when applied in situations such as percolation, and it is in this context that Lucio's name is prominent. In a typical application to percolation, one uses the geometrical characteristics of the event $\{e$ is pivotal for $A\}$ to derive differential inequalities for $P_{p}(A)$.

Russo's formula is key to the study of geometrical probability governed by a product measure. It has so many applications that it is a challenge to single out any one. We mention here its use in the derivation of exact values for critical exponents in two dimensions, [38; 46].

Similarly, extensions of Russo's formula have been central in several related fields, including but not limited to the contact model [10, Thm 2.13], continuum percolation [21;34], and the random-cluster model [11, Prop. 4].

\section{Russo-Seymour-Welsh inequalities}

For twenty years from about 1960 to 1980, mathematicians attempted to prove that the critical probability $p_{\mathrm{c}}$ of bond percolation on the square lattice satisfies $p_{\mathrm{c}}=\frac{1}{2}$. This prominent open problem was in the spirit of that of the critical temperature of the Ising model, resolved in 1944 by Onsager, [42]. Harris [31] showed how to use duality to obtain $p_{\mathrm{c}} \geq \frac{1}{2}$, but the corresponding upper bound was elusive. Then, in 
1978, a powerful technique emerged in independent and contemporaneous work of Lucio, [R12], and Seymour and Welsh, [43]. It has come to be known simply as 'RSW'.

Consider bond percolation with density $p$ on the square lattice $\mathbb{Z}^{2}$. A left-right crossing of a rectangle $B$ is an open path in $B$ which joins some vertex on its left side to some vertex on its right side. For positive integers $m$ and $n$, we define the rectangle

$$
B(m, n)=[0,2 m] \times[0,2 n],
$$

and let $\operatorname{LR}(m, n)$ be the event that there exists a left-right crossing of $B(m, n)$.

Lemma 4.1 (Russo-Seymour-Welsh (RSW), [R12; 43]). Let $p \in(0,1)$. We have

$$
P_{p}\left(\operatorname{LR}\left(\frac{3}{2} n, n\right)\right) \geq(1-\sqrt{1-\tau})^{3},
$$

where $\tau=P_{p}(\operatorname{LR}(n, n))$.

This fundamental but superficially innocuous lemma implies that, if the chance of crossing a square is bounded from 0 uniformly in its size, then so is the chance of crossing a rectangle with aspect ratio $\frac{3}{2}$. Using the self-duality of $\mathbb{Z}^{2}$, we have as input to the RSW lemma that

$$
P_{\frac{1}{2}}(\operatorname{LR}(n, n)) \geq \frac{1}{2} .
$$

Let $A_{n}$ be the event that the annulus $[-3 n, 3 n]^{2} \backslash[-n, n]^{2}$ contains an open cycle with the origin in the bounded component of its complement in $\mathbb{R}^{2}$. Using elementary geometrical arguments and the FKG inequality, it follows by the RSW lemma and (4.1) that there exists $\sigma>0$ such that $P_{p}\left(A_{n}\right) \geq \sigma$ for $n \geq 1$ and $p \geq \frac{1}{2}$.

The RSW lemma and the ensuing annulus inequality have proved to be key to the study of percolation in two dimensions. In common with other useful methods of mathematics, there is now a cluster of related inequalities, see for example [12], [27, Sect. 5.5], and [50, Chap. 5].

RSW methods were used by their discoverers to make useful but incomplete progress towards proving that $p_{\mathrm{c}}=\frac{1}{2}$, and they played a role in Kesten's full proof, [37]. (The principle novelty of Kesten's paper was a bespoke theory of sharp threshold, see Section 5.) More precisely, they led to the following result, which is presented in terms of site percolation on the square lattice $\mathbb{Z}^{2}$ and its matching lattice $\mathbb{Z}_{*}^{2}$, derived by adding the two diagonals to each face of $\mathbb{Z}^{2}$.

Theorem 4.2 (Russo, [R12]). Consider site percolation on the square lattice $\mathbb{Z}^{2}$. The critical points

$$
p_{\mathrm{c}}=\sup \{p: \theta(p)=0\}, \quad \pi_{\mathrm{c}}=\sup \{p: \chi(p)<\infty\},
$$


satisfy

$$
p_{\mathrm{c}}+\pi_{\mathrm{c}}^{*}=1, \quad p_{\mathrm{c}}^{*}+\pi_{\mathrm{c}}=1,
$$

where an asterisk denotes the corresponding values on the matching lattice.

The parallel work of Seymour and Welsh, [43], was directed at the bond model on $\mathbb{Z}^{2}$, of which the dual model lies on a translate of $\mathbb{Z}^{2}$. Following Kesten's proof of $p_{\mathrm{c}}\left(\mathbb{Z}^{2}\right)=\frac{1}{2}$ for bond percolation, Lucio revisited Theorem 4.2 in [R14] with a proof that $\pi_{\mathrm{c}}^{*}=p_{\mathrm{c}}^{*}$, and the consequent improvement of (4.2), namely $p_{\mathrm{c}}+$ $p_{\mathrm{c}}^{*}=1$. He also completed the proof, begun in [R12], that $\theta$ (and, similarly, the dual percolation probability $\left.\theta^{*}\right)$ is a continuous function on $[0,1]$. Continuity in two dimensions has since been extended to general percolation models (see, for example, [25, Sect. 8.3]).

RSW theory is now recognised as fundamental to rigorous proofs of conformal invariance of critical two-dimensional percolation and all that comes with that. The proof of Cardy's formula, [44; 45], provides a major illustration. It was observed by Aizenman and Burchard, [3], that certain connection probabilities belong to a space of uniformly Hölder functions. Since this space is compact, such functions have subsequential limits as the mesh of the lattice approaches 0 . The above Hölder property is proved using annulus inequalities.

Indeed the power of RSW arguments extends beyond percolation to a host of problems involving two-dimensional stochastic geometry, such as the FK-Ising model [18] and Voronoi percolation [49]. In addition, RSW theory provides one of the main techniques for the proof by Beffara and Duminil-Copin, [9], that the random-cluster model on $\mathbb{Z}^{2}$ with cluster-weighting parameter $q \geq 1$ has critical value $p_{\mathrm{c}}(q)=\sqrt{q} /(1+\sqrt{q})$. We retrieve Kesten's theorem by setting $q=1$.

\section{Approximate zero-one law}

Kolmogorov's zero-one law may be stated as follows. Consider the infinite product space $\Omega=\{0,1\}^{\mathbb{N}}$ endowed with the product $\sigma$-algebra and the product measure $P_{p}$. If $A$ is an event that is independent of any finite subcollection $\{\omega(e): e \in E\}$, $E \subseteq \mathbb{N},|E|<\infty$, then $P_{p}(A)$ equals either 0 or 1 . It follows that, for an increasing event $A$, there exists $p_{0} \in[0,1]$ such that

$$
P_{p}(A)= \begin{cases}0 & \text { if } p<p_{0} \\ 1 & \text { if } p>p_{0}\end{cases}
$$

This law is intrinsically an infinite-volume effect, in that the index set is the infinite set $\mathbb{N}$. Lucio posed the farsighted question in [R15] of whether there exists a finite volume version of this result, and this led him to his 'approximate zero-one law', following. 
Let $\Omega=\{0,1\}^{E}$ where $E$ is finite, and let $P_{p}$ be product measure on the partially ordered set $\Omega$ with density $p \in[0,1]$. The influence $I_{A, p}(e)$ of $e \in E$ on the event $A \subseteq \Omega$ is defined by

$$
I_{A, p}(e)=P_{p}\left(1_{A}\left(\omega_{e}\right) \neq 1_{A}\left(\omega^{e}\right)\right),
$$

where $1_{A}$ denotes the indicator function of $A$. When $A$ is increasing, this may be written

$$
I_{A, p}(e)=P_{p}\left(\omega_{e} \notin A, \omega^{e} \in A\right)=P_{p}(e \text { is pivotal for } A) .
$$

Theorem 5.1 (Russo's approximate zero-one law, [R15]). For $\epsilon>0$, there exists $\eta>0$ such that, if $A$ is an increasing event and

$$
I_{A, p}(e)<\eta, \quad e \in E, p \in[0,1],
$$

then there exists $p_{0} \in[0,1]$ such that

$$
P_{p}(A) \begin{cases}\leq \epsilon & \text { if } p<p_{0}-\epsilon \\ \geq 1-\epsilon & \text { if } p>p_{0}+\epsilon .\end{cases}
$$

This result was motivated by a desire to generalise certain results for box-crossing probabilities in percolation. Its impact extends far beyond percolation, and it is a precursor of a more recent theory, pioneered by Kahn, Kalai, Linial, [35] and Talagrand, [47; 48], of influence and sharp threshold. It is proved at [47, Thm 1.1] that there exists an absolute constant $c>0$ such that, for $p \in(0,1)$ and an increasing event $A$,

$$
\sum_{e \in E} I_{A, p}(e) \geq\left(\frac{c}{p(1-p) \log [2 /(p(1-p))]}\right) P_{p}(A)\left(1-P_{p}(A)\right) \log \left(1 / m_{p}\right),
$$

where

$$
m_{p}=\max \left\{I_{A, p}(e): e \in E\right\} .
$$

It follows that, when $p \in(0,1)$ and $A$ is increasing,

$$
\sum_{e \in E} I_{A, p}(e) \geq c^{\prime} P_{p}(A)\left(1-P_{p}(A)\right) \log \left(1 / m_{p}\right),
$$

where $c^{\prime}>0$ is an absolute constant.

Amongst the implications of (5.5) is a quantification of the relationship between $\epsilon$ and $\eta$ in Theorem 5.1. Suppose (5.2) holds with $\eta \in(0,1)$, so that $m_{p} \leq \eta$. By (5.5) and Russo's formula,

$$
\frac{d}{d p} P_{p}(A) \geq c^{\prime} P_{p}(A)\left(1-P_{p}(A)\right) \log (1 / \eta) .
$$


Choose $p_{0}$ such that $P_{p_{0}}(A)=\frac{1}{2}$, and integrate (5.6) to obtain

$$
P_{p}(A) \begin{cases}\leq \epsilon_{p} & \text { if } p<p_{0} \\ \geq 1-\epsilon_{p} & \text { if } p>p_{0}\end{cases}
$$

with

$$
\epsilon_{p}=\frac{1}{1+(1 / \eta)^{c^{\prime}\left|p-p_{0}\right|}}
$$

Such inequalities have found numerous applications in percolation and related topics, see for example $[12 ; 19 ; 24]$. They have been extended to general product measures, $[13 ; 28]$, and to probability measures satisfying the FKG lattice condition, [23]. Recent overviews include [27, Chap. 4] and [36].

\section{Percolation in dimension $d \geq 3$}

In 1983, Lucio spent a sabbatical at Princeton University. His work during that period led to two significant publications [R1; R3] on aspects of percolation in three dimensions. The first of these caused quite a stir in the community at the time of its appearance, largely since most work until then had been for models in only two dimensions. Whereas the dual of a bond model in two dimensions is another bond model, the dual model in three or more dimensions is a 'plaquette' model. Since the topology of surfaces of plaquettes is much more complicated that that of paths, the ensuing percolation duality poses a number of challenging topological questions.

The authors of [R1] consider bond percolation on $\mathbb{Z}^{3}$ with density $p$, together with its dual 'plaquette' model on $\mathbb{Z}_{*}^{3}:=\mathbb{Z}^{3}+\left(\frac{1}{2}, \frac{1}{2}, \frac{1}{2}\right)$. A plaquette is a unit square with vertices in $\mathbb{Z}_{*}^{3}$, and its bounding lines are edges of $\mathbb{Z}_{*}^{3}$. Each edge $e$ of $\mathbb{Z}^{3}$ intersects a unique plaquette $\Pi_{e}$, and $\Pi_{e}$ is termed occupied if and only if $e$ is closed (and unoccupied otherwise). Thus, a plaquette is occupied with probability $1-p$. For any collection $F$ of plaquettes, the boundary $\partial F$ is defined to be set of edges of $\mathbb{Z}_{*}^{3}$ belonging to an odd number of members of $F$.

Let $\gamma$ be a cycle of $\mathbb{Z}_{*}^{3}$. The main results of [R1] concern the probability there exists a set $F$ of occupied plaquettes which spans $\gamma$ in the sense that $\partial F=\gamma$. For simplicity, we shall suppose here that $\gamma$ is a $m \times n$ rectangle of the $x / y$ plane, and we denote the above event as $W_{\gamma}$. Note that $\gamma$ has area $m n$ and perimeter $2(m+n)$.

Theorem 6.1 (Aizenman, Chayes, Chayes, Fröhlich, Russo, [R1]). There exist constants $\pi_{\mathrm{c}}, \rho_{\mathrm{c}} \in(0,1)$ such that

$$
-\log P_{p}\left(W_{\gamma}\right) \sim \begin{cases}\alpha m n & \text { if } 1-p<\pi_{\mathrm{c}}, \\ \beta(m+n) & \text { if } 1-p>\rho_{\mathrm{c}},\end{cases}
$$

where $\alpha, \beta>0$ depend on $p$, and the asymptotic relation is as $m, n \rightarrow \infty$. 
The constants $\pi_{\mathrm{c}}, \rho_{\mathrm{c}}$ are the critical densities of the bond percolation model on $\mathbb{Z}^{3}$ given by

$$
\pi_{\mathrm{c}}=\sup \{p: \chi(p)<\infty\}, \quad \rho_{\mathrm{c}}=\lim _{k \rightarrow \infty} \widehat{p}_{\mathrm{c}}(k),
$$

where $\widehat{p}_{\mathrm{c}}(k)$ is the slab critical point

$$
\widehat{p}_{\mathrm{c}}(k)=\sup \left\{p: P_{p}\left(0 \leftrightarrow \infty \text { in }[0, \infty)^{2} \times[0, k]\right)=0\right\} .
$$

It was conjectured in [R1] that $\pi_{\mathrm{c}}=p_{\mathrm{c}}=\rho_{\mathrm{c}}$. The first equality was proved later in $[2 ; 41]$, and the second in $[8 ; 30]$.

There are only few percolation models on finite-dimensional lattices for which the numerical values of the critical probabilities are known exactly, and all such exact results are in two dimensions only (see, for example, [29]). In contrast, quite a lot of work has been devoted to obtaining rigorous upper and lower bounds for critical probabilities, and there is a host of numerical estimates.

Consider site percolation on the simple cubic lattice $\mathbb{Z}^{3}$. By a comparison with the site model on the triangular lattice, Lucio has shown (with Campanino, in [R3]) that $p_{\mathrm{c}} \leq \frac{1}{2}$. (See also [40].) They obtained also the strict inequality, with a distinctly more complicated argument.

Theorem 6.2 (Campanino, Russo, [R3]). The critical probability of site percolation on $\mathbb{Z}^{3}$ satisfies $p_{\mathrm{c}}<\frac{1}{2}$.

The point of this work was to show that, in a neighbourhood of $p=\frac{1}{2}$, there is coexistence of infinite open and infinite closed clusters in $\mathbb{Z}^{3}$. The corresponding statement for $d=2$ is, of course, false, in that coexistence occurs for no value of $p$.

Theorem 6.2 may still be the best rigorous upper bound that is currently known for $p_{\mathrm{c}}$. By examining its proof, one may calculate a small $\epsilon>0$ such that $p_{\mathrm{c}}<\frac{1}{2}-\epsilon$. It is expected that $p_{\mathrm{c}} \approx 0.31$.

\section{Uniqueness of the infinite open cluster}

Let $I$ be the number of infinite open clusters of a percolation model in a finitedimensional space. For a period in the 1980 s, the 'next' problem was to prove that $P_{p}(I=1)=1$ in the supercritical phase (when $p>p_{\mathrm{c}}$ ). This problem was solved by Aizenman, Kesten, and Newman [5] in 1987. Their proof seemed slightly mysterious at the time, and it was simplified by Lucio in the jointly written paper [R9]. The key step was to show, using a large-deviation estimate present already in [5], that there is density 0 of sites that are adjacent to two distinct infinite clusters.

This useful argument was soon overshadowed by the beautiful proof of uniqueness by Burton and Keane, [15], of which a key step is a novel argument to show 
there is density 0 of sites that are adjacent to three distinct infinite clusters. The proof of [15] uses translation-invariance of the underlying measure together with a property of so called 'finite energy', and may thus be extended to more general measures than product measures. On the other hand, since the proof uses no quantitative estimate, it yields no 'rate'. The methods of [5; R9] provide a missing rate, and this has been useful in the later work $[16 ; 17]$.

The question of uniqueness for dependent models is potentially harder, since the large-deviation estimate of [5; R9] is not available. In joint work [R10] with Gandolfi and Keane, Lucio used path-intersection arguments to show uniqueness for ergodic, positively associated measures in two dimensions, satisfying certain translation and reflection symmetries. Unlike the Burton-Keane proof, they needed no finite-energy assumption. An application of this work to quantum spin systems may be found in [6].

\section{Ising model}

Lucio has written three papers on the geometry of the $d$-dimensional Ising model, [R4; R5; R13]. In this work, he (and his coauthors) studied the relationship between properties of the infinite-volume Gibbs measures and the existence or not of an infinite cluster of either + or - spins (that is, of percolation in the Ising model).

The first two of these papers [R4; R5] explore a relationship between the Ising magnetization and the above percolation probability, and yield the non-existence of percolation in the high-temperature phase. This is complemented when $d=2$ with the proof that percolation (of the corresponding spin) exists in the low-temperature phase for either of the pure infinite-volume limits $\mu_{+}, \mu_{-}$, obtained respectively as the weak limits with $+/-$ boundary conditions. These methods were developed further in [R5], where a phase diagram was proposed for the existence of infinite clusters in the two-dimensional ferromagnetic Ising model, as a function of external field $h$ and temperature $T$. The principal features of this diagram were later proved by Higuchi, [33].

One of the central problems in two dimensions of the late 1970s was to prove or disprove the statement that every infinite-volume Gibbs measure is a convex combination of the two extremal measures $\mu_{+}, \mu_{-}$. Lucio obtained the following important result for this problem.

Theorem 8.1 (Russo, [R13]). Any infinite-volume Gibbs measure $\mu$, which is translation-invariant in one or both of the axial directions, is a convex combination of $\mu_{+}$and $\mu_{-}$.

Lucio proved this by considering the existence (or not) of infinite $+/-$ clusters on $\mathbb{Z}^{2}$ and its matching lattice. The full conclusion, without an assumption of partial translation-invariance, was obtained later in independent work of Aizenman, 
[1], and Higuchi, [32] (see also [22]). Therefore, in two dimensions (unlike three dimensions) there exists no non-translation-invariant Gibbs measure.

More recent work on the geometrical properties of the Ising model has been centred around the random-cluster model and the random-current representation, rather than the more fundamental percolation model. See, for example, [4; 26].

\section{Acknowledgements}

This work was supported in part by the Engineering and Physical Sciences Research Council under grant EP/I03372X/1. The author thanks Alberto Gandolfi for his comments and suggestions.

\section{Mathematical publications of Lucio Russo}

[R1] M. Aizenman, J. T. Chayes, L. Chayes, J. Fröhlich, and L. Russo, "On a sharp transition from area law to perimeter law in a system of random surfaces", Comm. Math. Phys. 92:1 (1983), 19-69.

[R2] C. Cammarota and L. Russo, "Bernoulli and Gibbs probabilities of subgroups of $\{0,1\}$ ", Forum Math. 3:4 (1991), 401-414.

[R3] M. Campanino and L. Russo, "An upper bound on the critical percolation probability for the three-dimensional cubic lattice”, Ann. Probab. 13:2 (1985), 478-491.

[R4] A. Coniglio, C. R. Nappi, F. Peruggi, and L. Russo, "Percolation and phase transitions in the Ising model”, Comm. Math. Phys. 51:3 (1976), 315-323.

[R5] A. Coniglio, C. R. Nappi, F. Peruggi, and L. Russo, "Percolation points and critical point in the Ising model”, J. Phys. A 10:2 (1977), 205-218.

[R6] F. di Liberto, G. Gallavotti, and L. Russo, "Markov processes, Bernoulli schemes, and Ising model”, Comm. Math. Phys. 33 (1973), 259-282.

[R7] G. Facchinetti and L. Russo, "A one-dimensional case of stochastic homogenization”, Boll. Un. Mat. Ital. C (6) 2:1 (1983), 159-170.

[R8] V. Franceschini and L. Russo, "Stable and unstable manifolds of the Hénon mapping", J. Statist. Phys. 25:4 (1981), 757-769.

[R9] A. Gandolfi, G. Grimmett, and L. Russo, "On the uniqueness of the infinite cluster in the percolation model”, Comm. Math. Phys. 114:4 (1988), 549-552.

[R10] A. Gandolfi, M. Keane, and L. Russo, "On the uniqueness of the infinite occupied cluster in dependent two-dimensional site percolation”, Ann. Probab. 16:3 (1988), 1147-1157.

[R11] G. Monroy and L. Russo, "A family of codes between some Markov and Bernoulli schemes", Comm. Math. Phys. 43:2 (1975), 155-159.

[R12] L. Russo, “A note on percolation”, Z. Wahrsch. Verw. Gebiete 43:1 (1978), 39-48.

[R13] L. Russo, "The infinite cluster method in the two-dimensional Ising model", Comm. Math. Phys. 67:3 (1979), 251-266.

[R14] L. Russo, “On the critical percolation probabilities”, Z. Wahrsch. Verw. Gebiete 56:2 (1981), 229-237.

[R15] L. Russo, “An approximate zero-one law”, Z. Wahrsch. Verw. Gebiete 61:1 (1982), 129-139. 


\section{References}

[1] M. Aizenman, "Translation invariance and instability of phase coexistence in the two-dimensional Ising system”, Comm. Math. Phys. 73:1 (1980), 83-94.

[2] M. Aizenman and D. J. Barsky, "Sharpness of the phase transition in percolation models", Comm. Math. Phys. 108:3 (1987), 489-526.

[3] M. Aizenman and A. Burchard, "Hölder regularity and dimension bounds for random curves", Duke Math. J. 99:3 (1999), 419-453.

[4] M. Aizenman, H. Duminil-Copin, and V. Sidoravicius, "Random currents and continuity of Ising model's spontaneous magnetization", Comm. Math. Phys. 334:2 (2015), 719-742.

[5] M. Aizenman, H. Kesten, and C. M. Newman, "Uniqueness of the infinite cluster and continuity of connectivity functions for short and long range percolation”, Comm. Math. Phys. 111:4 (1987), $505-531$.

[6] M. Aizenman and B. Nachtergaele, "Geometric aspects of quantum spin states", Comm. Math. Phys. 164:1 (1994), 17-63.

[7] R. E. Barlow and F. Proschan, Mathematical theory of reliability, Wiley, New York, 1965.

[8] D. J. Barsky, G. R. Grimmett, and C. M. Newman, "Percolation in half-spaces: equality of critical densities and continuity of the percolation probability", Probab. Theory Rel. Fields 90:1 (1991), 111-148.

[9] V. Beffara and H. Duminil-Copin, "The self-dual point of the two-dimensional random-cluster model is critical for $q \geq 1$ ”, Probab. Theory Rel. Fields 153:3-4 (2012), 511-542.

[10] C. E. Bezuidenhout and G. R. Grimmett, "Exponential decay for subcritical contact and percolation processes", Ann. Probab. 19:3 (1991), 984-1009.

[11] C. E. Bezuidenhout, G. R. Grimmett, and H. Kesten, "Strict inequality for critical values of Potts models and random-cluster processes", Comm. Math. Phys. 158:1 (1993), 1-16.

[12] B. Bollobás and O. Riordan, "The critical probability for random Voronoi percolation in the plane is 1/2", Probab. Theory Rel. Fields 136:3 (2006), 417-468.

[13] J. Bourgain, J. Kahn, G. Kalai, Y. Katznelson, and N. Linial, "The influence of variables in product spaces", Israel J. Math. 77:1-2 (1992), 55-64.

[14] S. R. Broadbent and J. M. Hammersley, "Percolation processes, I: Crystals and mazes", Proc. Cambridge Philos. Soc. 53 (1957), 629-641.

[15] R. M. Burton and M. Keane, "Density and uniqueness in percolation", Comm. Math. Phys. 121:3 (1989), 501-505.

[16] R. Cerf, "A lower bound on the two-arms exponent for critical percolation on the lattice", Ann. Probab. 43:5 (2015), 2458-2480.

[17] S. Chatterjee and S. Sen, "Minimal spanning trees and Stein's method", 2013. arXiv 1307.1661

[18] D. Chelkak, H. Duminil-Copin, and C. Hongler, "Crossing probabilities in topological rectangles for the critical planar FK-Ising model”, Electron. J. Probab. 21 (2016), 1-28.

[19] H. Duminil-Copin and I. Manolescu, "The phase transitions of the planar random-cluster and Potts models with $q \geq 1$ are sharp", Probab. Theory Related Fields 164:3-4 (2016), 865-892.

[20] R. Fitzner and R. van der Hofstad, "Generalized approach to the non-backtracking lace expansion”, (2015). arXiv 1506.07969

[21] M. Franceschetti, M. D. Penrose, and T. Rosoman, "Strict inequalities of critical values in continuum percolation”, J. Stat. Phys. 142:3 (2011), 460-486. 
[22] H.-O. Georgii and Y. Higuchi, "Percolation and number of phases in the two-dimensional Ising model", J. Math. Phys. 41:3 (2000), 1153-1169.

[23] B. T. Graham and G. R. Grimmett, "Influence and sharp-threshold theorems for monotonic measures”, Ann. Probab. 34:5 (2006), 1726-1745.

[24] B. T. Graham and G. R. Grimmett, "Sharp thresholds for the random-cluster and Ising models", Ann. Appl. Probab. 21:1 (2011), 240-265.

[25] G. R. Grimmett, Percolation, 2nd ed., Grundlehren der Mathematischen Wissenschaften 321, Springer, Berlin, 1999.

[26] G. R. Grimmett, The random-cluster model, Grundlehren der Mathematischen Wissenschaften 333, Springer, Berlin, 2006.

[27] G. R. Grimmett, Probability on graphs, Institute of Mathematical Statistics Textbooks 1, Cambridge University Press, 2010.

[28] G. R. Grimmett, S. Janson, and J. R. Norris, "Influence in product spaces", Adv. Appl. Probab. 48A (2016), 145-152.

[29] G. R. Grimmett and I. Manolescu, "Bond percolation on isoradial graphs: criticality and universality", Probab. Theory Rel. Fields 159:1-2 (2014), 273-327.

[30] G. R. Grimmett and J. M. Marstrand, "The supercritical phase of percolation is well behaved", Proc. Roy. Soc. London Ser. A 430:1879 (1990), 439-457.

[31] T. E. Harris, "A lower bound for the critical probability in a certain percolation process", Proc. Cambridge Philos. Soc. 56 (1960), 13-20.

[32] Y. Higuchi, "On the absence of non-translation invariant Gibbs states for the two-dimensional Ising model”, pp. 517-534 in Random fields (Esztergom, Hungary, 1979), vol. I, edited by J. Fritz et al., Colloq. Math. Soc. János Bolyai 27, North-Holland, Amsterdam, 1981.

[33] Y. Higuchi, "A sharp transition for the two-dimensional Ising percolation", Probab. Theory Rel. Fields 97:4 (1993), 489-514.

[34] J. Jiang, S. Zhang, and T. Guo, "Russo's formula, uniqueness of the infinite cluster, and continuous differentiability of free energy for continuum percolation", J. Appl. Probab. 48:3 (2011), 597-610.

[35] J. Kahn, G. Kalai, and N. Linial, "The influence of variables on Boolean functions", pp. 68-80 in Proceedings of the 29th annual symposium on foundations of computer science, IEEE Computer Society, Washington, D.C., 1988.

[36] G. Kalai and S. Safra, "Threshold phenomena and influence: perspectives from mathematics, computer science, and economics”, pp. 25-60 in Computational complexity and statistical physics, edited by A. G. Percus, G. Istrate, and C. Moore, Oxford University Press, 2006.

[37] H. Kesten, "The critical probability of bond percolation on the square lattice equals $\frac{1}{2}$ ", Comm. Math. Phys. 74:1 (1980), 41-59.

[38] H. Kesten, "Scaling relations for 2D-percolation", Comm. Math. Phys. 109:1 (1987), 109-156.

[39] G. A. Margulis, "Probabilistic characteristics of graphs with large connectivity", Problemy Peredachi Informatsii 10:2 (1974), 101-108.

[40] M. V. Menshikov, "Estimates for percolation thresholds for lattices in $\mathbb{R}^{n}$ ", Dokl. Akad. Nauk SSSR 284: 1 (1985), 36-39. In Russian; translated in Soviet Math. Dokl. 32:02 (1985), 368-370.

[41] M. V. Menshikov, "Coincidence of critical points in percolation problems", Dokl. Akad. Nauk SSSR 288:6 (1986), 1308-1311. In Russian; translated in Soviet Math. Dokl. 33:03 (1986), 856-859.

[42] L. Onsager, "Crystal statistics, I: A two-dimensional model with an order-disorder transition", Phys. Rev. (2) 65 (1944), 117-149. 
[43] P. D. Seymour and D. J. A. Welsh, "Percolation probabilities on the square lattice", pp. 227245 in Advances in graph theory, vol. 3, Annals of Discrete Mathematics 3, edited by B. Bollobás, North-Holland, Amsterdam, 1978.

[44] S. Smirnov, "Critical percolation in the plane: conformal invariance, Cardy's formula, scaling limits”, C. R. Acad. Sci. Paris Sér. I Math. 333:3 (2001), 239-244.

[45] S. Smirnov, "Critical percolation in the plane", (2009). arXiv 0909.4499

[46] S. Smirnov and W. Werner, "Critical exponents for two-dimensional percolation", Math. Res. Lett. 8:5-6 (2001), 729-744.

[47] M. Talagrand, “On Russo's approximate zero-one law”, Ann. Probab. 22:3 (1994), 1576-1587.

[48] M. Talagrand, "Concentration and influences", Israel J. Math. 111 (1999), 275-284.

[49] V. Tassion, "Crossing probabilities for Voronoi percolation", Ann. Probab. 44:5 (2016), 33853398.

[50] W. Werner, Percolation et modèle d'Ising, Cours Specialisés 16, Société Mathématique de France, Paris, 2009.

Received 17 Apr 2016. Accepted 19 May 2016.

GEOFFREY R. GRIMMETT: g.r.grimmett@statslab.cam.ac.uk

Statistical Laboratory, Centre for Mathematical Sciences, University of Cambridge,

Wilberforce Road, Cambridge, CB3 OWB, United Kingdom

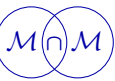





\title{
"MATHEMATICS" AND "PHYSICS" IN THE SCIENCE OF HARMONICS
}

\author{
STEFANO ISOLA
}

\begin{abstract}
Some aspects of the role that the science of harmonics has played in the history of science are discussed in light of Russo's investigation of the history of the concepts of "mathematics" and "physics".
\end{abstract}

\section{The rambling route of the ancient scientific method}

In several places in Russo's writings on the history of science, one can find enlightening discussions about the meanings of the concepts of "physics" and "mathematics", along with the particular notions of truth involved in them; see, e.g., [58, Chapter 6.6; 60, Chapter 15; 56; 57]. Both terms derive from the Greek: the original meaning of the former was the investigation of everything that lives, grows or, more generally, comes into existence, whereas the latter referred to all that is studied, thus deriving its meaning not from its content but from its method. In the Hellenistic period, the term "physics" continued to be used to indicate that sector of philosophy that addressed nature (the other sectors being ethics and logic), thus corresponding to what came to be called "natural philosophy" in modern times. On the other hand, the term "mathematics" was used to indicate all the disciplines (including geometry, arithmetic, harmonics, astronomy, optics, mechanics, hydrostatics, pneumatics, geodesy and mathematical geography) that shared the same method of investigation, based on the construction of theories by which "theorems" are proved, leaning on explicitly stated initial assumptions. Its meaning thus corresponded to what we call "exact sciences" and refers to a unitary body of scientific disciplines alien to the modern distinction between physical and mathematical sciences.

In antiquity, how the scope of mathematics contrasted with that of physics was a topic of much debate. According to some key testimonials reported and discussed in [56] — in particular that due to Geminus (and reported by Simplicius) in the first

\section{Communicated by Raffaele Esposito.}

MSC2010: 01A20.

Keywords: harmonic theory, mathematics, physics. 
century B.C. ${ }^{1}$ and, much later, that of Thomas Aquinas ${ }^{2}$ - the "physicist" would be able to grasp the "substance" of reality using philosophical categories, whereas a characteristic feature of the work of the astronomer, that is, the "mathematician", is its incapability to assert absolute truths, in that he is able to rigorously deduce/construct a number of consequences from previously stated hypotheses whose ultimate validity remains however out of control.

To better understand this discrepancy, let's step back again to highlight another important methodological difference between natural philosophy and exact science in antiquity, in that the former operates on a single level of discourse, where data from experience and thoughts are organized so as to produce "directly" a rational account of the perceptions themselves. ${ }^{3}$ In particular, natural philosophy starts "from the things which are more knowable and obvious to us and proceeds towards those which are clearer and more knowable by nature" [4, p. 184a], thus revealing the alleged genuine, mind-independent nature of things. This is also reflected in the use of language. As reported by the fifth-century Alexandrian scholar Ammonius, “... Aristotle teaches what the things principally and immediately signified by sounds [e.g., names and verbs] are, and these are thoughts. Through these as means

1“"The physicist will prove each fact by considerations of essence or substance, of force, of its being better that things should be as they are, or of coming into being and change; the astronomer will prove them by the properties of figures or magnitudes, or by the amount of movement and the time that is appropriate to it. Again, the physicist will in many cases reach the cause by looking to creative force; but the astronomer, when he proves facts from external conditions, is not qualified to judge of the cause... sometimes he invents by way of hypothesis, and states certain expedients by the assumption of which the phenomena will be saved. For example, why do the sun, the moon, and the planets appear to move irregularly? We may answer that, if we assume that their orbits are eccentric circles or that the stars describe an epicycle, their apparent irregularity will be saved; and it will be necessary to go further and examine in how many different ways it is possible for these phenomena to be brought about..." [34, p. 276].

2 "Reason may be employed in two ways to establish a point: firstly, for the purpose of furnishing sufficient proof of some principle, as in natural science, where sufficient proof can be brought to show that the movement of the heavens is always of uniform velocity. Reason is employed in another way, not as furnishing a sufficient proof of a principle, but as confirming an already established principle, by showing the congruity of its results, as the theory of eccentrics and epicycles is considered as established in astronomy, because thereby the sensible appearances of the heavenly movements can be explained; not, however, as if this proof were sufficient, forasmuch as some other theory might explain them" [1, pp. 63-64].

${ }^{3}$ In criticizing Protagoras's statement that man is the measure of all things, Aristotle says, "We say that knowledge and sense-perception are the measure of things because our recognition of something is due to them" [41, p. 184]. To him, therefore, sense-perception and knowledge are the faculties that furnish all our understanding of things and thus exhausted all possible meanings of the expression "criterion of truth". In Hellenistic practice, however, other meanings of this expression were put forward (of which Protagoras's dictum could be considered a likely precursor) including the Stoics' infallible act of cognition based on kataleptic impressions (self-certifying acts of sense-perceptions) as well as the hypothetico-deductive method of exact sciences. 
we signify things; and it is not necessary to consider anything else as intermediate between the thought and the thing, as the Stoics do, who assume what they name to be the meaning [lekton]" [61, p. 77].

Thus, at variance with the Aristotelian point of view, the early Stoics considered it necessary to distinguish between the pronounced sound and the meaning of what is pronounced as an intermediate link between a thought and a sound. The same kind of epistemological attitude characterized the exact sciences - which flourished in the same period of the early Stoic school - with their specific effort to overcome the illusion of being able to build intellectual schemes based directly on perceptible reality and the elaboration of abstract languages capable of describing not only aspects of the sensible world but also other designable realities; see [58], in particular Chapter 6. The existence of a double level of discourse seems therefore an essential feature of exact sciences, in that their assertions do not directly concern the things of the natural world but rather theoretical entities which are obtained by a procedure of "pruning" which allows one to focus on certain aspects of the phainomena - that is, what appears to the senses and calls for an explanation and to ignore those considered unessential. In brief, the methodological mark of exact sciences consists in the construction of simplified models of aspects of reality which, starting from suitable but "unjustified" hypotheses, operate on their internal entities in a logically rigorous way and then move back to the real world. Note that, by its very nature, every hypothesis is somehow "false", so nothing prevents different models based on different hypotheses of being capable of "saving" the same phenomena. In addition, while the assertions obtained at the theoretical level are "objective" and universally valid, the correspondence rules which transform the entities involved in the real world and the claims about them into theoretical entities and theoretical statements are instead historically determined. For example, in Hellenistic scientific theories dealing with phenomena related to the sense of sight, devices such as ruler and compass, designed to assist in the construction of the straight line and the circle, as well as in the measurement of their parts, incorporate the correspondence rules relating theoretical statements of geometry or optics to concrete objects. In theories of acoustic-musical phenomena, this role was played instead by the canon (see below). In both cases, the "concrete objects" — drawings with ruler and compass and pitches produced by a plucked string, respectively are not rough natural data: rather, they are the result of a somewhat refined human activity, which in turn is rooted in the historical and cultural context.

Although rarely acknowledged, the scientific method, as a cultural product of earlier Hellenistic times, underwent a rapid decline in the context of a more general cultural collapse that occurred during the second century B.C. ${ }^{4}$ Notwithstanding

\footnotetext{
${ }^{4}$ Particularly dramatic were the years 146-145 B.C., with the sharp hardening of the Roman policy in the Mediterranean that had among its consequences the reduction of Macedonia to a Roman
} 
the loss of a major part of ancient knowledge, the memory of Hellenistic science survived thanks to a series of geographically localized revival periods. ${ }^{5}$ On the other hand, a peculiar feature of these revivals was the insertion of individual contents, recovered from ancient science or derived from it, into foreign overarching systems of thought which provided their main motivating framework. ${ }^{6}$ In particular, "in the Age of Galileo", Russo says, "the exact science preserved the unity that distinguished the Greek models, from which it drew the terminology, but the ancient method was rarely understood. Not that the explanation reported by Simplicus had been forgotten, but few, as Stevin, used the freedom of choice of the hypotheses to build models; much more frequently the relative arbitrariness of the initial assumptions appeared (as it had appeared to Simplicius and Thomas Aquinas) as a particularity (as well as an oddity) of the method of the 'mathematician', which determined its inferiority with respect to philosophers and theologians, who knew how to distinguish "truth' from "falsehood" [56, p. 37]. The idea that the hypothetico-deductive method was mostly a limit that prevented approaching the absolute truth peaked with Newton. In the well known General Scholium added to the Principia in 1713, he writes, "But hitherto I have not been able to discover the cause of those properties of gravity from phenomena, and I frame no hypotheses [hypotheses non fingo]. For whatever is not deduced from the phenomena, is to be called an hypothesis; and hypotheses, whether metaphysical or physical, whether of occult qualities or mechanical, have no place in experimental philosophy. In this philosophy particular propositions are inferred from the phenomena, and afterwards rendered general by induction" [45, p. 392].

It is worth stressing that the term "phenomena" is used here with a meaning which differs considerably from the ancient one, in that it refers to something which lies beyond our perception. ${ }^{7}$ Likewise, the term "hypothesis" was given the new meaning — still in use — of a statement lying at the beginning of our interpretation of the external world but waiting to be corroborated or refuted as soon as the "facts" are known with sufficient detail. Thus, in every genuine search for the

province, the razing of Carthage and Corinth and the heavy political interference in Egypt with persecution and extermination of the Greek intellectual class [59, Chapter 5].

${ }^{5}$ The first of them was the resumption of scientific studies in imperial times, whose main protagonists were Heron, Ptolemy and Galen. The next ones occurred in the sixth-century Byzantine world, then in the medieval Islamic world (eighth to ninth centuries) and finally in Western Europe, from the "twelfth-century Renaissance" until the Renaissance par excellence of early modern times [58, Chapter 11].

${ }^{6}$ We shall discuss below an example which illustrates this fact in connection with Ptolemy's work.

${ }^{7}$ Think of the absolute motions of material bodies with respect to the immovable space which, coexisting with Aristarchan heliocentrism, cannot correspond to any observable datum (see the discussion given in [58, Chapter 11.7]). In a letter of 1698, Newton affirmed, "I am inclined to believe some general laws of the Creator prevailed with respect to the agreeable or unpleasing affections of all our senses" [46, Letter XXIX]. 
truth, hypotheses cannot be anything but a hindrance. ${ }^{8}$ As it is well known, Newtonianism was presented in the European continent as the philosophy of progress. The most famous of his supporters was Voltaire, who in the preface of the French translation of the Principia dismissed as "foolish" the followers of vortices formed by the "thin matter" of Descartes and Leibniz and affirmed that only a follower of Newton could be truly called a "physicist". Indeed, according to Russo, the spread of Newtonian mechanics has brought with it the way of reasoning on the basis of which "the exact science got broken into two stumps: 'mathematics' and 'physics'. Both of them inherited from the ancient 'mathematics' the quantitative approach and several technical results, and from the ancient 'physics' (that is from natural philosophy) the idea of producing statements which are absolutely 'true'. The essential difference was lying in the nature of such truth. While the truth of the assumptions of 'mathematics' (called postulates) was considered immediately evident, the assumptions of 'physics' (called principles) were regarded true inasmuch as they are 'proven by the phenomena'... It is plain that these differences were strictly connected to the diverse nature attributed to the entities studied by the two disciplines: the 'mathematical' entities, although usable to describe concrete objects, were considered abstract, whereas the 'physical' entities were considered as concrete as the objects they were referring to" [56, pp. 42-43]. In both cases, the "truth" of a scientific theory (e.g., a theory of the planetary motions) does not lie in its capability to "save the phenomena" (e.g., to determine with some accuracy the observable position of a planet at any time) but becomes something that one can "prove" by means of its own instruments, in the same way in which one can prove a statement on the entities internal to the theory itself. If so, a scientific theory would cease to be a theoretical model, instead becoming a system of statements set to describe the true nature of the real world. ${ }^{9}$

${ }^{8}$ As d'Alembert wrote, "it is not at all by vague and arbitrary hypotheses that we can hope to know nature; it is by thoughtful study of phenomena, by the comparisons we make among them, by the art of reducing, as much as that may be possible, a large number of phenomena to a single one that can be regarded as their principle" [23, p. 22], and a little further, "let us conclude that the single true method of philosophizing as physical scientists consists either in the application of mathematical analysis to experiments, or in observation alone, enlightened by the spirit of method, aided sometimes by conjectures when they can furnish some insights, but rigidly dissociated from any arbitrary hypotheses" [23, p. 25]. As an aside, this semantic transformation may have played a role in claiming a "historic mission" to human knowledge, from the naivety of the myth towards the final enlightenment, passing through an increasing control of the sources of error which allows one to progressively overcome all "false hypotheses" (that is, "prejudices"): a kind of secularized version of the medieval millenarianism, of which, among others, Newton was an ardent supporter.

${ }^{9}$ Such a prescientific position is proudly maintained by Voltaire in the entry "System" of [65, p. 224], which starts by stating, "We understand by system a supposition; for if a system can be proved, it is no longer a system, but a truth. In the meantime, led by habit, we say the celestial system, although we understand by it the real position of the stars". By the way, and not surprisingly, 
Following this reshaping of the scientific enterprise, some disciplines have been counted on one side and others on the other; still others have been somehow internally divided or eventually disappeared, as we shall see below in a particular example. Referring to the already cited writings of Russo for a discussion of the splitting between "mathematics" and "physics" in nineteenth and twentieth centuries, let us just remark that the final failure of the efforts towards a methodological reunification of the exact science, of which Poincare was a prominent exponent, and the prevailing of powerful trends towards specialization and fragmentation of the scientific disciplines, if on one side has led some to wonder what mystery lies behind the "unreasonable effectiveness" of mathematics in providing accurate descriptions of the phenomena [67], on the other side prompted one of the greatest contemporary mathematicians to acknowledge in this trend a severe crisis of science itself: "In the middle of the twentieth century it was attempted to divide physics and mathematics. The consequences turned out to be catastrophic. Whole generations of mathematicians grew up without knowing half of their science and, of course, in total ignorance of any other sciences. They first began teaching their ugly scholastic pseudomathematics to their students, then to schoolchildren (forgetting Hardy's warning that ugly mathematics has no permanent place under the Sun)" [7]; see also [57] for a further discussion.

\section{Acoustic-musical phenomena}

"Ho detto che la nostra scienza o arte musicale fu dettata dalla matematica. Doveva dire costruita. Essa scienza non nacque dalla natura,... ma ebbe origine ed ha il suo fondamento in quello che è giustamente chiamato seconda natura, ma che altrettanto a torto quanto facilmente e spesso è confuso e scambiato... colla natura medesima, voglio dire nell' assuefazione. Le antiche assuefazioni de' greci... furono l'origine e il fondamento della scienza musicale da' greci determinata, fabbricata e a noi ne' libri e nell'uso tramandata, dalla qual greca scienza vien per comun consenso e confessione la nostra europea" (G. Leopardi, Zibaldone [40, 3125-3126]).

Today musical theory is mainly "the study of the structure of music", whereas originally it was part of mathematics. What happened in the meantime? In order to get an idea, it is necessary to go back again to the rambling route of the ancient scientific method through the subsequent history. Resuming what was said in the previous section in a concise albeit vague way, we can say that the general objects of Greek science were not so much the "laws" of the natural world viewed as

this entry proceeds by strengthening the idea of a necessary progression of the knowledge by denying that Aristarchus introduced heliocentrism. 
an entity independent from the man who observes it but rather those indubitable epistemological data provided by the phainomena resulting from the interaction between subject and object through active perception. In particular, the models of Hellenistic exact science were primarily suited for that purpose: the creation of theoretical entities as intermediate utterances between the real objects and abstract truths has the effect of making that interaction available to conscious manipulation. This gets a peculiar meaning within the context of music theory which, as such, establishes sound, the material aspect of music, as something which can be knowingly investigated in connection with human experience. Although music - perhaps the most unfathomable expression of psychic activity - might not seem properly suited to scientific analysis, the investigation of acoustic-musical phenomena nonetheless provides an example where the epistemological opposition sketched in the previous section occurred with a striking character within the same domain, as we shall now briefly outline.

It is rather well known that Pythagorean music theory - as a part of their program of liberation of the soul by means of the intellectual perception of proportions in all things - starts from the recognition that the harmonic intervals can be expressed as simple numerical ratios. The following "Pythagorean principle" has been viewed as the first "natural law" expressed in terms of numerical entities (see, e.g., [11]): if two sounding bodies, such as stretched strings or sounding pipes, have lengths which are in simple proportions, and all other aspects are kept fixed, together they will produce musical intervals which are judged by the ear to be in harmonious agreement, or "consonant". Conversely, all intervals that the ear accepts as consonant can be represented as ratios of numbers from the tetrad 1, 2, 3, 4. ${ }^{10}$ The harmonic system of Philolaus (see, e.g., [18; 19]), for example, is a structure of intervals externally limited by the octave (diapason), whose ratio is $2: 1$, and internally articulated by intervals of fifths (diapente), with ratio $3: 2$, and fourths (diatessaron), with ratio $4: 3$. If we want to find four quantities for instance the lengths of the strings of a four-string lyre - that, taken in pairs, reproduce these ratios, then we can choose a unit of measure so that the longest string is 12 units, the intermediate ones 9 and 8 and the shortest 6 . It is clear that the system of reciprocal ratios, and therefore the whole harmonic structure, does not change if the strings have lengths $12,9,8$ and 6 meters, centimeters, stadiums, etc. Finally, observing that $(3: 2):(4: 3)=9: 8$, the interval of a tone, equivalent to the difference between a fifth and a fourth, is represented by the ratio 9:8. The octave is thus "harmonically" divided into two fourths spaced by a tone. ${ }^{11}$

${ }^{10}$ The question of which observations lay behind the detection of these ratios and when this happened is hard to answer [13].

${ }^{11}$ Note that $6 \cdot 12=8 \cdot 9$, i.e., the four numbers are in geometrical proportion. Moreover, $8=$ $2:\left(\frac{1}{6}+\frac{1}{12}\right)$ and $9=(6+12): 2$; namely, 8 and 9 are the harmonic mean and the arithmetic mean, 
Let us point out that in the transition from the Hellenic to the Hellenistic period mathematics becomes an exact science, in the sense specified above, not only by distinguishing theoretical entities from concrete objects but also from pure abstractions in the "platonic" sense. While discussing the subjects for the education of the "Guardians" of the Republic, Plato lets Socrates conceive that "as the eyes are designed to look up at the stars, so are the ears to hear harmonic motions", therefore agreeing that astronomy and music theory are sister sciences, as the Pythagoreans said $[50,530 \mathrm{~d}]$. On the other hand, those scholars are judged inadequate to reach the "true knowledge" beyond the sensible world in that "their method exactly corresponds to that of the astronomer; for the numbers they seek are those found in these heard concords, but they do not ascend to generalized problems and the consideration which numbers are inherently concordant and which not and why in each case" [50, 531c]. Clearly, the just mentioned "ascension" above experience does not need to be embedded in a theory. Rather, it would rely on "evidences" per se.

In a different direction, music theory, or at least that part of it dealing with tuning systems, was set to become a scientific discipline by putting together the arithmetic theory of proportions and the recognition of the proportionality between the pitch of the sounds and the speed of the vibrations that produce them, ${ }^{12}$ a conceptual step that according to some sources had been made in the circle of Archytas in about 400 B.C. [13; 36]. The "experimental device" enabling the establishment of a correspondence between concords and numerical ratios was the canon (kanon harmonikos), an instrument that in its simplest form is made of a single string stretched between two bridges fixed on a rigid base and equipped with another movable bridge by which one may divide the string into two parts, yielding sounds of variable pitch. One can further imagine a row fixed at its base on which the positions of the movable bridge corresponding to the notes can be marked. The name of the entire device is then a metonym for the line segment that represents it as a theoretical entity. The theory outlined in the Sectio canonis, attributed to Euclid, deals precisely with the harmonic divisions of this segment, i.e., with those divisions corresponding to musical intervals judged to be consonant [25]; see also [26]. In this work, far away from any mystical efflorescence about the music of the cosmos, a scheme of division of the octave by means of the theory of proportions contained in the Elements is proposed with the aim of producing patterns of consonant intervals adoptable in practice, e.g., when tuning musical

respectively, of the extremes 6 and 12. The exclusion of the geometric mean in "Pythagorean" music theory is justified by an impossibility result due to Archytas (see below).

${ }^{12}$ The recognition of the nature of sound as vibration of air, with alternation of rarefaction and compression, can already be found in Aristotle's Problemata [3] as well as in the Peripatetic De audibilibus [5], whereas the idea of a sound wave is attested at least as early as in the Stoa. 
instruments. Along this path, the branch of Greek music theory referred to as the science of harmonics entered the unitary body of Hellenistic mathematics, along with astronomy, arithmetic, geometry, optics, topography, pneumatics, mechanics and other disciplines [58, Chapter 3].

In the short introduction of the Sectio canonis, the author establishes a correspondence between musical intervals and numerical ratios and states the main hypothesis underlying the model: consonant intervals correspond to multiple or epimoric ratios. ${ }^{13}$ The rationale of this postulate relies on the observation that, as consonant intervals produce a perception of unity or tonal fusion between the notes, they must correspond to numbers which are given a "single name" in relation to one another. ${ }^{14}$ On the other hand, this postulate is clearly false not only because it includes among the concords also intervals considered dissonant by the Pythagorean principle stated above, such as the tone $9: 8$ or the ratio $5: 4$ (natural major third), but also because it counts as dissonant the interval composed by an octave plus a fourth, represented by the ratio $8: 3$, unanimously recognized as consonant by the music theorists of antiquity (exactly as an octave plus a fifth, that is, $3: 1)$. However, this is not a problem in itself, for all hypotheses are somehow "false": what matters is that the theory based on them is consistent and suited to save the phenomena which it aims to model. The introduction is then followed by twenty propositions: the first nine, of pure "number theory", provide a deductive construction of the Philolaus harmonic system sketched above, whereas the remaining ones form the part properly relevant to tuning systems. Of particular interest is the third proposition, which states that neither one nor more mean proportionals can be inserted within an epimoric interval. In particular, it is not possible to divide the octave into equal parts that form a rational relationship with the octave itself. ${ }^{15}$

The consequences of this simple result have been the subject of a controversy which has lasted for over two millennia, at the basis of which there is the distinction

${ }^{13}$ Greek arithmetic classified ratios into three basic types, which reduced to lowest terms corresponding to $n: 1$ (multiple), $(n+1): n$ (epimoric or superparticular) or $(n+m): n, n>m>1$ (epimeric or superpartient). Note that the first two are in a one-to-one correspondence: $p: q$ is multiple if and only if $p:(p-q)$ is epimoric so that $q$ is the greatest common divisor of both $p$ and $p-q$. In particular, the octave $2: 1$, participating to both consonant classes, is the "consonance of the consonances".

${ }^{14}$ The interpretation of this seemingly arbitrary correspondence is controversial. According to some scholars [11], the "single name" has to be ascribed to the fact that, unlike the epimeric ratios, multiple and epimoric ratios were indicated with a one-word name, like epitritos, "third in addition", for $4: 3$. According to a different interpretation [26] (based on [54, §I.5]), the "single name" is not a linguistic unity but a numerical one, corresponding to the greatest common "part" which composes the notes in both multiple and epimoric ratios (see footnote 13).

${ }^{15}$ Nor would it be possible to divide in this way the fifth, the fourth or the whole tone. This seems to be the first impossibility result surely ascribable to an author as Boethius [12, §III.11] reports a proof of it given by Archytas. 
between natural and tempered tunings [10; 37]. We'll not dwell here on the ways in which the different music theorists have conceived the division of the tonal continuum; see, e.g., $[9 ; 14 ; 20]$. Rather, we shall briefly discuss how in this domain the epistemological opposition between natural philosophy and mathematics manifested itself. To this end, we recall that the first writings of some importance dealing with Greek music theory are those of Aristoxenus and Theophrastus, both students of Aristotle and both harshly critical of the doctrine according to which the pitch can be conceived as a quantitative attribute of sound, representable by numbers. As representatives of the Peripatetic school, they were mainly interested in the "natural qualities" of the object of investigation. For instance, Theophrastus claims that differences of pitch are due to differences in the "shape" of the sounds' movement, not to differences of velocity, frequency of impact or the like, inasmuch as high notes travel in a straight line from the object to the ear while low ones spread more evenly all around the object; see his "De musica" excerpt in [52, pp. 61-65] and also [63]. In criticizing the "Pythagorean approach", he maintains that "if every interval were a quantity, and if melody arose from differences between notes, the melody would be as it is because it is a number. But if it were nothing but a number, everything numerable would participate in melody too, to the extent that it does in number". This illustrates in some way the single level of discourse maintained by natural philosophy about which we were talking in the previous section, where there is no space for intermediate entities in between the concrete objects and the abstract thoughts about them. A rather similar position is held by Aristoxenus, the leading musical theorist of antiquity. His Harmonic elements opens with the subject of vocal motion within the musical topos in which it moves, which is the continuum whose maximal range and minimal internal intervals are defined solely by what the human voice is capable of doing and by what the human ear can apprehend the moving voice to be doing. In particular, he claims that harmonic properties such as consonance are firstly subjects of experience by a musically trained ear and cannot be traced to numerical ratios. He wrote indeed, "we endeavor to supply proofs that will be in agreement with the phenomena, in this unlike our predecessors. For some of these introduced extraneous reasoning, and rejecting the senses as inaccurate fabricated rational principles, asserting that height and depth of pitch consist in certain numerical ratios and relative rates of vibration - a theory utterly extraneous to the subject and quite at variance with the phenomena" [6, pp. 188-189]. Although one may argue that the polemical target here is mostly the Platonic treatment of Pythagorean music theory, in the rejection of rational arguments based on assumptions external to the musical experience itself, we can see the demand that every assumption must be justified by the phenomena, that is, a substantial disclaiming of the scientific method. ${ }^{16}$

\footnotetext{
${ }^{16}$ In particular, Aristoxenus and his followers "admitted" that it was possible to divide the tone into two equal parts. This, of course, does not "contradict" the third proposition of the Sectio
} 
Be that as it may, with the crisis of Hellenistic civilization and in particular after the dramatic cultural collapse which occurred midway through the second century B.C., the scientific methodology rapidly disappeared together with the very possibility of understanding the need of the lekta - the conceptual constructions intermediate between the thoughts and the things - in the devising of meaningful representations of the relationships between human activity and the natural world. As Russo says, "in the imperial age, when the notion of theoretical models had been lost, such entities were conceivable only as real objects: the alternative between 'bodies' and 'incorporeal beings' thus became ineluctable. Some such entities were indeed made corporeal - witness the crystalline celestial spheres which replaced the spheres of Eudoxus of Cnidus and the epicycles of Apollonius of Perga. Likewise, the 'visual rays' of optics reacquired the character of physical objects emitted by the eyes, which was not present in Euclid's theory. ... Other entities, such as those of geometry, were given an incorporeal reality. This placed geometry in the realm of Platonic thought, a position that Hellenistic mathematics had left behind" [58, p. 232].

A similar fate befell the basic entities of the science of harmonics, such as the musical intervals, which lost the character of theoretical entities gained within Hellenistic science to be identified (again) either with corporeal items - such as the discrete movements of a melodic voice - or else with purely ideal abstractions, entities considered as much real as they are not attainable — such as the harmonic ratios composing the Zodiac or the human soul — both deemed to possess quantitative features to which reason can be "directly" applied by assigning them appropriate numbers.

An important example is provided by Ptolemy in the first book of his Harmonica, where, no longer being able to grasp the methodological tenets maintained by his Hellenistic sources, he falls back upon epistemological bases close to the Peripatetic ones, without thereby giving up the claim of employing refined mathematical tools inherited from his predecessors (yet conceived in the Platonic sense). For example, with a kind of inversion of the Hellenistic rule that requires a theory to save the phainomena, he says, "The purpose of the harmonicist would be to preserve in every way the reasoned hypotheses of the canon which do not in any way at all conflict with the perceptions as most people interpret them, just as the purpose of the astronomer is to preserve the hypotheses

canonis - implying that no (rational) mean proportional can be inserted between 9 and 8 - which characterizes intervals as elements of a theoretical model (hence defined solely by the hypotheses underlying the model itself). Rather, it results from the direct experience of placing one's finger on the string at the point corresponding to the division into equal semitones. A theoretical construction of the equal temperament has been made almost two millennia later by Stevin (see below). 
of the heavenly movements concordant with observable paths. Even these hypotheses are themselves assumed from what is clear and roughly apparent, but with the help of reason they discover detail with as much accuracy as is possible. For in every subject it is inherent in observation and knowledge to demonstrate that the works of nature have been crafted with some reason and prearranged cause and completed not at all in random" [54, §I.2, §§I.5.13-21]. ${ }^{17}$ Like other scholars of his time, Ptolemy's "criterion of truth" is dictated by a strange kind of "concord" between theory and observation, where the model, although highly mathematized, has lost its meaning as a theoretical entity and taken over the former prescientific meaning of direct representation of the known reality. In this regard, he seems to want to frame Hellenistic scientific results within philosophical arguments of the classical period. Although one might regard this as a dialectical strategy to give maximum credibility to the position he wants to hold, in this way he ends up denying the method of his Hellenistic predecessors, deeming legitimate only one theory: that whose assumptions are entirely justified by the phenomena and at the same time reflect the "rationality" of nature's works. ${ }^{18}$

Akin to Galen's craving to strike a balance between the "rationalist" and "empiricist" schools of medicine [30], ${ }^{19}$ Ptolemy loudly distinguishes his approach to the study of consonances from that of the "excessively rationalist" Pythagoreans - accused of accepting rationally justifiable statements even when they are contradicted by the senses ${ }^{20}$ — and that of the "overly empirical" Aristoxeneans, for whom audible harmonies are not subject to mathematical analysis at all. Since for him the objects of sense-perception and thought are (again) identical (cf. footnote 3), though apprehended in different ways, he feels entitled to set his "hypotheses" in the form of alleged mathematical counterparts of the relevant perceptual impressions. In doing so, Ptolemy assigns to the science of harmonics the task of explaining the audible and inaudible harmonies by reference to the formal, quantitative attributes of the different pitches, as to astronomy that of explaining the movements of the

${ }^{17}$ This goes hand in hand with some passages of the Almagest, for example where he says, "Now it is our purpose to demonstrate for the five planets, just as we did for the sun and moon, that all their anomalies can be represented by uniform circular motion, since these are proper to the nature of divine beings, while disorder and nonuniformity are alien [to such beings]" [53, §9.2].

${ }^{18}$ One should perhaps also include evidence that these narrow epistemological bases will actively operate in the development of modern science.

${ }^{19}$ A discussion on Ptolemy's epistemological affinity with his contemporary Galen can be found in [41].

${ }^{20}$ His concern focused in particular on the fact - already mentioned above - that the interval corresponding to the ratio $8: 3$ (diapason plus diatessaron), although unanimously recognized as consonant, was "deductively" counted as dissonant on the basis of the postulate opening the Sectio canonis [54, §I.5, §§I.12.4-8]. 
observable heavenly bodies by reference to the formal features of the spheres or other bodies on which they are physically carried. In this regard, the use of mathematics would serve mostly as a "rational criterion" to assist the senses in making fine discriminations.

As an aside, in the Alexandrian milieu of the imperial age, where the lingua franca was still Greek but life and thought were dominated by a cohort consisting of astrological fatalism, gnostic dualism and transcendent monotheism, even mathematics was mostly plunged into an atmosphere of irrationalism, with the distancing from the deductive method and the return of numerology. At the same time, among the objects of musical "perception", the prototype was considered the "music of the spheres", an old conception dating back at least to Plato's Timaeus [49] and Republic, resumed by Nicomachus [47] and subsequently by Ptolemy himself in the third book of his Harmonica. ${ }^{21}$ Thereafter, the Platonic connection between planetary motion and music became a cornerstone of the musica speculativawhich together with musica poetica and musica practica constituted the quadrivial discipline of musica. ${ }^{22}$ The regaining of a corporeal nature of the crystalline spheres to which the heavenly bodies were said to be attached goes hand in hand with the resumption of the celestial harmony as a "perceptible" datum, although emanating from an incorporeal and inaudible reality. ${ }^{23}$ Both subjects were then transmitted through centuries by sheer copying ${ }^{24}$ until they were taken seriously

${ }^{21}$ After comparing the various harmonic functions with several aspects of the human soul [54, $\S \S I I I .4-7]$, he proceeds by regarding the zodiac circle as a vibrating string and comparing the principal astrological "aspects" (angles between heavenly bodies that were believed to modify their degree of influence) with musical consonances, thereby explaining their differing "effectiveness" [54, $\S \S I I I .8-9]$.

${ }^{22}$ This tripartition of music reflected Aristotle's division of knowledge (epistēmēe into theōrētike poietike and praktike and was codified by the sixth-century Roman philosopher Boethius [12] as musica mundana, musica humana and musica instrumentalis. More generally, the resumption of a pre-Hellenistic classification of knowledge (in particular that outlined in Plato's Republic) becomes glaringly obvious with the reduction of the manifold Hellenistic sciences to the quadrivium, consisting of arithmetic, geometry, music and astronomy, which along with the trivium (made by grammar, logic and rhetoric) constituted the seven "liberal arts" that articulated the preparatory training for the study of theology in medieval times.

${ }^{23}$ Note that Aristotle, who believed in the existence of the rigid sidereal sphere [2, Chapter 6], refuted the conception of celestial music on the basis of physical arguments [2, Chapter 9]. On the other hand, in the cultural context to which we are referring, it would have seemed vain to refute on physical basis such Platonic mythological representations.

${ }^{24}$ Or else they were transmitted by anthological syntheses of the prisca sapientia, such as the commentary on Cicero's Somnium scipionis by the fifth-century neoplatonist Macrobius [42], where he drew comprehensively on the whole body of Pythagorean, Orphic and Platonic teachings and cosmology. How deep the decline of science at the end of the ancient world was can be grasped from the fact that, although Macrobius faithfully reports the ratios corresponding to the Pythagorean consonances, he does not even understand that they are ratios. For instance, he justifies the fact that the tone $9: 8$ cannot be divided into equal parts (a consequence of the Archytas impossibility result 
again in early modern times. For example, Kepler's "estimate" of the thickness of the crystal sphere of fixed stars ${ }^{25}$ went together with his tentative attempts to improve Ptolemy's harmonic investigations by searching for musical proportions in various quantities in the Solar system, such as the periods of the (heliocentric) planetary motions. ${ }^{26}$ Note however that although the faith in the "harmony of the world" had played an indubitable role in the reappearance of mathematics as the pivotal language of the resurgent sciences in early modern times, when at the time of Newton the terms "physics" and "mathematics" got the new meanings we have discussed above, science had begun to need different images; hence, celestial music became old-fashioned as a scientific subject and eventually became a purely literary metaphor. ${ }^{27}$

Altogether, the early modern resumption of studies on the science of harmonics took different forms, sometimes in open conflict with each other, often revealing with particular vividness the prevailing beliefs on the more general meaning of the scientific enterprise $[17 ; 33 ; 16]$. A well known example is the harsh conflict between Vincenzo Galilei (the father of Galileo) and Zarlino, where, among other things, to the "well ordered" Nature of Zarlino, which whispers to the human ear the true consonances, Galilei opposed the image of a Nature which proceeds "without cognition" (senza cognitione), with principles and purposes unrelated to man, and against which man takes advantage of the mechanical arts to an end that nature cannot achieve [31; 69]; see also [66, Chapter 2; 48]. Among the seventeenth-century scholars who took an active role in producing musical theories, like Simon Stevin, Kepler, Isaac Beeckman, Descartes, Mersenne, Francis Bacon, Galileo Galilei, Lord Brouncker, John Wallis, Christian Huygens, Robert Hooke and others, only the first one seems to have retained the option to build a model based on a free choice of hypotheses. ${ }^{28}$

discussed above) not with the nonexistence of a rational square root of 2 (and hence of 8 ) but with the fact that 9 is not divisible by 2 [42, Book Two, Chapter I.21-23].

${ }^{25}$ He estimated about two German miles [38, p. 288].

${ }^{26}$ We have to recognize that, unlike the first, the second concern was fruitful, as it is well known that the search for a harmonic correspondence between the periods of revolution and the radii of planets' orbits eventually led to the celebrated "third law" [39].

${ }^{27}$ Nevertheless, Newton himself had imagined recovering the lost prisca sapientia in which, among other things, the inverse square law of gravitational attraction between the planets would have been encrypted within Pythagoras' music of the spheres [43].

${ }^{28}$ This is the subject of a short treatise written in Dutch where, among other things, the equal temperament (i.e., the geometrical division of the octave into twelve equal parts, each corresponding to a ratio $1: \sqrt[12]{2}$ ) is constructed on the basis of two postulates. The first says that, as one part of a string is to another, so is the coarseness of the sound of the one to that of the other. The second says that natural singing is in the major diatonic scale, and in this scale all whole tones are equal and so are the semitones [62]. 
Somewhat later, in the age of Lights, and thus after the splitting between "physics" and "mathematics", an interesting confrontation about the science of harmonics took place between Euler and d'Alembert; see, e.g., [8, Chapter 4]. The swiss mathematician - perhaps the last representative of the conception of music as a part of mathematics - at age 24 finished writing his major work on the subject, the Tentamen novae theoriae musicae ex certissimis harmoniae principiis dilucide expositae (1731), whose main goal was to give an answer to the old question of why certain sounds are pleasant and others are not, an answer which would feature not only the perception of single intervals but also sequences of chords or even of a complete musical piece. He pursued this goal by assuming that any pleasure comes from the perception of a "perfection", which in turn is embodied in a notion of order that can be measured by an exponent calculated only in terms of the arithmetic proportions associated to the pitches of the tones involved. ${ }^{29}$ As a consequence, the fact that some people appreciate the use of some chords and others not is explained by saying that the latters' ear is not trained enough to perceive the order hidden in them. As we have pointed out previously about the Sectio canonis, the assumptions underlying this construction also cannot exempt themselves from being somehow "false": for instance, the same "exponent", and thus the same degree of pleasantness, is associated to a musical piece regardless if it is played forward or backward, which is in general something far from usual experience. But as we have seen, this is not a problem in itself, at least as long as things remain consistent with the ancient scientific method. It is not clear (to me) whether Euler considered the problem dealt with in his treatise as one of defining the value of something which was not defined before or as a Platonic search for some "true" value. Be that as it may, even only in his ambition to model far more than just a system of tuning, he exposed himself to the criticism that Johann Bernoulli leveled against him in 1731: “... you have derived the rule which establishes how the notes are to be combined, so that an intelligent ear can take delight in them. I think that this is appropriate for a musician who is more concerned about the accuracy of a piece of music than its effect, which satisfies the listener; a person of this kind will undoubtedly find enjoyment and delight, if you have written this down and examine it and find that it is well composed in accordance with the fundamental rules; but as a piece of music is usually played to ears that are devoid of understanding, and are not able to recognize the ratio between the beats of the intervals produced by the strings, and are even less able to count, then I believe that the same ears will appreciate or refuse the same piece

${ }^{29}$ See [27, pp. 197-427], where he starts from a masterful generalization of some previous ideas of Galileo [32] and Mersenne [44] according to which a chord of two sounds is all the more consonant when the "coinciding" blows resulting from the two sounds are in higher proportion in the whole of the produced blows. An example of calculation of this exponent is given below. 
of music, depending on whether they are used to this or that kind of music" [28, pp. 146-150].

But a critique of a different tenor was put forward by d'Alembert who, having in mind the example, bad for him, of the "mathematician" Euler, embodied the role of the "physicist", in the sense advocated by his mentor, Voltaire. In particular, he maintained that in music theory there is no place for "demonstration" — insofar as that term is reserved to "mathematics" - and one should adopt an "empiricaldeductive" methodology modeled on that of Newton. As he made clear in the preface of his widely read treatise on music theory, ${ }^{30}$ issued in 1752 , his main purpose in writing the work was "to show how one may deduce from a single experiment the laws of harmony which artists had arrived at only, so to speak, by groping" [22, p. vi]. The single experiment had to do in this case with Rameau's corps sonore, that is, any resonating system which, besides the fundamental frequency (sounding pitch), also generates a series of harmonically related overtones, such as the octave, the perfect twelfth (the octave of the perfect fifth), the major seventeenth (the double octave of the major third) and so on. To d'Alembert, the resonance of the sonorous body was the "most probable origin of harmony, and the cause of that pleasure which we receive from it". He thus strove to structure music as a science based on a single "principle" which is somehow "dictated by nature" and from which one should deduce "by an easy operation of reason, the chief and most essential laws of harmony".

The different positions embodied by the two scholars resulted in several controversies, among which the one about the possible solutions of the wave equation is perhaps the best known, although its current reconstructions usually neglect musical motivations and implications. In particular, the last account on the subject written by d'Alembert ends with a polemical stance against the music theory maintained by Euler, in which the very possibility of dealing with a musical phenomenon in terms of theoretical entities seems denied: "It is clear from the preceding formulae that, given an equal tension and thickness, the number of vibrations in the same time is inversely proportional to the length of the strings. As the higher or lower sound of the strings depends on their larger or smaller number of vibrations in the given time, it is undoubtedly for this reason that some very capable modern authors have considered it possible to represent the sounds by means of the logarithms of the ratios between the lengths of the strings. This idea is ingenious, and would appear to be based equally on figures of speech in acoustics and music, when we say that if four strings $a, b, c, d$ are geometrically proportional, the interval formed by sounds $a$ and $b$ will be equal to the interval formed by $c$ and $d$; hence it was

${ }^{30}$ It is a kind of résumé of the music-theoretic writings of the great composer Jean-Philippe Rameau [55], where he thought to find a paradigm of systematic method and synthetic structure which somehow confirmed his own scientific ideas [22]; see also [8; 15]. 
considered possible to conclude that the logarithms of the relationships $a: b$ and $c: d$ represented the intervals between the sounds. But undoubtedly this conclusion was not claimed to be anything more than a purely arbitrary supposition; the words interval between sounds, equality and difference of intervals are only abbreviated figures of speech, which should not be given a wider meaning than they really have. Sounds are merely sensations, and consequently they do not in reality have any ratio with one another; sounds cannot be compared, any more than colours can; all that is needed is a little attention to hear this..." [21] (cited in [64]). A further interesting confrontation between Euler and d'Alembert's methodologies concerned the interpretation of the (widely used) dominant seventh chord, namely a chord made out of a root, a major third, a perfect fifth and a minor seventh. Its name comes from the fact that it occurs naturally in the seventh chord built upon the fifth degree - the dominant - of a given major diatonic scale. For example, in the case of the C-major diatonic scale, we get the aggregate G-B-d-f. To d'Alembert, this aggregate was a nice major triad G-B-d to which the dissonant seventh $\mathrm{f}$ is added to unambiguously mark the root tone. Differently said, the dissonance GB-d-f is there just to indicate to the listeners that the piece being played must be in the key of C. Euler discussed this topic in one of the three or four articles that he devoted to music theory in his later years; see "Conjectures sur la raison de quelques dissonances généralement reçues dans la musique" (1766) [27, pp. 508515]. ${ }^{31}$ To briefly review Euler's argument, we start by recalling that he worked with "just intonation", i.e., the system of ratios described by Ptolemy and revived in the sixteenth century by Zarlino to account for the intervals used in polyphonic music. ${ }^{32}$ A portion of this system, covering a perfect twelfth interval, and reduced to a series of whole numbers, is presented in the following table:

\begin{tabular}{|cccccccccccc|}
\hline C & D & E & F & G & A & B & c & d & e & f & g \\
24 & 27 & 30 & 32 & 36 & 40 & 45 & 48 & 54 & 60 & 64 & 72 \\
\hline
\end{tabular}

The seventh chord G-B-d-f is then expressed by the ratios $36: 45: 54: 64$, to which Euler assigns the exponent given by their least common multiple, that is, $2^{6} \cdot 3^{3} \cdot 5=8640$. One recognizes that it is the tone $f$ that troubles this chord. Indeed, if we omit this tone, we obtain the much simpler ratios $4: 5: 6$, whose exponent is $2^{2} \cdot 3 \cdot 5=60$. From this, "it seems that the addition of the note $f$ ruins the harmony of this consonance too much for it to have a place in music. However,

${ }^{31}$ We shall use the English translation [29].

${ }^{32}$ Thus, the system replaced the so-called Pythagorean system - constructed with the perfect fifth $3: 2$ as the only reference interval besides the octave - which remained in use until the late Middle Ages, meeting the needs of the monophonic composition and medieval parallel singing. The subsequent invention of polyphony claimed an increasingly frequent use of intervals of third and sixth, which in the Pythagorean scale are not very consonant $[68 ; 10]$. 
to the ear's judgment, this dissonance is at worst disagreeable and has been used in music with great success. It even seems that musical composition acquires a certain force from it, and without it would bee too smooth and dull. Here we have quite a paradox, where the theory seems to be in contradiction with the practice, to which I will try to give an explanation" [29, §4]. The explanation of Euler is based on the following hypothesis: the organ of hearing is accustomed to taking as simple proportions all proportions that differ very little from it so that the difference is almost imperceptible. For example, in equal temperament, the fifth is expressed by the (irrational) ratio $1: \sqrt[12]{2}$, which hardly differs from the proportion of $2: 3$, but the ear is not bothered too much by this small discrepancy ${ }^{33}$ and in hearing the interval $\mathrm{C}: \mathrm{G}$ one may safely "think" the ratio $2: 3$. More generally, if the proportions expressing a combination of tones are too complicated, the ear will "substitute" a close approximation that is simpler. "Thus the heard proportions are different than the true, and it is from them that we must judge the true harmony and not from the actual numbers" $[29, \S 12]$. According to this assumption, the effect of listening to the dominant seventh chord, which corresponds to the tones $36,45,54,64$, is absolutely the same as listening to the tones $36,45,54,63$, which yield the proportion $4: 5: 6: 7$, whose exponent is $2^{2} \cdot 3 \cdot 5 \cdot 7=420$, about twenty times smaller than the "true" one.

In the perspective of the present work, we can recognize in this construction a way of "saving the phenomena" (the strange acceptance by the ear of a "dissonant" acoustic aggregate) in the same spirit as the ancient exact sciences and therefore the product of the scientific activity of someone who has not yet introjected the division between "mathematics" and "physics" as was vogue in his time. Euler's explanation is often presented as the legacy of an outdated attitude, still attached to a calcified "Pythagorean tradition", whereas d'Alembert would belong to "the right side of History"; see, e.g., [64, p. 289; 8, pp. 139-141]. Indeed, unlike Newtonian mechanics, which, although leaning on outlandish foundations, rather quickly has developed into a true scientific theory, the science of harmonics eventually has fallen apart, in that the subsequent evolution has gradually ousted music theory from the field of direct interest of the majority of scientists. On the one hand, musica theorica has been largely absorbed into musica practica, written by musicians for musicians and mainly focused on the empirical problems of harmony and counterpoint; on the other hand, the theoretical work on the phenomena regarding musical perception was broken up into several branches, with the result that the acoustic problems that traditionally were part of music theory were detached from

${ }^{33}$ Adopting the tempered fifth amounts to using the convergent $\frac{7}{12}$ of (the continued fraction expansion of $\log _{2}\left(\frac{3}{2}\right)$. The next convergent being $\frac{24}{41}$, the error is smaller than $(12 \cdot 41)^{-1}$, that is, about a hundredth of a tone. 
their musical context to become subjects treated separately by the "natural sciences", such as physics ${ }^{34}$, physiology ${ }^{35}$ and psychology ${ }^{36}$. Nowadays the scenario is rather involved, with the coexistence of several tendencies which mostly ignore each other. On the one side, we witness a significant renewal of interest on mathematical modeling of some aspects of music theory, with the search for structural, and to some extent universal, principles in the formation of musical scales; see, e.g., [37] and the references therein. In other directions, the massive advent of new information technologies in the last decades has created an unprecedented situation in which quantitative methods based on the automatic processing of large masses of data invade all fields, including music. Besides the indubitable enrichment with new sound media and new composing techniques (often directly inspired by mathematical constructions such as probability theory or game theory), as far as the new quantitative treatments of musical-acoustical phenomena are concerned - with the related conceptualizations and cultural trends - we have to say that the aims and the methodologies adopted in this context are often placed quite far from those embodied by the exact sciences. This calls for a critical analysis which is still to come.

\section{Acknowledgments}

I wish to thank Lucio Russo for his warm advice and Sara Munday for her careful reading of the manuscript and the many valuable corrections.

\section{References}

[1] T. Aquinas, "Question 32", pp. 60-71 in Summa theologica, I, 2nd ed., vol. 2, Burns Oates \& Washbourne, London, 1921.

[2] Aristotle, "Of the heavenly bodies (continued)", Book 2, pp. 283b-297b in De caelo, edited by J. L. Stocks, Oxford, London, 1922.

[3] Aristotle, The works of Aristotle, vol. 7: Problemata, edited by W. D. Ross, Oxford, London, 1927.

[4] Aristotle, "Physica", pp. i-267b in The works of Aristotle, vol. 2, edited by W. D. Ross, Oxford, London, 1930.

\footnotetext{
${ }^{34}$ Such treatments mainly revolved around investigations on the "nature of sound", in some aspects analogous to that in which the ancient optics, a science of vision, became the study of the "nature of light".

${ }^{35}$ This includes major achievement on the physiological analysis of the tonotopic structure of the inner ear, starting with the important work of Helmholtz [35] until more recent findings on the band structure of the basilar membrane [51], and the study of the auditory cortex by the so-called "neurosciences". By the way, this research can be viewed as providing a physiological basis both to the ancient principle of consonance and to the Eulerian principle mentioned above.

${ }^{36}$ Mainly focused on the empirical study of perceptive and cognitive aspects as well as social and therapeutic applications (see, e.g., [24]).
} 
[5] Aristotle, "The Peripatetic De audibilibus", Chapter 5, pp. 98-109 in Greek musical writings, vol. II: Harmonic and acoustic theory, edited by A. Barker, Cambridge University, 1989.

[6] Aristoxenus, The Harmonics of Aristoxenus, edited by H. S. Macran, Oxford, London, 1902.

[7] V. I. Arnold, "On teaching mathematics", Russ. Math. Surv. 53:1 (1998), 229-236.

[8] P. Bailhache, Une histoire de l'acoustique musicale, CNRS Éditions, Paris, 2001.

[9] C. A. Barbera, "Arithmetic and geometric divisions of the tetrachord", J. Music Theory 21:2 (1977), 294-323.

[10] J. M. Barbour, Tuning and temperament: a historical survey, Michigan State College, East Lansing, 1951.

[11] A. Barker, The science of harmonics in classical Greece, Cambridge University, 2007.

[12] A. M. S. Boethius, Fundamentals of music, edited by C. V. Palisca, Yale University, New Haven, CT, 1989.

[13] W. Burkert, Lore and science in ancient Pythagoreanism, Harvard University, Cambridge, MA, 1972.

[14] N. Cazden, "Pythagoras and Aristoxenos reconciled", J. Am. Music Soc. 11:2-3 (1958), 97105.

[15] T. Christensen, "Music theory as scientific propaganda: the case of d'Alembert's Élémens de musique”, J. Hist. Ideas 50:3 (1989), 409-427.

[16] V. Coelho (editor), Music and science in the age of Galileo, University of Western Ontario Series in Philosophy of Science 51, Kluwer Academic, Dordrecht, 1992.

[17] H. F. Cohen, Quantifying music: the science of music at the first stage of scientific revolution, 1580-1650, University of Western Ontario Series in Philosophy of Science 23, Springer, Dordrecht, 1984.

[18] R. L. Crocker, "Pythagorean mathematics and music", J. Aesthet. Art Critic. 22:2 (1963), 189198.

[19] R. L. Crocker, "Pythagorean mathematics and music, II", J. Aesthet. Art Critic. 22:3 (1964), 325-335.

[20] R. L. Crocker, "Aristoxenus and Greek mathematics", pp. 96-110 in Aspects of medieval and Renaissance music: a birthday offering to Gustave Reese, edited by J. LaRue, Norton, New York, 1966.

[21] J. 1. R. d'Alembert, "Addition au memoire sur la courbe que forme une corde tendüe, mise en vibration”, Hist. Acad. Roy. Sci. Belles Lettres 6 (1750), 355-360.

[22] J. 1. R. d'Alembert, Élémens de musique, théorique et pratique, suivant les principes de M. Rameau, 2nd ed., Charles-Antoine Jombert, Paris, 1759.

[23] J. 1. R. d'Alembert, Preliminary discourse to the encyclopedia of Diderot, edited by R. N. Schwab and W. E. Rex, University of Chicago, 1995.

[24] D. Deutsch (editor), The psychology of music, 3rd ed., Academic, London, 2013.

[25] Euclid, "The Euclidean Sectio canonis", Chapter 8, pp. 190-208 in Greek musical writings, vol. II: Harmonic and acoustic theory, edited by A. Barker, Cambridge University, 1989.

[26] Euclid, "Sectio canonis", pp. 677-703 in Tutte le opere, edited by F. Acerbi, Bompiani, Milano, 2007.

[27] L. Euler, Commentationes physicae ad physicam generalem et ad theoriam soni pertinentes, edited by E. Bernoulli et al., Opera Omnia (3): Opera physica, miscellanea 1, Birkhäuser, Basel, 1926. 
[28] L. Euler, Briefwechsel von Leonhard Euler mit Johann I Bernoulli und Niklaus I Bernoulli, edited by E. A. Fellmann and G. K. Mikhajlov, Opera Omnia (4A): Commercium epistolicum 2, Birkhäuser, Basel, 1998.

[29] L. Euler, "Conjecture sur la raison de quelques dissonances generalement recues dans la musique", translation, 2010, Available at http://eulerarchive.maa.org/docs/translations/E314en.pdf.

[30] M. Frede, “On Galen's epistemology”, pp. 65-86 in Galen: problems and prospects (Cambridge, 1979), edited by V. Nutton, Wellcome Institute for the History of Medicine, London, 1981.

[31] V. Galilei, Discorso intorno alle opere di Gioseffo Zarlino et altri importanti particolari attenenti alla musica, Bollettino Bibliografico Musicale, Milano, 1933.

[32] G. Galilei, Discorsi e dimostrazioni matematiche intorno a due nuove scienze, edited by E. Giusti, Nuova raccolta di classici italiani annotati 12, Einaudi, Turin, 1990.

[33] P. Gozza (editor), Number to sound: the musical way to the Scientific Revolution, Western Ontario Series in Philosophy of Science 64, Kluwer Academic, Dordrecht, 2000.

[34] T. Heath (editor), Aristarchus of Samos, the ancient Copernicus: a history of Greek astronomy to Aristarchus, together with Aristarchus's treatise on the sizes and distances of the sun and moon, Oxford, London, 1913.

[35] H. L. F. Helmholtz, On the sensations of tone as a physiological basis for the theory of music, 2nd ed., edited by A. J. Ellis, Dover, New York, 1954.

[36] C. A. Huffman, Archytas of Tarentum: Pythagorean, philosopher and mathematician king, Cambridge University, 2005.

[37] S. Isola, "Su alcuni rapporti tra matematica e scale musicali", Mat. nella Soc. e nella Cult. Rivista dell'Unione Mat. Italiana (I) 1:1 (2016), 31-50.

[38] J. Kepler, "Epitome astronomiae Copernicanae", Liber Quartus, Pars Prima, pp. 256-289 in Gesammelte Werke, vol. 7, edited by W. von Dyck and M. Caspar, Beck, Munich, 1953.

[39] J. Kepler, The harmony of the world, edited by E. J. Aiton et al., Memoirs of the American Philosophical Society 209, American Philosophical Society, Philadelphia, 1997.

[40] G. Leopardi, Pensieri di varia filosofia e di bella letteratura, vol. 5, Successori Le Monnier, Florence, 1900.

[41] A. A. Long, "Ptolemy On the Criterion: an epistemology for the practicing scientists", pp. 176207 in The question of "eclecticism": studies in later Greek philosophy, edited by J. M. Dillon and A. A. Long, University of California, Berkeley, California, 1988.

[42] A. A. T. Macrobius, Commentary on the dream of Scipio, edited by W. H. Stahl, Columbia University, New York, 1952.

[43] J. E. McGuire and P. M. Rattansi, "Newton and the "Pipes of Pan"”, Notes Rec. Roy. Soc. 21:2 (1966), 108-143.

[44] M. Mersenne, Harmonie universelle, contenant la théorie et la pratique de la musique, Cramoisy, Paris, 1636.

[45] I. Newton, The mathematical principles of natural philosophy, vol. 2, edited by A. Motte, Motte, London, 1729.

[46] I. Newton and R. Cotes, Correspondence of Sir Isaac Newton and Professor Cotes including letters of other eminent men, edited by J. Edleston, Parker, London, 1850.

[47] Nicomachus, Manual of harmonics of Nicomachus the Pythagorean, edited by F. R. Levin, Phanes, Grand Rapids, MI, 1993.

[48] C. V. Palisca, "Scientific empiricism in musical thought", pp. 91-137 in Seventeenth century science and the arts, edited by H. H. Rhys, Princeton University, 1961. 
[49] Plato, The Timaeus of Plato, edited by R. D. Archer-Hind, Macmillan, London, 1888.

[50] Plato, "Republic", pp. i-338 in The dialogues of Plato, 3rd ed., vol. 3, edited by B. Jowett, Oxford, London, 1892.

[51] R. Plomp and W. J. M. Levelt, "Tonal consonance and critical bandwidth", J. Acoust. Soc. Am. 38:4 (1965), 548-560.

[52] Porphyrios, Kommentar zur Harmonielehre des Ptolemaios, edited by I. Düring, Göteborgs Högskolas Årsskrift 38:2, Elanders Boktryckeri Aktiebolag, Göteborg, 1932.

[53] Ptolemy, Ptolemy's Almagest, 2nd ed., edited by G. J. Toomer, Princeton University, 1998.

[54] Ptolemy, Ptolemy Harmonics: translation and commentary, edited by J. Solomon, Brill, Leiden, 1999.

[55] J.-P. Rameau, Traité de l'harmonie réduite à ses principes naturels, Ballard, Paris, 1722.

[56] L. Russo, "Appunti per una storia dei concetti di 'matematica' e 'fisica", Punti Critici 1 (1999), $25-56$.

[57] L. Russo, "L'articolo di Arnold e i rapporti tra storia della cultura e scienza esatta", Punti Critici 3 (1999), 87-98.

[58] L. Russo, The forgotten revolution: how science was born in $300 \mathrm{BC}$ and why it had to be reborn, edited by S. Levy, Springer, 2004.

[59] L. Russo, L'America dimenticata, Mondadori Università, Milan, 2013.

[60] L. Russo, Stelle, atomi e velieri: percorsi di storia della scienza, Mondadori Università, Milan, 2015.

[61] J. L. Saunders (editor), Greek and Roman philosophy after Aristotle, Free Press, New York, 1966.

[62] S. Stevin, "On the theory of the art of singing", pp. 413-464 in The principal works of Simon Stevin, vol. 5: Engineering, music and civic life, edited by R. J. Forbes et al., C. V. Swets \& Zeitlinger, Amsterdam, 1966.

[63] Theophrastus, Theophrastus of Eresus: sources for his life, writings, thought and influence, edited by W. W. Fortenbaugh et al., Brill, Leiden, 1992.

[64] T. M. Tonietti, "Is music relevant for the history of science?", pp. 281-291 in The application of mathematics to the sciences of nature: critical moments and aspects, edited by P. Cerrai et al., Kluwer Academic, Dordrecht, 2002.

[65] Voltaire, A philosophical dictionary, vol. VI, 2nd ed., Hunt, London, 1824.

[66] D. P. Walker, Studies in musical science in the late Renaissance, Studies of the Warburg Institute 37, University of London, 1978.

[67] E. P. Wigner, "The unreasonable effectiveness of mathematics in the natural sciences", Commun. Pur. Appl. Math. 13:1 (1960), 1-14.

[68] G. Zarlino, Istitutioni harmoniche, de'Franceschi, Venice, 1558.

[69] G. Zarlino, Sopplimenti musicali, de'Franceschi, Venice, 1588.

Received 1 Apr 2016. Accepted 30 Jun 2016.

STEFANO Isola: stefano.isola@gmail.com

Scuola di Scienze e Tecnologie, Università di Camerino, I-62032 Camerino, Italy 


\title{
FROM QUANTUM TO CLASSICAL WORLD: EMERGENCE OF TRAJECTORIES IN A QUANTUM SYSTEM
}

\author{
Rodolfo Figari AND Alessandro TetA
}

\section{Dedicated to Lucio}

\begin{abstract}
This note deals with models of quantum systems where the emergence of a classical behavior can be concretely analyzed. We first briefly review some well known difficulties arising in the classical limit of quantum mechanics according to the Copenhagen interpretation. Then we discuss the seminal contribution by Mott (1929) on the tracks observed in a cloud chamber, where the problem can be approached in a particularly transparent way. Finally, we propose a model Hamiltonian, with interaction described by spin dependent point interactions, where Mott's analysis can be rephrased and the result can be rigorously formulated.
\end{abstract}

\section{Introduction}

Quantum mechanics is a theory of extraordinary success meant to describe the behavior of microscopic systems, i.e., systems with a typical action of the order of the Planck's constant $\hbar$. On the other hand, in the nonrelativistic regime, quantum mechanics is expected to be a universal theory and therefore should apply to the macroscopic world.

Its final formulation is due to Heisenberg, Born, Jordan [Born and Jordan 1925], Schrödinger [1982], Born [1926] and, for the mathematical aspects involved, to von Neumann [1932]. In fact, the quantum mechanical description of physical phenomena is rather abstract and counterintuitive, being based on the evolution of the wave function, i.e., a complex probability amplitude defined on the configuration space of the system with no direct physical meaning. This is in contrast with Newtonian mechanics, where physical objects are described through their positions and velocities, evolving in time in the phase space. The radically different approaches are the origin of some conceptual difficulties encountered when one tries to reconcile the two descriptions in some concrete physical situations. The aim of the present

\section{Communicated by Raffaele Esposito.}

PACS2010: 03.65.-w.

Keywords: environment induced decoherence, cloud chamber, transition quantum to classical. 
note is to discuss this delicate conceptual point through the analysis of concrete physical models. More precisely, the paper is organized as follows.

In this section we briefly review the basic rules of quantum mechanics in the realm of the so-called Copenhagen interpretation.

In Section 2 we analyze a specific case study where the problem of the emergence of a classical behavior in a quantum system appears in a clear way. In particular, we discuss the problem proposed by Mott [1929] concerning the emergence of a classical trajectory of a quantum particle in a cloud chamber.

In Section 3 we illustrate a model, based on a spin dependent point interaction Hamiltonian, where Mott's result can be rephrased in a more explicit and rigorous way.

Let us summarize the basic assumptions postulated for a system of $n$ quantum particles in $\mathbb{R}^{3}$. We avoid generality and technical difficulties and in particular we neglect the specific requests needed to describe systems of identical particles.

(1) State: The state of the system at time $t$ is described by the wave function $\psi_{t}\left(x_{1}, \ldots, x_{n}\right), x_{j} \in \mathbb{R}^{3}$, which is an element of the Hilbert space $L^{2}\left(\mathbb{R}^{3 n}\right)$ with $\left\|\psi_{t}\right\|=1$.

(2) Evolution of the state: Given the initial state $\psi_{0}$, the state at time $t$ is the solution of the Schrödinger equation

$$
i \hbar \frac{\partial \psi_{t}}{\partial t}=-\sum_{j=1}^{n} \frac{\hbar^{2}}{2 m_{j}} \Delta_{j} \psi_{t}+V\left(x_{1}, \ldots, x_{n}\right) \psi_{t},
$$

with initial datum $\psi_{0}$, where $m_{j}$ is the mass of the $j$-th particle, $\Delta_{j}$ denotes the Laplace operator relative to the coordinates of the $j$-th particle and $V$ is the interaction potential.

(3) Observables: An observable $\mathcal{A}$ relative to the system is represented by a selfadjoint operator $A$ in $L^{2}\left(\mathbb{R}^{3 n}\right)$. In a system made of a single quantum particle, simple examples of quantum observables are position and momentum. The position is represented by $\hat{x}_{k}$, defined as the multiplication operator by $x_{k}, k=1,2,3$, where $x_{k}$ denotes the $k$-th component of the position of the particle. Analogously, the momentum is represented by the differential operator

$$
\hat{p}_{k}=-i \hbar \frac{\partial}{\partial x_{k}}, \quad k=1,2,3 .
$$

One can easily check that the two observables do not commute and in fact, at least formally, they satisfy the Heisenberg commutation relations $\left[\hat{x}_{k}, \hat{p}_{l}\right]=i \hbar \delta_{k l} I$, where $I$ denotes the identity operator.

(4) Predictions: The predictions of the theory are given by Born's rule and, in general, are of probabilistic nature. In the special case of the position observable 
relative to a system made of one quantum particle, Born's rule reduces to

$$
\mathcal{P}(x \in \Omega ; \psi)=\int_{\Omega} \mathrm{d} x|\psi(x)|^{2},
$$

where the left hand side denotes the probability that the position of the particle described by the state $\psi$ is found in a set $\Omega \subset \mathbb{R}^{3}$. The prescription can be easily extended to the case of other observables making use of the spectral theorem for selfadjoint operators.

We list here few comments.

(i) As we already mentioned, the predictions of the theory are in excellent agreement with experiments. Furthermore, quantum mechanics is formulated as a universal theory (in the nonrelativistic regime) and therefore it can be used to describe both micro- and macrosystems.

(ii) Except in some special cases, Born's rule gives only probabilistic predictions. In particular, formula (1-2) means that the theory can only predict the statistical distribution of the detected positions in a large number of experiments made in identical conditions. We notice that a quantum particle, when a position measurement is performed in a single experiment, always appears localized in a well defined position which, in general, cannot be predicted by the theory. It should be emphasized that quantum mechanics is the first fundamental theory which explicitly renounces to a deterministic description of the physical phenomena.

(iii) For a single observable $\mathcal{A}$ represented by the operator $A$, let $\Delta_{\psi} A$ denote the mean square deviation of the statistical distribution of the possible values of $\mathcal{A}$ in the state $\psi$. It is always possible to find a state $\psi$ such that $\Delta_{\psi} A$ is arbitrarily small. This means that the values of the observable $\mathcal{A}$ in the state $\psi$ can be predicted with arbitrary accuracy. On the other hand, the noncommutative character of the algebra of observables implies that one cannot predict, with arbitrary accuracy, the value of all the observables relative to a given system. In particular, for a quantum particle in the state $\psi$ one can prove the Heisenberg uncertainty relations

$$
\Delta_{\psi} \hat{x}_{k} \Delta_{\psi} \hat{p}_{l} \geq \hbar / 2 \delta_{k l}
$$

It is evident that this inequality makes the notion of trajectory for a quantum particle problematic.

(iv) A crucial point of the theory is the linearity. This means that if $\psi_{1}(x)$ and $\psi_{2}(x)$ are two states then also the sum $\psi_{1}(x)+\psi_{2}(x)$, suitably normalized, is a possible state (superposition principle) and this property is preserved by the (linear) evolution. This apparently trivial fact has major physical consequences, because the predictions (see (1-2)) are given by a quadratic expression with respect to the 
state. In particular the probability density for the position is

$$
\left|\psi_{1}(x)+\psi_{2}(x)\right|^{2}=\left|\psi_{1}(x)\right|^{2}+\left|\psi_{2}(x)\right|^{2}+2 \operatorname{Re}\left(\psi_{1}(x) \overline{\psi_{2}(x)}\right) .
$$

From formula (1-4) it is clear that the situation described by $\psi_{1}(x)+\psi_{2}(x)$ cannot be considered in any sense as the "sum" of the situations described by $\psi_{1}(x)$ and $\psi_{2}(x)$ separately. In particular the last term in (1-4) is responsible for the appearance of interference effects, typical of waves, in the statistical distribution of the detected positions in a large number of identical experiments. Such effects can be directly observed in the so-called two-slit experiment.

We notice that a completely different situation occurs when we know that the system is in the state $\psi_{1}(x)$ with probability $p_{1}$ and in the state $\psi_{2}(x)$ with probability $p_{2}$, where $p_{1}+p_{2}=1$. In such a case the probability involved is due to our ignorance about the state and the system is described by a so-called classical statistical mixture of the two pure states $\psi_{1}(x)$ and $\psi_{2}(x)$.

(v) Another important aspect is the fact that the wave function is a "wave" in the configuration space of the system rather than in the three dimensional physical space. This implies the occurrence of "entanglement" for systems composed by more than one particle. An entangled state is a state that cannot be factorized in a product of one-particle states. At a kinematical level, this means that if a system is described by an entangled state $\psi\left(x_{1}, \ldots, x_{n}\right)$, it is not possible to associate a definite (pure) state to each subsystem. The situation is again radically different from the classical case and it is the origin of the "nonlocal effects" which can be produced on a subsystem $S_{1}$ acting on another spatially separated subsystem $S_{2}$.

We emphasize that there is a general and complete agreement in the physics community on the validity of the above rules, in the minimal formulation we have given. They are sufficient to give an accurate description of the physical phenomena and they can be considered the basis of the "pragmatic" view of the majority of the physicists working with quantum mechanics and without a specific interest in foundational problems.

On the other hand, one can strive for a better understanding of various ideas associated with the rules, such as the nature of the notion of probability arising in the theory, the meaning of an object's physical properties, the role of the measurement process and so on.

When one attempts to give an answer to such questions one enters the field of the so-called interpretational problem. Here, since the birth of quantum theory, many different views have been proposed that have stimulated a long and intense epistemological debate which is still active. We are not going to discuss here the different opinions and we limit ourselves to detail some aspect of the so-called standard or Copenhagen interpretation which, more or less consciously, is the point 
of view accepted by the majority of physicists. The aim is to highlight a conceptual difficulty arising in such an interpretation when one describes the measurement process or, more generally, the connection between the quantum and the classical description of the world.

The basic assumption in the Copenhagen interpretation is the completeness of the wave function. This means that the maximal information about a specific quantum system is encoded in its wave function. Therefore, the probabilistic predictions have an ontological character, i.e., they do not depend on our ignorance about some property of the system. If the system is in a state $\psi$ such that we cannot predict the value of an observable $\mathcal{A}$ with probability one, then the system does not have a definite, even if unknown, value of $\mathcal{A}$.

A consequence of this assumption makes the role of observation problematic. We recall that in classical physics a measurement of an observable is an innocuous process that reveals the value of the observable (which was already possessed by the system before the measurement). In the quantum case, and according to the Copenhagen interpretation, the situation is different. Let us assume that the system is in a state $\psi$ such that the observable $\mathcal{A}$ does not have a definite value. Assume that we perform a measurement of $\mathcal{A}$ and find a value $a_{0}$. If, after a very short time, we repeat the measurement of $\mathcal{A}$ we can reasonably expect to obtain the same value $a_{0}$. In other words, immediately after the first measurement we can predict with probability one the value $a_{0}$ for $\mathcal{A}$. This means that the measurement process is a strange mechanism that contributes to assign the value $a_{0}$ to the observable and it necessarily produces an instantaneous transition of the state $\psi \rightarrow \psi_{0}$, where $\psi_{0}$ is a state in which $\mathcal{A}$ has the definite value $a_{0}$ (typically an eigenvector of the self-adjoint operator $A$ representing $\mathcal{A}$ ).

The instantaneous (stochastic and nonlinear) transition $\psi \rightarrow \psi_{0}$ is called wave packet collapse.

Bohr [1928] explained the transition claiming that a measurement apparatus is a classical object and therefore it always possesses a definite value of its observables; he assumed that the interaction between the classical apparatus and the quantum system determines the collapse, inducing the transition of the quantum system state.

Bohr's explanation can be criticized in many respects. We simply mention the following observations.

It is not clear where the border line between the measurement apparatus (showing a classical behavior) and the system (showing a quantum behavior) should be fixed. The problem is usually solved pragmatically for each specific situation, but, at the conceptual level, there is ambiguity.

An even more relevant point is the fact that it is not explained why a measurement apparatus, despite being made of atoms, cannot be described by quantum 
mechanics and it is a priori considered as a classical object with well-defined classical properties.

On the other hand, taking a different point of view, one can insist to describe also the measurement apparatus as a quantum system using the Schrödinger equation. As a matter of fact, in this case one can arrive at the unpleasant situation in which the apparatus' pointer has no definite position. This fact, as clearly explained by von Neumann [1932], happens when the system is in a superposition state of two eigenstates of the observable to be measured. Due to the linearity of the evolution and to the interaction system-apparatus, such a superposition state is transferred to the pointer which, as a consequence, has no definite position after the measurement.

It should be underlined once again that the above difficulty arises only at a conceptual level. Roughly speaking, in concrete physical models describing the system-apparatus interaction the "difference" between the situation "the pointer has no definite position" and the situation "the pointer has a definite, even if possibly unknown, position" is so small that it is practically undetectable.

To summarize the situation, J. Bell [1987] wrote that the quantum description entirely based on the Schrödinger equation works perfectly well for all practical purposes (a phrase used often enough that he abbreviated it as FAPP), but he also insisted that a conceptual inconsistency in the Copenhagen interpretation of the formalism related to the measurement problem undoubtedly exists.

\section{Mott's analysis of the cloud chamber problem}

Here we briefly recall the basic principles of a cloud chamber [Leone and Robotti 2004; Gupta and Ghosh 1946] and the first theoretical explanation of the observed tracks proposed by Mott [1929]. For a more detailed historical analysis we refer to [Figari and Teta 2013; 2014].

The air contained in the cloud chamber, saturated with water vapor, is brought into a supersaturated state by means of a fast expansion that suddenly lowers its temperature. An $\alpha$-particle, released by a radioactive source in the center of the chamber, interacts with the atoms of the gas, inducing ionization. The ionized atoms then act as condensation nuclei, giving rise to the formation of small drops of water. The sequences of these drops form visible tracks which are naturally interpreted as magnifications of the $\alpha$-particle "trajectories". In fact, they are accurately characterized as trajectories of a charged classical particle (relativistic or nonrelativistic according to the initial particle velocity) in a classical electromagnetic field. And, in particular, they are straight lines whenever no electromagnetic field is present.

The first quantum theoretical analysis of the radioactive decay of a nucleus with the emission of an $\alpha$-particle was given by Gamow [1928] and by Condon and 
Gurney [1928]. The authors concluded that the initial state of the emitted $\alpha$-particle has to be described by a spherical wave, with center in the nucleus and isotropically propagating in space.

It was immediately noticed that the spherical shape of the initial state was apparently in contrast with the observed tracks in the cloud chamber. In particular, in the words of Mott [1929]: "it is a little difficult to picture how it is that an outgoing spherical wave can produce a straight track; we think intuitively that it should ionise atoms at random throughout space." In an attempt to obtain a satisfactory theoretical explanation of the tracks, it was realized that a crucial role must be played by an act of measurement responsible for the collapse of the spherical wave. This requires an establishment as to where a line of separation between the quantum system and the measuring device must be fixed. In this sense, the debate on the cloud chamber can be considered as the prototype of any further discussion about the measurement problem and the appearance of a classical behavior in a quantum system in the context of the Copenhagen interpretation of quantum mechanics.

In a first possible approach, the $\alpha$-particle is the quantum system under consideration and the gas of the chamber acts as the measurement device by which we observe the particle. Therefore, an atom of the gas "measures" the position of the $\alpha$-particle which must be considered a particle immediately after the disintegration process, since at that moment the gas (i.e., the device) reduces the initial spherical wave to a narrow wave packet with a definite momentum.

Another approach consists of considering the $\alpha$-particle and the gas as the whole quantum system under consideration. In this case, one observes the ionized atoms and the wave function $\psi$ of the system should provide the ionization probability.

According to this second point of view, the above mentioned intuitive difficulty can be overcome, since it arises from our erroneous "tendency to picture the wave as existing in ordinary three dimensional space, whereas we are really dealing with wave functions in multispace formed by the coordinates both of the $\alpha$-particle and of every atom in the Wilson chamber" [Mott 1929].

In his paper Mott proposes a simplified model to obtain a theoretical explanation following the second point of view. He considers a three particle system consisting of the $\alpha$-particle, initially described by a spherical wave centered at the origin, and two hydrogen atoms, initially in their ground state, whose nuclei are fixed at the positions $\boldsymbol{a}_{1}, \boldsymbol{a}_{2}$, with $\left|\boldsymbol{a}_{1}\right|<\left|\boldsymbol{a}_{2}\right|$. The $\alpha$-particle interacts with the electrons of the atoms while the repulsion between the two electrons is neglected. Moreover, he introduces some assumptions on the physical parameters characterizing the model: first, the observation point is far away from the origin and the atoms; secondly, the $\alpha$-particle has a high momentum and the collision with the atoms is almost elastic. Then he looks for a solution to the corresponding time-independent Schrödinger equation. Using perturbation theory up to second order and standard stationary 
phase arguments, he computes the probability that both atoms are excited. His important result can be formulated as follows: the probability that both atoms are excited is (nearly) zero unless $\boldsymbol{a}_{1}, \boldsymbol{a}_{2}$ and the origin lie on the same straight line.

Let us comment on the meaning of this statement.

Since the observed phenomenon in the experiment is the excitation of the atoms, Mott's result says that one can only observe straight tracks. Therefore, it provides a clear physical explanation of the straight tracks observed in the chamber entirely based on the Schrödinger equation, without any use of the collapse postulate. On the contrary, the explanation given following the first approach appears physically obscure, since it is not a priori clear how an atom (i.e., a microscopic system) can act as measurement device.

It should be stressed that the above result holds under specific physical assumptions on the parameters of the model. This means that the observed behavior of the $\alpha$-particle in a cloud chamber is far from being universal.

It is worth mentioning that Mott's analysis is the first example of an approach typical of the modern theory of environment-induced decoherence [Blanchard et al. 2000; Joos et al. 2003; Hornberger 2009; Adami et al. 2004; 2006]. In fact, the classical behavior (the trajectory) of the system ( $\alpha$-particle) emerges as an effect of the interaction with the environment (vapor atoms in the chamber) under suitable assumptions on the physical parameters of the model.

This approach is particularly interesting since it is based on a quantitative description of the phenomenon, with a possible explicit control of the approximations. This is a crucial aspect in a detailed comparison between theory and experiment, which certainly would not be possible following an approach based on the idea of wave packet reduction.

It should also be noted that from the mathematical point of view some aspects of Mott's analysis can be improved. For instance, the stationary phase theorem is used without an accurate control of the conditions of applicability. Another unsatisfactory aspect is the use of the stationary Schrödinger equation, which prevents a time-dependent description of the evolution of the whole system. The use of the time-dependent Schrödinger equation, with a clear definition of the initial state and an explicit description of the successive interactions of the $\alpha$-particle with the first and the second atom, is required to make both the analysis and the result more transparent. We refer to [Dell'Antonio et al. 2008; 2010; Finco and Teta 2011; Recchia and Teta 2014; Teta 2010] for results in this direction.

\section{A model of cloud chamber}

In this section we present a model of a quantum environment inside which a quantum particle evolves. The environment should mimic the supersaturated vapor of 
a cloud chamber where ionization takes place when a fast and massive particle is passing by. The model we propose consists of a quantum particle interacting via point interactions with localized finite-dimensional quantum subsystems (twolevel atoms or spins). Details of the construction of the Hamiltonian of the whole system can be found in [Cacciapuoti et al. 2007] (see also [Albeverio et al. 2005] for a comprehensive analysis of point interactions Hamiltonians).

We consider $N$ spins placed in fixed positions $\boldsymbol{y}_{1}, \boldsymbol{y}_{2}, \ldots, \boldsymbol{y}_{N}$. The state space of the system is the Hilbert space

$$
\mathcal{H}=L^{2}\left(\mathbb{R}^{3}\right) \otimes \mathbb{S}_{N} \equiv L^{2}\left(\mathbb{R}^{3}\right) \otimes\left(\mathbb{C}^{2}\right)_{1} \otimes \cdots \otimes\left(\mathbb{C}^{2}\right)_{N},
$$

where $\left(\mathbb{C}^{2}\right)_{j}$ denotes the $j$-th copy of $\mathbb{C}^{2}$ in which the $j$-th spin state is represented (note that the particle itself is assumed to have no spin). To describe the $j$-th spin state in $\left(\mathbb{C}^{2}\right)_{j}$ we take the basis made up of the first Pauli matrix eigenvectors

$$
\hat{\sigma}_{j}^{(1)} \chi_{\sigma_{j}}=\sigma_{j} \chi_{\sigma_{j}}, \quad \hat{\sigma}_{j}^{(1)}=\left(\begin{array}{rr}
1 & 0 \\
0 & -1
\end{array}\right), \quad \sigma_{j}= \pm 1 .
$$

Any state in $\mathcal{H}$ can be written according to the following decomposition

$$
\begin{gathered}
\Psi \in \mathcal{H}, \quad \Psi=\sum_{\sigma} \psi_{\sigma} \otimes \mathcal{X}_{\sigma}, \\
\sigma=\left\{\sigma_{1}, \sigma_{2}, \ldots, \sigma_{N}\right\}, \quad \mathcal{X}_{\sigma}=\chi_{\sigma_{1}} \otimes \chi_{\sigma_{2}} \otimes \cdots \otimes \chi_{\sigma_{N}},
\end{gathered}
$$

where $\psi_{\sigma} \in L^{2}\left(\mathbb{R}^{3}\right)$. Using such a decomposition, the scalar product in $\mathcal{H}$ reads

$$
\langle\Psi, \Phi\rangle=\sum_{\sigma}\left(\psi_{\sigma}, \phi_{\sigma}\right),
$$

where $(\cdot, \cdot)$ is the scalar product in $L^{2}\left(\mathbb{R}^{3}\right)$ and the induced norm will be denoted by $\|\cdot\|$. With this notation, the normalization condition in $\mathcal{H}$ reads

$$
\|\Psi\|_{\mathcal{H}}^{2}=\langle\Psi, \Psi\rangle=\sum_{\sigma}\left\|\psi_{\sigma}\right\|^{2}=1
$$

The Hamiltonian $H_{0}$ acting in $\mathcal{H}$ generating the free dynamics of the system reads

$$
\begin{aligned}
& D\left(H_{0}\right)=H^{2}\left(\mathbb{R}^{3}\right) \otimes \mathbb{S}_{N} \equiv H^{2}\left(\mathbb{R}^{3}\right) \otimes\left(\mathbb{C}^{2}\right)_{1} \otimes \cdots \otimes\left(\mathbb{C}^{2}\right)_{N}, \\
& H_{0}=-\frac{\hbar^{2}}{2 m} \Delta \otimes \mathbb{\rrbracket}_{\mathbb{S}_{N}}+\sum_{j=1}^{N} \rrbracket_{L^{2}} \otimes \alpha_{j} \mathbf{S}_{j}, \quad \alpha_{j} \in \mathbb{R}^{+}, \\
& \rrbracket_{\mathbb{S}_{N}}=\rrbracket_{\left(\mathbb{C}^{2}\right)_{1}} \otimes \cdots \otimes \rrbracket_{\left(\mathbb{C}^{2}\right)_{N}},
\end{aligned}
$$

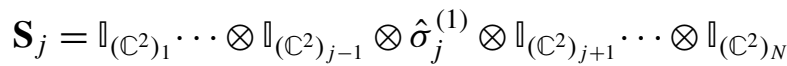

In (3-7), $\square_{\left(\mathbb{C}^{2}\right)_{j}}$ and $\square_{L^{2}}$ denote the identity operators in $\mathbb{C}^{2}$ and in $L^{2}\left(\mathbb{R}^{3}\right)$ respectively. The system energy is the sum of the kinetic energy of the particle and the energy of the spins. The $j$-th spin has an energy $\pm \alpha_{j}$ respectively in the states $\chi_{ \pm 1}$. In 
order to simplify notation, we will take in the following $\hbar=1,2 m=1$. The action of $H_{0}$ on functions of its domain is

$$
H_{0} \Psi=\sum_{\sigma}(-\Delta+\boldsymbol{\alpha} \cdot \boldsymbol{\sigma}) \psi_{\sigma} \otimes \mathcal{X}_{\sigma}
$$

where $\boldsymbol{\alpha}=\left(\alpha_{1}, \ldots, \alpha_{N}\right), \boldsymbol{\alpha} \cdot \boldsymbol{\sigma}=\alpha_{1} \sigma_{1}+\cdots+\alpha_{N} \sigma_{N}$.

The spectrum of $H_{0}$ is easily derived from the spectrum of the free Laplacian

$$
\sigma_{\mathrm{p}}\left(H_{0}\right)=\varnothing, \quad \sigma_{\mathrm{ess}}\left(H_{0}\right)=\sigma_{\mathrm{ac}}\left(H_{0}\right)=[\mu, \infty), \quad \mu=\min _{\boldsymbol{\sigma}}(\boldsymbol{\alpha} \cdot \boldsymbol{\sigma}) .
$$

Under the unitary group $e^{-i t H_{0}}$, the particle evolves freely with no interaction with the spins. The evolution of the $j$-th spin first component eigenstate corresponding to the eigenvalue \pm 1 is given by $e^{\mp i \alpha_{j} t} \chi_{ \pm 1}$. In fact, $e^{-i t H_{0}}$ is explicitly known and the solution of the Cauchy problem for the Schrödinger equation with initial datum $\Psi^{0}=\sum_{\sigma} \psi_{\sigma}^{0} \otimes \mathcal{X}_{\sigma} \in \mathcal{H}$ is

$$
\Psi^{t}=e^{-i t H_{0}} \Psi^{0}=\sum_{\sigma}\left(U^{t} \psi_{\sigma}^{0}\right) \otimes e^{-i \sigma \cdot \alpha t} \mathcal{X}_{\sigma},
$$

where

$$
\left(U^{t} f\right)(\boldsymbol{x})=\int_{\mathbb{R}^{3}} \mathrm{~d} \boldsymbol{x}^{\prime} \frac{e^{i\left|\boldsymbol{x}-\boldsymbol{x}^{\prime}\right|^{2} /(2 t)}}{(4 \pi i t)^{3 / 2}} f\left(\boldsymbol{x}^{\prime}\right)
$$

In order to define a Hamiltonian with spin-dependent point interactions placed at $\boldsymbol{y}_{1}, \ldots, \boldsymbol{y}_{N}$, one can start from the following consideration. Since the interaction must be nontrivial only at $\boldsymbol{y}_{1}, \ldots, \boldsymbol{y}_{N}$, such a Hamiltonian should act as the free Hamiltonian on wave functions vanishing at the points $\boldsymbol{y}_{1}, \ldots, \boldsymbol{y}_{N}$. This suggests considering the operator $\tilde{H}_{0}$ defined as the restriction of $H_{0}$ to the set of states whose spatial support does not contain $\boldsymbol{y}_{1}, \ldots, \boldsymbol{y}_{N}$ and to define the spin-dependent point interaction Hamiltonian any (nontrivial) self-adjoint extension of $\tilde{H}_{0}$.

In the following theorem we summarize the definition and properties of the Hamiltonians in the subfamily of the "local" self-adjoint extensions, characterized by local singular boundary conditions on each point $\boldsymbol{y}_{j}$. In order to state the theorem we introduce the notation

$$
\Phi_{j \sigma}^{z}=G^{z-\boldsymbol{\alpha} \cdot \boldsymbol{\sigma}}\left(\cdot-\boldsymbol{y}_{j}\right) \otimes \mathcal{X}_{\sigma}, \quad z \in \mathbb{C} \backslash \mathbb{R}
$$

where

$$
G^{w}(\boldsymbol{x})=\frac{e^{i \sqrt{w}|\boldsymbol{x}|}}{4 \pi|\boldsymbol{x}|}, \quad w \in \mathbb{C} \backslash \mathbb{R}^{+}, \quad \operatorname{Im} \sqrt{w}>0
$$

Then we have 
Theorem 3.1 [Cacciapuoti et al. 2007]. Define the operator $H^{A}$ with domain

$$
\begin{aligned}
D\left(H^{A}\right)=\left\{\Psi=\sum_{\sigma} \psi_{\sigma} \otimes \mathcal{X}_{\sigma} \in \mathcal{H} \mid \Psi=\Psi^{z}+\sum_{j \sigma} q_{j \sigma} \Phi_{j \sigma}^{z} ;\right. \\
\left.\Psi^{z} \in D\left(H_{0}\right), \operatorname{Im} \sqrt{z}>0, \sum_{j^{\prime} \boldsymbol{\sigma}^{\prime}} A_{j \sigma, j^{\prime} \boldsymbol{\sigma}^{\prime}} q_{j^{\prime} \boldsymbol{\sigma}^{\prime}}=f_{j \sigma}\right\},
\end{aligned}
$$

and action

$$
H^{A} \Psi=H_{0} \Psi^{z}+z \sum_{j, \sigma} q_{j \sigma} \Phi_{j \sigma}^{z}, \quad \Psi \in D\left(H^{A}\right),
$$

where

$$
\begin{aligned}
q_{j \sigma} & =\lim _{\left|\boldsymbol{x}-\boldsymbol{y}_{j}\right| \rightarrow 0} 4 \pi\left|\boldsymbol{x}-\boldsymbol{y}_{j}\right| \psi_{\boldsymbol{\sigma}}(\boldsymbol{x}), \\
f_{j \sigma} & =\lim _{\left|\boldsymbol{x}-\boldsymbol{y}_{j}\right| \rightarrow 0}\left[\psi_{\boldsymbol{\sigma}}(\boldsymbol{x})-\frac{q_{j \boldsymbol{\sigma}}}{4 \pi\left|\boldsymbol{x}-\boldsymbol{y}_{j}\right|}\right], \\
A_{j \boldsymbol{\sigma}, j^{\prime} \boldsymbol{\sigma}^{\prime}} & =0 \text { if } j \neq j^{\prime}, \\
A_{j \boldsymbol{\sigma}, j \boldsymbol{\sigma}^{\prime}} & =0 \text { if } \sigma_{k} \neq \sigma_{k}^{\prime} \text { for some } k \neq j, \\
A_{j \boldsymbol{\sigma}, j \boldsymbol{\sigma}^{\prime}} & =a_{j \sigma_{j}, j \sigma_{j}^{\prime}} \text { otherwise, with } \\
a_{j \sigma_{j}, j \sigma_{j}^{\prime}} & =\beta \delta_{\sigma_{j}, \sigma_{j}^{\prime}}+\sigma_{j} i \rho\left(1-\delta_{\sigma_{j}, \sigma_{j}^{\prime}}\right) \text { with } \beta, \rho \in \mathbb{R} .
\end{aligned}
$$

Then $H^{A}$ is self-adjoint and its resolvent, $R^{A}(z)=\left(H^{A}-z\right)^{-1}$, is the finite rank perturbation of the free resolvent $R(z)$ given by

$$
R^{A}(z)=R(z)+\sum_{j \boldsymbol{\sigma}, j^{\prime} \boldsymbol{\sigma}^{\prime}}\left(\left(\Gamma_{\beta, \rho}(z)\right)^{-1}\right)_{j \boldsymbol{\sigma}, j^{\prime} \boldsymbol{\sigma}^{\prime}}\left\langle\Phi_{j^{\prime} \boldsymbol{\sigma}^{\prime}}^{\bar{z}}, \cdot\right\rangle \Phi_{j \boldsymbol{\sigma}}^{z}, \quad z \in \rho\left(H^{A}\right),
$$

where $\Gamma_{\beta, \rho}(z)$ is the $N 2^{N} \times N 2^{N}$ matrix defined as

$$
\Gamma_{\beta, \rho}(z)=\Gamma(z)+A,
$$

with

$$
\begin{array}{rlrl}
\Gamma(z)_{j \sigma, j^{\prime} \boldsymbol{\sigma}^{\prime}} & =0, & & \text { if } \boldsymbol{\sigma} \neq \boldsymbol{\sigma}^{\prime}, \\
\Gamma(z)_{j \sigma, j \boldsymbol{\sigma}} & =\sqrt{z-\boldsymbol{\alpha} \cdot \boldsymbol{\sigma}} /(4 \pi i), & \\
\Gamma(z)_{j \sigma, j^{\prime} \boldsymbol{\sigma}} & =-G^{z-\boldsymbol{\alpha} \cdot \boldsymbol{\sigma}}\left(\boldsymbol{y}_{j}-\boldsymbol{y}_{j^{\prime}}\right), & & \text { if } j \neq j^{\prime},
\end{array}
$$

and $A$ is the matrix defined in (3-19)-(3-21).

Some particular features in the definition of $H^{A}$ are noteworthy:

- For the sake of convenience, the domain and action of $H^{A}$ are represented using the complex number $z$ but one can check that the definition of the operator is independent of the choice of $z$. 
- Functions in the domain of $H^{A}$ are either singular or zero in each scattering center $\boldsymbol{y}_{j}, j=1,2, \ldots, N$. The "charges" $q$ 's are the coefficients of the singular part whereas the $f$ 's are the values assumed in the scattering centers by the "regular part" of functions in the domain (i.e., the function minus its singular part in that point). The relation $\sum_{j^{\prime} \sigma^{\prime}} A_{j \sigma, j^{\prime} \sigma^{\prime}} q_{j^{\prime} \sigma^{\prime}}=f_{j \sigma}$ (see (3-15)) is then a singular boundary condition satisfied by the functions in the domain in each point $\boldsymbol{y}_{i}$. Relation (3-19) guarantees that the boundary condition satisfied in each point by functions in the domain do not depend on the boundary conditions satisfied elsewhere ("locality" of the self-adjoint extension). Notice that the matrix $A_{j \sigma, j^{\prime} \sigma^{\prime}}$ is defined in terms $\beta, \rho \in \mathbb{R}$ (see (3-21)), i.e., the two relevant parameters characterizing the interaction.

- The Hamiltonians one obtains for $\rho=0$ do not show any term indicating interaction between particle and spins. They correspond to point interaction Hamiltonians for the particle (see [Albeverio et al. 2005]) together with free evolution of the spins. Among the self-adjoint extensions of $\tilde{H}_{0}$ there are Hamiltonians where $\beta$ is taken as spin-dependent $(\beta(\sigma))$. Always in cases with $\rho=0$, the spins are still free, whereas the particle feels a point force in each $\boldsymbol{y}_{j}$ whose strength depends on the spin state in that same point. The latter were the Hamiltonians used to analyze neutron scattering by (fixed) nuclei.

- $\rho$ is the coupling constant of the particle-spin interaction. If $\rho$ is different from zero, the particle, in addition to the zero-range interaction with the points, can exchange energy with the spins. Condition (3-20) guarantees locality of the boundary conditions in this case.

- The spectrum of $H^{A}$ can have a very rich structure. In particular, several eigenstates embedded in the continuum when $\rho=0$ turn into resonances when $\rho \neq 0$ as a consequence of the interaction particle-spin. For our scope only the spectral structure at high energy will be relevant. It is easy to check that the spectrum of $H^{A}$ is purely continuous with no embedded eigenvalues for energies larger then $\max _{\sigma}(\boldsymbol{\alpha} \cdot \boldsymbol{\sigma})$.

The generalized eigenfunctions of $H^{A}$ are computable using the explicit form of the resolvent operator (3-22). For positive energies $\lambda>\max _{\sigma}(\boldsymbol{\alpha} \cdot \sigma)$ their $\sigma$ components are

$$
\begin{aligned}
& \varphi_{\beta, \rho}^{\boldsymbol{\sigma}}(\boldsymbol{x}, \lambda, \boldsymbol{\omega}) \otimes \mathcal{X}_{\boldsymbol{\sigma}}=\frac{(\lambda-\boldsymbol{\alpha} \cdot \boldsymbol{\sigma})^{1 / 4}}{(4 \pi)^{3 / 2}}\left[e^{i \sqrt{\lambda-\boldsymbol{\alpha} \cdot \boldsymbol{\sigma}} \boldsymbol{\omega} \cdot \boldsymbol{x}} \otimes \mathcal{X}_{\boldsymbol{\sigma}}\right. \\
&\left.+\sum_{j, j^{\prime}, \boldsymbol{\sigma}^{\prime}}\left(\Gamma_{\beta, \rho}(\lambda)\right)_{j, \boldsymbol{\sigma}, j^{\prime} \boldsymbol{\sigma}^{\prime}}^{-1} e^{i \sqrt{\lambda-\boldsymbol{\alpha} \cdot \boldsymbol{\sigma} \omega \cdot \boldsymbol{y}_{j}}} G^{\lambda-\boldsymbol{\alpha} \cdot \boldsymbol{\sigma}^{\prime}}\left(\boldsymbol{x}-\boldsymbol{y}_{j^{\prime}}\right) \otimes \mathcal{X}_{\boldsymbol{\sigma}^{\prime}}\right]
\end{aligned}
$$


where $\omega \in S^{2}$ is the unit vector representing the momentum direction. In fact, (3-25) remains valid for all values of $\lambda$ provided that the imaginary part of $\sqrt{\lambda-\boldsymbol{\alpha} \cdot \boldsymbol{\sigma}}$ is chosen positive for all $\sigma$ such that $\lambda-\boldsymbol{\alpha} \cdot \sigma<0$.

We consider the normalized initial condition

$$
\Psi^{0}=\psi_{0} \otimes \mathcal{X}_{\sigma_{0}},
$$

where $\sigma_{0}$ is the spin configuration in which $\sigma_{j}=-1$ for all $j$,

$$
\psi_{0}(\boldsymbol{x})=\hat{\mathcal{N}} \frac{e^{-x^{2} /\left(2 \gamma^{2}\right)}}{x} \sin \left(P_{0} x\right),
$$

with $x=|\boldsymbol{x}|$ and

$$
\hat{\mathcal{N}}=\frac{1}{\pi^{3 / 4} \sqrt{\gamma}\left(1-e^{-P_{0}^{2} \gamma^{2}}\right)^{1 / 2}},
$$

is a normalization factor making the norm of $\Psi^{0}$ equal to one. In momentum space the initial particle wave packet reads

$$
\hat{\psi}_{0}(\boldsymbol{k})=\hat{\mathcal{N}}^{\prime} / k\left[e^{-\left(\gamma^{2} / 2\right)\left(k-P_{0}\right)^{2}}-e^{-\left(\gamma^{2} / 2\right)\left(k+P_{0}\right)^{2}}\right]
$$

where $k=|\boldsymbol{k}|$ and $\hat{\mathcal{N}}^{\prime}=\gamma / 2 \hat{\mathcal{N}}$.

The meaning of the choice made above relates to the physical model: the spins are initially in the lowest energy state and are ready to detect the particle position during time evolution; the particle is propagating isotropically from the origin, with initial average radial momentum $P_{0}$ (corresponding to a wavelength $1 / P_{0}$ ). The parameter $\gamma$ represents the radial spread of the initial wave packet.

For simplicity, we fix $\alpha_{j}=\alpha>0$, for all $j$ in the Hamiltonian $H^{A}$ of the system. In this way $2 \alpha$ is the energy needed to flip the $j$-th spin from the down $\left(\sigma_{j}=-1\right)$ to the up state $\left(\sigma_{j}=1\right)$.

We consider situations in which the spin positions $y_{j}$ are distributed uniformly on a portion $\Sigma$ of linear dimension $D$ of a sphere of radius $L$ centered at the origin.

We assume that $\gamma \ll L$ in such a way that the initial wave packet is concentrated around the origin far from the scattering centers.

The parameter $\rho$ in $H^{A}$ has the dimension of inverse length and the role of the strength of the coupling between the particle and the spins; $1 / \beta$ is the scattering length of the scatterers.

We assume that the following inequalities between the order of magnitudes of relevant physical parameters hold:

$$
\begin{gathered}
1 / \beta \ll 1 / P_{0}<D, \\
\alpha \ll P_{0}^{2} \text { specifically } P_{0}^{2} \gtrsim N \alpha .
\end{gathered}
$$

The meaning of the assumptions above can be summarized as follows: 
The average initial energy of the particle is larger than the energy needed to produce any number of spin flips and the particle de Broglie wavelength is much larger than the scattering length of the point scatterers. The relation $1 / P_{0}<D$ will imply that two spherical waves

$$
\frac{\sin P_{0}\left|\boldsymbol{x}-\boldsymbol{y}_{j}\right|}{4 \pi\left|\boldsymbol{x}-\boldsymbol{y}_{j}\right|}, \quad j=j_{1}, j_{2},
$$

centered in two scatterers will show constructive interference only on the axis of the segment connecting the two points.

With the assumptions made above, Mott's conjecture can be rephrased as follows: consider the cone $C_{\Sigma}$ with apex at the origin and intersecting $S^{2}$ in $\Sigma$. Let us denote with $\chi_{\Sigma}$ the characteristic function of the cone. It is convenient to write the initial condition as the following superposition state

$$
\hat{\psi}_{0}(\boldsymbol{k})=\left(1-\chi_{\Sigma}(\boldsymbol{k})\right) \hat{\psi}_{0}(\boldsymbol{k})+\chi_{\Sigma}(\boldsymbol{k}) \hat{\psi}_{0}(\boldsymbol{k}) .
$$

We can show that the first term in (3-31) evolves almost freely giving a negligible contribution to any change in the configuration of spins in $\Sigma$. Only the second term in (3-31) can produce relevant changes in the spin configuration in $\Sigma$.

The precise statement will be formulated in terms of the long term behavior of the whole system (particle and spins). This requires the use of standard techniques in time-dependent and independent scattering theory (see [Reed and Simon 1979]). The wave operators $\Omega_{ \pm}$associated to the couple $H^{A}, H_{0}$ are defined as

$$
\begin{aligned}
& \Psi_{+}=\Omega_{+} \Psi=s-\lim _{t \rightarrow \infty} e^{i t H^{A}} e^{-i t H_{0}} \Psi, \\
& \Psi_{-}=\Omega_{-} \Psi=s-\lim _{t \rightarrow-\infty} e^{i t H^{A}} e^{-i t H_{0}} \Psi .
\end{aligned}
$$

As a consequence of the fact that the resolvent of $H^{A}$ is a finite rank perturbation of the resolvent of $H_{0}$, the wave operators exist and are complete. In particular one has

$$
\lim _{t \rightarrow \infty}\left\|e^{-i t H^{A}} \Psi-e^{-i t H_{0}} \Omega_{+}^{-1} \Psi\right\|_{\mathcal{H}}=0 .
$$

This means that $\Omega_{+}^{-1}$ applied to the initial state gives a modified initial state whose free evolution approximates the long term evolution of the system. In terms of $\Omega_{+}^{-1}$ our main result can be stated as follows:

Theorem 3.2. For any $\sigma \neq \sigma_{0}$, under the assumptions (3-30),

$$
\left\|\left(\Omega_{+}^{-1} \hat{\psi}^{0}\right)_{\sigma}(\boldsymbol{k})-\chi_{\Sigma}(\boldsymbol{k}) \hat{\psi}_{0}(\boldsymbol{k})\right\|_{L^{2}\left(\mathbb{R}^{3}\right)}<e^{-C P_{0}},
$$

where $C$ is a positive real constant and, as an abuse of notation, we use the same symbol $\Omega_{+}^{-1}$ for the wave operator acting on the particle Fourier space. 
Notice that the free evolution of the approximate modified initial state, denoted by $\chi_{\Sigma}(\boldsymbol{k}) \hat{\psi}_{0}(\boldsymbol{k})$, in (3-35) is characterized by a particle momentum always concentrated inside the cone and by an unaltered spin configuration. The result then states the following (conceivable) result: when the particle initial energy is very large compared to the energy necessary to flip a large number of spins

(1) the evolution of the particle state is not affected significantly by the interaction with the environment, and

(2) spins in a particular direction with respect to the source have negligible probability to be flipped by components of the initial wave packet heading in other directions.

The main steps heading to the result are sketched below. Only the proof of the main technical lemma will be detailed in the following.

The relation between wave operators and generalized eigenfunctions implies that the modified initial condition in a specific spin configuration reads

$$
\left(\Psi_{+}^{0}\right)_{\sigma}:=\left(\Omega_{+}^{-1} \Psi^{0}\right)_{\sigma}=\int_{\alpha \cdot \sigma}^{\infty} \mathrm{d} \lambda \int_{S^{2}} \mathrm{~d} \omega \phi_{\sigma}(\cdot, \lambda, \omega)\left(\varphi_{\beta, \rho}^{\sigma}(\lambda, \omega), \psi_{\sigma}^{0}\right),
$$

where

$$
\phi_{\sigma}(\boldsymbol{x}, \lambda, \omega)=\frac{(\lambda-\alpha \cdot \sigma)^{1 / 4}}{(4 \pi)^{3 / 2}} e^{i \sqrt{\lambda-\alpha \cdot \sigma} \omega \cdot \boldsymbol{x}}
$$

- The first step in the proof consists in carrying out an explicit computation of the scalar product of the initial conditions with the generalized eigenfunctions appearing in the integral on the right hand side of (3-36). If $\boldsymbol{y}$ is the position of a spin in $\Sigma$ and $\sigma \neq \sigma_{0}$ then

\section{Lemma 3.1.}

$$
\left\|\left(G^{\lambda-\widehat{\alpha \cdot \sigma}(\cdot-y)}, \hat{\psi_{0}}\right)-\left(G^{\lambda-\widehat{\alpha \cdot \sigma}(\cdot-y)}, \chi_{\Sigma} \hat{\psi}_{0}\right)\right\|<e^{C^{\prime} P_{0}}
$$

where $C^{\prime}$ is a positive real constant.

Proof. Let us write

$$
\begin{aligned}
F_{\boldsymbol{\sigma}, \Sigma}(\lambda,|\boldsymbol{y}|) \equiv\left(\left(G^{\lambda-\widehat{\boldsymbol{\alpha \cdot \sigma}(\cdot}}-\boldsymbol{y}\right), \chi_{\Sigma} \hat{\psi}_{0}\right) \\
=\int_{\mathbb{R}^{3}} \mathrm{~d} \boldsymbol{k} \overline{G^{\lambda-\widehat{\boldsymbol{\alpha} \cdot \boldsymbol{\sigma}}(\cdot-\boldsymbol{y})}} \hat{\psi}_{0}(\boldsymbol{k}) \chi_{\Sigma}(\boldsymbol{k})
\end{aligned}
$$


for the specific initial state of interest (3-27). The spherical wave Fourier transform is the distribution

$$
\begin{aligned}
\left.G^{\lambda-\widehat{\boldsymbol{\alpha} \cdot \boldsymbol{\sigma}}(\cdot-}-\boldsymbol{y}\right)=\frac{1}{(2 \pi)^{3 / 2}} \int_{\mathbb{R}^{3}} e^{-i \boldsymbol{k} \cdot \boldsymbol{x}} \frac{e^{i \sqrt{\lambda-\boldsymbol{\alpha} \cdot \boldsymbol{\sigma}}|\boldsymbol{x}-\boldsymbol{y}|}}{4 \pi|\boldsymbol{x}-\boldsymbol{y}|} \mathrm{d} \boldsymbol{x} \\
\quad=\frac{e^{-i \boldsymbol{k} \cdot \boldsymbol{y}}}{(2 \pi)^{3 / 2} k} \int_{0}^{\infty} \sin (k \xi) e^{i \sqrt{\lambda-\boldsymbol{\alpha} \cdot \boldsymbol{\sigma}} \xi} \mathrm{d} \xi,
\end{aligned}
$$

where $\sigma$ is a generic spin configuration $\sigma \neq \sigma_{0}$.

First, we compute explicitly the integral with respect to the momentum modulus $\mathrm{k}$. Neglecting terms exponentially decreasing faster then $e^{-c P_{0}^{2}}$ for any $c>\gamma^{2} / 2$, we perform the gaussian integral

$$
I(\xi) \equiv \int_{0}^{\infty} e^{i k|y| \cos \theta_{k, y}} \sin (k \xi) e^{-\gamma / 2\left(k-P_{0}\right)^{2}} \mathrm{~d} k=I_{+}(\xi)-I_{-}(\xi)
$$

where

$$
I_{ \pm}(\xi) \equiv \frac{1}{2 i} \int_{0}^{\infty} e^{i k|y| \cos \theta_{k, y}} e^{ \pm i k \xi} e^{-\gamma / 2\left(k-P_{0}\right)^{2}} \mathrm{~d} k
$$

and $\theta_{\boldsymbol{k}, \boldsymbol{y}}$ is the angle between the vectors $\boldsymbol{k}$ and $\boldsymbol{y}$. The result of the integration reads

$$
I_{ \pm}(\xi)=1 /(2 i \gamma) \sqrt{\pi / 2} e^{-\gamma^{2} / 2 P_{0}^{2}} e^{-1 /(2 \gamma 2) z_{ \pm}^{2}} \operatorname{Erfc}\left(-i /(\sqrt{2} \gamma) z_{ \pm}\right),
$$

where $z_{ \pm}= \pm \xi+|\boldsymbol{y}| \cos \theta_{\boldsymbol{k}, \boldsymbol{y}}-i \gamma^{2} P_{0}$ and Erfc denotes the complementary error function.

Defining $b=\sqrt{\lambda+N \alpha}$, we have

$$
\begin{aligned}
F_{\boldsymbol{\sigma}, \Sigma}(\lambda,|\boldsymbol{y}|) & \\
= & \frac{1}{(2 \pi)^{3 / 2}} \hat{\mathcal{N}}^{\prime} \int \chi_{\Sigma}\left[\int_{0}^{\infty}\left(I_{+}(\xi)-I_{-}(\xi)\right) e^{-i b \xi} \mathrm{d} \xi\right] \mathrm{d} \Sigma \\
= & D e^{-\left(b-P_{0}\right)^{2} \gamma^{2} / 2} \int_{S^{2}} \chi_{\Sigma}(\boldsymbol{k}) e^{i P_{0}|\boldsymbol{y}| \cos \theta_{\boldsymbol{k}, \boldsymbol{y}}} e^{-|\boldsymbol{y}|^{2} /\left(2 \gamma^{2}\right) \cos ^{2} \theta_{\boldsymbol{k}, \boldsymbol{y}}} \\
& {\left[\int_{0}^{\infty} e^{-\left(\xi /(\sqrt{2} \gamma)+i\left(b-P_{0}\right) \gamma / \sqrt{2}\right)^{2}} e^{-\xi / \gamma^{2}|\boldsymbol{y}| \cos \theta_{\boldsymbol{k}, \boldsymbol{y}}} \operatorname{Erfc}\left(-i /(\sqrt{2} \gamma) z_{+}\right) \mathrm{d} \xi\right] \mathrm{d} \Sigma } \\
+ & D e^{-\left(b+P_{0}\right)^{2} \gamma^{2} / 2} \int_{S^{2}} \chi_{\Sigma}(\boldsymbol{k}) e^{i P_{0}|\boldsymbol{y}| \cos \theta_{\boldsymbol{k}, \boldsymbol{y}}} e^{-|\boldsymbol{y}|^{2} /\left(2 \gamma^{2}\right) \cos ^{2} \theta_{\boldsymbol{k}, \boldsymbol{y}}} \\
& {\left[\int_{0}^{\infty} e^{-\left(\xi /(\sqrt{2} \gamma)+i\left(b+P_{0}\right) \gamma / \sqrt{2}\right)^{2}} e^{+\xi / \gamma^{2}|\boldsymbol{y}| \cos \theta_{\boldsymbol{k}, \boldsymbol{y}}} \operatorname{Erfc}\left(-i /(\sqrt{2} \gamma) z_{-}\right) \mathrm{d} \xi\right] \mathrm{d} \Sigma, }
\end{aligned}
$$


where $D=1 /(2 \pi)^{3 / 2} \hat{\mathcal{N}}^{\prime} 1 /(2 i \gamma) \sqrt{\pi / 2}$. Being the integral in the variable $\xi$ uniformly bounded for any finite value of $b, P_{0}$ and $\theta_{\boldsymbol{k}, \boldsymbol{y}}$, it is possible to integrate over the momentum directions which lie inside the cone $\mathcal{C}_{\Sigma}$.

In order to give an estimate for large $P_{0}|\boldsymbol{y}|$ of the integral we will make use of a stationary phase argument. We will take the axis of the cone to be the polar axis $\theta_{\boldsymbol{k}}=0$. Both integrals in (3-39) have the form

$$
\int_{0}^{\theta_{\max }} e^{i P_{0}|\boldsymbol{y}| \cos \theta_{\boldsymbol{k}, \boldsymbol{y}}} G\left(\cos \theta_{\boldsymbol{k}, \boldsymbol{y}}\right) \sin \theta_{\boldsymbol{k}} \mathrm{d} \theta_{\boldsymbol{k}} \mathrm{d} \phi_{\boldsymbol{k}},
$$

with $\cos \theta_{\boldsymbol{k}, \boldsymbol{y}}=\cos \theta_{\boldsymbol{k}} \cos \theta_{\boldsymbol{y}}+\sin \theta_{\boldsymbol{k}} \sin \theta_{\boldsymbol{y}} \cos \left(\phi_{\boldsymbol{k}}-\phi_{\boldsymbol{y}}\right)$.

It is easy to check that the phase $\cos \theta_{\boldsymbol{k}, \boldsymbol{y}}$ is stationary only if $\theta_{\boldsymbol{k}}=\theta_{\boldsymbol{y}}$ and $\phi_{k}=\phi_{y}$ and that the Hessian matrix determinant computed at the point of stationary phase is $\sin ^{2} \theta_{\boldsymbol{y}}$. We deduce that the case of stationary phase applies only if $\boldsymbol{y} /|\boldsymbol{y}|$ belongs to the cone $\mathcal{C}_{\Sigma}$ and that, in such a case, the leading term of (3-40) reads

$$
\frac{2 \pi}{i} \frac{e^{i P_{0}|\boldsymbol{y}|}}{P_{0}|\boldsymbol{y}|} G\left(\cos \theta_{\boldsymbol{k}, \boldsymbol{y}}=1\right) \frac{\sin \theta_{\boldsymbol{y}}}{\left|\sin \theta_{\boldsymbol{y}}\right|} .
$$

If $y$ is inside the cone $\mathcal{C}_{\Sigma}$ and $\theta_{\max }<\pi / 2$, we then have

$$
\begin{aligned}
F_{\boldsymbol{\sigma}, \Sigma}(\lambda,|\boldsymbol{y}|) \simeq- & \frac{\hat{\mathcal{N}}}{8} \frac{e^{i P_{0}|\boldsymbol{y}|}}{P_{0}|\boldsymbol{y}|} e^{-\left(b-P_{0}\right)^{2} \gamma^{2} / 2} e^{-|\boldsymbol{y}|^{2} /\left(2 \gamma^{2}\right)} \\
& {\left[\int_{0}^{\infty} e^{-\left(\xi /(\sqrt{2} \gamma)+i\left(b-P_{0}\right) \gamma / \sqrt{2}\right)^{2}} e^{-\xi / \gamma^{2}|\boldsymbol{y}|} \operatorname{Erfc}\left(-i /(\sqrt{2} \gamma) v_{+}\right) \mathrm{d} \xi\right] } \\
- & \frac{\hat{\mathcal{N}}}{8} \frac{e^{i P_{0}|\boldsymbol{y}|}}{P_{0}|\boldsymbol{y}|} e^{-\left(b+P_{0}\right)^{2} \gamma^{2} / 2} e^{-|\boldsymbol{y}|^{2} /\left(2 \gamma^{2}\right)} \\
& {\left[\int_{0}^{\infty} e^{-\left(\xi /(\sqrt{2} \gamma)+i\left(b+P_{0}\right) \gamma / \sqrt{2}\right)^{2}} e^{+\xi / \gamma^{2}|\boldsymbol{y}|} \operatorname{Erfc}\left(-i /(\sqrt{2} \gamma) v_{-}\right) \mathrm{d} \xi\right], }
\end{aligned}
$$

where $v_{ \pm}= \pm \xi+|\boldsymbol{y}|-i \gamma^{2} P_{0}$.

If the scatterer position lies outside the cone, standard results in approximation of integrals in the nonstationary case proves the exponential decay stated in the lemma.

Notice that, as a by-product, the lemma proves that the main contribution to the integral with respect to $\lambda$ in (3-36) is attained for $b \approx P_{0}$ which, under our assumption, means $\sqrt{\lambda} \approx P_{0}$.

- As a second step, one has to examine the coefficients in the sum of spherical waves $G^{\lambda-\alpha \cdot \sigma}$ in (3-25). In particular, it is necessary to check that the matrix $\Gamma_{\beta, \rho}(\lambda)$ is invertible and to control the norm of its inverse. Under the assumptions of the dynamical and geometrical parameters of the system 
listed above it is always possible to find a $\beta$, independent on the number of spins, for which the matrix $\Gamma_{\beta, \rho}(\lambda)$ is invertible and the norm of its inverse is bounded uniformly in the number of spins. Details about the invertibility and the continuum limit of the inverse of the $\Gamma$ matrix are given in [Figari and Teta 2014] (see also [Figari et al. 1988] for a similar result in the case of point interactions without spin dependence).

Theorem 3.2 specifies where the main contribution to the flipping probability comes from, yet does not give quantitative indications about the transition probabilities to any specific final configuration of the environment. In order to investigate thoroughly the asymptotic behavior of the particle and the environment and characterize its dependence on the number of flipped spins, it is necessary to go one step further:

- Prove a kind of Huygen's principle stating that the spherical waves centered in a large number of points uniformly distributed on $\Sigma$ interfere constructively only inside the cone $C_{\Sigma}$. The situation is analogous to the case of electromagnetic wave propagation through a hole when the linear dimensions of the hole are much larger than the wavelength (absence of diffraction).

- Give an estimate of the probability that a significant fraction of spins turns out to be flipped.

These two last technical steps, requiring detailed combinatorial estimates, will be examined in a paper in preparation.

To conclude, we want to summarize what we obtained in the language of the environment induced decoherence: if a large number of "clouds" of spin dependent point scatterers are present on the sphere of radius $L$, the above stated result amounts to saying that the long term behavior of the whole system state is the incoherent sum of states with support in distant regions of the configuration space, each characterized by a sharply defined particle momentum direction and a significant change in the spin configuration only relative to spins in that specific direction.

\section{References}

[Adami et al. 2004] R. Adami, R. Figari, D. Finco, and A. Teta, "On the asymptotic behaviour of a quantum two-body system in the small mass ratio limit", J. Phys. A 37:30 (2004), 7567-7580.

[Adami et al. 2006] R. Adami, R. Figari, D. Finco, and A. Teta, "On the asymptotic dynamics of a quantum system composed by heavy and light particles", Comm. Math. Phys. 268:3 (2006), 819852.

[Albeverio et al. 2005] S. Albeverio, F. Gesztesy, R. Høegh-Krohn, and H. Holden, Solvable models in quantum mechanics, 2nd ed., AMS Chelsea Publishing, Providence, RI, 2005.

[Bell 1987] J. S. Bell, Speakable and unspeakable in quantum mechanics, Cambridge University Press, 1987. 
[Blanchard et al. 2000] P. Blanchard, D. Giulini, E. Joos, C. Kiefer, and I.-O. Stamatescu (editors), Decoherence: theoretical, experimental and conceptual problems, Lecture Notes in Physics 538, Springer, 2000.

[Bohr 1928] N. Bohr, "The quantum postulate and the recent development of atomic theory", Nature, London 121 (1928), 580-590.

[Born 1926] M. Born, “Zur Quantenmechanik der Stoßvorgänge”, Z. Phys. 37:12 (1926), 863-867.

[Born and Jordan 1925] M. Born and P. Jordan, "Zur Quantenmechanik", Z. Phys. 34:1 (1925), $858-888$.

[Cacciapuoti et al. 2007] C. Cacciapuoti, R. Carlone, and R. Figari, "Spin-dependent point potentials in one and three dimensions", J. Phys. A 40:2 (2007), 249-261.

[Condon and Gurney 1928] E. Condon and R. Gurney, "Wave mechanics and radioactive disintegration", Nature 122 (1928), 439.

[Dell'Antonio et al. 2008] G. Dell'Antonio, R. Figari, and A. Teta, "Joint excitation probability for two harmonic oscillators in one dimension and the Mott problem", J. Math. Phys. 49:4 (2008), 042105, 20.

[Dell'Antonio et al. 2010] G. Dell'Antonio, R. Figari, and A. Teta, "A time-dependent perturbative analysis for a quantum particle in a cloud chamber", Ann. Henri Poincaré 11:3 (2010), 539-564.

[Figari and Teta 2013] R. Figari and A. Teta, "Emergence of classical trajectories in quantum systems: the cloud chamber problem in the analysis of Mott (1929)", Archive for History of Exact Sciences 67:2 (2013), 215-234.

[Figari and Teta 2014] R. Figari and A. Teta, Quantum dynamics of a particle in a tracking chamber, Springer Briefs in Physics, Springer, 2014.

[Figari et al. 1988] R. Figari, H. Holden, and A. Teta, "A law of large numbers and a central limit theorem for the Schrödinger operator with zero-range potentials", J. Statist. Phys. 51:1-2 (1988), $205-214$.

[Finco and Teta 2011] D. Finco and A. Teta, "Asymptotic expansion for the wave function in a one-dimensional model of inelastic interaction", J. Math. Phys. 52:2 (2011), 022103, 19.

[Gamow 1928] G. Gamow, "Quantum theory of atomic nucleus”, Z. Phys. 51:204 (1928).

[Gupta and Ghosh 1946] N. N. D. Gupta and S. K. Ghosh, "A report on the Wilson cloud chamber and its applications in physics", Rev. Mod. Phys. 18 (1946), 225-290.

[Hornberger 2009] K. Hornberger, "Introduction to decoherence theory", pp. 221-276 in Entanglement and decoherence, edited by A. Buchleitner et al., Lecture Notes in Phys. 768, Springer, Berlin, 2009.

[Joos et al. 2003] E. Joos, H. D. Zeh, C. Kiefer, D. Giulini, J. Kupsch, and I.-O. Stamatescu, Decoherence and the appearance of a classical world in quantum theory, 2nd ed., Springer, Berlin, 2003.

[Leone and Robotti 2004] M. Leone and N. Robotti, "A note on the Wilson cloud chamber (1912)", Eur. J. Phys. 25:6 (2004), 781.

[Mott 1929] N. F. Mott, "The wave mechanics of $\alpha$-ray tracks", Proc. R. Soc. Lond., Ser. A 126 (1929), 79-84.

[von Neumann 1932] J. von Neumann, Mathematische Grundlagen der Quantenmechanik, Grundlehren der math. Wiss. 38, Springer, Berlin, 1932. Translated as Mathematical Foundations of Quantum Mechanics, Princeton University Press, 1955.

[Recchia and Teta 2014] C. Recchia and A. Teta, "Semiclassical wave-packets emerging from interaction with an environment", J. Math. Phys. 55:1 (2014), 012104, 24. 
[Reed and Simon 1979] M. Reed and B. Simon, Methods of modern mathematical physics III, Academic Press, New York, 1979.

[Schrödinger 1982] E. Schrödinger, Collected papers on wave mechanics, Chelsea, New York, 1982.

[Teta 2010] A. Teta, "Classical behaviour in quantum systems: the case of straight tracks in a cloud chamber”, Eur. J. Phys. 31:1 (2010), 215.

Received 4 May 2016. Revised 13 Jun 2016. Accepted 1 Aug 2016.

RODOLFO FIGARI: figari@na.infn.it

Dept. of Physics "Ettore Pancini" and National Institute of Nuclear Physics,

University of Napoli Federico II, Complesso Univ. Monte Sant'Angelo Ed. 6, Via Cintia,

I-80126 Napoli, Italy

Alessandro Teta: teta@mat.uniroma1.it

Dipartimento di Matematica G. Castelnuovo, Sapienza Università di Roma, P.le A. Moro 5, I-00185 Roma, Italy 


\title{
PROPAGATION OF CHAOS AND EFFECTIVE EQUATIONS IN KINETIC THEORY: A BRIEF SURVEY
}

\author{
Mario Pulvirenti And Sergio Simonella
}

\begin{abstract}
We review some historical highlights leading to the modern perspective on the concept of chaos from the point of view of kinetic theory. We focus in particular on the role played by the propagation of chaos in the mathematical derivation of effective equations.
\end{abstract}

\section{The paradigm of kinetic theory}

Propagation of chaos is a central topic in kinetic theory and certainly exhibits interesting features from the point of view of probability theory and mathematical physics.

This contribution is dedicated to our friend and colleague Lucio Russo, who gave and is giving important contributions to these fields and to the history of science. The purpose is to review some important steps in the mathematical understanding of kinetic equations and of the notion of chaos.

We do not pretend to be exhaustive and limit ourselves to a selection of arguments which played a key role from a modern outlook. We also comment on some perhaps less known historical aspects underlining the long and difficult path of the scientific progress.

Many interesting systems in physics and applied sciences consist of a large number of identical components so that they are difficult to analyze from a mathematical point of view. On the other hand, quite often, we are not interested in a detailed description of the system but rather in its collective behavior. Therefore, it is necessary to look for all procedures leading to simplified models, retaining the interesting features of the original system, cutting away redundant information. This is exactly the methodology of statistical mechanics and kinetic theory. Here we want to outline the limiting procedure leading from the microscopic description of a large particle system (based on the fundamental laws like the Newton or Schrödinger equations) to the more practical picture dictated by kinetic theory.

\section{Communicated by Raffaele Esposito.}

MSC2010: 82-03.

Keywords: propagation of chaos, Boltzmann, Landau and Vlasov equations. 
Although recently the methodology of kinetic theory has been applied to a large variety of complex systems (consisting of a huge number of individuals), we will discuss here only models arising in physics and more precisely in classical mechanics. The starting point is a system of $N$ identical particles in the space $\mathbb{R}^{3}$. A microscopic state of the system is a sequence $z_{1}, \ldots, z_{N}$ where $z_{i}=\left(x_{i}, v_{i}\right)$ denotes position and velocity of the $i$-th particle. The particles interact via the (smooth) two-body interaction $\varphi: \mathbb{R}^{3} \rightarrow \mathbb{R}$, and the equations of motion are

$$
\left\{\begin{array}{l}
\dot{x}_{i}=v_{i} \\
\dot{v}_{i}=-\sum_{j: j \neq i} \nabla \varphi\left(x_{i}-x_{j}\right)
\end{array}\right.
$$

Particles have unit mass, and $\varphi$ depends on the distance $\left|x_{i}-x_{j}\right|$ so that the force of particle $j$ acting on particle $i$ (that is, $-\nabla \varphi\left(x_{i}-x_{j}\right)$ ) is directed along $x_{i}-x_{j}$.

We are interested in a situation where $N$ is very large (for instance, a cubic centimeter of a rarefied gas contains approximately $10^{19}$ molecules). The knowledge of the microscopic states becomes useless, and we turn to a statistical description. We introduce a probability measure $W^{N}\left(Z_{N}\right) d Z_{N}$ (absolutely continuous with respect to the Lebesgue measure), defined on the phase space of the system $\mathbb{R}^{3 N} \times \mathbb{R}^{3 N}$, where

$$
Z_{N}=\left(z_{1}, \ldots, z_{N}\right)=\left(x_{i}, v_{i}, \ldots, x_{N}, v_{N}\right)
$$

$W^{N}$ assigns the same statistical weight to two different vectors $Z_{N}$ and $Z_{N}^{\prime}$ differing only for the order of particles, i.e., identifying the same physical configuration. Physically relevant measures are symmetric with respect to permutations of the sequence $z_{1}, \ldots, z_{N}$.

The time-evolved measure is defined by

$$
W^{N}\left(Z_{N}, t\right)=W^{N}\left(\Phi^{-t}\left(Z_{N}\right)\right) .
$$

Here $\Phi^{t}\left(Z_{N}\right)$ denotes the dynamical flow constructed by solving the equations of motion; namely, $\Phi^{t}\left(Z_{N}\right)$ solves (1) with initial datum $Z_{N}$.

We can establish a partial differential equation, called the Liouville equation, describing the evolution of the measure (2). However, this equation is also not tractable from a practical point of view. To have an efficient reduced description, one can focus on the time evolution for the probability distribution of a given particle (say particle 1), all the particles being identical.

To this end, we define the $j$-particle marginals

$$
f_{j}^{N}\left(Z_{j}, t\right)=\int d z_{j+1} \cdots d z_{N} W^{N}\left(Z_{j}, z_{j+1}, \ldots, z_{N}, t\right), \quad j=1, \ldots, N,
$$


and we look for an equation describing the evolution of $f_{1}^{N}$. Roughly, we establish an evolution equation of the form

$$
\partial_{t} f_{1}^{N}=-v \cdot \nabla f_{1}^{N}+Q .
$$

The first term in the right-hand side denotes the contribution to the evolution of $f_{1}^{N}$ due to the free transport of particles, while the term $Q$ should describe the interaction of particle 1 with the rest of the system.

We now face a big difficulty. Since the interaction is binary, $Q$ will depend on $f_{2}^{N}$, namely the two-particle marginal. In other words, (4) is still useless because to know $f_{1}^{N}$ we need to know $f_{2}^{N}$ and to know $f_{2}^{N}$ we need to know $f_{3}^{N}$ and so on. We handle a hierarchy of equations, called BBGKY hierarchy [Bogolyubov 1946] (from the names of the physicists Bogolyubov, Born, Green, Kirkwood, and Yvon).

Here enters the property called propagation of chaos, that is,

$$
f_{2}^{N}\left(x_{1}, v_{1}, x_{2}, v_{2}, t\right)=f_{1}^{N}\left(x_{1}, v_{1}, t\right) f_{1}^{N}\left(x_{2}, v_{2}, t\right) .
$$

Accepting (5), $Q$ becomes a bilinear operator of $f_{1}^{N}$ and (4) is a closed equation. We have thus replaced a huge ordinary differential system with a single PDE. The price we pay is that (4) is nonlinear.

Strictly speaking, (5) is certainly false since it expresses the statistical independence of particle 1 and particle 2, which even if assumed at time 0 cannot hold at later times. Indeed, the dynamics creates correlations. Nevertheless, one can hope to recover this property in some asymptotic situation described by a suitable scaling limit. This is what happens in three different physical contexts: the meanfield, the low-density and the weak-coupling limits, yielding three different kinetic equations, namely the Vlasov, Boltzmann and Landau equations, respectively.

\section{Mean-field limit and Vlasov equation}

The simplest example in which the methods of kinetic theory apply is the meanfield limit. Let us suppose that the particle system we are considering interacts through a very small (possibly long-range) potential $O(1 / N)$, where the number of particles $N$ is going to diverge. The equation of motion becomes

$$
\left\{\begin{array}{l}
\dot{x}_{i}=v_{i}, \\
\dot{v}_{i}=-(1 / N) \sum_{j: j \neq i} \nabla \varphi\left(x_{i}-x_{j}\right) .
\end{array}\right.
$$

Consider also an initial distribution $W^{N}$ fully factorized, i.e., $W^{N}=f_{0}^{\otimes N}$. In this situation, the dynamics creates correlations at each positive time. However, given two particles, say 1 and 2 , the dynamics of particle 1 is influenced by the presence of particle 2 by a factor $O(1 / N)$. The same happens for particle 2 regarding the influence of particle 1 . Therefore, we expect that the correlations are negligible 
and in the limit $N \rightarrow \infty$

$$
f_{2}^{N} \approx\left(f_{1}^{N}\right)^{\otimes 2}
$$

We shall see that, in our context, (4) becomes

$$
\left(\partial_{t}+v_{1} \cdot \nabla_{x_{1}}\right) f_{1}^{N}(t)=\frac{(N-1)}{N} \int d x_{2} \int d v_{2} \nabla \varphi\left(x_{1}-x_{2}\right) \cdot \nabla_{v_{1}} f_{2}^{N}\left(x_{1}, v_{1}, x_{2}, v_{2}, t\right)
$$

so that, using (7) and formally taking the limit $N \rightarrow \infty$, we arrive at the following equation for the one-particle distribution $f$ :

$$
\left(\partial_{t}+v \cdot \nabla_{x}\right) f(x, v, t)=\int d y \int d w f(y, w, t) \nabla \varphi(x-y) \cdot \nabla_{v} f(x, v, t) .
$$

Equation (9) is called the Vlasov equation (from the name of the physicist who introduced it) and describes a large system of weakly interacting particles.

The rigorous analysis of the mean-field limit and the Vlasov equation are a well understood subject in the case of smooth potentials (see for instance [Dobrušin 1979]). The interesting case of the Coulomb interaction is still a challenging open problem.

\section{The Boltzmann equation}

Much more subtle are the limiting physical situations leading to the Boltzmann equation.

Ludwig Boltzmann established an evolution equation to describe the behavior of a rarefied gas in 1872, starting from the mathematical model of elastic balls and using mechanical and statistical considerations. The importance of this equation is twofold. On one side, it provides (as well as the hydrodynamical equations) a reduced description of the microscopic world. On the other, it is also an important tool for applications, especially for dilute fluids when the hydrodynamical equations fail to hold.

According to the general paradigm of kinetic theory, the starting point of Boltzmann's analysis is to renounce the study of gas in terms of the detailed motion of the molecules of the full system. It is preferable to investigate a function $f(x, v)$ which is the probability density of a given particle, where $x$ and $v$ denote its position and velocity.

Following the original approach proposed by Boltzmann, $f(x, v) d x d v$ is to rather be interpreted as the fraction of molecules falling in the cell of the phase space of size $d x d v$ around $(x, v)$. The two concepts are not exactly the same, but they are asymptotically equivalent (when the number of particles diverges) if a law of large numbers holds. 
More precisely, Boltzmann considered the occupation numbers of the cells of a grid in the phase space, when the side of the cell is macroscopically small but sufficiently large to contain a huge number of particles. From a historical point of view, Boltzmann's analysis was really remarkable. Probability theory was not well developed from a mathematical point of view, and even the possibility of describing the macroscopic world in terms of atoms and molecules was still doubtful.

Boltzmann considered a gas as microscopically described by a system of elastic (hard) balls, colliding according to the laws of classical mechanics.

The Boltzmann equation for the one-particle distribution function reads

$$
\left(\partial_{t}+v \cdot \nabla_{x}\right) f=Q(f, f)
$$

where $Q$, the collision operator, is defined by $Q(f, f)(x, v)=\int_{\mathbb{R}^{3}} d v_{1} \int_{S_{+}^{2}} d n\left(v-v_{1}\right) \cdot n\left[f\left(x, v^{\prime}\right) f\left(x, v_{1}^{\prime}\right)-f(x, v) f\left(x, v_{1}\right)\right]$,

with

$$
\begin{aligned}
v^{\prime} & =v-n\left[n \cdot\left(v-v_{1}\right)\right], \\
v_{1}^{\prime} & =v_{1}+n\left[n \cdot\left(v-v_{1}\right)\right]
\end{aligned}
$$

and $n$ a unitary vector varying in $S_{+}^{2}=\left\{n: n \cdot\left(v-v_{1}\right) \geq 0\right\}$.

Note that $v^{\prime}$ and $v_{1}^{\prime}$ are the outgoing velocities after a collision of two elastic balls with incoming velocities $v$ and $v_{1}$ and centers $x$ and $x+\varepsilon n$, with $\varepsilon$ the diameter of the spheres. Clearly the collision takes place if $n \cdot\left(v-v_{1}\right)>0$. Formulas (12) are consequences of the conservation of energy and momentum. Note that $\varepsilon$ does not enter (10) as a parameter.

As a fundamental feature of (10), one has the formal conservation (in time) of the five quantities

$$
\int d x \int d v f(x, v ; t) v^{\alpha}
$$

with $\alpha=0,1,2$, expressing conservation of probability, momentum and energy, respectively. From now on, we shall set $\int=\int_{\mathbb{R}^{3}}$ for notational simplicity.

Moreover, Boltzmann introduced the (kinetic) entropy defined by

$$
H(f)=\int d x \int d v f \log f(x, v)
$$

and proved the famous $H$ theorem asserting the decrease of $H(f(t))$ along the solutions of (10).

Finally, in the case of bounded domains or homogeneous solutions $(f=f(v, t)$ is independent of $x$ ), the distribution defined for some $\beta>0, \rho>0$ and $u \in \mathbb{R}^{3}$ by

$$
M(v)=\frac{\rho}{(2 \pi / \beta)^{3 / 2}} e^{-(\beta / 2)|v-u|^{2}},
$$


called the Maxwellian distribution, is stationary for the evolution given by (10). In addition, $M$ minimizes $H$ among all distributions with given total mass $\rho$, mean velocity $u$ and mean energy. The parameter $\beta$ is interpreted as the inverse temperature.

In conclusion, Boltzmann was able to introduce an evolution equation with the remarkable properties of expressing mass, momentum and energy conservation and also the tendency to thermal equilibrium. In other words, he tried to conciliate Newton's laws with the second principle of thermodynamics.

The $H$ theorem is in contrast with the laws of mechanics, which are timereversible. This fact caused skepticism among the scientific community, and the work of Boltzmann was attacked by several scientists. We refer the reader to the beautiful monograph by C. Cercignani [1998], which is a marvelous compromise between historical and high-level scientific divulgation, to have a faithful idea of the debate at the time.

To formally derive (10), let us consider a system of $N$ identical hard spheres of diameter $\varepsilon$ and unitary mass, interacting by means of the collision law (12). We denote by $\varepsilon$ the diameter of the particles in view of the fact that $\varepsilon$ is very small compared with typical macroscopic lengths.

The phase space $\Gamma_{N}$ of the system is the subset of $\left(\mathbb{R}^{6}\right)^{N}$ satisfying the hard-core condition, namely

$$
\left|x_{i}-x_{j}\right| \geq \varepsilon \quad \text { for } i \neq j .
$$

The dynamical flow is defined as the free flow, i.e.,

$$
Z_{N}(t)=\left(x_{1}+v_{1} t, v_{1}, \ldots, x_{N}+v_{N} t, v_{N}\right)
$$

up to the first impact time, namely when $\left|x_{i}-x_{j}\right|=\varepsilon$. Then an instantaneous collision takes place according to the law (12), and the flow goes on up to the next collision instant.

We denote by $Z_{N} \rightarrow \Phi^{t}\left(Z_{N}\right)$ the dynamical flow constructed in this way. The well-posedness of the hard-sphere dynamics is not obvious, due to the occurrence of multiple collisions or to the a priori possibility that collision times accumulate at a finite limiting time. However, such pathologies cannot occur outside a set of initial conditions $Z_{N}$ of vanishing measure. Therefore, the flow $Z_{N} \rightarrow \Phi^{t}\left(Z_{N}\right)$ can be defined almost everywhere with respect to the Lebesgue measure, and this is enough for our purposes.

Given a probability measure with density $W_{0}^{\varepsilon}$ on $\Gamma_{N}$, thanks to the invariance of the Lebesgue measure under the above evolution, we define the time-evolved measure as the measure with density

$$
W^{\varepsilon}\left(Z_{N}, t\right)=W_{0}^{\varepsilon}\left(\Phi^{-t} Z_{N}\right) .
$$


We recall that we consider probability distributions $W_{0}^{\varepsilon}$ which are initially (and hence at any positive time) symmetric in the exchange of the particles. The probability density of the first $j$ particles is given by the $j$-particle marginal

$$
f_{j}^{\varepsilon}\left(Z_{j}, t\right)=\int d z_{j+1} \cdots d z_{N} W^{\varepsilon}\left(Z_{j}, z_{j+1}, \ldots, z_{N}, t\right), \quad j=1, \ldots, N .
$$

Notational remark. Up to now, the two parameters $N$ and $\varepsilon$ have been introduced independently, and the definitions $\Gamma_{N}, Z_{N}, W^{\varepsilon}, f_{j}^{\varepsilon}$, etc., should exhibit a double dependence. However, in a moment, we shall fix a precise dependence $\varepsilon=\varepsilon(N)$ so that the notation is unambiguous.

Cercignani [1972] derived a hierarchy of equations for the marginals, and the first of such equations, for the one-particle distribution, is

$$
\left(\partial_{t}+v \cdot \nabla_{x}\right) f_{1}^{\varepsilon}=\text { Coll, }
$$

where Coll denotes the variation of $f$ due to the collisions. It takes the form

$$
\text { Coll }=(N-1) \varepsilon^{2} \int d v_{2} \int_{S^{2}} d n f_{2}^{\varepsilon}\left(x, v, x+n \varepsilon, v_{2}\right)\left(v_{2}-v\right) \cdot n .
$$

Let us argue the physical significance of (19) and (18). In absence of collisions, the probability density of a given particle would be conserved, that is,

$$
\frac{d}{d t} f^{\varepsilon}(x+v t, v, t)=0 .
$$

The presence of collisions and the total conservation of the probability imply that

$$
\frac{d}{d t} f^{\varepsilon}(x+v t, v, t)=\text { flux }
$$

where the flux is computed on the boundary of the spherical surface of the ball of radius $\varepsilon$ around $x$. Therefore, the probability flux due to a collision with a given particle, say particle 2 , having velocity $v_{2}$, is given by

$$
-\varepsilon^{2} \int_{S^{2}} f_{2}^{\varepsilon}\left(x, v, x+n \varepsilon, v_{2}\right) V \cdot n
$$

where $V=v_{2}-v$ is the relative velocity and $-n$ is the inward normal to the considered surface.

Integrating with respect to $d v_{2}$ and summing over all the possible choices of particles, we arrive to (18) and (19).

This is, basically, Boltzmann's original argument, except for an important conceptual difference. The basic object of investigation considered by Boltzmann is not the probability density $f$ but rather the quantity

$$
f^{\varepsilon}(x, v, t) \approx \frac{N_{\Delta}(t)}{|\Delta| N}
$$


where $\Delta$ is a small cell on the phase space around the point $(x, v),|\Delta|$ is its volume and $N_{\Delta}(t)$ is the occupation number of the cell $\Delta$ (number of particles falling in $\Delta$ ) at time $t$. Clearly, $\Delta$ must be small compared with the macroscopic lengths, e.g., the size of the box in which the gas is confined, but large with respect to the typical microscopic lengths, for instance $\varepsilon$ (molecular diameter).

The two concepts introduced are not exactly the same, but in view of a limit $N \rightarrow \infty$, it is possible to conceive a law of large numbers allowing one to identify the empirical sample with the a priori probability.

Next we shall tackle the problem of getting a closed equation. Apparently, we are in a situation analogous to the one discussed for the Vlasov equation, but there is a deep difference. Indeed for the hard-sphere system, one can write a hierarchy of equations which plays the role of the BBGKY hierarchy for smooth potentials. However, the interaction among the particles is strong and the mean-field argument used to invoke the propagation of chaos fails.

Boltzmann's most important assumption enters here, namely that two given particles should be (almost) uncorrelated if the gas is rarefied enough. This leads to the propagation of chaos

$$
f_{2}^{\varepsilon}\left(x, v, x_{2}, v_{2}\right)=f^{\varepsilon}(x, v) f^{\varepsilon}\left(x_{2}, v_{2}\right),
$$

which is however much more delicate in the present context. In fact, if two particles collide, correlations are created. Even assuming (20) at some time, if particle 1 collides with particle 2 , such an equation cannot be satisfied at any time after the collision.

Before discussing the propagation of chaos further, we notice that, in practical situations, for a rarefied gas, the combination $N \varepsilon^{3} \approx 10^{-8} \mathrm{~cm}^{3}$ (total volume occupied by the particles) is very small, while $N \varepsilon^{2}=O(1)$. This implies that the collision operator given by (19) is $O(1)$. Therefore, since we are dealing with a huge number of particles, we are tempted to perform the limit $N \rightarrow \infty$ and $\varepsilon \rightarrow 0$ in such a way that $\varepsilon^{2}=O\left(N^{-1}\right)$. As a consequence, the probability that two tagged particles collide (which is of the order of the surface of a ball, that is, $O\left(\varepsilon^{2}\right)$ ) is negligible. Instead, the probability that a given particle collides with any of the remaining $N-1$ particles $\left(O\left(N \varepsilon^{2}\right)=O(1)\right)$ is not negligible. On the other hand, condition (20) refers to two preselected particles (say 1 and 2) and it is not unreasonable to conceive that it holds in the limiting situation in which we are working.

Nevertheless, we cannot insert (20) into (19) because the integral operator refers to times both before and after the collision. Let us assume (20) only when the pair of velocities $v$ and $v_{2}$ are incoming $\left(\left(v-v_{2}\right) \cdot n>0\right)$. If the two particles are initially uncorrelated, it is unlikely that they have collided before a given time $t$ so that we assume their statistical independence. 
This is a standard argument in textbooks of kinetic theory, but some extra comments are needed. If particles 1 and 2 have not collided directly before a given time $t$, this does not imply that they are uncorrelated. Indeed there may exist a chain of collisions involving a group $i_{1}, i_{2}, \ldots$ of particles

$$
1 \rightarrow i_{1} \rightarrow i_{2} \rightarrow \cdots \rightarrow 2
$$

correlating particles 1 and 2 . The occurrence of this event must be excluded by a rigorous mathematical analysis.

Coming back to (19), for the outgoing pair of velocities $v$ and $v_{2}\left(\left(v_{2}-v\right) \cdot n>0\right)$, we make use of the continuity property

$$
f_{2}^{\varepsilon}\left(x, v, x+n \varepsilon, v_{2}\right)=f_{2}^{\varepsilon}\left(x, v^{\prime}, x+n \varepsilon, v_{2}^{\prime}\right)
$$

where the pair $v^{\prime}$ and $v_{2}^{\prime}$ is precollisional. On the two-particle distribution expressed in terms of precollisional variables, we apply condition (20), obtaining

$$
\begin{aligned}
\text { Coll }=(N-1) \varepsilon^{2} \int d v_{2} & \int_{S_{+}^{2}} d n\left(v-v_{2}\right) \cdot n \\
& \times\left[f\left(x, v^{\prime}\right) f\left(x-n \varepsilon, v_{2}^{\prime}\right)-f(x, v) f\left(x+n \varepsilon, v_{2}\right)\right]
\end{aligned}
$$

after a change $n \rightarrow-n$ in the positive part of Coll, using the notation $S_{+}^{2}$ for the hemisphere $S_{+}^{2}=\left\{n: n \cdot\left(v-v_{2}\right) \geq 0\right\}$.

Finally, in the limit as $N \rightarrow \infty$ and $\varepsilon \rightarrow 0$ with $N \varepsilon^{2}=\lambda^{-1}$, we find

$$
\left(\partial_{t}+v \cdot \nabla_{x}\right) f=\lambda^{-1} \int d v_{2} \int_{S_{+}} d n\left(v-v_{2}\right) \cdot n\left[f\left(x, v^{\prime}\right) f\left(x, v_{2}^{\prime}\right)-f(x, v) f\left(x, v_{2}\right)\right] .
$$

The parameter $\lambda$ represents, roughly, the typical length a particle can cover without undergoing any collision (mean free path). (In (10), we just chose $\lambda=1$.)

Remark. After having taken the limit $N \rightarrow \infty$ and $\varepsilon \rightarrow 0$, there is no way to distinguish between incoming and outgoing pair velocities because no trace of the parameter $\varepsilon$ is left in (23) and $n$ plays the role of a random parameter. However, keeping in mind the way we derived the Boltzmann equation, we shall conventionally maintain the name "incoming" for velocities satisfying the condition $\left(v-v_{2}\right) \cdot n \geq 0$ and consequently the pair $v^{\prime}$ and $v_{2}^{\prime}$ is outgoing in (23).

Equation (23) (or equivalently (10)-(11)) is the Boltzmann equation for hard spheres. Such an equation has a statistical nature, and it is not equivalent to the Hamiltonian dynamics from which it has been derived. Indeed the $H$ theorem shows that it is not reversible in time in contrast with the laws of mechanics. We note, incidentally, that this is not the case for the Vlasov equation, which inherits all the properties of the Hamiltonian systems. 
By the analysis on the order of magnitude of the quantities in the game, we deduced that the Boltzmann equation works in special situations only. The condition $N \varepsilon^{2}=O(1)$ means that we consider a rarefied gas, with almost vanishing volume density. After Boltzmann established the equation, Harold Grad [1949; 1958] postulated its validity in the limit $N \rightarrow \infty$ and $\varepsilon \rightarrow 0$ with $N \varepsilon^{2} \rightarrow$ const. as discussed above (this is often called, indeed, the Boltzmann-Grad limit).

There is no contradiction in the irreversibility or in the trend to equilibrium obtained after the limit, when they are strictly speaking false for mechanical systems. However, the arguments above are delicate and require a rigorous, deeper analysis. If the Boltzmann equation is not a purely phenomenological model derived by assumptions ad hoc and justified by its practical relevance, but rather a consequence of a mechanical model, we should derive it rigorously. In particular, the propagation of chaos should not be a hypothesis but the statement of a theorem.

After the formulation of the mathematical problem by Grad, Cercignani [1972] obtained the evolution equation (hierarchy) for the marginals of a hard-sphere system, and this was the starting point to rigorously derive the Boltzmann equation, as accomplished by Lanford [1975] in his famous paper, even though only for a short time interval.

Lanford's theorem is probably the most relevant result regarding the mathematical foundations of the kinetic theory. In fact, it dispelled the many previous doubts on the validity of the Boltzmann equation (although some authors refuse a priori the problem of deriving the equation starting from mechanical systems [Truesdell and Muncaster 1980]).

Unfortunately, the short-time limitation is serious. Only for special systems, as the case of a very rarefied gas expanding in a vacuum, can we obtain a global validity result [Illner and Pulvirenti 1986; 1989]. The possibility of deriving the Boltzmann equation globally in time, at least in cases when we have a good global existence of solutions, is still an open and challenging problem.

We conclude this section with some historical remarks.

Before Boltzmann, Maxwell proposed a kinetic equation that is nothing other than the Boltzmann equation integrated against test functions [Maxwell 1867; 1995]. He considered also more general potentials, in particular inverse-power-law potentials, essentially for the special properties of their cross-sections.

After Lanford's result, the case of smooth short-range potentials has been studied by other authors [King 1975; Gallagher et al. 2014; Pulvirenti et al. 2014], but the validity (or nonvalidity) of the Boltzmann equation in the case of genuine longrange potentials is open.

A rigorous derivation of the hierarchy of equations for hard spheres formally established by Cercignani is obtained in [Spohn 1991; Cercignani et al. 1994; Simonella 2014]. 


\section{The weak-coupling limit and the Landau equation}

The Boltzmann equation is suited to the description of rarefied gases, and one can ask whether a useful kinetic analysis can be applied also to the case of a dense gas. To introduce the problem, let us revisit first the Boltzmann-Grad limit in an alternative way. Let $\varepsilon$ be a small scale parameter denoting the ratio between the microscopic and macroscopic scales, for instance the inverse number of atomic diameters necessary to cover 1 meter or the inverse number of atomic characteristic times necessary to cover 1 second. Then scale space and time by $\varepsilon$ in the equations of motion (in our case, the hard-sphere hierarchy). We need to specify the number of particles $N$. In a box of side 1 , there should be $N \approx \varepsilon^{-3}$ particles if one assumes that the intermolecular distance is of the same order as the molecular diameter. The number of collisions of a given particle per macroscopic unit time would be $\varepsilon^{-1}$. As we have seen, in a low-density regime, $N$ scales differently, namely $N \approx \varepsilon^{-2}$, the number of collisions per unit time is finite and the one-particle distribution function satisfies the Boltzmann equation.

A variety of possible scalings describes different physical situations. For instance, the gas may be dense, $N=O\left(\varepsilon^{-3}\right)$ and the particles weakly interacting via a smooth two-body potential $\varphi$. To express the weakness of the interaction, we assume that $\varphi$ is rescaled by $\sqrt{\varepsilon}$. Since $\varphi$ varies on a scale $\varepsilon$ (in macroscopic units), the force will be $O(1 / \sqrt{\varepsilon})$ and act on a time interval $O(\varepsilon)$. The variation of momentum due to the single scattering is $O(\sqrt{\varepsilon})$, and the number of particles met by a typical particle is $O(1 / \varepsilon)$. Hence, the total momentum variation for unit time is $O(1 / \sqrt{\varepsilon})$. However, in the case of a homogeneous gas and symmetric forces, this variation should be zero in the average. The computation of the variance leads to a result $(1 / \varepsilon) O(\sqrt{\varepsilon})^{2}=O(1)$. Therefore, based on a central-limit type of argument, we expect that in the kinetic limit a diffusion equation in the velocity variable holds. Moreover, even though the force induced by a given particle on a test particle is $O(1 / \sqrt{\varepsilon})$ (i.e., not small as in the mean-field limit), the fact that it produces a small variation on the momentum should be sufficient to ensure propagation of chaos.

At the level of the kinetic equation (that is, assuming propagation of chaos), consider a model of collision with an operator of the form

$$
Q(f, f)=\int d v_{1} \int d p w(p) \delta\left(p^{2}+\left(v-v_{1}\right) \cdot p\right)\left[f^{\prime} f_{1}^{\prime}-f f_{1}\right]
$$

where

$$
f^{\prime}=f(v+p), \quad f_{1}^{\prime}=f\left(v_{1}-p\right) .
$$

Here $p$ is the transferred momentum in the collision, and $w$ is the probability density of having $p$ as an effect of the collision. The $\delta$ expresses energy conservation. 
To express the fact that the transferred momentum is small, let us rescale $w$ as $\left(1 / \varepsilon^{3}\right) w(p / \varepsilon)$ (so that the transferred momenta are $O(\varepsilon)$ ). In addition, let us rescale the inverse mean-free path by a factor $1 / \varepsilon$ to take into account the high density of particles. The collision operator becomes

$$
\begin{aligned}
& Q_{\varepsilon}(f, f)= \frac{1}{\varepsilon^{4}} \int d v_{1} \int d p w\left(\frac{p}{\varepsilon}\right) \delta\left(p^{2}+\left(v-v_{1}\right) \cdot p\right)\left[f^{\prime} f_{1}^{\prime}-f f_{1}\right] \\
&=\frac{1}{2 \pi \varepsilon^{2}} \int d v_{1} \int d p w(p) \int_{-\infty}^{+\infty} d s e^{i s\left(p^{2} \varepsilon+\left(v-v_{1}\right) \cdot p\right)} \\
& \times\left[f(v+\varepsilon p) f\left(v_{1}-\varepsilon p\right)-f(v) f\left(v_{1}\right)\right] \\
&=\frac{1}{2 \pi \varepsilon} \int d v_{1} \int d p w(p) \int_{0}^{1} d \lambda \int_{-\infty}^{+\infty} d s e^{i s\left(p^{2} \varepsilon+\left(v-v_{1}\right) \cdot p\right)} \\
& \times p \cdot\left(\nabla_{v}-\nabla_{v_{1}}\right) f(v+\varepsilon \lambda p) f\left(v_{1}-\varepsilon \lambda p\right) .
\end{aligned}
$$

Here the smooth function $w$, which modulates the collision, is assumed to depend on $p$ through its modulus only. Note that we used a change of variables $p / \varepsilon \rightarrow p$ and the representation formula in $\mathbb{R}^{1}$

$$
\delta(x)=\frac{1}{2 \pi \varepsilon} \int_{-\infty}^{+\infty} d s e^{i s x / \varepsilon} .
$$

To outline the behavior of $Q_{\varepsilon}(f, f)$ in the limit $\varepsilon \rightarrow 0$, we introduce a test function $u$ for which, after a change of variables (here $(\cdot, \cdot)$ denotes the scalar product in $\left.L^{2}(d v)\right)$,

$$
\begin{aligned}
\left(u, Q_{\varepsilon}(f, f)\right)=\frac{1}{2 \pi \varepsilon} & \int d v \int d v_{1} \int d p w(p) \int_{0}^{1} d \lambda \int_{-\infty}^{+\infty} d s \\
& \times e^{i s\left(p^{2}(\varepsilon-2 \varepsilon \lambda)+\left(v-v_{1}\right) \cdot p\right)} u(v-\varepsilon \lambda p) p \cdot\left(\nabla_{v}-\nabla_{v_{1}}\right) f f_{1} \\
=\frac{1}{2 \pi \varepsilon} & \int d v \int d v_{1} \int d p w(p) \int_{0}^{1} d \lambda \int_{-\infty}^{+\infty} d s \\
& \times e^{i s\left(v-v_{1}\right) \cdot p}\left[u(v)-\varepsilon \lambda p \cdot \nabla_{v} u(v)\right] p \cdot\left(\nabla_{v}-\nabla_{v_{1}}\right) f f_{1} \\
+ & \frac{1}{2 \pi} \int d v \int d v_{1} \int d p w(p) \int_{-\infty}^{+\infty} d s e^{i s\left(v-v_{1}\right) \cdot p} u(v) \\
& \times i s p^{2} \int_{0}^{1} d \lambda(1-2 \lambda) p \cdot\left(\nabla_{v}-\nabla_{v_{1}}\right) f f_{1}+O(\varepsilon) .
\end{aligned}
$$

Note now that the term $O\left(\varepsilon^{-1}\right)$ vanishes because of the symmetry $p \rightarrow-p(w$ is even). The last term also vanishes since the integral in $d \lambda$ is zero. As a result,

$$
\begin{aligned}
&\left(u, Q_{\varepsilon}(f, f)\right)=-\frac{1}{4 \pi} \int d v \int d v_{1} \int d p w(p) \int_{-\infty}^{+\infty} d s e^{i s\left(v-v_{1}\right) \cdot p} \\
& \times p \cdot \nabla_{v} u p \cdot\left(\nabla_{v}-\nabla_{v_{1}}\right) f f_{1}+O(\varepsilon) .
\end{aligned}
$$


Therefore, we have recovered (formally) the kinetic equation

$$
\left(\partial_{t}+v \cdot \nabla_{x}\right) f=Q_{L}(f, f)
$$

with a new collision operator

$$
Q_{L}(f, f)=\int d v_{1} \nabla_{v} a\left(\nabla_{v}-\nabla_{v_{1}}\right) f f_{1},
$$

where $a=a\left(v-v_{1}\right)$ denotes the matrix

$$
a_{i, j}(V)=\frac{1}{2} \int d p w(p) \delta(V \cdot p) p_{i} p_{j} .
$$

This matrix can be handled in a better way by introducing polar coordinates:

$$
\begin{aligned}
a_{i, j}(V) & =\frac{1}{2|V|} \int d p|p| w(p) \delta(\hat{V} \cdot \hat{p}) \hat{p}_{i} \hat{p}_{j} \\
& =\frac{B}{|V|} \int d \hat{p} \delta(\hat{V} \cdot \hat{p}) \hat{p}_{i} \hat{p}_{j},
\end{aligned}
$$

where $\hat{V}$ and $\hat{p}$ are the versors of $V$ and $p$, respectively, and

$$
B=\frac{1}{2} \int_{0}^{+\infty} d r r^{3} w(r) .
$$

Note that $B$ is the only parameter describing the interaction appearing in the equation. Finally a straightforward computation yields

$$
a_{i, j}(V)=\frac{B}{|V|}\left(\delta_{i, j}-\hat{V}_{i} \hat{V}_{j}\right) .
$$

The collision operator $Q_{L}$ was introduced by Landau in 1936 [Landau 1965; Lifshitz and Pitaevskiı 1981] for the study of a weakly interacting dense plasma, and (28) is called the Landau equation (sometimes Landau-Fokker-Planck).

The qualitative properties of the solutions to the Landau equation are the same as for the Boltzmann equation regarding the basic conservation laws and the $H$ theorem.

Following the paradigm of kinetic theory, we would like to derive the Landau equation from particle systems. A rigorous proof is however missing, even for short time intervals. We refer to [Boblylev et al. 2013] for a partial result.

\section{Some historical remarks}

The first attempt to implement the program of kinetic theory was due to Boltzmann, who derived his celebrated equation for rarefied gases in 1872 [Boltzmann 1964]. This followed some previous work of Maxwell. He wrote a system of equations 
in 1867 for the moments of the velocity distribution in order to justify the equilibrium measure which inherits his name [Maxwell 1867; 1995].

Boltzmann's work was attacked by several physicists and mathematicians due to the apparent basic contradiction between the $H$ theorem and the reversible nature of Newton's equations. In particular, the Poincaré recurrence theorem seemed to be in contrast with convergence toward an equilibrium state. Boltzmann replied to the criticisms asserting that the equation has a statistical meaning. Of course, he did not have at his disposal the mathematical tools suitable to make this statement more precise. We do not further discuss this interesting aspect and refer the reader to [Cercignani 1998].

In spite of the success of the Boltzmann equation in solving practical problems concerning rarefied gases, the issue of a rigorous justification of the equation remained open for a long time.

As already mentioned, a significant step forward was taken by Grad [1949; 1958], who figured out the scaling limit in which the equation is expected to hold, in the framework of classical mechanics. A second important contribution along the lines of Grad's approach was then made by Cercignani [1972], who established the hierarchy for the hard-sphere system whose first equation was written in Section 3. His analysis was formal, but it opened the way for Lanford's [1975] rigorous result on the short time validity of the Boltzmann equation.

Lanford's result solved the problem of conciliating the Boltzmann equation with the laws of classical mechanics.

On the other hand, even in recent times, the Boltzmann equation has often been considered a useful and successful tool of investigation and not necessarily as a direct consequence of the principles of mechanics. This is, for instance, the position of Truesdell and Muncaster [1980] in their famous monograph:

"We will make no attempt to trace the source of this irreversibility in more general theories or physico-philosophical speculations. Rather, in the spirit of rational mechanics, we shall attempt to determine its specific and rigorous mathematical nature and consequences."

Another attitude was the one of the great probabilist Kac [1956; 1959], who conceived the stochastic dynamics for an $N$-particle system yielding rigorously, in the limit $N \rightarrow \infty$, the homogeneous Boltzmann equation. His work is contemporary with the one of Grad; however, the point of view is very different. A footnote in [Kac 1956] reads,

"This formulation led to the well-known paradoxes which were fully discussed in the classical article of P. and T. Ehrenfest. These writers made it clear 
(a) that the 'Stosszahlansatz' cannot be strictly derivable from purely dynamic considerations and

(b) that the 'Stosszahlansatz' has to be interpreted probabilistically.

The recent attempts of Born and Green, Kirkwood and Bogoliubov to derive Boltzmann's equation from Liouville's equation and hence to justify the 'Stosszahlansatz' dynamically are, in our opinion, incomplete, inasmuch as they do not make it clear at what point statistical assumptions are introduced.

"The 'master equation' approach which we have chosen seems to us to follow closely the intentions of Boltzmann."

The works quoted after point (b) were the first to attempt a justification of kinetic equations based on a hierarchical technique, and the "Stosszahlansatz" is the property of propagation of chaos necessary to close the hierarchy (we refer in particular to [Bogolyubov 1946] for a pioneering analysis including the three classical kinetic equations).

Therefore, Kac's purpose is not just to provide a toy model as it is intended to be strongly related to the physics. A further quotation from the same paper is,

"Since the master equation is truly descriptive of the physical situation, and since existence and uniqueness of the solution of the master equation are almost trivial, the preoccupation with existence and uniqueness theorems for the Boltzmann equation appears to be unjustified on grounds of physical interest and importance."

An important point is that Kac's model is restricted to homogeneous situations (no dependence on positions). Interestingly enough, in the completely different context of numerical simulations of rarefied gases, Bird [1976] constructed the successful scheme known as DSMC (direct-simulation Monte Carlo), which splits the dynamics of a particle system into two parts: free motion and a stochastic interaction closely related to the one of Kac. In other words (without knowing Kac's work), Bird was providing an inhomogeneous stochastic model approximating the Boltzmann equation (see also [Cercignani et al. 1994] and references quoted therein).

Kac was greatly influenced by the famous treatise of Paul and Tatjana Ehrenfest [1959], where the conceptual bases of statistical mechanics are discussed. Here the authors try to explain the nature of the Boltzmann equation and the emergence of irreversibility with the aid of simple examples.

We note, incidentally, that in [Ehrenfest and Ehrenfest 1959] a model is introduced (often called the wind-tree model) in which a light (point) particle collides with a random distribution of square obstacles in the whole plane, in such a way that only four velocities are possible. The set of velocities is $\mathscr{V}=\left\{ \pm e_{1}, \pm e_{2}\right\}$, where 
$e_{i}, i=1,2$, are the versor of the coordinate axes in the plane. An elastic collision of the light particle with an obstacle with sides oriented at $\pi / 4$ is a rotation of the incoming velocity by $\pm \pi / 2$. The corresponding kinetic equation is linear and has the form

$$
\left(\partial_{t}+v \cdot \nabla_{x}\right) f=\frac{1}{2}\left(f\left(v^{\perp}\right)+f\left(-v^{\perp}\right)\right)-f(v)
$$

where $\mathscr{V} \ni v \rightarrow v^{\perp}$ is the rotation of $\pi / 2$.

For the more realistic "Lorentz model" with circular obstacles and velocity set $S^{1}$, a rigorous derivation of the linear Boltzmann equation was obtained by Gallavotti [1999] in a remarkable paper. His approach applies as well to the Ehrenfest wind-tree model.

We also mention a nonlinear version of the wind-tree model, namely the Broadwell model with kinetic equation

$$
\left(\partial_{t}+v \cdot \nabla_{x}\right) f=\left(f\left(v^{\perp}\right) f\left(-v^{\perp}\right)-f(v) f(-v)\right) .
$$

Surprisingly, this equation cannot be derived from the mechanical system of colliding square particles in the plane in the Boltzmann-Grad limit [Uchiyama 1988; Cercignani et al. 1994]. This counterexample shows how delicate a rigorous study of the low-density limit of deterministic systems can be.

As mentioned in Section 4, Landau proposed his kinetic equation for dense gases and plasmas in 1936. He started by assuming the Boltzmann equation with Coulomb cross-section, cutting divergences at short and at long distances. His argument is similar to the one presented here in Section 4, and the problem of the propagation of chaos is pragmatically avoided.

Bogolyubov [1946] works instead with the BBGKY hierarchy and asserts that it would be necessary to obtain the Landau equation starting from particle systems under a suitable scaling limit instead of starting from the Boltzmann equation directly. His discussion amounts to what is nowadays called the "weak-coupling limit" for the Landau equation (see also [Balescu 1975]) and includes an attempt to outline the various regimes in which the kinetic equations are expected to be valid, starting again from the hierarchy.

The Vlasov equation was introduced first in 1938 [Vlasov 1967] to study the time evolution of the distribution function of plasmas consisting of charged particles and long-range forces (for example, Coulomb) in contrast with the Landau equation, which is suited for particles interacting weakly via short-range forces. Actually both equations are needed to retain different aspects of the complicated dynamics of plasmas.

We shall conclude by recalling the famous speech by Hilbert [1902] at the International Congress of Mathematicians in Paris in 1900, where he posed twentythree problems as the basis of mathematical research in the forthcoming century. 
Among these, the sixth is perhaps a less definite problem but rather a broad field of investigation and a prophecy of the modern role of mathematics in physics. It is titled "Mathematical of the Axioms of Physics" and reads,

"The investigations on the foundation of geometry suggest [...] to treat in the same manner, by means of axioms, those physical sciences in which mathematics play an important part; in the first rank are the theory of probabilities and mechanics.

"As to the axioms of the theory of probabilities, it seems to me desirable that their logical investigation should be accompanied by a rigorous and satisfactory development of the method of mean values in mathematical physics, and in particular in the kinetic theory of gases.

"Important investigations by physicists on the foundations of mechanics are at hand [...]. Thus Boltzmann's work on the principles of mechanics suggests the problem of developing mathematically the limiting processes, there merely indicated, which lead from the atomistic view to the laws of the motion of continua."

The necessity of a rigorous approach to the scaling limits starting from fundamental particle models is clearly expressed. Moreover, the role of mathematics in investigating how different mathematical models of reality are connected is outlined:

"[...] Further, the mathematician has the duty to test exactly in each instance whether the new axioms are compatible with the previous ones. The physicist, as his theories develop, often finds himself forced by the results of the experiments to make new hypotheses, while he depends, with respect to the compatibility of the new hypotheses with the old axioms, solely upon these experiments or upon certain physical intuition, a practice which in the rigorously logical building up of a theory is not admissible."

Clarifying the scopes and methodologies of a physicist and a mathematician establishes the role of modern mathematical physics, in which the concept of a mathematical model, as a noncontradictory system of axioms, is fundamental.

The sixth problem of Hilbert can nowadays be further specified; namely there are at least three kinds of convergence which can be analyzed: (1) derive the Boltzmann equation from particle systems, (2) derive the Euler and/or Navier-Stokes equations from the Boltzmann equation and (3) derive the Euler and/or NavierStokes equations from particle systems.

We have remarkable progress regarding points (1) and (2). The first point has been discussed in this note. Regarding point (2), Hilbert [1912] himself introduced 
an expansion which is the basic tool for deriving the Euler equation for compressible fluids, in a suitable scaling limit, starting from the Boltzmann equation. Many rigorous results deriving hydrodynamic laws from the Boltzmann equation have been obtained over recent years. We underline that the hydrodynamic laws are the ones of a perfect gas since we start from a low-density regime. A more challenging problem is the derivation of the Euler equation from particle systems (point (3)). The laws relating density, pressure and temperature are not those of a perfect gas, but they may be computed through the Gibbs state associated with the interacting potential of the system. Such a difficult problem is unsolved on the mathematical side. We mention only the formal computations of the pioneering work by Morrey [1955]. Here the author gives a list of necessary steps to prove that the Euler equation can be obtained from the Newton laws. It is a notable work, having the merit of showing in a logically clear way what the link between the microscopic and macroscopic descriptions of fluids is. See [Esposito and Pulvirenti 2004] for a review of the argument.

\section{Acknowledgment}

We thank Thierry Paul for fruitful discussions.

\section{References}

[Balescu 1975] R. Balescu, Equilibrium and nonequilibrium statistical mechanics, Wiley-Interscience, New York, 1975.

[Bird 1976] G. A. Bird, Molecular gas dynamics, Clarendon, Oxford, 1976.

[Boblylev et al. 2013] A. V. Boblylev, M. Pulvirenti, and C. Saffirio, "From particle systems to the Landau equation: a consistency result", Comm. Math. Phys. 319:3 (2013), 683-702.

[Bogolyubov 1946] N. N. Bogolyubov, Problems of a dynamical theory in statistical physics, Gostekhisdat, Moscow, 1946. In Russian; translated in Studies in statistical mechanics, I, edited by J. de Boer and G. E. Uhlenbeck, North-Holland, Amsterdam, 1962.

[Boltzmann 1964] L. Boltzmann, Lectures on gas theory, University of California, Berkeley, CA, 1964.

[Cercignani 1972] C. Cercignani, "On the Boltzmann equation for rigid spheres", Transport Theory Statist. Phys. 2:3 (1972), 211-225.

[Cercignani 1998] C. Cercignani, Ludwig Boltzmann: the man who trusted atoms, Oxford University, 1998.

[Cercignani et al. 1994] C. Cercignani, R. Illner, and M. Pulvirenti, The mathematical theory of dilute gases, Applied Mathematical Sciences 106, Springer, New York, 1994.

[Dobrušin 1979] R. L. Dobrušin, "Vlasov equations", Funktsional. Anal. i Prilozhen. 13:2 (1979), 48-58. In Russian; translated in Funct. Anal. Appl. 13:2 (1979), 115-123.

[Ehrenfest and Ehrenfest 1959] P. Ehrenfest and T. Ehrenfest, The conceptual foundations of the statistical approach in mechanics, Cornell University, Ithaca, NY, 1959. 
[Esposito and Pulvirenti 2004] R. Esposito and M. Pulvirenti, "From particles to fluids", pp. 1-82 in Handbook of mathematical fluid dynamics, vol. III, edited by S. Friedlander and D. Serre, NorthHolland, Amsterdam, 2004.

[Gallagher et al. 2014] I. Gallagher, L. Saint-Raymond, and B. Texier, From Newton to Boltzmann: hard spheres and short-range potentials, Zurich Lectures in Advanced Mathematics 18, European Mathematical Society, Zürich, 2014.

[Gallavotti 1999] G. Gallavotti, “Grad-Boltzmann limit and Lorentz's gas”, Appendix 1.A2, pp. 48-55 in Statistical mechanics: a short treatise, Springer, Berlin, 1999.

[Grad 1949] H. Grad, "On the kinetic theory of rarefied gases", Comm. Pure Appl. Math. 2:4 (1949), 331-407.

[Grad 1958] H. Grad, "Principles of the kinetic theory of gases", pp. 205-294 in Handbuch der Physik, Band XII: Thermodynamik der Gase, edited by S. Flügge, Springer, Berlin, 1958.

[Hilbert 1902] D. Hilbert, "Mathematical problems", Bull. Amer. Math. Soc. 8:10 (1902), 437-479.

[Hilbert 1912] D. Hilbert, "Begründung der kinetischen Gastheorie", Math. Ann. 72:4 (1912), 562577.

[Illner and Pulvirenti 1986] R. Illner and M. Pulvirenti, "Global validity of the Boltzmann equation for a two-dimensional rare gas in vacuum”, Comm. Math. Phys. 105:2 (1986), 189-203.

[Illner and Pulvirenti 1989] R. Illner and M. Pulvirenti, "Global validity of the Boltzmann equation for two- and three-dimensional rare gas in vacuum: erratum and improved result", Comm. Math. Phys. 121:1 (1989), 143-146.

[Kac 1956] M. Kac, "Foundations of kinetic theory", pp. 171-197 in Proceedings of the Third Berkeley Symposium on Mathematical Statistics and Probability (Berkeley, CA, 1954-1955), vol. III, edited by J. Neyman, University of California, Berkeley, CA, 1956.

[Kac 1959] M. Kac, Probability and related topics in physical sciences: proceedings of the summer seminar (Boulder, CO, 1957), Lectures in Applied Mathematics 1, Interscience, London, 1959.

[King 1975] F. G. King, BBGKY hierarchy for positive potentials, Ph.D. thesis, University of California, Berkeley, 1975, Available at http://search.proquest.com/docview/302766232.

[Landau 1965] L. D. Landau, "The transport equation in the case of Coulomb interactions", Chapter 24, pp. 163-170 in Collected papers of L. D. Landau, edited by D. ter Haar, Pergamon, Oxford, 1965.

[Lanford 1975] O. E. Lanford, III, "Time evolution of large classical systems", pp. 1-111 in Dynamical systems, theory and applications (Seattle, 1974), edited by J. Moser, Lecture Notes in Physics 38, Springer, Berlin, 1975.

[Lifshitz and Pitaevskiı̌ 1981] E. M. Lifshitz and L. P. Pitaevskiı̆, Course of theoretical physics, vol. 10: Physical kinetics, edited by L. D. Landau and E. M. Lifshitz, Pergamon, Oxford, 1981.

[Maxwell 1867] J. C. Maxwell, "On the dynamical theory of gases", Philos. Trans. Roy. Soc. London 157 (1867), 49-88.

[Maxwell 1995] J. C. Maxwell, The scientific letters and papers of James Clerk Maxwell, vol. II: 1862-1873, edited by P. M. Harman, Cambridge University, 1995.

[Morrey 1955] C. B. Morrey, Jr., "On the derivation of the equations of hydrodynamics from statistical mechanics”, Comm. Pure Appl. Math. 8:2 (1955), 279-326.

[Pulvirenti et al. 2014] M. Pulvirenti, C. Saffirio, and S. Simonella, "On the validity of the Boltzmann equation for short range potentials”, Rev. Math. Phys. 26:2 (2014), 1450001.

[Simonella 2014] S. Simonella, "Evolution of correlation functions in the hard sphere dynamics", $J$. Stat. Phys. 155:6 (2014), 1191-1221. 
[Spohn 1991] H. Spohn, Large scale dynamics of interacting particles, Springer, Berlin, 1991.

[Truesdell and Muncaster 1980] C. Truesdell and R. G. Muncaster, Fundamentals of Maxwell's kinetic theory of a simple monatomic gas: treated as a branch of rational mechanics, Pure and Applied Mathematics 83, Academic, New York, 1980.

[Uchiyama 1988] K. Uchiyama, "On the Boltzmann-Grad limit for the Broadwell model of the Boltzmann equation”, J. Statist. Phys. 52:1-2 (1988), 331-355.

[Vlasov 1967] A. A. Vlasov, "The vibrational properties of an electron gas", Usp. Fiz. Nauk. 93 (1967), 444-470. In Russian; translated in Phys. Usp. 10:6 (1968), 721-733.

Received 28 Jul 2016. Accepted 3 Oct 2016.

MARIO PULVIRENTI: pulviren@mat.uniroma1.it

Dipartimento di Matematica, Università di Roma La Sapienza, Piazzale Aldo Moro 5, I-00185 Rome, Italy

SERGio Simonella: s.simonella@tum.de

Zentrum Mathematik, Technische Universität München, Boltzmannstraße 3, D-85748 Garching, Germany 


\title{
WHAT DECIDES THE DIRECTION OF A CURRENT?
}

\author{
CHRISTIAN MAES \\ Dedicated in honor of Lucio Russo
}

\begin{abstract}
Nonequilibria show currents that are maintained as the result of steady driving. We ask here what decides their direction. It is not only the second law or the positivity of the entropy production; nondissipative aspects also often matter and sometimes completely decide the direction.
\end{abstract}

1. Introduction

2. Traditional arguments 277

3. Problematic cases 282

4. Low temperature analysis 290

5. Conclusion 294

Acknowledgment 294

References $\quad 294$

\section{Introduction}

Predicting the course of events given the present state is part of scientific practice. In what direction things will evolve is however not always so evident. In thermodynamics, there are a number of general rules of thumb derived from the principal laws. For instance, macroscopic systems tend to equilibrate at the same temperature, chemical potential and pressure as the surroundings, relaxation (or time itself) flows in the direction of increasing entropy, etc. In mechanics, the ambition is even higher; we compute trajectories given the present state. Statistical mechanics is supposed to transfer mechanical laws to thermodynamic behavior, with the attenuation that some thermodynamic principles are not absolute but become statistical. For example, the Boltzmann equation for a dilute gas has a direction of time, but for mesoscopic systems, fluctuations can be expected, and as Maxwell emphasized,

\section{Communicated by Raffaele Esposito.}

MSC2010: 82C03.

Keywords: direction current, nonequilibrium statistical mechanics. 
"The truth of the second law is ... a statistical, not a mathematical, truth, for it depends on the fact that the bodies we deal with consist of millions of molecules ... Hence the second law of thermodynamics is continually being violated, and that to a considerable extent, in any sufficiently small group of molecules belonging to a real body" [Maxwell 1878].

That is, statistical mechanics will not only derive thermodynamics, it will also correct it and extend it. That is especially true for nonequilibrium statistical mechanics as there we are necessarily dealing with atypical behavior from the point of view of the microcanonical ensemble. It therefore becomes both a major inspiration and application of probability theory, exactly in the way Lucio Russo has been enjoying and contributing to it.

Going to irreversible thermodynamics [de Groot and Mazur 1962], that is, the thermodynamics for irreversible phenomena, the main guiding principle that survives for the direction of currents is the positivity of the entropy production. We are for example considering an open macroscopic system which is being steadily frustrated by contacts with different equilibrium baths. Currents will be maintained, at least on the time scales where the environment is kept at the same intensive values (e.g., temperature). The directions of these currents can and will vary with different arrangements, but the entropy production $\Sigma$ is positive. We denote by $\Sigma=\sum_{\alpha} J_{\alpha} F_{\alpha}$ a sum over all possible types of channels of transport of the product of currents (or displacements) $J_{\alpha}$ and thermodynamic forces $F_{\alpha}$. For predicting the current directions, we just see what is compatible with $\Sigma \geq 0$, nothing more. In the linear regime, where currents are proportional to forces, $J_{\alpha}=\sum_{\gamma} L_{\alpha \gamma} F_{\gamma}$ with symmetric ${ }^{1}$ Onsager linear response coefficients $L_{\alpha \gamma}=L_{\gamma \alpha}$, and the positivity of $\Sigma$ is the positivity of the matrix $\left(L_{\alpha \gamma}\right)$. Here again, statistical mechanics will derive and extend that scheme, but now it should be nonequilibrium statistical mechanics. That is very much unfinished business and could certainly go beyond the linear regime around zero thermodynamic forces. In fact, nonequilibrium statistical mechanics is far behind the equilibrium version:

"My inclination is to postpone the study of the large-system limit: Since it is feasible to analyze the nonequilibrium properties of finite systems - as Gibbs did for their equilibrium properties - it seems a good idea to start there. That may not answer all questions, but it advances nonequilibrium statistical mechanics to the point equilibrium had reached after Gibbs" [Ruelle 2004].

In other words, a general theory of nonequilibrium phase transitions or of universality is still nonexistent, and even a systematic way of dealing with many-body

\footnotetext{
${ }^{1}$ We ignore here the Casimir correction that takes into account the parity under time-reversal of the physical quantity being transported.
} 


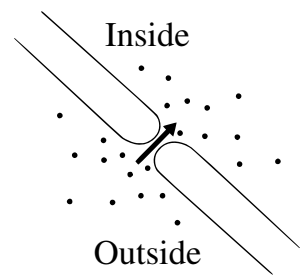

Figure 1. Example of a simple stationary current for which the direction is decided by the positivity of the entropy production.

effects is largely lacking. We certainly have no percolation or geometric picture of nonequilibrium collective phenomena, and remembering the crystal-clear and perfectly elegant contributions of Lucio to percolation theory and to mathematical statistical mechanics, we can only hope that the day will soon come when such a mathematical framework and geometric interpretation will also become available for nonequilibrium physics to match Lucio's standards.

In what follows, we are asking about what determines the direction of a nonequilibrium current. The main point will be that it is certainly not always the case that the current direction is decided by the positivity of the entropy production; nondissipative effects will be important and sometimes crucial. We refer to the pedagogical introduction [Maes 2015] on nondissipative aspects of nonequilibrium statistical mechanics. For the moment, it suffices to add that transition rates in a process also have time-symmetric parameters and, quite obviously, that we need to understand how they contribute to deciding the direction of the current.

\section{Traditional arguments}

2A. Phenomenology. The media inside and outside of a biological cell can be very different. These are connected via thin pores through which ions of various chemicals can be transported. Consider such a pore or channel in the membrane separating outside and inside; see Figure 1. Because of different concentrations at its ends, there will be a current through the pore. In fact, ions will be traveling from the region of higher chemical potential to the region of lower chemical potential. The same thing happens with many types of currents, whether the channel is connected to thermal, chemical or mechanical reservoirs. At the appropriate scale of time, the system is in steady nonequilibrium, not changing its macroscopic appearance. There is a constant production $\Sigma$ of entropy in the environment, which is positive,

$$
\Sigma=-\beta \mu_{1} J_{1}-\beta \mu_{2} J_{2}
$$

with $J_{i}$ the particle flux into the $i$-th reservoir at chemical potential $\mu_{i}$ and inverse temperature $\beta$. Stationarity (and bulk conservation of particles) implies $J_{1}+J_{2}=0$ 


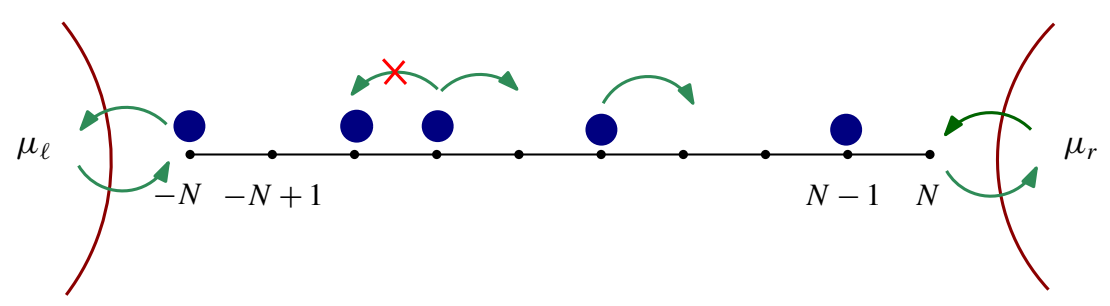

Figure 2. Stochastic lattice gas, symmetric in the bulk and governed by local interactions, driven by contact with particle reservoirs at different chemical potentials.

so that we can find the direction of the particle current $J_{1}$ by requiring

$$
\Sigma=\beta J_{1}\left(\mu_{2}-\mu_{1}\right) \geq 0 \quad \text { (second law). }
$$

By bulk conservation of particles, we still have $J_{1}=J$, the stationary particle current through the channel or pore from the second towards the first reservoir, and hence, $J \geq 0$ whenever $\mu_{2} \geq \mu_{1}$.

Similar scenarios can be written for thermal and mechanical baths that frustrate the system. Those are the typical cases where finding the direction of the current amounts to applying the second law in the form such that the stationary entropy production is positive.

While the previous case was treated rather phenomenologically, precise mathematical arguments can be provided for simple particle model systems following the same physics. Here comes an example.

2B. Stochastic lattice gas. We consider identical particles that can jump from site $i$ to the nearest neighbor site $j=i \pm 1$ on the finite linear chain $\Lambda_{N}=$ $\{-N,-N+1, \ldots, 0,1, \ldots, N-1, N\}$; see Figure 2. The endpoints $i= \pm N$ in $\Lambda_{N}$ are called the boundary of the system; the other sites are in the bulk. There is at most one particle per site $i$ so that a site $i$ can be vacant or occupied, and we write $\eta(i) \in\{0,1\}$ for the occupation at site $i \in \Lambda_{N}$. The state space is $K=\{0,1\}^{\Lambda_{N}}$ with elements $\eta, \eta^{\prime}, \xi, \ldots \in K$. The reasoning below is outlined in [Maes et al. 2009].

The energy function on $K$ is chosen as

$$
H(\eta)=-B \sum_{i=-N}^{N} \eta(i)-\kappa \sum_{i=-N}^{N-1} \eta(i) \eta(i+1),
$$

where $B$ and $\kappa$ are some real constants. The system is imagined in thermal contact with a very large heat bath at inverse temperature $\beta$ (Boltzmann's constant is set equal to 1). The energy change in that bath over the transition $\eta \rightarrow \eta^{\prime}$ gives a first contribution $\beta\left(H(\eta)-H\left(\eta^{\prime}\right)\right)$ to the change of entropy in the reservoir. Another 
important quantity here is the particle number

$$
\mathcal{N}_{[j, k]}(\eta)=\sum_{i=j}^{k} \eta(i)
$$

in the lattice interval $[j, k] \cap \Lambda_{N},-N \leq j \leq k \leq N$. The total number of particles inside the system is $\mathcal{N}=\mathcal{N}_{[-N, N] \text {. }}$

We now also imagine that the system is in contact with a particle reservoir at each of its boundary sites. There can be a birth or a death of a particle at these sites, which amounts to the entrance from and the exit to the corresponding reservoir of a particle. In that sense, we write $J_{\ell}=\Delta \mathcal{N}_{\ell}$ and $J_{r}=\Delta \mathcal{N}_{r}$ as the changes in particle number in the left and right particle reservoirs, respectively. The flow of particles in and out of the system can also contribute to the dissipated heat in the reservoir and hence to changes in entropy:

$$
S\left(\eta, \eta^{\prime}\right)=\beta\left[H(\eta)-H\left(\eta^{\prime}\right)\right]-\beta \mu_{\ell} \Delta \mathcal{N}_{\ell}\left(\eta, \eta^{\prime}\right)-\beta \mu_{r} \Delta \mathcal{N}_{r}\left(\eta, \eta^{\prime}\right)
$$

is the change of entropy in the environment for $\mu_{\ell}$ and $\mu_{r}$, the chemical potentials (up to some factor $\beta$ that we have ignored) of the left and right particle reservoirs, respectively. We will make mathematical sense of (2) entirely in terms of variables inside the system.

For the dynamics, we choose a continuous-time Markov process on $K$. Write the transformation

$$
\eta^{i, j}(k)= \begin{cases}\eta(k) & \text { if } k \neq i \text { and } k \neq j \\ \eta(i) & \text { if } k=j \\ \eta(j) & \text { if } k=i\end{cases}
$$

for the state obtained from $\eta$ after exchanging the occupation of the sites $i$ and $j$, only allowed for $j=i \pm 1$. The rate for that transition is taken to be

$$
C(i, j, \eta)=\exp \left[-\frac{\beta}{2}\left(H\left(\eta^{i, j}\right)-H(\eta)\right)\right], \quad|i-j|=1 .
$$

Similarly, the rate of birth and death for the transition $\eta \rightarrow \eta^{i}$ with

$$
\eta^{i}(k)= \begin{cases}1-\eta(k) & \text { if } k=i, \\ \eta(k) & \text { if } k \neq i,\end{cases}
$$

only occurring at sites $i=-N, N$, is

$$
C(i, \eta)=e^{-a_{i} \eta(i)} \exp \left[-\frac{\beta}{2}\left(H\left(\eta^{i}\right)-H(\eta)\right)\right] .
$$

The relevant parameters are the values $a_{-N}=\beta \mu_{\ell}$ and $a_{N}=\beta \mu_{r}$ representing the (different) chemical potentials of the two reservoirs at the outer edges. 
One observes from the definition (3) that

$$
\frac{C(i, j, \eta)}{C\left(i, j, \eta^{i, j}\right)}=\frac{\exp \left[-\beta H\left(\eta^{i, j}\right)\right]}{\exp [-\beta H(\eta)]}
$$

Furthermore, from (4),

$$
\frac{C(i, \eta)}{C\left(i, \eta^{i}\right)}=\frac{\exp \left[-a_{i} \eta(i)\right]}{\exp \left[-a_{i}(1-\eta(i))\right]} \frac{\exp \left[-\beta H\left(\eta^{i}\right)\right]}{\exp [-\beta H(\eta)]}, \quad i= \pm N .
$$

For $a_{-N}=a_{N}=a$, when the left and right particle reservoirs have equal concentration, then the system dynamics satisfies the condition of detailed balance: for all allowed transitions $\eta \rightarrow \eta^{\prime}$ and corresponding transition rates $W\left(\eta \rightarrow \eta^{\prime}\right)$,

$$
\frac{W\left(\eta \rightarrow \eta^{\prime}\right)}{W\left(\eta^{\prime} \rightarrow \eta\right)}=\frac{\mathbb{P}^{\beta, a}\left[\eta^{\prime}\right]}{\mathbb{P}^{\beta, a}[\eta]}
$$

for the grand-canonical equilibrium probabilities

$$
\mathbb{P}^{\beta, a}[\eta]=\frac{1}{\mathscr{L}} e^{a \sum \eta(i)} e^{-\beta H(\eta)}
$$

where $\mathscr{E}=\mathscr{L}(a, \beta, N)$ is a normalization factor. Thus, (8) is a reversible stationary measure when $a_{-N}=a_{N}=a$.

We now consider $a_{1} \neq a_{N}$ (different chemical potentials). At the left boundary of the system (see (6)),

$$
\frac{C(-N, \eta)}{C\left(-N, \eta^{-N}\right)}=e^{-\beta\left[H\left(\eta^{-N}\right)-H(\eta)\right]-a_{-N} J_{\ell}\left(\eta, \eta^{-N}\right)}
$$

where $J_{\ell}\left(\eta, \eta^{-N}\right)=1$ when the particle leaves the system via the site $-N$, i.e., $\eta(-N)=1$, and $J_{\ell}\left(\eta, \eta^{-N}\right)=-1$ when a new particle enters, i.e., $\eta(-N)=0$. Analogously, the current $J_{r}\left(\eta, \eta^{\prime}\right)=1$ when $\eta(N)=1$ and $\eta^{\prime}=\eta^{N}$ and $J_{\ell}\left(\eta, \eta^{\prime}\right)=$ -1 when $\eta(N)=0$ and $\eta^{\prime}=\eta^{N}$. The currents are zero otherwise.

As a consequence,

$$
\frac{W\left(\eta \rightarrow \eta^{\prime}\right)}{\left.W\left(\eta^{\prime} \rightarrow \eta\right)\right)}=e^{-\beta\left[H\left(\eta^{\prime}\right)-H(\eta)\right]-a_{-N} J_{\ell}\left(\eta, \eta^{\prime}\right)-a_{N} J_{r}\left(\eta, \eta^{\prime}\right)}
$$

where we see the change of entropy (2). In other words,

$$
\frac{W\left(\eta \rightarrow \eta^{\prime}\right)}{W\left(\eta^{\prime} \rightarrow \eta\right)}=e^{S\left(\eta, \eta^{\prime}\right)}
$$

(which is known as the condition of local detailed balance), and

$$
J_{\ell}\left(\eta, \eta^{\prime}\right)+J_{r}\left(\eta, \eta^{\prime}\right)=\mathcal{N}(\eta)-\mathcal{N}\left(\eta^{\prime}\right)
$$


or, with $a_{N}=a$ and $a_{-N}=a+\delta$,

$$
\frac{W\left(\eta \rightarrow \eta^{\prime}\right)}{W\left(\eta^{\prime} \rightarrow \eta\right)}=\frac{\mathbb{P}^{\beta, a}\left[\eta^{\prime}\right]}{\mathbb{P}^{\beta, a}[\eta]} e^{-\delta J_{\ell}\left(\eta, \eta^{\prime}\right)}
$$

with $\delta$ thus quantifying the amplitude of breaking of detailed balance.

As above, we define the bulk currents $J_{i}\left(\eta, \eta^{\prime}\right)$ to be +1 if in the transition $\eta \rightarrow \eta^{\prime}$ a particle moves over the bond $i \rightarrow i+1$ and equal to -1 if a particle moves $i \leftarrow i+1$. In fact, throughout, we confuse current with what is more like a time-integrated current or a change of particle number.

We have piecewise-constant paths $\omega$ over the time interval $[0, \tau]$, starting from some initial configuration $\eta_{0}$ after which it changes into $\eta_{t_{1}}, \eta_{t_{2}}, \ldots$ at random times $t_{1}, t_{2}, \ldots$ At the jump times, we take $\eta_{t_{k-1}}=\eta_{t_{k}^{-}}$and $\eta_{t_{k}}=\eta_{t_{k}^{+}}$for having right-continuous paths with left limits. The time-reversal transformation on pathspace $\Theta$ is defined via $(\Theta \omega)_{t}=\omega_{\tau-t}$, up to irrelevant modifications at the jump times making $\Theta \omega$ right-continuous again.

We consider a path $\omega=\left(\eta_{t}\right)_{t=0}^{\tau}$ and currents $J_{i}(\omega), i=-N, \ldots, N$, defined by

$$
J_{i}(\omega)=J_{i}\left(\eta_{0}, \eta_{t_{1}}\right)+J_{i}\left(\eta_{t_{1}}, \eta_{t_{2}}\right)+\cdots+J_{i}\left(\eta_{t_{n-1}}, \eta_{\tau}\right) .
$$

In particular, $J_{r}=J_{N}$ and for $i \leq k$

$$
\begin{aligned}
J_{i}(\omega)-J_{k}(\omega) & =\mathcal{N}_{[i+1, k]}\left(\eta_{\tau}\right)-\mathcal{N}_{[i+1, k]}\left(\eta_{0}\right), \\
J_{\ell}(\omega)+J_{-N}(\omega) & =\eta_{0}(-N)-\eta_{\tau}(-N) .
\end{aligned}
$$

Observe that the currents $J_{i}$ are extensive in the time $\tau$.

All of that is related to the process, be it transient or steady. We concentrate on the steady-state regime. It is easy to verify that we have here a unique stationary distribution $\rho$. That stationary distribution is only implicitly known and a solution of the (time-independent) master equation. Corresponding to $\rho$ is then a stationary process with distribution $\boldsymbol{P}_{\rho}$. If we look at expectations in the stationary process, we write $\langle\cdot\rangle_{\rho}$.

From the conservation laws (12) and (13),

$$
\left\langle J_{\ell}\right\rangle_{\rho}=-\left\langle J_{r}\right\rangle_{\rho}=-\left\langle J_{i}\right\rangle_{\rho}, \quad i \in \Lambda_{N} .
$$

Proposition 2.1. The direction of the current is from higher to lower chemical potential; i.e., assuming that $\delta \geq 0\left(\right.$ or $\left.a_{-N}=\mu_{\ell} \geq a_{N}=\mu_{r}\right)$, we have $\left\langle J_{i}\right\rangle_{\rho} \geq 0$.

Proof. The path density of $\boldsymbol{P}_{\rho}$ with respect to $\boldsymbol{P}_{\rho} \Theta$, both started in the stationary distribution $\rho$, is

$$
\frac{\mathrm{d} \boldsymbol{P}_{\rho}}{\mathrm{d} \boldsymbol{P}_{\rho} \Theta}(\omega)=\frac{\rho\left(\omega_{0}\right)}{\rho\left(\omega_{\tau}\right)} \exp \left[-\beta\left(H\left(\omega_{\tau}\right)-H\left(\omega_{0}\right)\right)+a \Delta \mathcal{N}-\delta J_{\ell}(\omega)\right]
$$


By normalization,

$$
\int \mathrm{d} \boldsymbol{P}_{\rho}(\omega) \frac{\mathrm{d} \boldsymbol{P}_{\rho} \Theta}{\mathrm{d} \boldsymbol{P}_{\rho}}(\omega)=1
$$

and hence, by concavity,

$$
\int \mathrm{d} \boldsymbol{P}_{\rho}(\omega) \log \frac{\mathrm{d} \boldsymbol{P}_{\rho} \Theta}{\mathrm{d} \boldsymbol{P}_{\rho}}(\omega) \leq 0 .
$$

But from (14) and by stationarity,

We conclude that

$$
0 \leq \int \mathrm{d} \boldsymbol{P}_{\rho} \log \frac{\mathrm{d} \boldsymbol{P}_{\rho}}{\mathrm{d} \boldsymbol{P}_{\rho} \Theta}(\omega)=-\delta\left\langle J_{\ell}\right\rangle_{\rho}=\delta\left\langle J_{i}\right\rangle_{\rho} .
$$

$$
\delta\left\langle J_{i}\right\rangle_{\rho} \geq 0
$$

which shows that the average direction of the particle current depends only on the sign of $\delta$.

Getting a strict inequality $\left\langle J_{i}\right\rangle_{\rho}>0$ is also possible for $\delta>0$; it suffices to see that there is a nonzero probability that the current $J_{i}$ as a function of the path $\omega$ is not identically equal to 0 even when $\omega_{0}=\omega_{\tau}$.

Looking back at the proof, we see that the main inequality has been the positivity (15) of the relative entropy between the forward and backward stationary processes. The latter coincides with the stationary entropy production, as is in fact visible from (11). Hence, the proof above, as in [Maes et al. 2009], is a nonperturbative statistical mechanical argument or the physical analogue for the phenomenology in Section 2A; there is nothing really new here.

\section{Problematic cases}

We collect a number of situations where either previous phenomenological or statistical mechanical arguments, based on the positivity of the entropy production, do not work. From a general perspective comparable to the so-called Curie principle, currents may appear whenever they are not forbidden by some symmetry. It is then not wholly surprising that we cannot always apply the same physical arguments. Yet the examples below are specifically relevant in the context of nonequilibrium physics, for which we may hope to develop some framework.

\section{A. Ratchet currents.}

3A1. Triangula. In [Van den Broeck et al. 2005], a number of versions of harddisk microscopic ratchets are introduced and studied with molecular dynamics and with some low-density expansions. A directed systematic motion appears when a temperature difference is applied to different units of a motor. One of the simple 


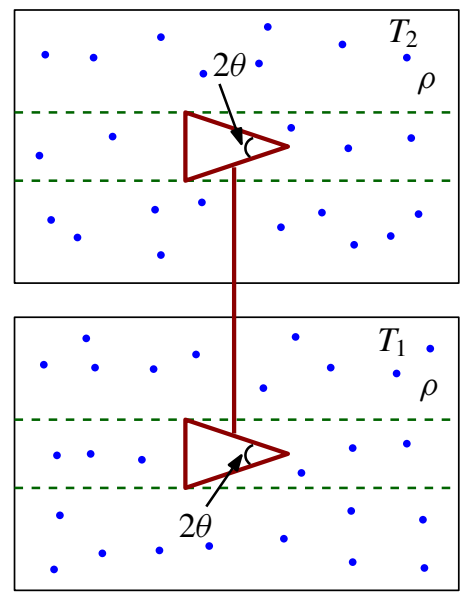

Figure 3. Triangula: the triangles can only move horizontally and are connected. They are in thermal baths at different temperatures.

examples is there called the Triangula: it is a motor consisting of two identical triangular units, each sitting in a gas (reservoir) consisting of hard disks whose centers collide elastically with the triangles; see Figure 3. The two triangles are rigidly connected along a rod, with their base parallel to it, and the whole motor is constrained to move along the horizontal direction without rotation or vertical displacement. When the temperatures in the two reservoirs are different, a systematic motion appears which turns out to be in the direction of the triangles' apices - to the right in Figure 3. The speed $V$ of the Triangula depends on the difference in temperatures $T_{1}$ and $T_{2}$ and on the apex angle $2 \theta$ and to some good approximation for low-density reservoirs is given by

$$
V=(1-\sin \theta) \frac{\sqrt{2 \pi k_{B} m}}{4 M}\left(T_{1}-T_{2}\right) \frac{\left(\sqrt{T_{1}}-\sqrt{T_{2}}\right)}{\left(\sqrt{T_{1}}+\sqrt{T_{2}}\right)^{2}}
$$

for $m$ the mass of the gas particle and $M$ the mass of the triangle (see (22) in [Van den Broeck et al. 2005] for the case of equal densities). If we write $T_{2}=$ $T(1+\varepsilon)$ and $T_{1}=T$, the leading order for that formula with $\varepsilon \downarrow 0$ becomes

$$
M V \simeq \frac{1-\sin \theta}{32} \sqrt{2 \pi k_{B} T m} \varepsilon^{2}
$$

and we see that the speed or current is second-order in the temperature difference. That is not attainable with linear response theory around equilibrium. The reason is that the translation current is orthogonal to the heat current (through the rod). Since we are thus in the regime of nonlinear response, that should already tell us that nondissipative features play a role [Basu et al. 2015]. As far as we know, nobody has a good heuristic or simple argument to explain that indeed $V>0$. Equation (16) 
connects the momentum of the Triangula with the thermal momentum of the gas, which appears rectified depending on the apex angle and is second-order between the temperatures, and is basically left without further explanation.

3A2. Parrondo game. The following is a paradoxical game invented by Juan Parrondo in 1996; see [Parrondo et al. 2000] for more explanations and references.

The state space is $K=\{1,2,3\}$, and the state at time $n$ is $x_{n}$. The Markov chain uses a different rule ( $A$ or $B$ ) at even and odd times $n$. Alternating, the following two games are played. Game $A$ is fair coin tossing: we simply move $x \rightarrow x \pm 1 \bmod 3$ with equal probability at even times. Game $B$ is played at odd times and with two biased coins, a good one and a bad one. In game $B$, the good coin is tossed when $x_{n} \in\{1,2\}$ and the bad coin is used each time when $x_{n}=3$. Winning takes $x_{n+1}=x_{n}+1$; losing at time $n$ means $x_{n+1}=x_{n}-1$, always modulo 3 . The transition probabilities are then

$$
\begin{array}{ll}
\operatorname{Prob}\left[x_{n+1}=x \pm 1 \mid x_{n}=x\right]=\frac{1}{2} & \text { when } n \text { is even, } \\
\operatorname{Prob}\left[x_{n+1}=x+1 \mid x_{n}=x\right]=\frac{3}{4} & \text { when } n \text { is odd and } x \neq 3, \\
\operatorname{Prob}\left[x_{n+1}=x+1 \mid x_{n}=x\right]=\frac{1}{10} & \text { when } n \text { is odd and } x=3 .
\end{array}
$$

Both games, when played separately at all times, are reversible. For example, for game $B$ (at all times), consider the cycle $3 \rightarrow 1 \rightarrow 2 \rightarrow 3$. Its stationary probability (always for game $B$ alone) is $\operatorname{Prob}[3 \rightarrow 1 \rightarrow 2 \rightarrow 3]=\rho(3) \times \frac{1}{10} \times \frac{3}{4} \times \frac{3}{4}=\frac{9}{160} \rho(3)$. For the reversed cycle, the probability $\operatorname{Prob}[3 \rightarrow 2 \rightarrow 1 \rightarrow 3]=\rho(3) \times \frac{9}{10} \times \frac{1}{4} \times \frac{1}{4}=$ $\frac{9}{160} \rho(3)$ is the same. The equilibrium distribution for game $B$ is then found to be $\rho(1)=\frac{2}{13}, \rho(2)=\frac{6}{13}$ and $\rho(3)=\frac{5}{13}$. Obviously then, there is no current when playing game $B$ and clearly the same is trivially verified for game $A$ when tossing with the fair coin. Yet, and here is the paradox, when periodically playing game $B$ after game $A$, a current arises.

As in the previous case of the Triangula, the very fact that a current arises is again not so strange, but the question is what really decides its direction. We will show how to solve that question for a continuous-time version at low temperature in Section 4.

3B. Multiple cycles. It is not uncommon in nonequilibrium to have multiple cycles in state space along which the dynamics can proceed. We give here two examples of one-dimensional random walks, visualized in Figures 4-6. Look first at Figure 4 , where we denote the states by $\{0, u, 1\}$ in an elementary triangle. For horizontal motion, there are two "channels" to move to the right, $0 \rightarrow u \rightarrow 1$ and $0 \rightarrow 1$, and two "channels", $0 \rightarrow u \rightarrow-1$ and $0 \rightarrow-1$, to move to the left. Going right, the system prefers the "channel" $0 \rightarrow u \rightarrow 1$, and for going left, the system 


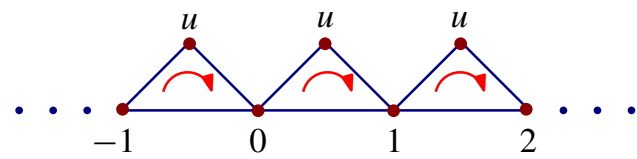

Figure 4. Necklace of three-state cycles with rotational current inducing a horizontal current.

prefers the channel $0 \rightarrow-1$ as we take the transition rates

$$
\begin{array}{lll}
k(1,0)=\varphi e^{\varepsilon / 2}, & k(0, u)=e^{\varepsilon / 2}, & k(u, 1)=e^{\varepsilon / 4}, \\
k(0,1)=\varphi e^{-\varepsilon / 2}, & k(u, 0)=1, & k(1, u)=e^{-\varepsilon / 4}
\end{array}
$$

for parameters $\varphi, \varepsilon>0$. That is periodically repeated to obtain a random walk on the line. We are interested in the physical "translational" current towards the right, that is,

$$
J=\rho(0)\left[e^{\varepsilon / 2}+\varphi e^{-\varepsilon / 2}\right]-\left[\rho(u)+\rho(0) \varphi e^{\varepsilon / 2}\right]
$$

where we restrict ourselves to translation-invariant stationary occupations satisfying the normalization $\rho(0)+\rho(u)=1$ and

$$
\rho(0)\left[e^{\varepsilon / 2}+e^{-\varepsilon / 4}\right]=\rho(u)\left[1+e^{\varepsilon / 4}\right] .
$$

We claim that entropy production decides the orientation of the rotational current within each triangle but not the direction of the induced translational current $J$. The $\varepsilon$ in (18) decides the direction of the rotational current and is responsible for the breaking of detailed balance. It stands for an entropy flux (per $k_{B}$ ). For example, the trajectory $0 \rightarrow u \rightarrow 1$ (taking the walker one step to the right) expends an entropy flux $\varepsilon$ (e.g., in the sense of [Maes et al. 2000]), as seen from the calculation

$$
\frac{k(0, u) k(u, 1)}{k(u, 0) k(1, u)}=\frac{e^{\varepsilon / 2} e^{\varepsilon / 4}}{1 \cdot e^{-\varepsilon / 4}}=e^{\varepsilon},
$$

but so does the step $0 \rightarrow-1$ exactly: taking the walker one step to the left,

$$
\frac{k(1,0)}{k(0,1)}=e^{\varepsilon} .
$$

In all, there is no entropic preference to go right or left. In other words, the effective bias is also decided by the parameter $\varphi$. We see that in Figure 5.

The current $J$ is plotted in Figure 5, left, as a function of $\varepsilon$ for two different choices of $\varphi$. Fixing say $\varepsilon=6$, we see a positive current for $\varphi=0.75$ and a negative current for $\varphi=0.90$. In other words, the direction of the current is not simply decided. The current diverges like $(1-\varphi) \exp [\varepsilon / 4]$ for $\varphi \neq 1$ as $\varepsilon \uparrow \infty$. If $\varphi$ is large, the current is to the left, and if $\varphi$ is small, the current becomes positive. For $\varphi>\frac{1}{2}$, there is a sign-reversal in the current as a function of the entropy flux $\varepsilon$. 


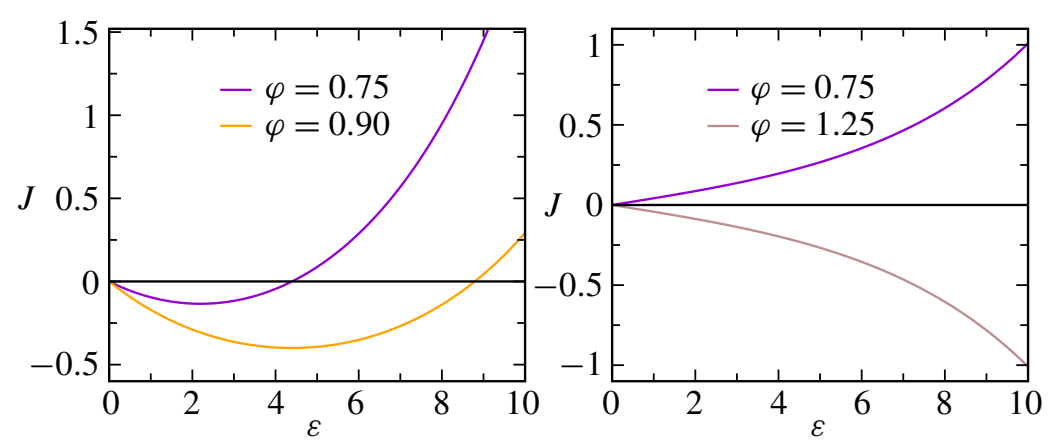

Figure 5. The horizontal current towards the right as a function of $\varepsilon$ corresponding to (left) Figure 4 for $\varphi=0.75$ (upper) and $\varphi=0.90$ (lower curve) and to (right) Figure 6 for $\varphi=0.75$ (upper) and $\varphi=1.25$ (lower curve).

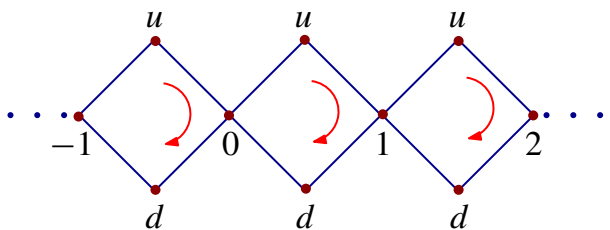

Figure 6. Four-state necklace with top versus bottom symmetry for $\varphi=1$.

We can add more symmetry in the construction by considering, minimally, a four-state Markov process as an elementary unit. Then we look at Figure 6, denoting the states by $\{0, u, 1, d\}$ (where $u$ stands for "up" and $d$ stands for "down"), and the transition rates are

$$
\begin{array}{llll}
k(1, d)=\varphi e^{\varepsilon / 4}, & k(d, 0)=\varphi e^{\varepsilon / 4}, & k(0, u)=e^{\varepsilon / 4}, & k(u, 1)=e^{\varepsilon / 4}, \\
k(d, 1)=\varphi e^{-\varepsilon / 4}, & k(0, d)=\varphi e^{-\varepsilon / 4}, & k(u, 0)=e^{-\varepsilon / 4}, & k(1, u)=e^{-\varepsilon / 4} .
\end{array}
$$

We again have two elementary paths in the opposite direction, which are now $R_{1}$ : $0 \rightarrow u \rightarrow 1$ and $R_{2}: 0 \rightarrow d \rightarrow-1$, for which the entropy fluxes are both equal to $\varepsilon$. (Of course, $R_{2}$ can be identified with the path $1 \rightarrow d \rightarrow 0$.) It will again be the "reactivity" $\varphi>0$ that also decides the direction of the current; see Figure 5, right. Or what starts out as a time-symmetric parameter turns out to give rise to time-asymmetry.

The above scenario has natural realizations, e.g., in the motion of some molecular motors like Myosin V studied in [Maes and O'Kelly de Galway 2015]. The $\varphi$ then corresponds to the activity of the leading head. Because it is lower than that of the trailing head, the motor moves forward. The relation between dynamical activity or "happy feet" (of Paulo Conte's song) and the direction of current has been anticipated in the Inferno (Canto I) of the Divine Comedy, where Dante writes, 

"E come quei che con lena affannata,
uscito fuor del pelago a la riva, si volge a l'acqua perigliosa e guata,
così l'animo mio, ch'ancor fuggiva, si volse a retro a rimirar lo passo che non lasciò già mai persona viva.
Poi ch'èi posato un poco il corpo lasso, ripresi via per la piaggia diserta, sì che 'l piè fermo sempre era 'l più basso" [Dante 1787, p. 2].

In Figure 6, one should imagine the top corresponding to lifting the trailing foot and the bottom to lifting the leading foot; $\varphi>1$ corresponds to a more "active" trailing foot which easily moves you forward. In the case of Dante leaving the valley and climbing the mountain, as the firm or more stable foot was always the lower $(\varphi<1)$, it becomes very difficult to go forward and not to retreat.

3C. Nonequilibrium internal degrees of freedom. We are used to thinking of internal degrees of freedom as an equilibrium reservoir. When a ball bounces off the ground, it slightly deforms and warms up, indicating a restitution coefficient which is less than 1 [Maes and Tasaki 2007]; the entropy gets dissipated in these many internal degrees of freedom. But what if the ball is "alive" or "active", or to put it in less suggestive language, what if the internal degrees of freedom are in steady nonequilibrium? Can that not produce extra interesting effects? The problematic case of the Triangula in Section 3A1 can be seen as an example. The two triangles connected by the vertical rod shown in Figure 3 make one extended object which internally is subject to heat conduction (vertical energy current from higher to lower temperature).

Look now at Figure 7 for greater simplification. Our object has position $q \in S^{1}$ on the ring suspended in a thermal bath at inverse temperature $\beta$. For its dynamics, we assume the overdamped Langevin dynamics

$$
\gamma \dot{q}=-\frac{\partial}{\partial q} E(x, q)+\sqrt{\frac{2 \gamma}{\beta}} \xi_{t}
$$

in the usual physics notation with $\xi_{t}$ standard white noise, $\gamma$ the damping coefficient and $E(x, q)$ some interaction potential with an "internal" degree of freedom, here a four-state Markov process with $x=1,2,3,4$. We assume that the $x$ relaxes fast to stationarity compared to the walker where the time scale is set by $\gamma$, and we take transition rates

$$
k^{q}\left(x, x^{\prime}\right)=e^{-(\beta / 2)\left[E\left(x^{\prime}, q\right)-E(x, q)\right]} \varphi\left(x, x^{\prime}\right) e^{s\left(x, x^{\prime}\right) / 2} .
$$




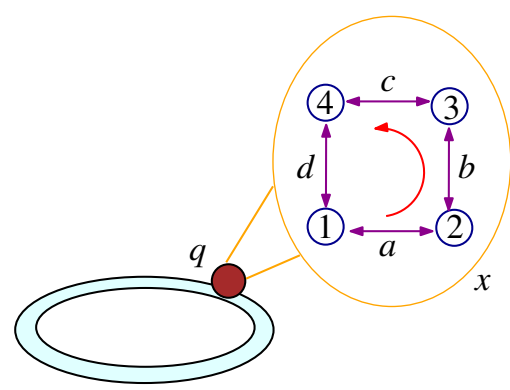

Figure 7. Walker (probe or colloid) on a ring with position $q$ with rotating stomach $x \in\{1,2,3,4\}$. The joint dynamics is specified in (19)-(20). The colloid's position is the slow degree of freedom.

The driving or nonequilibrium sits in $s(1,2)=s(2,3)=s(3,4)=s(4,1)=\beta \varepsilon$ and the symmetric $\varphi\left(x, x^{\prime}\right)$ are $\varphi(1,2)=a, \varphi(2,3)=b, \varphi(3,4)=c$ and $\varphi(4,1)=d$; see the "stomach" in Figure 7. Under the hypothesis of infinite time-scale separation, the colloid is subject to the mean force

$$
f(q)=-\sum_{x} \rho_{q}(x) \frac{\partial}{\partial q} E(x, q)
$$

which can be calculated exactly from the stationary distribution $\rho_{q}(x)$ for the internal degree of freedom $x$. When the rotational part of the force $f_{\text {rot }}=\oint f(q) \mathrm{d} q$ is nonzero, then the colloid will start moving around the circle. In fact, the steady current $J$, as plotted in Figure 8, is essentially just given by it. Obviously, there are many parameters: the form of the potential $E(x, q)$ but also the coefficients $a$, $b, c$ and $d$. We ask here what determines the sign of that rotational force, which of course determines the direction of the current of the walker.

We observe here that we can get a sign-reversal of the current by solely varying the kinetic factors $a, b, c$ and $d$. More specifically, we consider the energy function $E(x, q)$ for $q \in[-1,1], E(2, q)=E(4, q)=0$,

$$
E(1, q)=\left\{\begin{array}{ll}
\frac{4}{7}(1+q) & \text { for } q \leq \frac{3}{4}, \\
4(1-q) & \text { for } q \geq \frac{3}{4}
\end{array} \quad \text { and } \quad E(3, q)= \begin{cases}4(1+q) & \text { for } q \leq-\frac{3}{4} \\
\frac{4}{7}(1-q) & \text { for } q \geq-\frac{3}{4}\end{cases}\right.
$$

In Figure 8, left, we see the rotational current $J=f_{\text {rot }}$ as a function of $c$ for $a=b=d=1$ (first negative then positive) and as a function of $a$ for $b=c=$ $d=1$ (first positive then negative), both at driving $\varepsilon=5$ and $\beta=1$. The same is represented in the right panel but now as a function of the driving $\varepsilon$. We clearly get information about the time-symmetric part in the transition rates (20) from coupling that process $x_{t}$ to the position $q_{t}$ in (19) of the walker and measuring its induced current. 

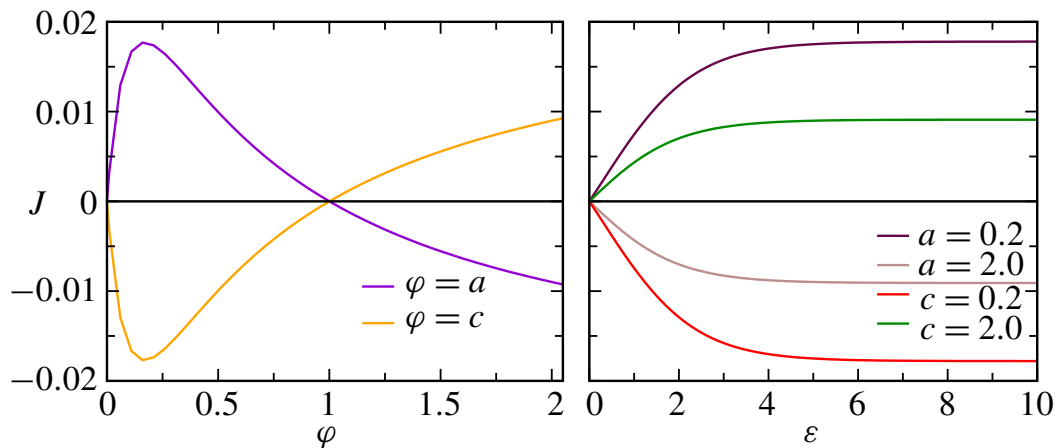

Figure 8. The rotational current $J=f_{\text {rot }}=\oint f(q) \mathrm{d} q$ of the colloid as it depends on the reactivities $\varphi$ and the driving $\varepsilon$ of the internal nonequilibrium in Figure 7; see (20). Left: the direction of the current can change as a function of $a, b, c$ and $d$; we see the current at $\varepsilon=5$ and $\beta=1$ as a function of $\varphi=a$ and as a function of $\varphi=c$, while the other reactivities equal 1 when not specified. Right: the current as a function of the driving $\varepsilon$ for various choices of $\varphi\left(x, x^{\prime}\right)$. From low to high, the curves correspond to $c=0.2$, $a=2.0, c=2.0$ and $a=0.2$ again with all other reactivities fixed to the value 1 when not specified.

3D. Wrong direction! The response to an external field can be negative. It is then the case that by pushing harder the particle gets slower. It could even happen that by pushing in one direction the particle moves in the opposite direction making negative absolute conductivity. In [Cleuren and Van den Broeck 2002], one uses memory to achieve that result, but one also gets it from considering the Markov models of Section 3B.

Consider again the setup of Figure 4 and the resulting Figure 5, left, for the horizontal current in the positive direction. There are possible stalling points $\left(\varepsilon^{*}, \varphi^{*}\right)$ where that current vanishes. Taking these values or, more generally, fixing arbitrary $(\varepsilon, \varphi)$, we perturb the rates $(18)$ as

$$
\begin{array}{lll}
k_{E}(1,0)=[\varphi+E] e^{\varepsilon / 2}, & k_{E}(0, u)=e^{[\varepsilon+E] / 2}, & k_{E}(u, 1)=e^{[\varepsilon+E] / 4}, \\
k_{E}(0,1)=[\varphi+E] e^{-\varepsilon / 2}, & k_{E}(u, 0)=1, & k_{E}(1, u)=e^{-\varepsilon / 4},
\end{array}
$$

pushing a bit harder with $E>0$ in the upper channel (only) and also changing the time-symmetric coefficient $\varphi \rightarrow \varphi+E$. We get a new value of the horizontal current $J_{E}$, and we can ask how it changes, that is, find the conductivity

$$
\sigma=\left.\frac{\mathrm{d} J_{E}}{\mathrm{~d} E}\right|_{E=0} .
$$




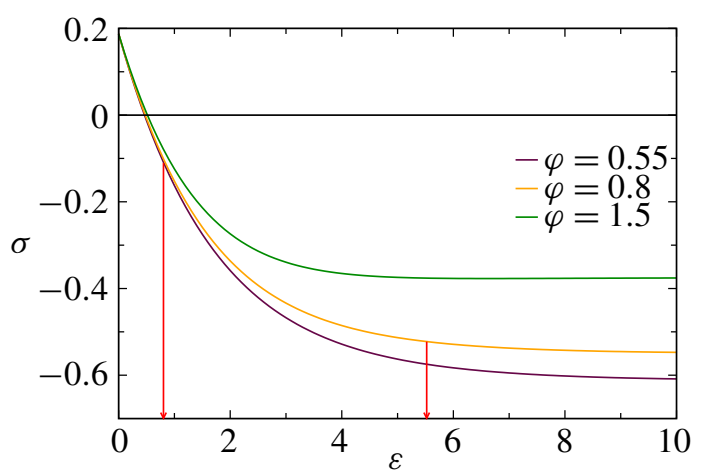

Figure 9. The conductivity $\sigma$ (22), rescaled by a factor of $\exp [-\varepsilon / 4]$, as a function of $\varepsilon$ for $\varphi=0.55$ (lowest curve) having its stalling point at $\varepsilon^{*} \simeq 0.80$, for $\varphi=0.8$ with stalling point at $\varepsilon^{*} \simeq 5.54$ and $\varphi=1.5$ (upper curve). There is negative conductivity $\sigma<0$, including at stalling points where the pushing makes the walker go back instead of forward.

We see in Figure 9 that $\sigma$ gets negative for large-enough values of $\varepsilon$, including at stalling values. Thus, there, the current actually goes backward while pushing forward.

\section{Low temperature analysis}

We consider here a continuous-time version of the Parrondo game of Section 3A2 with random flipping between a flat potential and a nontrivial energy landscape. It gives an approach to the problems of Sections 3A2-3B by considering lowtemperature asymptotics. In particular, we use the Freidlin-Wentzell theory of [Maes et al. 2014] to obtain an expression for the low-temperature ratchet current. Its direction is not determined by entropic considerations (only) but involves the reactivities.

Look at Figure 10. States of a continuous-time Markov process are on two rings, each having $N>2$ of states, denoted by $x=(i, n)$ where $i \in\{1=N+1,2, \ldots, N\}$ and $n=0,1$.

On the outer ring $(n=0)$, energies $E_{1}<\cdots<E_{N}$ are associated to the states and transition rates are thermal:

$$
k((i, 0),(i+1,0))=e^{(\beta / 2)\left(E_{i}-E_{i+1}\right)}, \quad k((i+1,0),(i, 0))=e^{(\beta / 2)\left(E_{i+1}-E_{i}\right)}
$$

for inverse temperature $\beta$. The inner ring $(n=1)$ corresponds to a walker in a flat potential landscape so that

$$
k((i, 1),(i+1,1))=k((i+1,1),(i, 1))=1 .
$$




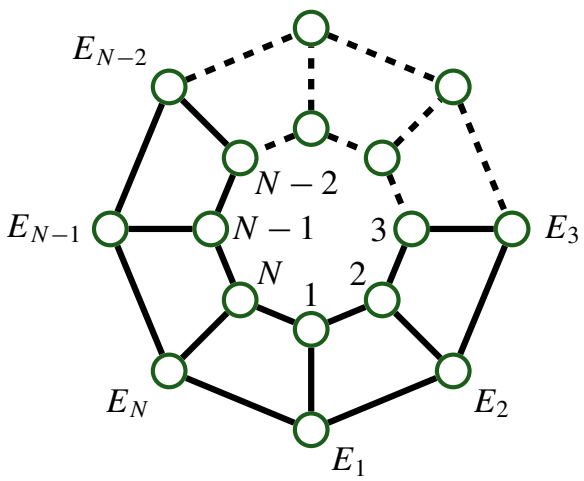

Figure 10. Continuous-time Parrondo game.

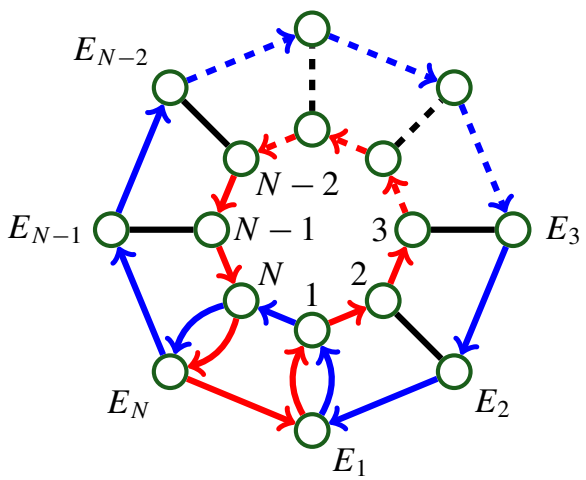

Figure 11. Trajectories $\omega_{1}$ (clockwise, blue) and $\omega_{2}$ (counterclockwise, red) with the same entropy flux yet in opposite directions.

The random flipping between the two potentials is realized by moves between the rings $n=0,1$, at transition rates $k((i, n),(i, 1-n))=a$ for some $a>0$. There is no explicit driving except that for $a=0$ there is detailed balance of course and for very strong coupling $a \gg 1$ the model is effectively running on a single ring. In the limit $a \uparrow \infty$, there is again detailed balance with inverse temperature $\beta / 2$.

The question for the nonequilibrium situation is in what sense the walker will typically move: either clockwise of counterclockwise. Again, the direction of that current, which we now call the ratchet current, is not decided by the positivity of the entropy production. Consider for example Figure 11, where two trajectories $\omega_{1}=((N, 0),(N-1,0), \ldots,(1,0),(1,1),(N, 1),(N, 0))$ and $\omega_{2}=$ $((N, 0),(1,0),(1,1),(2,1), \ldots,(N, 1),(N, 0))$ are depicted that wind in opposite directions yet whose entropy fluxes are exactly identical, equal to $s\left(\omega_{1}\right)=$ $s\left(\omega_{2}\right)=\beta\left(E_{N}-E_{1}\right)>0$. 
The stationary ratchet current $J_{R}$ in the clockwise direction is

$$
J_{R}=j((i+1,0),(i, 0))+j((i+1,1),(i, 1))
$$

with $j(x, y)=k(x, y) \rho(x)-k(y, x) \rho(y)$ where $\rho$ is the stationary probability law for the Markov dynamics (23)-(24). $J_{R}$ is the current over both rings together, and of course, that current also depends on the size $N$, on the energies and on temperature. We will look at the case $a=1$ but at low temperatures so that the transitions $(i, 0) \rightarrow(i+1,0)$ are exponentially damped. It simplifies the structure of the stationary distribution $\rho$; see [Maes et al. 2014], where a type of FreidlinWentzell analysis is applied to find its low-temperature asymptotics. The following combines proofs in [Maes et al. 2014] and in [Louis 2015] to show that the ratchet current is clockwise and saturates:

Proposition 4.1. $J_{R}=0$ for $N=3, J_{R}>0$ for all $N>3$ and $\lim _{N \uparrow \infty} J_{R}=\frac{1}{2}-\frac{1}{\sqrt{5}}$.

Proof. Consider the set $\mathscr{D}:=\{(1,0),(i, 1): i=1, \ldots, N\}$, and let $M(x)$ be the number of in-spanning trees in the digraph obtained from Figure 10 by keeping only the oriented bonds $(v, w)$ where $w$ is one of the most likely successors of $v$. From [Maes et al. 2014], we learn that at low temperatures $\rho(x) \propto|M(x)|$ for $x \in \mathscr{D}$ and $\rho(y) \simeq|M(y)| e^{\beta \Gamma(y)} / \mathscr{E}$, with some $\Gamma(y)<0$ for $y \notin \mathscr{D}$. By the matrix-tree theorem (see, e.g., [Tutte 1984]), we need the Laplacian matrix $L$ on the digraph $K^{D}$, and we erase the row and the column corresponding to vertex $x$ to obtain the matrix $L_{x}$. Then

$$
|M(x)|=\operatorname{det} L_{x} .
$$

The Laplacian of the digraph $K^{D}$ has a rather simple structure:

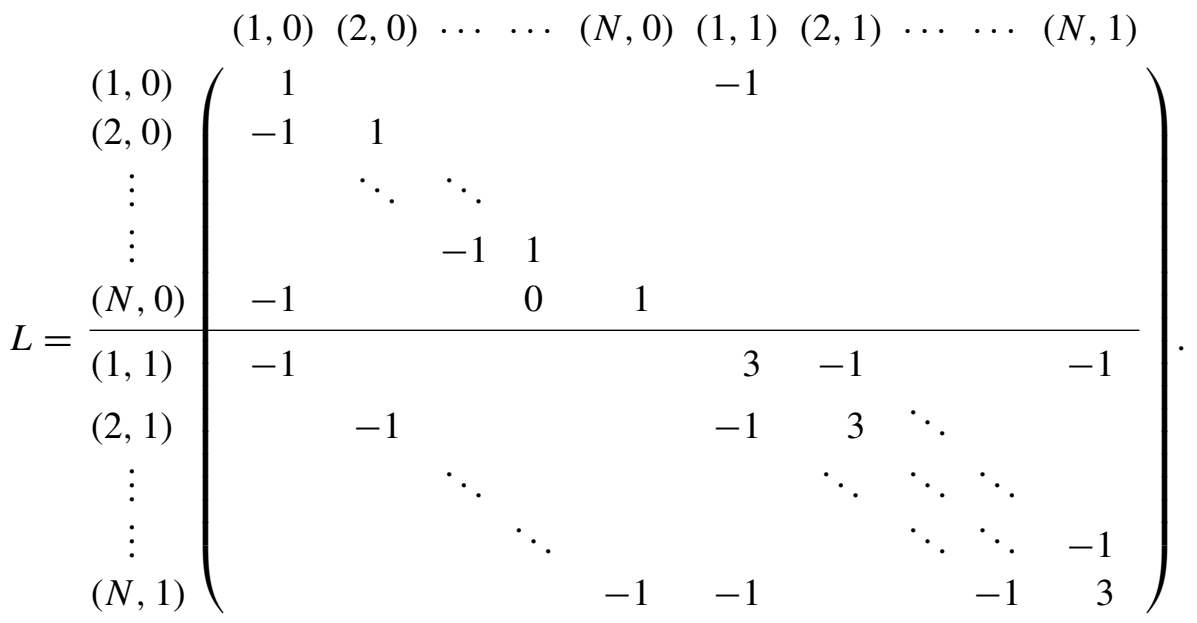


The state for which the number of in-trees becomes maximal is $(1,0)$ : there are more combinations to form an in-tree to $(1,0)$ than to any other state $(i, 1)$ on the inner ring.

To compute the ratchet current, we take $x=(1,1)$ with $\rho(1,1) \simeq A((1,1)) / \mathscr{E}$. Then

$$
j((2,1),(1,1)) \simeq \frac{1}{\mathscr{L}}(A((2,1))-A((1,1))) .
$$

Moreover,

$$
j((2,0),(1,0)) \simeq \frac{A((2,0))}{\mathscr{L}} .
$$

As a consequence,

$$
J_{R} \simeq \frac{1}{\mathscr{L}}\left(\operatorname{det} L_{(2,1)}+\operatorname{det} L_{(2,0)}-\operatorname{det} L_{(1,1)}\right) .
$$

Furthermore, by inspecting the Laplacian $L$, one finds that

- $\operatorname{det} L_{(2,0)}=2 \operatorname{det} B_{N-1}-3 \operatorname{det} B_{N-2}-3$,

- $\operatorname{det} L_{(1,1)}=\operatorname{det} B_{N-1}$ and

- $\operatorname{det} L_{(2,1)}=\operatorname{det} B_{N-2}+1$

with

$$
B_{N}=\left(\begin{array}{rrrr}
3 & -1 & & \\
-1 & 3 & \ddots & \\
& \ddots & \ddots & -1 \\
& & -1 & 3
\end{array}\right) \text {. }
$$

$B_{N}$ satisfies the recursion relation $\operatorname{det} B_{N}=3 \operatorname{det} B_{N-1}-\operatorname{det} B_{N-2}$, where det $B_{2}=8$ and $\operatorname{det} B_{1}=3$. Hence, by solving the recurrence, we get

$$
\operatorname{det} B_{N}=\frac{5-3 \sqrt{5}}{10}\left(\frac{3-\sqrt{5}}{2}\right)^{N}+\frac{5+3 \sqrt{5}}{10}\left(\frac{3+\sqrt{5}}{2}\right)^{N}
$$

to be used in

$$
J_{R} \simeq \frac{\operatorname{det} B_{N-1}-2 \operatorname{det} B_{N-2}-2}{\mathscr{L}}
$$

which already proves that $J_{R}>0$ for all $N \geq 4$ and $J_{R}=0$ when $N=3$; the direction is clockwise. For the $N$-asymptotics, we also need the normalization $\mathscr{L}$. In fact, $\mathscr{L} \simeq \sum_{x \in \mathscr{D}}|M(x)|=\sum_{x \in \mathscr{D}} \operatorname{det} L_{x}$. In [Louis 2015], it is shown that

$$
\mathscr{L} \simeq 2\left(\frac{3-\sqrt{5}}{2}\right)^{N}+2\left(\frac{3-\sqrt{5}}{2}\right)^{N}-4,
$$

which concludes the proof by a simple computation. 
Note that adding particles and interactions we can get direction-reversal of currents as we had in the previous Sections 3B-3C. An experimentally accessible example is described in [de Souza Silva et al. 2006]. That constitutes a big challenge in the discussion of the direction of currents that has not been touched upon here: how density and interactions can modify it.

\section{Conclusion}

To discover what decides the direction of a current under steady nonequilibrium conditions is a major challenge of statistical mechanics. In the present paper, we have seen that many effects are possible, not least from the variation of timesymmetric parameters in transition rates defining the process. That dependence on nondissipative aspects thus provides a method to obtain kinetic parameters from measuring the direction of the current.

\section{Acknowledgment}

I thank Urna Basu for many discussions and help.

\section{References}

[Basu et al. 2015] U. Basu, M. Krüger, A. Lazarescu, and C. Maes, "Frenetic aspects of second order response", Phys. Chem. Chem. Phys. 17 (2015), 6653-6666.

[Van den Broeck et al. 2005] C. Van den Broeck, P. Meurs, and R. Kawai, "From Maxwell demon to Brownian motor", New J. Phys. 7 (2005), 10.

[Cleuren and Van den Broeck 2002] B. Cleuren and C. Van den Broeck, "Random walks with absolute negative mobility”, Phys. Rev. E 65 (2002), 030101(R).

[Dante 1787] Dante, Inferno, Paris, C. A. I. Jacob, 1787.

[de Groot and Mazur 1962] S. R. de Groot and P. Mazur, Non-equilibrium thermodynamics, NorthHolland, Amsterdam, 1962.

[Louis 2015] J. Louis, "Low temperature ratchet current”, preprint, 2015. arXiv 1508.01189v1

[Maes 2015] C. Maes, “Non-dissipative effects in nonequilibrium systems”, lecture notes, 2015. arXiv 1603.05147v1

[Maes and O'Kelly de Galway 2015] C. Maes and W. O'Kelly de Galway, "On the kinetics that moves Myosin V”, Phys. A 436 (2015), 678-685.

[Maes and Tasaki 2007] C. Maes and H. Tasaki, "Second law of thermodynamics for macroscopic mechanics coupled to thermodynamic degrees of freedom", Lett. Math. Phys. 79:3 (2007), 251-261.

[Maes et al. 2000] C. Maes, F. Redig, and A. Van Moffaert, "On the definition of entropy production, via examples", J. Math. Phys. 41:3 (2000), 1528-1554.

[Maes et al. 2009] C. Maes, K. Netočný, and B. Shergelashvili, "A selection of nonequilibrium issues", pp. 247-306 in Methods of contemporary mathematical statistical physics, edited by R. Kotecký, Lecture Notes in Math. 1970, Springer, Berlin, 2009.

[Maes et al. 2014] C. Maes, K. Netočný, and W. O’Kelly de Galway, "Low temperature behavior of nonequilibrium multilevel systems", J. Phys. A Math. Theor. 47:3 (2014), 035002. 
[Maxwell 1878] J. C. Maxwell, “Tait's 'Thermodynamics', II”, Nature 17:432 (1878), 278-280.

[Parrondo et al. 2000] J. M. R. Parrondo, G. P. Harmer, and D. Abbott, "New paradoxical games based on Brownian ratchets", Phys. Rev. Lett. 85:24 (2000), 5226-5229.

[Ruelle 2004] D. Ruelle, "Conversations on nonequilibrium physics with an extraterrestrial", Phys. Today 57:5 (2004), 48-53.

[de Souza Silva et al. 2006] C. C. de Souza Silva, J. Van de Vondel, M. Morelle, and V. V. Moshchalkov, "Controlled multiple reversals of a ratchet effect", Nature 440:7084 (2006), 651-654.

[Tutte 1984] W. T. Tutte, Graph theory, Encyclopedia of Mathematics and its Applications 21, Addison-Wesley, Menlo Park, CA, 1984.

Received 1 Apr 2016. Revised 19 Aug 2016. Accepted 26 Sep 2016.

Christian MAES: christian.maes@fys.kuleuven.be

Instituut voor Theoretische Fysica, Katholieke Universiteit Leuven, Celestijnenlaan 200D, 3001 Leuven, Belgium 



\title{
A REMARK ON EIGENVALUE PERTURBATION THEORY AT VANISHING ISOLATION DISTANCE
}

\author{
FIORELla BARONE AND SANDRO GRAFFI
}

Let $T$ be a self-adjoint operator in a separable Hilbert space $X$, admitting compact resolvent and simple eigenvalues with possibly vanishing isolation distance, and let $V$ be symmetric and bounded. Consider the self-adjoint operator family $T(g): g \in \mathbb{R}$ in $X$ defined by $T+g V$ on $D(T)$. A simple criterion is formulated ensuring, for any eigenvalue of $T(g)$, the existence to all orders of its perturbation expansion and its asymptotic nature near $g=0$, with estimates independent of the eigenvalue index. An application to a class of Schrödinger operators is described.

\section{Introduction and formulation of the result}

The standard Rellich-Kato regular perturbation theory [Kato 1976] applies to isolated eigenvalues of finite multiplicity of a densely defined, closed operator $T$ in a Banach space $X$. We consider here only the particular case in which

- $X$ is a separable Hilbert space,

- $T$ is a self-adjoint operator in $X$ with compact resolvent and simple spectrum, and

- the perturbation is symmetric, regular and linear on the perturbation parameter.

Let the operator $V: D(V) \rightarrow X$ be symmetric and $T$-bounded with relative bound $b$; i.e., let $D(T) \subset D(V)$, and let there exist $a>0$ and $b>0$ such that

$$
\|V u\| \leq b\|T u\|+a\|u\| \quad \text { for all } u \in D(T) .
$$

With $g \in \mathbb{C}$, consider the operator family in $X$ defined as

$$
g \mapsto T(g):=T u+g V u, \quad D(T(g))=D(T) .
$$

Then $T(g)$ is closed with nonempty resolvent set for $|g|<1 / b$ and $T(g)^{*}=T(\bar{g})$ so that $T(g)=T(g)^{*}$ if $g \in \mathbb{R}$. Let $\lambda \in \mathbb{R}$ be an isolated eigenvalue of $T$ (simple by the above assumption), with isolation distance $d(\lambda)>0$. Here, let us recall that

$$
d(\lambda):=\operatorname{dist}(\operatorname{Spec}(T) \backslash\{\lambda\}, \lambda)>0 .
$$

Communicated by Raffaele Esposito.

MSC2010: 81Q05, 81Q10, 81Q15.

Keywords: isolation distance, eigenvalue perturbation theory. 
Then for $|g|$ suitably small, $T(g)$ has one and only one simple eigenvalue $\lambda(g)$ such that $\lim _{g \rightarrow 0} \lambda(g)=\lambda$ (see, e.g., [Kato 1976, §§VII.2-3] or [Reed and Simon 1978, §XII.1]). The function $g \mapsto \lambda(g)$ is holomorphic in a disk centered at the origin because its Taylor expansion at $g=0$

$$
\lambda(g)=\lambda+\sum_{\ell=1}^{\infty} \lambda_{\ell} g^{\ell}
$$

exists and converges for $|g|<r_{d}(\lambda)$, with $r_{d}(\lambda)>0$. The coefficients $\lambda_{\ell}$ are generated by (Rayleigh-Schrödinger) perturbation theory. This existence and convergence result depends in a critical way on the positivity of $d(\lambda)$ and therefore does not apply to nonisolated eigenvalues.

To the best of our knowledge, a simple, explicit criterion ensuring existence, let alone convergence, of (Rayleigh-Schrödinger) perturbation theory when $r_{d}(\lambda) \rightarrow 0$ is still missing, even under much stronger assumptions such as $r_{d}(\lambda) \rightarrow 0$ only if $\lambda \rightarrow \infty$ and boundedness of $V$. (For related questions involving the behavior of $r_{d}(\lambda)$ as $\lambda \rightarrow \infty$, we refer the reader to [Reed and Simon 1978, §XIII.5] and to [Brownell and Clark 1961; McLeod 1961; Tamura 1974]). Within this last class of Hilbert space operators, we formulate and prove here a similar criterion, working out the necessary estimates on the behavior of $\lambda_{\ell}$ uniform with respect to the eigenvalue index $\underline{n}$. Under more restrictive assumptions on the vanishing of $r_{d}(\lambda)$, the explicit dependence on $\underline{n}$ of the above estimates is actually determined.

Our hypotheses are formulated as follows.

(A1) $T$ is a nonnegative self-adjoint operator in the separable Hilbert space $X$, with compact resolvent and simple spectrum. Its eigenvalues are denoted by $\left\{\lambda_{\underline{n}}: \underline{n} \in \mathbb{N}^{s}\right\}, s \geq 1$, and the corresponding (normalized) eigenvectors by $\left\{\psi_{\underline{n}}: \underline{n} \in \mathbb{N}^{s}\right\}$.

(A2) $d(\underline{m}, \underline{n}):=\left|\lambda_{\underline{m}}-\lambda_{\underline{n}}\right| \rightarrow 0$ if and only if $|\underline{m}-\underline{n}| \rightarrow \infty$. Here $|n|:=n_{1}+\cdots+n_{s}$, $\underline{n} \in \mathbb{N}^{s}$.

(A3) There are $\Lambda>0$ and $\gamma>s-1$ such that

$$
\left|\lambda_{\underline{m}}-\lambda_{\underline{n}}\right|^{-1} \leq \Lambda|\underline{m}-\underline{n}|^{\gamma}, \quad \underline{m} \neq \underline{n} .
$$

Here $|\underline{x}|^{\gamma}:=x_{1}^{\gamma}+\cdots+x_{s}^{\gamma}$.

(A4) $V: X \rightarrow X$ is symmetric and bounded (hence self-adjoint). Moreover, there exist $A>\alpha$ and $\alpha>0$ such that

$$
\left|\left\langle\psi_{\underline{m}}, V \psi_{\underline{n}}\right\rangle\right| \leq A e^{-\alpha|\underline{m}-\underline{n}|}, \quad|\underline{m}-\underline{n}| \rightarrow \infty .
$$

Since $V$ is bounded, it is a fortiori $T$-bounded with $T$-bound 0 . Thus, the operator family $g \mapsto T(g)=T+g V$ with $D(T(g))=D(T)$ is type-A real-holomorphic in the sense of Kato [1976, §VII.2] for all $g \in \mathbb{C}$. 
Consider now the Rayleigh-Schrödinger perturbation expansion near any eigenvalue $\lambda_{\underline{n}}(g)$ of $H(g)$. The initial point of $\lambda_{\underline{n}}(g)$ is the eigenvalue $\lambda_{\underline{n}}$ of $T, \underline{n} \in \mathbb{Z}^{s}$. Thus,

$$
\lambda_{\underline{n}}(g)=\lambda_{\underline{n}}+\sum_{\ell=1}^{\infty} B_{\ell}(\underline{n}) g^{\ell} .
$$

The expansion (1-7) has positive radius of convergence $r_{n}, \underline{n} \in \mathbb{Z}^{s}$, by the boundedness of $V$, which implies

$$
r_{\underline{n}} \geq \frac{d\left(\lambda_{\underline{\underline{n}}}\right)\|V\|}{2}, \quad d\left(\lambda_{\underline{n}}\right)=\operatorname{dist}\left(\operatorname{Spec}\left[(T) \backslash\left\{\lambda_{\underline{n}}\right\}\right], \lambda_{\underline{n}}\right) .
$$

The vanishing of the convergence radius $r(\underline{n}) \downarrow 0$ as $d\left(\lambda_{\underline{n}}\right) \downarrow 0$ not only may cause the divergence of the perturbation expansion but may prevent its very existence also for a bounded perturbation $V$; see Remarks 1.3 and 2.1. Then the purpose of this paper is to explicit determine, under the above assumptions, the dependence of the perturbation series on the vanishing rate of the isolation distance $d\left(\lambda_{n}\right)$ by proving the following quantitative estimate.

Theorem 1.1. Let $T$ and $V$ fulfill assumptions (A1)-(A3). Set

$$
R(\Lambda, \alpha, \gamma):=\frac{\Lambda}{\alpha^{\gamma}} .
$$

Then the following $\underline{n}$-independent estimate holds:

$$
\left|B_{\ell}(\underline{n})\right|<R(\Lambda, \alpha, \gamma)^{\ell}(4 \ell)^{\ell+1}[\gamma(\ell-1)] \text { ! for all } \underline{n} \in \mathbb{Z}^{s} .
$$

The uniform estimate (1-10) makes it possible to establish the uniform asymptotic nature to all orders of the perturbation expansion.

Corollary 1.2. The perturbation expansion $\lambda_{\underline{n}}+\sum_{\ell=1}^{\infty} B_{\ell}(\underline{n}) g^{\ell}$ represents an asymptotic expansion to all orders of the eigenvalue $\lambda_{\underline{n}}(g)$ uniformly with respect to $\underline{n} \in \mathbb{N}^{s} ;$ i.e, for any fixed $N \in \mathbb{N}$,

$$
\lim _{|g| \rightarrow 0} \frac{\left|\lambda_{\underline{n}}(g)-\sum_{\ell=1}^{N} B_{\ell}(\underline{n}) g^{\ell}\right|}{|g|^{N}}=0
$$

uniformly with respect to $\underline{n} \in \mathbb{N}^{s}$.

Remark 1.3. The very existence of perturbation theory at the vanishing of the isolation distance, i.e., at the limit $\underline{n} \rightarrow \infty$ in the present case, requires the validity of estimates independent of $\underline{n}$ on the coefficients $B_{\ell, \underline{n}}$. The conditions (1-5) and (1-6) imply the existence of $\bar{g}(\underline{n})>0$ such that

$$
|g| \sum_{\substack{\underline{m} \in \mathbb{Z}^{s} \\ \underline{m} \neq \underline{n}}} \frac{\left|\left\langle\psi_{\underline{m}}, V \psi_{\underline{n}}\right\rangle\right|}{\left|\lambda_{\underline{m}}-\lambda_{\underline{n}}\right|}<1, \quad|g|<\bar{g}(\underline{n}) .
$$


Now

$$
\sup _{\underline{m} \neq \underline{n} \underline{\underline{n}}} \frac{1}{\left|\lambda_{\underline{m}}-\lambda_{\underline{n}}\right|} \leq \frac{2}{d_{\underline{n}}} .
$$

Moreover, since $V$ is bounded and symmetric,

$$
\|V\|=\max \left(\sup _{\underline{m} \in \mathbb{Z}^{s}} \sum_{n \in \mathbb{Z}^{s}}\left|\left\langle\psi_{\underline{m}}, V \psi_{\underline{n}}\right\rangle\right|, \sup _{\underline{n} \in \mathbb{Z}^{s}} \sum_{\underline{m} \in \mathbb{Z}^{s}}\left|\left\langle\psi_{\underline{m}}, V \psi_{\underline{n}}\right\rangle\right|\right),
$$

whence

$$
\sum_{\substack{m \in \mathbb{Z}^{s} \\ \underline{m} \neq \underline{\underline{n}}}} \frac{\left|\left\langle\psi_{\underline{m}}, V \psi_{\underline{n}}\right\rangle\right|}{\left|\lambda_{\underline{m}}-\lambda_{\underline{n}}\right|} \leq \frac{2}{d_{\underline{n}}}\|V\| .
$$

Thus, if $\underline{n}$ is fixed, i.e., if $\lambda_{\underline{n}}$ is isolated with isolation distance $d_{\underline{n}}>0$, the standard convergence criterion valid for the perturbation series of the isolated eigenvalue $\lambda_{\underline{n}}$ under the bounded perturbation $V$ (see, e.g., [Kato 1976, §VII.2])

$$
\frac{2|g|}{d_{\underline{n}}}\|V\|<1
$$

implies a fortiori the inequality (1-12). On the other hand, as $\underline{n} \rightarrow \infty$, i.e., $d_{\underline{n}} \rightarrow 0$, in general the inequality (1-13) has a meaning only for $g=0$, while (1-12) can be rewritten (again by (1-5) and (1-6), which are an adaptation of the small-denominator conditions of classical perturbation theory) in the form of an inequality independent of $\underline{n}$ :

$$
|g| A \Lambda\left[\frac{d^{\gamma}}{d \alpha^{\gamma}}\left(2 e^{\alpha \gamma}\right)\right]^{s}<1 .
$$

This inequality is the starting point for the $\underline{n}$-independent estimates of Theorem 1.1. Remark 1.4 (notation). The underlining operation always transforms into vector indices with $s$ components the corresponding scalar ones. Namely,

$$
\underline{m}:=\left(m_{1}, \ldots, m_{s}\right) \in \mathbb{Z}^{s}, \quad m_{i} \in \mathbb{Z}, i=1, \ldots, s .
$$

The star operation transforms a positive integer index into a nonnegative one; i.e., $q \in \mathbb{N}_{*}$ means $q=0,1, \ldots$. Furthermore, $|\underline{m}|$ denotes the length of the multiindex $\underline{m}$ :

$$
|\underline{m}|:=\left|m_{1}\right|+\cdots+\left|m_{s}\right| .
$$

Products and powers of multi-indices abbreviate products and powers of the composing indices:

$$
\begin{aligned}
\underline{m} ! & :=m_{1} ! \cdots m_{s} !, \\
\underline{z}^{\underline{m}} & :=z_{1}^{m_{1}} \cdots z_{s}^{m_{s}}, \\
\Gamma(\alpha \underline{z}) & :=\Gamma\left(\alpha z_{1}\right) \cdots \Gamma\left(\alpha z_{s}\right), \quad \alpha \in \mathbb{R} .
\end{aligned}
$$


Example 1.5. Let $T$ be the Schrödinger operator in $L^{2}\left(\mathbb{R}^{s}\right)$ with domain and action defined as

$$
\begin{aligned}
D(T) & =H^{2}\left(\mathbb{R}^{s}\right) \cap L_{2}^{2}\left(\mathbb{R}^{s}\right), \\
T u & =-\frac{1}{2} \Delta u+\frac{1}{2} \sum_{k=1}^{s}\left[\omega_{k}^{2} x_{k}^{2}-s / 2\right] u, \quad u \in D(T) .
\end{aligned}
$$

$T$ is the self-adjoint, compact-resolvent Schrödinger operator generated by the $p$ dimensional quantum harmonic oscillator, with frequencies $1 \geq \omega_{k}>0, k=1, \ldots, s$. Thus, condition (A1) is fulfilled.

The rescaling map $\left(U_{\underline{\omega}} f\right)(\underline{x})=\left(\omega_{1} \cdots \omega_{s}\right)^{1 / 2} f(\underline{\omega x}), \underline{\omega x}:=\left(\omega_{1} x_{1}, \ldots, \omega_{s} x_{s}\right)$, is unitary in $L^{2}\left(\mathbb{R}^{s}\right)$, and by an abuse of notation, we still denote by $T$ the unitary image $U_{\underline{\omega}} T U_{\omega}^{-1}$. Hence, the action $T u$ becomes

$$
T u=\frac{1}{2} \sum_{k=1}^{s} \omega_{k}\left[-\frac{d^{2} u}{d x_{k}^{2}}+x_{k}^{2} u-I u\right] .
$$

The corresponding eigenvalues are

$$
\lambda_{\underline{n}}(\omega)=\sum_{k=1}^{s} \omega_{k} n_{k}:=\langle\underline{\omega}, \underline{n}\rangle, \quad \underline{n} \in\left(\mathbb{N}_{*}\right)^{s} .
$$

Since $\omega_{i}>0$ and $n_{i}>0, i=1, \ldots, s$, the difference $\left|\lambda_{\underline{r}}-\lambda_{\underline{n}}\right|=\langle\underline{\omega},(\underline{r}-\underline{n})\rangle$ can vanish only if $|\underline{r}-\underline{n}| \rightarrow \infty$, with at least two of the components $r_{j}-n_{j}$, $j=1, \ldots, s$, having different sign. Hence, condition (A2) is fulfilled. Assume now irrational independence of the frequencies, i.e.,

$$
\omega_{1} v_{1}+\cdots+\omega_{s} v_{s}=0, v_{k} \in \mathbb{Z}, \quad \text { if and only if } v_{k}=0, k=1, \ldots, s .
$$

Then all eigenvalues $\lambda_{n}(\underline{\omega})$ are simple, and condition (A3) is equivalent to requiring the diophantine condition

$$
|\langle\underline{\omega}, \underline{v}\rangle|>\Lambda^{-1}|\underline{v}|^{-\gamma}, \quad \gamma>s-1, \underline{m}-\underline{n}:=\underline{v} \neq 0
$$

on the frequencies $\underline{\omega}$. The set of the diophantine values of $\underline{\omega}$ is dense in $[0,1]^{s}$.

By condition (1-20), the eigenvalues $\lambda_{\underline{n}}(\underline{\omega})$ are simple. The corresponding normalized eigenvectors are

$$
\psi_{\underline{n}}(\underline{x})=\frac{1}{\sqrt{2^{\underline{n}} \underline{n} !}} e^{-\underline{x}^{2} / 2} H_{\underline{n}}(\underline{x}):=|\underline{n}\rangle,
$$

where $x \mapsto H_{n}(x), n=0,1, \ldots$, is the $n$-th Hermite polynomial in $\mathbb{R}$ and

$$
\begin{aligned}
\underline{x} & =\left(x_{1}, \ldots, x_{s}\right) \in \mathbb{R}^{s}, & 2^{\underline{n}} & =2^{n_{1}+\cdots+n_{s}}, \\
e^{-\underline{x}^{2}} & =e^{-x_{1}^{2}-\cdots-x_{s}^{2}}, & H_{\underline{n}}(\underline{x}) & =H_{n_{1}}\left(x_{1}\right) \cdots H_{n_{s}}\left(x_{s}\right) .
\end{aligned}
$$


Now let $2<q<6$. Consider the function $\mathcal{V}(\underline{x}) \in C^{\infty}\left(\mathbb{R}^{s} ; \mathbb{R}\right)$ such that

$$
\mathcal{V}(\underline{x})=e^{-|\underline{x}|^{q}} \Phi(\underline{x}), \quad \sup _{\underline{x} \in \mathbb{R}^{s}}|\Phi(\underline{x})| \leq 1,|\underline{x}|^{q}:=\left|x_{1}\right|^{q}+\cdots+\left|x_{s}\right|^{q} .
$$

Denote by $V(\underline{x})$ the maximal multiplication operator by $\mathcal{V}(\underline{x})$ in $L^{2}\left(\mathbb{R}^{s}\right)$. Then $\|V\|_{L^{2} \rightarrow L^{2}} \leq 1$. Thus, the operator $H=T+V$ defined on $D(T)$ is self-adjoint in $L^{2}\left(\mathbb{R}^{s}\right)$ with compact resolvent. In Proposition 2.6 below we will prove the estimate

$$
\left|\left\langle\psi_{\underline{m}}, V \psi_{\underline{n}}\right\rangle_{L^{2}\left(\mathbb{R}^{s}\right)}\right| \leq e^{-\Sigma(|\underline{m}|+|\underline{n}|)},
$$

with $\Sigma>0$ independent of $(\underline{m}, \underline{n})$. Hence, (A4) is fulfilled in this example.

\section{Proof of the result}

We recall the basic definitions of (Rayleigh-Schrödinger) eigenvalue perturbation theory [Kato 1976, §II.1.5 and §§VII.1-3] in this context. Consider an eigenvalue $\lambda_{\underline{n}}, \underline{n} \in \mathbb{Z}^{s}$, of $T$ corresponding to the (normalized) eigenvector $\psi_{\underline{n}}$, henceforth abbreviated $|\underline{n}\rangle$.

- $P_{\underline{n}}$ denotes the (one-dimensional) orthogonal projection operator from $X$ to the one-dimensional subspace spanned by $|\underline{n}\rangle$.

- $S$ denotes the reduced resolvent of $T$, i.e., $S(\lambda):=\sum_{\underline{k} \neq \underline{n} \in \mathbb{Z}^{s}} \frac{P_{\underline{k}}}{\lambda_{\underline{k}}-\lambda}$.

- $S_{\underline{n}}$ is the reduced resolvent evaluated at $\lambda=\lambda_{\underline{n}}$, i.e., $S_{\underline{n}}:=\sum_{\underline{k} \neq \underline{n} \in \mathbb{Z}^{s}} \frac{P_{\underline{k}}}{\lambda_{\underline{k}}-\lambda_{\underline{n}}}$.

$$
\left[S_{\underline{n}}\right]^{\ell}:=\sum_{\underline{k} \neq \underline{n} \in \mathbb{Z}^{s}} \frac{P_{\underline{k}}}{\left(\lambda_{\underline{k}}-\lambda_{\underline{n}}\right)^{\ell}}, \quad \ell \geq 1, \quad\left[S_{\underline{n}}\right]^{0}=P_{\underline{n}} .
$$

In this situation we can use the explicit expressions (2.32)-(2.33) in [Kato 1976, Chapter II] for the coefficients $B_{\ell}(\underline{n})$ of the perturbation series (1-7):

$$
B_{\ell}(\underline{n})=\sum_{p=1}^{\ell} \frac{(-1)^{p}}{p} \sum_{\substack{k_{1}+\ldots+k_{p}=p-1 \\ k_{i}=0,1, \ldots \\ i=1, \ldots, p-1}} \operatorname{Tr}\left[V\left[S_{\underline{n}}\right]^{k_{1}} V \cdots V\left[S_{\underline{n}}\right]^{k_{p}}\right] .
$$

Since

$$
\begin{gathered}
{\left[S_{\underline{n}}\right]^{k_{p}}|\underline{n}\rangle=0, \quad k_{p}>0, \quad\left[S_{\underline{n}}\right]^{0}|\underline{n}\rangle=|\underline{n}\rangle,} \\
\operatorname{Tr}\left[V\left[S_{\underline{n}}\right]^{k_{1}} V \cdots V\left[S_{\underline{n}}\right]^{k_{p}}\right]=\left\langle n, V\left[S_{\underline{n}}\right]^{k_{1}} V \cdots V^{k_{p}} n\right\rangle,
\end{gathered}
$$


(2-2) becomes

$$
B_{\ell}(\underline{n})=\sum_{p=1}^{\ell} \frac{(-1)^{p}}{p} B_{\ell, p}(\underline{n}),
$$

where

$$
B_{\ell, p}(\underline{n})=\sum_{k_{1}+\cdots+k_{p-1}=p-1}\left\langle\underline{n}, V\left[S_{\underline{n}}\right]^{k_{1}} V \cdots V\left[S_{\underline{n}}\right]^{k_{p-1}} V \underline{n}\right\rangle .
$$

Remark 2.1. For $\ell=2$, we have $p=2$ and the above formulas yield the standard second-order term of the Rayleigh-Schrdinger expansion:

$$
B_{2,2}(\underline{n})=\sum_{\underline{k} \neq \underline{n}} \frac{|\langle\underline{n}, V \underline{k}\rangle|^{2}}{\lambda_{\underline{k}}-\lambda_{\underline{n}}} .
$$

In the absence of a condition controlling the vanishing of $\left|\lambda_{\underline{k}}-\lambda_{\underline{n}}\right|$ as $|\underline{k}-\underline{n}| \rightarrow \infty$, the above series can of course diverge even if

$$
\sup _{\underline{n} \in \mathbb{N}^{s}} \sum_{\underline{k} \in \mathbb{N}^{s}}\langle|\underline{n}, V \underline{k}|\rangle^{2}<+\infty
$$

On the other hand, this last inequality is implied by the standard Schur condition ensuring the boundedness of $V^{2}$ and hence of $V$.

We can rewrite in more detail the factor $\left\langle\underline{n}, V S_{\underline{n}}^{k_{1}} \cdots V S_{\underline{n}}^{k_{p-1}} V \underline{n}\right\rangle$ making explicit all factors with at least one $k_{i}=0$. Namely, consider the $q$-partition

$$
p-1=j_{1}+\cdots+j_{q}, \quad j_{s} \geq 1,1 \leq q \leq p-1 .
$$

The number of the $q$-partitions is (see, e.g., [Andrews 1976]) $N(p, q)=\left(\begin{array}{c}p-1 \\ q-1\end{array}\right)$. Furthermore, let $m(p, q)=p-1-q$ be the total number of zeros in the $q$-partition of $\left(k_{1}, \ldots, k_{p-1}\right)$. We can thus rearrange the sequence $\left(k_{1}, \ldots, k_{p-1}\right)$ as

$$
k_{1}+\cdots+k_{p-1}=p-1=m_{1}+j_{1}+m_{2}+j_{2}+m_{q}+j_{q}, \quad m=m_{1}+\cdots+m_{q} .
$$

As a consequence,

$$
\begin{aligned}
V\left[S_{\underline{n}}\right]^{k_{1}} \cdots V\left[S_{\underline{n}}\right]^{k_{p-1}} V= & \sum_{q=1}^{(p-1)} N(p, q) \prod_{h=1}^{m_{1}}\left[V P_{\underline{n}}\right]^{h} V\left[S_{\underline{n}}\right]^{j_{1}} \\
& \times \prod_{h=1}^{m_{2}}\left[V P_{\underline{n}}\right]^{h} \cdot V\left[S_{\underline{n}}\right]^{j_{2}} \cdots \prod_{h=1}^{m_{q}}\left[V P_{\underline{n}}\right]^{h} \cdot V\left[S_{\underline{n}}\right]^{j_{q}} V,
\end{aligned}
$$

whence: 
Lemma 2.2. Let $k_{1}, \ldots, k_{p}, m_{1}, \ldots, m_{q}, j_{1}, \ldots, j_{q}$ be related through (2-7). Then

$$
\begin{aligned}
& B_{\ell, p}(\underline{n})=\sum_{k_{1}+\cdots+k_{p-1}=p-1}\left\langle\underline{n}, V\left[S_{\underline{n}}\right]^{k_{1}} \cdots V\left[S_{\underline{n}}\right]^{k_{p-1}} V \underline{n}\right\rangle \\
& =\sum_{q=1}^{p-1} N(p, q)\langle\underline{n}, V \underline{n}\rangle^{m_{1}+\cdots+m_{q}} \sum_{\substack{\underline{r}_{1} \neq n, \ldots, \underline{r}_{q} \neq \underline{n} \\
j_{1}+\cdots+j_{q}=p-1}} \frac{\left|\left\langle\underline{n}, V \underline{r}_{1}\right\rangle\right|^{2}}{\left(\lambda_{\underline{r}_{1}}-\lambda_{\underline{n}}\right)^{j_{1}}} \cdot \frac{\left|\left\langle\underline{n}, V \underline{r}_{2}\right\rangle\right|^{2}}{\left(\lambda_{\underline{r}_{2}}-\lambda_{\underline{n}}\right)^{j_{2}}} \cdots \frac{\left|\left\langle\underline{n}_{\underline{n}}, V \underline{r}_{q}\right\rangle\right|^{2}}{\left(\lambda_{\underline{r}_{q}}-\lambda_{\underline{n}}\right)^{j_{q}}} \\
& =\sum_{q=1}^{p-1} N(p, q)\langle\underline{n}, V \underline{n}\rangle^{p-1-q} \sum_{\substack{\underline{r}_{1} \neq \underline{n}, \ldots, \underline{r}_{q} \neq \underline{n} \\
j_{1}+\cdots+j_{q}=p-1}} \frac{\left|\left\langle\underline{n}, V \underline{r}_{1}\right\rangle\right|^{2}}{\left(\lambda_{\underline{r}_{1}}-\lambda_{\underline{n}}\right)^{j_{1}}} \cdot \frac{|\langle\underline{n}, V \underline{\underline{r}} 2\rangle|^{2}}{\left(\lambda_{\underline{r}_{2}}-\lambda_{\underline{n}}\right)^{j_{2}}} \cdots \frac{\left|\left\langle\underline{n}, V \underline{r}_{q}\right\rangle\right|^{2}}{\left(\lambda_{\underline{r}_{q}}-\lambda_{\underline{\underline{n}}}\right)^{j_{q}}} .
\end{aligned}
$$

Proof. The product (2-8) is unchanged if $P_{\underline{n}}$ is replaced by $P_{n}^{2}$. Recalling that

$$
S^{j}=\sum_{\underline{r} \neq \underline{n}}^{\infty} \frac{P_{\underline{r}}}{\left(\lambda_{\underline{r}}-\lambda_{\underline{n}}\right)^{j}}
$$

and denoting

$$
\begin{aligned}
\Omega_{1}(m, j ; n) & :=\prod_{h=1}^{m}\left[V P_{\underline{n}}^{2}\right]^{h} V S^{j}, \\
\Omega_{2}(m-1, j ; n) & :=\prod_{h=1}^{m-1}\left[V P_{\underline{n}}^{2}\right]^{h} V P_{\underline{n}} P_{\underline{n}} V S^{j}, \\
\Omega_{3}(m-1, j ; n, r) & :=\prod_{h=1}^{m-1}\left[V P_{\underline{n}}^{2}\right]^{h} V P_{\underline{n}} P_{\underline{n}} V P_{\underline{r}} P_{\underline{r}},
\end{aligned}
$$

this yields

$$
\begin{aligned}
& B_{\ell, p}(\underline{n})=\sum_{q=1}^{p-1} N(p, q)\left\langle\underline{n}, \Omega_{1}\left(m_{1}, j_{1} ; n\right) \cdots \Omega_{1}\left(m_{q}, j_{q} ; n\right) V \underline{n}\right\rangle \\
& =\sum_{q=1}^{p-1} N(p, q)\left\langle\underline{n}, \prod_{h=1}^{m_{1}-1} \Omega_{2}\left(m_{1}-1, j_{1} ; n\right) \cdots \Omega_{2}\left(m_{q}-1, j_{q} ; n\right) V \underline{n}\right\rangle \\
& =\sum_{q=1}^{p-1} N(p, q)\left(\lambda_{\underline{r}_{1}}-\lambda_{\underline{n}}\right)^{-j_{1}} \cdot\left(\lambda_{\underline{r}_{2}}-\lambda_{\underline{n}}\right)^{-j_{2}} \cdots\left(\lambda_{\underline{r}_{q}}-\lambda_{\underline{n}}\right)^{-} j_{q} \\
& \times \sum_{\substack{\underline{r}_{1} \neq \underline{n}, \ldots, \underline{r}_{q} \neq \underline{n} \\
j_{1}+\cdots+j_{q}=p-1}}\left\langle\underline{n}, \Omega_{3}\left(m_{1}-1, j_{1} ; n, \underline{r}_{1}\right) \cdots \Omega_{3}\left(m_{q}-1, j_{q} ; n, \underline{r}_{q}\right) V \underline{n}\right\rangle .
\end{aligned}
$$


Hence, by (2-8),

$$
\begin{aligned}
& \sum_{k_{1}+\cdots+k_{p-1}=p-1}\left\langle n, V\left[S_{\underline{n}}\right]^{k_{1}} \cdots V\left[S_{\underline{n}}\right]^{k_{p-1}} V n\right\rangle \\
& =\sum_{q=1}^{p-1} N(p, q)\left\langle\underline{n}, \prod_{h=1}^{m_{1}-1}\left[V P_{\underline{n}}^{2}\right]^{h} V P_{\underline{n}} P_{\underline{n}} V P_{\underline{r}_{1}} P_{\underline{r}_{1}}\right. \\
& \left.\times \prod_{h=1}^{m_{2}-1}\left[V P_{\underline{n}}^{2}\right]^{h} V P_{\underline{n}} P_{\underline{n}} \cdot V P_{\underline{r}_{2}} P_{\underline{r}_{2}} \cdots \prod_{h=1}^{m_{q}} V P_{\underline{r}_{q}} P \underline{r}_{q} V \underline{n}\right) \\
& =\sum_{q=1}^{p-1} N(p, q)\langle\underline{n}, V \underline{n}\rangle^{m_{1}} \cdots\langle\underline{n}, V \underline{n}\rangle^{m_{q}} \\
& \times \sum_{\underline{r}_{1} \neq \underline{n}, \ldots, \underline{r}_{q} \neq \underline{n}} \frac{\left\langle\underline{n}, V \underline{r}_{1}\right\rangle\left\langle\underline{r}_{1}, V \underline{n}\right\rangle}{\left(\lambda_{\underline{r}_{1}}-\lambda_{\underline{n}}\right)^{j_{1}}} \cdot \frac{\left\langle\underline{n}, V \underline{r}_{2}\right\rangle\left\langle\underline{r}_{2}, V \underline{n}\right\rangle}{\left(\lambda_{\underline{r}_{2}}-\lambda_{\underline{n}}\right)^{j_{2}}} \cdots \frac{\left\langle\underline{n}, V \underline{r}_{q}\right\rangle\left\langle\underline{r}_{q}, V \underline{n}\right\rangle}{\left(\lambda_{\underline{r}_{q}}-\lambda_{\underline{n}}\right)^{j_{q}}} \\
& =\sum_{q=1}^{p-1} N(p, q)\langle\underline{n}, V \underline{n}\rangle^{p-1-q} \sum_{\substack{r_{1} \neq \underline{n}, \ldots, r_{q} \neq \underline{n} \\
j_{1}+\cdots+j_{q}=p-1}} \frac{\left|\left\langle\underline{n}, V \underline{r}_{1}\right\rangle\right|^{2}}{\left(\lambda_{\underline{r_{1}}}-\lambda_{\underline{n}}\right)^{j_{1}}} \cdot \frac{\left|\left\langle\underline{n}, V \underline{r}_{2}\right\rangle\right|^{2}}{\left(\lambda_{\underline{r}_{2}}-\lambda_{\underline{n}}\right)^{j_{2}}} \cdots \frac{\left|\left\langle\underline{n}, V \underline{\underline{r}_{q}}\right\rangle\right|^{2}}{\left(\lambda_{\underline{r}_{q}}-\lambda_{\underline{n}}\right)^{j_{q}}} .
\end{aligned}
$$

This concludes the proof.

The first step in estimating the coefficients $B_{\ell}(n)$ is therefore estimating the fractions $|\langle\underline{n}, V \underline{r}\rangle|^{2} /\left(\lambda_{\underline{r}}-\lambda_{\underline{n}}\right)^{j}$. In turn, this requires an analysis of the vanishing mechanism of the denominators $\left(\lambda_{\underline{r}}-\lambda_{\underline{r}}\right)^{j}$. A preliminary remark is:

Lemma 2.3. With the assumptions of Lemma 2.2,

$$
\sum_{\underline{r} \neq \underline{n} \in \mathbb{Z}^{s}} \frac{\left|\langle\underline{n}, V \underline{r}\rangle^{2}\right|}{\left|\lambda_{\underline{r}}-\lambda_{\underline{n}}\right|^{j}} \leq \frac{p A}{\alpha^{p}}\left(\frac{p \Lambda}{\alpha^{\gamma}}\right)^{j}(\gamma j) !
$$

Proof. Equation (2-9) is a direct consequence of assumptions (A3) and (A4) because

$$
\sum_{\underline{r} \neq \underline{n} \in \mathbb{Z}^{s}} \frac{\left|\langle\underline{n}, V \underline{r}\rangle^{2}\right|}{\left|\lambda_{\underline{r}}-\lambda_{\underline{n}}\right|^{j}} \leq A \Lambda^{j} \sum_{\underline{r} \neq \underline{n} \in \mathbb{Z}^{s}}|\underline{r}-\underline{n}|^{\gamma j} e^{-\alpha|\underline{r}-\underline{n}|}=A \Lambda^{j} \sum_{\underline{x} \neq 0}|\underline{x}|^{\gamma j} e^{-\alpha|\underline{x}|},
$$

where

$\sum_{\underline{x} \neq 0}|\underline{x}|^{\gamma j} e^{-\alpha|\underline{x}|}:=\sum_{\underline{x} \neq 0}\left[\left|x_{1}\right|^{\gamma}+\cdots+\left|x_{p}\right|^{\gamma}\right]^{j} e^{-\alpha|\underline{x}|} \leq p^{j}\left(\left|x_{1}\right|^{\gamma j}+\cdots+\left|x_{p}\right|^{\gamma j}\right) e^{-\alpha|\underline{x}|}$.

Hence,

$$
\sum_{\underline{x} \neq 0}|\underline{x}|^{\gamma j} e^{-\alpha|\underline{x}|} \leq p^{j+1} \sum_{x_{1} \neq 0}\left|x_{1}\right|^{\gamma j} e^{-\alpha|\underline{x}|} \leq \frac{p^{j+1}(\gamma j) !}{\alpha^{\gamma j+p}}
$$


and summing up we get

$$
\sum_{\underline{r} \neq \underline{n} \in \mathbb{Z}^{s}} \frac{\left|\langle\underline{n}, V \underline{r}\rangle^{2}\right|}{\left|\lambda_{\underline{r}}-\lambda_{\underline{n}}\right|^{j}} \leq \frac{p A}{\alpha^{p}}\left(\frac{p \Lambda}{\alpha^{\gamma}}\right)^{j}(\gamma j) !
$$

and this proves the lemma.

Corollary 2.4. Recalling that $k_{1}+\cdots+k_{p-1}=p-1, p=2, \ldots$, in the assumptions of Lemma 2.3, the following bounds hold:

$$
\begin{aligned}
\left|B_{\ell, p}(\underline{n})\right| & \leq \sum_{k_{1}+\cdots+k_{p-1}=p-1}\left|\left\langle n, V\left[S_{\underline{n}}\right]^{k_{1}} \cdots V\left[S_{\underline{n}}\right]^{k_{p-1}} V n\right\rangle\right| \\
& \leq p\left(\frac{2 A}{\alpha}\right)^{p}\left(\frac{p \Lambda}{\alpha^{\gamma}}\right)^{p-1} \sum_{q=1}^{p-1} p^{q} \sum_{j_{1}+\cdots+j_{q}=p-1}\left(\gamma j_{1}\right) ! \cdots\left(\gamma j_{q}\right) ! .
\end{aligned}
$$

Proof. It is enough to insert (2-9) in the statement of Lemma 2.2 on account of the bounds $N(p, q)<2^{p},|\langle\underline{n}, V \underline{n}\rangle| \leq 1$ and the fact that $j_{1}+\cdots+j_{q}=p-1$.

We can now state and prove the main estimate.

Proposition 2.5. Under assumptions (A1)-(A3),

$$
\left|B_{\ell}(\underline{n})\right| \leq\left(4 \ell^{2}\right)^{\ell+1} R(\Lambda, \alpha, \gamma)^{\ell}[\gamma(\ell-1)] ! .
$$

Proof. We have, by (2-4),

$$
\left|B_{\ell}(\underline{n})\right| \leq \sum_{p=1}^{\ell} \frac{B_{\ell, p}(\underline{n})}{p}
$$

Clearly,

Moreover,

$$
\sum_{j_{1}+\cdots+j_{q}=p-1}\left(\gamma j_{1}\right) ! \cdots\left(\gamma j_{q}\right) ! \leq(p-1)[\gamma(p-1)] ! .
$$

$$
\sum_{q=1}^{p-1} p^{q} \leq p^{p}
$$

Therefore, by Corollary 2.4,

$$
\left|B_{\ell, p}(\underline{n})\right|<(2 p)^{p}(p-1) p\left(\frac{2 A}{\alpha}\right)^{p}\left(\frac{p \Lambda}{\alpha^{\gamma}}\right)^{p-1}[\gamma(p-1)] !,
$$

whence since $A<\alpha$

$$
\begin{aligned}
\left|B_{\ell}(\underline{n})\right| & \leq \sum_{p=1}^{\ell} B_{\ell, p}(\underline{n}) \leq(2 \ell)^{\ell} \ell^{3} 2^{\ell} \ell^{\ell-1}\left(\frac{\Lambda}{\alpha^{\gamma}}\right)^{\ell-1}[\gamma(\ell-1)] ! \\
& \leq\left(4 \ell^{2}\right)^{\ell+1} R(\Lambda, \alpha, \gamma)^{\ell}[\gamma(\ell-1)] !,
\end{aligned}
$$


where

$$
R(\Lambda, \alpha, \gamma):=\frac{\Lambda}{\alpha^{\gamma}}
$$

Thus, the proof of the proposition is complete.

Proof of Theorem 1.1. The assertion is just (2-11).

Proof of Corollary 1.2. The validity of (1-11) is a direct consequence of the existence of the perturbation expansion for all $\underline{n} \in \mathbb{N}^{p}$. The uniformity with respect to $\underline{n}$ follows from the $\underline{n}$-independent bound (1-10).

Example 1.5 (continued). Consider again the normalized eigenvectors of $T$ :

$$
\psi_{\underline{n}}(\underline{x})=\prod_{k=1}^{s} \psi_{n_{k}}\left(x_{k}\right), \quad \psi_{n}(x):=\frac{1}{\sqrt{2^{n} n !}} e^{-x^{2} / 2} H_{n}(x), n=0,1, \ldots,
$$

where $H_{n}(x), x \in \mathbb{R}$, is the $n$-th Hermite polynomial. The vectors $\left\{\psi_{n}(\underline{x}): \underline{n} \in \mathbb{N}^{s}\right\}$ form an orthonormal basis in $L^{2}\left(\mathbb{R}^{s}\right)$. Recall that

$$
\mathcal{V}(\underline{x}):=e^{-|\underline{x}|^{q}} \Phi(\underline{x}) .
$$

Consequently, considering the potential $\mathcal{V}(\underline{x})$ and the corresponding maximal multiplication operator $V$ in $L^{2}\left(\mathbb{R}^{s}\right)$, we have:

Proposition 2.6. Condition (A4) is fulfilled in this example; i.e., there is $\Sigma(q)>0$ such that

$$
\left\langle\psi_{\underline{m}}, V \psi_{\underline{n}}\right\rangle:=\langle\underline{m}, \mathcal{V}(\underline{x}) \underline{n}\rangle \leq e^{-\Sigma[|\underline{m}|+|\underline{n}|]} .
$$

Proof. Consider first the case $s=1$. Recall the formula

$$
H_{n}(x)=n ! \sum_{k=0}^{[n / 2]} \frac{(-1)^{k}(2 x)^{n-2 k}}{k !(n-2 k) !}
$$

where as usual $[n / 2]$ is the integer part of $n$. We can thus write

$$
\psi_{m}(x) \psi_{n}(x)=\sqrt{2^{-(m+n) / 2} m ! n !} \sum_{h=0}^{[m / 2]} \sum_{k=0}^{[n / 2]} \frac{(-1)^{h+k}(2 x)^{m-2 h}(2 x)^{n-2 k}}{h !(m-2 h) ! k !(n-2 k) !},
$$

whence

$$
\begin{aligned}
|\langle m, \mathcal{V} n\rangle| & \leq \sqrt{2^{(m+n) / 2} m ! n !} \sum_{h=0}^{[m / 2]} \sum_{k=0}^{[n / 2]} \frac{\int_{R} x^{m+n-2(h+k)} e^{-|x|^{q}} d x}{h !(m-2 h) ! k !(n-2 k) !} \\
& <\sqrt{2^{(m+n) / 2} m ! n !} \sum_{h=0}^{[m / 2]} \sum_{k=0}^{[n / 2]} \frac{\Gamma([m+n-2(h+k)+1] / q)}{h !(m-2 h) ! k !(n-2 k) !} .
\end{aligned}
$$


Now,

$$
\begin{aligned}
\min _{0 \leq h \leq[m / 2]}[h !(m-2 h) !] & =\Gamma(m / 3+1)^{2}, \\
\min _{0 \leq k \leq[n / 2]}[h !(n-2 k) !] & =\Gamma(n / 3+1)^{2}, \\
\max _{\substack{0 \leq h \leq[m / 2] \\
0 \leq k \leq[n / 2]}}[\Gamma([m+n-2(h+k)+1] / q)] & =\Gamma([m+n+1] / q),
\end{aligned}
$$

and this implies

$$
|\langle m, \mathcal{V} n\rangle| \leq \sqrt{2^{(m+n) / 2} m ! n !} \cdot\left[\frac{m}{2}\right] \cdot\left[\frac{n}{2}\right] \frac{\Gamma([m+n+1] / q)}{\Gamma(m / 3+1)^{2} \cdot \Gamma(n / 3+1)^{2}} .
$$

Now apply the Stirling formula. Since

$$
6 \pi 2^{(m+n) / 4} e^{-(m+n) / 2} e^{-[m+n+1] / q} e^{(m+n) / 3} \cdot\left[\frac{m}{2}\right] \cdot\left[\frac{n}{2}\right] \leq 1
$$

for $m+n$ large enough,

$$
|\langle m, \mathcal{V} n\rangle| \leq \frac{((m+q) / q)^{(m+q) / q}}{m^{m / 6} n^{n / 6}} .
$$

Without loss, we can take $m=n+k, k \geq 0$. Then

$$
|\langle m, \mathcal{V} n\rangle|=|\langle n, \mathcal{V}(n+k)\rangle| \leq \frac{[(2 n+k) / q]^{(2 n+k) / q}}{(n+k)^{(n+k) / 6} n^{n / 6}}
$$

Now $(n+k)>(2 n+k) / q>(n+k) / 6$ if $2<q<6$ and hence there is $0<L<1$ such that

$$
|\langle n, \mathcal{V}(n+k)\rangle| \leq \frac{[(2 n+k) / q]^{(n+k) / 6}}{(n+k)^{(n+k) / 6} n^{n / 6}}<\frac{L^{n+k}}{n^{n / 6}}=\frac{L^{m}}{n^{n / 6}},
$$

whence, a fortiori, with $L=e^{-\Sigma}$

$$
|\langle m, \mathcal{V} n\rangle| \leq e^{-\Sigma(m+n)} .
$$

This concludes the proof for $s=1$. The general case follows through an immediate product argument.

\section{References}

[Andrews 1976] G. E. Andrews, The theory of partitions, Cambridge University, 1976.

[Brownell and Clark 1961] F. H. Brownell and C. W. Clark, "Asymptotic distribution of the eigenvalues of the lower part of the Schrödinger operator spectrum”, J. Math. Mech. 10:1 (1961), 31-70.

[Kato 1976] T. Kato, Perturbation theory for linear operators, 2nd ed., Grundlehren der mathematischen Wissenschaften 132, Springer, Berlin, 1976.

[McLeod 1961] J. B. McLeod, "The distribution of the eigenvalues for the hydrogen atom and similar cases", Proc. London Math. Soc. (3) 11 (1961), 139-158. 
[Reed and Simon 1978] M. Reed and B. Simon, Methods of modern mathematical physics, IV: Analysis of operators, Academic, San Diego, 1978.

[Tamura 1974] H. Tamura, "The asymptotic distribution of the lower part eigenvalues for elliptic operators”, Proc. Japan Acad. 50 (1974), 185-187.

Received 26 Oct 2015. Revised 28 Oct 2015. Accepted 9 May 2016.

FIORELLA BARONE: fiorella.barone@uniba.it

Dipartimento di Matematica, Università di Bari, 70122 Bari, Italy

SANDRO GRAFFI: sandro.graffi@unibo.it

Dipartimento di Matematica, Università di Bologna, 40127 Bologna, Italy

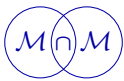





\title{
SOME RESULTS ON THE ASYMPTOTIC BEHAVIOR OF FINITE CONNECTION PROBABILITIES IN PERCOLATION
}

\author{
Massimo Campanino And Michele Gianfelice
}

\begin{abstract}
We review results of two previous papers on the asymptotic behavior of finite connection probabilities in three or more dimensions for Bernoulli percolation and the Fortuin-Kasteleyn random-cluster model. In the introduction, we prove a multidimensional renewal theorem that is needed for these results and previous results on Ornstein-Zernike behavior; the proof is significantly simpler than that originally derived by Doney (1966) and those of other subsequent works on this subject.
\end{abstract}

\section{Introduction}

In the last few decades, much progress has been made in the rigorous study of the asymptotic behavior of connection functions in percolation outside the critical point. This problem is related to that of typical fluctuations of clusters and, in two dimensions, of interfaces [Gallavotti 1972; Greenberg and Ioffe 2005].

In the case of the subcritical regime for Bernoulli percolation or the FortuinKasteleyn (FK) random-cluster model on a regular lattice, connection functions, i.e., the probabilities that two points are connected, decay exponentially as the distance between the points tends to infinity [Grimmett 1999; 2006]. In [Campanino and Ioffe 2002; Campanino et al. 2008], the exact asymptotic behavior of this decay has been established. It is called Ornstein-Zernike behavior [1914] since they derived it in statistical mechanics for systems outside the critical point. Moreover, in [Campanino and Ioffe 2002; Campanino et al. 2008], the strict convexity with positive Gaussian curvature and the analyticity of equidecay surfaces were established.

An extension of the techniques developed in [Campanino and Ioffe 2002] has been developed in [Campanino and Gianfelice 2009], where the exact asymptotics

\section{Communicated by Raffaele Esposito.}

Campanino and Gianfelice are partially supported by the Gruppo Nazionale per l'Analisi Matematica, la Probabilità e le loro Applicazioni (GNAMPA) and thank the referee for useful suggestions.

MSC2010: 60K35, 82B43, 60K15, 60F17.

Keywords: random-cluster model, Ornstein-Zernike behavior for connectivities, renormalization,

Ruelle operator, local limit theorem, invariance principle. 
for the probability that three points are connected has been derived. We refer the reader to [Coquille et al. 2014] for an application of this result.

In this paper, we review some recent results related to the case of finite connections in the supercritical regime. Here an infinite cluster exists and ordinary connection functions between two points converge to a positive constant as their distance tends to infinity. Indeed, in order to be connected between each other, it is enough for them to be both connected to the infinite cluster that is spread everywhere. One is therefore led to consider finite connection functions, i.e., the probabilities that two points are connected between each other but not to the infinite cluster. In this case, the points are surrounded by a surface in the dual lattice, where plaquettes of the dual lattice are occupied when the dual bond is vacant. One can expect in three or more dimensions that these surfaces have similar fluctuations as the connecting clusters in the subcritical regime and therefore that finite connection functions also exhibit Ornstein-Zernike behavior. The situation in two dimensions, where the dual surface is basically composed of two random paths that can freely fluctuate with the only constraint being no intersection, is different. In this case, for Bernoulli percolation on the square lattice above the critical point, [Campanino et al. 2010] established that finite connection functions exhibit a different behavior, corresponding to that of two independent random paths.

Here we expose some recent results on the asymptotic behavior of finite connection functions in three or more dimensions that appeared in [Campanino and Gianfelice 2011; 2015]. These results refer both to Bernoulli percolation and to the FK random-cluster model in the highly supercritical regime, i.e., when the parameter $p$ related to the occupation probability of a bond is close to 1 . It would be desirable to extend the validity of the results to a larger region of parameter values.

1.1. Notation. We denote $|x|:=\sum_{i=1}^{d}\left|x_{i}\right|$, by $\langle\cdot, \cdot\rangle$ the scalar product in $\mathbb{R}^{d}$ and by $\|\cdot\|:=\sqrt{\langle\cdot, \cdot\rangle}$ the associated Euclidean norm. We then set, for $x \neq 0$, $\hat{x}:=x /\|x\|$ and $\mathbb{S}^{d-1}:=\left\{z \in \mathbb{R}^{d}:\|z\|=1\right\}$, and denoting by $B$ the closed unit ball in $\mathbb{R}^{d}$, for $r>0$, we let $r B:=\left\{x \in \mathbb{R}^{d}:\|x\| \leq r\right\}$ and $B_{r}(x):=x+r B$.

For any $t \in \mathbb{R}^{d}$, we define

$$
\mathscr{H}_{x}^{t}:=\left\{y \in \mathbb{R}^{d}:\langle t, y\rangle=0\right\}
$$

to be the $(d-1)$-dimensional hyperplane in $\mathbb{R}^{d}$ orthogonal to the vector $t$ passing through $x$.

In the sequel, we will omit the dependence on $x$ in the notation if the point is taken to be the origin.

1.2. A note on the multidimensional renewal process. We first consider a simple model of a "multidimensional renewal process" that, with suitable adaptations, is at the basis of the arguments used in most of these works. 
Let $\left\{X_{i}\right\}_{i \geq 1}$ be a sequence of independent and identically distributed random vectors with values in $\mathbb{Z}^{d}$. We assume that $\mathbb{E}\left[e^{t\left|X_{1 \mid}\right|}\right]<\infty$ for some $t>0$. Moreover, we assume that the support of the law of $X_{1}$ spans all of $\mathbb{Z}^{d}$ and that $\mu:=\mathbb{E}\left[X_{1}\right]$ is such that $\mu \neq 0$.

The exact asymptotics of the expected number of visits to a point $x \in \mathbb{Z}^{d}$ by the random walk just defined, as $\|x\|$ tends to infinity, was derived for the first time in [Doney 1966] under the hypothesis of the existence of the first $l$ moments, with $l$ an explicit function of the lattice dimensions. Extensions of this result to the renewal measure of $x+B$, with $B$ a bounded subset of $\mathbb{R}^{d}$, are given in [Stam 1969], as well as in [Carlsson and Wainger 1984] where the exponential decay of the tail of $X_{1}$ is also assumed.

Here, under this last assumption, we present a proof of the multidimensional theorem on which Theorem 1 relies; the proof is considerably simpler than that originally derived in [Doney 1966].

Under the hypotheses just given on the law of $X_{1}$, the following properties are satisfied:

Local limit theorem: There exists $\alpha \in\left(0, \frac{1}{2}\right)$ such that, uniformly in $x \in \mathbb{Z}^{d}$ with $\|x-n \mu\|<n^{1 / 2+\alpha}$ as $n$ tends to infinity,

$$
\mathbb{P}\left\{\sum_{i=1}^{n} X_{i}=x\right\}=\frac{\exp \left\{-\left\langle A^{-1}(x-n \mu),(x-n \mu)\right\rangle / 2 n\right\}}{\sqrt{(2 \pi n)^{d} \operatorname{det} A}}(1+o(1)),
$$

where $A$ is the covariance matrix of $X_{1}$.

Large deviation estimate from the mean: This follows from the exponential tail of the distribution of $X_{1}$.

To estimate the asymptotic behavior in $n$ of the series

$$
\sum_{k \geq 1} \mathbb{P}\left\{\sum_{i=1}^{k} X_{i}=n \mu\right\}
$$

we decompose it as

$$
\begin{aligned}
& \sum_{k \geq 1} \mathbb{P}\left\{\sum_{i=1}^{k} X_{i}=n \mu\right\}= \sum_{k=1}^{n-\left\lfloor n^{1 / 2+\alpha}\right\rfloor} \mathbb{P}\left\{\sum_{i=1}^{k} X_{i}=n \mu\right\} \\
&+\sum_{k=n-\left\lfloor n^{1 / 2+\alpha}\right\rfloor+1}^{n+\left\lfloor n^{1 / 2+\alpha}\right\rfloor-1} \mathbb{P}\left\{\sum_{i=1}^{k} X_{i}=n \mu\right\} \\
&+\sum_{k \geq n+\left\lfloor n^{1 / 2+\alpha}\right\rfloor} \mathbb{P}\left\{\sum_{i=1}^{k} X_{i}=n \mu\right\} .
\end{aligned}
$$


Then we estimate the second term on the right-hand side by using the local limit theorem estimate and the sum of the first and the third terms by using the large deviation upper bound.

Therefore, we get

$$
\begin{aligned}
& \sum_{k \in \mathbb{N}:|k-n|<n^{1 / 2+\alpha}} \frac{\exp \left\{-\left\langle A^{-1}(k \mu-n \mu),(k \mu-n \mu)\right\rangle / 2 k\right\}}{\sqrt{(2 \pi k)^{d} \operatorname{det} A}}(1+o(1)) \\
& =\sum_{k \in \mathbb{N}:|k-n|<n^{1 / 2+\alpha}} \frac{\exp \left\{-|k-n|^{2}\left\langle A^{-1} \mu, \mu\right\rangle /\left(2 n\left(1+O\left(n^{\alpha-1 / 2}\right)\right)\right)\right\}}{\sqrt{\left(2 \pi n\left(1+O\left(n^{\alpha-1 / 2}\right)\right)^{d} \operatorname{det} A\right.}}(1+o(1)) \\
& =\frac{1}{\sqrt{(2 \pi n)^{d-1}(\operatorname{det} A)\left\langle A^{-1} \mu, \mu\right\rangle}}(1+o(1)) .
\end{aligned}
$$

Moreover, by the standard large deviation estimate for the sums of independent and identically distributed random variables with exponential tails, we obtain

$$
\sum_{k \in \mathbb{N}:|k-n| \geq n^{1 / 2+\alpha}} \mathbb{P}\left\{\sum_{i=1}^{k} X_{i}=n \mu\right\} \leq e^{-c_{1} n^{2 \alpha}} .
$$

Summing up (7) and (8),

$$
\sum_{k \geq 1} \mathbb{P}\left\{\sum_{i=1}^{k} X_{i}=n \mu\right\}=\frac{c_{2}}{n^{(d-1) / 2}}(1+o(1)),
$$

with $c_{2}$ a positive constant.

1.3. Possible developments. It would be desirable to extend the results of [Campanino and Gianfelice 2011;2015] to values of $p$ larger than some critical $p_{c}$. Moreover, it should be possible to extend the results of [Campanino et al. 2010] from independent percolation to the FK random-cluster model.

\section{Ornstein-Zernike behavior for the finite connectivity function in highly supercritical percolation models}

2.1. Bernoulli percolation and the FK random-cluster model. Let $\mathbb{L}^{d}$ denote the graph associated to $\left(\mathbb{Z}^{d}, \mathbb{E}^{d}\right)$, with

$$
\mathbb{E}^{d}:=\left\{\{x, y\} \in \mathscr{P}_{2}\left(\mathbb{Z}^{d}\right):|x-y|=1\right\} .
$$

Let $\mathfrak{L}_{0}$ be the collection of subgraphs of $\llbracket^{d}$ of finite order. If $G \in \mathfrak{L}_{0}, V(G)$ is the set of vertices and $E(G)$ is the set of edges of $G$. We denote by $\bar{G}$ the graph 
induced by the union of $V(G)^{1}$ with the sets of vertices of the components of $\llbracket^{d} \backslash G$ of finite size. We define the boundary of $G$ as the set

$$
\partial G:=\left\{e \in \mathbb{E}^{d} \backslash E(G):|e \cap V(G)|=1\right\} \subset \mathbb{E}^{d}
$$

and the external boundary of $G$ to be $\bar{\partial} G:=\partial \bar{G}$.

Considering the realization of $\mathbb{L}^{d}$ as a geometric graph embedded in $\mathbb{R}^{d}$, which with abuse of notation we still denote by $\mathbb{L}^{d}$, we can look at it as a cell complex, i.e., as the union of $\mathbb{Z}^{d}$ and $\mathbb{E}^{d}$ representing the collections of 0 -cells and of 1-cells, respectively. We denote by $\left(\mathbb{Z}^{d}\right)^{*}$ the collection of $d$-cells dual to 0 -cells in $\mathbb{L}^{d}$, that is, the collection of Voronoi cells of $\mathbb{L}^{d}$, and by $\left(\mathbb{E}^{d}\right)^{*}$ the collection of $(d-1)$-cells dual to 1-cells in $\mathbb{\llbracket}^{d}$, usually called plaquettes in the physics literature.

A bond percolation configuration on $\mathbb{L}^{d}$ is a map $\mathbb{E}^{d} \ni e \mapsto \omega_{e} \in\{0,1\}$. Setting $\Omega:=\{0,1\}^{\mathbb{E}^{d}}$, we define

$$
\Omega \ni \omega \mapsto \boldsymbol{E}(\omega):=\left\{e \in \mathbb{E}^{d}: \omega_{e}=1\right\} \in \mathscr{P}\left(\mathbb{E}^{d}\right) .
$$

Denoting by $\mathbb{G}:=\left\{G \subseteq \mathbb{L}^{d}: G=G(E), E \in \mathscr{P}\left(\mathbb{E}^{d}\right)\right\}$ the collection of spanning subgraphs of $\mathbb{L}^{d}$, we define the random graph

$$
\Omega \ni \omega \mapsto \boldsymbol{G}(\omega):=G(\boldsymbol{E}(\omega)) \in \mathbb{G}
$$

and by $\kappa(\omega)$ the number of its components. Then, given $l \geq 1$ and $x_{1}, \ldots, x_{l} \in \mathbb{Z}^{d}$, we denote by

$$
\Omega \ni \omega \mapsto \boldsymbol{C}_{\left\{x_{1}, \ldots, x_{l}\right\}}(\omega) \in \mathscr{P}\left(\mathbb{Z}^{d}\right)
$$

the common open cluster of the points $x_{1}, \ldots, x_{l} \in \mathbb{Z}^{d}$, that is, the set of vertices of the component of the random graph $\boldsymbol{G}$ to which these points belong, provided it exists, and set

$$
\Omega \ni \omega \mapsto \boldsymbol{E}_{\left\{x_{1}, \ldots, x_{l}\right\}}(\omega):=E\left(\boldsymbol{C}_{\left\{x_{1}, \ldots, x_{l}\right\}}(\omega)\right) \cap \boldsymbol{E}(\omega) \subseteq \mathbb{E}^{d} .
$$

We also define, in case $\boldsymbol{C}_{\left\{x_{1}, \ldots, x_{l}\right\}}$ is finite, the random set $\bar{\partial} \boldsymbol{C}_{\left\{x_{1}, \ldots, x_{l}\right\}}$ to be equal to $\bar{\partial} G$ if $G$ is the component of $\boldsymbol{G}$ whose set of vertices is $\boldsymbol{C}_{\left\{x_{1}, \ldots, x_{l}\right\}}$ and the random set

$$
\boldsymbol{S}_{\left\{x_{1}, \ldots, x_{l}\right\}}:=\left(\bar{\partial} \boldsymbol{C}_{\left\{x_{1}, \ldots, x_{l}\right\}}\right)^{*} .
$$

Let $\mathscr{F}$ be the $\sigma$-algebra generated by the cylinder events of $\Omega$. If $\Lambda$ is a finite subset of $\mathbb{Z}^{d}$, let $\mathbb{E}^{\Lambda}$ be the subset of $\mathbb{E}^{d}$ such that $V\left(\mathbb{E}^{\Lambda}\right)=\Lambda$ and denote $\Omega_{\Lambda}:=$ $\{0,1\}^{\mathbb{E}^{\Lambda}}$, by $\mathscr{F}_{\Lambda}$ the corresponding product $\sigma$-algebra and by $\mathscr{T}_{\Lambda}$ the $\sigma$-algebra generated by the cylinder events $\left\{\omega \in \Omega: \omega_{\Delta} \in A\right\}$, where $\Delta \subset \Lambda^{c}$ and $A \in \mathscr{F}_{\Delta}$. The random-cluster measures on $\mathbb{Z}^{d}$ [Fortuin and Kasteleyn 1972; Edwards and Sokal

${ }^{1}$ We refer the reader to [Campanino and Gianfelice 2011; 2015] for the notions of graph theory needed in the study of percolation theory. 
1988] with parameters $q \geq 1$ and $p \in[0,1]$ are the dependent bond percolation probability measures $\mathbb{P}$ on $(\Omega, \mathscr{F})$ specified by

$$
\mathbb{P}\left(A \mid \mathscr{T}_{\Lambda}\right)=\mathbb{P}_{\Lambda ; q, p}(A) \mathbb{P}-\text { a.s., } \quad A \in \mathscr{F},
$$

where, setting, for any $\pi \in \Omega_{\Lambda}^{c}, \Omega_{\Lambda}^{\pi}:=\left\{\omega \in \Omega: \omega_{e}=\pi_{e}, e \in \mathbb{E}^{d} \backslash \mathbb{E}^{\Lambda}\right\}, \mathbb{P}_{\Lambda ; q, p}^{\pi}$ is the probability measure on $(\Omega, \mathscr{F})$ with density

$$
\mathbb{P}_{\Lambda ; q, p}^{\pi}(\omega):=\frac{1}{\mathscr{E}_{\Lambda}^{\pi}(q ; p)} p^{|\boldsymbol{E}(\omega)|}(1-p)^{\left|\mathbb{E}^{\Lambda} \backslash \boldsymbol{E}(\omega)\right|} q^{\kappa_{\Lambda}(\omega)} \mathbf{1}_{\Omega_{\Lambda}^{\pi}}(\omega),
$$

where $\kappa_{\Lambda}(\omega)$ is the number of the components of $\boldsymbol{G}(\omega)$ intersecting $\Lambda$.

For $q=1$, the FK random-cluster model coincides with Bernoulli percolation. Therefore, we omit in this case the value of $q$ in the specification of $\mathbb{P}$.

Random-cluster measures satisfy the FKG inequality; that is, for any couple $f, g$ of random variables increasing with respect to the natural partial order defined on $\Omega, \mathbb{P}(f g) \geq \mathbb{P}(f) \mathbb{P}(g)$. Moreover, the partial order of $\Omega$ induces a stochastic ordering on the elements of the collection of probability measures defined by (18); namely, for any increasing random variable $f, \mathbb{P}_{\Lambda ; q, p}^{\pi_{1}}(f) \leq \mathbb{P}_{\Lambda ; q, p}^{\pi_{2}}(f)$ if $\pi_{1} \leq \pi_{2}$. Hence, denoting by $\preceq$ such an ordering, for all $\pi \in \Omega_{\Lambda}^{c}$, $\mathbb{P}_{\Lambda ; q, p}^{\mathrm{f}} \preceq \mathbb{P}_{\Lambda ; q, p}^{\pi} \preceq \mathbb{P}_{\Lambda ; q, p}^{\mathrm{W}}$, where $\mathbb{P}_{\Lambda ; q, p}^{\mathrm{f}}$ and $\mathbb{P}_{\Lambda ; q, p}^{\mathrm{W}}$ stand for the probability measure with density (18) corresponding to the free $(\pi \equiv 0)$ and to the wired $(\pi \equiv 1)$ boundary conditions, respectively. Since, for \# =f, w, the (weak) limit of the sequence $\left\{\mathbb{P}_{\Lambda ; q, p}^{\#}\right\}$ along any exhaustion $\{\Lambda\} \uparrow \mathbb{Z}^{d}$ exists (see, e.g., [Grimmett 2006, Theorem 4.19]) and is the random-cluster measure that we denote by $\mathbb{P}_{q, p}^{\#}$, the ordering $\preceq$ extends as well to random-cluster measures and $\mathbb{P}_{q, p}^{\mathrm{f}} \preceq \mathbb{P} \preceq \mathbb{P}_{q, p}^{\mathrm{W}}$.

Furthermore, denoting by $\mathbb{P}_{p^{\prime}}:=\mathbb{P}_{1, p^{\prime}}$ the independent Bernoulli bond percolation probability measures on $\mathbb{Z}^{d}$ with parameter $p^{\prime}$, by Theorem 3.21 of [Grimmett 2006, p. 43], we obtain the stochastic domination inequalities

$$
\mathbb{P}_{p(q)} \preceq \mathbb{P}_{q, p}^{\mathrm{f}} \preceq \mathbb{P}_{q, p}^{\mathrm{w}} \preceq \mathbb{P}_{p},
$$

where $p(q):=p /(p+q(1-p))$.

In the following, we assume the random-cluster measure $\mathbb{P}_{q, p}$ to be translationinvariant.

\subsection{Results.}

Theorem 1. For any $d \geq 3$ and any $q \geq 1$, there exists $p_{0}=p_{0}(q, d)$ such that, for all $p>p_{0}$, uniformly in $x \in \mathbb{Z}^{d}$ as $\|x\| \rightarrow \infty$,

$$
\mathbb{P}_{q, p}\left\{0 \leftrightarrow x,\left|\boldsymbol{C}_{\{0, x\}}\right|<\infty\right\}=\frac{\Phi_{q, p}(\hat{x})}{\sqrt{(2 \pi\|x\|)^{d-1}}} e^{-\tau_{q, p}(x)}(1+o(1)),
$$


where $\Phi_{q, p}$ is a positive real analytic function on $\mathbb{S}^{d-1}$ and $\tau_{q, p}$ an equivalent norm in $\mathbb{R}^{d}$.

As a byproduct of the proof, we also obtain the following result.

Theorem 2. For any $d \geq 3$ and any $q \geq 1$, there exists $p_{0}=p_{0}(q, d)$ such that, for all $p>p_{0}$, the equidecay set of the two-point finite connectivity function is locally analytic and strictly convex. Moreover, the Gaussian curvature of the equidecay set is uniformly positive.

These theorems are proven for the independent percolation case in [Campanino and Gianfelice 2011] and for the general case in [Campanino and Gianfelice 2015].

2.3. Analysis of connectivities. The following result appears as Proposition 4 in [Campanino and Gianfelice 2015].

Proposition 3. Given $q \geq 1$ and $p \in(0,1)$, let $\mathbb{P}_{q, p}$ be a translation-invariant random-cluster measure on $\mathbb{Z}^{d}$ with parameters $q$ and $p$. Then for any $x \in \mathbb{R}^{d}$,

$$
\tau_{q, p}(x):=-\lim _{n \rightarrow \infty} \frac{1}{n} \log \mathbb{P}_{q, p}\left\{0 \leftrightarrow\lfloor n x\rfloor,\left|\boldsymbol{C}_{\{0,\lfloor n x\rfloor\}}\right|<\infty\right\}
$$

exists and is a convex and homogeneous-of-order-1 function on $\mathbb{R}^{d}$.

Proof. For any $\Delta \subseteq \mathbb{Z}^{d}$, let us denote by $\boldsymbol{E}_{\Delta}:=\bigcup_{x \in \Delta} \boldsymbol{E}_{\{x\}} \subseteq \mathbb{E}^{d}$ the set of edges belonging to open paths starting at the vertices of $\Delta$.

Now let $\Lambda$ be a finite subset of $\mathbb{Z}^{d}$ such that $\Lambda \ni 0$. For any two distinct lattice points $x, y \in \Lambda$, looking at $\mathbf{1}_{\left\{0 \leftrightarrow x, 0 \leftrightarrow \Lambda^{c}\right\}}$ and $\mathbf{1}_{\left\{x \leftrightarrow y, y \leftrightarrow \Lambda^{c}\right\}}$ as functions of $\left(\boldsymbol{E}_{\{x\}}, \boldsymbol{E}_{\Lambda^{c}}\right)$, they are both nondecreasing on $\boldsymbol{E}_{\{x\}}$ and nonincreasing on $\boldsymbol{E}_{\Lambda^{c}}$. Therefore, by Theorem 2.1 in [van den Berg et al. 2006],

$$
\begin{aligned}
& \mathbb{P}_{q, p}\left(\left\{0 \leftrightarrow x, 0 \leftrightarrow \Lambda^{c}\right\} \cap\left\{x \leftrightarrow y, y \leftrightarrow \Lambda^{c}\right\} \mid\left\{x \leftrightarrow \Lambda^{c}\right\}\right) \\
& \geq \mathbb{P}_{q, p}\left(\left\{0 \leftrightarrow x, 0 \leftrightarrow \Lambda^{c}\right\} \mid\left\{x \leftrightarrow \Lambda^{c}\right\}\right) \mathbb{P}_{q, p}\left(\left\{x \leftrightarrow y, y \leftrightarrow \Lambda^{c}\right\} \mid\left\{x \leftrightarrow \Lambda^{c}\right\}\right) ;
\end{aligned}
$$

that is,

$$
\begin{aligned}
\mathbb{P}_{q, p}\left\{x \leftrightarrow \Lambda^{c}\right\} \mathbb{P}_{q, p}\left\{0 \leftrightarrow x, x \leftrightarrow y, x \leftrightarrow \Lambda^{c}\right\} \\
\\
\quad \geq \mathbb{P}_{q, p}\left\{0 \leftrightarrow x, x \leftrightarrow \Lambda^{c}\right\} \times \mathbb{P}_{q, p}\left\{x \leftrightarrow y, x \leftrightarrow \Lambda^{c}\right\},
\end{aligned}
$$

which implies

$$
\begin{aligned}
\mathbb{P}_{q, p}\left\{0 \leftrightarrow x, x \leftrightarrow y, \boldsymbol{C}_{\{0, x, y\}} \cap \Lambda^{c}=\varnothing\right\} \\
\\
\quad \geq \mathbb{P}_{q, p}\left\{0 \leftrightarrow x, \boldsymbol{C}_{\{0, x\}} \cap \Lambda^{c}=\varnothing\right\} \times \mathbb{P}_{q, p}\left\{x \leftrightarrow y, \boldsymbol{C}_{\{x, y\}} \cap \Lambda^{c}=\varnothing\right\} .
\end{aligned}
$$

But

$$
\mathbb{P}_{q, p}\left\{0 \leftrightarrow y, \boldsymbol{C}_{\{0, y\}} \cap \Lambda^{c}=\varnothing\right\} \geq \mathbb{P}_{q, p}\left\{0 \leftrightarrow x, x \leftrightarrow y, \boldsymbol{C}_{\{0, x, y\}} \cap \Lambda^{c}=\varnothing\right\}
$$


hence,

$$
\begin{aligned}
\mathbb{P}_{q, p}\{0 & \left.\leftrightarrow y, \boldsymbol{C}_{\{0, y\}} \cap \Lambda^{c}=\varnothing\right\} \\
& \geq \mathbb{P}_{q, p}\left\{0 \leftrightarrow x, \boldsymbol{C}_{\{0, x\}} \cap \Lambda^{c}=\varnothing\right\} \times \mathbb{P}_{q, p}\left\{x \leftrightarrow y, \boldsymbol{C}_{\{x, y\}} \cap \Lambda^{c}=\varnothing\right\} .
\end{aligned}
$$

Taking the limit $\Lambda \uparrow \mathbb{Z}^{d}$,

$$
\begin{aligned}
\mathbb{P}_{q, p}\left(\left\{0 \leftrightarrow y,\left|\boldsymbol{C}_{\{0, y\}}\right|<\infty\right\}\right) \\
\quad \geq \mathbb{P}_{q, p}\left(\left\{0 \leftrightarrow x,\left|\boldsymbol{C}_{\{0, x\}}\right|<\infty\right\}\right) \times \mathbb{P}_{q, p}\left(\left\{x \leftrightarrow y,\left|\boldsymbol{C}_{\{x, y\}}\right|<\infty\right\}\right) .
\end{aligned}
$$

Proceeding as in the proof of Proposition 15 in [Campanino and Gianfelice 2011], we obtain the thesis.

2.4. The independent percolation case. We will show, without going too much into details for which we refer the reader to [Campanino and Gianfelice 2011], how the proof of Theorem 1 in the independent percolation case can be reduced to that of an estimate of the form presented in (9).

Given $x, y \in \mathbb{Z}^{d}$, we set

$$
\varphi(x, y):=\left\{\begin{array}{cc}
\min \left\{\left|S_{\{x, y\}}(\omega)\right|: \omega \in\{x \leftrightarrow y\}\right\}, & x \neq y, \\
0, & x=y .
\end{array}\right.
$$

Here $\varphi$ is symmetric and translation-invariant, so we write $\varphi(x, y)=\varphi(x-y)$.

We remark that, given $G_{i}:=\left(V_{i}, E_{i}\right), i=1,2$, two connected subgraphs of $\mathbb{L}^{d}$ of finite size, by (11), $\partial\left(G_{1} \cup G_{2}\right) \subseteq \partial G_{1} \cup \partial G_{2}$. Moreover,

$$
\bar{\partial}\left(G_{1} \cup G_{2}\right)=\partial\left(\overline{G_{1} \cup G_{2}}\right) \subseteq \partial \overline{G_{1}} \cup \partial \overline{G_{2}} .
$$

This allowed us to prove (see Lemma 4 and Proposition 5 in [Campanino and Gianfelice 2011]) that the sequence $\left\{\bar{\varphi}_{n}\right\}_{n \in \mathbb{N}}$, where, for any $n \in \mathbb{N}, \mathbb{R}^{d} \ni x \mapsto \bar{\varphi}_{n}(x):=$ $\varphi(\lfloor n x\rfloor) / n \in \mathbb{R}^{+}$, converges pointwise to $\bar{\varphi}$, which is a convex, homogeneous-oforder-1 function on $\mathbb{R}^{d}$. Furthermore, $\left\{\bar{\varphi}_{n}\right\}_{n \in \mathbb{N}}$ converges uniformly on $\mathbb{S}^{d-1}$.

Conjecture 4. We conjecture that $\bar{\varphi}$ is the $l_{1}$ norm in $\mathbb{R}^{d}$, i.e., $\bar{\varphi}(x)=|x|:=$ $\sum_{i=1}^{d}\left|x_{i}\right|$.

In the limit of $p$ tending to 1 , the support of the probability distribution of the finite cluster containing two sites $x$ and $y$, conditioned to its existence, is given by the configurations minimizing $\varphi(x-y)$. Therefore, given $p$ sufficiently close to 1 , we can estimate the probability that the finite cluster of two points has an external boundary of size larger than $(1+\delta) \varphi(x-y)$, for $\delta$ larger than a given value $\delta^{*}(p, d)$ that tends to 0 as $p$ tends to 1 . Indeed, we have:

Proposition 5. There exist $c_{3}=c_{3}(d)>1$ and $\delta^{*}=\delta^{*}(p, d)$, with $\lim _{p \uparrow 1} \delta^{*}(p, d)=$ 0 , such that, for any $p \in\left(1-1 / c_{3}, 1\right)$ and any $\delta>\delta^{*}$,

$$
\mathbb{P}_{p}\left(\left\{\left|\boldsymbol{S}_{\{0, x\}}\right| \geq(1+\delta) \varphi(x)\right\} \mid\left\{\left|\boldsymbol{C}_{\{0, x\}}\right|<\infty\right\}\right) \leq c_{4} e^{-c_{5} \varphi(x)},
$$


with $c_{4}=c_{4}(d, p)>1$ and $c_{5}=c_{5}(d, p, \delta)>0$.

Hence, we are left with the estimate of the probability that the external boundary of $\boldsymbol{C}_{\{0, x\}}$ has size smaller than $(1+\delta) \varphi(x)$ for $\delta>\delta^{*}$.

Renewal structure of connectivities. Given $t \in \mathbb{S}^{d-1}$, we define

$$
\mathscr{H}_{y}^{t}:=\left\{x \in \mathbb{R}^{d}:\langle t, x\rangle=\langle t, y\rangle\right\}, \quad y \in \mathbb{R}^{d},
$$

to be the $(d-1)$-dimensional hyperplane in $\mathbb{R}^{d}$ orthogonal to the vector $t$ passing through a point $y \in \mathbb{R}^{d}$ and the corresponding half-spaces

$$
\begin{aligned}
& \mathscr{H}_{y}^{t,-}:=\left\{x \in \mathbb{R}^{d}:\langle t, x\rangle \leq\langle t, y\rangle\right\}, \\
& \mathscr{H}_{y}^{t,+}:=\left\{x \in \mathbb{R}^{d}:\langle t, x\rangle \geq\langle t, y\rangle\right\} .
\end{aligned}
$$

Let $t \in \mathbb{S}^{d}$. Given two points $x, y \in \mathbb{Z}^{d}$ such that $\langle x, t\rangle \leq\langle y, t\rangle$, we denote by $\boldsymbol{C}_{\{x, y\}}^{t}$ the cluster of $x$ and $y$ inside the strip $\mathscr{S}_{\{x, y\}}^{t}:=\mathscr{H}_{x}^{t,+} \cap \mathscr{H}_{y}^{t,-}$ provided it exists.

Let $u$ be the first of the unit vectors in the direction of the coordinate axes $u_{1}, \ldots, u_{d}$ such that $\langle t, u\rangle$ is maximal

Definition 6. Given $t \in \mathbb{S}^{d-1}$, let $x, y \in \mathbb{Z}^{d}$ such that $\langle x, t\rangle \leq\langle y, t\rangle$ be connected in $\mathscr{S}_{\{x, y\}}^{t}$. The points $b \in \boldsymbol{C}_{\{x, y\}}^{t}$ such that

(1) $\langle t, x+u\rangle \leq\langle t, b\rangle \leq\langle t, y-u\rangle$ and

(2) $\boldsymbol{C}_{\{x, y\}}^{t} \cap \mathscr{S}_{\{b-u, b+u\}}^{t}=\{b-u, b, b+u\}$

are said to be $t$-break points of $\boldsymbol{C}_{\{x, y\}}$. The collection of such points, which we remark is a totally ordered set with respect to the scalar product with $t$, will be denoted by $\boldsymbol{B}^{t}(x, y)$.

Definition 7. Given $t \in \mathbb{S}^{d-1}$, let $x, y \in \mathbb{Z}^{d}$ such that $\langle x, t\rangle \leq\langle y, t\rangle$ be connected in $\mathscr{S}_{\{x, y\}}^{t}$. An edge $\{b, b+u\}$ such that $b, b+u \in \boldsymbol{B}^{t}(x, y)$ is called a $t$-bond of $\boldsymbol{C}_{\{x, y\}}$. The collection of such edges will be denoted by $\boldsymbol{E}^{t}(x, y)$ while $\boldsymbol{B}_{e}^{t}(x, y) \subset$ $\boldsymbol{B}^{t}(x, y)$ will denote the subcollection of $t$-break points $b$ of $\boldsymbol{C}_{\{x, y\}}$ such that the edge $\{b, b+u\} \in \boldsymbol{E}^{t}(x, y)$.

Definition 8. Given $t \in \mathbb{S}^{d-1}$, let $x, y \in \mathbb{Z}^{d}$ such that $\langle t, x\rangle \leq\langle t, y\rangle$ be connected. Then $x, y \in \mathbb{Z}^{d}$ are said to be $h_{t}$-connected if

(1) $x$ and $y$ are connected in $\mathscr{Y}_{\{x, y\}}^{t}$ and $\left|\boldsymbol{C}_{\{x, y\}}^{t}\right|<\infty$ and

(2) $x+u, y-u \in \boldsymbol{B}^{t}(x, y)$.

Moreover, denoting by $\left\{x \stackrel{h_{t}}{\leftrightarrow} y\right\}$ the event that $x$ and $y$ are $h_{t}$-connected, we set

$$
h_{t}^{(p)}(x, y):=\mathbb{P}_{p}\left\{x \stackrel{h_{t}}{\leftrightarrow} y\right\} .
$$


Notice that, by translation invariance, $h_{t}^{(p)}(x, y)=h_{t}^{(p)}(y-x, 0)$, so in the sequel, we will denote it simply by $h_{t}^{(p)}(y-x)$. We also define by convention $h_{t}^{(p)}(0)=1$.

Definition 9. Let $t \in \mathbb{S}^{d-1}$ and $x, y \in \mathbb{Z}^{d}$ be $h_{t}$-connected. If $\boldsymbol{B}^{t}(x+u, y-u)$ is empty, then $x$ and $y$ are said to be $f_{t}$-connected and the corresponding event is denoted by $\left\{x \stackrel{f_{t}}{\leftrightarrow} y\right\}$. We then set

$$
f_{t}^{(p)}(y-x):=\mathbb{P}_{p}\left\{x \stackrel{f_{t}}{\leftrightarrow} y\right\} .
$$

We define by convention $f_{t}^{(p)}(0)=0$.

Definition 10. Given $t \in \mathbb{S}^{d-1}$, let $x, y \in \mathbb{Z}^{d}$ such that $\langle t, x\rangle \leq\langle t, y\rangle$ be connected. Then:

(1) $x, y$ are called $\bar{h}_{t}$-connected and the corresponding event is denoted by $\left\{x \stackrel{\bar{h}_{t}}{\leftrightarrow} y\right\}$, if $\boldsymbol{C}_{\{x, y\}} \cap \mathscr{Y}_{\{y-u, y\}}^{t}=\{y-u, y\}$ and $\left|\boldsymbol{C}_{\{x, y\}} \cap \mathscr{H}_{y}^{t,-}\right|<\infty$.

(2) $x, y$ are called $\bar{f}_{t}$-connected and the corresponding event is denoted by $\left\{x \stackrel{\bar{f}_{t}}{\leftrightarrow} y\right\}$, if they are $\bar{h}_{t}$-connected and $\boldsymbol{B}^{t}(x, y)=\varnothing$.

Definition 11. Given $t \in \mathbb{S}^{d-1}$, let $x, y \in \mathbb{Z}^{d}$ such that $\langle t, x\rangle \leq\langle t, y\rangle$ be connected. Then:

(1) $x$ and $y$ are called $\tilde{h}_{t}$-connected, and the corresponding event is denoted by $\left\{x \stackrel{\tilde{h}_{t}}{\leftrightarrow} y\right\}$ if

(a) $\boldsymbol{C}_{\{x, y\}} \cap \mathscr{Y}_{\{x, x+u\}}^{t}=\{x, x+u\}$ and

(b) $\left|\boldsymbol{C}_{\{x, y\}} \cap \mathscr{H}_{x}^{t,+}\right|<\infty$.

(2) $x$ and $y$ are called $\tilde{f}_{t}$-connected, and the corresponding event is denoted by $\left\{x \stackrel{f_{t}}{\leftrightarrows} y\right\}$ if they are $\tilde{h}_{t}$-connected and $\boldsymbol{B}^{t}(x, y)=\varnothing$.

The functions $\bar{h}_{t}^{(p)}(x, y):=\mathbb{P}_{p}\left\{x \stackrel{\bar{h}_{t}}{\leftrightarrow} y\right\}$ and $\tilde{h}_{t}^{(p)}(x, y):=\mathbb{P}_{p}\left\{x \stackrel{\tilde{h}_{t}}{\leftrightarrow} y\right\}$ are translation invariant.

Denoting by $g_{t}^{(p)}(x, y)$, for $t \in \mathbb{S}^{d-1}$, the probability of the event

$$
\left\{x \stackrel{g_{t}}{\leftrightarrow} y\right\}:=\left\{x \leftrightarrow y,\left|\boldsymbol{C}_{\{x, y\}}\right|<\infty,\left|\boldsymbol{B}_{e}^{t}(x, y)\right| \leq 1\right\},
$$

which is also translation invariant, we obtain

$$
\begin{aligned}
\mathbb{P}_{p}\left\{0 \leftrightarrow x,\left|\boldsymbol{C}_{\{0, x\}}\right|<\infty\right\} & =g_{t}^{(p)}(x)+\sum_{z_{1}, z_{2} \in \mathbb{Z}^{d}} \bar{f}_{t}^{(p)}\left(z_{1}\right) h_{t}^{(p)}\left(z_{2}-z_{1}\right) \tilde{f}_{t}^{(p)}\left(x-z_{2}\right), \\
h_{t}^{(p)}(x) & =\sum_{z \in \mathbb{Z}^{d}} f_{t}^{(p)}(z) h_{t}^{(p)}(x-z) .
\end{aligned}
$$


Proposition 12. Given $t \in \mathbb{S}^{d-1}$, for any $p \in(0,1)$ and $x \in \mathbb{R}^{d}$ such that $\langle t, x\rangle>0$,

$$
\tau_{p}^{t}(x):=-\lim _{n \rightarrow \infty} \frac{1}{n} \log h_{t}^{(p)}(\lfloor n x\rfloor)
$$

exists and is a convex and homogeneous-of-order-1 function on $\mathbb{R}^{d}$. Moreover, for $p \in\left(1-1 / c_{3}, 1\right)$,

$$
\tau_{p}^{t}(x) \geq \bar{\varphi}(x) \log \frac{1}{c_{3}(1-p)} .
$$

The proof follows from the supermultiplicativity property of the function $h_{t}^{(p)}$; we refer the reader to Proposition 15 in [Campanino and Gianfelice 2011] for the details.

Since, by Proposition 3, for any $p \in(0,1)$ and $d \geq 2, \tau_{p}:=\tau_{1, p}$ is an equivalent norm in $\mathbb{R}^{d}$, there exists $c_{-}=c_{-}(p, d)>0$ such that

$$
\mathbb{P}_{p}\left\{0 \leftrightarrow x,\left|\boldsymbol{C}_{\{0, x\}}\right|<\infty\right\} \leq e^{-c_{-}\|x\|},
$$

while as a byproduct of the proof of Proposition 5 we get that there exists $c_{+}=$ $c_{+}(p, d)>0$ such that

$$
h_{t}^{(p)}(x) \geq e^{-c_{+}\|x\|},
$$

it follows that $\tau_{p}^{t} \geq \tau_{p}$ is finite and is an equivalent norm in $\mathbb{R}^{d}$.

Renormalization. We define

$$
\mathscr{W}:=\bigcap_{\hat{x} \in \mathbb{S}^{d-1}}\left\{w \in \mathbb{R}^{d}:\langle w, \hat{x}\rangle \leq \bar{\varphi}(\hat{x})\right\} .
$$

Given $x \in \mathbb{Z}^{d}$, let $t \in \mathfrak{d} W(x):=\{w \in \mathfrak{d} W:\langle w, x\rangle=\bar{\varphi}(x)\}$.

For $N \in \mathbb{N}$ larger than 1 , let us set $\mathfrak{t}_{N}=\mathfrak{t}_{N}(x):=\lfloor\|x\| / N\rfloor-1$ and

$$
\begin{aligned}
& y_{i}:=\lfloor i N \hat{x}\rfloor, \mathscr{H}_{i}^{t}:=\mathscr{H}_{y_{i}}^{t}, \mathscr{H}_{i}^{t,-}:=\mathscr{H}_{y_{i}}^{t,-}, \mathscr{H}_{i}^{t,+}:=\mathscr{H}_{y_{i}}^{t,+}, \quad i=0, \ldots, \mathfrak{t}_{N}, \\
& y_{\mathfrak{t}_{N}+1}:=x, \mathcal{H}_{y_{t_{N}+1}^{t}}^{t}:=\mathscr{H}_{x}^{t}, \mathcal{H}_{y_{\mathfrak{t}_{N}+1}^{t,-}}^{t}:=\mathcal{H}_{x}^{t,-}, \\
& \mathscr{S}_{i}^{t}:=\mathscr{H}_{i}^{t,+} \cap \mathscr{H}_{i+1}^{t,-} \text {. }
\end{aligned}
$$

With slight notational abuse, we still denote by $S_{\{0, x\}}$ its representation as a hypersurface in $\mathbb{R}^{d}$ and define

$$
\boldsymbol{C}_{i}^{t}:=\boldsymbol{C}_{\{0, x\}} \cap \mathscr{S}_{i}^{t}, \quad \boldsymbol{S}_{i}^{t}:=\boldsymbol{S}_{\{0, x\}} \cap \mathscr{S}_{i}^{t} .
$$

Hence, $\boldsymbol{C}_{\{0, x\}}=\bigcup_{i=0}^{\mathfrak{t}_{N}} \boldsymbol{C}_{i}^{t}$ and $\boldsymbol{S}_{\{0, x\}} \cap \mathcal{S}_{0, x}^{t} \subseteq \bigcup_{i=0}^{\mathrm{t}_{N}} \boldsymbol{S}_{i}^{t}$.

We call crossing any connected component $s$ of $\boldsymbol{S}_{i}^{t}$ such that, denoting by $\mathscr{K}(\boldsymbol{s})$ the compact subset of $\mathscr{S}_{i}^{t}$ whose boundary is $s$, there exist $y \in \mathscr{H}_{i}^{t,-} \cap \mathbb{Z}^{d}$ and $y^{\prime} \in \mathscr{H}_{i+1}^{t,+} \cap \mathbb{Z}^{d}$, both belonging to $\boldsymbol{C}_{\{0, x\}}$, which are connected by an open path in $\mathbb{L}^{d} \cap \mathscr{K}(\boldsymbol{s})$. 
We remark that, since $\boldsymbol{C}_{\{0, x\}}$ is connected, the existence of two crossings in $\mathcal{Y}_{i}^{t}$ implies the existence of two disjoint paths connecting $\mathscr{H}_{i}^{t}$ and $\mathscr{H}_{i+1}^{t}$ while the converse does not hold true in general.

We say that a slab $\mathscr{Y}_{i}^{t}$ is good if $\boldsymbol{S}_{i}^{t}$ is connected and made by just a single crossing of size smaller than twice the minimal one; otherwise, we call it bad.

In Section 3 of [Campanino and Gianfelice 2011], making use of a deterministic statement, we proved that the number of bad slabs is at most $2 \delta\|x\| / N$. Therefore, it is possible to modify the configuration of at most $c_{6} N^{d}$ bonds, with $c_{6}=$ $c_{6}(d, \delta)$, inside any 3 -tuple of consecutive slabs containing a single crossing in such a way that the resulting cluster will have at least one $t$-bond inside each of these slabs. Since these modifications can be performed independently, this fact and Proposition 5 imply the mass-gap condition $f_{t}^{(p)}(x) \leq e^{-c_{7}\|x\|} h_{t}^{(p)}(x)$ with $c_{7}=c_{7}(p)>0$, uniformly in $t \in \mathbb{S}_{x}^{d-1}$. Thus, from (37), we have $\tau_{p}^{t}=\tau_{p}$ for any $t \in \mathbb{S}_{x}^{d-1}$.

Extending $f_{t}^{(p)}$ to a function defined on the whole lattice by setting it equal to 0 , where it is undefined, set

$$
\begin{aligned}
& \mathbb{R}^{d} \ni s \mapsto H_{t}^{(p)}(s):=\sum_{x \in \mathbb{Z}^{d}} h_{t}^{(p)}(x) e^{\langle s, x\rangle} \in \overline{\mathbb{R}}, \\
& \mathbb{R}^{d} \ni s \mapsto F_{t}^{(p)}(s):=\sum_{x \in \mathbb{Z}^{d}} f_{t}^{(p)}(x) e^{\langle s, x\rangle} \in \overline{\mathbb{R}} .
\end{aligned}
$$

The renewal equation (38) implies

$$
H_{t}^{(p)}(s)=\frac{1}{1-F_{t}^{(p)}(s)} .
$$

Since (41) implies that, for all $p \in\left(p_{c}(d), 1\right)$, the effective domain of $H_{t}(s)$,

$$
\mathscr{D}_{t}^{p}:=\left\{s \in \mathbb{R}^{d}: H_{t}^{(p)}(s)<\infty\right\},
$$

is not empty since $\stackrel{\mathscr{D}}{t}_{t}^{p} \supseteq \mathscr{\Upsilon}_{t}^{p} \ni 0$, where

$$
\mathscr{K}_{t}^{p}:=\bigcap_{\hat{x} \in \mathbb{S}^{d-1}}\left\{s \in \mathbb{R}^{d}:\langle s, \hat{x}\rangle \leq \tau_{p}^{t}(\hat{x})\right\}
$$

is the convex body polar with respect to $u_{t}^{p}:=\left\{x \in \mathbb{R}^{d}: \tau_{t}^{p}(x) \leq 1\right\}$.

For $s \in \mathcal{K}_{t}^{p}$, since

$$
\langle s, x\rangle \leq \max _{s \in \mathscr{K}_{t}^{p}}\langle s, x\rangle=\tau_{p}^{t}(x) \leq 1,
$$

$h_{t}^{(p)}(x) \leq e^{-\tau_{p}^{t}(x)}$ and $F_{t}^{(p)}(s)$ is finite, moreover continuous, then, for all $s \in \partial \mathcal{K}_{t}^{p}$,

$$
\mathbb{Z}^{d} \ni x \mapsto q_{t ; s}^{(p)}(x):=f_{t}^{(p)}(x) e^{\langle s, x\rangle} \in \mathbb{R}
$$


is the density of the probability measure $Q_{t ; s}^{(p)}$ on $\left(\mathbb{Z}^{d}, \mathscr{B}\left(\mathbb{Z}^{d}\right)\right)$, which has exponentially decaying tails:

$$
f_{t}^{(p)}(x) e^{\langle s, x\rangle} \leq e^{-c_{7}\|x\|} h_{t}^{(p)}(x) e^{\langle s, x\rangle} \leq e^{-c_{7}\|x\|} .
$$

If $X$ is a random vector with probability distribution $Q_{t ; s}^{(p)}$, denoting by $\mathbb{E}_{p}^{t ; s}$ the expectation of a random variable under $Q_{t ; s}^{(p)}$, we set

$$
\mu_{t}^{p}(s):=\mathbb{E}_{p}^{t ; s}[X]=\operatorname{grad} \log F_{t}^{(p)}(s),
$$

while

$$
C_{t}^{p}(s):=\text { Hess } \log F_{t}^{(p)}(s)
$$

denotes the covariance matrix of $X$. Since $f_{t}^{(p)}(x)>0$ on a whole half-space, $C_{t}^{p}(s)$ is nondegenerate. Hence,

$$
\partial \mathcal{K}_{t}^{p}=\left\{s \in \mathbb{R}^{d}: F_{t}^{(p)}(s)=1\right\} \subseteq \mathbb{R}^{d} \backslash \mathscr{D}_{t}^{p}
$$

is a real analytic strictly convex surface with Gaussian curvature uniformly bounded away from 0 , and therefore, because $Q_{t ; s}^{(p)}$ is supported on $\mathscr{H}_{0}^{t,+} \cap \mathbb{Z}^{d}, \mu_{t}^{p}(s) \neq 0$ and $\left(s, \mu_{t}^{p}(s)\right)>0$ for any $s \in B_{r}(t) \cap \partial \mathscr{K}_{t}^{p}$ with $r$ sufficiently small.

Then let $s \in B_{r}(t) \cap \partial \mathscr{K}_{t}^{p}$; for any $\mu \in B\left(\mu_{t}^{p}(s)\right) \cap \mathscr{H}_{\mu_{t}^{p}(s)}^{s}$, if $\left\{X_{i}\right\}_{i \geq 1}$ is a sequence of independent and identically distributed random vectors distributed according to $Q_{t ; s}^{(p)}$, for $n \in \mathbb{N}$, we can rewrite (38) as

$$
h_{t}^{(p)}(\lfloor n \mu\rfloor)=\delta_{0}(\lfloor n \mu\rfloor)+e^{-\langle\lfloor n \mu\rfloor, s\rangle} \sum_{k \geq 1} \bigotimes_{i=1}^{k} Q_{t ; s}^{(p)}\left\{\sum_{i=1}^{k} X_{i}=\lfloor n \mu\rfloor\right\} .
$$

Then the proof of the Theorem 1 follow from (9).

2.5. The case of the FK random-cluster model. One can extend the previous result to finite connections of the FK random-cluster model when the parameter $p$ related to the occupation probability of a bond is close to 1 . As in the independent percolation case, an estimate on the size of the surface in the dual lattice surrounding a finite cluster of two points, as the one given in Proposition 5, is still in force due to the stochastic domination inequalities given in (19).

This led us to consider only realizations of the cluster $\boldsymbol{C}_{\{0, x\}}$ whose external boundary has size smaller than or equal to $(1+\delta) \varphi(x)$ for sufficiently small $\delta$. Moreover, a suitably modified deterministic statement, as the one presented before to prove mass-gap condition, allows us to give a probabilistic description of $\boldsymbol{C}_{\{0, x\}}$ in terms of a concatenation of subclusters stretching along the direction dual to $\hat{x}$ with respect to $\tau_{p, q}$, whose sizes, under the conditional measure $\mathbb{P}_{q, p}\left(\cdot \mid\left\{0<\left|\boldsymbol{C}_{\{0, x\}}\right|<\infty\right\}\right)$, have exponentially decaying tails. 
When the parameter $p$ is close to 1 , one can perform a polymer expansion for the supercritical random-cluster model. In this way, we can reduce the analysis of the exact asymptotics of the finite two-point connection function to the proof of a local limit theorem result for a random process via thermodynamic formalism as in [Campanino et al. 2003].

We refer the reader to [Campanino and Gianfelice 2015] for the details.

\section{References}

[van den Berg et al. 2006] J. van den Berg, O. Häggström, and J. Kahn, "Some conditional correlation inequalities for percolation and related processes", Random Structures Algorithms 29:4 (2006), $417-435$.

[Campanino and Gianfelice 2009] M. Campanino and M. Gianfelice, "A local limit theorem for triple connections in subcritical Bernoulli percolation”, Probab. Theory Related Fields 143:3-4 (2009), 353-378.

[Campanino and Gianfelice 2011] M. Campanino and M. Gianfelice, "On the Ornstein-Zernike behaviour for the Bernoulli bond percolation on $\mathbb{Z}^{d}, d \geq 3$, in the supercritical regime", J. Stat. Phys. 145:6 (2011), 1407-1422.

[Campanino and Gianfelice 2015] M. Campanino and M. Gianfelice, "On the Ornstein-Zernike behaviour for the supercritical random-cluster model on $\mathbb{Z}^{d}, d \geq 3$ ”, J. Stat. Phys. 159:6 (2015), $1456-1476$.

[Campanino and Ioffe 2002] M. Campanino and D. Ioffe, "Ornstein-Zernike theory for the Bernoulli bond percolation on $\mathbb{Z}^{d}$ ", Ann. Probab. 30:2 (2002), 652-682.

[Campanino et al. 2003] M. Campanino, D. Ioffe, and Y. Velenik, "Ornstein-Zernike theory for finite range Ising models above $T_{c}$ ", Probab. Theory Related Fields 125:3 (2003), 305-349.

[Campanino et al. 2008] M. Campanino, D. Ioffe, and Y. Velenik, "Fluctuation theory of connectivities for subcritical random cluster models", Ann. Probab. 36:4 (2008), 1287-1321.

[Campanino et al. 2010] M. Campanino, D. Ioffe, and O. Louidor, "Finite connections for supercritical Bernoulli bond percolation in 2D", Markov Process. Related Fields 16:2 (2010), 225-266.

[Carlsson and Wainger 1984] H. Carlsson and S. Wainger, "On the multidimensional renewal theorem”, J. Math. Anal. Appl. 100:1 (1984), 316-322.

[Coquille et al. 2014] L. Coquille, H. Duminil-Copin, D. Ioffe, and Y. Velenik, "On the Gibbs states of the noncritical Potts model on $\mathbb{Z}^{2}$ ", Probab. Theory Related Fields 158:1-2 (2014), 477-512.

[Doney 1966] R. A. Doney, “An analogue of the renewal theorem in higher dimensions", Proc. London Math. Soc. (3) 16 (1966), 669-684.

[Edwards and Sokal 1988] R. G. Edwards and A. D. Sokal, "Generalization of the Fortuin-KasteleynSwendsen-Wang representation and Monte Carlo algorithm”, Phys. Rev. D (3) 38:6 (1988), 2009_ 2012.

[Fortuin and Kasteleyn 1972] C. M. Fortuin and P. W. Kasteleyn, "On the random-cluster model, I: Introduction and relation to other models", Physica 57 (1972), 536-564.

[Gallavotti 1972] G. Gallavotti, "The phase separation line in the two-dimensional Ising model", Comm. Math. Phys. 27 (1972), 103-136.

[Greenberg and Ioffe 2005] L. Greenberg and D. Ioffe, "On an invariance principle for phase separation lines”, Ann. Inst. H. Poincaré Probab. Statist. 41:5 (2005), 871-885. 
[Grimmett 1999] G. Grimmett, Percolation, 2nd ed., Grundlehren der mathematischen Wissenschaften 321, Springer, Berlin, 1999.

[Grimmett 2006] G. Grimmett, The random-cluster model, Grundlehren der mathematischen Wissenschaften 333, Springer, Berlin, 2006.

[Ornstein and Zernike 1914] L. S. Ornstein and F. Zernike, "Accidental deviations of density and opalescence at the critical point of a single substance", Nederl. Akad. Wetensch. Proc. 17:2 (1914), 793-806.

[Stam 1969] A. J. Stam, "Renewal theory in $r$ dimensions, I", Compositio Math. 21 (1969), 383399.

Received 1 Apr 2016. Revised 1 May 2016. Accepted 31 May 2016.

MASSIMO CAMPANINO: massimo.campanino@unibo.it

Dipartimento di Matematica, Università degli Studi di Bologna, I-40127 Bologna, Italy

MiCHELE GianfeliCE: gianfelice@mat.unical.it

Dipartimento di Matematica e Informatica, Università della Calabria, Campus di Arcavacata, I-87036 Arcavacata di Rende, Italy 



\title{
CORRELATION INEQUALITIES FOR THE POTTS MODEL
}

\author{
GEOFFREY R. GRIMMETT
}

Dedicated in friendship to Lucio Russo

\begin{abstract}
Correlation inequalities are presented for ferromagnetic Potts models with external field, using the random-cluster representation of Fortuin and Kasteleyn, together with the FKG inequality. These results extend and simplify earlier inequalities of Ganikhodjaev and Razak, and also of Schonmann, and include GKS-type inequalities when the spin space is taken as the set of $q$-th roots of unity.
\end{abstract}

\section{Introduction}

Correlation inequalities are key to the classical theory of interacting systems in statistical mechanics. The Ising model, especially, has a plethora of associated inequalities that have played significant roles in the development of a coherent theory of phase transition (see, for example, the books [7; 22]). These inequalities are frequently named after their discoverers, and include inequalities of Griffiths [14; 15; 16], Griffiths, Kelly, and Sherman (GKS) [20], Griffiths, Hurst, and Sherman (GHS) [17], Ginibre [13], Simon and Lieb [21; 24], and so on.

A more probabilistic theory of Ising/Potts models has emerged since around 1970 , initiated partly by the work of Fortuin and Kasteleyn $[8 ; 9 ; 10]$ on the random-cluster representation of the Potts model and the random-current method championed by Aizenman [1] and co-authors. Probably the principle inequality in the probabilistic formulation is that of Fortuin, Kasteleyn, and Ginibre (FKG) [11].

Inequalities are rarer for the Potts model, and our purpose in this note is to derive certain correlation inequalities for a ferromagnetic Potts model with external field, akin to the GKS inequalities for the Ising model. The main technique used here is the random-cluster representation of this model and particularly the FKG inequality.

Our results generalize and simplify the work of Ganikhodjaev and Razak [12], who have shown how to formulate and prove GKS-type inequalities for the Potts

\section{Communicated by Raffaele Esposito.}

MSC2010: 82B20, 60K35.

Keywords: Griffiths inequality, GKS inequality, Ising model, Potts model, random-cluster model, angular spins. 
model with a general number $q$ of local states. Furthermore, our Theorems 3.5 and 3.7 extend the two correlation inequalities of Schonmann [23], which in turn extended inequalities of [6]. Some of the arguments given here may be known to others.

The structure of this paper is as follows. The Potts and random-cluster models are introduced in Section 2, and the results of the paper (Theorems 3.5-3.7) follow in Section 3. The proofs are given in Sections 4, 5, and 6.

\section{The Potts model with external field}

Let $G=(V, E)$ be a finite graph, and let $J=\left(J_{e}: e \in E\right)$ and $h=\left(h_{v}: v \in V\right)$ be vectors of nonnegative reals and $q \in\{2,3, \ldots\}$. An edge $e \in E$ joins two distinct vertices $x$ and $y$, and we write $e=\langle x, y\rangle$.

We take the "local state space" for the $q$-state Potts model to be the set $\mathcal{Q}:=$ $\{0,1, \ldots, q-1\}$ of "spins". The configuration space of the model is the product space $\Sigma:=\mathcal{Q}^{V}$, and a typical configuration is written $\sigma=\left(\sigma_{v}: v \in V\right) \in \Sigma$. The Potts measure on $G$ with parameters $J$ and $h$ has sample space $\Sigma$ and probability measure given by

$$
\pi(\sigma)=\frac{1}{Z} \exp \left\{\sum_{e=\langle x, y\rangle \in E} J_{e} \delta_{e}(\sigma)+\sum_{v \in V} h_{v} \delta_{v}(\sigma)\right\}, \quad \sigma \in \Sigma,
$$

where $\delta_{e}(\sigma)=\delta_{\sigma_{x}, \sigma_{y}}$ and $\delta_{v}(\sigma)=\delta_{\sigma_{v}, 0}$ are Kronecker delta functions and $Z$ is the appropriate normalizing constant. Thus, the $J_{e}$ are edge-coupling constants, and the $h_{v}$ are external fields relative to the local state 0 . The Potts measure is said to be ferromagnetic since $J_{e} \geq 0$ for $e \in E$.

We shall make use of the random-cluster representation, for a recent account and bibliography of which we refer the reader to [18]. The graph $G$ is augmented by adding a "ghost" vertex $g$, which is joined by edges $\langle g, v\rangle$ to each vertex $v \in V$; the ensuing graph is denoted $G^{+}=\left(V^{+}, E^{+}\right)$. The relevant sample space is the product space $\Omega:=\{0,1\}^{E^{+}}$. For $\omega=\left(\omega_{e}: e \in E^{+}\right) \in \Omega$, an edge $e$ is called open if $\omega_{e}=1$ and closed otherwise.

An edge $e \in E$ is assigned parameter $p_{e}=1-e^{-J_{e}}$, and an edge of the form $\langle g, v\rangle$ is assigned parameter $p_{v}=1-e^{-h_{v}}$. The random-cluster probability measure $\phi$ on $G$ has sample space $\Omega$ and is given by

$$
\phi(\omega)=\frac{1}{Z_{\mathrm{RC}}}\left\{\prod_{e=\langle x, y\rangle \in E^{+}} p_{e}^{\omega_{e}}\left(1-p_{e}\right)^{1-\omega_{e}}\right\} q^{k(\omega)}, \quad \omega \in \Omega,
$$

where $k(\omega)$ is the number of connected components of the graph with vertex set $V^{+}$ and edge set $\eta(\omega):=\left\{e \in E^{+}: \omega_{e}=1\right\}$. 
The relationship between the Potts model and the random-cluster model is explained in [18, §1.4], where it is shown in particular that $Z_{\mathrm{RC}}=e^{-|E|} Z$.

The measures $\pi$ and $\phi$ may be coupled as follows. Suppose $\omega$ is sampled from $\Omega$ according to $\phi$, and let $C_{v}$ be the connected component of $(V, \eta(\omega))$ containing $v \in V^{+}$; the $C_{v}$ are called open clusters. Every vertex in $C_{g}$ is allocated spin 0 . To an open cluster of $\omega$ other than $C_{g}$, we allocate a uniformly chosen spin from $\mathcal{Q}$ such that every vertex in the cluster receives this spin and the spins of different clusters are independent. The ensuing spin vector $\sigma=\sigma(\omega)$ has law $\pi$. See [18, Theorem 1.3] for a proof of this standard fact and for references to the original work of Fortuin and Kasteleyn.

This paper will make use of the FKG inequality and the comparison inequalities for the random-cluster model. These are presented in a number of places already and are not repeated here. The reader is referred instead to [18, Theorem 3.8] for the FKG inequality and to [18, Theorem 3.21$]$ for the comparison inequalities.

\section{The correlation inequalities}

We begin with a space of functions. Let $\mathcal{F}_{q}$ be the set of functions $f: \mathcal{Q} \rightarrow \mathbb{C}$ such that, for all integers $m, n \geq 0$,

$$
\begin{aligned}
& \mathbb{E}\left(f(X)^{m}\right) \text { is real and nonnegative, } \\
& \mathbb{E}\left(f(X)^{m+n}\right) \geq \mathbb{E}\left(f(X)^{m}\right) \mathbb{E}\left(f(X)^{n}\right),
\end{aligned}
$$

where $X$ is a uniformly distributed random variable on $\mathcal{Q}$. The above conditions may be written out as follows. We have that $f \in \mathcal{F}_{q}$ if, for $m, n \geq 0$,

$$
\begin{aligned}
& S_{m}:=\sum_{x \in \mathcal{Q}} f(x)^{m} \text { is real and nonnegative, } \\
& q S_{m+n} \geq S_{m} S_{n} .
\end{aligned}
$$

For $I \in \mathcal{Q}$, let $\mathcal{F}_{q}^{I}$ be the subset of $\mathcal{F}_{q}$ containing all $f$ such that

$$
f(I)=\max \{|f(x)|: x \in \mathcal{Q}\} .
$$

This condition entails that $f(I)$ is real and nonnegative.

Let $f: \mathcal{Q} \rightarrow \mathbb{C}$. For $\sigma \in \Sigma$, let

$$
f(\sigma)^{R}:=\prod_{v \in R} f\left(\sigma_{v}\right), \quad R \subseteq V .
$$

Thinking of $\sigma$ as a random vector with law $\pi$, we write $\left\langle f(\sigma)^{R}\right\rangle$ for the mean value of $f(\sigma)^{R}$. 
Theorem 3.5. Let $f \in \mathcal{F}_{q}^{0}$. For $R \subseteq V$, the mean $\left\langle f(\sigma)^{R}\right\rangle$ is real-valued and nondecreasing in the vectors $J$ and $h$ and satisfies $\left\langle f(\sigma)^{R}\right\rangle \geq 0$. For $R, S \subseteq V$, we have

$$
\left\langle f(\sigma)^{R} f(\sigma)^{S}\right\rangle \geq\left\langle f(\sigma)^{R}\right\rangle\left\langle f(\sigma)^{S}\right\rangle .
$$

If there is no external field, in that $h \equiv 0$, it suffices for the above that $f \in \mathcal{F}_{q}$ in place of $f \in \mathcal{F}_{q}^{0}$.

Here are three classes of functions belonging to $\mathcal{F}_{q}^{0}$.

Theorem 3.6. Let $q \geq 2$. The following functions $f: \mathcal{Q} \rightarrow \mathbb{C}$ belong to $\mathcal{F}_{q}^{0}$ :

(a) $f(x)=\frac{1}{2}(q-1)-x$,

(b) $f(x)=e^{2 \pi i x / q}$, a $q$-th root of unity, and

(c) $f: \mathcal{Q} \rightarrow[0, \infty)$, with $f(x) \leq f(0)$ for $x \in \mathcal{Q}$.

When combined with Theorem 3.5, case (a) yields the inequalities of Ganikhodjaev and Razak [12], but with simpler proofs. When $q=2$, the latter reduce to the GKS inequalities for the Ising model; see $[14 ; 15 ; 16 ; 20]$. We do not know if the implications of Theorem 3.5 with case (b) are either known or useful. Perhaps they are examples of the results of Ginibre [13]. In case (c) with $f(x)=\delta_{x, 0}$, Theorem 3.5 yields the first correlation inequality of Schonmann [23].

Our second main result follows next.

Theorem 3.7. Let $q \geq 2$ and $f_{0} \in \mathcal{F}_{q}^{0}$, and let $f_{1}: \mathcal{Q} \rightarrow \mathbb{C}$ satisfy (3.1). If $f_{0}$ and $f_{1}$ have disjoint support in that $f_{0} f_{1} \equiv 0$, then for $R, S \subseteq V$,

$$
\left\langle f_{0}(\sigma)^{R} f_{1}(\sigma)^{S}\right\rangle \leq\left\langle f_{0}(\sigma)^{R}\right\rangle\left\langle f_{1}(\sigma)^{S}\right\rangle .
$$

If $h \equiv 0$, it is enough to assume $f_{0} \in \mathcal{F}_{q}$ in place of $f_{0} \in \mathcal{F}_{q}^{0}$.

Two correlation inequalities were proved in [23]: a "positive" inequality that is implied by Theorems 3.5 and 3.6(c) and a "negative" inequality that is obtained as a special case of Theorem 3.7 on setting $f_{0}(x)=\delta_{x, 0}$ and $f_{1}(x)=\delta_{x, 1}$. Recall that Schonmann's inequalities were themselves (partial) generalizations of correlation inequalities of [6].

Amongst the feasible extensions of the above theorems that come to mind, we mention the classical space-time models used to study the quantum Ising/Potts models $[2 ; 3 ; 4 ; 5 ; 19]$.

\section{Proof of Theorem 3.5}

We use the coupling of the random-cluster and Potts model described in Section 2. Let $\omega \in \Omega$, and let $A_{g}, A_{1}, A_{2}, \ldots, A_{k}$ be the vertex sets of the open clusters of $\omega$, where $A_{g}$ is that of the open cluster $C_{g}$ containing $g$. 
Let $R \subseteq V$, and let $f \in \mathcal{F}_{q}^{0}$. By (3.4),

$$
f(\sigma)^{R}=f(0)^{\left|R \cap A_{g}\right|} \prod_{r=1}^{k} f\left(X_{r}\right)^{\left|R \cap A_{r}\right|},
$$

where $X_{r}$ is the random spin assigned to $A_{r}$. This has conditional expectation $g_{R}: \Omega \rightarrow \mathbb{C}$ given by

$$
\begin{aligned}
g_{R}(\omega) & :=\mathbb{E}\left(f(\sigma)^{R} \mid \omega\right) \\
& =f(0)^{\left|R \cap A_{g}\right|} \prod_{r=1}^{k} \mathbb{E}\left(f(X)^{\left|R \cap A_{r}\right|} \mid \omega\right) .
\end{aligned}
$$

By (3.1) and (3.3), $g_{R}(\omega)$ is real and nonnegative, whence so is its mean $\phi\left(g_{R}\right)=$ $\left\langle f(\sigma)^{R}\right\rangle$. (It will be convenient to use $\phi(Y)$ to denote the expectation of a random variable $Y: \Omega \rightarrow \mathbb{R}$.)

We show next that $g_{R}$ is a nondecreasing function on the partially ordered set $\Omega$. It suffices to consider the case when the configuration $\omega^{\prime}$ is obtained from $\omega$ by adding an edge between two clusters of $\omega$. In this case, by (3.2) and (3.3), $g_{R}\left(\omega^{\prime}\right) \geq g_{R}(\omega)$. That $\left\langle f(\sigma)^{R}\right\rangle=\phi\left(g_{R}\right)$ is nondecreasing in $J$ and $h$ follows by the appropriate comparison inequality for the random-cluster measure $\phi[18$, Theorem 3.21].

Now,

$$
\mathbb{E}\left(f(\sigma)^{R} f(\sigma)^{S} \mid \omega\right)=f(0)^{\left|R \cap A_{g}\right|+\left|S \cap A_{g}\right|} \prod_{r=1}^{k} \mathbb{E}\left(f(X)^{\left|R \cap A_{r}\right|+\left|S \cap A_{r}\right|} \mid \omega\right) .
$$

By (3.2),

$$
\mathbb{E}\left(f(\sigma)^{R} f(\sigma)^{S} \mid \omega\right) \geq g_{R}(\omega) g_{S}(\omega) .
$$

By the FKG property of $\phi[18$, Theorem 3.8],

$$
\begin{aligned}
\left\langle f(\sigma)^{R} f(\sigma)^{S}\right\rangle & =\phi\left(\mathbb{E}\left(f(\sigma)^{R} f(\sigma)^{S} \mid \omega\right)\right) \\
& \geq\left\langle f(\sigma)^{R}\right\rangle\left\langle f(\sigma)^{S}\right\rangle,
\end{aligned}
$$

as required.

When $h \equiv 0$, the terms in $f(0)$ do not appear in the above, and it therefore suffices that $f \in \mathcal{F}_{q}$.

\section{Proof of Theorem 3.6}

We shall use the elementary fact that, if $T$ is a nonnegative random variable,

$$
\mathbb{E}\left(T^{m+n}\right) \geq \mathbb{E}\left(T^{m}\right) \mathbb{E}\left(T^{n}\right), \quad m, n \geq 0 .
$$


This trivial inequality may be proved in several ways, one of which is the following. Let $T_{1}$ and $T_{2}$ be independent copies of $T$. Clearly,

$$
\left(T_{1}^{m}-T_{2}^{m}\right)\left(T_{1}^{n}-T_{2}^{n}\right) \geq 0
$$

since either $0 \leq T_{1} \leq T_{2}$ or $0 \leq T_{2} \leq T_{1}$. Inequality (5.1) follows by multiplying out (5.2) and averaging.

(a) Inequality (3.3) with $I=0$ is a triviality. Since $f(X)$ is real-valued, with the same distribution as $-f(X), \mathbb{E}\left(f(X)^{m}\right)=0$ when $m$ is odd and is positive when $m$ is even. When $m+n$ is even, (3.2) follows from (5.1) with $T=f(X)^{2}$, and both sides of (3.2) are 0 otherwise.

(b) An easy calculation shows that

$$
\mathbb{E}\left(f(X)^{m}\right)= \begin{cases}1 & \text { if } q \text { divides } m \\ 0 & \text { otherwise }\end{cases}
$$

and (3.1) and (3.2) follow.

(c) Inequality (3.2) follows by (5.1) with $T=f(X)$.

\section{Proof of Theorem 3.7}

We may as well assume that $f_{0} \not \equiv 0$ so that $f_{0}(0)>0$ and $f_{1}(0)=0$. We use the notation of Section 4 , and let $F_{i}: \Omega \rightarrow \mathbb{C}$ be given by

$$
\begin{aligned}
& F_{0}(\omega)=f_{0}(0)^{\left|R \cap A_{g}\right|} \prod_{r=1}^{k} \mathbb{E}\left(f_{0}(X)^{\left|R \cap A_{r}\right|} \mid \omega\right), \\
& F_{1}(\omega)=\prod_{r=1}^{k} \mathbb{E}\left(f_{1}(X)^{\left|S \cap A_{r}\right|} \mid \omega\right) .
\end{aligned}
$$

By (3.1), $F_{0}$ and $F_{1}$ are real-valued and nonnegative. Since $f_{0} \in \mathcal{F}_{q}^{0}, F_{0}$ is nondecreasing (as in Section 4).

Since $f_{0} f_{1} \equiv 0$,

$$
\mathbb{E}\left(f_{0}(\sigma)^{R} f_{1}(\sigma)^{S} \mid \omega\right)=1_{Z}(\omega) F_{0}(\omega) F_{1}(\omega),
$$

where $1_{Z}$ is the indicator function of the event $Z=\{S \leftrightarrow R \cup\{g\}\}$. Here, as usual, we write $A \leftrightarrow B$ if there exists an open path in $\omega$ from some vertex of $A$ to some vertex of $B$. Let $T$ be the subset of $V^{+}$containing all vertices joined to $S$ by open paths, and write $\omega_{T}$ for the configuration $\omega$ restricted to $T$. Using conditional expectation,

$$
\begin{aligned}
\left\langle f_{0}(\sigma)^{R} f_{1}(\sigma)^{S}\right\rangle & =\phi\left(1_{Z} F_{0} F_{1}\right) \\
& =\phi\left(1_{Z} F_{1} \phi\left(F_{0} \mid T, \omega_{T}\right)\right),
\end{aligned}
$$


where we have used the fact that $1_{Z}$ and $F_{1}$ are functions of the pair $T, \omega_{T}$ only. On the event $Z, F_{0}$ is a nondecreasing function of the configuration restricted to $V^{+} \backslash T$. Furthermore, given $T$, the conditional measure on $V^{+} \backslash T$ is the corresponding random-cluster measure. It follows that

$$
\phi\left(F_{0} \mid T, \omega_{T}\right) \leq \phi\left(F_{0}\right) \quad \text { on the event } Z
$$

by [18, Theorem 3.21]. By (6.3),

$$
\begin{aligned}
\left\langle f_{0}(\sigma)^{R} f_{1}(\sigma)^{S}\right\rangle & \leq \phi\left(1_{Z} F_{1} \phi\left(F_{0}\right)\right) \\
& \leq \phi\left(F_{0}\right) \phi\left(F_{1}\right) \\
& =\left\langle f_{0}(\sigma)^{R}\right\rangle\left\langle f_{1}(\sigma)^{S}\right\rangle,
\end{aligned}
$$

and the theorem is proved.

When $h \equiv 0, A_{g}=\{g\}$ in (6.1), and it suffices that $f_{0} \in \mathcal{F}_{q}$.

\section{Acknowledgements}

This work was supported in part by the Engineering and Physical Sciences Research Council under grant EP/I03372X/1. The author is grateful to Jakob Björnberg for proposing the model with angular spins and also to Chuck Newman and Aernout van Enter for their comments and suggestions.

\section{References}

[1] M. Aizenman, "Geometric analysis of $\varphi^{4}$ fields and Ising models, I-II", Comm. Math. Phys. 86:1 (1982), 1-48.

[2] M. Aizenman and B. Nachtergaele, "Geometric aspects of quantum spin states", Comm. Math. Phys. 164:1 (1994), 17-63.

[3] J. E. Björnberg, "Vanishing critical magnetization in the quantum Ising model", Comm. Math. Phys. 337:2 (2015), 879-907.

[4] J. E. Björnberg and G. R. Grimmett, "The phase transition of the quantum Ising model is sharp", J. Stat. Phys. 136:2 (2009), 231-273.

[5] N. Crawford and D. Ioffe, "Random current representation for transverse field Ising model", Comm. Math. Phys. 296:2 (2010), 447-474.

[6] J. De Coninck, A. Messager, S. Miracle-Sole, and J. Ruiz, "A study of perfect wetting for Potts and Blume-Capel models with correlation inequalities", J. Statist. Phys. 52:1-2 (1988), 45-60.

[7] R. Fernández, J. Fröhlich, and A. D. Sokal, Random walks, critical phenomena, and triviality in quantum field theory, Springer, Berlin, 1992.

[8] C. M. Fortuin, "On the random-cluster model, II: The percolation model”, Physica 58:3 (1972), 393-418.

[9] C. M. Fortuin, "On the random-cluster model, III: The simple random-cluster model", Physica 59:4 (1972), 545-570.

[10] C. M. Fortuin and P. W. Kasteleyn, "On the random-cluster model, I: Introduction and relation to other models", Physica 57:4 (1972), 536-564. 
[11] C. M. Fortuin, P. W. Kasteleyn, and J. Ginibre, "Correlation inequalities on some partially ordered sets”, Comm. Math. Phys. 22:2 (1971), 89-103.

[12] N. Ganikhodjaev and F. A. Razak, "Griffith-Kelly-Sherman correlation inequalities for generalized Potts model", Math. Phys. Anal. Geom. 13:1 (2010), 1-18.

[13] J. Ginibre, "General formulation of Griffiths' inequalities", Comm. Math. Phys. 16:4 (1970), 310-328.

[14] R. B. Griffiths, "Correlations in Ising ferromagnets, I", J. Math. Phys. 8:3 (1967), 478-483.

[15] R. B. Griffiths, "Correlations in Ising ferromagnets, II: External magnetic fields", J. Math. Phys. 8:3 (1967), 484-489.

[16] R. B. Griffiths, "Rigorous results for Ising ferromagnets of arbitrary spin", J. Math. Phys. 10:9 (1969), 1559-1565.

[17] R. B. Griffiths, C. A. Hurst, and S. Sherman, "Concavity of magnetization of an Ising ferromagnet in a positive external field", J. Math. Phys. 11:3 (1970), 790-795.

[18] G. R. Grimmett, The random-cluster model, Grundlehren der math. Wissenschaften 333, Springer, Berlin, 2006.

[19] G. R. Grimmett, "Space-time percolation”, pp. 305-320 in In and out of equilibrium, 2 (Rio de Janeiro, 2006), edited by V. Sidoravicius and M. E. Vares, Progr. Probab. 60, Birkhäuser, Basel, 2008.

[20] D. G. Kelly and S. Sherman, "General Griffiths' inequalities on correlations in Ising ferromagnets”, J. Math. Phys. 9:3 (1968), 466-484.

[21] E. H. Lieb, “A refinement of Simon's correlation inequality”, Comm. Math. Phys. 77:2 (1980), $127-135$.

[22] B. M. McCoy and T. T. Wu, The two-dimensional Ising model, Harvard University, Cambridge, MA, 1973.

[23] R. H. Schonmann, "On two correlation inequalities for Potts models", J. Statist. Phys. 52:1-2 (1988), 61-67.

[24] B. Simon, "Correlation inequalities and the decay of correlations in ferromagnets", Comm. Math. Phys. 77:2 (1980), 111-126.

Received 3 Dec 2015. Accepted 28 Mar 2016.

GEOFFREY R. GRIMMETT: g.r.grimmett@statslab.cam.ac.uk

Statistical Laboratory, Centre for Mathematical Sciences, University of Cambridge, Cambridge, CB3 OWB, United Kingdom 


\title{
QUANTUM MECHANICS: \\ SOME BASIC TECHNIQUES FOR SOME BASIC MODELS I: THE MODELS
}

\author{
VINCENZO GRECCHI
}

\begin{abstract}
This is a short review of the main results on the oscillators considered basic models in quantum mechanics. Since the potential is polynomial, it is possible to extend the stationary states to entire functions on the complex plane where the semiclassical theory works better. The control on the energy levels is based on the isolation of the nodes, the zeros stable at the unperturbed limit. A new and simple model for the process of racemization of chiral molecules is added.
\end{abstract}

\section{Introduction}

For me it is a great pleasure to contribute to a deserved homage to Professor Lucio Russo. I have to offer only this flower from my garden, a review of results on the spectrum of basic operators in quantum mechanics.

I remember conversations with Lucio about the existence of gambling experts ( $x \cup \beta \varepsilon \cup \tau \varkappa \varkappa_{\text {) }}$ in Roman times, as reported by Plotinus (204-270 AD), who regarded them as professional scientists. ${ }^{1}$ Does this mean there were probabilist mathematicians in those times? In any case, it seems that only discrete probability was known, since Plotinus apparently rejected the possibility of anything like what we call statistical mechanics:

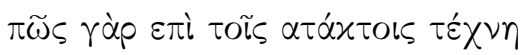

(what science can operate where there is no order?) ${ }^{2}$

This memory is appropriate as an introduction, since probability theory was Lucio's first interest and it is one of the bases of quantum mechanics.

The reason for this review is the difficulty of reading all the single papers considered here. The problems considered in such papers are not unrelated and need

\section{Communicated by Francesco dell'Isola.}

PACS2010: 03.65.-w.

Keywords: quantum oscillators, $P T$ symmetry, isospectral Hamiltonians, spectral analysis, analyticity of the eigenvalues.

${ }^{1}$ Enneads, III, 1, 6, lines 9-10.

${ }^{2}$ Enneads, III, 1, 3, line 15. 
the same techniques to be solved. In particular, I recall the methods for summing divergent series, going back to Guido Grandi [1710].

This review is almost entirely restricted to one-dimensional problems with polynomial potentials (oscillators), with a focus on the cubic case.

Among the points of interest in such research is the meaning of perturbation series in quantum field theory, where the summability test for perturbation series is relevant.

Also of interest from the viewpoint of rigorous atomic and molecular physics are the possible models for molecular structures. In some quantum models presented here there are resonances and associated metastable states. The notion of resonance in quantum mechanics is similar to the same notion in acoustics as discussed by Pietro Mengoli [1670] (see also [Martinez and Nédélec 2012]).

I recall that Lucio has studied Mengoli's contribution to the beginnings of modern mathematical analysis.

All the molecules with a structure [Woolley 1982] and the atoms in an external uniform electric field (the Stark effect) are in a metastable state [Graffi and Grecchi 1978].

In the case of an analytic potential, a resonance is a complex eigenvalue of a non-self-adjoint operator related in a definite way to the original Hamiltonian of the model [Aguilar and Combes 1971].

A particular kind of non-self-adjoint operator is known as PT or PT-symmetric. An operator $H$ is PT if $(P T) H(P T)^{-1}=H$, where $P$ and $T$, the parity and timereversal operators, are defined in (2) below. New interest in such problems started from a collaboration with the late professor Vladimir Buslaev of Saint Petersburg. In [Buslaev and Grecchi 1993] we solved a conjecture of Zinn-Justin about the coincidence of two perturbation series: one in a double-well problem and the other in an unstable single-well problem (where there are expected resonances). We proved the isospectrality of the Hamiltonian (coincidence of the eigenvalues) of the first problem with a PT extension of the formal Hamiltonian of the other one.

Actually also the operator for the resonances of the linear potential (free Stark effect), previously considered by Herbst and Simon [1978], and an analytic continuation of the operator for the resonances in the sense of Gamow [Caliceti and Maioli 1983] of the cubic potential, previously considered by Caliceti, Graffi and Maioli [Caliceti et al. 1980], are PT operators.

I have considered analytic continuations of the resonances of the cubic oscillator in collaboration with André Martinez and Marco Maioli [Grecchi and Martinez 2013; Grecchi et al. 2009; 2010] and with Riccardo Giachetti [Giachetti and Grecchi 2011]. In this last study, we proved the existence of level crossings in a doublewell problem. During the crossing process, the corresponding states pass from the bilocalization in both the wells to the localization in a single well (not the same 
for the two states). This is the proof of the existence of singularities (similar to the Bender-Wu singularities of the levels of the quartic oscillator [Bender and $\mathrm{Wu}$ 1969; Harrell and Simon 1980; Benassi and Grecchi 1980; Alvarez 1995]) of the resonances as analytic functions of the parameter.

Section 2 recaps the essentials of quantum mechanics to fix some notions. In Section 3 we discuss solvable oscillators as examples. In Section 4 is a short exposition on the quartic oscillator. Section 5 briefly cover PT oscillators. Section 6 is devoted to the cubic oscillator with a single well. In Section 7 is the cubic PT oscillator with a double well. In Section 8 a simple model for the racemization effect is discussed.

\section{Essentials of quantum mechanics}

Sir William Rowan Hamilton [1834; 1835] laid the basis for the understanding tht classical mechanics, in the Hamilton-Jacobi formalism, is a short-wavelength limit of a possible theory of matter waves. Actually, he obtained the Hamilton-Jacobi method in mechanics by analogy with the eikonal approximation of the equations of electromagnetic waves.

Schrödinger (1926) formalized this theory, quantum mechanics, by his famous equation.

In $d$-dimensional quantum mechanics, the Hamiltonian of a particle of mass $m=\frac{1}{2}$ is a linear operator in $\mathscr{H}=L^{2}\left(\mathbb{R}^{d}\right)$ of the kind

$$
H=p^{2}+V, \quad \text { where } p^{2}=-\nabla^{2}
$$

and where the Planck constant $\hbar=h / 2 \pi$ is unitary and $V$ is the formal multiplication operator by the real function $V(x)$ defined by $(V \psi)(x)=V(x) \psi(x)$ for a vector $\psi \in \mathscr{H}$ represented by a function $\psi(x)$. In the sequel the multiplication operator $V$ is identified with its corresponding function $V(x)$. We call unstable a model with a potential not bounded below at infinity. The basic operators of interest are self-adjoint (s.a.) (observables) restricted to their domains [Kato 1966]. The possible eigenvalues of a self-adjoint operator are necessarily real. We call $D(A)$ the domain of the operator $A$, and the domain of the Hamiltonian $H$, defined as a sum of two operators by (1), satisfies the condition

$$
D(V) \cap D\left(p^{2}\right) \subset D(H) \subset \mathscr{H} .
$$

The spectral theorem [Kato 1966; Reed and Simon 1975; 1978] allows one to associate the self-adjoint Hamiltonian with the real energy spectrum. A state is a class of equivalence of vectors $\psi \in \mathscr{H}$ of unitary norm, with $\psi \sim \lambda \psi,|\lambda|=1$. At any $t \in \mathbb{R}$ the vector becomes $\psi(t)=U_{t} \psi$, where $U_{t}:=\exp (-i t H)$ is a unitary operator conserving the norm. We define a (energy) level to be an eigenvalue $E$ 
and a stationary state, the class of equivalence of eigenvectors $\psi$ of $H$, where the stationarity is characterized by the behavior $\psi(t)=\exp (-i E t) \psi \sim \psi$.

The parity $P$ and the time-reversal $T$ operators are defined respectively by

$$
P \psi(x)=\psi(-x) \text { and } T \psi(x)=\bar{\psi}(x) \text { for all } \psi \in \mathcal{H} .
$$

Since the operator $H$ is real, we have $T U_{t} T^{-1}=U_{-t}$, justifying the name of time reversal for the operator $T$.

Given a unitary operator $U$, we get the change of representation of the states $\psi \rightarrow U \psi$ for all $\psi \in \mathscr{H}$. In this representation the Hamiltonian becomes the unitarily equivalent Hamiltonian $H_{U}=U H U^{-1}$, with the same spectrum. In the case of the Fourier transform, $\mathscr{F}=U$, we pass from the position to the momentum representation. For the continuation of the resonances in the complex plane of the parameter, we use generalized changes of representation (see Section 1d of the sequel).

In the case of the hydrogen atom we are in $3 \mathrm{D}(d=3)$ :

$$
H_{0}=p^{2}+V(x), \quad x \in \mathbb{R}^{3}, p^{2}=-\nabla^{2}, V(x)=-K /|x|,
$$

where $K>0$ is fixed and

$$
D\left(H_{0}\right)=D\left(p^{2}\right) \subset \mathscr{H}=L^{2}\left(\mathbb{R}^{3}\right) .
$$

There are infinite eigenvalues of $H_{0}$, (energy) levels $E_{n}<0$, where the eigenfunctions are the stationary states $\psi_{n}$, since $\psi_{n}(t)=\exp \left(-i E_{n} t\right) \psi_{n} \sim \psi_{n}$. The absolute continuous spectrum $R_{+}$is related to the range of the mean energies $((\psi, H \psi)=E \geq 0)$ of the unstable (scattering) states $\psi \in D(H)$.

Now we consider the hydrogen Stark effect, with a perturbed hydrogen Hamiltonian

$$
H_{\epsilon}=H_{0}+\epsilon x_{1}, \quad \epsilon>0 .
$$

In this case there are resonances, complex numbers $E_{n}(\epsilon)$, such that $E_{n}(\epsilon) \rightarrow E_{n}$ as $\epsilon \rightarrow 0$. As introduced above, the corresponding states $\psi_{n}(\epsilon) \notin \mathscr{H}$, if suitably cut at infinity, become metastable states. This fact was proven by the complex scaling of the variables [Aguilar and Combes 1971] $x \rightarrow \lambda x$ and $p \rightarrow(1 / \lambda) p, \lambda \notin \mathbb{R}$ and $\Im \ln (\lambda)>0$, getting the non-s.a. operators

$$
H_{\epsilon}(\lambda)=\lambda^{-2}\left(p^{2}-\lambda(K /|x|)+\epsilon \lambda^{3} x_{1}\right) \quad \text { and } \quad D\left(H_{\epsilon}\right)=D\left(p^{2}\right) \cap D\left(x_{1}\right),
$$

with complex eigenvalues $E_{n}(\epsilon)$, invariant for $0<\Im \ln (\lambda)<\pi / 6$, representing the resonances of the Stark effect [Graffi and Grecchi 1978; Herbst and Simon 1978]. Notice that, by the use of the parabolic coordinates, the Stark effect in hydrogen can be decomposed into two anharmonic oscillators, one stable and the other one unstable. The perturbation series in $\epsilon$ of the resonances are divergent but summable 
by the distributional Borel method [Caliceti et al. 1986] to the real part of the resonances. Also the mean life of the metastable states is given by the same method.

\section{Some solvable models}

As basic solvable models, we consider the linear and the quadratic potentials.

We start with the Hamiltonian with linear potential

$$
H_{1}(\lambda)=\lambda^{-2}\left(p^{2}+\lambda^{3} x\right), \quad \lambda \neq 0, \Im \ln (\lambda)=\theta .
$$

In the case of $\lambda=1$, we have the self-adjoint Hamiltonian $H_{1}(1)$ with continuous spectrum $\sigma_{c}=\mathbb{R}$ [Kato 1966]. The resonances are given by the eigenvalues of the operators $H_{1}(\lambda)$ for $0<\theta<\pi / 3$ and domain $D\left(H_{1}(\lambda)\right)=D\left(p^{2}\right) \cap D(x)$. In particular, for $\theta=\pi / 6$, we have $H_{1}(\exp (i \pi / 6))=\exp (-i \pi / 3) O_{1}$ where

$$
O_{1}=p^{2}+i x
$$

is a PT operator with discrete spectrum [Herbst and Simon 1978]. Actually, because of the invariance of the spectrum to any translation $x \rightarrow x+\epsilon, \epsilon \in \mathbb{R}$, an eigenvalue $E_{n}$ should satisfy the impossible condition that there exists $m=m(n, \epsilon)$ such that $E_{n}=E_{m}+i \epsilon$. Thus, there are no resonances in this problem $H_{1}(1)$, as can be understood by the absence of wells or traps of the potential.

The other solvable Hamiltonians are

$$
H_{2}^{ \pm}(\lambda)=\lambda^{-2}\left(p^{2} \pm \lambda^{4} x^{2}\right), \quad \lambda \neq 0 .
$$

In particular,

$$
H_{2}^{+}(1):=O_{2}=p^{2}+x^{2}
$$

is the harmonic oscillator with simple levels $E_{n}=2 n+1, n \in \mathbb{N}$, and states $\psi_{n}(x)$. For $E=E_{n}$, the interval $[-\sqrt{E}, \sqrt{E}]$ is the internal Stokes line [Giller 2011] (oscillatory range) where all the zeros (nodes) of $\psi_{n}(x)$ lie.

In the case of $H_{2}^{-}(1)=p^{2}-x^{2}$, we have the repulsive harmonic oscillator with continuous spectrum $\sigma_{c}=\mathbb{R}$. The eigenvalues $E_{n}(\lambda)$ of the operator

$$
H_{2}^{-}(\lambda)=\lambda^{-2}\left(p^{2}-\lambda^{4} x^{2}\right), \quad \lambda \neq 0,
$$

are independent of $\lambda$ for $0<\Im \ln (\lambda)<\pi / 2$. Moreover, the eigenfunctions $\psi_{n}(\lambda, z)$, extended as entire functions in $z$, are simple dilations [Aguilar and Combes 1971] of $\psi_{n}(1, z): \psi_{n}(\lambda, z)=\lambda^{1 / 2} \psi_{n}(1, \lambda z)$. In particular, the spectrum of $H_{2}^{-}(\sqrt{i})=$ $-i\left(p^{2}+x^{2}\right)$ is the set of the top resonances of $H_{2}^{-}(1)$ :

$$
\sigma_{d}=\{-i(2 n+1)\}_{n \in \mathbb{N}} \text {. }
$$


Associated with these resonances are metastable states trapped about the top of the potential at $x=0$. Remark that the top of the potential is an unstable equilibrium point in classical mechanics.

Translations in the complex plane $x \rightarrow x+\alpha$ allow one to extend the family of operators. In particular, for $\lambda=1, \alpha=i$,

$$
H_{2}^{+}(1, i)=p^{2}+(x+i)^{2}=p^{2}+x^{2}+2 i x-1 \sim H_{2}^{+}(1)
$$

is a nontrivial PT operator with real levels $E_{n}=2 n+1$.

Now we consider some nonsolvable oscillators.

\section{The quartic oscillator}

The quartic oscillator is the first of the nonsolvable oscillators to be studied rigorously. It is defined by the family of Hamiltonians

$$
H_{1}(\beta)=p^{2}+V(x), \quad V(x)=x^{2}+\beta x^{4}, x \in \mathbb{R}, p^{2}=-\frac{d^{2}}{d x^{2}},
$$

where $\beta$ is in the cut plane

$$
\mathbb{C}_{c}=\{z \in \mathbb{C}: z \neq 0, z=|z| \exp (i \theta),|\theta|<\pi\},
$$

with domain $D\left(H_{1}\right)=D\left(p^{2}\right) \cap D\left(x^{4}\right)$.

For $\beta$ positive, $H_{1}$ is self-adjoint and the potential has a single well on the real axis. Actually, on the real axis the force $-V^{\prime}(x)=-2 x\left(1+4 \beta x^{2}\right)$ vanishes only at the origin.

For the corresponding levels $E_{n}(\beta)$, the Stieltjes or Padé summability of the perturbation series was proven in [Loeffel et al. 1969]. The main technique used was the Loeffel-Martin method of control of the zeros of the states in the complex plane. Later, the Borel summability of the perturbation series was proven in [Graffi et al. 1970]. Analytic continuations of the levels $E_{n}(\beta)$ beyond $\mathbb{C}_{c}$ suggested the existence of crossings (the Bender-Wu singularities [1969]).

We consider also the two-dimensional quartic oscillator by the reduction of the Hamiltonian to the radial operators:

$$
H_{2}(\beta, j)=p_{r}^{2}+\frac{j^{2}-1}{4 r^{2}}+r^{2}+\beta r^{4}, \quad r \in \mathbb{R}_{+}, p_{r}^{2}=-\frac{d^{2}}{d r^{2}},
$$

for $\beta \in \mathbb{C}_{c}$ and $j \geq 0$. The level $E_{n}(\beta, j)$, at the limit of the rim of the cut defined by $\arg (\beta)=\pi^{-},|\beta|=b>0$, is the resonance in the sense of the boundary conditions of Gamow [Caliceti and Maioli 1983] of the formal Hamiltonian $H_{2}(\beta, j)$ at $\beta=-b$. Since the hydrogen Stark effect can be decomposed by the parabolic coordinates in two operators of this kind, the existence of the resonances of the hydrogen Stark effect was also proven [Graffi and Grecchi 1978]. Moreover, 
the Borel distributional summability of the perturbation series of the same Stark resonances was proven in [Caliceti et al. 1993].

Of great interest is the double-well quartic oscillator defined by the families of self-adjoint Hamiltonians

$$
Q(\beta, j)=p^{2}+x^{2}(\beta x-1)^{2}-j\left(\beta x-\frac{1}{2}\right), \quad x \in \mathbb{R}, \beta \in \mathbb{R}_{+},
$$

for a fixed $j \in \mathbb{R}$. In particular, for $j=0$ we have the case of a symmetric double well. The states are of even or odd parity, and the levels are classified in doublets of opposite parity with a splitting of exponential order. The perturbation series are the same for both levels of a doublet so that the distributional sum of the series cannot give both the levels and in fact neither. In [Caliceti et al. 1988; 1996] a sequence of approximants to the levels up to the first exponential order was given by the distributional Borel sum [Caliceti et al. 1986] of the perturbation series.

\section{PT operators: a short story}

For the study of the resonances in the case of analytic potential, the introduction of non-self-adjoint Hamiltonians [Aguilar and Combes 1971] is useful. Sometimes the resonance Hamiltonian can be put in $P T$-symmetric form [Herbst and Simon 1978]. A PT operator is a real operator in the momentum representation, and its spectrum can be real.

I recall that some numerical investigations by Zinn-Justin suggested the coincidence of the perturbation series of the levels of two different problems: the unstable, rotationally symmetric, anharmonic oscillator in 2D and the double-well oscillator. Since usually a coincidence is not causal, the people of the scientific world looked for a stronger fact: the isospectrality of two Hamiltonian families. Many attempts were made without a full success. Eventually Buslaev and Grecchi [1993] proved the isospectrality of the self-adjoint double-well Hamiltonian $Q(\beta, j)$ of (6) and the radial reduction of the unstable anharmonic operator defined by a complex translation on the full axis as a PT operator:

$$
H_{\epsilon}(\beta, j)=\frac{1}{2}\left(p_{r}^{2}+\frac{j^{2}-1}{4 r_{\epsilon}^{2}}+r_{\epsilon}^{2}\right)-\beta^{2} r_{\epsilon}^{4}, \quad r \in \mathbb{R},
$$

where $p_{r}=-i \partial_{r}$ and $r_{\epsilon}=r-i \epsilon$, for an $\epsilon>0$. By some transformations on the variable and on the wave function, and also by the Fourier transform, it was proven that the two operators are isospectral, or equivalent in our terminology (the notion of equivalence in this case is not standard but was shared by others).

Scaling the variable, we also proved the isospectrality of the PT Hamiltonian

$$
O_{4}(\epsilon, \alpha, j)=\frac{1}{2}\left(p_{r}^{2}+\frac{j^{2}-1}{4 r_{\epsilon}^{2}}+\alpha r_{\epsilon}^{2}\right)-r_{\epsilon}^{4}, \quad r \in \mathbb{R}, r_{\epsilon}=r-i \epsilon,
$$


for an $\epsilon>0$, and the double-well one,

$$
Q(\alpha, j)=p^{2}+\left(x^{2}-\frac{1}{4} \alpha^{2}\right)^{2}-j x
$$

self-adjoint for $\alpha$ and $j$ real. As a consequence, we have proven the realness of the spectrum of families of PT Hamiltonians.

Our proofs are rigorous and obviously independent of the Zinn-Justin conjecture. For people interested in the paper [Benassi and Grecchi 1980], let me say that it is synthetic and very precise in the operatorial implementation of the analytic transforms.

Later, Bender and Boettcher [1998] discussed and Shin [2002] proved the realness of the spectrum of a class $O_{N}$ of $P T$-symmetric operators, containing our (7) only in the case of $j=1$.

\section{The cubic single-well Hamiltonian}

The cubic resonance problem is one of the simplest, not explicitly solvable ones in quantum mechanics [Caliceti et al. 1980; Grecchi and Martinez 2013; Grecchi et al. 2009; 2010; Caliceti 2000]. We consider the single (real) well Hamiltonian

$$
H(\beta)=p^{2}+x^{2}+i \sqrt{\beta} x^{3}, \quad \beta \in \mathbb{C}_{c} .
$$

We define nodes to be the $n$ zeros of state $\psi_{n}(\beta)$ stable at the unperturbed limit $\beta \rightarrow 0$.

Remark. The stability of the nodes at $\beta=0$ is equivalent to the stability of the levels because of the Cauchy theorem.

Also our results on the analyticity of the levels for large $|\beta|$ depend on the stability and isolation of the nodes.

(a) The first thing to prove is the absence of the essential spectrum. Thus, we prove the compactness of the resolvent.

Let us consider the Hamiltonian $H(\beta)$, for any fixed $\beta \in \mathbb{C}_{c}$. For any fixed $E \in \mathbb{C}$, the semiclassical behavior of the fundamental states [Sibuya 1975] implies that the Green's function $G(x, y)$ behaves as $|G(x, y)| \sim|x y|^{-3 / 4}$ for both $|x|,|y| \rightarrow \infty$ and is exponentially small if only one of the coordinates $(x, y)$ diverges, so that is in $L^{2}\left(\mathbb{R}^{2}\right)$. The corresponding integral operator, the inverse of $H(\beta)$, is HilbertSchmidt.

(b) The second thing to prove is the isolation of the nodes or the confinement and the stability of the nodes for the parameter on the cut plane.

(b1) The first part of the proof regards the existence of a no-vanishing zone [Delabaere and Trinh 2000]. 
The proof is based on the method of Loeffel and Martin [Loeffel et al. 1969] for the confinement of the zeros (9).

We consider the stripe of the complex plane of the variable $\beta$

$$
A(\beta)=\left\{z=x+i y \in \mathbb{C}: x, y \in \mathbb{R}, 0 \leq y \leq \frac{2}{3 \sqrt{b}} \cos \frac{\theta}{2}\right\},
$$

where $\beta=b \exp (i \theta)$.

Proposition. For all $\beta \in \Omega$, the eigenfunction $\psi_{\beta}$ does not admit any zeros on the stripe $A(\beta)$.

Let $V_{\beta}(z)=z^{2}+i \sqrt{\beta} z^{3}, z=x+i y$ [Loeffel et al. 1969]. Then

$$
\begin{aligned}
\Im \psi_{\beta}^{\prime}(x+i y) \overline{\psi_{\beta}(x+i y)} & =\int_{-\infty}^{x} \Im\left(V_{\beta}(r+i y)-E(\beta)\right)\left|\psi_{\beta}(r+i y)\right|^{2} d r \\
& =-\int_{x}^{\infty} \Im\left(V_{\beta}(r+i y)-E(\beta)\right)\left|\psi_{\beta}(r+i y)\right|^{2} d r
\end{aligned}
$$

It is enough to prove that the integrand changes sign at most once in $\mathbb{R}$ for any $z \in A(\beta)$. In this case at least one, and thus both, of the integrals in (9) are nonzero. Since $\left|\psi_{\beta}(z)\right|^{2} \geq 0$ and positive almost everywhere, we look for the monotonicity of the factor of the integrand

$$
\Im\left(V_{\beta}(r+i y)-E(\beta)\right)=2 r y+r^{3} \sqrt{b} c-3 r^{2} y \sqrt{b}-3 r y^{2} c-\Im(E(\beta)),
$$

where $c=\cos (\theta / 2)$ and $s=\sin (\theta / 2)$, or the nonvanishing of the derivative

$$
\begin{aligned}
\frac{d}{d r} \Im\left(V_{\beta}(r+i y)\right) & =3 \sqrt{b} c r^{2}-6 \sqrt{b} y s r+2 y-3 y^{2} \sqrt{b} c \\
& =3 \sqrt{b} c\left(r^{2}-2 y r \tan +\frac{2 y-3 y^{2} \sqrt{b} c}{3 \sqrt{b} c}\right),
\end{aligned}
$$

where $c=\cos (\theta / 2), s=\sin (\theta / 2)$ and $\tan =\tan (\theta / 2)$. The reduced discriminant is

$$
\Delta=9 y b\left(y-\frac{2}{3 \sqrt{b}} \cos \frac{\theta}{2}\right) \text {. }
$$

(b2) The second part of the proof regards the absence of zeros far from the origin in a large sector.

The eigenfunctions are $L^{2}$ on the real axis so that they are vanishing at infinity in two Stokes sectors

$$
S_{ \pm}(\beta)=\left\{x \in \mathbb{C}:\left|\arg (i x)+\frac{\theta}{10} \mp \frac{2 \pi}{5}\right|<\frac{\pi}{5}\right\} .
$$


This means that [Sibuya 1975] they are free of zeros (for large $|x|$ ) on two extended sectors

$$
S^{ \pm}(\beta)=\left\{x \in \mathbb{C}:\left|\arg (i x)+\frac{\theta}{10} \mp \frac{2 \pi}{5}\right|<\frac{3 \pi}{5}\right\} .
$$

Thus, the sequence of zeros $N_{j}$ of $\psi_{n}(x, \beta)$ have the asymptotic phase $\pi / 2+\theta / 10$, as $\left|N_{j}\right| \rightarrow \infty$.

\section{(c) The third thing to prove is the absence of nonperturbative levels.}

It is worth recalling this proof since it looks difficult but needs only a careful analysis.

Let $E(\beta)$ be an eigenvalue defined as a multiple-valued analytic function on $\mathbb{C}_{c}$, with normalized eigenfunction $\psi_{\beta}$, and let $E_{n}(\beta)$ be a perturbative eigenvalue for $b$ small.

Proposition. Let $\widetilde{E}(\beta)$ be a determination of $E(\beta)$; then on a disk $|\beta|<b_{0}, \beta \in \mathbb{C}_{c}$, $\widetilde{E}(\beta)=E_{n}(\beta)$ for $a b_{0}>0$ and $n \in \mathbb{N}$.

Remark. A consequence of this proposition is that the branch points of $\widetilde{E}(\beta)$ in $\mathbb{C}_{c}$ do not accumulate at 0 .

Proof. Let $\gamma$ be a simple path in $\mathbb{C}_{c} \cup\{0\}$ such that $\widetilde{E}(\beta)$ is analytic in a neighborhood of $\gamma$ in $\mathbb{C}_{c}$, bypassing the possible sequence of positive branch points on $\gamma$, with $\beta_{k} \in \gamma$ and $\beta_{k} \rightarrow 0$ as $k \rightarrow \infty$.

Let us distinguish two cases.

Case 1. The sequence $\left(\left|\widetilde{E}\left(\beta_{k}\right)\right|\right)_{k}$ is bounded. In this case, we have a subsequence converging to a limit $\widetilde{E}\left(\beta_{k}\right) \rightarrow E_{n}$, where $E_{n}=E_{n}(0)$ is an eigenvalue of the unperturbed Hamiltonian because of the stability. Moreover, for the stability and the perturbation theory, we have $\widetilde{E}(\beta)=E_{n}(\beta)$ for $\beta \in \gamma,|\beta|<b_{0}$, for a $b_{0}>0$.

Case 2. $\left|\widetilde{E}\left(\beta_{k}\right)\right| \rightarrow \infty$ as $k \rightarrow \infty$.

Let us split $B:=\left(\beta_{k}\right)_{k}$ into

$$
B_{ \pm}=\left\{\beta \in B: \pm|\widetilde{E}(\beta)| \leq \pm \beta^{-1}\right\} .
$$

(2a) When $\beta \in B_{-}$we make the change of variable $x=\lambda y$, with $\lambda:=\sqrt{|\widetilde{E}(\beta)|}$, and we set

$$
\phi(y)=\sqrt{\lambda} \psi_{\beta}(\lambda y), \quad h:=\frac{1}{\lambda^{2}}, \quad b^{\prime}=\lambda^{2} \sqrt{\beta} \in(0,1] \quad \text { and } \quad u:=\widetilde{E}(\beta) /|\widetilde{E}(\beta)|
$$

so that we have

$$
\left(H_{h}\left(b^{\prime}\right)-u\right) \phi(y)=-h^{2} \phi^{\prime \prime}(y)+\left(y^{2}+i \sqrt{b^{\prime}} y^{3}-u\right) \phi(y)=0,
$$

and we can use the complex semiclassical method for $\alpha$ and $\hbar$ small [Voros 1994]. Since $b^{\prime}>0$, the numerical range of $H_{h}\left(b^{\prime}\right)$ and $u$ are in the half-plane $\Re z \geq 1$. Thus, $\arg (u)<\pi / 2$ and two turning points $y_{ \pm}\left(b^{\prime}, u\right)$ of (10) are located in the 
half-plane $\Im y \leq 1 / \sqrt{2}$, while the third one is in $\Im y \geq 1$. In this case there are no

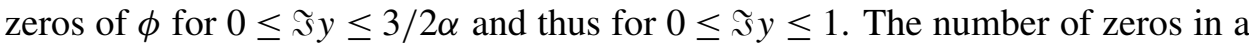
disk of fixed radius $\left|y_{+}\left(b^{\prime}, u\right)-y\right| \leq \delta$ diverges as $O(1 / h)$ in contradiction with the finite constant number of the nodes.

(2b) When $\beta \in B_{+}$, with $\lambda:=(|\widetilde{E}(\beta)| / \sqrt{\beta})^{1 / 3}$, we make the change of variable $x=\lambda y$, and we define the new function

$$
\phi(y)=\sqrt{\lambda} \psi_{\beta}(\lambda y)
$$

to be the solution of the equation

$$
-h^{2} \phi^{\prime \prime}(y)+\left(\alpha y^{2}+i y^{3}-u\right) \phi(y)=0,
$$

with $u=E(\beta) /|E(\beta)|, h=1 / \lambda \sqrt{|E(\beta)|} \rightarrow 0$ as $\beta \rightarrow 0$ and $0<\alpha=(\beta|\widetilde{E}(\beta)|)^{-1 / 3} \leq$ 1 as $\beta \rightarrow 0$.

In this case, where $\beta \in B_{+}$, we prove the same contradiction as in the case $B_{-}$. In particular, if $\alpha \rightarrow 0$ both $\Im y_{ \pm}$become negative as in the $\beta \rightarrow \infty$ case.

\section{(d) Now we prove that the levels are Stieltjes functions.}

The proof that

$$
f(\beta):=-\frac{E_{n}(\beta)-E_{n}(0)}{\beta}, \quad n \in \mathbb{N},
$$

is a Stieltjes function is based on its analyticity and boundedness on $\mathbb{C}_{c}$ and the definite sign of the imaginary part of $\Im E_{n}(-b-i 0) \leq 0$. The sign of $\Im E_{n}(-b-i 0)$ comes from the Gamow condition of the corresponding state $\phi(x)$ at $-\infty$. The state $\phi(x)$ and $\phi^{\prime}(x)$ have no zeros on the real axis. The level is a generalized resonance, or a limit of ordinary resonances, and its imaginary part, with changed sign, is related to the inverse mean life of the associated metastable states. The resonance eigenfunction satisfies the Gamow condition for $-x>0$ large, and by the semiclassical behavior [Sibuya 1975], we have

$$
\begin{gathered}
\hbar \frac{\phi^{\prime}(x)}{\phi(x) \sqrt{E-V(x)}} \rightarrow-i, \quad \sqrt{E-V(x)}|\phi(x)|^{2} \rightarrow|c|^{2}>0, \\
-\Im E=-\frac{\hbar^{2}}{\int_{x}^{\infty}|\phi(y)|^{2} d y} \Im\left(\frac{\phi^{\prime}(x)}{\phi(x)}|\phi(x)|^{2}\right) \rightarrow \hbar|c|^{2} .
\end{gathered}
$$

\section{The cubic $P T$-symmetric double well}

The single-well cubic oscillator discussed above can be analytically continued by generalized changes of representation [Aguilar and Combes 1971; Graffi and Grecchi 1978], obtaining the family of PT Hamiltonians

$$
H_{\hbar}=\hbar^{2} p^{2}+V(x), \quad V(x)=i\left(x^{3}-x\right), \hbar>0 .
$$


Since $V^{\prime}(x)=0$ at the two points $x_{ \pm}= \pm 1 / \sqrt{3} \in \mathbb{R}$ (wells), we have a double-well PT Hamiltonian, whose states possibly localize about one of the wells (localization) or about both (delocalization or bilocalization). The transition from one to the other kind of state is singular and happens at the level crossing.

Also in the case of the unstable double-well Stark effect, varying the field parameter, there is a double level crossing and the states are localized for large negative and large positive parameter, but not for small parameter [Grecchi et al. 1996].

The existence of the level crossings is the main issue of the research on this model. The levels at the crossings are near a critical energy, usually the top of the internal barrier. In our PT case the internal barrier doesn't exist and the critical energy is a singularity of the Stokes graph [Giller 2011; Delabaere and Trinh 2000].

The localization is a typical semiclassical effect, since the classical particles, at low energy, are localized in one of the wells.

Actually, in usual quantum mechanics with a family of self-adjoint Hamiltonians for all real parameters, the delocalization of the states is stable and the level crossings are forbidden [Kato 1966; Reed and Simon 1975; 1978]. Also in the case of single-well PT Hamiltonians [Shin 2002; Caliceti 2000; Grecchi and Martinez 2013; Grecchi et al. 2009; 2010] the states are always localized about the well and the crossings are absent.

The semiclassical theory provides good qualitative and quantitative results on the levels $E_{n}(\hbar)$ for lower parameter $\hbar$ up to the crossing value [Bender and $\mathrm{Wu}$ 1969; Benassi and Grecchi 1980; Harrell and Simon 1980; Alvarez 1995]. The complex semiclassical method [Voros 1994; Delabaere et al. 1997] has extended the results to larger, but not very large, values of the parameter [Delabaere and Trinh 2000; Delabaere and Pham 1997]. Recently, rigorous results were also obtained in [Eremenko and Gabrielov 2011] by different techniques. Such approaches are useful and complementary to our rigorous treatment based on the nodal analysis [Giachetti and Grecchi 2015; 2016a; 2016b]. The nodal analysis is found also in other papers [Grecchi and Martinez 2013; Grecchi et al. 2009; 2010; Eremenko et al. 2008a; 2008b; Shanley 1988a; 1988b; Eremenko and Gabrielov 2009].

As proven above, all the states $\tilde{\psi}_{n}(\beta)$, with corresponding levels $\widetilde{E}_{n}(\beta)$, are perturbative and their subscripts $n$ are the numbers of their zeros stable at $\beta=0$ (nodes). The result is relevant to our present context, as we may observe that the Hamiltonians (12) and (8) are related by a generalized change of representation (with a definite law of transformation of the levels). Indeed, making the unitary translations $x=x_{ \pm}+y$, we obtain

$$
H_{\hbar}^{ \pm}=\hbar^{2} p^{2}+i\left(y^{3} \pm \sqrt{3} y^{2}\right) \mp i E_{0}, \quad E_{0}=\frac{2}{3 \sqrt{3}} .
$$


In order to connect $H_{\hbar}^{ \pm}$with $H(\beta)$ in the sectors $\pi<\mp \arg (\beta)<3 \pi / 2$, respectively, we consider the analytic dilations [Aguilar and Combes 1971]

$$
y=\lambda^{ \pm}(\hbar) z, \quad \lambda^{ \pm}(\hbar)=3^{-1 / 8} \sqrt{\hbar} \exp (\mp i \pi / 8),
$$

conserving the spectrum. Letting $c^{ \pm}=3^{1 / 4} \sqrt{ \pm i}$ we find from (8)

$$
H\left(\beta^{ \pm}(\hbar)\right) \sim\left(1 / \hbar c^{ \pm}\right) H_{\hbar}^{ \pm} \pm i E_{0} \quad \text { and } \quad E_{n}\left(\beta^{ \pm}(\hbar)\right)=\left(1 / \hbar c^{ \pm}\right) E_{n}^{ \pm}(\hbar) \pm i E_{0}
$$

where

$$
\beta^{ \pm}(\hbar)=\exp (\mp i 5 \pi / 4) 3^{-5 / 4} \hbar
$$

Notice that

$$
E_{n}^{ \pm}(\hbar)=\hbar^{6 / 5} \widehat{E}_{n}\left(\alpha^{\mp}\right), \quad n \in \mathbb{N}, \quad \text { and } \quad \alpha^{ \pm}=\exp ( \pm i \pi) \hbar^{-4 / 5}, \quad \hbar>0 .
$$

We see that levels $E_{n}^{ \pm}(\hbar)$ are complex conjugates and are different for small $\hbar>0$, since their semiclassical behaviors are

$$
E_{n}^{ \pm}(\hbar)=\mp i E_{0}+\sqrt{ \pm i} \sqrt[4]{3}(2 n+1) \hbar+O\left(\hbar^{2}\right) \in \mathbb{C}_{\mp}=\{z \in \mathbb{C}: \mp \Im \mathfrak{I} z>0\} .
$$

The zeros of $\psi_{n}^{ \pm}(\hbar, z)$ for large $|z|$ are contained respectively in

$$
\mathbb{C}^{ \pm}=\{z \in \mathbb{C}: \pm \Re z>0\} .
$$

The large- $\hbar$ behavior of the levels $E_{m}(\hbar)$ is studied by using a different representation with the Hamiltonians

$$
O_{3}(\alpha)=p^{2}+W(\alpha, x), \quad W(\alpha, x)=i\left(x^{3}+\alpha x\right), \alpha \in \mathbb{C} .
$$

The eigenvalue $\widehat{E}_{m}(\alpha)$ of $O_{3}(\alpha)$ is holomorphic in a neighborhood of the origin. Thus, for large positive $\hbar$ the levels $E_{m}(\hbar)$ are defined by

$$
\hbar^{-6 / 5} E_{m}(\hbar)=\widehat{E}_{m}(\alpha), \quad \alpha=-\hbar^{-4 / 5} \leq 0,
$$

and they are connected with the eigenvalues of $H(\beta)$ by the relation

$$
\widehat{E}_{m}(\alpha(\beta))=\beta^{-1 / 5} \widetilde{E}_{m}(\beta)+\frac{2 \beta^{-1 / 5}}{27 \beta}, \quad \alpha(\beta)=\frac{1}{3 \beta^{4 / 5}} \geq 0,
$$

at $\alpha=0$. The level functions $\widehat{E}_{m}(\alpha)$ are real-analytic for $\alpha \in \mathbb{R},|\alpha|$ small. Thus, all such levels $E_{m}(\hbar)$ are given by analytic continuations of the perturbative levels $\widetilde{E}_{m}(\beta)$ through $(21)$ and $(22)$, and are extensible as many-valued functions (taking different names), to the sector

$$
\mathbb{C}^{0}=\{z \in \mathbb{C}: z \neq 0,|\arg (z)|<\pi / 4\}
$$

of the $\hbar$ complex plane. The corresponding states $\psi_{m}(\hbar)$ are delocalized (or bilocalized about both wells). 
The crossing selection rule is given in simple terms: the two positive levels $E_{2 n+(1 / 2) \pm(1 / 2)}(\hbar)$ with delocalized states $\psi_{2 n+(1 / 2) \pm(1 / 2)}(\hbar), \hbar>\hbar_{n}$, cross at $\hbar_{n}>0$ and become the two complex levels $E_{n}^{ \pm}(\hbar)$ with localized states $\psi_{n}^{ \pm}(\hbar)$ for $\hbar<\hbar_{n}$. This selection rule completely describes the full crossing process.

The two levels $E_{n}^{ \pm}(\hbar)$ of (15) and (17) are complex-conjugated, and the two states $\psi_{n}^{ \pm}(\hbar)$ are $P T$-conjugated and concentrated about the wells $x_{ \pm}$, respectively.

This process is possible because of the instability of the delocalization of both the states $\psi_{2 n+(1 / 2) \pm(1 / 2)}(\hbar)$ for decreasing $\hbar>0$.

Conclusion. The nodes of the states $\psi_{2 n+(1 \pm 1) / 2}(\hbar)$ for large $\hbar>0$, with positive levels $E_{2 n+(1 \pm 1) / 2}(\hbar)$, are in the half-plane $\mathbb{C}_{-}$. Only the state $\psi_{2 n+1}(\hbar)$ has an unstable imaginary node. The number of nonimaginary nodes of both the states $\psi_{2 n+(1 \pm 1) / 2}(\hbar)$ are $2 n, n$ of them in $\mathbb{C}^{ \pm}$.

The nodes of the states $\psi_{n}^{ \pm}(\hbar)$ for $h b<\hbar_{n}$ are in $\mathbb{C}^{ \pm}$, respectively.

\section{A simple model for the racemization}

Many years ago Friedrich Hund proposed a model [Dennison and Uhlenbeck 1932] for the ammonia molecule $\mathrm{NH}_{3}$. Supposing the hydrogen atoms lie in a plane, he considered the motion of the nitrogen atom along an orthogonal axis. The forces acting on the $\mathrm{N}$ atom should correspond to a double-well potential because of the symmetry and the typical interatomic forces. In this quantum model the $\mathrm{N}$ atom state is delocalized or localized about both the wells. Actually, we know that the molecule in a dense gas has a structure and the $\mathrm{N}$ atom is localized. It is possible to represent this effect by a nonlinear model [Grecchi and Martinez 1995].

A molecule with a structure is a metastable state of its system [Woolley 1982]. Here we consider a linear model with a PT Hamiltonian in order to define the resonant state related to the metastable one. The delocalized state of the $\mathrm{N}$ atom for low density of the gas can be represented by our $P T$ states $\psi_{2 n+(1 \pm 1) / 2}(\hbar)$ of (12) for large $\hbar>0$. The splitting $\Delta E=E_{2 n+1}(\hbar)-E_{2 n}(\hbar)$ gives the pulsation of the inversion line $\omega=\Delta E / \hbar$ and the beating effect. The dynamics of the packet

$$
\psi=C\left(\psi_{2 n+1}(\hbar)+\psi_{2 n}(\hbar)\right),
$$

is

$$
\psi(t)=C(t) \exp (-i E t / \hbar)\left(\psi_{2 n+1}(\hbar)+\exp (-i \omega t) \psi_{2 n}(\hbar)\right),
$$

where $E=E_{2 n+1}(\hbar)$, showing the beating effect with the pulsation of the so-called inversion line.

For large density of the gas, we expect a localization of the nitrogen atom in one side of the plane of the hydrogen atoms, so that the structure of the molecule is pyramidal. If two of the hydrogen atoms are substituted by different isotopes $\mathrm{NH}_{3} \rightarrow$ NHDT, the pyramidal structure of the molecule gets a definite chirality. 
Actually, the model is not complete since there exists the racemization effect: a slow change of chirality.

In our model we can represent the state by one of the eigenfunctions $\psi(0)=$ $\psi_{n}^{ \pm}(\hbar), \hbar<\hbar_{n}$, localized about one of the wells $x_{ \pm}$, respectively. This state is stationary:

$$
\psi(t)=C(t) \exp (-i E t / \hbar) \psi(0), \quad E=E_{n}^{ \pm}(\hbar),
$$

where the coefficient $C(t)>0$ is given by the unitary normalization $\|\psi(t)\|=1$.

But it is also possible to represent the racemization.

The dynamics of a state is given by $\psi(t)=C(t) \exp \left(-i H_{\hbar} t\right) \psi(0)$, where $C(t)>$ 0 is given by the unitary normalization $\|\psi(t)\|=1$. Let us consider the $P T$ state as a wave packet at $t=0$,

$$
\psi(0)=C(0)\left(\psi_{n}^{-}(\hbar)+\psi_{n}^{+}(\hbar)\right)
$$

with unitary norm, for $\hbar>0$ small. This packet is $P T$-symmetric and is delocalized, or better, it is bilocalized about both the wells. The dynamics of the packet is

$$
\psi(t)=C(t)\left(\exp (-i \bar{E} t / \hbar) \psi_{n}^{-}(\hbar)+\exp (-i E t / \hbar) \psi_{n}^{+}(\hbar)\right),
$$

where $E=E_{n}^{+}(\hbar)$. Since $\Im E<0$, we have $|\psi(t)| \rightarrow\left|\psi_{n}^{\mp}(\hbar)\right|$ as $t \rightarrow \pm \infty$. Thus, we have a process of localization of the packet about the well $x_{\mp}$ in the limit $t \rightarrow \pm \infty$, respectively. The full process for $t$ going from $-\infty$ to $\infty$ is a slow change of localization of the $\mathrm{N}$ atom from a well to the other one. This corresponds to a change of chirality of the molecule or a racemization [Streitwieser and Heathcock 1985, p. 122-124].

In this case it is evident that the $P$ and $T$ symmetry of the process breaks, conserving the $P T$ symmetry. If $\hbar_{n}-\hbar$ is small enough, the time of the racemization process is much longer than the period of the beating effect.

I don't attempt to justify the model from a physical point of view. As a physical comment, I would say that, in general, time symmetry breaking is a consequence of space symmetry breaking. A rigid body is not a system in a stationary state since it breaks the space symmetry of the potential [Woolley 1982]. Thus, it breaks translational time symmetry.

\section{References}

[Aguilar and Combes 1971] J. Aguilar and J. M. Combes, "A class of analytic perturbations for one-body Schrödinger Hamiltonians”, Comm. Math. Phys. 22:4 (1971), 269-279.

[Alvarez 1995] G. Alvarez, "Bender-Wu branch points in the cubic oscillator", J. Phys. A 28:16 (1995), 4589-4598.

[Benassi and Grecchi 1980] L. Benassi and V. Grecchi, "Resonances in the Stark effect and strongly asymptotic approximants", J. Phys. B 13:5 (1980), 911-930. 
[Bender and Boettcher 1998] C. M. Bender and S. Boettcher, "Real spectra in non-Hermitian Hamiltonians having $\mathscr{P} \mathscr{T}$ symmetry", Phys. Rev. Lett. 80:24 (1998), 5243-5246.

[Bender and Wu 1969] C. M. Bender and T. T. Wu, "Anharmonic oscillator", Phys. Rev. (2) 184:5 (1969), 1231-1260.

[Buslaev and Grecchi 1993] V. Buslaev and V. Grecchi, "Equivalence of unstable anharmonic oscillators and double wells", J. Phys. A 26:20 (1993), 5541-5549.

[Caliceti 2000] E. Caliceti, "Distributional Borel summability of odd anharmonic oscillators", $J$. Phys. A 33:20 (2000), 3753-3770.

[Caliceti and Maioli 1983] E. Caliceti and M. Maioli, "Odd anharmonic oscillators and shape resonances”, Ann. Inst. H. Poincaré Sect. A (N.S.) 38:2 (1983), 175-186.

[Caliceti et al. 1980] E. Caliceti, S. Graffi, and M. Maioli, "Perturbation theory of odd anharmonic oscillators", Comm. Math. Phys. 75:1 (1980), 51-66.

[Caliceti et al. 1986] E. Caliceti, V. Grecchi, and M. Maioli, "The distributional Borel summability and the large coupling $\Phi^{4}$ lattice fields", Comm. Math. Phys. 104:1 (1986), 163-174.

[Caliceti et al. 1988] E. Caliceti, V. Grecchi, and M. Maioli, "Double wells: perturbation series summable to the eigenvalues and directly computable approximations", Comm. Math. Phys. 113:4 (1988), 625-648.

[Caliceti et al. 1993] E. Caliceti, V. Grecchi, and M. Maioli, "Stark resonances: asymptotics and distributional Borel sum", Comm. Math. Phys. 157:2 (1993), 347-357.

[Caliceti et al. 1996] E. Caliceti, V. Grecchi, and M. Maioli, "Double wells: Nevanlinna analyticity, distributional Borel sum and asymptotics", Comm. Math. Phys. 176:1 (1996), 1-22.

[Delabaere and Pham 1997] E. Delabaere and F. Pham, "Unfolding the quartic oscillator", Ann. Physics 261:2 (1997), 180-218.

[Delabaere and Trinh 2000] E. Delabaere and D. T. Trinh, "Spectral analysis of the complex cubic oscillator", J. Phys. A 33:48 (2000), 8771-8796.

[Delabaere et al. 1997] E. Delabaere, H. Dillinger, and F. Pham, "Exact semiclassical expansions for one-dimensional quantum oscillators", J. Math. Phys. 38:12 (1997), 6126-6184.

[Dennison and Uhlenbeck 1932] D. M. Dennison and G. E. Uhlenbeck, "The two-minima problem and the ammonia molecule", Phys. Rev. 41:3 (1932), 313-321.

[Eremenko and Gabrielov 2009] A. Eremenko and A. Gabrielov, "Analytic continuation of eigenvalues of a quartic oscillator", Comm. Math. Phys. 287:2 (2009), 431-457.

[Eremenko and Gabrielov 2011] A. Eremenko and A. Gabrielov, "Singular perturbation of polynomial potentials with applications to PT-symmetric families", Mosc. Math. J. 11:3 (2011), 473-503, 629-630.

[Eremenko et al. 2008a] A. Eremenko, A. Gabrielov, and B. Shapiro, "High energy eigenfunctions of one-dimensional Schrödinger operators with polynomial potentials", Comput. Methods Funct. Theory 8:2 (2008), 513-529.

[Eremenko et al. 2008b] A. Eremenko, A. Gabrielov, and B. Shapiro, "Zeros of eigenfunctions of some anharmonic oscillators", Ann. Inst. Fourier 58:2 (2008), 603-624.

[Giachetti and Grecchi 2011] R. Giachetti and V. Grecchi, " $P T$-symmetric operators and metastable states of the 1D relativistic oscillators", J. Phys. A 44:9 (2011), 095308.

[Giachetti and Grecchi 2015] R. Giachetti and V. Grecchi, "Localization of the states of a $P T$ symmetric double well”, Internat. J. Theoret. Phys. 54:11 (2015), 3889-3899.

[Giachetti and Grecchi 2016a] R. Giachetti and V. Grecchi, "Bender-Wu singularities", J. Math. Phys. 57:12 (2016), 122109. 
[Giachetti and Grecchi 2016b] R. Giachetti and V. Grecchi, "Level crossings in a $P T$-symmetric double well", J. Phys. A 49:10 (2016), 105202.

[Giller 2011] S. Giller, "The semiclassical small- $\hbar$ limit of loci of roots of subdominant solutions for polynomial potentials", J. Math. Phys. 52:6 (2011), 063514, 44.

[Graffi and Grecchi 1978] S. Graffi and V. Grecchi, "Resonances in Stark effect and perturbation theory", Comm. Math. Phys. 62:1 (1978), 83-96.

[Graffi et al. 1970] S. Graffi, V. Grecchi, and B. Simon, "Borel summability: application to the anharmonic oscillator", Phys. Lett. B 32:7 (1970), 631-634.

[Grandi 1710] G. Grandi, Quadratura circuli, et hyperbola per infinitas hyperbolas, et parabolas quadrabiles geometricè exhibita, et demonstrata, Francisci Bindi, Pisa, 1710.

[Grecchi and Martinez 1995] V. Grecchi and A. Martinez, "Non-linear Stark effect and molecular localization”, Comm. Math. Phys. 166:3 (1995), 533-548.

[Grecchi and Martinez 2013] V. Grecchi and A. Martinez, "The spectrum of the cubic oscillator", Comm. Math. Phys. 319:2 (2013), 479-500.

[Grecchi et al. 1996] V. Grecchi, A. Martinez, and A. Sacchetti, "Double well Stark effect: crossing and anticrossing of resonances", Asymptotic Anal. 13:4 (1996), 373-391.

[Grecchi et al. 2009] V. Grecchi, M. Maioli, and A. Martinez, "Padé summability of the cubic oscillator", J. Phys. A 42:42 (2009), 425208.

[Grecchi et al. 2010] V. Grecchi, M. Maioli, and A. Martinez, "The top resonances of the cubic oscillator", J. Phys. A 43:47 (2010), 474027.

[Hamilton 1834] W. R. Hamilton, "On a general method in dynamics, by which the study of the motions of all free systems of attracting or repelling points is reduced to the search and differentiation of one central relation, or characteristic function", Phil. Trans. R. Soc. Lond. 124 (1834), 247-308.

[Hamilton 1835] W. R. Hamilton, "On the application to dynamics of a general mathematical method previously applied to optics", pp. 513-518 in Report of the Fourth Meeting of the British Association for the Advancement of Science (Edinburgh, 1834), John Murray, London, 1835.

[Harrell and Simon 1980] E. Harrell and B. Simon, "The mathematical theory of resonances whose widths are exponentially small”, Duke Math. J. 47:4 (1980), 845-902.

[Herbst and Simon 1978] I. W. Herbst and B. Simon, "Stark effect revisited", Phys. Rev. Lett. 41:2 (1978), 67-69.

[Kato 1966] T. Kato, Perturbation theory for linear operators, Grundlehren math. Wissenschaften 132, Springer, Berlin, 1966.

[Loeffel et al. 1969] J. J. Loeffel, A. Martin, B. Simon, and A. S. Wightman, "Pade approximants and the anharmonic oscillator", Phys. Lett. B 30:9 (1969), 656-658.

[Martinez and Nédélec 2012] A. Martinez and L. Nédélec, "Optimal lower bound of the resonance widths for a Helmholtz tube-shaped resonator", J. Spectr. Theory 2:2 (2012), 203-223.

[Mengoli 1670] P. Mengoli, Speculationi di musica, Herede del Benacci, Bologna, 1670.

[Reed and Simon 1975] M. Reed and B. Simon, Methods of modern mathematical physics, II: Fourier analysis, self-adjointness, Academic, New York, 1975.

[Reed and Simon 1978] M. Reed and B. Simon, Methods of modern mathematical physics, IV: Analysis of operators, Academic, New York, 1978.

[Shanley 1988a] P. E. Shanley, "Nodal properties of the scaled quartic anharmonic oscillator", Ann. Physics 186:2 (1988), 325-354.

[Shanley 1988b] P. E. Shanley, "Spectral properties of the scaled quartic anharmonic oscillator", Ann. Physics 186:2 (1988), 292-324. 
[Shin 2002] K. C. Shin, "On the reality of the eigenvalues for a class of $\mathscr{P} \mathcal{T}$-symmetric oscillators", Comm. Math. Phys. 229:3 (2002), 543-564.

[Sibuya 1975] Y. Sibuya, "Distribution of zeros", Chapter 7, pp. 152-165 in Global theory of a second order linear ordinary differential equation with a polynomial coefficient, Math. Studies 18, North-Holland, Amsterdam, 1975.

[Streitwieser and Heathcock 1985] A. Streitwieser and C. H. Heathcock, Introduction to organic chemistry, 3rd ed., Macmillian, New York, 1985.

[Voros 1994] A. Voros, "Exact quantization condition for anharmonic oscillators (in one dimension)", J. Phys. A 27:13 (1994), 4653-4661.

[Woolley 1982] R. G. Woolley, "Natural optical activity and the molecular hypothesis", pp. 1-35 in Structures versus special properties, edited by M. J. Clarke et al., Struct. Bond. 52, Springer, Berlin, 1982.

Received 25 Mar 2016. Revised 29 Sep 2016. Accepted 31 Oct 2016.

VINCENZO GRECCHI: vincenzo.grecchi@unibo.it

Dipartimento di Matematica, Università di Bologna, I-40126 Bologna, Italy

and

Gruppo Nazionale di Fisica Matematica, Istituto Nazionale di Alta Matematica Francesco Severi, I-00185 Roma, Italy 


\title{
QUANTUM MECHANICS: SOME BASIC TECHNIQUES FOR SOME BASIC MODELS II: THE TECHNIQUES
}

\author{
VINCENZO GRECCHI
}

\begin{abstract}
This paper is a continuation of the previous one: "Quantum mechanics: some basic techniques for some basic models, I: The models". The main subject is the perturbation theory in quantum mechanics giving diverging perturbation series for the energy levels of the oscillators. Thus, a short historical introduction on the methods of the sum of diverging power series is given.
\end{abstract}

\section{Introduction and some techniques}

This is the second part of an article in honor of Professor Lucio Russo. In Section 2 there is a short history of the regular methods of the sum of divergent series. It starts with the formal series $\tilde{S}(a)=\sum_{n} a_{n}$, where $a=a_{0}, a_{1}, \ldots$ is considered a vector of components $a_{n}=(-1)^{n}$, with a possible sum $S(a)=\frac{1}{2}$ as suggested by Guido Grandi. The best proof of the value of this sum, if existent, is based on the assumption that $S(a)$ is a linear functional. The same linearity was used by Pietro Mengoli for the sum of some convergent series.

1a. Quadratic estimates. A family of analytic operators of type A should have constant domain [Kato 1966; Reed and Simon 1975; 1978]. For the control on the domain, the quadratic estimates can be used. We consider the simple closed $P T$ operator $H:=O_{3}(0)=p^{2}+i x^{3}$, and we want to prove that $D(H)=D\left(p^{2}\right) \cap D\left(x^{3}\right)$, knowing that $D\left(p^{2}\right) \cap D\left(x^{3}\right) \subset D(H)$. We consider the quadratic norm as a quadratic form

$$
\|H \psi\|^{2}=\left\langle\psi, H^{*} H \psi\right\rangle \quad \text { for } \psi \in D\left(p^{2}\right) \cap D\left(x^{3}\right),
$$

where

$$
H^{*} H=p^{4}+x^{6}+3\left[x^{2}, p\right]_{+}=p^{4}+x^{6}+3\left[\left(p+x^{2}\right)^{2}-p^{2}-x^{4}\right],
$$

\section{Communicated by Francesco dell'Isola.}

PACS2010: 03.65.-w.

Keywords: confinement of the nodes and exact quantization condition, Stokes lines, Stokes sectors, generalized change of representation, Riccati equation and semiclassical series, divergent perturbation series, sum of divergent series. 
and we have the inequality

$$
\begin{aligned}
\|H \psi\|^{2} & \geq\left\|p^{2} \psi\right\|^{2}-3\|p \psi\|^{2}+\left\|x^{3} \psi\right\|^{2}-3\left\|x^{2} \psi\right\|^{2} \\
& >(1-\epsilon)\left(\left\|p^{2} \psi\right\|^{2}+\left\|x^{3} \psi\right\|^{2}\right)-\frac{C}{\epsilon}\|\psi\|^{2}, \quad \epsilon>0,(1-\epsilon)>0, C>0,
\end{aligned}
$$

so that $D(H)$ cannot be extended and $D(H)=D\left(p^{2}\right) \cap D\left(x^{3}\right)$.

1b. The method of Loeffel and Martin for the confinement of the zeros. We explain the Loeffel-Martin method [Loeffel et al. 1969] with a useful example. We consider a positive level $E=\hat{E}_{m}(0)$ with state $\psi(z)=\hat{\psi}_{m}(z)$, of the operator $O_{3}(0)=K(0)$ [Giachetti and Grecchi 2016a] transformed by the analytic translation $x \rightarrow x+i y$ :

$$
O_{3}(y, 0)=p^{2}+i(x+i y)^{3}=p^{2}+i\left(x^{2}-3 y^{2}\right) x+y^{3}-3 y x^{2}=p^{2}+V_{y}(x) .
$$

Then

$$
\begin{aligned}
& -\Im\left[\bar{\psi}(x+i y) \partial_{x} \psi(x+i y)\right]=\int_{x}^{\infty} \Im\left(V_{y}(s)-E\right)|\psi(s+i y)|^{2} d s \\
& =\int_{x}^{\infty}\left(s^{2}-3 y^{2}\right) s|\psi(s+i y)|^{2} d s=-\int_{-\infty}^{x}\left(s^{2}-3 y^{2}\right) s|\psi(s+i y)|^{2} d s \neq 0
\end{aligned}
$$

for $|x| \geq \sqrt{3}|y|, y \in \mathbb{R}$. In this case we have a rigorous confinement of the nodes in the region

$$
\mathbb{C}_{\sigma}=\{z=x+i y: y<0,|x|<-\sqrt{3} y\} \subset \mathbb{C}_{-}=\{z \in \mathbb{C}: \Im z<0\} .
$$

Now we extend the Loeffel-Martin method to the case $\Im E \neq 0, x=0$, obtaining the equation

$$
\Im\left(\hbar^{2} \bar{\phi}(y) \partial_{y} \phi(y)\right)=-\Im E \int_{y}^{\infty}|\phi(s)|^{2} d s \quad \text { for all } y \in \mathbb{R},
$$

where $\phi(y)=\psi(i y)$, if the integral in (1-2) exists and is bounded. Thus, the state of a nonreal level is free of imaginary zeros.

1c. The exact quantization condition [Giachetti and Grecchi 2016b]. We consider the semiclassical Hamiltonian

$$
H_{\hbar}=\hbar^{2} p^{2}+V(x), \quad V(x)=i\left(x^{3}-x\right), p^{2}=-\frac{d^{2}}{d x^{2}}, \hbar>0 .
$$

Let us fix $\hbar>0$ small, the level $E=E_{n}^{+}(\hbar) \in \mathbb{C}_{-}$(the case $E_{n}^{-}(\hbar)$ is perfectly analogous $)$ and the state $\psi_{E}(z)=\psi_{n}^{+}(\hbar, z)$ with $n$ nodes in $\mathbb{C}^{+}=\{z \in \mathbb{C}: \Re z>0\}$. We have the exact quantization condition

$$
J(E, \hbar):=\frac{\hbar}{2 i \pi} \oint_{\gamma} \frac{\psi_{E}^{\prime}(z)}{\psi_{E}(z)} d z+\frac{\hbar}{2}=\hbar\left(n+\frac{1}{2}\right),
$$


where $\gamma=\partial \Omega \subset \mathbb{C}^{+}$and $\Omega$ is a connected domain large enough to contain all the nodes. The fixed number $n$ and the isolation of the nodes allow us to bound the energy value.

In particular, for small $\hbar>0$, and fixed $n \in \mathbb{N}$, the quantization rules (1-4) become the semiclassical quantization conditions for $E=E_{n}^{+}(\hbar)$

$$
J(E, \hbar)=\frac{1}{2 i \pi} \oint_{\gamma} p_{0}(E, z) d z+O\left(\hbar^{2}\right)=\hbar\left(n+\frac{1}{2}\right),
$$

where $p_{0}(E, z)=\sqrt{V(z)-E}$, and the path $\gamma$ shrinks around the short Stokes line.

1d. The generalized changes of representation [Aguilar and Combes 1971]. A change of representation is given by a unitary transform $U_{\delta} \neq U_{0}=I$, where $\delta$ is any nonzero real number and $I$ is the identity operator, giving a new Hamiltonian $H_{\delta}=U_{\delta} H U_{\delta}^{*}$ unitarily equivalent to the original one. In this case, a level of the Hamiltonian is the same as that of the original one, $E_{n}(\delta)=E_{n}(0)$, and the state is transformed into $\psi_{n}(\delta)=U_{\delta} \psi_{n}$. Let $H_{\delta}$ be an analytic family of operators of type A in the parameter $\delta \in \Omega \subset \mathbb{C}$, with $\mathbb{R} \subset \Omega$, and let $H$ have discrete spectrum $\left\{E_{n}\right\}_{n}$. Then the operator $H_{\delta}, \delta \in \Omega \subset \mathbb{C}$, is isospectral to $H:=H_{0}$ so that

$$
E_{n}(\delta)=E_{n},
$$

and the vector $\psi_{n}(\delta)=U_{\delta} \psi_{n}, \delta \in \Omega$ and $\delta=i \theta, \theta \in \mathbb{R}$, is the state $\psi_{n}$ in a generalized representation.

Actually, there are more general changes of representation with definite transformations of the eigenvalues

$$
E_{n}(\delta)=f_{\delta}\left(E_{n}\right)
$$

1e. The Stokes sectors, the asymptotic behavior, the Riccati equation [1761] and the semiclassical series expansion. We recall the asymptotic behavior of the states at infinity, the Stokes sectors and the Carlini semiclassical series expansion [Plana 1832; Fröman and Fröman 1965].

Let us fix a direction in the complex plane of the variable $\arg (z)=\alpha$. The asymptotic behavior of a fundamental solution of $H_{\hbar}$ (see (1-3)) at infinity in this direction is given by [Sibuya 1975]

$$
\hbar \lim _{\substack{|z| \rightarrow \infty \\ \arg z=\alpha}} \frac{\ln \psi_{n}(z)}{\int^{z} \sqrt{V(w)} d w}= \pm 1 .
$$

The Stokes directions $\alpha_{j}$ are defined by the oscillatory condition of the fundamental solutions at infinity:

$$
\lim _{|z| \rightarrow \infty} \arg \left(\ln \psi_{n}(z)\right)=\pi / 2+j \pi, \quad j=-2,-1,0,1,2 .
$$


A Stokes sector of the complex plane is a minimal sector between two Stokes directions. In the case of the cubic oscillator $H_{\hbar}$, the Stokes directions $\arg (z)=\alpha_{j}$ are

$$
\lim _{|z| \rightarrow \infty} \arg \left(\ln \psi_{n}(z)\right)=5 \arg (i z) / 2=\pi / 2+j \pi, \quad j=-2,-1,0,1,2,
$$

and the Stokes sectors

$$
S_{j}=\{z \in \mathbb{C}:|\arg (i z)-2 j \pi / 5|<\pi / 5\}, \quad j=-2,-1,0,1,2 .
$$

A state $\psi_{n}(\hbar)$ of the Hamiltonian $H_{\hbar}$ is exponentially decreasing at infinity in the sectors $S_{ \pm 1}$ and exponentially increasing in the sectors $S_{0}$ and $S_{ \pm 2}$.

This state has large zeros only in the imaginary asymptotic direction. If we label the zeros $Z_{j}$ such that $\left|Z_{j}\right| \rightarrow \infty$ for $j \rightarrow \infty$,

$$
\arg \left(Z_{j}\right) \rightarrow \alpha_{ \pm 2}=\pi / 2 \quad \text { as } j \rightarrow \infty .
$$

Remark. In the case of a double-well cubic oscillator, this result is not sufficient for our purposes. We have proven that in the case of positive levels $E_{m}$ the large zeros of $\psi_{m}$ are exactly imaginary. Moreover, we have $\mp \Im E_{n}^{ \pm}>0$ and the large zeros of $\psi_{n}^{ \pm}$are in $\mathbb{C}^{\mp}$.

We express two fundamental solutions, in a given sector $S_{j}$, of the Schrödinger equation

$$
\left(\hbar^{2} p^{2}+p_{0}^{2}(z)\right) \psi(z)=0, \quad p^{2}=-\frac{d^{2}}{d z^{2}}, p_{0}^{2}(z)=V(z)-E,
$$

in the form

$$
\psi_{ \pm}(z)=\exp \left( \pm \frac{1}{\hbar} \int_{0}^{z} p_{\hbar}(w) d w\right)
$$

where $p_{\hbar}(z)$ satisfies the Riccati equation

$$
p_{\hbar}^{2}(z)+\hbar p_{\hbar}^{\prime}(z)=p_{0}^{2}(z), \quad p_{0}^{2}(z)=V(z)-E .
$$

We formally solve by the Carlini series

$$
p_{\hbar}(z) \sim \sum_{n} p_{n}(z) \hbar^{n},
$$

where the coefficients are computed recursively starting from the positive definition of the classical momentum: $p_{0}(z)=\sqrt{V(z)-E}$ and

$$
\begin{aligned}
& p_{1}(z)=\frac{i p_{0}^{\prime}(z)}{2 p_{0}(z)} \\
& p_{n}(z)=-\frac{1}{2 p_{0}(z)}\left(\sum_{j=1}^{n-1} p_{n-j}(z) p_{j}(z)+i p_{n-1}^{\prime}(z)\right), \quad n=2,3, \ldots
\end{aligned}
$$


Defining

$$
p_{\hbar}(z)=P_{\chi}(z)+\hbar Q_{\chi}(z), \quad \chi=\hbar^{2},
$$

we get the equation for $P_{\chi}(z)$

$$
P_{\chi}^{2}(z)-p_{0}^{2}(z)=-\chi\left(Q_{\chi}^{2}(z)+Q_{\chi}^{\prime}(z)\right)
$$

where

$$
Q_{\chi}(z)=-\frac{P_{\chi}^{\prime}(z)}{2 P_{\chi}(z)}
$$

We thus have the equivalent expression of the solutions

$$
\psi_{ \pm}(z)=\frac{1}{\sqrt{P_{\chi}(z)}} \exp \left( \pm \frac{1}{\hbar} \int_{0}^{z} P_{\chi}(w) d w\right),
$$

where the Riccati solution $P_{\chi}(z)$ has the even part of the Carlini expansion:

$$
P_{\chi}(z) \sim \sum_{j \in \mathbb{N}} \chi^{j} p_{2 j}(z) \quad \text { with truncations } P_{\chi}^{N}(z) \sim \sum_{j}^{N} \chi^{j} p_{2 j}(z) .
$$

Thus, the semiclassical momentum $P_{\chi}(z)$ is an asymptotic notion, and the coefficients of the Carlini series are singular near the turning points. In certain cases, the exact momentum $P_{\chi}(z)$ is the Borel sum of the series in (1-13) [Voros 1994; Delabaere et al. 1997]. Notice that the behaviors of the fundamental solutions, for large $|z|$ in a Stokes sector, are given by (1-12) at a zeroth order of approximation with $p_{0}(z)$ in place of $P_{\chi}(z)$ [Sibuya 1975].

1f. The Stokes lines and the classical trajectories. Here we also examine the critical energies at the semiclassical limit $\hbar=0$.

Let us consider the real cubic oscillator

$$
H_{r}(\hbar):=\hbar^{2} p^{2}+V^{r}(x), \quad V^{r}(x)=x^{3}-x, \hbar>0,
$$

and the energy range $R_{0}=\left[-E_{0}, E_{0}\right]$, where $E_{0}=2 / 3 \sqrt{3}$. The union of the trajectories of the classical motion $M(E)$ consists of the oscillation range $\sigma(E):=$ $\left[I_{-}, I_{+}\right]$and the escape route $B(E):=\left(-\infty, I_{0}\right], I_{0}<-1 / \sqrt{3}<I_{-}<I_{+}$. We recall the definition of the Stokes lines at energy $E$. A Stokes line is tangent at each one of its points $z$ to the direction $d z^{2}(z)$, where $p(z)^{2} d z^{2}(z)<0, p(z)^{2}=V^{r}(z)-E$, and starts from a turning point $I$. From the complex semiclassical point of view, $\sigma(E)$ is the internal Stokes line and $B(E)$ is the exceptional Stokes line [Giller 2011].

The fundamental state $\psi_{E}^{0}(\hbar, x), E \in R_{0}$, subdominant in the positive semiaxis,

$$
\psi_{E}^{0}(\hbar, x) \sim \frac{1}{\sqrt{p(x)}} \exp \left(-\frac{1}{\hbar} \int_{I_{+}}^{x} p(w) d w\right),
$$


for large $x>0$, can be continued onto the complex plane cut along $\sigma(E)$ and $B(E)$. We extend the approximate fundamental state by setting $\psi_{E}^{0}(\hbar, x)=\left(\psi_{E}^{0}(\hbar, x+i 0)+\right.$ $\left.\psi_{E}^{0}(\hbar, x-i 0)\right) / 2$ for all $x \in M(E)$. This approximation oscillates in $M(E)$ as the exact fundamental state. In the limit $\hbar \rightarrow 0$ all the zeros of the exact fundamental state $\psi_{E}(\hbar)$ tend to $M(E)$ as $\hbar \rightarrow 0$ [Giller 2011]. The union of the trajectories of the classical motion $M(E)$ is singular at $E=E_{0}=2 / 3 \sqrt{3}$, where the escape route $B(E)$ touches the oscillation range $\sigma(E)$.

Now we consider the $P T$-symmetric double well with Hamiltonian $H_{\hbar}=\hbar^{2} p^{2}+$ $i\left(x^{3}-x\right)$, for $\hbar>0$. For $E>0$ large, the escape route is part of the imaginary axis, while the oscillation range should be computed numerically.

The union of the trajectories of the classical motion $M(E)$ has the singular point $E^{c}=0.352268 \ldots$ where the escape route touches the oscillation range at its middle point. For larger $E>E^{c}$ the escape route and the oscillation range are separated. The energy levels $E_{n}(\hbar)$ are positive for large $\hbar>0$ and are imaginary at the limit $\hbar \rightarrow 0$. This means the existence of (at least) a singularity and a crossing at $\hbar_{n}>0$ for each pair of functions $E_{n}^{ \pm}(\hbar), 0<\hbar<\hbar_{n}$. The crossing parameters $\hbar_{n}>0$ and the energy crossings $E_{n}^{c}>0$ have the limits $\hbar_{n} \rightarrow 0$ and $E_{n}^{c}=E_{n}^{ \pm}\left(\hbar_{n}\right) \rightarrow E^{c}$ as $n \rightarrow \infty$. The approximate actions around the oscillation range defined at the crossing, $J_{n}:=n \hbar_{n}$, have limit $J\left(E^{c}, 0\right)$ as $n \rightarrow \infty$ (see (1-5)), the action integral around the oscillation range $\sigma(E)$.

We now consider the two cases denoted by the signs \pm together. At energies $E_{n}^{\mp}(0)= \pm i E_{0}$, the oscillation range $\sigma(E)$ reduces to the points $I_{-}=I_{+}=\mp 1 / \sqrt{3}$, respectively. It is possible to prove that the escape route $B(E)$ starts at $I_{0}= \pm 2 / \sqrt{3}$ and goes toward $+i \infty$ staying in the half-plane $\mathbb{C}^{ \pm}$. Moreover, the levels $E_{n}^{ \pm}(\hbar)$ are nonreal for $\hbar<\hbar_{n}$ and the zeros of $\psi_{n}^{ \pm}(\hbar)$ cannot be on the imaginary axis. Thus, the $n$ nodes of $\psi_{n}^{ \pm}(\hbar)$ are stable in $\mathbb{C}^{\mp}$ for $0<\hbar<\hbar_{n}$.

1g. The theory of the regular perturbations. Let $H_{0}$ be a self-adjoint operator on the Hilbert space $\mathscr{H}$ with compact resolvent, so that the spectral theorem takes the expression

$$
H_{0}=\sum_{n} E_{n} P_{n}, \quad \mathbf{1}=\sum_{n} P_{n}, P_{n} P_{k}=\delta_{n}^{k} P_{n}, P_{n}^{*}=P_{n},
$$

where the set of real eigenvalues $\left\{E_{n}\right\}_{n}$ (the spectrum of $\left.H_{0}: \sigma\left(H_{0}\right)\right)$ have no accumulation points in $\mathbb{R}$ and the orthogonal projectors $P_{n}$ have a finite-dimensional image: $m_{n}=\operatorname{dim}\left(P_{n} \mathscr{H}\right) \in \mathbb{N}$.

Resolvent. For $z \in \mathbb{C}-\sigma\left(H_{0}\right)$ the resolvent of $H_{0}$ with parameter $z$,

$$
R_{0}(z)=\left(H_{0}-z\right)^{-1}=\sum_{n}\left(E_{n}-z\right)^{-1} P_{n}
$$


is a compact operator, with norm

$$
\left\|R_{0}(z) u\right\|^{2} \leq \max _{n}\left|E_{n}-z\right|^{-2} \sum_{n}\left\|P_{n} u\right\|^{2}=\|u\|^{2} / \rho\left(z, \sigma\left(H_{0}\right)\right)^{2},
$$

where $\rho\left(z, \sigma\left(H_{0}\right)\right)$ is the distance of $z$ from the spectrum of $H_{0}$. In particular, if $u=P_{j} u$, we have

$$
\left\|R_{0}(z) u\right\|=1 / \rho\left(z, E_{j}\right) .
$$

Operator $V$ relatively bounded with respect to $H_{0}(\mathrm{Kato})$. Let $V$ be another operator on $\mathscr{H}$ such that $D\left(H_{0}\right) \subset D(V)$ and

$$
\|V u\| \leq a\left\|H_{0} u\right\|+b\|u\| \quad \text { for all } u \in D\left(H_{0}\right)
$$

for positive constants $a$ and $b$. We use the notation $V<H_{0}$.

Theorem. With $V$ as above, the operator

$$
A:=A(z):=V R_{0}(z)
$$

for $z \notin \sigma\left(H_{0}\right)$ is a bounded operator.

Proof. Let us consider any $v \in \mathscr{H}$; then $A v=V u$, where $u=R_{0}(z) v$, with norm $\|u\| \leq\left\|R_{0}(z)\right\|\|v\| \equiv c\|v\|$. For the relative boundedness we have $\|V u\| \leq$ $a\left\|H_{0} u\right\|+b\|u\|$, where $\left\|H_{0} u\right\|=\left\|H_{0} R_{0}(z) v\right\|=\|v+z u\| \leq\|v\|+|z|\|u\|$; hence, $\|A v\| \leq(a(1+|z| c)+b c)\|v\|$, whence $A$ is a bounded operator with norm

$$
\|A\| \leq a(1+|z| c)+b c .
$$

Analytic family of operators. Let us consider the analytic family of operators $H_{\epsilon}=$ $H_{0}+\epsilon V$ for $\epsilon \in \mathbb{C}$.

Theorem. The resolvent of $H_{\epsilon}, R_{\epsilon}(z)=B_{\epsilon}$ for a fixed $z \notin \sigma\left(H_{\epsilon}\right)$, is an analytic family of bounded operators on the domain $|\beta|<b_{0}$.

Proof. We have

$$
B_{\epsilon}=R_{\epsilon}(z)=\left(\left(H_{0}-z\right)+\epsilon V\right)^{-1}=R_{0}(z)(1+\epsilon A)^{-1}=R_{0}(z) \sum_{n}(-\epsilon A)^{n},
$$

where $A$ is defined in (1-15) and the series of powers of operators converges in norm for $|\epsilon|<b_{0}=1 /\|A\|$.

Norm convergence of the resolvents. We have that

$$
\left\|B_{\epsilon}-B_{0}\right\| \leq\left\|R_{0}(z)\right\| \sum_{n=1}^{\infty}(|\epsilon|\|A\|)^{n}
$$

vanishes as $\epsilon \rightarrow 0$. Analogously, for $b_{1}>0$ small and $\left|\epsilon_{1}\right|<b_{1},\left\|B_{\epsilon}-B_{\epsilon_{1}}\right\| \rightarrow 0$ as $\epsilon \rightarrow \epsilon_{1}$. 
Perturbation not relatively bounded.

Quadratic estimate 1. For any $u \in D\left(H_{0}\right) \cap D(V)$, there exist $a, b>0$ such that

$$
\left\|H_{0} u\right\|^{2}+|\epsilon|^{2}\|V u\|^{2} \leq a\left\|H_{\epsilon} u\right\|^{2}+b\|u\|^{2} .
$$

Let $V=V_{0} V^{\prime}$ such that $V^{\prime}<H$ and $V_{0}=V_{0}^{*}<H_{0}$.

Quadratic estimate 2. For any $u \in D\left(H_{0}\right) \cap D(V)$, there exist positive constants $a$ and $b$ such that

$$
\left\|V^{\prime} u\right\|^{2} \leq a\left\|H_{\epsilon} u\right\|^{2}+b\|u\|^{2} .
$$

We have

$$
R_{\epsilon}-R_{0}=\epsilon R_{0} V R_{\epsilon}=\epsilon A_{0} A^{\prime},
$$

where the $z \notin \sigma\left(H_{0}\right)$ dependence is omitted. Both the operators $A_{0}=R_{0} V_{0}$ and $A^{\prime}=V^{\prime} R_{\epsilon}$ are uniformly bounded as $\epsilon \rightarrow 0$. Thus, we have the norm resolvent convergence

$$
\left\|R_{\epsilon}-R_{0}\right\| \leq|\epsilon|\|A\|\left\|A^{\prime}\right\| \rightarrow 0
$$

as $\epsilon \rightarrow 0$.

In our case, we have $H_{0}=p^{2}+\alpha x^{2},|\alpha|=1, V_{0}=x, V^{\prime}=i x^{2}$ and $\epsilon=\sqrt{b} \geq 0$.

Projector on an isolated part of the spectrum. Let $\gamma=\partial \Gamma$ be a regular closed positively oriented curve in $\mathbb{C}$ encompassing a simple isolated eigenvalue of $H_{0}$ once:

$$
\sigma_{\Gamma}:=E_{j}=\Gamma \cap \sigma\left(H_{0}\right), \quad \gamma \cap \sigma\left(H_{0}\right)=\varnothing .
$$

For instance, let $\gamma$ be the circle $\left|z-E_{j}\right|=d / 2$, where $d>0$ is the isolation distance of $E_{j}$. We have

$$
P_{j}=-(1 / 2 \pi i) \int_{\gamma} R_{0}(z) d z
$$

because of (1-14) and the residue theorem. Because of the norm convergence of the resolvents and $\gamma$ being a compact set, we have the norm convergence of the projectors $P_{j}(\epsilon):=P_{\epsilon}$,

$$
\left\|P_{\epsilon}-P_{j}\right\| \rightarrow 0
$$

for $|\epsilon| \rightarrow 0$, and there exists $b_{0}>0$ such that

$$
P_{j} \epsilon=-(1 / 2 \pi i) \int_{\gamma} R_{\epsilon}(z) d z
$$

for $0<\epsilon<b_{0}$. 
Perturbation expansion of $P_{\epsilon}$. The set of projectors $P_{\epsilon}$ is an analytic family of projectors, and its power series expansion is

$$
P_{\epsilon}=\sum_{n} \epsilon^{n} Q_{n}
$$

where

$$
Q_{n}=-(2 \pi i)^{-1} \int_{\gamma} R_{0}(z)\left(-V R_{0}(z)\right)^{n} d z .
$$

Continuity and stability of the spectrum. Let $m_{\epsilon}=\operatorname{dim}\left(P_{\epsilon} \mathscr{H}\right) \in \mathbb{N}$ be the total multiplicity of the eigenvalues of $H_{\epsilon}$ contained in $\Gamma$. This is a continued function for $0<\epsilon \leq \epsilon_{0}$ and, being discrete-valued, is constant. This means that in $\Gamma$ we always have the only eigenvalue $E_{j}(\epsilon)$ near $E_{j}$, for $\epsilon$ small, and we can compute this analytic eigenvalue by the perturbation method.

1h. Perturbation expansion of a simple isolated eigenvalue. Let $E(0)$ be a simple isolated eigenvalue of $H_{0}$, with normalized eigenvector $u_{0}$ and projector $P_{0}$, so that $H_{0} u_{0}=E(0) u_{0}$ and $P_{0} u_{0}=u_{0}$. We have $\left\|P_{\epsilon}-P_{0}\right\| \rightarrow 0$ as $\epsilon \rightarrow 0$ so that $u_{\epsilon}=P_{\epsilon} u_{0} \rightarrow u_{0}$ as $\epsilon \rightarrow 0$ is an eigenvector: $H_{\epsilon} u_{\epsilon}=E(\epsilon) u_{\epsilon},\left\|u_{\epsilon}\right\|>0$. Thus, $\left\langle u_{0}, P_{\epsilon} u_{0}\right\rangle=\left\langle P_{\epsilon} u_{0}, P_{\epsilon} u_{0}\right\rangle \rightarrow 1$ as $\epsilon \rightarrow 0$. We can compute $E(\epsilon)$ by the expression

$$
\begin{aligned}
E(\epsilon) & =\left\langle u_{0}, H_{\epsilon} P_{\epsilon} u_{0}\right\rangle /\left\langle u_{0}, P_{\epsilon} u_{0}\right\rangle \\
& =E(0)+\epsilon\left\langle u_{0}, V P_{\epsilon} u_{0}\right\rangle /\left\langle u_{0}, P_{\epsilon} u_{0}\right\rangle \\
& =E(0)+\epsilon \frac{\sum_{n} \epsilon^{n}\left\langle u_{0}, V Q_{n} u_{0}\right\rangle}{\sum_{n} \epsilon^{n}\left\langle u_{0}, Q_{n} u_{0}\right\rangle} \\
& =E(0)+\epsilon\left\langle u_{0}, V u_{0}\right\rangle+O\left(\epsilon^{2}\right)=\sum_{n} \epsilon^{n} c_{n},
\end{aligned}
$$

where the $\left\{c_{n}\right\}_{n}$ are the coefficients of the power series expansion given by the perturbation theory as a ratio of two power series. Since the two series are geometric, it is possible to evaluate the remainder, and it turns out to be of the same order as the next perturbation coefficient.

1i. Divergent perturbation series. If $V$ is not bounded with respect to $H_{0}$, but any vector $V Q_{n} u_{0}$ or $Q_{n} u_{0}, n \in \mathbb{N}$, is bounded, we have bounded coefficients of a divergent perturbation series. We do not use the estimates of the growing of the coefficients and the remainders made with the perturbation theory because we have the exact behavior from the Stieltjes property and the behavior of the imaginary part of the "resonances".

Now it is useful to recall the method of summing the divergent perturbation series. 


\section{Divergent power series regular sum: a short story}

The formal series [Akhiezer 1965] as a vector function with specified terms

$$
\tilde{S}(a):=\sum_{k=0}^{\infty} a_{k}:=\sum a_{k}, \quad a=\left\{a_{k}\right\}_{k \in \mathbb{N}}, a_{k} \in \mathbb{C},
$$

is convergent (Cauchy) with sum $S(a)$, if there exists

$$
S(a)=\lim _{n \rightarrow \infty} S_{n}(a) \in \mathbb{C}, \quad S_{n}(a)=\sum_{k=0}^{n} a_{k} .
$$

Actually Pietro Mengoli's [1650] idea of a convergent series was not so different.

For the simple formal series $\tilde{S}(a)$ as a function of the sequence of its terms

$$
a=\left\{a_{k}=(-1)^{k}\right\}_{k \in \mathbb{N}},
$$

Guido Grandi [1703], obviously unaware of the Cauchy condition, suggested that the only possible sum of the series $S(a)$ is $\frac{1}{2}$.

Let us recall a possible proof.

We assume that the sum $S(a)$ is a linear functional of the vector $a$. Now we recall some obvious facts.

The sum of a series with only one nonzero term must exist:

$$
\text { if } b=b_{0}, 0,0,0, \ldots, \quad S(b)=b_{0} .
$$

The invariance of the series by translation of the indexes is obvious:

$$
\tilde{S}(T a)=\tilde{S}(a), \quad a=a_{0}, a_{1}, \ldots, T a=0, a_{0}, a_{1}, \ldots
$$

So we must admit the same invariance for the sum

$$
S(T a)=S(a) .
$$

The proof that $S(a)$, for $a$ as in (2-2), if it exists, must be equal to $\frac{1}{2}$, is based on the extension of the linearity of the sum used by Pietro Mengoli for convergent series.

Hypothesis 1. The sum of a series $S(a)$ is a linear function of the vector of its elements $a$ :

$$
S(a)=S(b)+\lambda S(c), \quad a=b+\lambda c,
$$

if all the sums exist.

Proposition. Assuming the existence of the sum S(a) of the formal series (2-2),

$$
S(a)=\frac{1}{2} .
$$


Proof. We decompose the vector $a$ as $a=b+a_{0}^{\prime}$, where $b=a_{0}, 0,0, \ldots$ and $a_{0}^{\prime}=0, a_{1}, a_{2}, \ldots=-T a$. Thus, since $a=b+\lambda T a$ where $\lambda=-1$, by Hypothesis 1 ,

$$
S(c)=S(b)+\lambda S(T a)=b_{0}+\lambda S(a),
$$

if $\lambda \in \mathbb{C}, c=b+\lambda T a$ and $b$ is as above in (2-3). The sum of the series satisfies a linear equation

$$
S(a)=S(b)-S(T a)=a_{0}-S(a)=1-S(a),
$$

with the unique solution $S(a)=\frac{1}{2}$.

Notice that Mangione [1971] says that the statement of the existence of the sum of the series $\tilde{S}(a)$ is a mistake (obviously it is, assuming the Cauchy definition of the sum).

Later Cesaro proposed his method of the sum (1894-1897). The partial sums of $\tilde{S}(a)$ are

$$
S_{n}(a)=\frac{1}{2}+\frac{1}{2}(-1)^{n}, \quad n=0,1,2, \ldots,
$$

so that the mean values of the first sums are

$$
S_{N}^{c}(a)=\sum_{n=0}^{N-1} \frac{S_{n}(a)}{N}=\frac{1}{2}+\frac{1}{2 N}(-1)^{N-1}, \quad N=1,2, \ldots
$$

The Cesaro sum of the series, $\lim _{N \rightarrow \infty} S_{N}^{c}(a)$, exists and coincides with Grandi's.

2a. The Taylor expansion of a function. An analytic function is the sum of its power series expansion on a disk:

$$
f(z)=\sum_{k=0}^{\infty} c_{k} z^{k}, \quad c_{k}=\frac{f^{(k)}(0)}{k !},\left|c_{k}\right|=\frac{1}{r_{k}^{k}},|z|<r,
$$

where $r=\underline{\lim } r_{k}>0$.

Example 1. We have the expansion

$$
f(z)=\frac{1}{1+z}=\sum_{k=0}^{\infty} c_{k} z^{k}=\sum_{k=0}^{\infty} c_{k} z^{-k-1}, \quad c_{k}=(-1)^{k},
$$

on the open disk $|z|<1$. Extending the sum by continuity to the point $z=1$, we get the Grandi proof of the Grandi sum of the Grandi series.

We now state some definitions and results. 
2b. A divergent power series asymptotic to a function (Poincaré). A divergent power series is asymptotic to a function $f$ when

$$
\left|f(x)-\sum_{n=0}^{N-1} c_{n} x^{n}\right| \leq C_{N} x^{N}, \quad 0<x<X .
$$

The astronomer approximants. Let $C_{N}=a^{N} N$ !, where $a>0$. For any $x$ as above, we fix $N(x)=[1 / a x]$ and we take the approximant

$$
\sum_{n=0}^{N(x)-1} c_{n} x^{n}
$$

with an error exponentially small as $x \rightarrow 0$.

Remark. The asymptotic function $f(x)$ in (2-10) is not unique; there is also the family of functions $f_{\epsilon}(x)=f(x)+\epsilon \exp (-1 / a x)$ for $\epsilon \neq 0$ with $|\epsilon|$ small enough.

2c. Strong asymptotics and uniqueness of the function (Carleman). Consider the formal power series $\sum_{n}^{\infty} c_{n} z^{n}$ and the function $f(z)$. If there exist $X, C_{N}>0$ such that

$$
\sum_{N} \frac{1}{\sqrt[N]{C_{N}}}=\infty
$$

and $f(z)$ is analytic on

with

$$
D_{X}=\{z:|z| \leq X,|\arg (z)| \leq \pi / 2\}
$$

$$
\left|f(z)-\sum_{n}^{N-1} c_{n} z^{n}\right| \leq C_{N}|z|^{N}
$$

uniformly for $N \in \mathbb{N}$ and $z \in D_{X}$, then the function $f(z)$ is uniquely defined on $D_{X}$ by the formal series and is called the sum of the series.

2d. Direct methods of sum. We define more general approximants of the series (2-8), not only the polynomials $S_{n}(z)=\sum_{k=0}^{n} c_{k} z^{k}$ but also the rational functions called diagonal Padé approximants:

$$
\frac{Q_{j}(z)}{P_{j}(z)}=S_{2 j}(z)+O\left(z^{2 j+1}\right)
$$

where $P_{j}(z)$ and $Q_{j}(z)$ are polynomials of degree $j$, with $P_{j}(0)=1$. If they converge, we say that the series is summable, and the limit is called the Padé sum. Let us notice that in the case (2-9) the approximants are exact:

$$
\frac{Q_{j}(z)}{P_{j}(z)}=f(z), \quad z \neq-1,
$$

for all $j \geq 1$.

Another regular method of sum is the Borel one. 
For the series of Example 1, we call the Borel transform the entire function defined by the convergent series

$$
f^{B}(z)=\sum_{k=0}^{\infty} \frac{c_{k}}{k !} z^{k}=\sum_{k=0}^{\infty} \frac{1}{k !}(-z)^{k}=\exp (-z),
$$

and we get the sum of the original series by the integral

$$
f(z)=\int_{0}^{\infty} f^{B}(t z) \exp (-t) d t=\int_{0}^{\infty} \exp (-t(1+z)) d t
$$

on the half-plane $\mathfrak{R z}>-1$.

Example 2 (Euler function (1770)). We may take

$$
f(z)=\int_{0}^{\infty} \frac{1}{1+z t} \exp (-t) d t=\sum_{k=0}^{\infty} c_{k} z^{k}, \quad c_{k}=(-1)^{k} k ! .
$$

In this case, the power series converges nowhere, but we understand by inspection that it is the Borel sum of the Borel transform, $f^{B}(z)=(1+z)^{-1}$, on the half-plane $\Re z>0$.

Remark. In this case, the Borel transform is not entire, and the analytic continuation of the Borel transform on the half-axis is not automatic. In any case, the Borel summability does not directly give convergent approximants, but it is not difficult to find them by mapping the region of analyticity of the Borel transform on a disk containing the origin.

Criterion of Borel summability of a series to a function (Nevanlinna) [Caliceti et al. 1986]. Let us consider the formal power series $\sum_{n}^{\infty} c_{n} z^{n}$ and the function $f(z)$. If there exist $X, C_{N}>0$ such that

$$
C_{N}=O\left(A^{N} N !\right)
$$

and the function is analytic and

$$
\left|f(z)-\sum_{n}^{N-1} c_{n} z^{n}\right| \leq C_{N}|z|^{N}, \quad D_{X}=\{z:|z-X|<X\}
$$

uniformly for $N$ and $z$, then the function is uniquely defined as the Borel sum of the formal series.

Returning to the problem, is it possible for the Padé approximants to do better? Yes! Stieltjes (1894) proved the convergence of the Padé approximants on all of the cut plane $\mathbb{C}_{c}=\{z \in \mathbb{C}: z \neq 0,|\arg (z)|<\pi\}$. Our function can be written $f(z)=y g(y)=y \int_{0}^{\infty} \frac{1}{y+t} \exp (-t) d t=\int_{0}^{\infty} \frac{1}{1+t z} \exp (-t) d t=\sum_{j}(-1)^{j} c_{j} z^{j}$, 
where $y=1 / z$ and $g(y)$ is a Stieltjes function with the associated continued fraction (Laguerre) [Wall 1948]

$$
g(y)=1 /(y+1 /(1+1 /(y+2 /(1+2 /(y+3 /(\cdots)))))) .
$$

The approximants of the continued fraction are the Padé approximants of the series.

Definition. A Stieltjes function is a bounded analytic function on the cut plane, real for $z>0$, with $\overline{g(z)}=g(\bar{z})$, which satisfies the Herglotz property [Shohat and Tamarkin 1943, Lemma 2.2],

$$
\frac{\Im g(z)}{\Im z}<0, \quad \Im z \neq 0,
$$

vanishing at $\infty$. In this case, by the Stieltjes-Stone inversion formula, the nondecreasing measure $\mu(t)$ with $\mu(-\infty)=\mu(0)=0$ and $\mu(t)=(\mu(t-0)+\mu(t+0)) / 2$ is defined by

$$
\mu(b)-\mu(a)=\lim _{\epsilon \rightarrow 0}-\frac{1}{2 i \pi} \int_{a}^{b}(g(t+i \epsilon)-g(t-i \epsilon)) d t,
$$

for any $a$ and $b$ with $0 \leq a<b$, such that

$$
g(z)=\int_{0}^{\infty} \frac{1}{z+t} d \mu(t):=S\left(\frac{1}{z+t}\right) .
$$

Definition of the series of Hamburger (more general than the Stieltjes one) and the Hamburger moment problem. Let us consider the real sequence $c:=\left\{c_{n}\right\}_{n \in \mathbb{N}}$. There exists a nondecreasing function $\mu$, taking on infinitely many values in the interval $[-\infty, \infty)$, such that the sequence $c$ is the sequence of the moments of the measure $d \mu$ :

$$
c_{n}=\int_{-\infty}^{\infty} t^{n} d \mu(t):=S\left(t^{n}\right) \in \mathbb{R} \quad \text { for all } n \in \mathbb{N} .
$$

The existence of a measure is proved if and only if we have the positivity of the sequence of $n \times n$ matrices

$$
\left(A_{n}\right)_{j, k}=c_{j+k}
$$

so that the determinants

$$
D_{n}=\operatorname{det} A_{n}>0 \quad \text { for all } n \in \mathbb{N} \text {. }
$$

We can set $c_{0}=D_{0}=D_{-1}=1$. 
2e. The Stieltjes series and the Stieltjes moment problem. Let us consider the real sequence $c:=\left\{c_{n}\right\}_{n \in \mathbb{N}}$. There exists a nondecreasing function $\mu$, taking on infinitely many values in the interval $[0, \infty)$, such that the sequence $c$ is the sequence of the moments of the measure $d \mu$ :

$$
c_{n}=\int_{0}^{\infty} t^{n} d \mu(t):=S\left(t^{n}\right) \in \mathbb{R} \quad \text { for all } n \in \mathbb{N} .
$$

The existence is proved if and only if we have the positivity of the two sequences of $n \times n$ matrices

$$
\left(A_{n}\right)_{j, k}=c_{j+k}, \quad\left(B_{n}\right)_{j, k}=c_{j+k+1}
$$

so that we have

$$
D_{n}=\operatorname{det} A_{n}>0 \quad \text { for all } n \in \mathbb{N} .
$$

We can set $c_{0}=D_{0}=D_{-1}=1$.

2f. Definition of orthonormal polynomials. The orthonormal polynomials $P_{n}(t)$ for the measure $d \mu$ and the mean $S$,

$$
S\left(P_{n} P_{m}\right)=\delta_{m}^{n},
$$

have an explicit expression:

$$
P_{n}(t)=\frac{1}{\sqrt{D_{n-1} D_{n}}} \operatorname{det}\left(\begin{array}{cccc}
c_{0} & c_{1} & \cdots & c_{n} \\
c_{1} & c_{2} & \cdots & c_{n+1} \\
\vdots & \vdots & \ddots & \vdots \\
c_{n-1} & c_{n} & \cdots & c_{2 n-1} \\
1 & t & \cdots & t^{n}
\end{array}\right)=\frac{\sqrt{D_{n-1}}}{\sqrt{D_{n}}} t^{n}+R_{n-1}(t) .
$$

It is easy to prove that

$$
S\left(P_{n}(t) t^{m}\right)=\sqrt{\left(D_{n} / D_{n-1}\right)} \delta_{n}^{m}
$$

if $m \leq n$. Let us expand

$$
t P_{n}(t)=a_{n, n+1} P_{n+1}(t)+a_{n, n} P_{n}(t)+a_{n, n-1} P_{n-1}(t)+\cdots .
$$

Comparing the leading coefficients, we get

$$
a_{n, n+1}=\frac{\sqrt{D_{n-1} D_{n+1}}}{D_{n}} .
$$

Multiplying by $P_{m}(t)$ and taking the mean, we get

$$
a_{n, n-j}=S\left(t P_{n}(t) P_{n-j}(t)\right)=0 \quad \text { for all } j>1, \quad a_{n, n-1}=a_{n-1, n} .
$$


Setting $b_{n}=a_{n, n+1}$ and $a_{n}=a_{n, n}$, we get the difference equation

$$
t P_{n}(t)=b_{n} P_{n+1}(t)+a_{n} P_{n}(t)+b_{n-1} P_{n-1}(t), \quad n \in \mathbb{N},
$$

with the boundary conditions given by $b_{-1}=0$ and $P_{0}(t) \equiv 1$.

2g. The determination of the moment problem and the essential self-adjointness of an operator! The determination of the Stieltjes moment problem can be seen as the essential self-adjointness of a positive operator $B$ in $\mathscr{H}$. Let us consider a generating vector $e_{0}$ on the domain of all the operators $D=\bigcap_{n} B^{n}$ and the linear space $L=L\left(B, e_{0}\right)$ generated by the vectors $\left\{e_{n}^{\prime}:=B^{n} e_{0}\right\}_{n}$ and the Hilbert subspace $\mathscr{H}\left(B, e_{0}\right)$, the closure of $L\left(B, e_{0}\right)$ in $\mathscr{H} . \dot{A}$ is $B$ restricted to $L$. If $\dot{A}$ is essentially self-adjoint, we call $A$ its positive self-adjoint closure in $\mathscr{H}\left(A, e_{0}\right)$ with spectral function $E(x), x \geq 0$. In this case, the unique measure $\mu(x)$ is the mean value of the spectral function on the generating vector $e_{0},\left\langle e_{0}, E(x) e_{0}\right\rangle$, and the Stieltjes function given by the mean value of the resolvent for $-z \in \mathbb{C}_{c}$,

$$
\begin{aligned}
g(z) & =-\left\langle e_{0}, \frac{1}{A-z} e_{0}\right\rangle=\int_{0}^{\infty} \frac{1}{z-x} d \mu(x) \\
& =\frac{c_{0}}{z}+\frac{c_{1}}{z^{2}}+\cdots+\frac{c_{2 n-1}}{z^{2 n}}+O\left(1 / z^{2 n+1}\right),
\end{aligned}
$$

for $|z|$ large, is the Stieltjes sum of its asymptotic series. Let us consider the infinite real Jacobian matrix as the representation of the linear operator $\dot{A}$ on the canonical basis $\left\{e_{n}=P_{n}(\dot{A}) e_{0}\right\}_{n}$ so that

$$
\dot{A} e_{k}=b_{k-1} e_{k-1}+a_{k} e_{k}+b_{k} e_{k+1}, \quad b_{k}>0, k \in \mathbb{N}, b_{-1}=0 .
$$

The operator $\dot{A}$ is defined as a symmetric operator on the dense set of finite vectors, $g_{N}=\sum^{N} x_{k} e_{k}$. We define the adjoint operator $\dot{A}^{*}$ by the condition $g=\sum x_{k} e_{k} \in$ $D\left(\dot{A}^{*}\right)$ if there exists $g^{*}=\sum y_{k} e_{k}$ such that

$$
\left\langle\dot{A} e_{k}, g\right\rangle=\left\langle e_{k}, g^{*}\right\rangle \quad \text { for all } k \in \mathbb{N} \text { with } g^{*}:=\dot{A}^{*} g
$$

or

$$
b_{k-1} x_{k-1}+a_{k} x_{k}+b_{k} x_{k+1}=y_{k} \quad \text { for all } k \in \mathbb{N} .
$$

Thus, the vector $g \in D\left(\dot{A}^{*}\right)$ if $g^{*}$ is in the space $l^{2}$ :

$$
\sum_{k}\left|b_{k-1} x_{k-1}+a_{k} x_{k}+b_{k} x_{k+1}\right|^{2}<\infty .
$$

The deficiency index of the operator $\dot{A}$ is $(0,0)$ or $(1,1)$.

We look for complex eigenvalues of $\dot{A}^{*}$ :

$$
\begin{aligned}
\dot{A}^{*} g & =\sum\left(b_{k-1} x_{k-1}+a_{k} x_{k}+b_{k} x_{k+1}\right) e_{k} \\
& =\sum x_{k}\left(b_{k-1} e_{k-1}+a_{k} e_{k}+b_{k} e_{k+1}\right)=\lambda \sum x_{k} e_{k}:=\lambda g
\end{aligned}
$$


so that

$$
\lambda x_{k}=b_{k-1} x_{k-1}+a_{k} x_{k}+b_{k} x_{k+1}, \quad k \in \mathbb{Z}_{+},
$$

with boundary conditions $\lambda x_{0}=a_{0} x_{0}+b_{0} x_{1}$ or $x_{1}=\left(\lambda-a_{0}\right) x_{0} / b_{0}, x_{0}=c \neq 0$, so that $x_{k}=c P_{k}(\lambda)$. The same recurrence relation (2-12), with initial conditions $Q_{0}(\lambda)=0$ and $Q_{1}(\lambda)=1 / b_{0}$, defines the polynomials $Q_{k}(\lambda)$.

It is equivalent to consider the relation

$$
z Y_{k+1}(z)=\left(z-a_{k}\right) Y_{k}(z)-b_{k-1}^{2} Y_{k-1}(z), \quad k \in \mathbb{Z}_{+},
$$

with solutions $N_{k}$ and $M_{k}$ for boundary conditions $N_{0}=Q_{0}=0, N_{1}=1, M_{0}=$ $P_{0}=1$ and $M_{1}=z-a_{0}$ :

$$
\begin{aligned}
M_{k}(z) & =b_{0} b_{1} \cdots b_{k-1} P_{k}(z), \quad N_{k}(z)=b_{0} b_{1} \cdots b_{k-1} Q_{k}(z), \quad k>0, \\
g(z) & =1 /\left(z-a_{0}-b_{0}^{2} /\left(z-a_{1}-b_{1}^{2} /\left(z-a_{2}-b_{2}^{2} /\left(z-a_{3}-\cdots\right)\right)\right)\right) \\
& =\frac{Q_{k}(z)}{P_{k}(z)}+O\left(1 / z^{2 k+1}\right) .
\end{aligned}
$$

In the determined case the sequence of diagonal Padé approximants converge for $z \in \mathbb{C}_{c}$ to the function

$$
g(z)=\lim _{k \rightarrow \infty}\left(\frac{N_{k}(z)}{M_{k}(z)} \equiv \frac{Q_{k}(z)}{P_{k}(z)}\right) .
$$

Thus, we have deficiency index $(0,0)$ if and only if

$$
\sum_{k}\left|P_{k}(\lambda)\right|^{2}=\infty
$$

We have the analogue of the Liouville-Ostrogradskii formula for $k \in \mathbb{Z}_{+}$:

$b_{k-1} P_{k-1} Q_{k}-P_{k} b_{k-1} Q_{k-1}=-b_{k} P_{k+1} Q_{k}+P_{k} b_{k} Q_{k+1}=b_{0} P_{0} Q_{1}-P_{1} b_{0} Q_{0}=1$

or

$$
P_{k-1} Q_{k}-P_{k} Q_{k-1}=P_{k-1} Q_{k-1}^{\prime}-P_{k-1}^{\prime} Q_{k-1}=\frac{1}{b_{k-1}},
$$

where

$$
Q_{k-1}^{\prime}=Q_{k}-Q_{k-1}, \quad P_{k-1}^{\prime}=P_{k}-P_{k-1} .
$$

Theorem (Hellinger). Let us fix $z, n \in \mathbb{Z}_{+}$and

$$
w_{n}(t):=w_{n}(z, t)=-\frac{Q_{n}(z)-t Q_{n-1}(z)}{P_{n}(z)-t P_{n-1}(z)}, \quad \Im(z) \neq 0,
$$

where $w_{n}(z, \infty)=w_{n-1}(z, 0)$. The images of $w_{n}: \mathbb{R} \rightarrow \mathbb{C}$ are nested circles of $n$-decreasing radii

$$
r=r(n, z)=\frac{1}{|\Im z|} \frac{1}{\sum_{k=0}^{n-1}\left|P_{k}(z)\right|^{2}}
$$


In the case of zero deficiency index, we have the limit point case: $r(n, z) \rightarrow 0$, as $n \rightarrow \infty$, and $w_{n}(z, t)$ have a $t$-independent limit as $n \rightarrow \infty$.

Theorem (criterion). Let

$$
\sum_{n} \frac{1}{b_{n}}=\infty
$$

The J-matrix is determinate: we have a unique solution of the moment problem and convergence of the continued fraction.

Proof. Use the formula (2-15):

$$
\begin{aligned}
\sum_{k=1}^{n} \frac{1}{b_{k-1}} & =\left|\sum_{k=1}^{n}\left(P_{k-1} Q_{k}-P_{k} Q_{k-1}\right)(\lambda)\right| \\
& \leq 2 \sqrt{\sum_{k=0}^{n}\left|Q_{k}^{2}(\lambda)\right| \sum_{k=0}^{n}\left|P_{k}^{2}(\lambda)\right|}
\end{aligned}
$$

Theorem (Carleman criterion). If

$$
\sum_{n} \frac{1}{\sqrt[2 n]{c_{2 n}}}=\infty
$$

then the Hamburger moment problem

$$
c_{k}=\int_{-\infty}^{\infty} x^{k} d \mu(x)
$$

is determinate.

Proof. From (2-12) we have

$$
b_{0} b_{1} \cdots b_{n-1} P_{n}(\lambda)=\lambda^{n}+O\left(\lambda^{n-1}\right)
$$

for $\lambda$ large. Hence,

$$
b_{0} \cdots b_{n-1} S\left(P_{n}^{2}\right)=\int_{-\infty}^{\infty} x^{n} P_{n}(x) d \mu(x) \leq \sqrt{c_{2 n}} \sqrt{S\left(P_{n}^{2}\right)},
$$

or since $S\left(P_{n}^{2}\right)=1$,

$$
b_{0} \cdots b_{n-1} \leq \sqrt{c_{2 n}}
$$

and

$$
\infty=\sum_{n} \frac{1}{\sqrt[2 n]{c_{2 n}}} \leq \sum_{n} \frac{1}{\sqrt[n]{b_{0} \cdots b_{n-1}}}<e \sum_{n} \frac{1}{b_{n}}
$$

for a general inequality of Carleman. 
The determined Hamburger moment problem corresponds to a determined Stieltjes one:

$$
c_{n}^{\prime}=\int_{0}^{\infty} y^{n} d \mu^{\prime}(y)
$$

where $c_{n}^{\prime}=c_{2 n}, y=x^{2}$ and

$$
d \mu^{\prime}(y):=d \mu(\sqrt{y})+d \mu(-\sqrt{y})
$$

is the unique solution.

\section{References}

[Aguilar and Combes 1971] J. Aguilar and J. M. Combes, "A class of analytic perturbations for one-body Schrödinger Hamiltonians”, Comm. Math. Phys. 22:4 (1971), 269-279.

[Akhiezer 1965] N. I. Akhiezer, The classical moment problem and some related questions in analysis, Oliver \& Boyd, Edinburgh, 1965.

[Caliceti et al. 1986] E. Caliceti, V. Grecchi, and M. Maioli, "The distributional Borel summability and the large coupling $\Phi^{4}$ lattice fields", Comm. Math. Phys. 104:1 (1986), 163-174.

[Delabaere et al. 1997] E. Delabaere, H. Dillinger, and F. Pham, "Exact semiclassical expansions for one-dimensional quantum oscillators", J. Math. Phys. 38:12 (1997), 6126-6184.

[Fröman and Fröman 1965] N. Fröman and P. O. Fröman, JWKB approximation: contributions to the theory, North-Holland, Amsterdam, 1965.

[Giachetti and Grecchi 2016a] R. Giachetti and V. Grecchi, "Bender-Wu singularities", J. Math. Phys. 57:12 (2016), 122109.

[Giachetti and Grecchi 2016b] R. Giachetti and V. Grecchi, "Level crossings in a $P T$-symmetric double well”, J. Phys. A 49:10 (2016), 105202.

[Giller 2011] S. Giller, "The semiclassical small- $\hbar$ limit of loci of roots of subdominant solutions for polynomial potentials", J. Math. Phys. 52:6 (2011), 063514.

[Grandi 1703] G. Grandi, Quadratura circuli, et hyperbola per infinitas hyperbolas, et parabolas geometricè exhibita, Francisci Bindi, Pisa, 1703.

[Kato 1966] T. Kato, Perturbation theory for linear operators, Grundlehren math. Wissenschaften 132, Springer, Berlin, 1966.

[Loeffel et al. 1969] J. J. Loeffel, A. Martin, B. Simon, and A. S. Wightman, "Pade approximants and the anharmonic oscillator", Phys. Lett. B 30:9 (1969), 656-658.

[Mangione 1971] C. Mangione, "Logica e fondamenti della matematica", Chapter 6, in Storia del pensiero filosofico e scientifico, vol. III: Il settecento, edited by L. Geymonat, Garzanti, Milan, 1971.

[Mengoli 1650] P. Mengoli, Nova quadratura arithmetica, Iacobi Monti, Bologna, 1650.

[Plana 1832] J. Plana, Théorie du mouvement de la lune, Imprimerie Royale, Turin, 1832.

[Reed and Simon 1975] M. Reed and B. Simon, Methods of modern mathematical physics, II: Fourier analysis, self-adjointness, Academic Press, New York, 1975.

[Reed and Simon 1978] M. Reed and B. Simon, Methods of modern mathematical physics, IV: Analysis of operators, Academic Press, New York, 1978.

[Riccati 1761] J. Riccati, Opere, Jacopo Giusti, Lucca, Italy, 1761. 
[Shohat and Tamarkin 1943] J. A. Shohat and J. D. Tamarkin, The problem of moments, Math. Surveys 1, American Mathematical Society, Providence, RI, 1943.

[Sibuya 1975] Y. Sibuya, "Distribution of zeros", Chapter 7, pp. 152-165 in Global theory of a second order linear ordinary differential equation with a polynomial coefficient, Math. Studies 18, North-Holland, Amsterdam, 1975.

[Voros 1994] A. Voros, "Exact quantization condition for anharmonic oscillators (in one dimension)", J. Phys. A 27:13 (1994), 4653-4661.

[Wall 1948] H. S. Wall, Analytic theory of continued fractions, Van Nostrand, New York, 1948.

Received 29 Sep 2016. Accepted 31 Oct 2016.

VINCENZO GRECCHI: vincenzo.grecchi@unibo.it

Dipartimento di Matematica, Università di Bologna, I-40126 Bologna, Italy

and

Gruppo Nazionale di Fisica Matematica, Istituto Nazionale di Alta Matematica Francesco Severi, I-00185 Roma, Italy 


\title{
ON STOCHASTIC DISTRIBUTIONS AND CURRENTS
}

\author{
VinCENZO CAPASSO AND FRANCO FLANDOLI \\ Dedicated to Lucio Russo, on the occasion of his 70th birthday
}

\begin{abstract}
In many applications, it is of great importance to handle random closed sets of different (even though integer) Hausdorff dimensions, including local information about initial conditions and growth parameters. Following a standard approach in geometric measure theory, such sets may be described in terms of suitable measures. For a random closed set of lower dimension with respect to the environment space, the relevant measures induced by its realizations are singular with respect to the Lebesgue measure, and so their usual Radon-Nikodym derivatives are zero almost everywhere. In this paper, how to cope with these difficulties has been suggested by introducing random generalized densities (distributions) á la Dirac-Schwarz, for both the deterministic case and the stochastic case. For the last one, mean generalized densities are analyzed, and they have been related to densities of the expected values of the relevant measures. Actually, distributions are a subclass of the larger class of currents; in the usual Euclidean space of dimension $d$, currents of any order $k \in\{0,1, \ldots, d\}$ or $k$ currents may be introduced. In this paper, the cases of 0 -currents (distributions), 1-currents, and their stochastic counterparts are analyzed. Of particular interest in applications is the case in which a 1-current is associated with a path (curve). The existence of mean values has been discussed for currents too. In the case of 1-currents associated with random paths, two cases are of interest: when the path is differentiable, and also when it is the path of a Brownian motion or (more generally) of a diffusion. Differences between the two cases have been discussed, and nontrivial problems are mentioned which arise in the case of diffusions. Two significant applications to real problems have been presented too: tumor driven angiogenesis, and turbulence.
\end{abstract}

\section{Introduction: preliminaries and notation}

Many real phenomena may be modeled as random closed sets in $\mathbb{R}^{d}$, and in several situations as evolving random closed sets. Application areas include crystallization processes: Figures 1 and 2 (see [Capasso 2003; Capasso and Micheletti 2006], and

\section{Communicated by Raffaele Esposito.}

MSC2010: 28A75, 47B80, 60D05, 60H25, 46FXX, 52A22, 53C65.

Keywords: stochastic geometry, random distributions, random currents, mean geometric densities. 


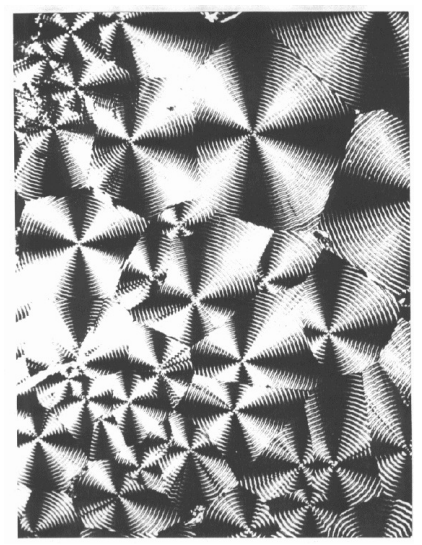

Figure 1. The final tessellation in a real experiment of a crystallization process of a polymer [MONTELL-Italy].

references therein; see also [Ubukata 2003] for the crystallization processes on sea shells, and [Callister Jr. 2007, p. 92; Hochrainer et al. 2007; Kassner et al. 2000] for dislocations: Figure 3); tumor growth [Anderson 2003]; angiogenesis: Figure 6 [Carmeliet and Jain 2000]; patterns in biology: Figure 4; the spread of a pollutant in an environment; etc.

All quoted processes may be described by time dependent random closed sets at different Hausdorff dimensions (for instance, crystallization processes are modeled in general by full dimensional growing sets and lower dimensional interfaces, while angiogenesis by systems of random curves). In many cases, because of the coupling with suitable underlying fields (such as temperature, nutrients, etc.), these kinds of phenomena may be modeled as space-time structured stochastic processes, whose geometric structure is of great relevance, as discussed in [Capasso et al. 2013].

A rigorous definition of the relevant geometric quantities in a stochastic setting of the above systems (fibers for angiogenesis, dislocations for crystalline materials, etc.) is very important for statistical applications (see, e.g., [Ambrosio et al. 2009; Camerlenghi et al. 2014]), and in mean field approximations (see, e.g., [Bonilla et al. 2017; Hochrainer et al. 2007; Bessaih et al. 2017]).

A presentation of an angiogenesis model will be offered later in Section 4.1.

For definitions and basic properties of Hausdorff measure and Hausdorff dimension see, e.g., [Ambrosio et al. 2000; Falconer 1986; Federer 1996; Morgan 1998].

We remind here the concepts and results of current literature which are relevant for our analysis. Actually, the subject of stochastic geometry, considered here in the direction of geometric measure theory, does have a nontrivial intersection with the literature on convex geometry for which the reader may refer to [Baddeley et al. 2007] and references therein. 


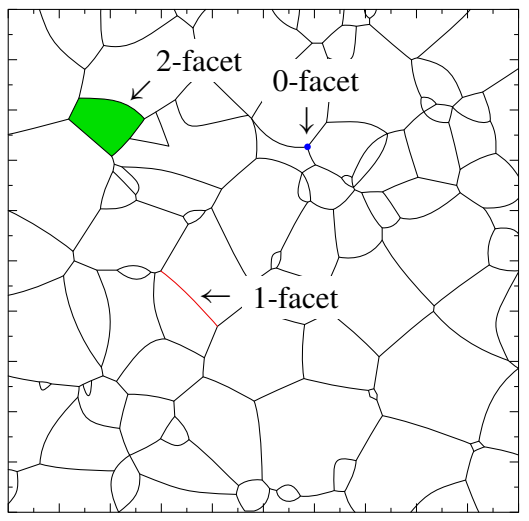

Figure 2. The final tessellation in a simulated experiment of a crystallization process of a polymer. This picture, together with the real one, shows the relevance of components at all integer Hausdorff dimensions for describing the final morphology [Burger et al. 2002].

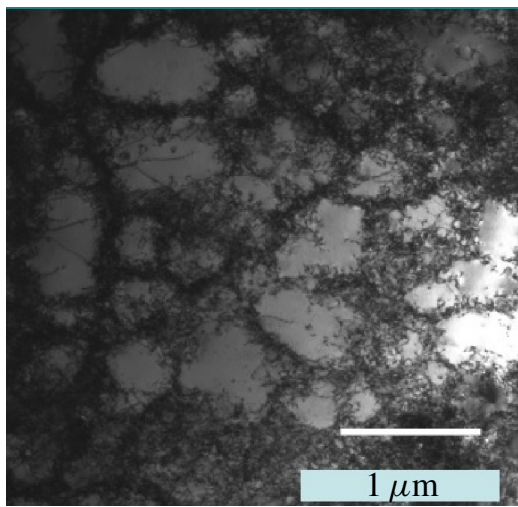

Figure 3. Dislocations in copper crystals [M. Kassner; private collection].

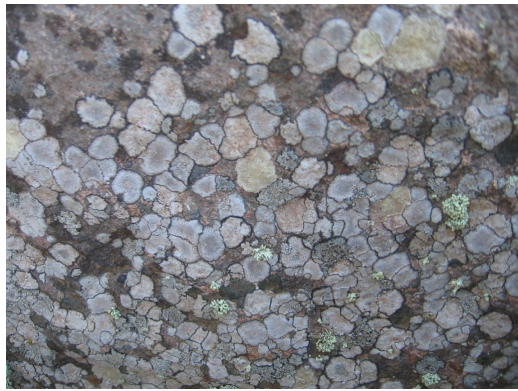

Figure 4. Pattern formation in a lichen colony [V. Capasso; private collection]. 


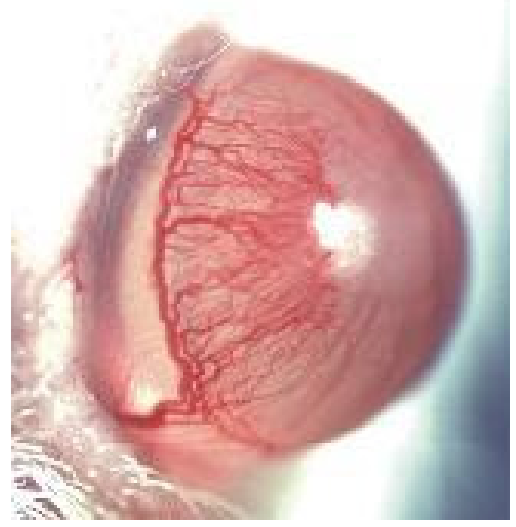

Figure 5. Angiogenesis on a rat cornea [Dejana, personal collection].

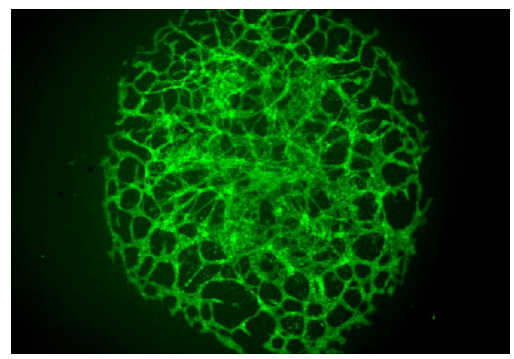

Figure 6. Vascularization of an allantoid. [Dejana, personal collection]. An important example of a fiber process of Hausdorff dimension 1 in a 3D space.

Let us consider the space $\mathbb{R}^{d}$ for $d>1$, and denote by $v^{d}$ the usual $d$-dimensional Lebesgue measure, and by $\mathcal{B}_{\mathbb{R}^{d}}$ the Borel $\sigma$-algebra of $\mathbb{R}^{d}$.

We know that every positive Radon measure $\mu$ on $\mathbb{R}^{d}$ can be represented as

$$
\mu=\mu_{\ll}+\mu_{\perp},
$$

where $\mu_{\ll}$ and $\mu_{\perp}$ are the absolutely continuous part with respect to $v^{d}$ and the singular part of $\mu$, respectively. We shall denote by $B_{r}(x)$ the $d$-dimensional closed ball centered in $x$ with radius $r$.

Let us denote by $\mathcal{H}^{s}$ the $s$-dimensional Hausdorff measure.

We will consider a class of subsets of $\mathbb{R}^{d}$ with integer Hausdorff dimension.

Definition 1. Given an integer $n \in[0, d]$, we say that a closed subset $A$ of $\mathbb{R}^{d}$ is $n$-regular if it satisfies the following conditions:

(i) $\mathcal{H}^{n}\left(A \cap B_{R}(0)\right)<\infty$ for any $R>0$; 
(ii) $\lim _{r \rightarrow 0} \frac{\mathcal{H}^{n}\left(A \cap B_{r}(x)\right)}{b_{n} r^{n}}=1$ for $\mathcal{H}^{n}$-a.e. $x \in A$.

We recall that when $n$ is integer, then $b(n)=b_{n}$, which is the volume of the unit ball in $\mathbb{R}^{n}$.

Note that condition (ii) is related to a characterization of the $\mathcal{H}^{n}$-rectifiability of the set $A$ [Falconer 1986, p.256, 267; Ambrosio et al. 2000, p.83].

Remark 2. We may observe that if $\Theta$ is an $n$-regular closed set in $\mathbb{R}^{d}$, we have

$$
\lim _{r \rightarrow 0} \frac{\mathcal{H}^{n}\left(\Theta \cap B_{r}(x)\right)}{b_{n} r^{n}}= \begin{cases}1 & \mathcal{H}^{n} \text {-a.e. } x \in \Theta \\ 0 & \text { for } x \notin \Theta .\end{cases}
$$

In fact, since $\Theta^{C}$ is open, for all $x \notin \Theta$ there exists $r_{0}>0$ such that for all $r \leq r_{0}$, we have $B_{r}(x) \subset \Theta^{C}$, that is $\mathcal{H}^{n}\left(\Theta \cap B_{r}(x)\right)=0$ for all $r \leq r_{0}$; thus the limit equals 0 for all $x \in \Theta^{C}$.

For a general set $A$, problems about " $\mathcal{H}^{n}$-a.e." and "for all" may arise when we consider a point $x \in \partial A$ where the boundary is not a regular manifold. For example, if $A$ is a closed square in $\mathbb{R}^{2}$, for all points $x$ on the edges,

$$
\lim _{r \rightarrow 0} \frac{\mathcal{H}^{2}\left(A \cap B_{r}(x)\right)}{b_{2} r^{2}}=\frac{1}{2},
$$

while for each of the four vertices the limit equals $\frac{1}{4}$.

Observe that in both cases the set of such points has $\mathcal{H}^{2}$-measure zero.

From now on we shall consider $n$-regular closed sets $\Theta$ in $\mathbb{R}^{d}$, with $0 \leq n \leq d$. As a consequence, for $n<d$ (by assuming $0 \cdot \infty=0$ ) by (1-1) we also have

$$
\lim _{r \rightarrow 0} \frac{\mathcal{H}^{n}\left(\Theta \cap B_{r}(x)\right)}{b_{d} r^{d}}=\lim _{r \rightarrow 0} \frac{\mathcal{H}^{n}\left(\Theta \cap B_{r}(x)\right)}{b_{n} r^{n}} \frac{b_{n} r^{n}}{b_{d} r^{d}}= \begin{cases}\infty & \mathcal{H}^{n} \text {-a.e. } x \in \Theta, \\ 0 & \text { for } x \notin \Theta .\end{cases}
$$

Note that in the particular case $n=0$, with $\Theta=X_{0}$ point in $\mathbb{R}^{d}\left(X_{0}\right.$ is indeed a 0 -regular closed set),

$$
\lim _{r \rightarrow 0} \frac{\mathcal{H}^{0}\left(X_{0} \cap B_{r}(x)\right)}{b_{d} r^{d}}= \begin{cases}\infty & \text { if } x=X_{0}, \\ 0 & \text { if } x \neq X_{0} .\end{cases}
$$

Note that if $\Theta$ is an $n$-regular closed set in $\mathbb{R}^{d}$ with $n<d$, then the Radon measure

$$
\mu_{\Theta}(\cdot):=\mathcal{H}^{n}(\Theta \cap \cdot)
$$

is a singular measure with respect to $v^{d}$.

It is then clear that the quantity

$$
\delta_{\Theta}(x):=\lim _{r \rightarrow 0} \frac{\mathcal{H}^{n}\left(\Theta \cap B_{r}(x)\right)}{b_{d} r^{d}}, \quad x \in \mathbb{R}^{d},
$$


associated with $\Theta$ cannot be considered as a classical function. But, analogously to the Dirac delta function $\delta_{X_{0}}(x)$ associated with a point $X_{0} \in \mathbb{R}^{d}$, we may refer to it as a generalized density (or the generalized Radon-Nikodym derivative of the measure $\mu_{\Theta}$ with respect to $v_{d}$ ), or better as a distribution á la Schwarz (see Section 2).

We may notice that in the case $\Theta=X_{0}$, the generalized density $\delta_{X_{0}}(x)$ coincides with the well-known Dirac delta function at a point $X_{0}$ that is the (generalized) density of the singular Dirac measure $\varepsilon_{X_{0}}$ [Kolmogorov and Fomin 1970].

The usefulness of introducing these generalized functions associated with sets of any dimension $n \in\{0, \ldots, d\}$, in particular in the stochastic case, has been discussed in various papers [Matheron 1965; Ambrosio et al. 2009; Burger et al. 2002; Capasso and Villa 2006; 2007; Vladimirov 1979].

The previous ideas extend to another framework, the one of currents. First, let us restrict ourselves to the so-called 1-currents, which heuristically are distributional generalizations of the concept of vector fields. In the smooth case, a 1-current in $\mathbb{R}^{d}$ is simply a smooth vector field $\xi: \mathbb{R}^{d} \rightarrow \mathbb{R}^{d}$. A smooth vector field $\xi(x)$ acts on test functions (above we have introduced objects using the language of measures, but here it is more natural to use directly the language of test functions) as

$$
(\xi, \theta)=\int_{\mathbb{R}^{d}}\langle\xi(x), \theta(x)\rangle \mathrm{d} x,
$$

for all smooth test vector fields $\theta: \mathbb{R}^{d} \rightarrow \mathbb{R}^{d}$. But, similarly to the case of measures concentrated on lower-dimensional subsets $\Theta$ of $\mathbb{R}^{d}$, we may consider vector fields concentrated on lower-dimensional subsets. Let us restrict further our discussion of 1-currents to the case of currents associated with curves. Given a smooth curve $\gamma:[a, b] \rightarrow \mathbb{R}^{d}$, the concept of current associates to it a distribution vector field concentrated along the curve, and having the direction tangent to the curve. The action on smooth test vector fields $\theta: \mathbb{R}^{d} \rightarrow \mathbb{R}^{d}$ is

$$
(\xi, \theta)=\int_{a}^{b}\left\langle\theta(\gamma(t)), \gamma^{\prime}(t)\right\rangle \mathrm{d} t,
$$

or, in the more intuitive language that we shall explain in the sequel,

$$
\xi(x)=\int_{a}^{b} \delta_{\gamma(t)}(x) \gamma^{\prime}(t) \mathrm{d} t,
$$

which emphasizes the fact that the vector field is localized on the curve, with the direction of the tangent.

In Section 2 we present densities of measures as linear functionals for both the deterministic and the stochastic case; special emphasis is given to random functionals associated with random closed sets and their mean values. Section 3 is devoted 
to 1-currents with their stochastic counterparts. Finally Section 4 presents examples of significant applications of real interest, such as tumor driven angiogenesis and turbulence.

\section{Densities as linear functionals}

2.1. The deterministic case. We know that the Dirac delta $\delta_{X_{0}}$ at a point $X_{0} \in \mathbb{R}^{d}$ can be defined as a linear functional associated with a finite Borel measure, the well-known Dirac measure $\varepsilon_{X_{0}}$, concentrated at $X_{0}$; as such it is the (generalized) density of $\varepsilon_{X_{0}}$. In fact, we recall that, according to the Riesz representation theorem (see, e.g., [Folland 1999, p. 212]), Radon measures in $\mathbb{R}^{d}$ (i.e., nonnegative and $\sigma$ additive set functions defined on the Borel $\sigma$-algebra $\mathcal{B}_{\mathbb{R}^{d}}$ that are finite on bounded sets) can be canonically identified with linear and order preserving functionals on $C_{c}\left(\mathbb{R}^{d}, \mathbb{R}\right)$, the space of continuous functions with compact support in $\mathbb{R}^{d}$. The identification is provided by the integral operator, i.e.,

$$
(\mu, f)=\int_{\mathbb{R}^{d}} f \mathrm{~d} \mu \quad \forall f \in C_{c}\left(\mathbb{R}^{d}, \mathbb{R}\right) .
$$

If $\mu \ll v^{d}$, it admits (as a Radon-Nikodym density) a classical function $\delta_{\mu}$ defined almost everywhere in $\mathbb{R}^{d}$, so that

$$
(\mu, f)=\int_{\mathbb{R}^{d}} f(x) \delta_{\mu}(x) \mathrm{d} x \quad \forall f \in C_{c}\left(\mathbb{R}^{d}, \mathbb{R}\right),
$$

in the usual sense of a Lebesgue integral.

If $\mu \perp v^{d}$, we may speak of a density $\delta_{\mu}$ only in the sense of distributions (formally, it is almost everywhere trivial, but it is $\infty$ on a set of $v^{d}$-measure zero). In this case, the symbol

$$
\int_{\mathbb{R}^{d}} f(x) \delta_{\mu}(x) \mathrm{d} x:=(\mu, f)
$$

can still be adopted, provided the integral on the left-hand side is understood in a generalized sense, and not as a Lebesgue integral.

In either case, from now on, we may denote by $\left(\delta_{\mu}, f\right)$ the quantity $(\mu, f)$. Accordingly, we say that a sequence of measures $\mu_{n}$ weakly* converges to a Radon measure $\mu$ if $\left(\delta_{\mu_{n}}, f\right)$ converges to $\left(\delta_{\mu}, f\right)$ for any $f \in C_{c}\left(\mathbb{R}^{d}, \mathbb{R}\right)$. A classical criterion (see, for instance, [Evans and Gariepy 1992, p. 54; Ambrosio et al. 2000]) states that $\mu_{n}$ weakly* converge to $\mu$ if and only if $\mu_{n}(A) \rightarrow \mu(A)$ for any bounded open set $A$ with $\mu(\partial A)=0$.

By the common integral representation for generalized functions,

$$
\int_{A} \delta_{X_{0}}(x) \mathrm{d} x:=\varepsilon_{X_{0}}(A)=\mathcal{H}^{0}\left(X_{0} \cap A\right),
$$


we have

$$
\left(\delta_{X_{0}}, f\right)=\int_{\mathbb{R}^{d}} \delta_{X_{0}}(x) f(x) \mathrm{d} x=f\left(X_{0}\right), \quad f \in C_{c}\left(\mathbb{R}^{d}, \mathbb{R}\right) .
$$

Now we are ready to introduce the delta function of an $n$-regular set $\Theta$ as the linear functional (the generalized function) $\delta_{\Theta}$ in a similar way.

Consider the measure defined on the Borel $\sigma$-algebra of $\mathbb{R}^{d}$, as follows:

$$
\mu_{\Theta}(A):=\mathcal{H}^{n}(\Theta \cap A), \quad A \in \mathcal{B}_{\mathbb{R}^{d}} .
$$

The linear functional associated with it is

$$
\left(\mu_{\Theta}, f\right):=\int_{\mathbb{R}^{d}} f(x) \mu_{\Theta} \mathrm{d} x
$$

for $f \in C_{c}\left(\mathbb{R}^{d}, \mathbb{R}\right)$.

In accordance with what we have said in the Introduction, the following holds.

Proposition 3. If $n$ is an integer strictly less than $d p$ the measure $\mu_{\Theta}$ is a singular measure with respect to the usual Lebesgue measure on $\mathbb{R}^{d}$.

In accordance with the usual representation of distributions in the theory of generalized functions, we formally write

$$
\int_{\mathbb{R}^{d}} f(x) \mu_{\Theta} \mathrm{d} x=\int_{\mathbb{R}^{d}} f(x) \delta_{\Theta}(x) \mathrm{d} x=:\left(\delta_{\Theta}, f\right) .
$$

Remark 4. We may notice that the classical Dirac delta $\delta_{X_{0}}(x)$ associated to a point $X_{0}$ is now a particular case corresponding to $n=0$. If $\Theta$ is a piecewise smooth surface $S$ in $\mathbb{R}^{d}$ (and so 2-regular), then by the definition in (2-2), it follows that for any test function $f$,

$$
\left(\delta_{S}, f\right)=\int_{S} f(x) \mathrm{d} S,
$$

which is the definition of $\delta_{S}$ in [Vladimirov 1979, p. 33].

In terms of the above arguments, we may state that $\delta_{\Theta}(x)$ is the (generalized) density of the measure $\mu_{\Theta}$, defined by (2-1), with respect to the usual Lebesgue measure $v^{d}$ on $\mathbb{R}^{d}$. Note that if $n=d$, then $\mu_{\Theta}$ is absolutely continuous with respect to $v^{d}$, so that $\delta_{\Theta}$ is its classical Radon-Nikodym derivative [Kolmogorov 1956].

\subsubsection{Paths: 1-dimensional sets.}

Definition 5. A path (or curve or line) in $\mathbb{R}^{d}$ is a continuous mapping $\gamma:[a, b] \subset$ $\mathbb{R} \rightarrow \mathbb{R}^{d}$. The point $\gamma(a)$ is called the initial point, and the point $\gamma(b)$ is called the final point. The image of the path $\gamma([a, b])$ is called the arc or the support of $\gamma$. A path $\gamma$ is closed if its end points coincide; it is simple if it has no multiple points (apart from possibly the end points for a closed path). 
If we denote by $e_{j}: j=1, \ldots, d$ the canonical basis of $\mathbb{R}^{d}$, we may introduce the components of the vector function $\gamma$ as

$$
\gamma_{j}=\left\langle e_{j}, \gamma\right\rangle:[a, b] \rightarrow \mathbb{R} .
$$

For $t \in(a, b)$, we put

$$
\gamma^{\prime}(t):=\lim _{h \rightarrow t ; h \in[a, b]} \frac{\gamma(h)-\gamma(t)}{h-t}
$$

whenever the limit exists. This is equivalent to state that all derivatives

$$
\gamma_{j}^{\prime}(t):=\lim _{h \rightarrow t ; h \in[a, b]} \frac{\gamma_{j}(h)-\gamma_{j}(t)}{h-t}, \quad j=1, \ldots, d,
$$

exist in $t$.

Definition 6. A path $\gamma:[a, b] \rightarrow \mathbb{R}^{d}$ is said to be smooth if it is a function of class $C^{1}([a, b])$ and $\gamma^{\prime}(t) \neq 0$ for any $t \in(a, b)$.

An additional piece of information related to the regularity of a path regards the evaluation of its arc length.

Definition 7. Let $\gamma:[a, b] \rightarrow \mathbb{R}^{d}$ be a path and let $\Pi$ the set of all finite partitions $\pi:=\left\{a=t_{1}<\cdots<t_{k}=b\right\}$ of the interval $[a, b]$. Denote by

$$
L(\gamma, \pi):=\sum_{i=1}^{k-1}\left\|\gamma\left(t_{i+1}\right)-\gamma\left(t_{i}\right)\right\| .
$$

The path $\gamma$ is said rectifiable or of bounded variation if

$$
L(\gamma):=\sup _{\pi \in \Pi} L(\gamma, \pi)<+\infty ;
$$

this quantity is referred to as the length of $\gamma$.

The following theorem holds [Galbis and Maestre 2012, p. 24].

Theorem 8. If $\gamma$ is a smooth curve whose domain is an interval $[a, b] \subset \mathbb{R}$, then it is rectifiable and its length is given by

$$
L(\gamma)=\int_{a}^{b}\left\|\gamma^{\prime}(t)\right\| \mathrm{d} t .
$$

Remark 9. The above theorem can be easily extended to a piecewise smooth path [Galbis and Maestre 2012, p. 26].

Example 10. The following are examples of smooth simple curves:

1. A segment in $\mathbb{R}^{d}$ with end points $x_{\text {ini }}$ and $x_{\text {fin }} \in \mathbb{R}^{d}$ :

$$
\Gamma=\left\{x_{\text {ini }} \cdot t+x_{\text {fin }} \cdot(1-t): t \in[0,1]\right\} .
$$


2. A circle in $\mathbb{R}^{2}$ :

$$
\Gamma=\{(\cos t, \sin t): t \in[0,2 \pi]\} .
$$

These sets are 1-regular, according to Definition 1.

If $\gamma:[a, b] \rightarrow \mathbb{R}^{d}$ is a smooth path, denote by $\Gamma:=\gamma([a, b]) \subset \mathbb{R}^{d}$ its support; we may associate to $\Gamma$ the Radon measure defined as

$$
\mu_{\Gamma}: A \in \mathcal{B}_{\mathbb{R}^{d}} \mapsto \mu_{\Gamma}(A):=\mathcal{H}^{1}(\Gamma \cap A) .
$$

The following theorem shows the action of the measure $\mu_{\Gamma}$ on test functions $f \in C_{c}\left(\mathbb{R}^{d}, \mathbb{R}^{d}\right)$ (see, e.g., [Evans and Gariepy 1992]).

Theorem 11. If $\gamma:[a, b] \rightarrow \mathbb{R}^{d}$ is a $C^{1}([a, b])$ simple curve such that $\Gamma=$ $\gamma([a, b])$, then for all $f \in C_{c}\left(\mathbb{R}^{d}, \mathbb{R}^{d}\right)$,

$$
\int f(x) \mu_{\Gamma} \mathrm{d} x=\int_{a}^{b} f(\gamma(t))\left\|\gamma^{\prime}(t)\right\| \mathrm{d} t .
$$

Similarly, for every $A \in \mathcal{B}\left(\mathbb{R}^{d}\right)$,

$$
\mu_{\Gamma}(A)=\int_{a}^{b} \epsilon_{\gamma(t)}(A)\left\|\gamma^{\prime}(t)\right\| \mathrm{d} t
$$

\subsection{The stochastic case.}

2.2.1. Random Radon measures. Consider the space $C_{c}\left(\mathbb{R}^{d}, \mathbb{R}\right)$ of continuous functions with compact support. This space is the union of the separable Banach spaces $C(K, \mathbb{R})$ over all compact sets $K \in \mathbb{R}^{d}$; let us write $\|\cdot\|_{K}$ for the norm on $C(K)$ :

$$
\|f\|_{K}=\sup _{x \in K}|f(x)| .
$$

The space $C_{c}\left(\mathbb{R}^{d}, \mathbb{R}\right)$ can be endowed with the direct limit topology associated to a family $C\left(K_{n}, \mathbb{R}\right)$ with $K_{n}$ increasing to $\mathbb{R}^{d}$; we do not need the details of this definition but we need to know the following useful fact: a linear functional $I: C_{c}\left(\mathbb{R}^{d}, \mathbb{R}\right) \rightarrow \mathbb{R}$ is continuous if and only if for every compact $K \in \mathbb{R}^{d}$ there is $C_{K}>0$ such that

$$
|I(f)| \leq C_{K}\|f\|_{K} \quad \text { for every } f \in C(K, \mathbb{R}) .
$$

Moreover, the dual of $C_{c}\left(\mathbb{R}^{d}, \mathbb{R}\right)$ is the space of signed Radon measures, which we shall denote by $\mathcal{M}$.

Definition 12. Given a probability space $(\Omega, \mathcal{F}, P)$, a random Radon measure is a map $\mu: \Omega \rightarrow \mathcal{M}$ such that for every $f \in C_{c}\left(\mathbb{R}^{d}, \mathbb{R}\right)$, the function $(\mu, f):=$ $\int_{\mathbb{R}^{d}} f(x) \mu \mathrm{d} x: \Omega \rightarrow \mathbb{R}$ is measurable. We shall write $\left(\mu_{\omega}, f\right)$ to emphasize the dependence on $\omega$ when $\mu$ is random. 
Proposition 13 (expected value of a random Radon measure). Let $\mu: \Omega \rightarrow \mathcal{M}$ be a random Radon measure. Assume that for every compact $K \in \mathbb{R}^{d}$ there is $C_{K}>0$ such that

$$
\int_{\Omega}\left|\left(\mu_{\omega}, f\right)\right| P \mathrm{~d} \omega \leq C_{K}\|f\|_{K},
$$

for every $f \in C(K, \mathbb{R})$. Then there exists an element $\hat{\mu} \in \mathcal{M}$ such that

$$
(\hat{\mu}, f)=\int_{\Omega}\left(\mu_{\omega}, f\right) P \mathrm{~d} \omega=\mathbb{E}[(\mu, f)],
$$

for every $f \in C_{c}\left(\mathbb{R}^{d}, \mathbb{R}\right)$.

Proof. Define the number $(\hat{\mu}, f)$ by the previous identity; for every $f \in C_{c}\left(\mathbb{R}^{d}, \mathbb{R}\right)$, since $f \in C(K, \mathbb{R})$ for some compact set $K$, we have $\int_{\Omega}\left|\left(\mu_{\omega}, f\right)\right| P \mathrm{~d} \omega<\infty$ by assumption, hence $\int_{\Omega}\left(\mu_{\omega}, f\right) P \mathrm{~d} \omega$ is well-defined.

Denote by $\Phi: f \in C_{c}\left(\mathbb{R}^{d}, \mathbb{R}\right) \mapsto \Phi(f):=(\mu, f) \in L^{1}(\Omega)$. We have to show that, as a function of $f$, the number $(\hat{\mu}, f)$ is linear continuous on $C_{c}\left(\mathbb{R}^{d}\right)$. Taking $f, g \in C_{c}\left(\mathbb{R}^{d}, \mathbb{R}\right)$ and $\alpha, \beta \in \mathbb{R}$, since $\Phi$ is linear, we have $\Phi_{\omega}(\alpha f+\beta g)=\alpha \Phi_{\omega}(f)+$ $\beta \Phi_{\omega}(g)$, hence

$$
\int_{\Omega} \Phi_{\omega}(\alpha f+\beta g) P \mathrm{~d} \omega=\alpha \int_{\Omega} \Phi_{\omega}(f) P \mathrm{~d} \omega+\beta \int_{\Omega} \Phi_{\omega}(g) P \mathrm{~d} \omega,
$$

which implies the linearity. For the continuity, for every compact set $K$ and $f \in$ $C(K, \mathbb{R})$, we have

$$
|(\hat{\mu}, f)| \leq \int_{\Omega}\left|\Phi_{\omega}(f)(\omega)\right| P \mathrm{~d} \omega \leq C_{K}\|f\|_{K}
$$

by assumption, hence $\hat{\mu}$ is a continuous functional.

Under the assumptions of Proposition 13, the measure $\hat{\mu} \in \mathcal{M}$, which satisfies the equality

$$
(\hat{\mu}, f)=\mathbb{E}[(\mu, f)]
$$

for every $f \in C_{c}\left(\mathbb{R}^{d}, \mathbb{R}\right)$ will be called the expected value of the measure $\mu$ and will be denoted by $\mathbb{E}[\mu]$.

2.2.2. Random linear functionals. Let $S$ be a separable linear metric space of test functions, for instance, $C_{c}\left(\mathbb{R}^{d}, \mathbb{R}\right)$ or $C_{c}^{\infty}\left(\mathbb{R}^{d}, \mathbb{R}\right)$ or $L^{2}(0, T)$. Denote by $S^{\prime}$ the space of continuous linear functionals on $S$. Let $(\Omega, \mathcal{F}, P)$ be a probability space. The following definition generalizes Definition 12.

Definition 14. We call random functional in the strict sense any map $\phi: \Omega \rightarrow S^{\prime}$ such that $(\phi, f)$ is measurable for every $f \in S$. 
Remark 15. The definition above is analogous to the well-known definition for Banach valued random variables (see, e.g., [Araujo and Giné 1980; Bharucha-Reid 1972; Bosq 2000]).

Later we will consider random functionals in a broad sense.

2.3. Random functionals associated with random closed sets. We recall that a random closed set $\Xi$ in $\mathbb{R}^{d}$ is a measurable map

$$
\Xi:(\Omega, \mathcal{F}, \mathbb{P}) \longrightarrow\left(\mathbb{F}, \sigma_{\mathbb{F}}\right),
$$

where $\mathbb{F}$ denotes the class of the closed subsets in $\mathbb{R}^{d}$, and $\sigma_{\mathbb{F}}$ is the $\sigma$-algebra generated by the so-called hit-or-miss topology (see [Matheron 1975]).

Definition 16. Given an integer $n$ with $0 \leq n \leq d$, we say that a random closed set $\Theta$ in $\mathbb{R}^{d}$ is $n$-regular if it satisfies the following conditions:

(i) for almost all $\omega \in \Omega$, the set $\Theta(\omega)$ is an $n$-regular closed set in $\mathbb{R}^{d}$; and

(ii) $\mathbb{E}\left[\mathcal{H}^{n}\left(\Theta \cap B_{R}(0)\right)\right]<\infty$ for any $R>0$.

Suppose now that $\Theta$ is a random $n$-regular closed set in $\mathbb{R}^{d}$. Thanks to the assumptions on the random set $\Theta$, it can be shown that the random measure $\mu_{\Theta}$ is $P$-a.s. a Radon measure on $\mathcal{B}_{\mathbb{R}^{d}}$; it can be further shown that, as a map $\mu_{\Theta}: \omega \in$ $\Omega \mapsto \mu_{\Theta(\omega)} \in \mathcal{M}$, it is a random Radon measure according to Definition 12 (see, e.g., [Baddeley and Molchanov 1997; Matheron 1965; Zähle 1982]).

In this case it makes sense to define, for any $\omega \in \Omega$, the linear functional $\delta_{\Theta(\omega)}$ such that, for any $f \in C_{c}\left(\mathbb{R}^{d}, \mathbb{R}\right)$ :

$$
\left(\delta_{\Theta}, f\right):=\int_{\mathbb{R}^{d}} f(x) \mu_{\Theta}(x) \mathrm{d} x, \quad P \text {-a.s. }
$$

According to Definition $14, \delta_{\Theta}$ is then a random linear functional in the strict sense.

By recollecting all of the above, we may finally state the following.

Proposition 17. Let $\Theta$ be a random closed n-regular set in $\mathbb{R}^{d}$. Then the associated random Radon measure $\mu_{\Theta}$ satisfies the assumption of Proposition 13, and therefore the expected value $\mathbb{E}\left[\mu_{\Theta}\right]$ is well-defined. We therefore define the expected value of the generalized density $\delta_{\Theta}$ by the following identities:

$$
\begin{aligned}
\left(\mathbb{E}\left[\delta_{\Theta}\right], f\right) & =\int_{\mathbb{R}^{d}} f(x) \mathbb{E}\left[\delta_{\Theta}\right](x) \mathrm{d} x \\
& :=\int_{\mathbb{R}^{d}} f(x) \mathbb{E}\left[\mu_{\Theta}\right] \mathrm{d} x=\mathbb{E}\left[\int_{\mathbb{R}^{d}} f(x) \mu_{\Theta}(x) \mathrm{d} x\right] \\
& =\mathbb{E}\left[\int_{\mathbb{R}^{d}} f(x) \delta_{\Theta} \mathrm{d} x\right]=\mathbb{E}\left[\left(\delta_{\Theta}, f\right)\right],
\end{aligned}
$$

for any $f \in C_{c}\left(\mathbb{R}^{d}, \mathbb{R}\right)$. 
Proof. Let $K$ be a compact in $\mathbb{R}^{d}$ and $f \in C_{c}(K, \mathbb{R})$. We have

$$
\begin{aligned}
\int_{\Omega}\left|\left(\mu_{\Theta(\omega)}, f\right)\right| P \mathrm{~d} \omega & =\int_{\Omega}\left|\int_{\mathbb{R}^{d}} f(x) \mu_{\Theta(\omega)} \mathrm{d} x\right| P \mathrm{~d} \omega \\
& \leq \int_{\Omega} \int_{\mathbb{R}^{d}}|f(x)| \mu_{\Theta(\omega)} \mathrm{d} x P \mathrm{~d} \omega \\
& =\int_{\Omega} \int_{K}|f(x)| \mu_{\Theta(\omega)} \mathrm{d} x P \mathrm{~d} \omega,
\end{aligned}
$$

because $f$ has support in $K$, and

$$
\begin{aligned}
\int_{\Omega}\left|\left(\mu_{\Theta(\omega)}, f\right)\right| P \mathrm{~d} \omega & \leq\|f\|_{K} \int_{\Omega} \int_{K} \mu_{\Theta(\omega)} \mathrm{d} x P \mathrm{~d} \omega \\
& =\|f\|_{K} \int_{\Omega} \mu_{\Theta(\omega)}(K) P \mathrm{~d} \omega \\
& =\|f\|_{K} \mathbb{E}\left[\mathcal{H}^{n}(\Theta \cap K)\right] \\
& \leq C_{K}\|f\|_{K},
\end{aligned}
$$

by (ii) of the previous definition (the set $K$ is included in a ball $B_{R}(0)$ ). Hence we may apply Proposition 13 for the existence of the expected value $\mathbb{E}\left[\mu_{\Theta}\right]$.

2.3.1. Absolutely continuous (in mean) random sets.

Remark 18. When $n=d$, the integral and expectation in (2-9) can be exchanged by Fubini's theorem, since in this case both $\mu_{\Theta}$ and $\mathbb{E}\left[\mu_{\Theta}\right]$ are absolutely continuous with respect to $v^{d}$ and $\delta_{\Theta}(x)=\mathbb{1}_{\Theta}(x), v^{d}$-a.s.

In particular, $\delta_{\Theta}(x)=\mathbb{1}_{\Theta}(x), v^{d}$-a.s. implies that

$$
\mathbb{E}\left[\delta_{\Theta}\right](x)=\mathbb{P}(x \in \Theta), \quad v^{d} \text {-a.s. }
$$

and the following chain of equalities are well-known (according to our definition of $\mathbb{E}\left[\delta_{\Theta}\right][$ Kolmogorov 1956, p. 46]):

$$
\begin{aligned}
\mathbb{E}\left[v^{d}(\Theta \cap A)\right] & =\mathbb{E}\left(\int_{\mathbb{R}^{d}} \mathbf{1}_{\Theta \cap A}(x) \mathrm{d} x\right)=\mathbb{E}\left(\int_{A} \mathbf{1}_{\Theta}(x) \mathrm{d} x\right) \\
& =\int_{A} \mathbb{E}\left(\mathbf{1}_{\Theta}(x)\right) \mathrm{d} x=\int_{A} \mathbb{P}(x \in \Theta) \mathrm{d} x .
\end{aligned}
$$

In materials science, the density

$$
\rho(x):=\mathbb{E}\left[\delta_{\Theta}\right](x)=\mathbb{P}(x \in \Theta)
$$

is known as the (degree of) crystallinity.

If $\Theta$ is not a pathological set, i.e., if $\mathcal{H}^{n}(\Theta)(\omega)>0$ with $\mathbb{P}$-a.e. for $\omega \in \Omega(n<d)$, we may notice that, even though for a.e. realization $\Theta$ of $\Theta$ the measure $\mu_{\Theta}$ is 
positive and singular (and so it is not absolutely continuous), and the expected measure $\mathbb{E}\left[\mu_{\Theta}\right]$ may be absolutely continuous with respect to $v^{d}$.

Example. Consider the case $n=0$. Let $\Theta=X_{0}$ be a random point in $\mathbb{R}^{d}$; in this case, $\mathcal{H}^{0}\left(X_{0} \cap A\right)=\mathbb{1}_{A}\left(X_{0}\right)$, and so

$$
\mathbb{E}\left[\mathcal{H}^{0}\left(X_{0} \cap A\right)\right]=\mathbb{P}\left(X_{0} \in A\right) .
$$

If $X_{0}$ is a continuous random point with the pdf $p_{X_{0}}$, then $\mathbb{E}\left[\mathcal{H}^{0}\left(X_{0} \cap \cdot\right)\right]$ is absolutely continuous and, in this case, $\mathbb{E}\left[\delta_{X_{0}}\right](x)$ is just the probability density function $p_{X_{0}}(x)$, so $\int_{A} \mathbb{E}\left[\delta_{X_{0}}\right](x) v^{d} \mathrm{~d} x$ is the usual Lebesgue integral. Note that we formally have

$$
\mathbb{E}\left[\delta_{X_{0}}\right](x)=\int_{\mathbb{R}^{d}} \delta_{y}(x) p_{X_{0}}(y) v^{d} \mathrm{~d} y=\int_{\mathbb{R}^{d}} \delta_{x}(y) p_{X_{0}}(y) v^{d} \mathrm{~d} y=p_{X_{0}}(x) ;
$$

and in accordance with Proposition 17,

$$
\begin{aligned}
\int_{A} \mathbb{E}\left[\delta_{X_{0}}\right](x) v^{d} \mathrm{~d} x & =\int_{A} p_{X_{0}}(x) v^{d} \mathrm{~d} x=\mathbb{P}\left(X_{0} \in A\right) \\
& =\mathbb{E}\left[\mathcal{H}^{0}\left(X_{0} \cap A\right)\right]=\mathbb{E}\left[\int_{A} \delta_{X_{0}}(x) v^{d} \mathrm{~d} x\right] .
\end{aligned}
$$

If $X_{0}$ is discrete, i.e., $X_{0}=x_{i}$ with probability $p_{i}$, only for an at most countable set of points $x_{i} \in \mathbb{R}^{d}$, then $\mathbb{E}\left[\mathcal{H}^{0}\left(X_{0} \cap \cdot\right)\right]$ is singular and, as in the previous case, we have that $\mathbb{E}\left[\delta_{X_{0}}\right](x)$ coincides with the probability distribution $p_{X_{0}}$ of $X_{0}$.

In fact, in this case $p_{X_{0}}(x)=\sum_{i} p_{i} \delta_{x_{i}}(x)$, and by computing the expectation of $\delta_{X_{0}}$, we formally obtain

$$
\mathbb{E}\left[\delta_{X_{0}}\right](x)=\delta_{x_{1}}(x) p_{1}+\delta_{x_{2}}(x) p_{2}+\cdots=\sum_{i} p_{i} \delta_{x_{i}}(x)=p_{X_{0}}(x) .
$$

Remark 19. By Remark 18 and the considerations in the above example, we may claim that, in the cases $n=d$ and $n=0$ with $X_{0}$ being continuous, the expected linear functionals $\mathbb{E}\left[\delta_{\Theta}\right]$ and $\mathbb{E}\left[\delta_{X_{0}}\right]$ are defined by the function $\rho(x):=\mathbb{P}(x \in \Theta)$ and by the pdf $p_{X_{0}}$ of $X_{0}$, respectively, in the following way:

$$
\left(\mathbb{E}\left[\delta_{\Theta}\right], f\right):=\int_{\mathbb{R}^{d}} f(x) \rho(x) \mathrm{d} x
$$

and

$$
\left(\mathbb{E}\left[\delta_{X_{0}}\right], f\right):=\int_{\mathbb{R}^{d}} f(x) p_{X_{0}}(x) \mathrm{d} x .
$$

In fact, let us consider the random point $X_{0}$; in accordance with Proposition 17,

$$
\left(\mathbb{E}\left[\delta_{X_{0}}\right], f\right):=\int_{\mathbb{R}^{d}} f(x) p_{X_{0}}(x) \mathrm{d} x=\mathbb{E}\left[f\left(X_{0}\right)\right]=\mathbb{E}\left[\left(\delta_{X_{0}}, f\right)\right] .
$$


Of particular interest is the case of fiber processes, i.e., 1-dimensional random sets that occur in medicine as models for vessels, nerves, etc., and in materials science as models for line dislocations (defects in a crystalline material) [Hochrainer et al. 2007], etc. In these cases, an empirical definition of line density is given as the total length of dislocation lines per unit volume (see, e.g., [Callister Jr. 2007, p. 177]). This has given a strong motivation for a rigorous definition of the mean density of random sets of any Hausdorff dimension; for a discussion about continuity and absolute continuity of random closed sets, we refer to [Capasso and Villa 2006; 2007].

2.3.2. Example: an absolutely continuous 1-regular random set. Let $\left(X_{t}, V_{t}\right)$ be the solution of the stochastic differential system in $\mathbb{R}^{d}$ :

$$
\begin{aligned}
\mathrm{d} X_{t} & =V_{t} \mathrm{~d} t, \\
\mathrm{~d} V_{t} & =b\left(X_{t}, V_{t}\right) \mathrm{d} t+\mathrm{d} W_{t},
\end{aligned}
$$

where $b$ is Lipschitz continuous and $W_{t}$ is a Brownian motion in $\mathbb{R}^{d}$. If the initial condition $\left(X_{0}, V_{0}\right)$ of the above system has a smooth density $p_{0}(x, v)$ with respect to the usual Lebesgue measure on $\mathbb{R}^{d}$, thanks to hypoellipticity, $\left(X_{t}, V_{t}\right)$ has (for every $t>0)$ a smooth density $p_{t}(x, v)$ with respect to the Lebesgue measure; it is a solution to the Fokker-Planck equation:

$$
\begin{aligned}
\frac{\partial p}{\partial t} & =\Delta_{v} p-v \cdot \nabla_{x} p-\operatorname{div}_{v}(b p), \\
\left.p\right|_{t=0} & =p_{0} .
\end{aligned}
$$

Given $T>0$, consider the random path in $\mathbb{R}^{d}$ given by $t \in[0, T] \mapsto X_{t} \in \mathbb{R}^{d}$ (not necessarily simple); in accordance with Theorem 11, the associated random Radon measure $\mu_{X}$ is such that

$$
\int f(x) \mu_{X} \mathrm{~d} x=\int_{0}^{T} f\left(X_{t}\right)\left\|V_{t}\right\| \mathrm{d} t, \quad f \in C_{c}\left(\mathbb{R}^{d}, \mathbb{R}\right) .
$$

This formula defines a random generalized density $\delta_{X}(x)$ by the identity

$$
\left(\delta_{X}, f\right)=\int_{0}^{T} f\left(X_{t}\right)\left\|V_{t}\right\| \mathrm{d} t, \quad f \in C_{c}\left(\mathbb{R}^{d}, \mathbb{R}\right) .
$$

It can be checked that we have

$$
\delta_{X}(x)=\int_{0}^{T} \delta_{X_{t}}(x)\left\|V_{t}\right\| \mathrm{d} t=\int_{0}^{T} \delta_{X_{t}}(x)\left\|X_{t}^{\prime}\right\| \mathrm{d} t .
$$

Proposition 20. Assume $\mathbb{E}\left[\left\|X_{0}\right\|+\left\|V_{0}\right\|\right]<\infty$. Then the random generalized density $\delta_{X}$, which is a.s. concentrated on the random curve $X$, has a smooth average 
density $\mathbb{E}\left[\delta_{X}\right](x)$ given by

$$
\mathbb{E}\left[\delta_{X}\right](x)=\int_{0}^{T} \int_{\mathbb{R}^{d}}\|v\| p_{t}(x, v) \mathrm{d} v \mathrm{~d} t .
$$

Proof. We only sketch the proof. We have, with $\Theta_{1}$ equal to the support of the curve $X$,

$$
\begin{aligned}
\mathbb{E}\left[\mathcal{H}^{1}\left(\Theta_{1} \cap B_{R}(0)\right)\right] & =\mathbb{E}\left[\int_{0}^{T} 1_{B_{R}(0)}\left(X_{t}\right)\left\|V_{t}\right\| \mathrm{d} t\right] \\
& \leq \mathbb{E}\left[\int_{0}^{T}\left\|V_{t}\right\| \mathrm{d} t\right]=\int_{0}^{T} \mathbb{E}\left[\left\|V_{t}\right\|\right] \mathrm{d} t .
\end{aligned}
$$

We continue the proof under the additional assumption that $b$ is bounded, otherwise it is sufficient to use a Gronwall-type argument (it is here that we use $\mathbb{E}\left[\left\|X_{0}\right\|\right]<\infty$ ). We have

$$
V_{t}=V_{0}+\int_{0}^{t} b\left(X_{s}, V_{s}\right) \mathrm{d} s+W_{t}
$$

hence

$$
\left\|V_{t}\right\| \leq\left\|V_{0}\right\|+T\|b\|_{\infty}+\left\|W_{t}\right\|
$$

which implies $\sup _{t \in[0, T]} \mathbb{E}\left[\left\|V_{t}\right\|\right]<\infty$ (because $\mathbb{E}\left[\left\|V_{0}\right\|\right]<\infty$ ), hence

$$
\int_{0}^{T} \mathbb{E}\left[\left\|V_{t}\right\|\right] \mathrm{d} t<\infty
$$

and therefore $\mathbb{E}\left[\mathcal{H}^{1}\left(\Theta_{1} \cap B_{R}(0)\right)\right]<\infty$. By the general criterion above, this implies that $\mathbb{E}\left[\delta_{X}\right]$ exists and

$$
\left(\mathbb{E}\left[\delta_{X}\right], f\right)=\int_{0}^{T} \mathbb{E}\left[f\left(X_{t}\right)\left\|V_{t}\right\|\right] \mathrm{d} t .
$$

It follows that

$$
\begin{aligned}
\left(\mathbb{E}\left[\delta_{X}\right], f\right) & =\int_{0}^{T} \int_{\mathbb{R}^{d}} \int_{\mathbb{R}^{d}} f(x)\|v\| p_{t}(x, v) \mathrm{d} x \mathrm{~d} v \mathrm{~d} t \\
& =\int_{\mathbb{R}^{d}}\left(\int_{0}^{T} \int_{\mathbb{R}^{d}}\|v\| p_{t}(x, v) \mathrm{d} v \mathrm{~d} t\right) f(x) \mathrm{d} x .
\end{aligned}
$$

The arbitrarity of $f$ gives us the existence of the average density $\mathbb{E}\left[\delta_{X}\right](x)$ and its formula.

Hence we may claim that the path $X$ is a 1-dimensional absolutely continuous (in mean) random set in $\mathbb{R}^{d}$. 
2.4. More on random functionals. Given a probability space $(\Omega, \mathcal{F}, P)$, we denote by $L^{0}(\Omega)$ the space of $P$-equivalence classes of random variables $X: \Omega \rightarrow \mathbb{R}$, endowed with the Ky Fan topology, i.e., the topology of the convergence in probability.

In the previous sections, we defined as random functionals all measurable mappings from $\Omega$ to the dual space $C_{c}\left(\mathbb{R}^{d}, \mathbb{R}\right)^{\prime}$ (we continue to restrict ourselves to functionals on $C_{c}\left(\mathbb{R}^{d}, \mathbb{R}\right)$ ). However, there exist examples of random objects which are natural to call random functionals, but do not enter in the previous definition. Let us then introduce a broader concept.

Definition 21. We use random functional in the broad sense of any continuous linear map $\Phi: C_{c}\left(\mathbb{R}^{d}, \mathbb{R}\right) \rightarrow L^{0}(\Omega)$. When there exists a random functional in the strict sense $\phi: \Omega \rightarrow C_{c}\left(\mathbb{R}^{d}, \mathbb{R}\right)^{\prime}$ such that

$$
\Phi(f)(\omega)=\left(\phi_{\omega}, f\right)
$$

for all $f \in C_{c}\left(\mathbb{R}^{d}, \mathbb{R}\right)$ and for a.a. $\omega \in \Omega$, we say that $\phi_{\omega}$ is a pathwise realization of $\Phi$.

Example 22. Let $W$ be a Brownian motion on $(\Omega, \mathcal{F}, P)$ and let $d=1$. To any function $f \in C_{c}(\mathbb{R}, \mathbb{R})$, we associate the random variable

$$
\Phi(f)=\int_{0}^{T} f\left(W_{t}\right) \mathrm{d} W_{t} .
$$

It is known that there is no simple pathwise meaning for this integral: we cannot fix $\omega \in \Omega$ and consider it as a map from $C_{c}(\mathbb{R}, \mathbb{R})$ to $\mathbb{R}$. This is an example of a random functional in the broad sense which has no pathwise realization.

Example 23. Referring to the above example, a pathwise realization exists if we restrict ourselves to more regular test functions $f$. Indeed, if $f \in C_{c}^{1}(\mathbb{R}, \mathbb{R})$, and $F^{\prime}=f$, by Itô's formula we have

$$
F\left(W_{T}\right)=F\left(W_{0}\right)+\int_{0}^{T} f\left(W_{t}\right) \mathrm{d} W_{t}+\frac{1}{2} \int_{0}^{T} f^{\prime}\left(W_{t}\right) \mathrm{d} t,
$$

and therefore the random functional in the strict sense $\phi_{\omega}$, defined as

$$
\left(\phi_{\omega}, f\right)=F\left(W_{T}(\omega)\right)-F\left(W_{0}(\omega)\right)-\frac{1}{2} \int_{0}^{T} f^{\prime}\left(W_{t}(\omega)\right) \mathrm{d} t
$$

(these expressions are all well-defined path by path) satisfies

$$
(\phi ., f)=\int_{0}^{T} f\left(W_{t}\right) \mathrm{d} W_{t}=\Phi(f), \quad P \text {-a.s. }
$$

hence $\phi_{\omega}$ is a pathwise realization of $\Phi$; but only on $f \in C_{c}^{1}(\mathbb{R}, \mathbb{R})$. 
Example 24. If we allow ourselves to use $L^{2}(0, T)$ as a set of test functions in place of $C_{c}\left(\mathbb{R}^{d}, \mathbb{R}\right)$, the classical Wiener integral

$$
\Phi(f)=\int_{0}^{T} f_{t} \mathrm{~d} W_{t}
$$

is another example of random functional in the broad sense which has no pathwise realization. Again, if we change the set of test functions and we take $f \in$ $W^{1,2}(0, T)$, the pathwise realization exists, given by

$$
\left(\phi_{\omega}, f\right)=f_{T} W_{T}-\int_{0}^{T} f_{t}^{\prime} W_{t} \mathrm{~d} t .
$$

The previous examples have mainly an academic character, so we do not insist on this notion here. However, in the context of random currents, there are examples of great potential interest for applications, so we shall enter in more detail.

\section{Currents}

In this section we wish to extend our analysis to vector fields in $\mathbb{R}^{d}$, hence to random currents. Motivations for the study of random currents are the same as anticipated in Section 2.3 for 1-dimensional random sets, i.e., fiber processes.

We will start by defining line integrals of differential forms (see, e.g., [Giaquinta et al. 1998; Giaquinta and Modica 2009; Galbis and Maestre 2012, p. 21; Buck 1956]).

3.1. Differential forms. Let $g: U \subset \mathbb{R}^{d} \rightarrow \mathbb{R}$ be a real function of class $C^{1}$ in the open set $U$, i.e., it has all partial derivatives of the first order continuous in $U$. The differential of $g$ at a point $x \in U$ is the linear function $\operatorname{dg}(x): \mathbb{R}^{d} \rightarrow \mathbb{R}$ such that

$$
h=\left(h_{1}, \ldots, h_{d}\right) \in \mathbb{R}^{d} \mapsto \operatorname{dg}(x)(h)=\sum_{j=1}^{d} \frac{\partial g}{\partial x_{j}}(x) h_{j} .
$$

It is usually suggestive to denote the partial increments $h_{j}=\left\langle e_{j}, h\right\rangle$ by $\mathrm{d} x_{j}$, so that the expression (3-1) is usually written as

$$
\mathrm{d} x=\left(\mathrm{d} x_{1}, \ldots, \mathrm{d} x_{d}\right) \in \mathbb{R}^{d} \mapsto \operatorname{dg}(x)(\mathrm{d} x)=\sum_{j=1}^{d} \frac{\partial g}{\partial x_{j}}(x) \mathrm{d} x_{j} .
$$

Expressions (3-1) or (3-2) are a particular case of a more general mathematical entity called differential form of degree one, or simply 1-form, defined below.

Definition 25. Let $U \subset \mathbb{R}^{d}$ be an open set. A differential form of degree one or simply a 1 -form on $U$ is a mapping

$$
\omega: U \rightarrow \mathcal{L}\left(\mathbb{R}^{d}, \mathbb{R}\right)=\left(\mathbb{R}^{d}\right)^{\prime},
$$


where $\mathcal{L}\left(\mathbb{R}^{d}, \mathbb{R}\right)$ denotes the space of all real valued linear functions on $\mathbb{R}^{d}$, i.e., the dual space $\left(\mathbb{R}^{d}\right)^{\prime}$ of $\mathbb{R}^{d}$.

Given a 1 -form $\omega$, a point $x \in U$, and an integer $j=1, \ldots, d$, we will denote the scalar $\omega(x)\left(e_{j}\right) \in \mathbb{R}$ by $f_{j}(x)$. Evidently $f_{j}: U \rightarrow \mathbb{R}$; by the linearity of $\omega(x) \in\left(\mathbb{R}^{d}\right)^{\prime}$, for any $h=\left(h_{1}, \ldots, h_{d}\right) \in \mathbb{R}^{d}$ we have

$$
\omega(x)(h)=\sum_{j=1}^{d} f_{j}(x) h_{j},
$$

or

$$
\omega(x)(\mathrm{d} x)=\sum_{j=1}^{d} f_{j}(x) \mathrm{d} x_{j} .
$$

This expression is usually abbreviated into

$$
\omega=\sum_{j=1}^{d} f_{j} \mathrm{~d} x_{j} .
$$

The functions $f_{j}$, where $j=1, \ldots d$, are called the component functions of the differential form $\omega$. We will usually assume that the component functions of a 1 -form are continuous; in such a case we say that the form is continuous.

Remark 26. The above discussion shows that the study of a 1-form is essentially equivalent to the study of a vector field, say

$$
F(x)=\left(f_{1}(x), \ldots, f_{d}(x)\right) ; \quad x \in U .
$$

3.2. Line integrals of differential forms. The line integral of a differential form was originally motivated by the calculation of the work done by a force field along a path.

Consider a smooth path $\gamma:[a, b] \rightarrow \mathbb{R}^{3}$ all contained in an open set $U \subset \mathbb{R}^{3}$; and let $F: U \rightarrow \mathbb{R}^{3}$ be a force field acting on a point object. The work done by the force field $F$ along the path $\gamma$ can be obtained by taking into account two facts:

1. The work done by a force field along a path depends only upon the component of the force along the tangent direction of the path at each point.

2. The work done by a constant field $F_{0}$ to move an object along a line segment in its direction is given by the product of $\left\|F_{0}\right\|$ and the length of the line segment.

Consider a very fine partition $\pi=\left\{a=t_{1}<\cdots<t_{k}=b\right\}$ of the interval $[a, b]$, so that the arc length of $\left.\gamma\right|_{\left[t_{j}, t_{j+1}\right]}$, according to Theorem 8 , can be approximated by

$$
\left\|\gamma^{\prime}\left(t_{j}\right)\right\|\left(t_{j+1}-t_{j}\right) \text {. }
$$


We know that the unit vector which is tangent to a smooth path at $\gamma(t), t \in[a, b]$ is given by

$$
T(t)=\frac{\gamma^{\prime}(t)}{\left\|\gamma^{\prime}(t)\right\|},
$$

so that we may assume, at a good approximation, that along the path $\left.\gamma\right|_{\left[t_{j}, t_{j+1}\right]}$ the force field has a constant value $F\left(\gamma\left(t_{j}\right)\right)$. Consequently, the work done by the force field $F$ along $\left.\gamma\right|_{\left[t_{j}, t_{j+1}\right]}$ can be approximated by

$$
\left\langle F\left(\gamma\left(t_{j}\right)\right), T\left(t_{j}\right)\right\rangle \| \gamma^{\prime}\left(t_{j} \|\left(t_{j+1}-t_{j}\right)=\left\langle F\left(\gamma\left(t_{j}\right)\right), \gamma^{\prime}\left(t_{j}\right)\right\rangle\left(t_{j+1}-t_{j}\right),\right.
$$

where $j=1, \ldots, k-1$. Summing up a good approximation of the work done in moving the object along $\gamma$ is given by

$$
\sum_{k=1}^{k-1}\left\langle F\left(\gamma\left(t_{j}\right)\right), \gamma^{\prime}\left(t_{j}\right)\right\rangle\left(t_{j+1}-t_{j}\right) ;
$$

the usual theorem of existence of the Riemann integral of a continuous function along a finite interval leads to the following proposition.

Proposition 27. The work done by a continuous force field $F: U \rightarrow \mathbb{R}^{3}$ along a piecewise smooth path $\gamma:[a, b] \rightarrow \mathbb{R}^{3}$ all contained in an open set $U \subset \mathbb{R}^{3}$ is given by

$$
\int_{\gamma} F=\int_{a}^{b}\left\langle F(\gamma(t)), \gamma^{\prime}(t)\right\rangle \mathrm{d} t
$$

The following definition is then meaningful.

Definition 28. Let $F: U \rightarrow \mathbb{R}^{d}$ be a continuous vector field along a piecewise smooth path $\gamma:[a, b] \rightarrow \mathbb{R}^{d}$ all contained in an open set $U \subset \mathbb{R}^{d}$. The line integral of $F$ along $\gamma$ is given by

$$
\int_{\gamma} F:=\int_{a}^{b}\left\langle F(\gamma(t)), \gamma^{\prime}(t)\right\rangle \mathrm{d} t
$$

It is clear that the line integral of a vector field depends upon the orientation of the path, i.e.,

$$
\int_{\gamma[a, b]} F:=-\int_{\gamma[b, a]} F .
$$

Thanks to the correspondence between 1-forms and vector fields, the following definition is appropriate.

Definition 29. Let $U \subset \mathbb{R}^{d}$ be an open set, let $\omega$ be a continuous 1 -form on $U$, and let $\gamma:[a, b] \rightarrow \mathbb{R}^{d}$ be a piecewise smooth path, all contained in $U$. If $F(x)=$ 
$\left(f_{1}(x), \ldots, f_{d}(x)\right)$, for $x \in U$, is the vector field associated with $\omega$, the line integral of $\omega$ along $\gamma$ is given by

$$
\begin{aligned}
\int_{\gamma} \omega:=\int_{a}^{b} \omega(\gamma(t)) \gamma^{\prime}(t) \mathrm{d} t & =\int_{a}^{b}\left\langle F(\gamma(t)), \gamma^{\prime}(t)\right\rangle \mathrm{d} t \\
& =\sum_{j=1}^{d} \int_{a}^{b} f_{j}(\gamma(t)) \gamma_{j}^{\prime}(t) \mathrm{d} t .
\end{aligned}
$$

3.3. 1-currents. In the sequel, a 1-current will be a continuous linear functional on $C_{c}\left(\mathbb{R}^{d}, \mathbb{R}^{d}\right)$ (more generally the literature considers a continuous linear functional on $\left.C_{c}^{\infty}\left(\mathbb{R}^{d}, \mathbb{R}^{d}\right)\right)$.

Typical examples of 1-currents are those induced by regular curves in $\mathbb{R}^{d}$. Let $\gamma:[a, b] \subset \mathbb{R} \rightarrow \mathbb{R}^{d}$ be a smooth curve in $\mathbb{R}^{d}$; the linear functional $\xi$ defined by

$$
\begin{aligned}
\theta \in C_{c}\left(\mathbb{R}^{d}, \mathbb{R}^{d}\right) \mapsto(\xi, \theta) & =\int_{a}^{b}\left\langle\theta(\gamma(t)), \gamma^{\prime}(t)\right\rangle \mathrm{d} t \\
& =\sum_{j=1}^{d} \int_{a}^{b} \theta_{j}(\gamma(t)) \gamma_{j}^{\prime}(t) \mathrm{d} t \in \mathbb{R}
\end{aligned}
$$

is a 1 -current.

Another case is one in which the 1-current is induced by a vector field. By an abuse of notation, let $\xi: \mathbb{R}^{d} \rightarrow \mathbb{R}^{d}$ be a vector field; the associated current is

$$
\theta \in C_{c}\left(\mathbb{R}^{d}, \mathbb{R}^{d}\right) \mapsto(\xi, \theta)=\int_{\mathbb{R}^{d}}\langle\theta(x), \xi(x)\rangle \mathrm{d} x=\sum_{j=1}^{d} \int_{\mathbb{R}^{d}} \theta_{j}(x) \xi_{j}(x) \mathrm{d} x .
$$

We may recover the case (3-10) as a particular case of (3-11) by the localization on the regular path $\{\gamma(t), t \in[a, b]\}$ made by a usual Dirac delta distribution

$$
\xi(x)=\int_{a}^{b} \delta_{\gamma(t)}(x) \gamma^{\prime}(t) \mathrm{d} t, \quad x \in \mathbb{R}^{d} ;
$$

i.e.,

$$
\xi=\int_{a}^{b} \delta_{\gamma(t)}(\cdot) \gamma^{\prime}(t) \mathrm{d} t
$$

3.4. Random 1-currents and their mean densities. We have said above that a deterministic 1-current is a linear continuous functional on $C_{c}^{\infty}\left(\mathbb{R}^{d}, \mathbb{R}^{d}\right)$. Since it is sufficient for our purposes, in order to stress the analogy with Radon measures, we consider afterwards only 1-currents which are linear continuous functionals on $C_{c}\left(\mathbb{R}^{d}, \mathbb{R}^{d}\right)$. There are two definitions of random 1-currents, as in the case of random functionals (see Section 2.2.2).

The most natural one is the following [Flandoli et al. 2009; Bessaih et al. 2017]. 
Definition 30. Given a probability space $(\Omega, \mathcal{F}, P)$, consider the space $C_{c}\left(\mathbb{R}^{d}, \mathbb{R}^{d}\right)$ of compact support continuous vector fields endowed with the topology of convergence on compact sets and let $C_{c}\left(\mathbb{R}^{d}, \mathbb{R}^{d}\right)^{\prime}$ be the space of 1-currents on $\mathbb{R}^{d}$, endowed with the dual topology, and the corresponding Borel sigma algebra. We call a random 1-current in the strict sense any measurable map

$$
\xi: \omega \in \Omega \mapsto \xi_{\omega} \in C_{c}\left(\mathbb{R}^{d}, \mathbb{R}^{d}\right)^{\prime} .
$$

The second, a weaker one, relates to the real value obtained when applying the current to a test vector field.

Definition 31. Given a probability space $(\Omega, \mathcal{F}, P)$, consider the space $L^{0}(\Omega)$ of real-valued random variables endowed with the Ky Fan topology, i.e., the topology of the convergence in probability, and the corresponding Borel sigma algebra. We call a random 1-current in the broad sense any continuous linear map $\Xi: C_{c}\left(\mathbb{R}^{d}, \mathbb{R}^{d}\right) \rightarrow L^{0}(\Omega)$. When there exists a random 1-current in the strict sense $\xi: \Omega \rightarrow C_{c}\left(\mathbb{R}^{d}, \mathbb{R}\right)^{\prime}$ such that

$$
\Xi(f)(\omega)=\left(\xi_{\omega}, f\right)
$$

for all $f \in C_{c}\left(\mathbb{R}^{d}, \mathbb{R}^{d}\right)$ and a.a. $\omega \in \Omega$, we say $\xi_{\omega}$ is a pathwise realization of $\Xi$.

One can show, as in the case of random functionals, that strict sense implies broad sense, but the opposite is not true. Below we provide a typical example for each category and compute the corresponding mean densities.

3.4.1. The mean of a 1-current. Consider a random 1-current in the strict sense $\xi_{\omega} \in C_{c}\left(\mathbb{R}^{d}, \mathbb{R}^{d}\right)^{\prime}$, and $\omega \in \Omega$. We say that it admits a mean value if a 1-current $\hat{\xi} \in C_{c}\left(\mathbb{R}^{d}, \mathbb{R}^{d}\right)^{\prime}$ exists such that, for any $\theta \in C_{c}\left(\mathbb{R}^{d}, \mathbb{R}^{d}\right)$,

$$
(\hat{\xi}, \theta)=\mathbb{E}[(\xi, \theta)]
$$

Whenever this happens, we will identify the current $\mathbb{E}[\xi]:=\hat{\xi}$ as the mean value of the current $\xi$.

We will say that the mean current $\mathbb{E}[\xi]$ is induced by a vector field if a locally integrable vector field $u: \mathbb{R}^{d} \rightarrow \mathbb{R}^{d}$ exists such that

$$
(\mathbb{E}[\xi], \theta)=\int_{\mathbb{R}^{d}}\langle\theta(x), u(x)\rangle \mathrm{d} x .
$$

3.5. Example of a random 1-current in the strict sense and its expectation. Let $\left(X_{t}, V_{t}\right)$ be the solution of the stochastic equation in $\mathbb{R}^{d} \times \mathbb{R}^{d}$ :

$$
\begin{aligned}
\mathrm{d} X_{t} & =V_{t} \mathrm{~d} t, \\
\mathrm{~d} V_{t} & =b\left(t, X_{t}, V_{t}\right) \mathrm{d} t+\mathrm{d} W_{t},
\end{aligned}
$$


where $b$ is Lipschitz continuous and $W_{t}$ is a Brownian motion in $\mathbb{R}^{d}$. Assume that $\left(X_{0}, V_{0}\right)$ has a smooth density $p_{0}(x, v)$ with respect to Lebesgue measure. Thanks to hypoellipticity, $\left(X_{t}, V_{t}\right)$ has, for every $t>0$, a smooth density $p_{t}(x, v)$ with respect to Lebesgue measure; it is a solution to the Fokker-Planck equation:

$$
\begin{aligned}
\frac{\partial p}{\partial t} & =\Delta_{v} p-v \cdot \nabla_{x} p-\operatorname{div}_{v}(b p), \\
\left.p\right|_{t=0} & =p_{0} .
\end{aligned}
$$

Given $T>0$, consider the random curve in $\mathbb{R}^{d}$ given by $t \in[0, T] \mapsto X_{t} \in \mathbb{R}^{d}$ (not necessarily simple) and consider the associated random 1-current $\xi_{X}$ formally defined as

$$
\xi_{X}(x)=\int_{0}^{T} \delta_{X_{t}}(x) V_{t} \mathrm{~d} t, \quad x \in \mathbb{R}^{d}
$$

namely

$$
\left(\xi_{X}, \theta\right)=\int_{0}^{T}\left\langle\theta\left(X_{t}\right), V_{t}\right\rangle \mathrm{d} t, \quad \theta \in C_{c}\left(\mathbb{R}^{d}, \mathbb{R}^{d}\right) .
$$

One can easily recognize that $\xi_{X}$ is a 1-current in the strict sense. We have

$$
\begin{aligned}
\mathbb{E}\left[\left(\xi_{X}, \theta\right)\right]=\mathbb{E}\left[\int_{0}^{T}\left\langle\theta\left(X_{t}\right), V_{t}\right\rangle \mathrm{d} t\right] & =\int_{0}^{T} \mathbb{E}\left[\left\langle\theta\left(X_{t}\right), V_{t}\right\rangle\right] \mathrm{d} t \\
& =\int_{0}^{T}\left(\int_{\mathbb{R}^{d} \times \mathbb{R}^{d}}\langle\theta(x), v\rangle p_{t}(x, v) \mathrm{d} x \mathrm{~d} v\right) \mathrm{d} t .
\end{aligned}
$$

We may also proceed as in Proposition 20, and see directly from (3-17) that, for any $x \in \mathbb{R}^{d}$, we do have formally

$$
\begin{aligned}
\mathbb{E}\left[\xi_{X}(x)\right] & =\int_{0}^{T} \mathbb{E}\left[\delta_{X_{t}}(x) V_{t}\right] \mathrm{d} t \\
& =\int_{0}^{T} \mathrm{~d} t \int_{\mathbb{R}^{d} \times \mathbb{R}^{d}} \delta_{y}(x) v p_{t}(y, v) \mathrm{d} y \mathrm{~d} v \\
& =\int_{0}^{T} \mathrm{~d} t \int_{\mathbb{R}^{d} \times \mathbb{R}^{d}} \delta_{x}(y) v p_{t}(y, v) \mathrm{d} y \mathrm{~d} v \\
& =\int_{0}^{T} \mathrm{~d} t \int_{\mathbb{R}^{d}} v p_{t}(x, v) \mathrm{d} v .
\end{aligned}
$$

In accordance with the above, we have proven the following.

Proposition 32. The 1-current $\xi_{X}$ admits a mean current $E\left[\xi_{X}\right]$ on $\mathbb{R}^{d}$, induced by the vector field

$$
u(x)=\int_{0}^{T} \int_{\mathbb{R}^{d}} v p_{t}(x, v) \mathrm{d} v \mathrm{~d} t, \quad x \in \mathbb{R}^{d}
$$


3.6. Example of a random 1-current in the broad sense and its expectation. Let $X_{t}$ be the solution of the stochastic equation in $\mathbb{R}^{d}$ :

$$
\mathrm{d} X_{t}=b\left(t, X_{t}\right) \mathrm{d} t+\mathrm{d} W_{t},
$$

where $b$ is Lipschitz continuous and $W_{t}$ is a Brownian motion in $\mathbb{R}^{d}$. Assume that $X_{0}$ has a smooth density $p_{0}(x)$ with respect to Lebesgue measure. Also $X_{t}$ has, for every $t>0$, a smooth density $p_{t}(x)$ with respect to Lebesgue measure; it is a solution to the Fokker-Planck equation:

$$
\begin{aligned}
\frac{\partial p}{\partial t} & =\Delta_{x} p-\operatorname{div}_{x}(b p), \\
\left.p\right|_{t=0} & =p_{0} .
\end{aligned}
$$

Given $T>0$, consider again the random curve $X_{t}$ in $\mathbb{R}^{d}$ (not necessarily simple) and consider the associated random 1-current $\xi_{X}$ formally defined as

$$
\xi_{X}(x)=\int_{0}^{T} \delta_{X_{t}}(x) \mathrm{d} X_{t}, \quad x \in \mathbb{R}^{d} ;
$$

namely,

$$
\left(\xi_{X}, \theta\right)=\int_{0}^{T}\left\langle\theta\left(X_{t}\right), \mathrm{d} X_{t}\right\rangle, \quad \theta \in C_{c}\left(\mathbb{R}^{d}, \mathbb{R}^{d}\right),
$$

where now the integral is understood as a stochastic integral. There are two main choices: Itô and Stratonovich integrals. Let us discuss only the Itô case, but the other one is also not difficult.

Given $\theta \in C_{c}\left(\mathbb{R}^{d}, \mathbb{R}^{d}\right),(\xi, \theta)$ is a well defined random variable, because the process $\theta\left(X_{t}\right)$ is adapted, $E\left[\int_{0}^{T}\left\|\theta\left(X_{t}\right)\right\|^{2} \mathrm{~d} t\right]<\infty$, and $\int_{0}^{T}\left\langle\theta\left(X_{t}\right), \mathrm{d} X_{t}\right\rangle$ is given by

$$
\int_{0}^{T}\left\langle\theta\left(X_{t}\right), \mathrm{d} X_{t}\right\rangle=\int_{0}^{T}\left\langle\theta\left(X_{t}\right), b\left(t, X_{t}\right)\right\rangle \mathrm{d} t+\int_{0}^{T}\left\langle\theta\left(X_{t}\right), \mathrm{d} W_{t}\right\rangle .
$$

So $\xi_{X}$ is a current in the broad sense. In general, it is not clear if it is a current also in the strict sense because we cannot "fix $\omega$ " and consider the map $\omega \in \Omega \mapsto$ $\int_{0}^{T}\left\langle\theta\left(X_{t}(\omega)\right), \mathrm{d} X_{t}(\omega)\right\rangle$ (the Itô integral is an equivalence class and its pointwise evaluation at $\omega$ is not a well-defined concept).

We have

$$
\mathbb{E}\left[\int_{0}^{T}\left\langle\theta\left(X_{t}\right), \mathrm{d} X_{t}\right\rangle\right]=\int_{0}^{T} \mathbb{E}\left[\left\langle\theta\left(X_{t}\right), b\left(t, X_{t}\right)\right\rangle\right] \mathrm{d} t+\mathbb{E}\left[\int_{0}^{T}\left\langle\theta\left(X_{t}\right), \mathrm{d} W_{t}\right\rangle\right] .
$$

Since for an Itô integral $E\left[\int_{0}^{T}\left\langle\theta\left(X_{t}\right), \mathrm{d} W_{t}\right\rangle\right]=0$, we have

$$
\int_{0}^{T} \mathbb{E}\left[\left\langle\theta\left(X_{t}\right), b\left(t, X_{t}\right)\right\rangle\right] \mathrm{d} t=\int_{0}^{T}\left(\int_{\mathbb{R}^{d}}\langle\theta(x), b(t, x)\rangle p_{t}(x) \mathrm{d} x\right) \mathrm{d} t .
$$

Hence we have proved the following. 
Proposition 33. The 1 -current $\xi_{X}$ admits a mean current $\mathbb{E}\left[\xi_{X}\right]$ on $\mathbb{R}^{d}$, induced by the vector field

$$
u(x)=\int_{0}^{T} b(t, x) p_{t}(x) \mathrm{d} t
$$

\section{Advanced applications and open problems}

4.1. A mathematical model for tumor induced angiogenesis. The main features of the process of formation of a tumor-driven vessel network are (see [Chaplain and Stuart 1993; Plank and Sleeman 2004; Bonilla et al. 2017])

(i) vessel branching;

(ii) vessel extension;

(iii) chemotaxis in response to a generic tumor angiogenic factor (TAF), released by tumor cells;

(iv) haptotactic migration in response to fibronectin gradient, emerging from the extracellular matrix and through degradation and production by endothelial cells themselves; and

(v) anastomosis, the coalescence of a capillary tip with an existing vessel.

We will limit ourselves to describe the dynamics of tip cells at the front of growing vessels, as a consequence of chemotaxis in response to a generic TAF released by tumor cells.

The $i$-th tip cell is characterized by its position and velocity $\left(X_{t}^{i}, V_{t}^{i}\right) \in \mathbb{R}^{2 d}$ for $t \geq 0$; also its history

$$
\left(X_{s}^{i}, V_{s}^{i}\right)_{s \in\left[T^{i}, \Theta^{i} \wedge t\right)}
$$

plays a role; the random variables $T^{i}$ and $\Theta^{i}$ are respectively the birth (branching) and death (anastomosis) times of the $i$-th tip cell. All random variables and processes are defined on a filtered probability space $\left(\Omega, \mathcal{F}, \mathcal{F}_{t}, P\right)$.

The number of tip cells changes in time, due to proliferation and death. We denote this random number by $N_{t}$ for $t \geq 0$.

The growth factor is a random function $C: \Omega \times[0, \infty) \times \mathbb{R}^{d} \rightarrow \mathbb{R}$, that we write as $C(t, x)$.

Tip cells and growth factor satisfy the system

$$
\begin{aligned}
\mathrm{d} X_{t}^{i} & =V_{t}^{i}, \\
\mathrm{~d} V_{t}^{i} & =\left[-k_{1} V_{t}^{i}+f\left(C\left(t, X_{t}^{i}\right)\right) \nabla C\left(t, X_{t}^{i}\right)\right] \mathrm{d} t+\sigma \mathrm{d} W_{t}^{i}, \\
\frac{\partial}{\partial t} C(t, x) & =k_{2} \delta_{A}(x)+d_{1} \Delta C(t, x)-\eta(t, x, S .) C(t, x),
\end{aligned}
$$


where $k_{1}, k_{2}, \sigma, d_{1}>0$ and $f: \mathbb{R} \rightarrow \mathbb{R}$ are given, $W_{t}^{i}$ for $i \in \mathbb{N}$ are independent Brownian motions, $A$ is a Borel set of $\mathbb{R}^{d}$ representing the tumoral region acting as a source of the TAF; initial conditions $X_{0}^{i}, V_{0}^{i}$ and $C(0, x)$ are also given.

Let us describe the term $\eta(t, x, S$.). For every $t \geq 0$, we introduce the measure

$$
S_{t}:=\sum_{i=1}^{N_{t}} 1_{t \in\left[T^{i}, \Theta^{i}\right)} \epsilon_{\left(X_{t}^{i}, V_{t}^{i}\right)},
$$

where $\epsilon$ denotes the usual Dirac measure. With these notations we may assume that, for every $t \geq 0$, the function $\eta(t, \cdot, \cdot)$ maps $\mathbb{R}^{d} \times C\left([0, t] ; \mathcal{M}\left(\mathbb{R}^{d}\right)\right)$ into $\mathbb{R}$ :

$$
\eta(t, \cdot, \cdot): \mathbb{R}^{d} \times C\left([0, t] ; \mathcal{M}\left(\mathbb{R}^{d}\right)\right) \rightarrow \mathbb{R}
$$

With the notation $\eta\left(t, x, S\right.$.), we understand $\eta\left(t, x,\left\{S_{s}\right\}_{s \in[0, t]}\right)$.

We may leave the function $\eta$ unspecified, with suitable assumptions. However, to help with intuition, we may assume the following structure:

$$
\eta(t, x, S .)=\int_{0}^{t} e^{-(t-s) / \tau}\left(\int_{\mathbb{R}^{d}} \int_{\mathbb{R}^{d}} K\left(x-x^{\prime}\right)\left|v^{\prime}\right| S_{S}\left(\mathrm{~d} x^{\prime}, \mathrm{d} v^{\prime}\right)\right) \mathrm{d} s
$$

for a suitable function $K: \mathbb{R}^{d} \rightarrow \mathbb{R}$.

In the SDE system (4-1), besides the friction force, there is a force due to the underlying TAF field $C(t, \mathbf{x})$; from the relevant literature we take [Plank and Sleeman 2004; Stéphanou et al. 2006]

$$
f(C)=\frac{d_{1}}{\left(1+\gamma_{1} C\right)^{q}} .
$$

The capillary network of endothelial cells $X(t)$ consists of the union of all random trajectories representing the extension of individual capillary tips from the random time of birth (branching) $T^{i}$ to the random time of death (anastomosis) $\Theta^{i}$,

$$
X(t)=\bigcup_{i=1}^{N_{t}}\left\{X^{i}(s), T^{i} \leq s \leq \min \left\{t, \Theta^{i}\right\}\right\}
$$

giving rise to a stochastic network. Thanks to the choice of a Langevin model for the vessel's extension, we may assume that the trajectories are sufficiently regular and have integer Hausdorff dimension 1.

Hence the random measure [Capasso and Villa 2008]

$$
A \in \mathcal{B}_{\mathbb{R}^{d}} \mapsto \mathcal{H}^{1}(X(t) \cap A) \in \mathbb{R}_{+}
$$


may admit a random generalized density $\delta_{X(t)}(x)$ with respect to the usual Lebesgue measure on $\mathbb{R}^{d}$ such that, for any $A \in \mathcal{B}_{\mathbb{R}^{d}}$,

$$
\mathcal{H}^{1}(X(t) \cap A)=\int_{A} \delta_{X(t)}(x) \mathrm{d} x .
$$

By Theorem 11, we may then state that

$$
\mathcal{H}^{1}(X(t) \cap A)=\int_{0}^{t} \sum_{i=1}^{N_{s}} \epsilon_{X^{i}(s)}(A)\left|\frac{\mathrm{d}}{\mathrm{d} s} X^{i}(s)\right| \rrbracket_{s \in\left[T^{i}, \Theta^{i}\right)} \mathrm{d} s .
$$

Hence,

$$
\delta_{X(t)}=\int_{0}^{t} \sum_{i=1}^{N_{s}} \delta_{X^{i}(s)}\left|\frac{\mathrm{d}}{\mathrm{d} s} X^{i}(s)\right| \mathbb{q}_{s \in\left[T^{i}, \Theta^{i}\right)} \mathrm{d} s .
$$

4.1.1. Vessel branching. Two kinds of branching have been identified; either from a tip or from a vessel.

The birth process of new tips can be described in terms of a marked point process (see, e.g., [Brémaud 1981]), by means of the random measure $\Phi$ on $\mathcal{B}_{\mathbb{R}^{+} \times \mathbb{R}^{d} \times \mathbb{R}^{d}}$ such that, for any $t \geq 0$ and any $B \in \mathcal{B}_{\mathbb{R}^{d}} \times \mathbb{R}^{d}$,

$$
\Phi((0, t] \times B):=\int_{0}^{t} \int_{B} \Phi(\mathrm{d} s \times \mathrm{d} x \times \mathrm{d} v),
$$

where $\Phi(\mathrm{d} s \times \mathrm{d} x \times \mathrm{d} v)$ is the random variable that counts those tips born either from an existing tip, or from an existing vessel, during times in $(s, s+\mathrm{d} s]$, with positions in $(x, x+\mathrm{d} x]$, and velocities in $(v, v+\mathrm{d} v]$.

Given the history $\mathcal{F}_{t^{-}}$of the whole process up to time $t^{-}$, we claim that the compensator of the random measure $\Phi(\mathrm{d} s \times \mathrm{d} x \times \mathrm{d} v)$ is $\alpha(C(s, x)) G_{v_{0}}(v) S_{s}(d(x, v)) \mathrm{d} s$

$$
+\beta(C(s, x)) G_{v_{0}}(v)\left(K_{1} * \delta_{X(t)}\right)(x) \mathrm{d} x \mathrm{~d} v \mathrm{~d} s,
$$

where $\alpha(C)$ and $\beta(C)$ are nonnegative functions; for example, we may take

$$
\alpha(C)=\beta(C)=\alpha_{1} \frac{C}{C_{R}+C},
$$

where $C_{R}$ is a reference density parameter [Capasso and Morale 2009]; $K_{1}: \mathbb{R}^{d} \rightarrow$ $\mathbb{R}$ is a suitable mollifying kernel.

As a technical simplification, we will further assume that the initial value of the state of a new tip is $\left(T^{N(t)+1}, X^{N(t)+1}, v^{N(t)+1}\right)$, where $T^{N(t)+1}$ is the random time of branching, $X^{N(t)+1}$ is the random point of branching, and $v^{N(t)+1}$ is a random velocity, selected out of a normal distribution $G_{v_{0}}$ with mean $v_{0}$, and some variance. 
4.1.2. Anastomosis. When a vessel tip meets an existing vessel, it joins at that point and time and it stops moving. This process is called tip-vessel anastomosis.

As in the case of the branching process, we may model this process via a marked counting process; anastomosis is modeled as a "death" process.

Let $\Psi$ denote the random measure on $\mathcal{B}_{\mathbb{R}^{+} \times \mathbb{R}^{d} \times \mathbb{R}^{d}}$ such that, for any $t \geq 0$ and any $B \in \mathcal{B}_{\mathbb{R}^{d} \times \mathbb{R}^{d}}$,

$$
\Psi((0, t] \times B):=\int_{0}^{t} \int_{B} \Psi(\mathrm{d} s \times \mathrm{d} x \times \mathrm{d} v),
$$

where $\Psi(\mathrm{d} s \times \mathrm{d} x \times \mathrm{d} v)$ is the random variable counting those tips that are absorbed by the existing vessel network during time $(s, s+\mathrm{d} s]$, with position in $(x, x+\mathrm{d} x]$, and velocity in $(v, v+\mathrm{d} v]$.

We assume that the compensator of the random measure $\Psi(\mathrm{d} s \times \mathrm{d} x \times \mathrm{d} v)$ is

$$
\gamma\left(K_{1} * \delta_{X(s)}\right)(x) S_{S}(d(x, v)) \mathrm{d} s,
$$

where $\gamma$ is a suitable constant.

4.2. Turbulence. The topic of turbulence is too wide and deep to be recalled here; let us mention a general reference [Frisch 1995] and one example to attempt to develop fragments of a rigorous theory based on the stochastic Navier-Stokes equations and their invariant measures [Flandoli et al. 2008]. Among the several ideas to approach turbulence, which is to a wide extent a statistical theory, there is one of Alexander Chorin [1994] which is particularly attractive. Based on some evidence that turbulent fluids are "made" (this sentence has to be taken in a very intuitive sense) of vortex structures, in particular filament-like structures (see [Frisch 1995] for a discussion), Chorin had the idea to describe such "vortex filaments" by means of paths of stochastic processes, and relate the statistical properties of these processes to the statistical properties of turbulent fluids. In particular, Chorin considered the so called self-avoiding walk and tried to connect its Flory exponents to the Kolmogorov exponents of K41 theory of turbulence - we cannot enter in further detail here, see [Chorin 1994]. Unfortunately, as Chorin admitted in his book, this connection is not clearly identified, so it was only a research suggestion; taken by some authors, like Gallavotti [2002], Lions and Majda [2000] and Flandoli and Gubinelli [2002] who tried to develop part of such arguments using Brownian motion instead of self-avoiding walk. The problem remains essentially open after these contributions.

The concept of a random 1-current described in the present work is strongly related to - in fact, it was strongly motivated by — stochastic vortex filaments. It does not solve in itself the question of connection with K41 or other statistical theories of turbulence, but it gives a precise language to approach it. Before we 
continue, let us say again that vortex filaments are an intuitive concept, since classical continuum mechanics allows us only to define the vorticity field of a fluid, and the identification of "structures" in it is not rigorous, at least at present. Having said this, the intuitive idea of a vortex filament is a curve, a vortex line (an integral curve of the vorticity field) over which the vorticity field is supported - maybe with the generalization of a finite number of such lines, not only one. This directly leads to the concept of a 1-current, a distributional vector field, concentrated along curves. Since a statistical theory should be developed, random 1-currents are the right objects. If we denote by $X$ the process whose paths represent the vortex filaments, the random distributional vorticity field is given by

$$
\xi_{X}=\int_{0}^{T} \delta_{X_{s}} \mathrm{~d} X_{s}
$$

The two main open questions are about statistical properties and about the realism of these random vortex structures. Concerning the realism, we mean the connection between these structures and more classical objects of fluid dynamics, typically the partial differential equations of continuum mechanics. The only partial result until now in this direction is the mean field result [Bessaih et al. 2017], where a smoothed version of 3D Euler equations is obtained as the mean field of interacting vortex filaments - also with a smoothed Biot-Savart kernel.

Concerning the statistical properties, first attempts have been made in [Chorin 1994; Lions and Majda 2000; Flandoli and Gubinelli 2002] by using Gibbs measures. The problem, not yet solved, is to identify a stochastic process $X$ such that the random distributional vorticity field $\xi_{X}$ written above has statistical properties like those of K41 theory. This means that the associated random velocity field $u_{X}=K * \xi_{X}$ has to be introduced ( $K$ denotes here the Biot-Savart kernel) and expected values like

$$
\mathbb{E}\left[|u(x+r e)-u(x)|^{2}\right],
$$

where $r$ is a real number and $e$ is a unitary vector, have to be computed and compared with the prescription of statistical theories of turbulence (K41 prescribes a behavior of the form $\mathrm{Cr}^{2 / 3}$ for small $r$ ).

A more modest but still quite open problem, very related to the machinery developed in the present work, is the following one: given a vorticity field $\xi(x)-$ generic, or typical of turbulent fluids - find a stochastic process $X$ such that

$$
\xi(x)=\mathbb{E}\left[\xi_{X}\right](x) .
$$

Solving this problem may increase some understanding of the statistical problem above and provide relevant initial conditions for the mean-field approach of [Bessaih et al. 2017]. 
4.3. Pathwise stochastic analysis. In recent years, a new direction in stochastic analysis grew up thanks to a number of outstanding contributions. The beginning of this direction is often traced back to a paper by Hans Föllmer [1981] where he derived an Itô formula for the composition $f\left(x_{t}\right)$ of a $C^{2}$ function $f: \mathbb{R}^{d} \rightarrow \mathbb{R}$ with a single deterministic path $x:[0, T] \rightarrow \mathbb{R}^{d}$, which possesses quadratic variation along a sequence of partitions $\left(\pi_{n}\right)$ of $[0, T]$, in the sense that

$$
\left[x_{.}^{(i)}\right]_{t}^{\left(\pi_{n}\right)}:=\lim _{n \rightarrow \infty} \sum_{t_{i} \in \pi_{n}}\left(x_{t_{i+1} \wedge t}^{(i)}-x_{t_{i} \wedge t}^{(i)}\right)^{2}
$$

exists finite and continuous in $t \in[0, T]$, for each $i=1, \ldots, d$, where $x_{t}^{(i)}$ denotes the $i$-coordinate of $x_{t}$. The stochastic integral $\int_{0}^{t}\left\langle\nabla f\left(x_{s}\right), \mathrm{d} x_{s}\right\rangle$ required in the formula,

$$
f\left(x_{t}\right)-f\left(x_{0}\right)-\frac{1}{2} \int_{0}^{t} \sum_{i, j=1}^{d} \partial_{i} \partial_{j} f\left(x_{s}\right) \mathrm{d}\left[x_{\cdot}^{(i)}, x_{\cdot}^{(j)}\right]_{s}^{\left(\pi_{n}\right)}=\int_{0}^{t}\left\langle\nabla f\left(x_{s}\right), \mathrm{d} x_{s}\right\rangle,
$$

is not a priori defined, but it exists from the formula itself: the limit or Riemann sums

$$
\lim _{n \rightarrow \infty} \sum_{t_{i} \in \pi_{n}}\left\langle\nabla f\left(x_{t_{i} \wedge t}\right), x_{t_{i+1} \wedge t}-x_{t_{i} \wedge t}\right\rangle
$$

exists since the sum of the other terms in the formula have a limit, thanks to the existence of the quadratic variation (the so-called joint quadratic variation between two coordinates $\left[x^{(i)}, x^{(j)}\right]_{t}^{\left(\pi_{n}\right)}$ exists by polarization and it is a bounded variation function, hence the integral on the left-hand side of (4-11) exists as limit of RiemannStieltjes sums). Probability is not totally excluded by this approach, but its role is localized: probability provides the existence of the quadratic variation $\left[x^{(i)}\right]_{t}^{\left(\pi_{n}\right)}$ for almost every path of relevant stochastic processes, like Brownian motion and more generally continuous semimartingales; it is otherwise very difficult to construct a deterministic function having nonzero quadratic variation $\left[x^{(i)}\right]_{t}^{\left(\pi_{n}\right)}$ (if it is zero, the formula above is just the usual chain rule). In a sense, a main topic in stochastic analysis, which previously has been treated by probability from the first to the last step (namely Itô's formula from the viewpoint of Itô's approach), is now decoupled: one half of the story is based on probability - the existence of the quadratic variation - and the other half is purely deterministic.

The question arisen by that paper is: to what extent can one develop a similar approach for other pieces of stochastic analysis? A breakthrough is rough path calculus developed by Terry Lyons [1998], which introduces new classes of paths, defines stochastic integrals for them and solves stochastic differential equations. Again, the theory is fully deterministic but at the foundation there is the concept of rough path, a sort of path $x$ enriched by its Lévy areas $\int_{0}^{t} x_{s}^{(i)} \mathrm{d} x_{s}^{(j)}$ (we refer here to 
a subclass for the general theory); the latter object exists for almost every path of Brownian motion and semimartingales using probability, namely using Itô's theory of stochastic integration.

The theory of rough paths has been revisited with a novel approach by Massimiliano Gubinelli [2004] and, in a sense based on this new viewpoint, a sort of multidimensional analog (namely for functions $x: \mathbb{R}^{d} \rightarrow \mathbb{R}$ or more precisely for distributions) has been developed in the outstanding work of Martin Hairer [2014], see also the alternative theory by Gubinelli et al. [2015]. Again in these theories, probability guarantees the existence of fundamental objects, after which the procedure to define other objects and solve (ordinary or partial) differential equations is fully deterministic. Let us also mention this kind of two-step approach in other recent directions of stochastic analysis, like stochastic homogenization [Gloria and Otto 2015].

After reviewing this introduction to the subject of "stochastic calculus without probability", let us mention an open research direction related to the topics of the present paper. Probability allows us to define stochastic integrals of the form

$$
\int_{0}^{t}\left\langle\theta\left(X_{s}\right), \mathrm{d} X_{s}\right\rangle
$$

for several stochastic processes $X$ and functions $\theta$. The map $\theta \mapsto \int_{0}^{t}\left\langle\theta\left(X_{s}\right), \mathrm{d} X_{s}\right\rangle$ is a random 1-current in the broad sense. In many cases, thanks to probabilistic estimates, there is a random 1-current in the strict sense $\xi_{X, t}(\omega)$ associated to it, given by

$$
\xi_{X, t}=\int_{0}^{t} \delta_{X_{s}} \mathrm{~d} X_{s}
$$

This is, for a given $\omega$, a deterministic 1-current associated to the deterministic path $X .(\omega)$. Conceptually, this is similar to the quadratic variation or the Lévy area associated to $X .(\omega)$ : concepts which are well-defined by probability, and would be extremely difficult to define without. The question then is: starting from the deterministic pair

$$
\left(X .(\omega), \xi_{X, \cdot}(\omega)\right)
$$

is it possible to develop, by purely deterministic methods, some pieces of stochastic calculus, as it was done by the theories recalled above starting from the pairs (path, quadratic variation), (path, Lévy area) and so on? For instance, is it possible to formulate and solve differential equations driven by single paths of Brownian motions? At present, this program has not been developed. Only the regularity, in terms of distributions, of $\xi_{X, \cdot}(\omega)$ has been partially understood, see [Flandoli et al. 2005; 2009]. 


\section{Acknowledgements}

It is our duty and pleasure to acknowledge the generous contributions of Elisabetta Dejana (Universitá degli Studi di Milano, Italy) for Figures 5, 6, and Michael E. Kassner (University of Southern California, USA) for Figure 3. We are grateful to the anonymous referee for comments and suggestions that have led to a significant improvement of the paper.

\section{References}

[Ambrosio et al. 2000] L. Ambrosio, N. Fusco, and D. Pallara, Functions of bounded variation and free discontinuity problems, Clarendon Press, New York, 2000.

[Ambrosio et al. 2009] L. Ambrosio, V. Capasso, and E. Villa, "On the approximation of mean densities of random closed sets", Bernoulli 15:4 (2009), 1222-1242.

[Anderson 2003] A. R. A. Anderson, "The effects of cell adhesion on solid tumour geometry", pp. 315-325 in Morphogenesis and pattern formation in biological systems: experiments and models, edited by T. Sekimura et al., Springer, Tokyo, 2003.

[Araujo and Giné 1980] A. Araujo and E. Giné, The central limit theorem for real and Banach valued random variables, Wiley, New York, 1980.

[Baddeley and Molchanov 1997] A. J. Baddeley and I. S. Molchanov, "On the expected measure of a random set", pp. 3-20 in Proceedings of the international symposium on advances in theory and applications of random sets (Fontainebleau, France), edited by D. Jeulin, World Scientific, River Edge, NJ, 1997.

[Baddeley et al. 2007] A. Baddeley, I. Bárány, R. Schneider, and W. Weil, Stochastic geometry, Lecture Notes in Mathematics 1892, Springer, Heidelberg, 2007.

[Bessaih et al. 2017] H. Bessaih, M. Coghi, and F. Flandoli, "Mean field limit of interacting filaments and vector valued non linear PDEs", J. Stat. Phys. (published online January 2017).

[Bharucha-Reid 1972] A. T. Bharucha-Reid, Random integral equations, Mathematics in Science and Engineering 26, Academic Press, New York, 1972.

[Bonilla et al. 2017] L. L. Bonilla, V. Capasso, M. Alvaro, M. Carretero, and F. Terragni, "On the mathematical modelling of tumor-induced angiogenesis", Math. Biosci. Eng. 14:1 (2017), 45-66.

[Bosq 2000] D. Bosq, Linear processes in function spaces: theory and applications, Lecture Notes in Statistics 149, Springer, New York, 2000.

[Brémaud 1981] P. Brémaud, Point processes and queues: martingale dynamics, Springer, New York, 1981.

[Buck 1956] R. C. Buck, Advanced calculus, McGraw-Hill, New York, 1956.

[Burger et al. 2002] M. Burger, V. Capasso, and C. Salani, "Modelling multi-dimensional crystallization of polymers in interaction with heat transfer", Nonlinear Anal-Real 3:1 (2002), 139-160.

[Callister Jr. 2007] W. Callister Jr., Materials science and engineering: an introduction, 7th ed., Wiley, New York, 2007.

[Camerlenghi et al. 2014] F. Camerlenghi, V. Capasso, and E. Villa, "On the estimation of the mean density of random closed sets”, J. Multivariate Anal. 125 (2014), 65-88.

[Capasso 2003] V. Capasso (editor), Mathematical modelling for polymer processing: polymerization, crystallization, manufacturing, Mathematics in Industry 2, Springer, Berlin, 2003. 
[Capasso and Micheletti 2006] V. Capasso and A. Micheletti, "Stochastic geometry and related statistical problems in biomedicine", pp. 35-69 in Complex systems in biomedicine, edited by A. Quarteroni et al., Springer, Milan, 2006.

[Capasso and Morale 2009] V. Capasso and D. Morale, "Stochastic modelling of tumour-induced angiogenesis", J. Math. Biol. 58:1-2 (2009), 219-233.

[Capasso and Villa 2006] V. Capasso and E. Villa, "On the continuity and absolute continuity of random closed sets", Stoch. Anal. Appl. 24:2 (2006), 381-397.

[Capasso and Villa 2007] V. Capasso and E. Villa, "On mean densities of inhomogeneous geometric processes arising in material science and medicine”, Image Anal. Stereol. 26:1 (2007), 23-36.

[Capasso and Villa 2008] V. Capasso and E. Villa, "On the geometric densities of random closed sets", Stoch. Anal. Appl. 26:4 (2008), 784-808.

[Capasso et al. 2013] V. Capasso, M. Gromov, A. Harel-Bellan, N. Morozova, and L. L. Pritchard (editors), Pattern formation in morphogenesis: problems and mathematical issues, Springer Proceedings in Mathematics 15, Springer, Heidelberg, 2013.

[Carmeliet and Jain 2000] P. Carmeliet and R. K. Jain, "Angiogenesis in cancer and other diseases", Nature 407:6801 (2000), 249-257.

[Chaplain and Stuart 1993] M. A. J. Chaplain and A. M. Stuart, "A model mechanism for the chemotactic response of endothelial cells to tumour angiogenesis factor", Math. Med. Bio. 10:3 (1993), $149-168$.

[Chorin 1994] A. J. Chorin, Vorticity and turbulence, Applied Mathematical Sciences 103, Springer, New York, 1994.

[Evans and Gariepy 1992] L. C. Evans and R. F. Gariepy, Measure theory and fine properties of functions, CRC Press, Boca Raton, FL, 1992.

[Falconer 1986] K. J. Falconer, The geometry of fractal sets, Cambridge Tracts in Mathematics 85, Cambridge University Press, 1986.

[Federer 1996] H. Federer, Geometric measure theory, Springer, Berlin, 1996.

[Flandoli and Gubinelli 2002] F. Flandoli and M. Gubinelli, "The Gibbs ensemble of a vortex filament", Probab. Theory Related Fields 122:3 (2002), 317-340.

[Flandoli et al. 2005] F. Flandoli, M. Gubinelli, M. Giaquinta, and V. M. Tortorelli, "Stochastic currents", Stoch. Process. Appl. 115:9 (2005), 1583-1601.

[Flandoli et al. 2008] F. Flandoli, M. Gubinelli, M. Hairer, and M. Romito, "Rigorous remarks about scaling laws in turbulent fluids", Comm. Math. Phys. 278:1 (2008), 1-29.

[Flandoli et al. 2009] F. Flandoli, M. Gubinelli, and F. Russo, "On the regularity of stochastic currents, fractional Brownian motion and applications to a turbulence model", Ann. Inst. Henri Poincaré Probab. Stat. 45:2 (2009), 545-576.

[Folland 1999] G. B. Folland, Real analysis: modern techniques and their applications, 2nd ed., Wiley, New York, 1999.

[Föllmer 1981] H. Föllmer, "Calcul d'Ito sans probabilités", Séminaire de probabilités de Strasbourg 15 (1981), 143-150.

[Frisch 1995] U. Frisch, Turbulence, Cambridge University Press, 1995.

[Galbis and Maestre 2012] A. Galbis and M. Maestre, Vector analysis versus vector calculus, Springer, New York, 2012.

[Gallavotti 2002] G. Gallavotti, Foundations of fluid dynamics, Springer, Berlin, 2002.

[Giaquinta and Modica 2009] M. Giaquinta and G. Modica, Mathematical analysis: an introduction to functions of several variables, Birkhäuser, Boston, 2009. 
[Giaquinta et al. 1998] M. Giaquinta, G. Modica, and J. Souček, Cartesian currents in the calculus of variations, I: Cartesian currents, Mathematics and Related Areas 37, Springer, Berlin, 1998.

[Gloria and Otto 2015] A. Gloria and F. Otto, "The corrector in stochastic homogenization: optimal rates, stochastic integrability, and fluctuations", 2015. arXiv 1510.08290

[Gubinelli 2004] M. Gubinelli, "Controlling rough paths”, J. Funct. Anal. 216:1 (2004), 86-140.

[Gubinelli et al. 2015] M. Gubinelli, P. Imkeller, and N. Perkowski, "Paracontrolled distributions and singular PDEs", Forum Math. Pi 3 (2015), e6, 75.

[Hairer 2014] M. Hairer, “A theory of regularity structures”, Invent. Math. 198:2 (2014), 269-504.

[Hochrainer et al. 2007] T. Hochrainer, M. Zaiser, and P. Gumbsch, "A three-dimensional continuum theory of dislocation systems: kinematics and mean-field formulation", Philos. Mag. 87:8-9 (2007), $1261-1282$.

[Kassner et al. 2000] M. Kassner, M.-T. Pérez-Prado, K. Vecchio, and M. Wall, "Determination of internal stresses in cyclically deformed copper single crystals using convergent-beam electron diffraction and dislocation dipole separation measurements", Acta Mater. 48:17 (2000), 4247-4254.

[Kolmogorov 1956] A. N. Kolmogorov, Foundations of the theory of probability, Chelsea Publishing, New York, 1956.

[Kolmogorov and Fomin 1970] A. N. Kolmogorov and S. V. Fomin, Introductory real analysis, Prentice-Hall, Englewood Cliffs, NJ, 1970.

[Lions and Majda 2000] P.-L. Lions and A. Majda, "Equilibrium statistical theory for nearly parallel vortex filaments", Comm. Pure Appl. Math. 53:1 (2000), 76-142.

[Lyons 1998] T. J. Lyons, "Differential equations driven by rough signals", Rev. Mat. Iberoamericana 14:2 (1998), 215-310.

[Matheron 1965] G. Matheron, Les Variables Regionalisées et leur Estimation, Masson, Paris, 1965.

[Matheron 1975] G. Matheron, Random sets and integral geometry, Wiley, New York, 1975.

[Morgan 1998] F. Morgan, Geometric measure theory: a beginner's guide, Academic Press, San Diego, CA, 1998.

[Plank and Sleeman 2004] M. J. Plank and B. D. Sleeman, "Lattice and non-lattice models of tumour angiogenesis", Bull. Math. Biol. 66:6 (2004), 1785-1819.

[Stéphanou et al. 2006] A. Stéphanou, S. R. McDougall, A. R. A. Anderson, and M. A. J. Chaplain, "Mathematical modelling of the influence of blood rheological properties upon adaptative tumourinduced angiogenesis", Math. Comput. Modelling 44:1-2 (2006), 96-123.

[Ubukata 2003] T. Ubukata, "Computer modeling of microscopic features of molluscan shells", pp. 355-367 in Morphogenesis and pattern formation in biological systems: experiments and models, edited by T. Sekimura et al., Springer, Tokyo, 2003.

[Vladimirov 1979] V. S. Vladimirov, Generalized functions in mathematical physics, Mir, Moscow, 1979.

[Zähle 1982] M. Zähle, "Random processes of Hausdorff rectifiable closed sets", Math. Nachr. 108 (1982), 49-72.

Received 7 Jun 2016. Revised 4 Sep 2016. Accepted 24 Oct 2016.

VINCENZO CAPASSO: vincenzo. capasso@unimi.it

ADAMSS and Department of Mathematics, University of Milan, Via Saldini 50, I-20133 Milano, Italy

FRANCO FLANDOLI: flandoli@dma.unipi.it

Department of Mathematics, University of Pisa, Largo Pontecorvo 5, I-56127 Pisa, Italy 


\title{
A NOTE ON GIBBS AND MARKOV RANDOM FIELDS WITH CONSTRAINTS AND THEIR MOMENTS
}

\author{
Alberto Gandolfi AND Pietro LenARdA
}

This paper focuses on the relation between Gibbs and Markov random fields, one instance of the close relation between abstract and applied mathematics so often stressed by Lucio Russo in his scientific work.

We start by proving a more explicit version, based on spin products, of the Hammersley-Clifford theorem, a classic result which identifies Gibbs and Markov fields under finite energy. Then we argue that the celebrated counterexample of Moussouris, intended to show that there is no complete coincidence between Markov and Gibbs random fields in the presence of hard-core constraints, is not really such. In fact, the notion of a constrained Gibbs random field used in the example and in the subsequent literature makes the unnatural assumption that the constraints are infinite energy Gibbs interactions on the same graph. Here we consider the more natural extended version of the equivalence problem, in which constraints are more generally based on a possibly larger graph, and solve it.

The bearing of the more natural approach is shown by considering identifiability of discrete random fields from support, conditional independencies and corresponding moments. In fact, by means of our previous results, we show identifiability for a large class of problems, and also examples with no identifiability. Various open questions surface along the way.

Personal acknowledgment. One of us (Gandolfi) learned about the theory of Gibbs and Markov random fields from Lucio Russo in a course based on [Ruelle 1978]. $\mathrm{He}$ is indebted to Lucio for his inspirational lectures and for many other things, such as an interest in percolation theory and statistical physics, a deep conviction of the close relation between abstract and applied mathematics, and an involvement in questions about the history of science.

This paper focuses on one instance of this close association between abstract and applied mathematics, namely the relation between Gibbs and Markov random fields; in spite of the great number of studies and applications of these models, this relationship has not been appropriately investigated in the literature.

\section{Communicated by Raffaele Esposito.}

MSC2010: primary 60J99, 82B20; secondary 44A60, 62B05, 62M40.

Keywords: Gibbs distributions, Markov random fields, hard-core constraints, moments,

Hammersley-Clifford, Moussouris. 


\section{Introduction}

Gibbs random fields are important models in equilibrium statistical mechanics, and Markov random fields are fundamental models in applications. They can both be defined in terms of a given graph, and they are almost two faces of the same phenomenon: Gibbs fields are defined from microscopic interactions and Markov fields by, in principle observable, conditional independencies.

Leaving the details for later, we can say in a nutshell that every Gibbs measure is Markov; the question is whether the opposite is also true. A celebrated result of Hammersley and Clifford [1971] states that with finite energy, i.e., the absence of zero probability configurations, every Markov random field is Gibbs. On the other hand, a famous example by Moussouris [1974] shows that in the presence of hard-core constraints, which is to say without the finite energy assumption, there are Markov fields which are not Gibbsian. Further studies have clarified that on a chordal graph Gibbsianity is equivalent to the global Markov property, regardless of finite energy [Lauritzen 1996], and that detailed algebraic conditions seem to be needed on nonchordal graphs [Geiger et al. 2006]. These results would seem to settle the issue.

There is, however, one weakness in this picture. When hard-core conditions, which can also be graph-based, appear in the results above, they are defined in terms of the same graph as the one used for the interactions. Such a choice seems to be justified by two simple remarks: first, one can always take the union of the two graphs, the one for interactions and the one for the hard-core conditions, as a common graph for both (as larger graphs accommodate more interactions or less conditional independence requirements); second, one can interpret the hard-core conditions as unbounded interactions, that are thus subject to the same geometrical dependence. In spite of these two remarks, however, the assumption of a unique graph is physically unwarranted; in general, the mechanisms which induce hardcore conditions are completely different from those generating interactions. For gravitational fields, for instance, the interaction is long-range while hard-core conditions can take care of the impenetrability of rigid bodies; on the other hand, in a canonical ensemble of short range interacting particles, the hard-core condition is long-range as opposed to the interaction. Even more importantly, the graph of Markov conditional independencies is naturally related to the one on which the interaction is based, which, as we just argued, has no relation to the one for hard-core conditions. Assuming a unique range for hard-core conditions and interactions hinders the more relevant relation between interactions and conditional independencies, and leads to confusing results.

The first consequence of the remarks above is the need of a more careful analysis, and of more explicit notation highlighting the importance of the graphs next to the 
notions of Markov or Gibbs; we present this in Section 2. Notice that a more careful distinction of the role of interactions and hard-core constraints already appears (albeit with less explicit notation) in the literature, chiefly in Ruelle's thermodynamic formalism [Ruelle 1978].

With the more explicit and natural identification of the graphs, it is still the case that a Gibbs random field is Markov, but the issue of whether a Markov random field on a given finite graph can always be obtained as a Gibbs field on the same graph with hard-core conditions based on a possibly larger graph falls outside the scope of past researches. In Section 5 we provide an answer to this question.

Prior to this, we give an alternative proof of the Hammersley-Clifford theorem, potentially more suitable for applications. One of the earlier proofs by Besag [1974] expresses the interaction in terms of products of spins, but works only for the binary case and has some problematic steps in the argument; the proof of Grimmett [1973], on the other hand, is valid for all finite and countable state spaces, but does not express the interaction as an explicit function of the spin values. The proof we present here expresses the interaction in terms of spin products and holds for all finite state spaces. In a sense, it exploits the fact that spin products are a basis of the interaction space. In so doing, we get an explicit calculation in terms of inverse Vandermonde matrices; we also get a more direct relation with the moments of the distribution.

Another noticeable consequence of our work is explored in Section 7, where we show that the statistical identifiability of a discrete random field by support, conditional independencies and moments can be analyzed by a combination of the moment related representation of Gibbs fields in Section 4, and of the clarification of the role of the graphs in the Markov-Gibbs relation in Section 5.

\section{Definitions}

Let $\Lambda$ be a finite set of vertices, $\Omega_{x}$ be a finite set for each $x \in \Lambda$, and $\Omega=\prod_{x \in \Lambda} \Omega_{x}$. For a subset $A \subseteq \Lambda, \omega_{A}$ is a configuration in $\Omega_{A}=\prod_{x \in A} \Omega_{x}$; the same notation is used for the restriction of a configuration $\omega \in \Omega$ to $A$. Later on, we use the notation

$$
\left[\bar{\omega}_{1}, *, \bar{\omega}_{2}, \ldots, *\right]=\left\{\omega \in \Omega: \omega_{i}=\bar{\omega}_{i}, \text { for all } i \text { such that } \bar{\omega}_{i} \neq *\right\}
$$

for cylinders.

In this paper we consider probabilities, generally denoted as $P$, on $(\Omega, \mathcal{P}(\Omega))$, where for every finite set $S, \mathcal{P}(S)$ indicates the set of all subsets of $S$.

To express the notions of interest here, we consider a graph $\mathcal{G}=(\Lambda, \mathcal{B})$ in which the set of undirected bonds is $\mathcal{B} \subseteq\{\{x, y\} \mid x \neq y, x, y \in \Lambda\}$. Given a probability $P$, we say that two sets $A, B \subseteq \Lambda$ are conditionally independent given a third set $C$, 
$A \Perp B \mid C$, if $\omega_{A}$ and $\omega_{B}$ are conditionally independent given $\omega_{C}$ for all $\omega_{S} \in \Omega_{S}$, and $S=A, B, C$.

A probability $P$ is pairwise Markov with respect to the bonds $\mathcal{B}$, or as we call it from now on, $\mathcal{B}$-pair-Markov, if

(1) for all pairs of vertices $x, y \in \Lambda$ which are not neighbors on $\mathcal{G}$, i.e., such that $\{x, y\} \notin \mathcal{B}, x \Perp y \mid(\Lambda \backslash\{x, y\})$,

and it is $\mathcal{B}$-global-Markov with respect to the bonds $\mathcal{B}$ if

(1') for all pairs of disjoint sets $A, B \subseteq \Lambda$ which are not neighbors on $\mathcal{G}$, i.e., such that there is no bond in $\mathcal{B}$ connecting a vertex of $A$ to a vertex of $B$, $A \Perp B \mid(\Lambda \backslash A \cup B)$.

$P$ is $\mathcal{B}$-pair-Markov $>$, or $\mathcal{B}$-global-Markov ${ }^{>}$, if in addition

(2) $P(\omega)>0$ for all $\omega \in \Omega$.

These are the notions of Markov probability or Markov random field generally used in the literature [Grimmett 1973; Lauritzen 1996; Geiger et al. 2006].

A $\mathcal{B}$-clique of the graph $\mathcal{G}$ is a maximal complete subgraph, possibly including single vertices, of $\mathcal{G}$. We denote by $\mathcal{C} \ell(\mathcal{B})$ the collection of subsets $A \subseteq \Lambda$ which are subsets of the vertex set of a clique of $\mathcal{G}=(\Lambda, \mathcal{B})$. A $\mathcal{B}$-interaction is a function $\phi: \cup_{A \in \mathcal{C} \ell(\mathcal{B})} \Omega_{A} \rightarrow \mathbb{R}$. Next, we consider a further collection $\mathcal{F} \subseteq \mathcal{P}(\Lambda)$ of subsets of $\Lambda$, and a (possibly empty) set $\bar{\Omega}_{A}$ of forbidden configurations for each $A \in \mathcal{F}$; notice that some authors focus on the set of allowed configurations (see [Ruelle 1978]), but our choice underlines the exceptionality of being forbidden and highlights the role of $\mathcal{F}$, as no restrictions can be imposed for sets not in $\mathcal{F}$. In greater generality, one can take as forbidden configurations those belonging to the set $\bar{\Omega}$ of zeros of a function $\rho$ defined on $\Omega$. It is convenient to deal with forbidden configurations by assigning them a probability anyway, which is then required to be zero.

A probability $P$ is Gibbs with respect to the graph $\mathcal{G}=(\Lambda, \mathcal{B})$ and the allowed configurations on $\mathcal{F}$, or $\mathcal{B}-\mathcal{F}$-Gibbs, as we call it from now on, if for all $\omega \in \Omega$,

$$
P(\omega)=\frac{1}{Z_{\phi}}\left(e^{\sum_{A \in \mathcal{C}(\mathcal{B})} \phi\left(\omega_{A}\right)} \prod_{B \in \mathcal{F}} \mathbb{q}_{\Omega_{B} \backslash \bar{\Omega}_{B}}\left(\omega_{B}\right)\right),
$$

where $\rrbracket_{S}$ the indicator function of the set $S, \phi$ is a $\mathcal{B}$-interaction, $\bar{\Omega}_{B}$ for $B \in \mathcal{F}$ is a collection of forbidden configurations, and $Z_{\phi}$ is a normalization factor. Notice that $\mathcal{B}-\varnothing$-Gibbs means that all configurations have positive probability; $\varnothing$ - $\varnothing$-Gibbs is a Bernoulli distribution; and $\varnothing-\mathcal{F}$-Gibbs is a Bernoulli distribution constrained to have some zero probabilities. Moreover, $\mathcal{B}-\Lambda$-Gibbs means that the hard-core constraints can be imposed on the entire configuration, and $\mathcal{B}-\mathcal{B}$-Gibbs indicates the fact that both interaction and hard-core constraints are assigned on configurations defined on subsets of the cliques of the same graph $(\Lambda, \mathcal{B})$. 
In abstract terms, $\phi$ and $\bar{\Omega}$ are measurable with respect to a $\sigma$-algebra of $\Omega$, but as the $\sigma$-algebra can be expressed in terms of set $\mathcal{B}$ of bonds (in the sense that the $\sigma$ algebra is the one generated by $\cup_{A \in \mathcal{C} \ell(\mathcal{B})} \Omega_{A}$ ), we focus on $\mathcal{B}$ and $\mathcal{F}$ in the notation.

Finally, we sometimes use indices to distinguish the various collection of bonds $\mathcal{B}$. In general, we indicate it by $\mathcal{B}_{m}$ if $m$ is the size of the largest clique. Notice that if $\mathcal{B} \subseteq \mathcal{B}^{\prime}$ and $\mathcal{F} \subseteq \mathcal{F}^{\prime}$, then $\mathcal{B}$-pair(global)-Markov implies $\mathcal{B}^{\prime}$-pair(global)-Markov, and $\mathcal{B}$ - $\mathcal{F}$-Gibbs implies $\mathcal{B}^{\prime}-\mathcal{F}^{\prime}$-Gibbs, so one is generally interested in the minimal such graphs and collections.

\section{Previous results and one ensuing question}

Global Markov implies pairwise Markov, but one can easily construct an example with enough configurations of zero probability showing that the opposite implication does not hold [Lauritzen 1996]. On the other hand, on some graphs there is no difference between pairwise and global Markov, as the only sets which can be separated are pairs. Such is, for instance, the graph with $\Lambda=\{1,2,3,4\}$ and $\mathcal{B}_{2}=\{(1,2),(2,3),(3,4),(4,1)\}$; this graph is used for several examples below.

$\mathcal{B}$ - $\mathcal{F}$-Gibbs implies $\mathcal{B}$-global-Markov for any $\mathcal{F}$, which then implies $\mathcal{B}$-pairMarkov. The reversed implication is given for the case in which finite energy holds by the celebrated Hammersley-Clifford theorem [1971], which in our terminology can be phrased as follows:

Theorem 3.1 (Hammersley-Clifford). Given a graph $\mathcal{G}=(\Lambda, \mathcal{B})$, a random field $P$ is $\mathcal{B}$-pair-Markov $>$ if and only if it is $\mathcal{B}-\varnothing$-Gibbs for some potential $\phi$.

There are various proofs of this result, probably starting from [Brook 1964] and the unpublished paper [Hammmersley and Clifford 1971] (see also [Grimmett 2010]). An explicit dependence of $\phi$ from the spin values appears for the binary case (i.e., $\left|\Omega_{x}\right|=2$ ) in [Besag 1974] (with some unclear steps in the proofs); a general version including countable state spaces was proven by Grimmett [1973], but without the explicit dependence of $\phi$ from the spin values. A simpler statement, in which conditional probabilities are known instead of unconditional ones, is presented in [Onural 2016] and very likely elsewhere. For completeness, we prove Theorem 3.1 once again in Section 4 below; the proof we give is for all finite $\Omega_{x}$ but with $\phi$ explicitly expressed in terms of spin products. Our results can also be indirectly obtained from [Grimmett 1973] by decomposing $\phi$ on the basis of spin products.

The Hammersley-Clifford theorem has been generalized by Lauritzen [1996] by means of chordal graphs: an undirected graph is said to be chordal if every cycle of length 4 or more has a chord.

Theorem 3.2. If the graph $\mathcal{G}=(\Lambda, \mathcal{B})$ is chordal, then a random field $P$ is $\mathcal{B}$ global-Markov if and only if it is $\mathcal{B}-\mathcal{B}$-Gibbs for some potential $\phi$. 
An algebraic interpretation and a slight generalization of these results is given in [Geiger et al. 2006].

In the opposite direction, Moussouris' counterexample [1974] shows that it is not true that every $\mathcal{B}$-global-Markov random field is $\mathcal{B}$ - $\mathcal{B}$-Gibbs. This happens necessarily on a nonchordal graph.

Example 3.3 (Moussouris). Take $\Lambda=\{1,2,3,4\}, \mathcal{B}_{2}=\{(1,2),(2,3),(3,4),(4,1)\}$ and let $P_{\mathcal{M}}$ be the uniform distribution on

$$
\begin{aligned}
& \Omega^{\prime}=\{(0,0,0,0),(1,0,0,0),(1,1,0,0),(1,1,1,0), \\
&(1,1,1,1),(0,1,1,1),(0,0,1,1),(0,0,0,1)\} .
\end{aligned}
$$

It is easily seen (and we will explicitly show a related statement in Lemma 5.2 below) that

$$
\{1\} \Perp\{3\} \mid\{2,4\} \quad \text { and } \quad\{2\} \Perp\{4\} \mid\{1,3\}
$$

so that $P_{\mathcal{M}}$ is $\mathcal{B}_{2}$-pair-Markov, and is (on this graph) also $\mathcal{B}_{2}$-global-Markov. On the other hand, $P_{\mathcal{M}}$ cannot be $\mathcal{B}_{2}-\mathcal{B}_{2}$-Gibbs; if it was so, then

$$
\begin{aligned}
P_{\mathcal{M}}(\omega) & =\frac{1}{Z} \prod_{A \in \mathcal{C} \ell\left(\mathcal{B}_{2}\right)} e^{\phi_{B}\left(\omega_{B}\right)} \prod_{B \in \mathcal{B}_{2}} \mathbb{\square}_{\Lambda \backslash \bar{\Omega}_{B}}\left(\omega_{B}\right) \\
& =c(\omega) \psi_{(1,2)}\left(\omega_{1}, \omega_{2}\right) \psi_{(2,3)}\left(\omega_{2}, \omega_{3}\right) \psi_{(3,4)}\left(\omega_{3}, \omega_{4}\right) \psi_{(4,1)}\left(\omega_{4}, \omega_{1}\right)
\end{aligned}
$$

for suitable, not necessarily nonnegative, functions $\psi_{(i, j)}$ and $c(\omega)>0$. But

$$
\begin{aligned}
& P_{\mathcal{M}}(0,1,1,0)=\psi_{(1,2)}(0,1) \psi_{(2,3)}(1,1) \psi_{(3,4)}(1,0) \psi_{(4,1)}(0,0)=0, \\
& P_{\mathcal{M}}(0,1,1,1)=\psi_{(1,2)}(0,1) \psi_{(2,3)}(1,1) \psi_{(3,4)}(1,1) \psi_{(4,1)}(1,0)=\frac{1}{8}, \\
& P_{\mathcal{M}}(0,0,0,0)=\psi_{(1,2)}(0,0) \psi_{(2,3)}(0,0) \psi_{(3,4)}(0,0) \psi_{(4,1)}(0,0)=\frac{1}{8}, \\
& P_{\mathcal{M}}(1,1,1,0)=\psi_{(1,2)}(1,1) \psi_{(2,3)}(1,1) \psi_{(3,4)}(1,0) \psi_{(4,1)}(0,1)=\frac{1}{8},
\end{aligned}
$$

are incompatible.

However, the probability in Moussouris example is $\mathcal{B}_{2}-\Lambda$-Gibbs, actually even $\varnothing$ - $\Lambda$-Gibbs: to see this, it is enough to take the uniform Bernoulli distribution $\hat{P}(\omega)=1 / Z e^{0}=1 / 2^{4}$, with $\mathcal{F}=\Lambda$ and $\bar{\Omega}_{\Lambda}=\Omega \backslash \Omega^{\prime} ;$ the graph of the hardcore constraints can actually be further reduced (see Section 6 below), although obviously not to $\mathcal{B}_{2}$. Moussouris' example is thus clearly not a counterexample to the following, more natural, question.

Question. Is every Markov random field a constrained Gibbs random field on the same graph, in the more natural sense that every $\mathcal{B}$-global-Markov random field is $\mathcal{B}-\Lambda$-Gibbs?

We take over this issue in Section 5 below. 


\section{Markov-Gibbs equivalence with no constraints: an explicit Hammersley-Clifford theorem}

Before tackling the main question, we give a new proof of Theorem 3.1. We actually prove the following more explicit version, assuming that the possible states $\Omega_{x}$ are real numbers. It amounts to an explicit expansion of the interaction on the basis of spin products.

Lemma 4.1. Let $\mathcal{G}=(\Lambda, \mathcal{B})$ be a given finite graph; $\Omega=\prod_{x \in \Lambda} \Omega_{x}$ for finite $\Omega_{x} \subseteq \mathbb{R} ;$ and $P$ be a $\mathcal{B}$-pair-Markov ${ }^{>}$random field on $(\Omega, \mathcal{P}(\Omega))$.

Next, let $\widetilde{\Omega}_{x}=\left\{0,1, \ldots,\left|\Omega_{x}\right|-1\right\} ; \widetilde{\Omega}=\prod_{x \in \Lambda} \widetilde{\Omega}_{x} ;$ and for $\sigma \in \widetilde{\Omega}$ and $\omega \in \Omega$, let $\omega^{\sigma}:=\prod_{x \in \Lambda} \omega_{x}^{\sigma_{x}}$ (with the convention $0^{0}=1$, if needed). Moreover, let $\widetilde{\Omega}(\mathcal{B})$ be the set of $\sigma$ such that $\left\{x: \sigma_{x} \neq 0\right\}$ is contained in a clique of $\mathcal{B}$. Then

$$
P(\omega)=\frac{1}{Z} e^{\sum_{\sigma \in \tilde{\Omega}(\mathcal{B})} J_{\sigma} \omega^{\sigma}}
$$

with

$$
J_{\sigma}=\sum_{\omega \in \Omega} V_{\sigma, \omega}^{-1} \log P(\omega),
$$

where $V_{\sigma, \omega}^{-1}=\prod_{x \in \Lambda} V_{\sigma_{x}, \omega_{x}}^{-1}(x)$ and $V_{\sigma_{x}, \omega_{x}}^{-1}(x)$ is the element in position $\left(\sigma_{x}, \omega_{x}\right)$ of the inverse $V^{-1}(x)$ of the Vandermonde matrix $V(x)=\left(r^{s}\right)_{r \in \Omega_{x}, s \in \widetilde{\Omega}_{x}}$.

Proof. The Vandermonde matrix is invertible as long as the elements of $\Omega_{x}$ are all different [Macon and Spitzbart 1958]. Next, for each $\omega \in \Omega$,

$$
\begin{aligned}
e^{\sum_{\sigma \in \tilde{\Omega}} J_{\sigma} \omega^{\sigma}} & =e^{\sum_{\sigma \in \tilde{\Omega}} \omega^{\sigma} \sum_{\bar{\omega} \in \Omega} V_{\sigma, \bar{\omega}}^{-1} \log P(\bar{\omega})} \\
& =e^{\sum_{\bar{\omega} \in \Omega} \log P(\bar{\omega}) \sum_{\sigma \in \tilde{\Omega}} \prod_{x \in \Lambda} \omega_{x}^{\sigma_{x}} V_{\sigma_{x}, \bar{\omega}_{x}}^{-1}(x)} \\
& =e^{\sum_{\bar{\omega} \in \Omega} \log P(\bar{\omega}) \prod_{x \in \Lambda}\left(\sum_{\sigma_{x} \in \tilde{\Omega}_{x}} \omega_{x}^{\sigma_{x}} V_{\sigma_{x}, \bar{\omega}_{x}}^{-1}(x)\right)} \\
& =e^{\sum_{\bar{\omega} \in \Omega} \log P(\bar{\omega}) \prod_{x \in \Lambda} \delta_{\omega_{x}, \bar{\omega}_{x}}} \\
& =e^{\log P(\omega)}=P(\omega),
\end{aligned}
$$

which gives (2) but with $\widetilde{\Omega}$ instead of $\widetilde{\Omega}(\mathcal{B})$. We now need to show that if $x \Perp y \mid$ $(\Lambda \backslash\{x, y\})$, then $J_{\sigma}=0$ for all $\sigma$ such that $\sigma_{x} \sigma_{y} \neq 0$; it would then follow that for all $\sigma \in \widetilde{\Omega}$ such that $J_{\sigma} \neq 0$ we have that $\left\{x: \sigma_{x} \neq 0\right\}$ is contained in a clique of $\mathcal{G}$, as required. Indeed, if $x \Perp y \mid(\Lambda \backslash\{x, y\})$ then for all $\omega_{x} \in \Omega_{x}, \omega_{y} \in \Omega_{y}$, $\omega_{\backslash x, y} \in \Omega_{\backslash x, y}=\prod_{z \in \Lambda \backslash\{x, y\}} \Omega_{z}$ and $\omega=\left(\omega_{x} \omega_{y} \omega_{\backslash x, y}\right)$ we have

$$
P\left(\omega_{x} \omega_{\backslash x, y}\right) P\left(\omega_{y} \omega_{\backslash x, y}\right)=P\left(\omega_{\backslash x, y}\right) P\left(\omega_{x} \omega_{y} \omega_{\backslash x, y}\right),
$$

i.e.,

$\log P(\omega)=\log P\left(\omega_{x} \omega_{y} \omega_{\backslash x, y}\right)=\log P\left(\omega_{x} \omega_{\backslash x, y}\right)+\log P\left(\omega_{y} \omega_{\backslash x, y}\right)-\log P\left(\omega_{\backslash x, y}\right)$.

Therefore, if $\sigma$ is such that $\sigma_{x} \sigma_{y} \neq 0$, we have 


$$
\begin{aligned}
& J_{\sigma}=\sum_{\omega \in \Omega} V_{\sigma, \omega}^{-1} \log P(\omega) \\
& =\sum_{\omega_{\backslash x, y} \in \Omega_{\backslash x, y}} \prod_{z \in \Lambda \backslash\{x, y\}} V_{\sigma_{z}, \omega_{z}}^{-1}(z) \sum_{\omega_{x} \in \Omega_{x}} \sum_{\omega_{y} \in \Omega_{y}} V_{\sigma_{x}, \omega_{x}}^{-1}(x) V_{\sigma_{y}, \omega_{y}}^{-1}(y) \\
& \left(\log P\left(\omega_{x} \omega_{\backslash x, y}\right)+\log P\left(\omega_{y} \omega_{\backslash x, y}\right)-\log P\left(\omega_{\backslash x, y}\right)\right),
\end{aligned}
$$

which vanishes for the following reason: We have

$$
\sum_{\omega_{x} \in \Omega_{x}} V_{\sigma_{x}, \omega_{x}}^{-1}(x)=\sum_{\omega_{x} \in \Omega_{x}} V_{\sigma_{x}, \omega_{x}}^{-1}(x) 1=\left[V^{-1}(x) V(x)\right]_{\left(\sigma_{x}, 1\right)}=\delta_{\sigma_{x}, 0}
$$

since the first column of $V(x)$ is constantly equal to 1 ; here, $[A]_{i, j}$ denotes the element $\{i, j\}$ of the matrix $A$. This way, if $\sigma_{x} \sigma_{y} \neq 0$ then both $\sum_{\omega_{x} \in \Omega_{x}} V_{\sigma_{x}, \omega_{x}}^{-1}(x)=0$ and $\sum_{\omega_{y} \in \Omega_{y}} V_{\sigma_{y}, \omega_{y}}^{-1}(y)=0$, so we always get 0 for the right-hand side of (3) by taking the last two sums in the appropriate order.

It follows that if $x$ and $y$ are conditionally independent, then $J_{\sigma}=0$ unless $\sigma_{x} \sigma_{y}=0$. This implies that all bonds between vertices in $\left\{x: \sigma_{x} \neq 0\right\}$ belong to $\mathcal{G}$, and hence $\left\{x: \sigma_{x} \neq 0\right\}$ is contained in a clique of $\mathcal{G}$. Therefore, only $\sigma \in \widetilde{\Omega}(\mathcal{B})$ appear in (2), and the result is proven.

Proof of Theorem 3.1. One direction is proven by Lemma 4.1. For the converse, if $P$ is $\mathcal{B}$ - $\varnothing$-Gibbs then $P(\omega)=1 / Z_{\phi}\left(e^{\sum_{A \in \mathcal{C}(\mathcal{B})} \phi\left(\omega_{A}\right)}\right)$ and if $A, B \subseteq \Lambda$ are two disjoint sets which are not neighbors on $\mathcal{G}$ then the probability factorizes, hence $A \Perp B \mid(\Lambda \backslash A \cup B)$.

The interaction thus identified is unique, except for the value of $J_{\sigma(0)}$, where $\sigma(0)$ denotes the configuration such that $(\sigma(0))_{x}=0$ for all $x \in \Lambda$.

Lemma 4.2. If $P(\omega)=\frac{1}{Z} e^{\sum_{\sigma \in \widetilde{\Omega}} J_{\sigma} \omega^{\sigma}}$, then

$$
\sum_{\omega \in \Omega} V_{\sigma, \omega}^{-1} \log P(\omega)=J_{\sigma} \text { for all } \sigma \neq \sigma(0) .
$$

Proof. For $\sigma \neq \sigma(0)$,

$$
\begin{aligned}
& \sum_{\omega \in \Omega} V_{\sigma, \omega}^{-1} \log P(\omega) \\
& =\sum_{\omega \in \Omega} V_{\sigma, \omega}^{-1}\left(\log e^{\sum_{\bar{\sigma} \in \tilde{\Omega}} J_{\bar{\sigma}} \omega^{\bar{\sigma}}}-\log Z\right)=\sum_{\omega \in \Omega} V_{\sigma, \omega}^{-1}\left(\sum_{\bar{\sigma} \in \widetilde{\Omega}} J_{\bar{\sigma}} \omega^{\bar{\sigma}}-\log Z\right) \\
& =\sum_{\bar{\sigma} \in \widetilde{\Omega}} J_{\bar{\sigma}} \sum_{\omega \in \Omega} V_{\sigma, \omega}^{-1} \omega^{\bar{\sigma}}-\sum_{\omega \in \Omega} V_{\sigma, \omega}^{-1} \log Z \\
& =\sum_{\bar{\sigma} \in \widetilde{\Omega}} J_{\bar{\sigma}} \sum_{\omega \in \Omega} \prod_{x \in \Lambda} V_{\sigma_{x}, \omega_{x}}^{-1}(x) \omega_{x}^{\bar{\sigma}_{x}}-(\log Z) \delta_{\sigma=\sigma(0)} \\
& =\sum_{\bar{\sigma} \in \widetilde{\Omega}} J_{\bar{\sigma}} \prod_{x \in \Lambda} \sum_{\omega_{x} \in \Omega_{x}} V_{\sigma_{x}, \omega_{x}}^{-1}(x) \omega_{x}^{\bar{\sigma}_{x}}=\sum_{\bar{\sigma} \in \widetilde{\Omega}} J_{\bar{\sigma}} \prod_{x \in \Lambda} \delta_{\sigma_{x}=\bar{\sigma}_{x}}=\sum_{\bar{\sigma} \in \widetilde{\Omega}} \delta_{\sigma=\bar{\sigma}} J_{\bar{\sigma}}=J_{\sigma} .
\end{aligned}
$$




\section{Markov-Gibbs with hard-core constraints}

We go back to our Question formulated on page 412 and answer it.

For a probability $P$ on some $(\Lambda, \mathcal{P}(\Lambda)), \hat{P}$ is a strictly positive extension of $P$ if $\hat{P}(\omega)>0$ for all $\omega \in \Omega$, and

$$
\hat{P}(\omega)=\frac{1}{\hat{Z}} P(\omega),
$$

for some constant $\hat{Z}$, for all $\omega \in \Omega$ for which $P(\omega)>0$.

Lemma 5.1. On a graph $\mathcal{G}=(\Lambda, \mathcal{B})$, a probability $P$ is $\mathcal{B}-\Lambda$-Gibbs if and only if it has a $\mathcal{B}$-global-Markov strictly positive extension $\hat{P}$.

Proof. If $P$ is $\mathcal{B}-\Lambda$-Gibbs, then define $\hat{P}(\omega):=1 /(Z(\hat{P})) e^{\sum_{A \in \mathcal{C} \ell(\mathcal{B})} \phi\left(\omega_{A}\right)}$, for a suitable constant $Z(\hat{P})$, which is $\mathcal{B}-\varnothing$-Gibbs (and hence $\mathcal{B}$-global-Markov). Moreover, $\hat{P}$ is strictly positive; if $P(\omega)>0$ then $P(\omega)=1 / Z_{\phi}\left(e^{\sum_{A \in \mathcal{C} \ell(\mathcal{B})} \phi\left(\omega_{A}\right)}\right)=$ $\left(Z(\hat{P}) / Z_{\phi}\right) \hat{P}(\omega)$ so that (4) holds with $\hat{Z}=Z(\hat{P}) / Z_{\phi}$.

Vice versa, if $P$ has a $\mathcal{B}$-global-Markov strictly positive extension $\hat{P}$, then by the Hammersley-Clifford Theorem, $\hat{P}$ is a $\mathcal{B}-\varnothing$-Gibbs random field, i.e., $\hat{P}(\omega)=$ $1 /(Z(\hat{P}))\left(e^{\sum_{A \in \mathcal{C} \ell(\mathcal{B})} \phi\left(\omega_{A}\right)}\right)$ for some suitable $\phi$. By (4),

$$
P(\omega)=\hat{Z} \frac{1}{Z(\hat{P})}\left(e^{\sum_{A \in \mathcal{C}(\mathcal{B})} \phi\left(\omega_{A}\right)} \prod_{B \in \mathcal{F}} \mathbb{\Omega}_{\Omega_{B} \backslash \bar{\Omega}_{B}}\left(\omega_{B}\right)\right),
$$

which is $\mathcal{B}-\Lambda$-Gibbs with $Z_{\phi}=Z(\hat{P}) / \hat{Z}$.

Our Question has a negative answer.

Lemma 5.2. There is a graph $\mathcal{G}=(\Lambda, \mathcal{B})$ and a $\mathcal{B}$-global-Markov random field (with hard-core constraints) which is not $\mathcal{B}-\Lambda$-Gibbs.

Proof. In fact, we can take the same graph as Moussouris, with bond set $\mathcal{B}_{2}$. As support of the probability we take

$$
\begin{aligned}
\Omega^{\prime \prime}=\{(1,1,1,1),(0,1,1,1),(1,0,1,1),(0,0,1,1), \\
(1,1,0,0),(1,0,0,0),(0,1,0,0),(0,0,0,0)\} ;
\end{aligned}
$$

hence, we assume that all configurations in $\bar{\Omega}=\Omega \backslash \Omega^{\prime \prime}$ have zero probability: this is a $\mathcal{F}=\Lambda$ (or possibly a subgraph, see the next section below) constraint. This time, however, the probability $P_{*}$ is taken as follows: $P_{*}(1,1,1,1)=\frac{2}{9}$, and $P_{*}(\omega)=\frac{1}{9}$ for all other $\omega \in \Omega^{\prime \prime} \backslash\{(1,1,1,1)\}$.

We first show that

$$
\{1\} \Perp\{3\} \mid\{2,4\} \quad \text { and } \quad\{2\} \Perp\{4\} \mid\{1,3\} .
$$


For each $x, y \in \Lambda$, if $\bar{\omega}=\omega_{\Lambda \backslash\{x, y\}}$ has nonzero probability, then $x \Perp y \mid \Lambda \backslash\{x, y\}$ under a probability $P$ is equivalent to

$$
\begin{aligned}
& P\left(\omega_{x}=1, \omega_{y}=1, \omega_{\Lambda \backslash\{x, y\}}=\bar{\omega}\right) P\left(\omega_{x}=0, \omega_{y}=0, \omega_{\Lambda \backslash\{x, y\}}=\bar{\omega}\right) \\
&=P\left(\omega_{x}=1, \omega_{y}=0, \omega_{\Lambda \backslash\{x, y\}}=\bar{\omega}\right) P\left(\omega_{x}=0, \omega_{y}=1, \omega_{\Lambda \backslash\{x, y\}}=\bar{\omega}\right),
\end{aligned}
$$

as easily seen by elementary calculations [Moussouris 1974; Lauritzen 1996]. To verify the claimed conditional independencies we the have to verify the following equalities [Lauritzen 1996; Geiger et al. 2006], in which $x=1, y=3$ in the first four equalities, and $x=2, y=4$ in the others:

$$
\begin{aligned}
\text { (I) } & P(1,1,1,1) P(0,1,0,1)=P(0,1,1,1) P(1,1,0,1), \\
(I I) & P(1,0,1,1) P(0,0,0,1)=P(0,0,1,1) P(1,0,0,1), \\
(I I I) & P(1,1,1,0) P(0,1,0,0)=P(0,1,1,0) P(1,1,0,0), \\
(I V) & P(1,0,1,0) P(0,0,0,0)=P(0,0,1,0) P(1,0,0,0), \\
(V) & P(1,1,1,1) P(1,0,1,0)=P(1,0,1,1) P(1,1,1,0), \\
(V I) & P(0,1,1,1) P(0,0,1,0)=P(0,0,1,1) P(0,1,1,0), \\
(V I I) & P(1,1,0,1) P(1,0,0,0)=P(1,0,0,1) P(1,1,0,0), \\
(V I I I) & P(0,1,0,1) P(0,0,0,0)=P(0,0,0,1) P(0,1,0,0),
\end{aligned}
$$

with none having four zero values, so that the condition $P(\bar{\omega})>0$ is valid. These relations are easily seen to hold for $P_{*}$, as in each row there is exactly one configuration in $\bar{\Omega}$ on the right-hand side and one on the left-hand side of the equality. So, $P_{*}$ is $\mathcal{B}_{2}$-pair-Markov; since on this graph the two notions coincide, $P_{*}$ is also $\mathcal{B}_{2}$-global-Markov.

We now verify that $P_{*}$ does not admit a $\mathcal{B}_{2}$-global-Markov strictly positive extension so that it cannot be $\mathcal{B}_{2}-\Lambda$-Gibbs. If such extension $\hat{P}$ existed, then all the above equalities would have to hold for $\hat{P}$ as well, as it would have to be $\mathcal{B}_{2}$ global-Markov; since $\hat{P}$ is an extension of $P_{*}$, it would have to be $\hat{P}(\omega) / \hat{P}\left(\omega^{\prime}\right)=$ $P_{*}(\omega) / P_{*}\left(\omega^{\prime}\right)$ for all $\omega, \omega^{\prime} \in \Omega^{\prime \prime}$. From the first equality above we would have

$$
\frac{\hat{P}(0,1,0,1)}{\hat{P}(1,1,0,1)}=\frac{\hat{P}(0,1,1,1)}{\hat{P}(1,1,1,1)}=\frac{P_{*}(0,1,1,1)}{P_{*}(1,1,1,1)}=\frac{1}{2} .
$$

On the other hand, from the seventh equality

$$
\frac{\hat{P}(1,1,0,1)}{\hat{P}(1,0,0,1)}=\frac{\hat{P}(1,1,0,0)}{\hat{P}(1,0,0,0)}=\frac{P_{*}(1,1,0,0)}{P_{*}(1,0,0,0)}=1,
$$

or $\hat{P}(1,1,0,1)=\hat{P}(1,0,0,1)$; the second and eighth equality would give

$$
\hat{P}(1,0,0,1)=\hat{P}(0,0,0,1) \quad \text { and } \quad \hat{P}(0,0,0,1)=\hat{P}(0,1,0,1),
$$


respectively. Hence, it would be $\hat{P}(1,1,0,1)=\hat{P}(0,1,0,1)$, which would be a contradiction with (6) above.

\section{Examples of minimal graphs}

Given a graph $\mathcal{G}=(\Lambda, \mathcal{B})$, it would be interesting to classify $\mathcal{B}$-global-Markov or $\mathcal{B}$-pair-Markov random fields $P$ in terms of the minimal graphs $\mathcal{B}^{\prime}=\mathcal{B}^{\prime}(\Omega, \mathcal{B}, P)$ and $\mathcal{B}^{\prime \prime}=\mathcal{B}^{\prime \prime}(\Omega, \mathcal{B}, P)$ so that $P$ is $\mathcal{B}^{\prime}-\mathcal{B}^{\prime \prime}$-Gibbs; this problem can be given an explicit algebraic form, following the lines of [Geiger et al. 2006]. We have not been able to develop relevant results in this direction though, and therefore we limit ourselves to a review of the previous examples from this point of view.

Example 6.1 (minimal graphs in Moussouris' example). $\bar{\Omega}=\Omega \backslash \Omega^{\prime}$ is measurable with respect to the $\sigma$-algebra generated by the cylinders

$$
[10 * 1],[01 * 0],[* 101],[* 010],
$$

where we use the previously introduced notation for cylinders. Therefore, a minimal collection $\mathcal{F}$ is $\{1,2,4\},\{2,3,4\}$, which corresponds to the graph with bonds $\mathcal{B}_{3}=\mathcal{B}_{2} \cup\{\{2,4\}\}$. As we already observed, to define the uniform probability we can take bonds $\mathcal{B}_{0}=\varnothing$; hence, $P_{\mathcal{M}}$ is $\mathcal{B}_{2}$-global-Markov and $\mathcal{B}_{0}$ - $\mathcal{B}_{3}$-Gibbs.

We see now that even changing the probability in Moussouris' example would not have yielded a counterexample to our Question.

Example 6.2 (minimal graphs in a modified Moussouris example). Consider the same graph with bonds $\mathcal{B}_{2}$ and $\Omega^{\prime}$ as in Moussouris' example, but with any probability $P$ strictly positive on $\Omega^{\prime}$. We can construct a $\mathcal{B}_{2}$-global-Markov strictly positive extension $\hat{P}$ as follows. Start from some configuration ([0101] for instance), let $\hat{P}(0,1,0,1)=c$, and notice that the conditions for $\mathcal{B}$-global-Markov are those in (5). From relation $(I)$, any $\mathcal{B}_{2}$-global-Markov strictly positive extension has

$$
\hat{P}(1,1,0,1)=\frac{P(1,1,1,1)}{P(0,1,1,1)} c ;
$$

next, from equality (VII), one gets

$$
\hat{P}(1,0,0,1)=\frac{P(1,0,0,0)}{P(1,1,0,0)} \hat{P}(1,1,0,1)=\frac{P(1,0,0,0)}{P(1,1,0,0)} \frac{P(1,1,1,1)}{P(0,1,1,1)} c ;
$$

recursively, we get all probabilities as function of $c$, and finally $c$ from normalization. This generates an extension $\hat{P}$ of $P$. We know $\hat{P}$ is strictly positive as $P$ was strictly positive on $\Omega^{\prime}$ and the above operations preserve positivity; furthermore, it is $\mathcal{B}_{2}$-global-Markov as the relations in (5) are all valid for $\hat{P}$ as well. As such, the Hammersley-Clifford theorem applies to $\hat{P}$, which is then $\mathcal{B}_{2}-\varnothing$-Gibbs. We have seen in Example 6.1 that the constraints are generated by the graphs with bonds $\mathcal{B}_{3}$; 
therefore, any absolutely continuous modification $P$ of Moussouris' example is $\mathcal{B}_{2}$ $\mathcal{B}_{3}$-Gibbs.

Example 6.3 (minimal graphs for $P_{*}$ ). The configurations in $\bar{\Omega}=\Omega \backslash \Omega^{\prime \prime}$ are generated by the cylinders

$$
[* * 10],[* * 01]
$$

hence, a minimal collection $\mathcal{F}$ is $\{3,4\}$, which corresponds to the graph with bonds $\mathcal{B}_{2}^{\prime}=\{\{3,4\}\}$.

Next, observe that is not possible to express the probability in terms of a $\mathcal{B}_{2}$ Markov probability, as otherwise $P_{*}$ would be $\mathcal{B}_{2}-\mathcal{B}_{2}$-Gibbs, and we know from Lemma 5.2 that it is not. On the other hand, as on $\mathcal{B}_{3}$ pairwise Markov is the same as global Markov, and $\mathcal{B}_{3}$ produces a separable graph, the result in [Lauritzen 1996] implies that $P_{*}$ is $\mathcal{B}_{3}-\mathcal{B}_{3}$-Gibbs. Hence, $P_{*}$ is certainly factorizable on $\mathcal{B}_{3}$. A simple calculation shows that all such factorizations have interactions

$$
\begin{aligned}
J_{\{3\}} & =x_{3}, & J_{\{4\}} & =x_{4}, \\
J_{\{4,1\}} & =\log \frac{3}{2}, & J_{\{3,4\}} & =-\left(x_{3}+x_{4}\right), \\
\{1,2,3\} & =\log 2, & J_{\{3,4,1\}} & =\log \frac{2}{3},
\end{aligned}
$$

and $J_{A}=0$ for all other sets $A$. Therefore, taking

$$
\mathcal{B}_{3}^{\prime}=\{\{3\},\{4\},\{3,4\},\{4,1\},\{1,2,3\},\{3,4,1\}\},
$$

we have that $P_{*}$ is $\mathcal{B}_{3}^{\prime}-\mathcal{B}_{3}$-Gibbs. As we have already noticed that the hard-core constraints are generated by $\mathcal{B}_{2}^{\prime}$, we have that $P_{*}$ is $\mathcal{B}_{3}^{\prime}-\mathcal{B}_{2}^{\prime}$-Gibbs.

Back to the question and notation at the beginning of this section, it is not easy at this point to elaborate on the relationship between $\mathcal{B}$ and $\mathcal{B}^{\prime}$ and $\mathcal{B}^{\prime \prime}$. For instance, in all the previous examples, one of the two graphs $\mathcal{B}^{\prime}$ or $\mathcal{B}^{\prime \prime}$ was always contained in $\mathcal{B}$; but not even this holds in general.

Example 6.4. Consider $\Lambda$ as in the examples above, and identical copies $\Lambda(1)$ and $\Lambda(2)$; in each copy consider a copy $\mathcal{B}_{2}(1)$ and $\mathcal{B}_{2}(2)$, respectively, of the edges in $\mathcal{B}_{2}$, in each copy between the appropriate vertices. The graph we consider is then $\mathcal{G}=\left(\Lambda(1) \cup \Lambda(2), \mathcal{B}_{2}(1) \cup \mathcal{B}_{2}(2)\right)$. The configuration space is $\Omega=\Omega(1) \times \Omega(2)$, where $\Omega(i)$ is the copy over $\Lambda(i)$ of the configurations of $\{0,1\}^{\Lambda}$. The probability $P$ is taken to be the product $P=P_{\mathcal{M}} \times P_{*}$. It is easily seen that $P$ is $\left(\mathcal{B}_{2}(1) \cup\right.$ $\mathcal{B}_{2}(2)$ )-global-Markov. On the other hand, the hard-core constraints are generated by cylinders in Examples 6.1 and 6.3 in the two copies, so that the graph of hardcore conditions is $\mathcal{B}_{3}^{\prime \prime}=\mathcal{B}_{3}(1) \cup \mathcal{B}_{2}^{\prime}(2)$. However, the interactions are generated by the graph $\mathcal{B}_{3}^{\prime \prime \prime}=\mathcal{B}_{0}(1) \cup \mathcal{B}_{3}^{\prime}(2)$. So, altogether, $P$ is $\left(\mathcal{B}_{2}(1) \cup \mathcal{B}_{2}(2)\right)$-global-Markov and $\mathcal{B}_{3}^{\prime \prime}-\mathcal{B}_{3}^{\prime \prime \prime}$-Gibbs, but neither $\mathcal{B}_{3}^{\prime \prime}$ nor $\mathcal{B}_{3}^{\prime \prime \prime}$ are contained in $\mathcal{B}_{2}(1) \cup \mathcal{B}_{2}(2)$. 


\section{Identifiability of statistical models by support, moments and conditional independencies}

As an application of our results, we turn to an identifiability problem in statistics. Suppose that of a discrete random field $P$ on the configuration space $\Omega_{\Lambda}$, for some finite set $\Lambda$, we have observations that determine the support of the distribution, the pairwise (or global) conditional independencies, leading to a dependency graph $\mathcal{G}=(\Lambda, \mathcal{B})$ with bonds $\mathcal{B}$, and finally the collection of moments determined by the cliques of $\mathcal{B}$, that is, all moments

$$
m_{\sigma}=E_{P}\left(\omega^{\sigma}\right)
$$

where $\sigma \in \tilde{\Omega}$ is such that $\left\{x: \sigma_{x} \neq 0\right\}$ is contained in a clique of $\mathcal{B}$. If (8) holds then we say that $P$ satisfies the $\mathcal{B}$-moments.

We start by combining the results of Sections 4 and 5 to show that these are, in general, sufficient statistics to determine the distribution. Later we verify, however, that the counterexample of Lemma 5.2 leads to exceptional cases in which identifiability by the above statistics breaks down.

The result about identifiability is given in two steps. First, we assume that $P$ is known to $\mathcal{B}$ - $\mathcal{F}$-Gibbs for some $\mathcal{F}$.

Lemma 7.1. Given $\mathcal{B}, \mathcal{F}$, the $\bar{\Omega}_{A}$ for $A \in \mathcal{F}$, and $\mathcal{B}$-moments $m_{\sigma}$, there is at most one $\mathcal{B}-\mathcal{F}$-Gibbs random field $P$ satisfying the $\mathcal{B}$-moments.

Proof. Suppose there are two $\mathcal{B}-\mathcal{F}$-Gibbs random fields $P$ and $P^{\prime}$ satisfying the $\mathcal{B}$ moments. Each has a $\mathcal{B}$-global-Markov strictly positive extension by Lemma 5.1, which can be expressed as in Lemma 4.1 with interactions $J$ and $J^{\prime}$, respectively. Now consider

$$
f(t)=E_{P(t)}\left(\sum_{\sigma \in \tilde{\Omega}(\mathcal{B})} \omega^{\sigma}\left(J_{\sigma}^{\prime}-J_{\sigma}\right)\right),
$$

where $P(t)=\frac{1}{Z} e^{\sum_{\sigma \in \tilde{\Omega}(\mathcal{B})} \omega^{\sigma}\left(J_{\sigma}+t\left(J_{\sigma}^{\prime}-J_{\sigma}\right)\right)}$ for $t \in[0,1]$. We have

$$
f(0)=\sum_{\sigma \in \tilde{\Omega}(\mathcal{B})} E_{P(0)}\left(\omega^{\sigma}\right)\left(J_{\sigma}^{\prime}-J_{\sigma}\right)=\sum_{\sigma \in \tilde{\Omega}(\mathcal{B})} E_{P(1)}\left(\omega^{\sigma}\right)\left(J_{\sigma}^{\prime}-J_{\sigma}\right)=f(1)
$$

by equality of $\mathcal{B}$-moments. Moreover, $f^{\prime}(t)=\operatorname{Var}_{P_{t}}\left(\sum_{\sigma \in \tilde{\Omega}(\mathcal{B})} \omega^{\sigma}\left(J_{\sigma}^{\prime}-J_{\sigma}\right)\right) \geq 0$. Combined with $f(0)=f(1)$ this implies $f^{\prime}(t)=0$ for all $t \in[0,1]$. Hence, $0=$ $f^{\prime}(0)=\operatorname{Var}_{P_{0}}\left(\sum_{\sigma \in \tilde{\Omega}(\mathcal{B})} \omega^{\sigma}\left(J_{\sigma}^{\prime}-J_{\sigma}\right)\right) \geq 0$, which implies $\sum_{\sigma \in \tilde{\Omega}(\mathcal{B})} \omega^{\sigma}\left(J_{\sigma}^{\prime}-J_{\sigma}\right) \equiv 0$ and that the two extensions of $P$ and $P^{\prime}$ coincide. This implies that also the two random fields coincide.

Theorem 7.2. Let $\mathcal{B}$ and $\mathcal{B}$-moments $m_{\sigma}$ be given. 
(1) If finite energy holds then there is at most one $\mathcal{B}$-global-Markov random field $P$ satisfying the $\mathcal{B}$-moments; in particular, $P$ is $\mathcal{B}-\varnothing$-Gibbs.

(2) If $\mathcal{G}=(\Lambda, \mathcal{B})$ is chordal then there is at most one $\mathcal{B}$-global-Markov random field $P$ satisfying the $\mathcal{B}$-moments; in particular, $P$ is $\mathcal{B}-\mathcal{B}$-Gibbs.

Proof. (1) If a random field is $\mathcal{B}$-global-Markov and completely positive then it is $\mathcal{B}-\varnothing$-Gibbs by Theorem 3.1, and uniqueness follows from Lemma 7.1.

(2) If $\mathcal{G}=(\Lambda, \mathcal{B})$ is chordal then $P$ is $\mathcal{B}-\mathcal{B}$-Gibbs by Theorem 3.2, hence uniqueness follows from Lemma 7.1.

Identifiability can break down. We first observe that in Moussouris' example identifiability still holds, and then show that it does not hold for $P_{*}$ in Lemma 5.2.

Example 7.3. $\mathcal{B}_{2}$-moments in Moussouris' example are $E\left(\omega_{i}\right)=\frac{1}{2}, i=1, \ldots, 4$; $E\left(\omega_{i} \omega_{i+1}\right)=\frac{3}{8}, i=1, \ldots, 3 ; E\left(\omega_{4} \omega_{1}\right)=\frac{1}{8}$. With some algebra one can see that if a random field $P$ satisfies these $\mathcal{B}_{2}$-moments then

$$
\begin{aligned}
P(1,0,1,1)=-P(0,0,1,0)-P(0,1,0,0)-P(0,1,0,1) & \\
& -P(0,1,1,0)-P(1,0,0,1)-P(1,0,1,0)-P(1,1,0,1),
\end{aligned}
$$

which implies that $\Omega^{\prime}$ is the support of $P$; some further algebra shows that the other linear relations imply then that $P$ is uniform on $\Omega^{\prime}$, so that $P=P_{\mathcal{M}}$.

Example 7.4. The random field $P_{*}$ in the proof of Lemma 5.2 has $\mathcal{B}_{2}$-moments $E\left(\omega_{i}\right)=\frac{5}{9}, i=1, \ldots, 4 ; E\left(\omega_{i} \omega_{i+1}\right)=\frac{3}{9}, i=1,2,4 ; E\left(\omega_{3} \omega_{4}\right)=\frac{5}{9}$. We see that there is not a unique random field which has the same support $\Omega^{\prime \prime}$ of $P_{*}$, is $\mathcal{B}_{2}$-global-Markov, and satisfies the above $\mathcal{B}_{2}$-moments. With a little algebra one can easily verify that every random field $P$ with support in $\Omega^{\prime \prime}$ and

$$
\begin{array}{lll}
P(1,0,1,1)=\frac{1}{3}-\lambda, & P(1,1,0,0)=\frac{1}{3}-\lambda, \quad P(1,0,0,0)=-\frac{1}{9}+\lambda, \\
P(0,1,1,1)=\frac{1}{3}-\lambda, & P(0,1,0,0)=-\frac{1}{9}+\lambda, & P(0,0,1,1)=-\frac{1}{9}+\lambda, \\
& P(0,0,0,0)=\frac{1}{3}-\lambda, &
\end{array}
$$

with $\lambda=P(1,1,1,1) \in\left[\frac{1}{9}, \frac{1}{3}\right]$, satisfies the above $\mathcal{B}_{2}$-moments and is also $\mathcal{B}_{2}$ global-Markov (as it is absolutely continuous with respect to $P_{*}$ ).

By Lemma 7.1 , there is a unique $\mathcal{B}_{2}-\Lambda$-Gibbs satisfying the above $\mathcal{B}_{2}$-moments, namely the one with $\lambda \approx 0.2119$ equal to the real root of $\lambda\left(\lambda-\frac{1}{9}\right)^{3}-\left(\frac{1}{3}-\lambda\right)^{4}=$ $-9+107 x-459 x^{2}+729 x^{3}=0$; one can get this equation directly by explicitly writing out the conditions for $P$ to be $\mathcal{B}_{2}-\Lambda$-Gibbs, or indirectly by noticing that this must be the only value of $\lambda$ which does not lead to a contradiction in (6) and (7). For all other values of $\lambda \in\left[\frac{1}{9}, \frac{1}{3}\right]$, including $\lambda=\frac{2}{9}$ as in Lemma 5.2, the random fields are not $\mathcal{B}_{2}-\Lambda$-Gibbs, so they constitute a family of counterexamples to our Question, all with the same $\mathcal{B}_{2}$-moments. 
Remarks. (1) The last example raises the question (related to that of the minimal graph mentioned in Section 6) of the minimal set of moments which can identify a $\mathcal{B}$-Markov random field with hard-core constraints.

(2) The results above about identifiability of random fields from Markov properties and moments can be interpreted as follows. $\mathcal{B}$-moment conditions identify a simplex of probability measures, as described in detail in [Pitowsky 1989] for the violation of correlation conditions in quantum mechanics. When the $\mathcal{B}$-Markov conditional independencies are added, then the resulting algebraic variety reduces to a point in the interior of the simplex, or to a (possibly nontrivial) variety contained in the boundary of the original simplex. We do not know which additional observations could guarantee uniqueness.

\section{Conclusions}

We have reviewed the Hammersley-Clifford Theorem, which states the equivalence of Markov and Gibbs random fields when there are no hard-core conditions, giving a more explicit proof than usual with the interaction expressed in terms of spin products.

We then addressed the same problem when there are hard-core constraints. We argued that the hard-core constraints are more naturally represented in terms of a separate graph from that used to determine the interactions; in this respect, the counterexample of Moussouris as well as the subsequent literature on constrained Markov random fields do not address the appropriate issues.

We have shown that even allowing the largest possible graph for the hard-core constraints, there are cases in which it is not possible to restrict the graph of the interactions to the one for the Markov conditional independence.

This, in turn, has opened the question of finding minimal graphs for the hardcore conditions and the interactions given the graph of the Markov conditional independencies. We have not been able to address this issue, but we have provided examples of minimal graphs.

Finally, we discussed the statistical identifiability of a random field in terms of support, conditional independencies and moments, with the last two requirements based on the same graph. Our proof of the Hammersley-Clifford theorem allows us to easily show identifiability if finite energy is ascertained or the graph is chordal; while our counterexample allows us to exhibit a case in which support, conditional independencies and moments do not uniquely determine the random field.

\section{References}

[Besag 1974] J. Besag, "Spatial interaction and the statistical analysis of lattice systems", J. Roy. Statist. Soc. Ser. B 36 (1974), 192-236. 
[Brook 1964] D. Brook, "On the distinction between the conditional probability and the joint probability approaches in the specification of nearest-neighbour systems", Biometrika 51 (1964), 481483.

[Geiger et al. 2006] D. Geiger, C. Meek, and B. Sturmfels, "On the toric algebra of graphical models", Ann. Stat. 34:3 (2006), 1463-1492.

[Grimmett 1973] G. R. Grimmett, "A theorem about random fields", Bull. Lond. Math. Soc. 5 (1973), 81-84.

[Grimmett 2010] G. R. Grimmett, Probability on graphs: random processes on graphs and lattices, Institute of Mathematical Statistics Textbooks 1, Cambridge University Press, 2010.

[Hammmersley and Clifford 1971] J. M. Hammmersley and P. Clifford, "Markov fields on finite graphs and lattices", 1971, www.statslab.cam.ac.uk/ grg/books/hammfest/hamm-cliff.pdf.

[Lauritzen 1996] S. L. Lauritzen, Graphical models, Oxford Statistical Science Series 17, Clarendon Press, New York, 1996.

[Macon and Spitzbart 1958] N. Macon and A. Spitzbart, "Inverses of Vandermonde matrices", Amer. Math. Monthly 65 (1958), 95-100.

[Moussouris 1974] J. Moussouris, "Gibbs and Markov random systems with constraints", J. Stat. Phys. 10 (1974), 11-33.

[Onural 2016] L. Onural, "Gibbs random fields and Markov random fields with constraints", preprint, 2016. arXiv 1603.01481

[Pitowsky 1989] I. Pitowsky, Quantum probability—quantum logic, Lecture Notes in Physics 321, Springer, Berlin, 1989.

[Ruelle 1978] D. Ruelle, Thermodynamic formalism: the mathematical structures of classical equilibrium statistical mechanics, Encyclopedia of Mathematics and its Applications 5, Addison-Wesley, Reading, MA, 1978.

Received 14 Sep 2016. Accepted 12 Jan 2017.

AlBERTO GANDOLFI: ag189@nyu.edu NYU Abu Dhabi, PO Box 129188, Abu Dhabi, United Arab Emirates and

Dipartimento di Matematica e Informatica "Ulisse Dini”, Università di Firenze, Viale Morgagni 67/a, 50134 Firenze, Italy

PIETRO LENARDA: pietro.lenarda@imtlucca.it

Multi-scale Analysis of Materials, IMT School for Advanced Studies Lucca, I-55100 Lucca, Italy 


\title{
QUANTUM MECHANICS: LIGHT AND SHADOWS (ONTOLOGICAL PROBLEMS AND EPISTEMIC SOLUTIONS)
}

\author{
Gianfausto Dell’'Antonio
}

\begin{abstract}
We discuss several problems that arise in the Copenhagen interpretation of quantum mechanics, in an attempt to come to grips with what E. T. Jaynes has called the quantum omelette. ${ }^{1}$
\end{abstract}

\section{Introduction}

In this contribution in honor of Lucio Russo, friend and admired colleague, I will present some remarks on the status of quantum mechanics, a theory through which we try to understand the world of atoms and molecules.

The research in this field has led to extraordinary successes; in fact, our present technology is to a large extent based on our description of this world.

We are convinced that we have found the key that opens the door to its full content, and that in the future our task is only (!) to unravel ways to solve complicated equations, maybe with the aid of a computer.

But quantum mechanics (QM) also poses conceptual problems and has been the arena of debates since the times of the founding fathers.

These problems are related to the meaning of QM as a physical theory.

The problematic relations between ontology and epistemology have been debated in western culture since the time of Plato, but the debate has seen a new life with QM since the basic foundational elements of classical physics are not valid in QM.

I will consider here only the traditional presentation of QM based on the formulation given by the Copenhagen school and developed among others by Born, Jordan, Heisenberg, and Schrödinger.

Generally speaking, this is the only presentation taught in universities and known to the majority of physicists.

Another presentation, which I will call pilot wave theory, originated by de Broglie and brought to a high mathematical standard by S. Goldstein and D. Dürr, gives

\section{Communicated by Raffaele Esposito.}

PACS2010: 03.65.Ta.

Keywords: quantum mechanics, observables, tracks, Born's rule.

${ }^{1}$ See [Jaynes 1990]. 
a totally different representation of the building blocks of theory and has its own conceptual difficulties. I shall come back briefly to this theory.

It is a basic assumption of western science that existence and reality can be represented through a metaphysical system which, while creating its own fundaments, serves the purpose of representing reality.

One should recognize that the origin of physics is the idea that reality exists and is at least partially accessible to our inspection.

At the same time, humans shape their experiences not only through their senses but also through their metaphysical and categorial presuppositions.

Causality, identity, and noncontradiction are not regarded as platonic concepts that humans discovered in the world as ideas but rather as prior conditions for human understanding.

The categorial representation of reality, in particular Newtonian space-time, limits and configures in a definite manner Newtonian physics.

On the other hand, there is David Hume's analysis of the inductive nature of science and the impossibility of grounding the notion of causation in experience. Causation as such is never found in the observable word; it is rather a metaphysical presupposition which allows the subject to make sense of observations.

In the same way, identity and noncontradiction are the conditions that constrain our observations.

Positivism relies on the distinction between empirical terms (empirically given in physical theories and experiments) and theoretical terms which are their translations into simple statements.

In this way, true knowledge (episteme) is replaced by objective knowledge with humans' shaping (experience).

One may say that the machian critic of Newton mechanics paved the way for quantum mechanics.

In fact, the multiple representations provided by different modern theories suggest that the successes of human understanding must be regarded as creations rather that discoveries.

When discussing the breakdown of the foundations of theories in the twentieth century, Wolfgang Pauli [1994] remarked that the modern physicist regards with skepticism philosophical systems which, while imagining that they have definitively recognized the a priori conditions of human understanding itself, have in fact succeeded only in setting up the a priori conditions of the system of mathematics and the exact sciences of a particular epoch.

Still we believe that physical theories are not only mirrors that reflect our own beliefs.

Within physical discourse, a cornerstone is counterfactual reasoning. If a theory makes predictions which agree reasonably well with experimental or observational 
results, scientists are inclined to believe that its logical and mathematical structure reflects the structure of the real world in some way, even if the philosophers remain permanently skeptical [Griffiths 2002].

In this regard, the classical representation of physics was produced when Newton related the mathematical theory of calculus with physical notions such as space, time, force, particles, and mass.

This representation was extended by Maxwell relating the theory of partial differential equations with electromagnetism and introducing in physics the notions of charge and fields.

This approach, contrary to the pythagorean-platonic view, regards mathematics as a nonrepresentative discipline. It is only physics which, making use of the mathematical formalism, attempts to discuss physical reality.

In this respect, it can be said that quantum mechanics represents a dissolution of this classical representation of the world.

\section{Origins of $\mathbf{Q M}$}

Conventionally one places the beginning of quantum mechanics at Planck's formulation of the quantum postulate. The theory soon went beyond its original formulation, which aimed to justify the spectrum of black-body radiation.

It was observed that in the quantum world some physical quantities, in particular the energy of the states of the atoms, seemed to be forced to have a discrete spectrum. This is of course totally different from the classical world as we perceive with our senses.

On the basis of the relation between energy and momentum of particles of light established by Einstein, this provided a quantization also of the frequency of the radiation emitted or absorbed.

The energy of the states of the atoms and some quantities related to a pair of states (emission or absorption of light) were the only information accessible to experimenters.

Following the positivistic rule that only observable quantities should be considered within a theory, W. Heisenberg advanced the first closed formulation of the theory, matrix mechanics.

The theory was not designed to talk about trajectories of particles; following the Einstein dictum "it is only the theory that tells you what you can observe", Heisenberg derived the uncertainty principle from matrix mechanics and the quantum postulate.

Notice that in this postulate enter quantities (position and momentum) that are well defined in classical physics, but are attributed to matrices in this formulation of QM. 
Through a more accurate analysis, for which the contributions of M. Born and P. Jordan and the reference to the dispersion relations of Kramers were essential, it was soon discovered that the theory could have a wider scope, still within atomic physics.

The theory of matrices was given a stronger mathematical flavor with the theory of operators in a Hilbert space. Notice that at the very same time and in the same place (Göttingen) Hilbert was developing his functional calculus (and Jordan was his assistant for some time).

Several other matrices entered in the relations that were derived with matrix mechanics. The ones that more frequently appeared in the analysis were powers of position matrices, powers of momentum matrices, and their real linear span.

If $a_{i, j}$ is a matrix that represents the observable $A$ and $\phi_{i}$ is the vector associated to the state $S$ in order to account for experimental data, $\sum_{i, j} \bar{\phi}_{i} a_{i, j} \phi_{j}$ has to be taken to be the expected value for a measurement of $A$ in the state $S$.

The measurements resulted in a real number, and therefore, all matrices which were used were hermitian. It was natural (and mathematically more convenient) to agree that all hermitian matrices represent observables.

Notice that observable is here understood as a definition, without reference to the instrumental apparatus that can be used to measure its value. This is a clear violation of the positivistic rule, since for very few observables can a prescription be given to construct an instrument which can be used to measure them.

Therefore, the term observable, often used in the mathematical formulation of $\mathrm{QM}$, refers to an ontological description.

In this formalism, it turns out that in order to represent something one is forced to consider matrices which are complex-valued. Therefore, observables can be represented by matrices with complex entries. Observations always lead to a real number, and therefore, not all matrices represent observables but only those that are hermitian.

Notice that the algebra of matrices is not commutative and the product of two hermitian matrices need not be hermitian. In the mathematical formulation of the theory, it was convenient to consider the entire algebra of matrices and not only the "observable" ones.

The theory of matrices developed by Born, Heisenberg, and Jordan gave a rational basis to very many problems in atomic physics.

At the same time, it made clear that classical mechanics and dynamics were not a valid instrument for the description of the structure of the atomic world, in spite of the fact that the description of the atomic reactions was given in classical terminology. Indeed it was heavily stressed by N. Bohr that all relevant information can only be transmitted and received within the formalism of classical mechanics. 
Roughly at the same time, E. Schrödinger developed wave mechanics adapting ideas of L. de Broglie.

L. de Broglie had remarked that a quantal fraction of reality seemed to have a particle-like behavior in some experimental instances and a wave-like behavior (diffraction) in others.

Following an analogy with the variational principles of classical mechanics both in the lagrangian and hamiltonian formulations, he proposed that a quantal fraction of reality be described by a complex wave. It was complex because the analogy with classical (hamiltonian) mechanics required the introduction of a "momentum" and by the formalism of the Fourier transform a fraction of reality with definite momentum was represented by a monochromatic wave and therefore complex-valued.

Schrödinger adapted the de Broglie formalism to describe the structure of the atoms, in particular the hydrogen atom. He modified the wave equation proposed by de Broglie to take into account that the dynamics is nonrelativistic, and he proposed an equation which replicates the hamiltonian structure of classical mechanics and takes into account the intuition of de Broglie, i.e., that plane waves should be representative of sharp values of the momentum.

The Fourier transform gives the relation between a representation in which the position has a relevant role in the description and a representation in which the relevant role is given to momentum.

Later Born postulated that the square of the modulus of the wave gives the density of the probability that at a given time the system is in a spacial configuration and that the square of the modulus of its Fourier transform gives the density of the probability that the system is in a specific momentum configuration.

A great success of the analysis of Schrödinger was the proof that his timeindependent equation gave exactly the energy levels found for the hydrogen atom. It was proven later by $\mathrm{W}$. Pauli that this result can be obtained by purely algebraic analysis.

For more complex atomic structure, the analysis is not so simple and requires several approximations and estimates. Still the analysis of the atomic spectra through a solution of the Schrödinger equation led to excellent results (and also to interesting developments in the theory of partial differential equations).

With de Broglie and Schrödinger begins the formulation of quantum mechanics as wave mechanics; immediately after it was proved by several people (including Schrödinger himself) that the two formulations are equivalent (as a theory of operators in a separable Hilbert space).

Schrödinger's formulation employs the representation of the Hilbert space as a space of square-integrable functions over a measure space; Heisenberg's formulation chooses a specific orthonormal complete basis and looks at the operators as "matrices" in this basis. 
Of course separable Hilbert spaces are isomorphic, and therefore, one can represent QM in any other realization of the Hilbert space, for example in the realization as square-integrable functions on the unit interval of the real line. But in this representation, the operators corresponding to simple physical quantities (e.g., position, momentum, and energy) have a very complicated presentation.

A major role in the theory is played by the rule that describes the value of an observable when the system is in a given state.

The structure of the formalism suggests that this operation be linear in the observables but sesquilinear in the wave function that represents the state, in order to ensure that the result of any measurement is a real number. This is formalized in Born's rule, which we will discuss shortly.

This leads, as Schrödinger immediately remarked, to a characteristic feature of the theory, the superposition principle which is better explained in the Schrödinger representation.

If $\phi$ and $\psi$ are unit vectors in the Hilbert space, $\Phi=(\phi+\psi) /|\phi+\psi|$ is also a unit vector but in general $(\phi, A \phi)+(\psi, A \psi) \neq(\Phi, A \Phi)$.

There is therefore interference between the waves. This phenomenon is very well known for water waves and for electromagnetic waves, but here it is counterintuitive since the waves are probability waves.

The superposition principle has a more involuted description in the formalism of Heisenberg, but the two formulations are equivalent, and therefore, the superposition principle also affects this representation. Independently of the formalism chosen, this implies a relationship between states that cannot be explained in terms of familiar classical physical concepts.

Some researchers tried to escape the problem posed by the superposition principle by searching for hidden variables (the wave functions does not fully describe the state of the system).

The naive hidden variables theory turned out to not be practicable because they lead to inequalities (Bell's inequalities) that are disproved by experiments.

A totally different way out is the pilot wave theory (also named Bohm theory), which was initiated by de Broglie himself. It is a non-Newtonian theory in which particles move under the action of a vector field (pilot wave) which itself satisfies a Schrödinger equation defined on the configuration space of all the particles.

It is a theory of particles and not a hidden variable theory because the (point) particles are fully described by their position and momentum. It is not a Newtonian theory because no reference is made to forces; the motion of the particles is ruled by a vector field which is not associated to the particles.

Its presence is perceived only through its action on the particles. The evolution of the field is independent of the particles and is determined by a Schrödinger equation on the configuration space of all the points. 
We shall not further discuss this interesting theory (which is also mathematically difficult as the vector field is singular) which, as remarked before, has its own interpretation problems.

\section{Ontology and epistemology: the quantum omelette}

Bohr's reaction to the difficulty of describing the ontological content of quantum mechanics was to abandon the physical representation of quantum mechanics, i.e., regard the formalism of quantum mechanics as solely epistemological.

There is a difference between discussing what reality is and how humans acquire knowledge from experience.

An ontological question is a question about the nature of existence and reality. It presupposes the existence of reality and the possibility to represent it.

An epistemological answer is related to the way in which humans connect to external reality. It is not regarded as a ground or goal of understanding. Its task is to describe how humans relate to experience. Physical theories are economics of human experience (Mach).

From this point of view, problems are not out there but are part of a definite viewpoint with definite metaphysical assumptions and presuppositions without which they cannot even be stated.

According to Jaynes [1990], our present the quantum-mechanical formalism is not entirely ontic but at the same time not entirely epistemological. It is a peculiar mixture describing in part nature and in part incomplete human information about nature, all scrambled up by Heisenberg and Bohr into an omelette that nobody knows how to unscramble.

Unscrambling the quantum omelette is a prerequisite for any advance in basic interpretation of the theory.

Quantum mechanics, in its Copenhagen interpretation, makes a process end with a choice, codified by Born's probability rule.

But if we take an ontological point of view, there can be no choice which determines what reality is: a subject cannot define by a choice, within a physical representation of a theory, what is physically real. Physical reality can be represented in an objective manner only if the subject (the experimenter) plays no role within that representation.

Einstein showed the inconsistencies with respect to physical reality (as understood in classical physics, the setting in which experiments and their outcomes are described) in which QM had been drawn through Bohr's complementarity approach.

What is considered physically real according to quantum mechanics? 
Bohr's epistemological approach escapes ontological debates. Bohr's explained how things had to be done. Following this set of rules, one could recover from QM a rational account of classical phenomena [Bohr 1935; 1963].

When asked whether the quantum theory can be considered as somehow mirroring an underlying quantum reality, Bohr declared, "There is no quantum world. There is only an abstract quantum physical description" [Petersen 1963]. It is wrong to think that the task of physics is to find out what nature is. Physics concerns what we can say about nature.

Later he wrote, "Physics is to be regarded not so much as the study of something a priori given but as development of methods of ordering and surveying human experience which can be unambiguously communicated in ordinary human language".

Bohr always stood on the epistemic side and never discussed questions related to the ontology of the quantum realm.

It was Heisenberg, and later Born and Pauli, who, when stressing the successes of the new theory, incoherently mixed the epistemological complementarity scheme of Bohr with an ontological (Platonistic) approach which assumed a direct relation between the mathematical formalism and reality itself.

This led to the cooking of the quantum omelette.

This quantum-unrealistic position was consolidated at the Solvay conference (1927) and is now part of what every physicist learns and practices. It is the conceptual background of all the brilliant successes of QM in atomic, nuclear, and solid-state physics over the past ninety years.

Physicist have learned to think about the theory in a highly unrealistic way, to be at ease with wave functions and operators. This (unrealistic) way has brought about the most marvelous predictive successes in the history of science.

The triumph of this approach is exemplified by the fact that the Copenhagen interpretation is taught in all universities around the globe, while the mathematically equivalent pilot wave theory of de Broglie is seldom taught and is considered a curiosity.

Our students manipulate and draw wave functions as if they had an objective reality.

\section{Copenhagen quantum mechanics}

The quantum theory of Planck and Bohr was the basis on which one had to construct a new theory to describe the dynamics of atomic systems.

Let us recall that a mathematical model, according to J. von Neumann, is a mathematical construction that, supplemented by a verbal language of correspondence, provides a coherent basis for the description of a class of physical phenomena. 
A model originates from a combination of experimental evidence, theoretical analysis, and mathematical analogies. A model obtains the status of a theory on the basis of the amount of physical phenomena it helps to organize in a coherent way.

When the class of phenomena described by a model in a somewhat unified way covers an entire field of physics, one speaks of $a$ theory. A theory in general provides a different perception of what is relevant, both conceptually and from the point of view of the experiments, a different paradigm.

The passage from a model to a theory is also conditioned by cultural background, versatility to adapt to applications, and also prejudgements.

A theory indicates what experiments are worth performing and what the questions that can be meaningfully asked are.

Form this point of view, QM deserves to be called a theory; it has changed our perception of the world at the atomic scale, providing a unified physical and mathematical picture, is at the basis of new technology, and has stimulated the development of a relevant part of modern mathematics.

When a theory reaches acceptance by a majority, it tends to dismiss as "false" or "irrelevant" any other attempt to construct an alternative model. Researchers working in QM tend to dismiss alternative theories as irrelevant and mental constructions and have the tendency to dismiss as futile the research on the foundation of the theory.

The consensus that comes from extraordinary successes is taken as a sign of truth.

Still there are conceptual problems that come partly, as N. Bohr emphasized, from the fact that the language which is used is borrowed from classical physics (N. Bohr went to the extreme of stating that classical physics is necessary to describe quantum mechanics) and partly from the difficulty of reconciling the intrinsic probabilistic aspects of quantum mechanics with the deterministic features which we are used to associating to physical phenomena.

For these reasons, quantum mechanics is a theory which is mathematically selfconsistent and very effective in its application, but not conceptually complete.

We have stated that the mathematical construction of a model (and of a theory) requires

- stating axioms (or postulates), in general derived from phenomenology and from some historical and cultural background (the structure of previous successful theories),

- deduction of some nontrivial consequences, typically under the form of theorems and equations, and

- determining a verbal language which associates the mathematical structures to measurable quantities; this empirical description is in everyday language and links the theory to experimental data. 
In classical physics, the mathematical constructions are, e.g., the variational principles, the equations of hamiltonian or lagrangian dynamics of material points, and the equations of the dynamics of the continuum and the equations of electromagnetism as formulated by Maxwell.

The (scientific) verbal language, i.e., the correspondence between mathematical entities and quantities that can be measured, is given for granted in classical physics; this common agreement is a result of centuries of "experience" and is also due to the fact that we have a daily experience of the classical world.

No one doubts the objective meaning of terms such as measure of a velocity or measure of a magnetic field, and we regard the result of a measurement as independent of the experimenter and of the apparatus used.

For the phenomena at the atomic scale as described by QM, this objectivity fails and the very concept of measurement can become problematic.

One can try to overcome this problem by stating that macroscopic objects, such as a measurement apparatus, must be regarded as classical objects, obeying the laws of classical physics.

But this would divide the physical world into two separate incompatible parts, and it would be difficult to make precise each time to which world one refers.

Many efforts have been made to solve the measurement problem (i.e., the detailed description in QM of the process of measurement), and various mechanisms have been proposed to explain why in (most) macroscopic bodies one does not perceive the typical structures of QM.

In particular, they explain why it is difficult to perceive (outside specialized laboratories) the superposition principle and the entanglement. Some of these attempts have led to a better understanding of the conceptual structure of the formalism and of its interpretation, but a satisfactory answer has yet to be found.

Let us stress again that from the empirical point of view QM has had outstanding success in organizing, describing, and also in some cases predicting results of experiments in its range of validity, namely the (nonrelativistic) physics of atoms and molecules and their aggregates.

At the same time, the refinement of the formalism of QM has contributed greatly to the development of modern mathematics.

Still it must be remembered that this theory has its own range of validity, in particular that it is nonrelativistic and it is not applicable to phenomena which occur at very high energies.

\section{States and observables in $Q M$}

We review the basic structure with a mathematical description of the concepts of states and observables. 
In general terms, a state of a system is the result of a preparation procedure. In order to construct a model, one must think of an idealized procedure that results in a well defined state.

One may think that the definition of a state is such that when a system is in state " $\mathrm{A}$ " any experiment gives the same result if performed by different observers.

This is true in classical physics; a measurement can be done, at least in principle, without altering the state of the system. And one can describe the state of a composite system by separately describing its parts. To give an example, the "state" of the solar system is described by giving the position and velocity of the single planets.

This has led those in classical mechanics to consider as elementary states the points in phase space $M$.

An observable is characterized by the value it takes on each state, i.e., by a realvalued function on the phase space: if $m \in \mathcal{M}$ and if $f$ is continuous, the number $f(m)$ represents the result of the measurement of the observable described by $f$ when performed on a system in the state described by $m$.

From a mathematical point of view, therefore, the states in classical mechanics are elements of the dual of the space of continuous functions, the duality given by $\{m, f\} \rightarrow f(m)$.

The meaning of the word measurement and the role of the measurement apparatus are not discussed further; their definitions are considered clearly established and universally accepted. And it does not depend on the observer.

In classical statistical mechanics, one also introduces more general states represented by positive measures $\mu$ that are absolutely continuous with respect to Lebesgue measure.

These states are linear positive functionals on essentially bounded functions; as a consequence one can include in the theory a larger class of observables, i.e., functions in $L^{1}(M)$.

As can be seen from this brief reminder, the definition of pure state in classical mechanics is linked to the possibility of considering continuous functions as observables.

Dynamics is given by means of differential equations for functions in phase space which are required to be differentiable.

When one tries to develop QM and its dynamics keeping some analogy with Hamiltonian mechanics, the first problem one faces is that in QM an equivalent of phase space does not exist, and therefore, it is difficult to decide a priori how to describe a pure state and characterize an observable.

This problem is solved differently in the two basic formulations of quantum mechanics, which we shall denote Schrödinger QM and Heisenberg QM. 
Schrödinger quantum mechanics. In the formulation of quantum mechanics due to Schrödinger, the primitive elements are the (pure) states which are represented by (normalized) vectors in a separable complex Hilbert space (for one particle, $\left.\mathscr{H} \equiv L^{2}\left(\mathbb{R}^{d}\right)\right)$ where $d$ is the number of degrees of freedom of the corresponding classical system.

This interpretation makes explicit use of the analogy between $|\phi(x)|^{2}, x \in \mathbb{R}^{d}$, and the classical Liouville distribution $\rho(x)$.

In Schrödinger's formulation, the observables are a dual structure; they are represented by operators on $\mathscr{H}$.

Since $\mathcal{H}$ is concretely represented as $L^{2}\left(\mathbb{R}^{d}\right)$, the observables are represented by operations on functions, typically by multiplication by another function and by differential operators.

In view of the analysis done by de Broglie, the operator $-i \frac{\partial}{\partial x_{m}}$ can be identified with the momentum of a particle. Also real functions of momentum space represent observables. For these observables, one can expect to define a possible measurement procedure.

Dynamics of the states is given by the Schrödinger equation; dynamics on the observables is defined by duality.

Since the set of operators which are sums of a function of position and a function of momentum is not invariant under time translations, in order to be able to describe the dynamics, one is forced to increase the number of observables.

If we require the average value of any observable in any state to be a real number, we should restrict ourselves to hermitian operators (more precisely to self-adjoint operators in order to have a functional calculus).

One is therefore led to state that the observables are in one-to-one correspondence with self-adjoint operators, in spite of the fact that for a generic self-adjoint operator one is not able to exhibit the experimental apparatus which may be used to measure the observable it represents.

According to Born's rule, $\int_{\Omega \subset \mathbb{R}^{3}}|\phi(x)|^{2} d x$ represents the probability that, performing a position measurement of a particle in the state described by $\phi(x)$, the outcome is that the particle is localized in the region $\Omega$.

This implies that, if the observable $A$ is represented by the function $A(x)$ in configuration space, then

$$
(\phi, A \phi) \equiv \int|\phi(x)|^{2} A(x) d x
$$

is the average of the results one obtains if one measures the outcomes of a measurement of $A$. 
In the same way, if the observable $B$ is represented by the function $B(p)$ in momentum space, then

$$
(\hat{\phi}, B \hat{\phi}) \equiv \int|\hat{\phi}|^{2}(p) B(p) d p
$$

(where $\hat{\phi}(p)$ is the Fourier transform of $\phi(x)$ ) is the average of the results of the measurements of the observable $B$.

By polarization, one obtains the value of $(\phi, A \psi)$ for every observable $A$ and for any pair $\phi, \psi \in \mathscr{H}$.

Heisenberg's quantum mechanics. In the formulation given by Born, Jordan, and Heisenberg (matrix mechanics), the primitive elements are the matrices that give the probability of transition from an atomic state $u_{n}$ to another state $u_{m}$ under the influence of an external field or under spontaneous decay

This leads one to consider as basic elements in the theory the observables represented by infinite matrices, i.e., linear operators on a separable Hilbert space.

The structure of the states plays a lesser role in this formulation of quantum mechanics. They are considered the result of an initialization and are distinguished by means of the value they give for the expected value of the observables.

This correspondence is linear for the matrices that represent observables, and so the states are linear functions of the observables continuous in a suitable topology, i.e., elements in a dual space.

Interference effects are not easy to describe in the Heisenberg formalism. A concrete analysis of entanglement and interference without reference to the Schrödinger representation is difficult.

On equivalence. We have already seen that the two representations are equivalent in the mathematical sense and correspond mathematically to dual structures.

The mathematical instruments used are different: mostly algebraic in Heisenberg's presentation and mostly function-theoretical in Schrödinger's.

A bridge between the physics of the two formulations of QM is given by Born's rule, which we will describe soon as an axiom.

In this rule, the states and the observables play equally important and symmetric roles.

But Schrödinger's formulation has special properties that come from the fact that one was naturally led to use the representation of the Hilbert space as (squareintegrable) functions on the configuration space.

This special presentation introduces spurious elements which make QM more visualizable in space (one draws on the blackboard the shape of the wave function) at the price of introducing misunderstandings. 
Indeed the wave function is not a measurable quantity; it is rather an abstract instrument which can be used to determine probabilities of real events.

Still some remnants of the visual picture survive. The use of periodic cells introduces homology, and smooth functions can belong to different homology classes.

If, as usually assumed in regular crystals, the wave functions are coherent over many cells, these homological properties are inherited by the state of the crystal and may influence the expectation values of specific observables.

Therefore, the homology class of a wave function (a priori an abstract object) may be measurable; indeed the topology of the wave function is at the root of the use of geometrical and topological methods in solid-state theory.

As a consequence, one must be prepared to recognize that there are "geometric" properties of the wave functions which correspond to measurable quantities.

\section{The axioms}

After these preliminaries, we can now state the axioms of quantum mechanics. In choosing the order of the axioms, we shall follow the point of view of Schrödinger.

Axiom 1. Pure states are represented by unit vectors in a separable Hilbert space $\mathscr{H}$. Vectors that differ by a phase represent the same pure state.

It follows that the (pure) states are represented by projection operators $P_{\phi}=$ $|\phi\rangle\langle\phi|$ (in Dirac's notation).

Since a Hilbert space is a vector space, the superposition principle holds: if $\phi, \psi \in \mathscr{H}$, then also $a \phi+b \psi \in \mathscr{H}$ for $a, b \in \mathbb{C}$. We assume here that all Hilbert space vectors represent states, i.e., there are no "superselection rules".

Also in quantum mechanics, one can introduce nonpure states, called statistical mixtures. They are represented by sums of projection operators

$$
\sigma=\sum_{n} c_{n} P_{\phi_{n}}, \quad c_{n}>0, \sum_{n} c_{n}=1 .
$$

Positive-trace class operators with trace 1 are called density matrices. Their relation with the pure states is the same as in classical mechanics.

Contrary to what happens in classical mechanics, no pure state is dispersionfree for all observables; this is due to the fact that their dual, the algebra $\mathscr{B}(\mathscr{H})$, is not commutative.

Recall that the dispersion of a state $\sigma$ relative to a (symmetric) operator $A$ is

$$
\Delta_{\sigma}(A) \equiv \sigma\left(A^{2}\right)-(\sigma(A))^{2} .
$$

A state $\sigma$ is dispersion-free relative to $A$ if and only if $\Delta_{\sigma}(A)=0$.

For comparison, notice that in classical mechanics, where the role of $\mathscr{B}(\mathscr{H})$ is taken by continuous functions, all pure states are dispersion-free with respect to each observable. 
The dual of the pure states, under the duality given by $P_{\phi}, A \rightarrow \operatorname{tr} P_{\phi} A$, is $\mathscr{B}(\mathscr{H})$, the set of bounded closed operators $\mathscr{H}$.

Axiom 2. - The observables in quantum mechanics are represented by the selfadjoint operators on a separable (complex) Hilbert space $\mathcal{H}$.

- The mean value of the measurement of the observable represented by the selfadjoint operator $A$ in the state represented by $P_{\phi}$ (the projection operator on the one-dimensional subspace spanned by the vector $\phi$ ) is given by

$$
\langle A\rangle_{\phi} \equiv(\phi, A \phi) \equiv \operatorname{tr}\left(A P_{\phi}\right)
$$

where the symbol tr stands for trace, a function defined as usual for finite-rank matrices and extended by sum convergence in the case of infinite matrices.

Notice that in this in the formulation of Born's rule we assumed that the measurement is an abstract procedure that requires no further analysis. In this respect also Axiom 2 is ontic.

Notice that if $A \in \mathscr{B}(\mathscr{H})$ the correspondence

$$
A \rightarrow \operatorname{tr}(\sigma A)
$$

defines a linear continuous functional on $\mathscr{B}(\mathscr{H})$.

Therefore, we could start, as in the Heisenberg point of view, with the definition of the observables as the real part of the algebra of all bounded operators on a complex Hilbert space $\mathscr{H}$ and consider the states as derived quantities by duality.

Axioms 1 and 2 describe the mathematical content of $Q M$ (and also its metaphysical content).

We introduce now two axioms that represent the verbal part of the model, i.e., the rules which must be used to associate measurable quantities to the mathematical entities in Axioms 1 and 2.

Axioms 3 and 4 connect the mathematical formalism to the outcome of laboratory experiments and are therefore of epistemic character.

A step in this direction has already been made in Axiom 2 by Born's rule, but nothing has been said so far about the description of a single measurement.

In particular until now we did not speak of the effect that has on a state described by $\sigma$, the measurement of an observable $a$ described by an operator $A$.

Axiom 3. Let the operator $A$ describe the observable $a$, and assume that $A$ has purely discrete simple spectrum, i.e., the eigenvalues are different from each other and the eigenfunctions $\psi_{i}^{A}$ form a complete orthonormal basis.

If one performs a measurement of the observable $a$ in a state represented by a vector $\phi \in \mathscr{H},|\phi|_{2}=1$, the outcome can be only one of the eigenvalues $a_{k}$ of $A$. The probability of the outcome $a_{k}$ is $p_{k}^{A}=\left|\left(\psi_{k}^{A}, \phi\right)\right|^{2}$, where $\psi_{k}^{A}$ is the eigenfunction of the operator $A$ associated to the $k$-th eigenvalue. 
We remark that this statement is compatible with Born's rule. Indeed from Axiom 3 it follows that the average of the results of the measurements of $a$ when the state is described by the vector $\phi$ is $(\phi, A \phi) \equiv \operatorname{tr}\left(P_{\phi} A\right)$ where we have denoted by $P_{\phi}$ the orthogonal projection on the vector $\phi$.

For observables which are represented by operators with partly continuous spectrum, the formulation of Axiom 3 is slightly more complicated; we don't detail here the obvious modifications. Axiom 3 is probably too ambitious as formulated. Given a generic symmetric bounded operator $A$, it is difficult even in principle to give a prescription for the construction of a measuring instrument which measures the observable associated to $A$.

For example it is difficult to indicate the instrument that measures the observable associated to $\xi_{\Omega} \hat{\xi}_{\Sigma} \xi_{\Omega}$, where $\xi_{\Omega}$ is the operator of multiplication by the indicator function of the domain $\Omega$ in configuration space and $\hat{\xi}_{\Sigma}$ is multiplication by the indicator function of the domain $\Sigma$ in Fourier space.

Axiom 3 refers to the possible results of a measurement and to the probability with which they are obtained.

There is no indication of the effect of the state of the system after measurement.

In classical mechanics, it is assumed that, at least in principle, it is possible to perform measurements on a system without altering its state. In quantum mechanics, this is not possible. The interaction with the measuring apparatus alters in general the state of the system in a way that cannot be predicted. But one assumes:

Axiom 4 (projection postulate). If $a_{i}$ is a nondegenerate eigenvalue, with eigenfunction $\psi_{i}^{A}$ of the operator $A$ associated to the observable $a$, and if the measurement of $a$ has given $a_{i}$ as a result, immediately after the measurement, the state of the system is described by the vector $\psi_{i}^{A}$.

The formulation immediately after, although imprecise, takes into account the fact that the operator $A$ may not commute with the hamiltonian and therefore the eigenstates are not invariant in time.

Since the evolution under the Schrödinger equation is a continuous process in time, this effect is negligible if the time elapsed between two measurements is negligible.

We notice that Axiom 4 is needed to give objective meaning to the measurement process, i.e., the measurement codifies an objective property of the system after the measurement.

But Axiom 4 has far-reaching consequences since the algebra of the observables is nonabelian in quantum mechanics.

Suppose that at time $t_{1}>t_{0}$ we perform a second measurement now of the observable $b$ associated to the operator $B$ which does not commute with $A$ and has eigenvectors $\left\{\psi_{k}\right\}$. 
According to the rules of quantum mechanics, we obtain the result $b_{k}$ with probability $\left|\left(\phi_{1}, \psi_{k}\right)\right|^{2}$; if the result is $b_{k}$, we conclude, by Axiom 4 , that the system immediately after the new measurement is in state $\psi_{k}$.

Now we perform again a measurement of $A$ at time $t_{2}>t_{1}$. The result will be $a_{h}$, $h \neq 1$, with probability $\left|\left(\psi_{h}, \phi_{1}\right)\right|^{2}<1$ : the system has a finite probability to be in a state different from $\phi_{1}$.

This implies that it is impossible to determine (even approximately) the state of the system if one does not have complete control of the environment.

For comparison, notice that in classical mechanics all observables (i.e., all functions on phase space) take at any given time a definite value on all pure states.

\section{The semiclassical limit}

The problem of finding a path relating the classical description and the quantum one is an ontological problem which aims to provide a physical explanation of what the relation between the classical and quantum realms is, both of which are presupposed to be physically real.

This problem cannot be understood in epistemological terms alone because there is no reference of the theory to something happening within physical reality. It is occasionally referred to as the problem of the quantum-to-classical limit.

Apart from the mathematical formulation which we shall outline shortly, the limit should be described in terms of a physical representation since it tries to explain what is going on beyond abstract mathematical formalism.

Therefore, the path must be represented in physical terms. The question analyzed from the point of view of Bohr's interpretation is ill posed: how can we possibly argue that there is a limit that can be explained with physical reality?

The quantum-to-classical limit in quantum mechanics is not so much a mathematical problem which seeks to relate incompatible formalisms but rather a physical problem which should provide a physical explanation for the connection between these seemingly incompatible descriptions.

The search of the physical explanation for the path from the classical to the quantum (or conversely) is a strictly representative enterprise [Jaynes 1990].

In some ways, this problem is similar to the problem of the description of a constrained system in mechanics. On one side is the classical microscopic point of view (world), where all systems satisfy the classical equations of Hamilton and Maxwell.

On the other side is the world in which constraints are considered as objective elements, worthy of a classification and an explicit (physical) description. 
In this case, within classical dynamics, the connection between the two physical worlds is made through approximations, stating the physical approximation scheme as carefully as one is able.

The same view is taken in classical mechanics about statistical mechanics or thermodynamics, which have their own strict internal rules. It is suggested that there is a bridge to the world of classical mechanics; the bridge is made of approximations and changes of scales.

One may wonder whether there is a comparable practical bridge between the classical world and the quantum one.

Mathematically one can construct such a bridge provided one is willing to play with Planck's constant $\hbar$, which appears in the quantum-mechanical formalism.

Since Planck's constant has a well defined value (in suitable units) in our physical world, any such limit must be seen as a mathematical exercise.

One can prove mathematically that in a very precise sense two classes of solutions of the Schrödinger equation have as its limit when $\hbar \rightarrow 0$ two classes of solutions of classical dynamics, of Liouville and Hamilton type, respectively.

Generally speaking, the initial data should be well localized both in configuration space and in momentum space, compatibly with the rules of the Fourier transform (semiclassical wave packets) or otherwise much more localized in momentum space, and depend smoothly on the spacial coordinates (WKB states)

Remark that due to the structure of the Schrödinger equation one can trade the smallness of Planck's constant for a large value of the mass.

As a consequence, the barycenter of a very massive quantum-mechanical body (e.g., the earth) moves in a gravitational field roughly in the same way as a classical point particle with the same mass. This saves classical celestial mechanics.

But in general the description of dynamics is entirely different in classical and quantum mechanics.

\section{Principle of decoherence}

Taking for granted that the description of the motion of a stone is totally different from that of very small bodies such as atoms and electrons, one may expect that this difference is due to the (relative) complexity of the stone and that the peculiar features of quantum mechanics, detectable at the level of atoms, is averaged out and therefore no longer relevant for the motion of the stone.

This would prove a physical way to connect the classical and quantum worlds and would imply that for all practical purposes quantum-mechanical bodies of macroscopic size can be correctly described by classical physics.

The first problem of this approach is that apparently it is not only the size of the body which determines whether it has a quantum behavior. 
Quantum-electronic devices, e.g., superconductors, can have the size of a meter and still must be described by quantum mechanics. It is still debated whether macromolecules can be described with classical mechanics or whether for them the quantum-mechanical properties must be taken into account.

As for superconductors, their coherence (tendency to show a quantum behavior) can be seen mathematically as a result of their almost pure periodic structure. Mathematically this is translated into the fact that the wave function is correlated over many elementary cells and therefore the analytic and topological structure is stable over a long distance.

Amorphous materials are more subject to interference effects so that the quantum structure is averaged out over a relatively short distance and is not effective over a long distance.

This averaging out is at the basis of the principle of decoherence which is often considered as a solution to the problem of the quantum-classical divide.

We shall briefly describe this principle through a typical example. We shall see that, contrary to a statement which is often made, some traces of the quantum behavior remain and in particular this principle does not solve the measurement problem (intrinsic indeterminacy in the measurement process).

Decoherence should be regarded as a consequence of a continuous process of correlations between the quantum system under study and the environment.

From a mathematical point of view, decoherence is linked to partial trace or conditioning.

Conditioning in quantum mechanics has properties similar to those of the operation with the same name in classical probability theory, but one should notice that, contrary to what happens in classical probability theory, in quantum mechanics complete information about the state of the system does not imply knowledge of the state of each component.

Suppression of information relative to the environment should lead to writing effective equations for a relevant subset of the measurable quantities of the subsystem.

If the subset can be described in classical terms, we expect that these equations are the equations of classical physics. The structure of the interaction should determine the subset of observables for which this reduction is possible.

The dynamics that one obtains should describe the evolution of these observables independently of the evolution of the environment for almost all its configurations and for a sufficiently long time.

It must be said at the onset that this program, on the mathematical side, is still in its infancy, in spite of its extreme conceptual interest. 
Roughly speaking, the mechanism of decoherence is as follows. Assume that the initial state of the total system observed object + environment is

$$
\Psi \in \mathscr{H}_{\mathrm{tot}}=\mathscr{H}_{\mathrm{obs}} \otimes \mathscr{H}_{\mathrm{env}}, \quad \Psi=\psi \otimes \phi, \psi \in \mathscr{H}_{\mathrm{obs}}, \phi \in \mathscr{H}_{\mathrm{env}} .
$$

If one measures an observable $A \in \mathscr{B}\left(\mathscr{H}_{\mathrm{obs}}\right)$, the mean of the values will be $(\Phi,(A \otimes I) \Phi)$ (we have introduced the natural immersion of $\mathscr{B}\left(\mathscr{H}_{\mathrm{obs}}\right)$ in $\mathscr{B}(\mathscr{H})$ ).

If the hamiltonian of the total system is $H$, at time $t>0$, the measurement of $A$ in the state $\Phi$ will give

$$
\left(\Phi, e^{i t H}(A \otimes I) e^{-i t H} \Phi\right)
$$

Due to the interaction between the two systems, there does not exist in general an operator $K \in \mathscr{B}\left(\mathscr{H}_{\mathrm{obs}}\right)$ such that for all $A \in \mathscr{B}\left(\mathscr{H}_{\mathrm{obs}}\right)$ and for a generic state $\Phi$

$$
\left(\Phi, e^{i t H}(A \otimes I) e^{-i t H} \Phi\right)=\left(\Phi,\left(e^{i t K} A e^{-i t K} \otimes I\right) \Phi\right) .
$$

One can hope (maybe even expect) that, if the environment has a large number of degrees of freedom and the interaction is very weak, the interaction has mainly the effect of modifying the state of the system, making it appear as a classical Liouville state.

In this case, the coherence which is at the root of the superposition principle is hidden by the lack of control of the environment. This would provide the bridge between the quantum world and the classical one.

This description scheme has not yet been developed; only special cases have been treated rigorously, and only strong qualitative arguments have been given in sufficiently general cases.

Strong qualitative arguments have been given, e.g., to show that decoherence for a quantum system can be produced by the interaction with a large number of light particles, and in this case position variables emerge as a "pointer basis" (variables which have a classical behavior).

One finds an overview of these considerations, e.g., in [Robert 1998; Wheeler and Zurek 1983].

Arguments have also been given to describe the decoherence for a quantum system in a thermal bath, i.e., interacting with a large number of particles in thermodynamic equilibrium at fixed temperature. In this direction, an approach to the mathematical description of decoherence has been pursued in [Hornberger 2009] in the framework of the algebraic formulation of quantum mechanics.

In spite of these developments, there are still many unsettled points in a mathematical theory of decoherence. 


\section{Mechanisms of decoherence}

As a possible mechanism of decoherence, consider the system composed of a case of a great number of quantum particles of very small mass $\epsilon$ scattering one after the other and independently off a quantum particle of mass 1 .

Let $\phi(x), x \in \mathbb{R}^{3}$, be the wave function of the quantum particle before collision, and denote by $y$ the coordinate of the first light particle.

The dynamics of the first collision is given by the Schrödinger equation (in units $\hbar=1)$

$$
i \frac{\partial \phi}{\partial t}=H \phi, \quad H=-\frac{1}{2} \Delta_{x}-\frac{1}{2 \epsilon} \Delta_{y}+V(y-x),
$$

where $\Delta$ is the laplacian and the potential $V$ is regular and compactly supported.

The initial state of the system is $\Phi \equiv \phi(x) \psi(y)$, and we are interested in

$$
\left(e^{i t H} \Phi,(A \otimes I) e^{i t H} \Phi\right)
$$

where $A$ is an observable of the system which is represented by an operator with kernel $A\left(x, x^{\prime}\right)$.

Setting $\xi=x-y$ and $\eta=x+y / \epsilon$ and noticing that all laplacians commute, it is not difficult to see that $e^{i t H}$ has the form

$$
e^{i t H}=e^{i(t / 2) \Delta_{x}} e^{i(t / 2 \epsilon) \Delta_{\eta}} e^{-i(t / 2 \epsilon) \Delta_{y}} e^{i t(1 / 2 M) \Delta_{y}+V_{x}(y)}, \quad V_{x}(y)=V(y-x) .
$$

Consider now the case in which the particle with coordinate $y$ is very light $(\epsilon$ is very small), and set $t=\epsilon s$.

Keeping into account that $A \otimes I$ commutes with $H_{y}^{0}$,

$$
\left(e^{i t H} \Phi,(A \otimes I) e^{i t H} \Phi\right)=\left(W_{s} \Phi,\left(e^{-i \epsilon s H_{x}^{0}} A e^{i \epsilon s H_{x}^{0}} \otimes I\right) W_{s, x} \Phi\right), \quad H_{x}^{0}=-\frac{1}{2} \Delta_{x},
$$

where

$$
W_{s, x}=e^{-i(s / \epsilon)(1 / 2) \Delta_{y}} e^{i(s / \epsilon)\left((1 / 2) \Delta_{y}+V_{x}(y)\right)} .
$$

When $\epsilon \rightarrow 0$, the operator $W_{s}$ converges to the wave operator $W_{x}$ for the scattering of the light particle off the heavy one with a potential $V_{x}(y)$.

One therefore has

$$
\left(e^{i t H} \Phi,(A \otimes I) e^{i t H} \Phi\right)=\int a_{t}\left(x, x^{\prime}\right) \zeta_{t}\left(x, x^{\prime}\right) \bar{\phi}(x) \phi_{t}(x) d x d x^{\prime}
$$

where $a_{t}\left(x, x^{\prime}\right)$ is the integral kernel of $e^{-i t H_{1}^{0}} A e^{i t H_{1}^{0}}$ and $\zeta_{t}\left(x, x^{\prime}\right)$ is a positive-type function (i.e., $\int \zeta\left(x, x^{\prime}\right) \bar{f}\left(x^{\prime}\right) f(x) d x d x^{\prime} \geq 0$ for all $f$ ).

The function $\zeta$ is given explicitly as

$$
\zeta_{t}\left(x, x^{\prime}\right)=\left(W_{x^{\prime}} \phi, W_{x}(t) \phi\right) .
$$


As a function of $u=x-y$ and $v=(x+y) / 2$, for each value it reaches its maximum when $u=0$.

Notice that $\zeta_{0}\left(x, x^{\prime}\right) \bar{\phi}\left(x^{\prime}\right) \phi(x)$ is the kernel of the density matrix before the interaction.

The function $\zeta_{t}$ has the following properties:

(a) $\zeta_{t}(x, x)=1$.

(b) If $t>0,\left|\zeta_{t}\left(x, x^{\prime}\right)\right|<1$ if $0<\left|x-x^{\prime}\right|<K_{t}$ where the constant $K_{t}$ depends on the initial datum and on the potential.

(c) $\bar{\zeta}_{t}\left(x, x^{\prime}\right)=\zeta_{t}\left(x^{\prime} x\right)$.

Property (b) indicates that due to the interaction the integral kernel is slightly ( $\epsilon$ is very small) more concentrated on the diagonal and slightly more spread out due to the dispersive properties of the Schrödinger equation.

After the interaction with the first particles, the kernel of the density matrix will be

$$
\rho^{\prime}\left(x^{\prime}, x\right)=\zeta_{t}\left(x^{\prime}, x\right) \rho\left(x^{\prime}, x\right) .
$$

Assume now that the successive interaction with the $N$ particles of the environment are independent and take place at times $\left\{T_{k}\right\}$ with intervals of order $\epsilon^{-1}$ in such a way that it is entirely a sequence of independent events.

In this case, the modifications to the kernel of the density matrix can be considered as independent and after $N$ collisions the kernel of the density matrix will be

$$
\rho_{N}\left(x,{ }^{\prime} x\right) \bar{\phi}\left(x^{\prime}\right) \phi(x) \zeta_{1}\left(x^{\prime}, x\right) \zeta_{2}\left(x^{\prime}, x\right) \cdots \zeta_{N}\left(x^{\prime}, x\right) .
$$

Since all functions $\zeta_{k}, k=1, \ldots, N$, have the properties (a), (b), and (c), we conclude that if $N$ is very large the kernel of the density matrix after the very many collisions is concentrated for all macroscopic times on the diagonal and therefore is represented by a classical Liouville measure.

It is not difficult to show that under these conditions the propagation of the heavy particle is described within a good approximation by the Lagrange equation in configuration space.

In the terminology of the theory of decoherence, this may be described as the choice of a preferred basis (the configuration space).

But notice that in general the support on the diagonal is not of the order $\sqrt{\hbar}$ and therefore the (approximate, classical) dynamics is that of a Liouville distribution.

The procedure we have described is largely heuristic, and to have a rigorous result one should proceed much more carefully, establishing exact formulae and giving accurate estimates of the terms which one neglects.

This is a very difficult task that nobody has completed so far. 


\section{Experiments on decoherence}

From the experimental point of view, interesting and very refined experiments have been performed in particular by the group of S. Haroche at the École Normale Supérieure. A typical experiment is described in [Raimond 2014].

In this experiment, rubidium atoms (mice) initially in a circular Rydberg state $f$ are injected one at a time in a photon box (cat), an open cavity with reflecting walls that can keep for 1 millisecond a specified number of photons of wavelength 6 millimeters.

The electromagnetic field in the cavity is prepared in a state of 9 or 10 photons; this state approximates reasonably well a coherent state for which a (semi)classical description is possible.

In particular one can define the frequency of the radiation field. In this state the cavity-cat is sleeping.

The field is prepared in a (quasi)monochromatic state with frequency resonating with that of the transition between the $f$ and the $g$ states of the rubidium.

The rubidium atom (mouse) which is injected in the cavity is in a state " $f$ "; this state has small dipole momentum and therefore does not disturb the cat (change the number of photons).

While crossing the cavity, the atom-mouse is subjected to a laser beam to induce a transition to another state " $g$ "; the superposition of the two states has a large electric dipole.

This produces emission and absorption of photons and modifies the distribution of the number of photons in the cavity-cat which is now suspended between two normal states $f$ and $g$ (and this makes its presence visible to the cat).

The environment is in this case represented by the walls of the cavity that "interact" with the field present in the cavity because of imperfections.

The number of photons in the cavity (the status of the cat) can be monitored by sending into the cavity a second rubidium atom. If the cat has remained in a suspended state, it will interact with the atom-mouse.

The apparatus therefore permits one to tell whether the cavity is in a coherent state and thereby permits one to measure the decoherence induced by the environment (by impurities in the walls of the photon box). The experiment reveals that the amount of decoherence depends on the delay with which the second atom is injected into the cavity.

Call decoherence time the time after which the description of the photon box as a coherent state produces a relative error of $1 / 10^{4}$. The results of the experiment which is roughly described above indicate that the decoherence time is on the order of 0.1 milliseconds. 
Decoherence is therefore effective in a small time even in this carefully organized experiment.

One can expect that in the case of a real cat the survival time of coherence is several orders of magnitude smaller and therefore coherence cannot be seen under normal everyday life conditions (i.e., for true cats).

This and related experiments show that a result (the cat suspended between two physical states) that seems to be counterintuitive when one has little control of the environment can be observed in a laboratory in which maximum control is possible.

\section{The measurement problem and tracks in a cloud chamber}

The difficulty in unscrambling the quantum omelette is clearly shown in the measurement problem, which cannot be solved by the theory of decoherence.

As Bohr emphasized, measurement is done with classical instruments and the result is expressed in classical language.

One often says that the instrument interacts with the object to be measured, but there is an ambiguity in this statement.

In mathematical terms, the term interaction refers to the description of the dynamics by means of the structure of the equations. It belongs therefore to the world of mathematical quantum mechanics.

On the other hand, the word measurement (distinct from interaction) must refer to a process that takes place in the real world and leads to unambiguous results.

According to Bohr [Robert 1998], the unambiguous interpretation of any measurement must be essentially framed in terms of classical physics theories, and we may say that the language of Newton and Maxwell remains the language of physicists for all time.

Therefore, the analysis of the quantum measurement process is the key to recovering a rational account of physical phenomena.

We have stated that the main problem in QM is the distinction between interaction (a mathematical structure) and measurement (a physical process).

We exemplify these difficulties by considering a simple phenomenon, the occurrence of tracks in a cloud chamber. It is simple enough to admit an almost complete mathematical description, and at the same time it contains all the interpretation problems in QM.

A Wilson cloud chamber is a vessel that contains vapor which is in a supersaturated state. Under a small local perturbation, it can locally make a transition to a liquid state (droplet). 
It is an experimental fact that an $\alpha$-decay produces in a cloud chamber at most one track (sequence of liquid droplets placed on a line that is straight or slightly curved if a magnetic field is present (a trajectory of a classical particle)).

Different decay events produce tracks that point in random directions. This seems to contradict the description of decay in QM: according to Gamow if the decay takes place at rest, a spherical wave is produced and moves radially according to Schrödinger's equation.

The presence of the track makes the result appear as if the interaction with the supersaturated vapor turned the wave into a particle. We want to place this effect in the context of Schrödinger's QM.

Notice that in this experiment the experimenter is the cloud chamber (or rather the supersaturated gas).

The problem of justifying the presence of a track of droplets within Schrödinger's quantum mechanics goes back to the early days of QM together with the question of whether the presence of the track can be considered proof that a real $\alpha$-particle is produced in the decay.

Mott [1929] was among the first to attempt a systematic description using properties of the solutions of the time-independent Schrödinger's equation. A rather detailed account of the history of the problem and of various attempts to find a solution can be found in a recent book by Figari and Teta [2014].

The analysis given by Mott is based on stationary and nonstationary phase techniques in the time-independent formulation of Schrödinger's equation; it goes in the right direction but is incomplete in several ways.

To improve the analysis, we rely on semiclassical theory [Dell'Antonio 2015].

We shall see that the properties of the initial wave function allow for the introduction of a semiclassical formalism in which the interaction of the wave with a single atom can be regarded as semiclassical inelastic scattering. We stress that this description does not have a universal character and depends essentially on the mathematical properties of the initial state.

In this mathematical formulation, the $\alpha$-wave before the production of the first droplet of the track can be regarded (mathematically) as fragmented into (coherent) semiclassical wavelets, each of high momentum, moving radially away from the point at which the decay has taken place.

The linear size of each wavelet is comparable to that of the atoms. Each wavelet moves according to the laws of QM: its barycenter moves on a classical path (curvilinear if a constant magnetic field is present), and its dispersion is of order $\sqrt{\hbar}$ (in natural units) and increases slowly in time.

No physical significance should be attached to this mathematical exercise. One can compare it to the description of light as composed of light rays with a major difference: the $\alpha$-wave is a probability wave. 
The $\alpha$-wavelets move coherently; the entire wave keeps its spherical structure in accordance to Huygens' principle.

When one of the wavelets interacts with an atom, the coherence with the other wavelets is lost: the combined system wavelet + ion $(+$ emitted electron) belongs now to a different subspace of the Hilbert space in which the entire system (emitted wave and atoms of the vapor) is described.

If one regards the resulting subsystem as isolated, the result is an entangled state of the wavelet, of the wave functions of an ion, and perhaps of the emitted electron.

The interaction wavelet-atom can be considered as independent of the environment, and mathematically it can be regarded as inelastic scattering.

Therefore, QM describes the system after the interactions but before the production of the first droplet, as a collection of very many coherent triples each composed of the wave function of an ionized atom, a semiclassical wavelet, and the wave function of an electron.

Each triplet belongs to a different sector of the Hilbert space, and there is no interference between them.

Note that mathematically the ionization of an atom is the result of an interaction described in QM by a unitary propagation within a huge Hilbert space.

On the other hand, the production of the first droplet is a random macroscopic event, the result of a chain of processes of magnification which can probably best be described within statistical mechanics. The local phase transition is due to the modification produced by the ion in the electronic structure of the nearby atoms. QM can at most be used to determine the probability that a droplet be formed.

Its relation with the Hilbert space description in QM is the measurement problem; how does the quantum-mechanical system choose the (probability) wavelet that produces the droplet? Is it a random choice?

After the production of the droplet, the remaining wavelets no longer enter the description of the system. It is improper to say that they have disappeared because as probability waves they had no physical existence even before the formation of the droplet.

The selection process is probability preserving (since the outcome occurs with probability 1) and nonlocal (since the initial wave function is extended and the final result is localized in a small cone). It is not described by the Schrödinger equation.

Since the interaction provides strong entanglement among the component of each of the triplets of probability waves indicated above, one may assume that the process of measurement selects not only an ion but also the corresponding $\alpha$-wavelet, although no actual measurement of the wavelet is done.

After the interaction, the chosen wavelet is still a (semiclassical) probability wave. It can interact with the atoms in its path. Since the wave function of the 
wavelet has support on the order of magnitude of the square root of Planck's constant, the interaction can be regarded by a macroscopic observer as having taken place at one point.

The momentum of the wavelet is essentially concentrated along a vector that is directed from the point of decay to the point at which the first droplet is formed.

The production of further droplets is again a macroscopic phenomenon not described by the Schrödinger equation. Since the interaction is local, the incoming (probability) wavelet is well localized and the exchange of momentum is negligible; its barycenter has essentially a classical motion.

The wavelet therefore moves as a classical object (an $\alpha$-particle) with roughly the same energy and momentum of the wave emitted in the decay.

The question of whether after the production of the first droplet the remaining droplets in the track are produced by a probability wavelet or by a particle is devoid of objective meaning.

In any case the interaction of the wavelet-particle with the other atoms leads to the formation of a straight line of droplets (or a curved line if a magnetic field is present). Due to the semiclassical nature of the wavelet, the direction of the track is determined by the position of the source and of the first droplet.

Notice that after each collision the shape of the wavelet may change and from the point of view of mathematics at every interaction the wavelet changes sector in the abstract Hilbert space.

The quantum aspects of this description are limited to the fact that, although the initial state is completely known, one can give only the probability that a track is produced in a given direction.

We stress that one sees droplets only if ionization takes place and the ion triggers the magnification mechanism. Without this mechanism (which can be described at most by quantum statistical mechanics), the event is not recognized by the macroscopic observer as a measurement and it must be considered only as an interaction.

One therefore has to invoke the presence of a step in which probability is turned into occurrence. This step is beyond $\mathrm{QM}$ and has not been understood so far. This ambiguity is at the heart of the measurement problem in QM.

Notice that also from a bayesian point of view (updating of information) this is a difficult problem since the updating is (presumably) done by the supersaturated gas. On the other hand, critics of the description of a measurement process have always remarked that a Ph.D. is not required to a make a measurement.

\section{Some mathematics}

For completeness we give some details of the mathematics involved. More details can be found in [Dell'Antonio 2015]. 
According to quantum mechanics, the $\alpha$-wave $\phi_{0}$ produced in the decay can be presented as a complex-valued spherically symmetric function with support in a small neighborhood of the origin and with a phase proportional to $M\left|v_{0}\right||x| / \hbar$ where $M \in \mathbb{R}^{+}$and $v_{0} \in \mathbb{R}^{3}$ are the parameters (mass and radial velocity) which characterize the wave produced in the decay.

We take natural units in which Planck's constant $\hbar$ is very small, and we assume that $\left|v_{0}\right|$ is very large.

It is convenient to have a different (equivalent) presentation of the initial data as a function on the product of a small interval $I \subset \mathbb{R}^{+}$and a fibered two-dimensional sphere $S^{2}$, with fibers perpendicular at each point to the sphere.

This presentation is particularly adapted to the introduction of a semiclassical structure since both the free evolution in time and the wave packets are obtained by the convolution of the wave function with a gaussian kernel.

For concreteness we shall write

$$
\phi_{0}(x)=C e^{-|x|^{2} /(2 \hbar)} \int_{S^{2}} d \omega e^{i M v_{0}(\zeta(\omega), x) / \hbar}, \quad x \in \mathbb{R}^{3},
$$

where $C$ is a normalization constant and $\zeta(\omega), \omega \in S^{2}$, is the unit vector orthogonal to $S^{2}$ at the point $\omega$ and directed opposite to the center of the sphere. The wave is produced with high momentum, and therefore, we take $1 \ll v_{0}$.

If there are no interactions, the wave evolves according to the free Schrödinger equation; the evolution is described by the convolution with a suitable gaussian kernel.

Taking into account that $\left|v_{0}\right|$ is very large, it is easy to see that at a later time $T$ the wave is approximately localized in a corona of mean radius $v_{0} T$ and width on the order of $\sqrt{\hbar}$.

Therefore, up to a small error, the wave function $\phi_{T}(x)$ at time $T$ can also be presented as a function on the product of an interval on the positive real axis and the fibered unit sphere.

We shall assume that the (mathematical) interaction of the wave with the atoms is of very short range and is nontrapping. This will allow us to consider the result of the interaction as an inelastic scattering event. If the atoms are sufficiently separated from each other, we can consider the interactions as independent scattering events.

The fact that several further ionizations are seen (forming a track of droplets) suggests that the interaction between the atom and the semiclassical wave is rather strong, and therefore, it is advisable to avoid using perturbation theory (a contact interaction may be a better choice).

The waves in the Schrödinger picture are probability waves and carry no objective reality; they are tools to give the probability distribution of the outcomes if 
a measurement of a given observable is performed. Understanding the process by which this probability is turned into a specific outcome constitutes the measurement problem that is still unsolved in spite of its conceptual relevance.

A detailed description in quantum mechanics of the interaction of the emitted wave with the atoms in the cloud chamber is beyond reach. We therefore make some simplifying assumptions and approximations. We use natural units in which Planck's constant $\hbar$ is very small.

Before the interaction, the wave satisfies the free Schrödinger equation and therefore the solution at time $T$ is given by the convolution of the initial data with a gaussian kernel. Under the assumption that $M v_{0}$ is very large, the presentation we have used provides the following description of the wave at any time $T>0$ :

$$
\phi_{T}(x)=F_{T}^{\hbar}(|x|) \int_{S^{2}} d \omega e^{i M v_{0}(\zeta(\omega), x) / \hbar}+R_{T}(x), \quad x \in \mathbb{R}^{3} .
$$

The function $F_{T}^{\hbar}(\rho)$ is supported in a spherical corona of depth $\sqrt{\hbar}$ and radius $\left|v_{0}\right| T$. The residual term $R_{T}(x)$ has $L^{2}$ norm of order $\sqrt{\hbar}$ and decreases fast in time. We will neglect this term in the following analysis and will take the $L^{2}$ norm of $\phi_{T}$ to be 1 .

We make use of natural units in which Planck's constant $h$ is very small. The essential support of the wave function of an atom has linear size of order $\sqrt{\hbar}$. All quantities will be evaluated up to a relative error of order $\sqrt{\hbar}$.

One can consider separately the evolution of small fragments, wavelets, of the $\alpha$-wave, of linear size $\sqrt{\hbar}$.

The density of the atoms in the cloud chamber is such that each fragment interacts with at most one of the atoms.

In the presentation of the $\alpha$-wave given in (2.2), the fragments are obtained using elements of a smooth partition of the unit sphere. Each element $\xi$ has support of linear size $O(\sqrt{\hbar})$, and its initial condition at time $T$ is

$$
\phi_{\xi}(x, T)=\Phi_{\xi}(x, T)+R_{T}, \quad \Phi_{\xi}(x, T)=F_{T}(|x|) \int_{S^{2}} \xi(\omega) e^{i M v_{0}(\zeta(\omega), x) / \hbar} d \omega .
$$

$F_{T}(\rho)$ has support in a neighborhood of $v_{0} T$ of linear dimension $O(\sqrt{\hbar})$, and we shall neglect $R_{T}$, which is smaller in norm by a factor $O(\sqrt{\hbar})$ with respect to $\Phi_{\xi}$.

We prove now that the solution with initial condition $\Phi_{\xi}(x, T)$ is localized together with its (quantum) Fourier transform in a domain of linear size $\sqrt{\hbar}$ and therefore represents a semiclassical wavelet. We take $\xi$ to be localized around the point $(0,0,1)$. By construction the function $\Phi_{\xi}(x, T)$ is then supported in a neighborhood of linear size $O(\sqrt{\hbar})$ of $\left(0,0, v_{0} T\right)$. Recall that the quantum Fourier transform is the Fourier transform written in units of $\hbar^{-1}$. Notice that we make an 
error of order $\hbar$ in substituting the support of $\xi$ on the sphere of radius $v_{0} T$ with its projection on the tangent plane. Up to an error of order $\hbar$, we can therefore write

$$
\tilde{\Phi}_{\xi}(x, T)=\int \tilde{\xi}(y) F_{T}(|x|) e^{i M\left(v_{0} x_{3}+y_{1} x_{1}+y_{2} x_{2}\right) / \hbar} d y_{1} d y_{2}
$$

where $\tilde{\xi}(y)$ has support in $|y|<C_{2} \sqrt{\hbar}$.

The Fourier transform is easy to compute; it has support in the ball of radius $O(\sqrt{\hbar})$ and center $\left(0,0, M v_{0}\right)$. Therefore, under free evolution, $\Phi_{\xi}(x, T)$ behaves as a semiclassical wave packet. Since $v_{0}$ is very large, if the interaction is not trapping, the wave remains in the cloud chamber for a very short time $\Delta$.

We can use this semiclassical picture during the time in which the interaction with the atoms takes place. Standard phase-space analysis shows that, if $\phi_{T}^{\xi}$ and $\phi_{T}^{\eta}$ have supports separated by a finite distance $d$, the same is true (up to an error of order $\hbar$ ) for a time $T \leq t \leq T+\Delta$.

If the phenomenon we describe were scattering of a semiclassical wavelet by a potential $V(x)$, regularity and no trapping properties of the potential would give a description of the event as semiclassical scattering. Regularity conditions on the potential must be imposed in order for dynamics to preserve the semiclassical structure. In our case the scattering is inelastic because the final state also contains an electron.

Consider first the interaction with a single atom with wave function $\psi_{Y}$ with essential support in a neighborhood of linear size of the order $\sqrt{\hbar}$ of a point $Y \in \mathbb{R}^{3}$. As a result of the interaction, ionization occurs; we assume that the wave function of the resulting ion remains localized in a neighborhood of $Y$ of linear size $O(\sqrt{\hbar})$.

We have assumed that the interaction is not trapping and its range is of order $\sqrt{\hbar}$. Since the speed with which the wave moves is very large, the interval of time $\Delta$ in which the interaction takes place is very short. Due to our assumption on the density of the atoms, we can assume that the fragmentation of the wave is such that during the interval of time $\Delta$ only one of the fragments interacts with the atom in $Y$. Under this assumption after the interaction, this fragment is localized again in a region of linear size $\sqrt{\hbar}$ near the atom in $Y$.

Since the momentum of the incoming fragment was localized around $M v_{0} \hat{Y}$ in units of $1 / \hbar$ and the loss of momentum in the interaction is very small on this scale (the ionization energy is comparatively small), energy-momentum conservation implies that also the momentum of the outgoing fragment is sharply localized around $M v_{0} \hat{Y}$.

The outgoing fragment is therefore represented by a semiclassical wavelet, with approximately the same mean momentum as the incoming wavelet and approximately the same variance.

Consider an atom localized near the point $P \in \mathbb{R}^{3} \equiv\left(0,0, r_{0}\right)$. Under free motion 
the wave function $F_{T}^{\hbar}$ overlaps the wave function of the atom for a very small time interval $\Delta_{0} T$. Since the interaction is of very short range and nontrapping, the wave function with initial data $F_{T}^{\hbar}$ overlaps the wave function for a very short time. Therefore, only a small part of the incoming wave contributes to the interaction with the atom in $P$.

This suggests a (mathematical) decomposition of the incoming spherical wave into fragments (wavelets), each of which can interact with only one of the atoms. We will prove that, due to the properties of the initial $\alpha$-wave, the wave can indeed be seen as decomposed into small (coherent) fragments (wavelets) each propagating as a semiclassical wave packet (its barycenter follows a classical path). The dispersion is of order $\sqrt{\hbar}$ both in space and momentum (the latter in units of $\sqrt{\hbar}$ ).

Notice that we are manipulating mathematical objects (probability amplitudes) that enter into the mathematical framework by which quantum mechanics describes outcomes of experimental observations.

Before the formation of the first droplet (and after very many interactions), the partition in wavelets is a mathematical exercise. The macroscopic production of the droplet selects one the ions (the seed for the production of the droplet). This selection process is nonlocal and is not described by the Schrödinger equation.

The measurement process also selects the wavelet associated to the ion (although one measurement is performed on it). The selected wavelet is still a probability wave. Momentum conservation together with the semiclassical approximation imply that also this outgoing $\alpha$-wavelet can be treated semiclassically (but its shape may have been changed by the interaction).

The outgoing wavelet interacts with the atoms on its path giving rise to further ionizations. Each ionized atom is a seed for production of a droplet; this originates the track. All other probability wavelets now have probability 0 ; therefore, there is only one track.

As already mentioned, the mathematical device of partitioning does not lead per se to anything physical. It reveals a detectable phenomenon due to the macroscopic mechanism of production of a droplet.

Notice that in this description the semiclassical wave packet entangled with the selected atom may lead to measurable effects although no measurement is performed on it.

As a result of the measurement (a probability-preserving nonlocal map), one of the ions is selected and the corresponding wavelet acquires probability 1 . This distinguishes measurement from interactions.

We now generalize the analysis to take into account that there are many atoms in the cloud chamber, uniformly distributed and sufficiently separated so that each of them interacts with at most one of the wavelets and the interactions can be regarded as independent. 
Each wavelet interacts with at most one atom. The outcome of the interaction is an entangled state made of the wave functions of an ion and of the outgoing wavelet (and of an electron).

We conclude that the interaction of the $\alpha$-wave with the atoms in the cloud chamber can be mathematically described before the measurement as a sequence of disjoint and independent interactions of semiclassical probability wavelets with the atoms of the cloud chamber.

After the interaction, the wavelets move incoherently and the wave functions of the atoms are turned into the wave function of an ion. The interaction time is so short that we are justified in substituting the interaction with the scattering map.

The act of measurement (the cloud chamber measures the position of the first droplet produced) selects, according to Born's rule, one and only one of the ions to be the seed of the process of formation of the first droplet of liquid. The exact mechanism behind this selection has not been understood so far; it can be best described within quantum statistical mechanics.

The ion selected modifies the wave function of the nearby atoms. Since the vapor is supersaturated, this gives rise locally to a phase transition with production of a liquid droplet.

We assume that the measurement process also has the effect of keeping, as part of the description of the system after the measurement, also the wavelet entangled with the selected ion (although no direct measurement is performed on the wavelet).

The wavelet which is selected may originate on its path further ionizations, and this gives the visible track. Notice that the (position) measurement of the first droplet in the path is the only one which is represented by a (unitary) nonlocal transformation. The process of production of the remaining part of the track is essentially local.

After the measurement only one of the wavelets enters in the description of the system; it has essentially the energy-momentum of the entire incoming wave. Since it is well localized in position, it can be described as a particle (the $\alpha$-particle). The remaining (probability) wavelets no longer enter the description of the system.

In conclusion, the analysis we have performed of the production of tracks in a cloud chamber shows that interaction should not be confused with measurement.

We briefly note the relation of this analysis with the problem of decoherence.

Before the interaction with the atoms, the semiclassical wavelets were coherent. After the interaction with an atom, the wavelet is entangled with the wave function of an ion. The coherence with the rest of the wave is no longer detectable (it would require a detailed knowledge of the wave functions of the atoms and of the emitted electrons).

If the environment contains $N$ atoms which are placed sufficiently far apart so that the interaction of the wave with each atom can be treated as independent, the 
interaction produces $N$ mutually incoherent triples each representing an entangled state of an ion, a wavelet, and the emitted electron.

This decoherence between the triples is entirely different from the decoherence of a slow-moving quantum wave as a result of very many interactions with the ambient space. Decoherence in the cloud chamber experiment is related to a single interaction with an atom.

\section{Quantum mechanics: Born's rule as conditional probability and information-theory analysis}

Combined with the projection postulate, Born's rule says that, when one knows that a measurement corresponding to an observable $a$ associated to a symmetric operator $A$ with discrete spectrum has taken place but one does not know the result, the following information is gained.

If the initial state is described by density matrix $\rho_{\text {in }}$, then the density matrix $\rho_{\text {fin }}$ of the final state is given by

$$
\rho_{\mathrm{fin}}=\sum_{i} \operatorname{tr}\left(P_{i} \rho_{\mathrm{in}} P_{i}\right)
$$

where $A=\sum_{i} \lambda_{i} P_{i}$ is the spectral decomposition of the operator $A$. One has by definition $\sum_{i} P_{i}=I$ and $A=\sum_{i} \lambda_{i} P_{i}$.

This formulation no longer requires that the initial state of the system be pure.

It is interesting to notice that the formula can be interpreted in information theory as saying that $\rho_{\text {fin }}$ represents the most probable state that one may have after a measurement of the observable $a$ in the state described by $\rho_{\text {in }}$ [Vedral 2002].

We clarify what this statement means.

According to von Neumann, information is measured by relative entropy, and the most probable state is the state which corresponds to minimal entropy relative to the initial state.

Following Wiener, we consider the amount of information to be the negative of the quantity defined as entropy.

We therefore take the negative of the relative entropy function $D(\rho, \sigma)$ as a measure of the relative information about the quantum state $\sigma$ that can be derived from knowledge of the quantum state $\rho$ :

$$
D(\rho, \sigma) \equiv \operatorname{tr}(\rho \ln \rho-\rho \ln \sigma) .
$$

The function $D$ is nonnegative and can be considered a nonsymmetric distance.

The most probable outcome state is by definition the state which minimizes the distance $D(\rho, \sigma)$ for all allowed $\sigma$.

In the case of measurement of the observable $a$ which is represented by the operator $A=\sum \lambda_{i} P_{i}$, the reference states are the density matrices which belong 
to the set

$$
\Sigma_{A} \equiv\left\{\sigma:\left[P_{i}, \sigma\right]=0\right\} \text { for all } P_{i} .
$$

The last equation is equivalent to the condition $[A, \sigma]=0$, i.e., the requirement that the state is obtained as a consequence of the measurement of the observable $a$.

We must minimize $D(\rho, \sigma)$ over $\Sigma$. This amounts to selecting the quantum state that is least distinguishable from the original state among all the states that satisfy the constraint of being produced by the measurement of $a$.

We consider only the case in which the Hilbert space is finite-dimensional. The same results are obtained if $A$ is compact.

The set $\Sigma_{A}$ is defined by a linear relation, so it is a simplex.

$D(\cdot, \cdot)$ is jointly convex in both arguments so that $(\cdot, \rho)$ is convex for all $\rho$.

Since the problem is finite-dimensional, the following holds: if the function $f$ is (Gateau) differentiable and strictly convex on a simplex, and the directional derivatives (we are in a finite-dimensional setting) at a point $b$ are all 0 , then $b$ is the global minimum of $f$.

We can parametrize $\Sigma_{A}$ noticing that every element is of the form

$$
\sigma=U \Lambda U^{*}
$$

where $\Lambda$ is a trace-1 matrix with positive entries and $U$ is a unitary operator $U=$ $\pi_{i} U_{i}$ where $U_{i}$ is the identity on the range of $\left(I-P_{i}\right)$.

This means that $\left[U, P_{i}\right]=0=\left[\Lambda, P_{i}\right]$.

Therefore, writing $\sigma_{i}$ for $\sigma$ restricted to the range of $P_{i}$, we have for every function $f$ on $\Sigma_{A}$

$$
f(\sigma)=\bigoplus_{i} f\left(\sigma_{i}\right)
$$

(i.e., functions act blockwise on $\Sigma_{A}$ ).

Consider first the variation along the directions parametrized by $U$.

We look for the variation in the direction parametrized by one-parameter subgroups. Call $L$ the generator.

We then compute

$$
\left.\frac{d}{d t} \phi_{t}^{*} \operatorname{tr} A U\right|_{t=0}=\left.\frac{d}{d t} \operatorname{tr}\left(A e^{t L}\right) U\right|_{t=0}=\sum_{i, j} A_{i, j} L U_{i, j}=\operatorname{tr}(A L U)
$$

where we have denoted by $\phi_{t}^{*}$ the adjoint action. In the same way, one computes

$$
\left.\frac{d}{d t} \phi^{*} \operatorname{tr}\left(A U B U^{*}\right)\right|_{t=0}=\operatorname{tr}\left(A L U B U^{*}\right)-\operatorname{tr}\left(A U B U^{*} L\right) .
$$

It is easy to verify that

$$
\left[L_{i}, P_{j}\right]=0, \quad L_{i} P_{j}=\delta_{i, j} L_{i} .
$$


The derivatives take the form

$$
\partial_{L_{i}} \sum_{j} \operatorname{tr}\left(P_{j} \rho P_{j} U_{j} \ln \lambda_{j} U_{j} \ln \lambda_{j} U_{j}^{*}\right)=\operatorname{tr} L_{i}\left[\ln \sigma_{i}, \rho_{i}\right] .
$$

If $\sigma$ and $\sum P_{i} \rho P_{i}$ can be diagonalized simultaneously, the derivatives vanish.

This is also a necessary condition since the commutator $\left[\ln \sigma_{i}, \rho_{i}\right]$ is traceless and $L_{k}$ and $i L_{k}$ span the all-traceless matrices in the $i$-th block.

We must consider next the variation with respect to $\Lambda$ restricting to the case when $\sigma$ and $\sigma_{i} P_{i} \rho P_{i}$ can be simultaneously diagonalized.

Let $\mu_{k}^{\sigma}$ and $\mu_{k}^{\rho}$ be the eigenvalues of $\sigma$ and of $\sum_{i} P_{i} \sigma P_{i}$.

If $\mu_{i}^{\rho} \neq 0$ and $\mu_{i}^{\sigma}=0$, one has $D(\rho, \sigma)=\infty$ so that this cannot be a minimum. One has

$$
\partial_{\lambda_{k}^{\sigma}}-\partial_{\lambda_{k}^{\mu}} \sum_{m} \lambda_{m}^{\rho} \ln \lambda_{m}^{\sigma}=0,
$$

which implies that $\lambda_{k}^{\rho} / \lambda_{k}^{\mu}$ is independent of $k$.

So the ratio of the eigenvalues of $\sigma$ and of $\sum_{i} P \sigma_{i} P$ is fixed. Since they are both of trace 1 , they coincide.

It follows that the state $\sigma=\sum P_{i} \rho P_{i}$ is the unique minimum of the relative entropy, i.e., it is the unique state that is least distinguishable from the original state among all states which are compatible with the observation of the observable $a$.

The results was later generalized by Kostecki [2014], who proves that minimization of the (Araki) quantum entropy is equivalent to the Lüders rule (a rule for updating information)

$$
\rho \rightarrow \frac{\sum_{j \in J} P_{j} \rho P_{j}}{\sum_{j} \operatorname{tr}\left(P_{j} \rho P_{j}\right)}
$$

where $J$ is a subset of a countable set corresponding to an orthogonal decomposition and $\sum_{i} P_{i}=I \in \mathscr{B}(\mathscr{H})$ (the spectral sequence of an operator $A$ on $\mathscr{H}$ ) and the domain of this equation is restricted by the condition that $\sum_{i} \operatorname{tr}\left(P_{i} \rho\right) \neq 0$ (the measurement of $A$ succeeded).

This result has a strong bayesian flavor, and this leads us to the next topic.

\section{Quantum bayesianism (QB)}

I close this paper with a brief discussion of quantum bayesianism.

This vision of quantum mechanics, put forward by C. Fuchs and A. Peres [2000] and then by C. Fuchs, N. Mermin, and A. Schack [Fuchs et al. 2015], explores further the path laid by Bohr.

The approach of these authors was to follow the Bayes-de Finetti interpretation of probability as updating of information in order to account for QM. They call this approach quantum bayesianism (in short QB-ism). 
They agree with Bohr that the primitive concept of experience is fundamental for the understanding of QM, but contrary to Bohr, QB-ism explicitly takes the subjective view of probability stressed by Bayes and de Finetti; i.e., probabilities are assigned to an event by an agent and are particular to that agent.

These authors state that QM does not describe physical reality. It provides every single agent with an algorithm for computing probabilities for macroscopic events (such as detector clicks) that are consequences of the agent's interactions with his world.

The agent has in general no control of the reaction, and the result of the experiences leads to an upgrading of the picture and of the expectations.

Still, an important component of the agent's experience is the impact of the efforts of other agents to communicate in speech or writing their own experiences. Science is a collaborative effort to find, through individual actions and verbal communications, a model for what is common to all our constructed external worlds.

To reify the (common) external world is a sound strategy for all practical purposes, but when subtle scientific concept are at stake, such as quantum state, it pays to trace back our description to our experience of the external world.

In a letter to Sommerfeld, Schrödinger [2011, p. 490] already stated, "Quantum mechanics forbids statements about what really exists - statements about the object. Its statements deal only with the object-subject relation".

And Niels Bohr [1934, p. 18] added, "in our description of nature the purpose is not to disclose the real essence of the phenomena but only to track down [...] relations between the manifold aspects of our experience".

Failing to recognize the foundational role of personal experience creates puzzles and paradoxes.

This strict definition of the scope of quantum theory is the only one ever needed whether by experimenters or by theorists.

In a letter to Peierls, J. Bell commented, "One can learn quantum mechanics the way one learns how to ride a bicycle, without really knowing what one is doing. But it is impossible to make sense of either without taking into account of what people actually do with them".

QB-ism shares with the Copenhagen interpretation the statement that the quantum state of a system is not an objective property of that system but only a mathematical tool to think about it.

A fundamental difference is that QB-ism (like Bayes) explicitly introduces each user of quantum mechanics into the story, together with the world external to the user.

A measurement is any action that an agent takes to elicit a response. Given a measurement outcome, the quantum formalism guides the agent in updating the probabilities for subsequent measurements. 
From this point of view a measurement does not, as the term unfortunately suggests, reveal a preexistent state of the system.

Quantum mechanics is a powerful tool that any agent can use to organize his own experience. That this tool is used with spectacular success is an important objective fact about the world we live in.

But quantum mechanics itself does not deal directly with the objective world: it belongs to our experience of that objective world.

This is entirely different from the standard versions of quantum mechanics; for example Landau and Lifshitz [1965, pp. 2-3] state, "By measurement [...] we understand any process of interaction between classical and quantum objects, occurring $[\ldots]$ independently of any observer".

Bohr renounces this extreme attitude, but still individuals enter the story only as proprietors of a large classical apparatus, and the apparatus objectifies the diverse family of users. Replacing the single user with the apparatus introduces the ill defined shifty split much criticized by J. Bell.

This is a split between classical and quantum, macroscopic and microscopic; the split is shifty because its location can be freely shifted.

Because the outcomes of the Copenhagen experiments are classical, they are considered automatically real. In this interpretation, words like macroscopic are used to indicate the objective, nonquantum character of the outcome of a measurement.

In QB-ism measurement has a broader meaning; every action constitutes a measurement, and every outcome is a private experience that can be communicated in classical terms. The famous story about Wigner's friend is transformed from a paradox to a basic dictum.

The Copenhagen school holds that a quantum state encapsulates our knowledge. QB-ism replaces knowledge with belief, the belief of the person who made a state assignment, the belief of the implications of further experiences.

An important difference is the meaning of "with probability 1". As in the theory of Bayes, it reflects only the willingness to accept bets. It does not imply the existence of a deterministic mechanism.

This point was made long ago by $\mathrm{D}$. Hume in his critique of induction: in physics we believe in induction only because it has worked over and over again.

That probability-1 assignments are as any other assignment is essential for the coherence of QB-ism.

\section{References}

[Bohr 1934] N. Bohr, Atomic theory and the description of nature, Cambridge University, 1934.

[Bohr 1935] N. Bohr, "Can quantum-mechanical description of physical reality be considered complete?”, Phys. Rev. 48:8 (1935), 696-702. 
[Bohr 1963] N. Bohr, "The unity of human knowledge", pp. 8-16 in Philosophical writings of Niels Bohr, vol. 3: Essays 1958-1962 on atomic physics and human knowledge, Ox Bow, Woodbridge, CT, 1963.

[Dell'Antonio 2015] G. F. Dell'Antonio, “On tracks in a cloud chamber”, Found. Phys. 45:1 (2015), 11-21.

[Figari and Teta 2014] R. Figari and A. Teta, Quantum dynamics of a particle in a tracking chamber, Springer, Berlin, 2014.

[Fuchs and Peres 2000] C. A. Fuchs and A. Peres, "Quantum theory needs no 'interpretation"', Phys. Today 53:3 (2000), 70-71.

[Fuchs et al. 2015] C. A. Fuchs, N. D. Mermin, and R. Schack, "Reply to Nauenberg", Amer. J. Phys. 83:3 (2015), 198.

[Griffiths 2002] R. B. Griffiths, Consistent quantum theory, Cambridge University, 2002.

[Hornberger 2009] K. Hornberger, "Introduction to decoherence theory", Chapter 5, pp. 221-276 in Entanglement and decoherence, edited by A. Buchleitner et al., Lecture Notes in Physics 768, Springer, 2009.

[Jaynes 1990] E. T. Jaynes, "Probability in quantum theory", pp. 381-403 in Complexity, entropy and the physics of information (Santa Fe, NM, 1989), edited by W. H. Zurek, Santa Fe Institute Studies in the Sciences of Complexity 8, Addison-Wesley, Redwood City, CA, 1990.

[Kostecki 2014] R. P. Kostecki, "Lüders' and quantum Jeffrey's rules as entropic projections", preprint, 2014. arXiv $1408.3502 \mathrm{v} 1$

[Landau and Lifshitz 1965] L. D. Landau and E. M. Lifshitz, Quantum mechanics: non-relativistic theory, 2nd ed., Course of Theoretical Physics 3, Pergamon, Oxford, 1965.

[Mott 1929] N. F. Mott, "The wave mechanics of $\alpha$-ray tracks", P. Roy. Soc. A 126 (1929), 79-84.

[Pauli 1994] W. Pauli, Writings on physics and philosophy, edited by C. P. Enz and K. von Meyenn, Springer, Berlin, 1994.

[Petersen 1963] A. Petersen, “The philosophy of Niels Bohr”, B. Atom. Sci. 19:7 (1963), 8-14.

[Raimond 2014] J.-M. Raimond, "Exploring the quantum world with photons trapped in cavities and Rydberg atoms", Chapter 11, pp. 395-426 in Quantum machines: measurement and control of engineered quantum systems (Les Houches, France, 2011), edited by M. Devoret et al., Lecture Notes of the Les Houches Summer School 96, Oxford University, 2014.

[Robert 1998] D. Robert, "Semi-classical approximation in quantum mechanics: a survey of old and recent mathematical results", Helv. Phys. Acta 71 (1998), 44-116.

[Schrödinger 2011] E. Schrödinger, Eine Entdeckung von ganz außerordentlicher Tragweite: Schrödingers Briefwechsel zur Wellenmechanik und zum Katzenparadoxon, edited by K. von Meyenn, Springer, Berlin, 2011.

[Vedral 2002] V. Vedral, "The role of relative entropy in quantum information theory", Rev. Modern Phys. 74:1 (2002), 197-234.

[Wheeler and Zurek 1983] J. A. Wheeler and W. H. Zurek (editors), Quantum theory and measurement, Princeton University, 1983.

Received 1 Sep 2016. Revised 6 Dec 2016. Accepted 12 Jan 2017.

Gianfausto Dell' Antonio: gianfa@sissa.it

Mathematics Department, University Sapienza, Rome, Italy

and

Mathematics Area, SISSA, Trieste, Italy 


\title{
LUCIO RUSSO: PROBABILITY THEORY AND CURRENT INTERESTS
}

\author{
Giovanni GaLlavotTi
}

We review Russo's original contributions to various fields of probability theory and his parallel interest in history and the influence of Hellenistic science.

The work of Lucio Russo started in the early 1970s. I met him in Naples, and it became immediately clear that I had met a very young and very promising scientist. And I had the privilege to collaborate with him on the problem of the isomorphism between Ising model equilibrium probability distributions in the convergence domain of the cluster expansion and Bernoulli schemes of the same entropy [di Liberto et al. 1973].

Soon afterwards I left Naples, but I continued to follow his work. His study of the isomorphism problem was pursued and led to a major result on coding theory; he proved the existence of a finitary code between the simplest Markov process (the one-dimensional Ising model with nearest neighbor interaction) and the Bernoulli shift with the same entropy [Monroy and Russo 1975]. I saw the impact that this work had on the complete solution of the general construction [Keane and Smorodinsky 1977] of a code of a Markov chain into a Bernoulli shift and the recognition that it received.

To carry out this work he learned in a short time the deep and innovative work of Ornstein on the theory of Bernoulli shifts and was able to give a substantial contribution to the applications of the theory.

He rapidly became internationally known as a leading probabilist, contributing to advances on the percolation problem beginning with studying the attractive Ising model, establishing a close relation between the existence of infinite clusters of spin + or - and the presence of spontaneous magnetization in the extremal states $\mu_{ \pm}$, with the remarkable result that in general dimension the spontaneous magnetization in the state $\mu_{+}$or $\mu_{-}$yields a lower bound to the percolation probability (i.e., existence of infinite clusters of + or - , respectively). Furthermore, in $2 \mathrm{D}$ coexistence of infinite clusters of both types was excluded in all pure states [Coniglio et al. 1976; 1977].

\section{Communicated by Francesco dell'Isola.}

Keywords: percolation, probability, Ising model, phase coexistence, coding theory, Markov processes, Hellenistic science, infinite clusters. 
In the course of about three years, he worked intensely on percolation (in Bernoulli sites distribution as well as in Ising model equilibria), employing the FKG and GHS inequalities, first extending a result by Harris on critical percolation and proving that mean size of the finite clusters is finite [Russo 1978]. This led, in a subsequent work, to the proof, in the case of percolation on $\mathbb{Z}^{2}$, of the Essam-Sykes conjecture on the critical probabilities of two "matching graphs" $\left(p_{c}+p_{c}^{*}=1\right)$ [Russo 1981].

A result on the difficult problem of the (site) percolation in $\mathbb{Z}^{3}$ followed after a series of studies (mostly in 2D). The first was on a proof that in 2D-Ising there is a unique infinite cluster for $T \leq T_{c}$ and that the critical point and the percolation point coincide (different from their already known noncoincidence in 3D) [Coniglio et al. 1976; 1977]; the smoothness, away from the critical point, of the 2D percolation probability dependence on the occupation probability followed [Russo 1978]; the Essam-Sykes conjecture was then proved [Russo 1981], obtaining also (on $\mathbb{Z}^{2}$ ) that the site percolation probability is a continuous function of the site probability $p$. Finally the methods, always based on inequalities known in statistical mechanics and on several extensions developed in Russo's works, have been applied to the 3D-percolation and to the 3D-Ising model: the critical site percolation is proved to be $<\frac{1}{2}$, and in 3D-Ising at high temperature (and small field), coexisting infinite clusters are shown to be possible [Campanino and Russo 1985].

The first results on percolation paved the way for a groundbreaking result, based on the Ising model inequalities and relying also on ideas developed in the first few percolation papers (which will also be further developed in the later works), on the uniqueness of the translation-invariant Gibbs states in the two-dimensional Ising model [Russo 1979]. It introduced a fresh view and a new method on the uniqueness problem of the pure phases and proved that the states that were translationinvariant in one direction were necessarily invariant under all translations. Immediately after publication of his work, his result has been basic to the final solution of the longstanding conjecture about the translation invariance of the equilibrium states of the Ising model at all temperature and field values (independently in [Higuchi 1981; Aizenman 1980]).

Percolation continued to be the focus of his interests; for the site percolation problem, with site probability $p$, Russo proved that the probability of a given positive event $A$ measurable at infinity (or close enough to be such) passes from 0 to 1 as $p$ grows through a value $p_{0}$, an extension of the Hewitt-Savage zero-one law [Russo 1982]. This result was also shown to be useful in determining, by a new method, the relation $p_{c}+p_{c}^{*}=1$ for the critical percolations on the square lattice and its matching lattice.

A theory of a different kind of percolation, the (independently occupied with probability $p$ ) plaquettes percolation on $\mathbb{Z}^{d}, d \geq 3$, is studied. It was shown that in $d=3$ the existence of a phase transition in the dependence of the probability that 
the occupied plaquettes form a surface with given boundary decays exponentially with the area of the surface for small $p$ but at high plaquettes density, i.e., $p$ close to 1 , the probability decays with the length of the boundary. The result, found in [Aizenman et al. 1983], is obtained employing also the duality between plaquette percolation and bond percolation, and the sharpness of the transition (as a function of $p$ ) is related to a conjecture on the $3 \mathrm{D}$ bond percolation.

The problem of the uniqueness of infinite percolating clusters is then reexamined first in the case of Bernoulli percolation and then in the 2D case of rather general site distribution, subject to conditions of translation ergodicity in each direction and to the monotonicity of $\mu(F \cap G) \geq \mu(F) \mu(G)$ [Gandolfi et al. 1988a; 1988b].

The attention to inequalities, an essential feature of most if not all of Russo's works, has generated a work on new inequalities related to the FKG inequalities which have new applications to a variant of the Ising model, the "plaquette model" [Cammarota and Russo 1991], considered in certain gauge theories.

Russo has also contributed to other fields of mathematics like dynamical systems and analysis, with the same uncompromising attention to mathematical rigor transpiring from his probability works, providing insights and suggestions in the normal seminars held in the departments where he has been a member [Franceschini and Russo 1981; 1983].

All the above results were obtained by Russo working alone or in collaboration with other scientists. His sharp understanding of the probability theory of Bernoulli distributions or of Ising distributions as always been an essential contribution. At the end of the 1980s, Russo's main interests switched to the history of science, revealing a less known aspect of his personality which had remained hidden to most of his colleagues. In a sense he really concentrated on the classic achievements from the Hellenistic science to the 1600s.

This choice left little room to continue developing probability theory of percolation (whether Bernoulli or Ising or other). I witnessed that this generated deep regrets from several leading probabilists.

His involvement in the new task that he assigned himself was totalizing: to study the few remnants of the Hellenistic age, he ended up gradually learning classic Greek (starting from brushing up the Latin and Greek he had learned in high school). The works that arise from this activity are for me difficult to comment on, not having really worked on the ancient or modern history of science, and I will try to mention briefly some of them; many are in Italian, and I expect that after translation into English they will generate an even wider debate.

He began, around 1990, with a critique of the authenticity of the geometric entities in the definitions at the beginning of Euclid's Elements. The analysis appeared in the Bollettino dei Classici of the Accademia dei Lincei, and a revised English version in the Archive for History of Exact Sciences [Russo 1992; 1998a]. The 
thesis is that, in the several centuries elapsed since Euclid to the present version of the Elements, the introductory definitions were added to the original work, or "simplified". The thesis is developed by a detailed logical analysis of the definitions; they are certainly captivating and lead to meditation on the subject. They are often not accepted on the grounds that some incompetent scholar should have dared to "simplify" Euclid's work [Artmann 1998]. Personally I see no reason to rule out the possibility of distortion in the transmission of the definitions: the work of Boltzmann (whose original sources are still available) is a compelling example of how scientific ideas may be misinterpreted, and nevertheless be useful, just a few years (not even centuries) after most scientists proceeded to make use of their consequences relying on expositions rather than relying on the original.

Several essays followed: with sharp critiques of modern interpretations of Hellenistic achievements in astronomy, geography, mechanics, dynamics [Russo 1993b; 1994], gravitation and tidal theory [Russo 1993a; 1993c]. In [Russo and Medaglia 1996] he convincingly refuted the common wisdom that Aristarchus was accused of "impiety" because of his heliocentric system.

His essays have often been collected and extended in books, starting with The Forgotten Revolution, whose Italian original [Russo 1996] went through three editions, was then revised as it was translated into English [Russo 2004], and from there into German and Polish. The book is captivating, with several new readings of Greek and Roman texts which are often very convincing (showing the contribution that practicing scientists can make to historical research). In the final chapters of the book, he defends the view that the development of science in the Renaissance benefited from access to ancient works that circulated in Europe after the fall of Constantinople - to a far greater extent that is usually recognized, because some of those works have since been lost. The notion that Renaissance scholars might have concealed their inspiration in ancient sources has faced skepticism or outright rejection in many quarters (see [Pambuccian 2001; Ewing 2004; Greene 2004; Rowan-Robinson 2004] for example), though others have been more open to that possibility [Graffi 1998; Netz 2002]. However controversial Russo's views on the development of modern science may be, his discussion of the classic achievements and of the imperial decay of science is fascinating and rich in new insights.

The Forgotten Revolution is a seminal and stimulating work whose theses will long generate debate and further studies. An example that illuminates the kind of critical examination of sources (here Pliny and Vitruvius) that Russo excels at considers the motion of the moon [Russo 1996, §10.6, p. 319; Russo 2004, §10.8]. He advanced this argument elsewhere as well: for instance in an essay on the astronomy of Hipparchus [Russo 1994] and other pre-Ptolemaic sources.

Russo has since written more essays, eventually coherently organized and collected into books. For instance, the book Flussi e riflussi on the history and the 
origins of the theory of tides, expanding [Russo 1995], gives a detailed and documented analysis of the developments in the modern period and its roots in antiquity [Bonelli and Russo 1996; Russo 2003], stressing that the theory was developed with apparently independent contributions repeatedly discovered and lost or forgotten, influencing many thoughts and ending to be eventually attributed only to particular scientists (Newton, Laplace, Kelvin and G. H. Darwin). And we list only a few essays: a discussion on the nature of and relation between mathematics and physics and its evolution from Hellenism to contemporary science or a paper on the "case" of Aristarchus [Russo 2002], which goes back to the impiety accusation and further clarifies its unfoundedness, shown to be due to a misunderstanding of a text of Plutarch. He discusses also the important contributions of Seleucus to heliocentrism and to theories of tides [Russo 2002] and an essay on Archimedes and on the myths about him [Russo 2013a].

A more unorthodox book is L'America dimenticata [Russo 2013b], where he develops the idea (briefly suggested in the Revolution) that America was visited over an extended period of time by sailors from the ancient Mediterranean, including the Carthaginians both before and after the downfall of their city. The book focuses on the thesis that civilization is not deterministically controlled by human genes, and independent human communities might evolve very differently rather than proceeding, deterministically, in parallel through the same stages, only up to random time delays. In particular major calamities might completely stop, pull back by centuries or divert the evolution: one of them was the Roman destruction of Carthage, which cut earlier links between Europe and Central America, as Russo contends, and caused the loss of knowledge of the Atlantic navigation practiced at least by Phoenicians and Carthaginians, with the result that scanty vestiges remain of the link between the evolution of European and American civilizations.

The case is discussed in detail, expanding [Russo 2013c], and taking the opportunity to analyze a debated geography question and to offer a new interpretation of the related work of Ptolemy. The highlight is a scientific analysis of the reduction of the Earth's circumference from 252000 to 180000 stadia: from the value established by Eratosthenes and used by Hipparchus to the value documented, more than three centuries later, by Ptolemy.

According to Russo, the problem was not, as often thought, that Ptolemy mistook the length of the "Eratosthenes stadium" (thinking it to be 1.43 times smaller than that used afterwards, well into the next millennium). Instead, the error can be traced to the misidentification of the extreme western point of the known world with the Canary Islands, rather than the Lesser Antilles.

To be precise, according to Ptolemy, the latitude of this western limit (expressed in today's coordinates) was around $5.5^{\circ}$, and it belonged to what he called the Blessed Isles. He identified them with the Canary Islands; but he states that the 
longitudinal extent of the Blessed Isles is $1^{\circ}$, while the Canaries archipelago is close to $5^{\circ}$ wide, and there is also a $15^{\circ}$ difference in latitude between the two. A better fit for the Blessed Isles of Ptolemy's sources, Russo contends, would be the Lesser Antilles, which match the latitude and longitudinal extent reported by Ptolemy, and also match the longitude deduced from other information that Ptolemy provides: that the western and eastern edges of the known world lie on opposite meridians, and that the eastern edge is at a certain (relatively short) distance from the capital of China, whole location in Hellenistic times we know (Xi'an).

Russo's conclusion and proposal is that Ptolemy, taking as given the $180^{\circ}$ width of the known world - a datum apparently inherited from earlier scientists (the evidence is analyzed) - and its eastern limit, unwittingly moved east its western limit, because the Blessed Isles had become associated with the Canary Islands, their earlier identity having been forgotten in the wake of Carthage's destruction (further arguments can be found in [Russo 2016]). A consequence seems to be that Ptolemy had several correct distances along (some) parallels but no accurate astronomical determination of longitudes for at least a few known sites ${ }^{1}$ to fit the known distances: giving the Canaries as the extreme limit and $180^{\circ}$ degrees the width of the inhabited world, a shorter Earth radius and a dilation of the longitudes resulted.

The analysis also solves another mystery arising from Ptolemy's Geography: the location of Thule. Showing that the most common accepted proposals do not fit all available facts, Russo places Thule, after reinterpreting Ptolemy's value of its longitude, on the east coast of Greenland, which is consistent with what we know of Pytheas' explorations.

The book contains a dense set of footnotes to document the statements taken from the literature (mostly classical) and a captivating analysis of them. Unfortunately the notes have been relegated to the end of the chapters (as done also in the next book by Russo, a choice increasingly made by printers), which is very inconvenient for the readers because the footnotes are really essential here.

In a third book Stelle, atomi e velieri (Stars, atoms and sailing ships) [Russo 2015], the basic idea on the nondeterministic evolution of science and its dependence on continuous interaction, through possibly tenuous temporal or spatial links between different scientists, is taken up again and further developed. Here Russo puts together all his previous arguments and offers a rapid and synthetic view of some of the major events in the history of science, always paying attention and

\footnotetext{
${ }^{1}$ Longitude could have been only determined by on-site astronomical observations, which require (good instruments and) a physical presence to observe the exact moment of an expected celestial event, e.g., a lunar eclipse or an equinox, and either the exact local solar hour or the concomitant equatorial coordinates of a star. Such observations were possible in antiquity (and positively mentioned and appreciated by Ptolemy [Russo 2013b, p. 158]) but there are few clear traces of them.
} 
adding evidence to reinforce the main idea that can be found in his earlier books and papers. For instance it is interesting to see collected and organized in a wider context the analysis of the roles of Copernicus and Bruno and the violent opinions of Voltaire on Aristarchus, which will make at least some readers wish that Voltaire had been more careful in his judgment; see also [Bonelli and Russo 1996; Russo 2002]. The book expands all themes treated earlier, adding substantial remarks on science after the Renaissance, with arguments in favor of one of his main theses, i.e., the cultural interdependence between many scientists, starting with Hellenic and Hellenistic science, and that major discoveries are often only attributed to "giants". He acutely examines the evolution of the disciplines called mathematics, physics and mathematical physics, expressing judgments which will, likely, generate lively debates.

Recently Russo has proposed a probabilistic approach to the determination of the dates of events known to have occurred in a time interval [Benedetto et al. 2016], a return to probability theory, applied to the date of birth of Hypatia and Theon. A further book is dedicated to the history of science in Italy [Russo and Santoni 2010]. The editorial activity of Russo also led him to write, perhaps under the influence of his personal history with parents deeply dedicated to high school teaching (hence with an early direct knowledge of the problems and issues), an essay on education criticizing, often harshly, the modern methods of teaching science in the Italian high schools [Russo 1998b], a critique which hopefully will be heard, sooner or later.

\section{References}

[Aizenman 1980] M. Aizenman, "Translation invariance and instability of phase coexistence in the two-dimensional Ising system”, Comm. Math. Phys. 73:1 (1980), 83-94.

[Aizenman et al. 1983] M. Aizenman, J. T. Chayes, L. Chayes, J. Fröhlich, and L. Russo, "On a sharp transition from area law to perimeter law in a system of random surfaces", Comm. Math. Phys. 92:1 (1983), 19-69.

[Artmann 1998] B. Artmann, Review of [Russo 1998a], Math Reviews, 1998, Available at http:// www.ams.org/mathscinet-getitem? $\mathrm{mr}=1612769$.

[Benedetto et al. 2016] C. Benedetto, S. Isola, and L. Russo, "Dating Hypatia's birth: a probabilistic model", preprint, 2016, Available at https://www.researchgate.net/publication/307593271. To appear in Math. Mech. Complex Syst.

[Bonelli and Russo 1996] F. Bonelli and L. Russo, "The origin of modern astronomical theories of tides: Chrisogono, de Dominis and their sources”, Brit. J. Hist. Sci. 29:4 (1996), 385-401.

[Cammarota and Russo 1991] C. Cammarota and L. Russo, "Bernoulli and Gibbs probabilities of subgroups of $\{0,1\}^{S}$, Forum Math. 3 (1991), 401-414.

[Campanino and Russo 1985] M. Campanino and L. Russo, "An upper bound on the critical percolation probability for the three-dimensional cubic lattice", Ann. Probab. 13:2 (1985), 478-491.

[Coniglio et al. 1976] A. Coniglio, C. R. Nappi, F. Peruggi, and L. Russo, "Percolation and phase transitions in the Ising model”, Comm. Math. Phys. 51:3 (1976), 315-323. 
[Coniglio et al. 1977] A. Coniglio, C. R. Nappi, F. Peruggi, and L. Russo, "Percolation points and critical point in the Ising model", J. Phys. A 10:2 (1977), 205-218.

[Ewing 2004] J. H. Ewing, Review of [Russo 2004], Math Reviews, 2004, Available at http:// www.ams.org/mathscinet-getitem?mr=2038833.

[Facchinetti and Russo 1983] G. Facchinetti and L. Russo, "A one-dimensional case of stochastic homogenization", Boll. Un. Mat. Ital. C (6) 2:1 (1983), 159-170.

[Franceschini and Russo 1981] V. Franceschini and L. Russo, "Stable and unstable manifolds of the Hénon mapping", J. Statist. Phys. 25:4 (1981), 757-769.

[Gandolfi et al. 1988a] A. Gandolfi, G. Grimmett, and L. Russo, "On the uniqueness of the infinite cluster in the percolation model”, Comm. Math. Phys. 114:4 (1988), 549-552.

[Gandolfi et al. 1988b] A. Gandolfi, M. Keane, and L. Russo, "On the uniqueness of the infinite occupied cluster in dependent two-dimensional site percolation", Ann. Probab. 16:3 (1988), 11471157.

[Graffi 1998] S. Graffi, Review of [Russo 1996], Notices Amer. Math. Soc. 45:5, 1998, Available at http://www.ams.org/notices/199805/review-graffi.pdf.

[Greene 2004] M. Greene, “The birth of modern science?”, Nature 430:7000 (2004), 614. Review of [Russo 2004].

[Higuchi 1981] Y. Higuchi, "On the absence of non-translation invariant Gibbs states for the twodimensional Ising model”, pp. 517-534 in Random fields (Esztergom, Hungary, 1979), vol. I, edited by J. Fritz et al., Colloq. Math. Soc. János Bolyai 27, North-Holland, Amsterdam, 1981.

[Keane and Smorodinsky 1977] M. Keane and M. Smorodinsky, "A class of finitary codes", Israel J. Math. 26:3-4 (1977), 352-371.

[di Liberto et al. 1973] F. di Liberto, G. Gallavotti, and L. Russo, "Markov processes, Bernoulli schemes, and Ising model", Comm. Math. Phys. 33:4 (1973), 259-282.

[Monroy and Russo 1975] G. Monroy and L. Russo, "A family of codes between some Markov and Bernoulli schemes”, Comm. Math. Phys. 43:2 (1975), 155-159.

[Netz 2002] R. Netz, "Review: La rivoluzione dimenticata. Il pensiero scientifico greco e la scienza moderna", Hist. Math. 29:1 (2002), 72-73.

[Pambuccian 2001] V. V. Pambuccian, Review of [Russo 1996], Math Reviews, 2001, Available at http://www.ams.org/mathscinet-getitem?mr=1629047.

[Rowan-Robinson 2004] M. Rowan-Robinson, "Praising Alexandrians to excess", Phys. World 17:4 (2004), 42. Review of [Russo 2004].

[Russo 1978] L. Russo, “A note on percolation”, Z. Wahrsch. Verw. Gebiete 43:1 (1978), 39-48.

[Russo 1979] L. Russo, "The infinite cluster method in the two-dimensional Ising model”, Comm. Math. Phys. 67:3 (1979), 251-266.

[Russo 1981] L. Russo, "On the critical percolation probabilities", Z. Wahrsch. Verw. Gebiete 56:2 (1981), 229-237.

[Russo 1982] L. Russo, “An approximate zero-one law”, Z. Wahrsch. Verw. Gebiete 61:1 (1982), 129-139.

[Russo 1992] L. Russo, "Sulla non autenticità delle definizioni degli enti geometrici fondamentali contenute negli Elementi di Euclide", Boll. Classici 13 (1992), 25-44.

[Russo 1993a] L. Russo, "Il contenuto scientifico di un brano di Lucrezio (IV, 387-396)", Boll. Classici 14 (1993), 93-95.

[Russo 1993b] L. Russo, "Un brano di Plutarco (Moralia, 923C-924A) e la storia della dinamica", Boll. Classici 14 (1993), 80-92. 
[Russo 1993c] L. Russo, "Vitruvio ('De architectura', IX, i, 11-14): un brano di argomento astronomico”, Boll. Classici 14 (1993), 96-102.

[Russo 1994] L. Russo, "The astronomy of Hipparchus and his time: a study based on pre-Ptolemaic sources", Vistas Astronom. 38:2 (1994), 207-248.

[Russo 1995] L. Russo, "L'astronomo Seleuco, Galileo e la teoria della gravitazione", Quaderni Urbinati Cultura Classica 49:1 (1995), 143-160.

[Russo 1996] L. Russo, La rivoluzione dimenticata: il pensiero scientifico greco e la scienza moderna, Feltrinelli, Milan, 1996.

[Russo 1998a] L. Russo, "The definitions of fundamental geometric entities contained in Book I of Euclid's Elements”, Arch. Hist. Exact Sci. 52:3 (1998), 195-219.

[Russo 1998b] L. Russo, Segmenti e bastoncini: dove sta andando la scuola?, Feltrinelli, Milan, 1998.

[Russo 2002] L. Russo, "Aristarco di Samo: uno scienzato isolato?", preprint, 2002, Available at http://www.academia.edu/5791148.

[Russo 2003] L. Russo, Flussi e riflussi: indagine sull'origine di una teoria scientifica, Feltrinelli, Milan, 2003.

[Russo 2004] L. Russo, The forgotten revolution: how science was born in 300 BC and why it had to be reborn, Springer, 2004.

[Russo 2013a] L. Russo, "Archimedes between legend and fact", Lettera Mat. 1:3 (2013), 91-95.

[Russo 2013b] L. Russo, L'America dimenticata: i rapporti tra le civiltà e un errore di Tolomeo, Mondadori Università, Milan, 2013.

[Russo 2013c] L. Russo, “Ptolemy's longitudes and Eratosthenes' measurement of the earth's circumference”, Math. Mech. Complex Syst. 1:1 (2013), 67-79.

[Russo 2015] L. Russo, Stelle, atomi e velieri: percorsi di storia della scienza, Mondadori Università, Milan, 2015.

[Russo 2016] L. Russo, "Far-reaching Hellenistic geographical knowledge hidden in Ptolemy's data”, preprint, 2016, Available at https://hal.archives-ouvertes.fr/hal-01275282.

[Russo and Medaglia 1996] L. Russo and S. M. Medaglia, "Sulla presunta accusa di empietà ad Aristarco di Samo", Quaderni Urbinati Cultura Classica 53:2 (1996), 113-121.

[Russo and Santoni 2010] L. Russo and E. Santoni, Ingegni minuti: una storia della scienza in Italia, Feltrinelli, Milan, 2010.

Received 7 Nov 2016. Accepted 19 Dec 2016.

GIOVANNI GALLAVOTTI: giovanni.gallavotti@roma1.infn.it

Dipartimento di Fisica, Universitá di Roma "La Sapienza”, 00185 Roma, Italy 



\title{
AN ATTEMPT TO LET THE “TWO CULTURES” MEET: RELATIONSHIP BETWEEN SCIENCE AND ARCHITECTURE IN THE DESIGN OF GREEK TEMPLES.
}

\author{
Claudio D’ Amato
}

\begin{abstract}
Editor's note: This unusual paper appears by invitation as an illustration of Lucio Russo's far-reaching influence. The author writes:

The present contribution is aimed at stimulating some reflections on the fracture existing (and enlarging) between what were famously called "the two cultures" by C. P. Snow in his influential book (Snow, C. P., The two cultures and the scientific revolution: The Rede Lecture. Cambridge Univ. P., 1959). The focus is on the architecture of Greek temples of Classical and Hellenistic age. At the same time, some possibility to fill the gap is described, based on my experience and my scientific interaction with Lucio Russo, to whom this editorial is dedicated.
\end{abstract}

\section{Introduction}

One of the theoretical issues which my cultural action in the Architecture School of Bari has been focused on, was the definition of a curriculum able to realize a conceptual unity of teachings of different nature (technical-scientific and historicalartistic) that contribute to train students. An action aimed at countering the globalization and the specialization of knowledge. In this strategy a major role was assigned, starting from the very first year of the course, to teaching classical architecture, in particular Greek, from which all of the theoretical principles and language of Western architecture until the nineteenth century derived. My relationship with Lucio Russo has to be considered within this cultural action: I owe to him a significant step in my teaching and research activities, about which I will speak in these pages.

I met Lucio Russo for the first time in January 2000, after reading his pamphlet Segmenti e Bastoncini, in which I immediately found a convergence of ideas on the importance of "classical" education in Higher School and on the criticism to the deconceptualization promoted by the reform of the (then) Minister Luigi Berlinguer. It was for me a very important meeting. He spoke me about the journal Punti 
critici (which he founded), and about the cultural action in which he was engaged at that time with many lectures in high schools. We also talked about La rivoluzione dimenticata, which I did not know, and that a few months later he came to present in Bari ${ }^{1}$.

In the following part of this editorial I will show some examples of the research approach I was used to before meeting Lucio Russo and later I will show how the scientific interaction with him changed this approach and the related results.

\section{My way of working before meeting Lucio Russo Case study 1: the replica of the capital 9A of the temple of Apollo Epicurius at Bassae ${ }^{2}$}

In 2000, from April to September, I was busy with my research group ${ }^{3}$ in the realization of the replica of the capital 9A of the temple of Apollo Epicurius (rescuer) in Bassae (Messenia). An experiment with which I was searching for a satisfactory transition from traditional techniques of cutting stones to those using numerical control machines. The goal was to improve the performance of architecture with load-bearing masonry systems (in terms of energy performance, cost, etc.) in comparison with that of reinforced concrete frame.

\footnotetext{
${ }^{1}$ May 22, 2000, Politecnico di Bari, Aula Magna "Attilio Alto".

$\mathbf{2}^{2}$ Tradition attributes its design to Ictinus (for a non-specialist approach to the problem, see the popular book by Rhys Carpenter, The Architects of the Parthenon, Penguin Books, 1970). The temple was dedicated to Apollo, who had come to the rescue (Epicurius $=$ rescuer) of the village of Bassae in Messenia victim of an epidemic. The temple constituted a prototype that spread in the following century beyond the geographical limits of the Peloponnesus, extending up to the boundaries of the Greek world. As concerns the exceptional nature of this temple W. B. Dinsmoor writes: "I believe we can say that within the perimeter of the peristyle may be found more fascinating problems than in any other building in the world of ancient Greece" (W. B. Dinsmoor, The temple of Apollo at Bassae, Metropolitan Museum Studies IV, New York, 1932-33). The temple is peripteral-hexastyle ( $6 \times 15$ columns) distinguished by the contemporary presence of the Doric order on the outside, in the peristyle, the pronaos and the opisthodome; and Ionic and Corinthian orders on the inside. Ionic are the columns standing against the spurs, which articulate the interior space of the cell and the frieze at the top, while the column placed on the axis of the cell is instead Corinthian.

${ }^{3}$ The experimental nature of the research project allowed us to:

- select as material Carrara marble, suitable for its degree of hardness to the technical characteristics of the set of tools (milling cutter and tips) available at the time of realization;

- produce a half capital, in consideration of its weight (approx. $1500 \mathrm{~kg}$ for a mean size of approx. $100 \times 60 \times 120)$.

The time required for study (defining the laws of composition and examination) and designing the three-dimensional model was approximately 30 days (April 2000); the time required for realization, about 5 days (September 2000). A large and detailed account of this experience is in C. D'Amato (2003). The formal unity of the Greek Temple. The realization of the 9A capital replica of Apollo epicurius' Temple at Bassai. In: First International Congress on Construction History, Madrid, 2024 January 2003, vol. I / 84-9728-071-7, p. 683-691, ISBN: 84-9728-070-9.
} 
We chose as a case study the Doric capital because in its seeming simplicity it is one of the most difficult and complex model, for its capacity to clearly demonstrate the thesis, since the apparent simplicity of its form, tolerates no imperfection in design or execution. And among the many possible, we choose that of the temple of Apollo Epicurius for its very urgent problems of restauration.

Replace it with a replica? We accepted the challenge, because we felt it was possible to accomplish the goal with the help of new technologies. But the real challenge were the conceptual problems beyond the specific case of the restoration until to the core of contemporary architectural design with its new modes of design and production.

In September, we presented the replica of the capital 9A at MarmoMacc of Verona ${ }^{4}$. We wanted to demonstrate the possibility of "replicating" (not copying) architectural elements irreparably damaged, of which it was perfectly known the shape and the geometric model.

We were convinced that it was possible to go back to the original design model, starting from the conceptual horizon and from the production way of classical Greece, that is from the design geometry and from their concrete "constructability"5.

The Greek Temple could be easily considered as a paradigm in this sense: here stone, used as unique material for the definition of the aesthetic character, expresses directly the structural and decorative geometrical texture, and also defines the formal unity of the architectural system as a whole. Moreover it is characterized by an exact design and perfection in execution, aspects that make it exemplary.

As I said, the damaged capital 9/A of Apollo Epicurius' Temple at Bassae, has been chosen for the elaboration of cad/cam processing software, and associated $3 \mathrm{D}$ virtual simulations for its realization with a $\mathrm{CNC}$ machining center.

This is a chance only if we know all the original project, not just its individual components; and also if we have the technology to run a perfect workmanship. Only if these conditions exist, we can speak of "replicas."

${ }^{4} 35$ th MarmoMacc, International Exhibition of Marble, Stone and Technology, Veronafiere, Saturday, September 30, 2000. Panel discussion: Stone-cutter of the twenty-first century. The replica of the capital 9A of the temple of Apollo at Bassae. Participants: C. D'Amato, S. Alevridis (Ephoria Z, Olympia), B. Cache (ETSAB, Barcelona), P. Marconi (Università Roma Tre), G. Margheriti (CMS), G. Rocco (Università di Chieti), J. Tzedakis (Ephoria Z, Olympia). This experience was the junction of all the research and practice of construction previously initiated by me in the School of Architecture of Bari (that can be defined "traditional") with the "stereotomic" one, favored by the use of numerical control machines and 3D modeling software.

${ }^{5}$ The geometric knowledge of the classical age was at the basis of Euclid's Elements. From it also were derived the instruments and methods used in the building site, such as e.g. the jigs and the rotation process. 


\section{Case study 2: the modelling of the capital 9A: structural geometries and computerized modelling techniques}

The "electronic" modelling of the capital 9A and of the eighth drum of its column, was conducted by processing the data derived from traditional survey (manual) on a scale of $1: 1$.

Capital 9A consists of a square-based parallelepiped abacus, an echinus whose contour is defined by a polycentric or spline curve, by a collarino and by the end of the fluting which is joined to the collarino through a complex surface.

The eighth drum 9A consists of a truncated cone with fluting and engraved end (hypotrachelion).

The identification of the different parts of the capital was done through computerized polygonal CAD modelling by "surfaces" and not by the parametrizing of "primitives" or "extrusion" of polygons, for the purpose of facilitating manipulation of the 3D object.

The abacus, a square-based parallelepiped volume, was obtained by the mutual and orthogonal composition of flat surfaces ("2D faces").

The echinus and the collarino, a single volume of rotation, are the result of a surface of revolution, obtained by rotating the profile of these elements for $360^{\circ}$ around an axis of radial symmetry.

The collarino, serving as union between the end of the fluting and the echinus, mathematically defined as quadric surface, was obtained through a "polar series" of a bilinear curved surface consisting of 20 elements through an angle of $360^{\circ}$. This surface was modelled in two successive stages: the first by interpolating a "Coon surface" for four spatial curves: the first of these is the portion of convex circumference termination of the annuli, contained in the horizontal plane included in an angle of $9^{\circ}$ (half of $360^{\circ} / 20$ ); the second is the profile of the fluting, contained in the vertical plane passing through the axis of radial symmetry; the third is the section measured at the centreline of the fluting, contained in the vertical plane passing through the axis of radial symmetry; the fourth is the portion of concave circumference at the base of the fluting contained in the horizontal plane. The surface determined in this way represents half of the global surface, which is completed, in the second stage, by "mirroring" the surface found according to the axis passing through the centre point of the fluting, perpendicular to the axis of radial symmetry.

The drum consists of a surface passing through three sections contained in horizontal and parallel planes, measured at different heights. The hypotrachelion has been obtained by generating a surface having as generatrix the raised profile (sawtooth section) contained in the vertical plane passing through the axis of radial symmetry, and as directrix the section of the drum, contained in the horizontal plane, at that height with the fluting. 


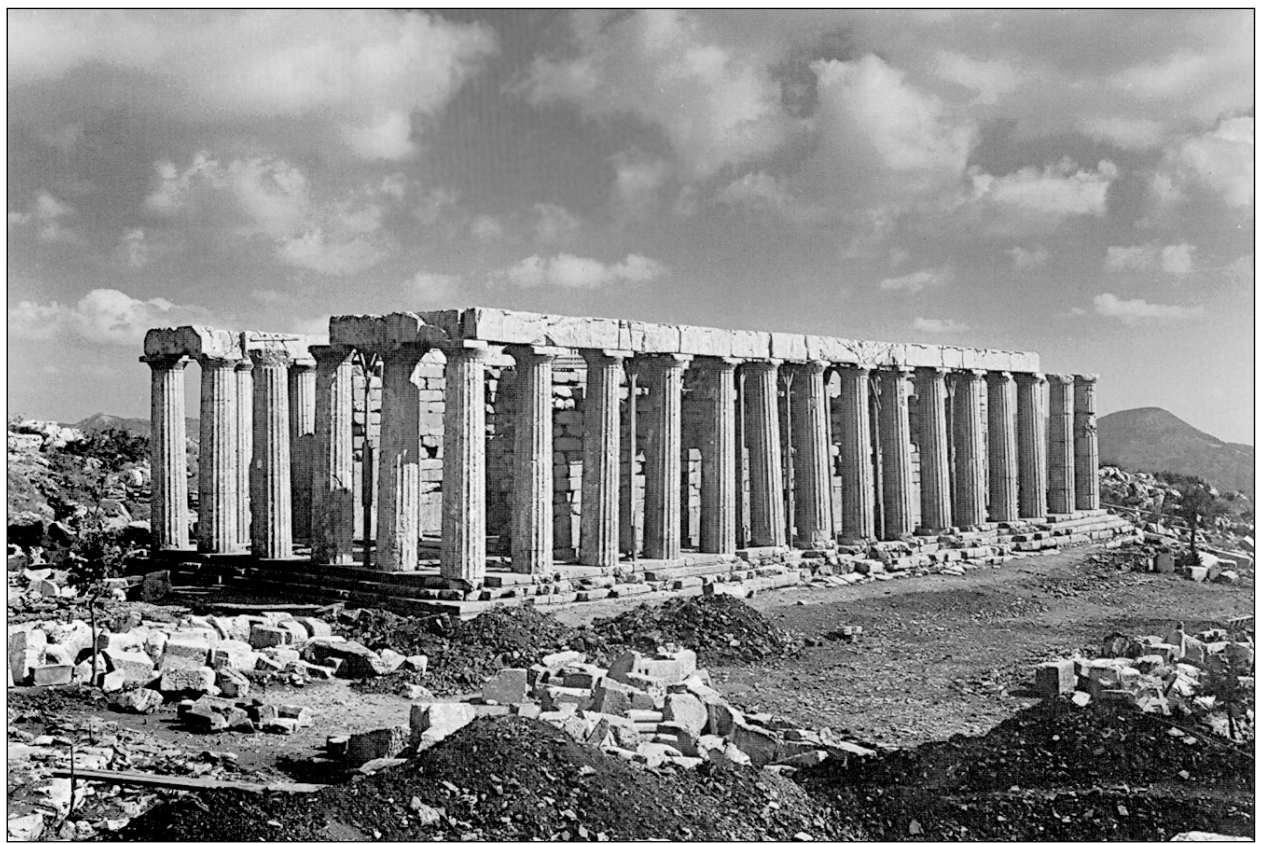

Figure 1. View of the temple of Apollo at Bassae from the northwest, before restoration work (Archive of the Ephoria Z, Olympia).

In October 2000 I gave start to a final synthesis course, whose topic was $D e$ signing and building with dry stone ${ }^{6}$ : the case study was the temple of Apollo at Bassae, and its theme of design was the remaking of its roof, in accordance with the procedures of anastylosis and integration with replicas of the missing pieces (whose geometric shape was known) or heavily damaged. On that occasion the idea of a seminar with Lucio Russo on design issues of the Greek temples of the classical age and the Hellenistic period was born.

\section{How my research approach changed after meeting Lucio Russo: the relationship between science and architecture in the design of Greek temples of the classical age and the Hellenistic period}

On 12 March 2001, Lucio Russo gave a seminar concerning the relationship between

\footnotetext{
${ }^{6}$ Tutors: Claudio D’Amato (Architectural design), Angelo Ambrosi (Architectural Drawing), Mauro Mezzina (Structure and construction); Students: M. Alicino, F. Aulicino, C. Carone, F. Cavone, V. Chieti, G. Dell'Aquila, S. Dentico, L. Donatelli, A. Paresce with the assistance of the PhD students Giuseppe Fallacara and Annalisa Di Roma. A campaign of surveys was carried out on April 9-12, 2001 in agreement with Ephoria Z of Olympia and in collaboration with the architect Sofoklis Alevridis of the Temple Commission.
} 
science and architecture in the design of Greek temples ${ }^{7}$. It was mainly focused on the "rules of correspondence" between the architectural project and its transfer, first "on paper" and then in its construction. So, a seminar that explored the Euclidean geometry as a tool which admits only geometric entities constructible with ruler and compass, on the basis of postulates.

Below there is a synthesis of some excerpts from the seminar of Lucio Russo.

The theorems: deductive method and theoretical entities of thinking. Let us focus on some methodological characteristics of the Hellenistic scientific theories, in the belief that some of these features are still valid in contemporary culture. Hellenistic science is an absolute novelty in the history of mankind, but if you take the books of history of science this novelty does not always emerge.

The scientific method has its roots in the culture of classical period, but emerges substantially in the 4th century B.C., and is characterized by two elements which are the deductive method and the existence of a purely theoretical level of thinking.

A typical case is that of the rational geometry of Euclid's Elements, where there are theoretical entities (triangles, circles, etc. in which no one stumbles walking in the street), and where the propositions can be proved with theorems, i.e. by means of arguments that logically link the statements to previously accepted ones, starting from the well-known five postulates. It is very interesting to think to the origin of the deductive method, which is an absolute novelty compared to earlier civilizations. In Greek civilization, the deductive method was prepared by other events that have to do with logic, in particular the analysis of the syllogism in Aristotle.

Deductive method and rhetoric. The aspect that should be emphasized is that there is a direct relationship between the demonstrative method and rhetoric. It is very interesting to read Aristotle's Rhetoric, in which it is clear that rhetorics gave rise to logic and not the converse. The syllogism, in particular, is identified as the only reasoning which resists to any confutation attempt. This was indeed an essential step in the development of logic.

Theoretical entities. There is a close relationship between the two components of Hellenistic science that are the logical reasoning within the theoretical model, i.e. the systematic use of the demonstrative method, and the theoretical nature of the entities. How were the theoretical entities thought? It is important to reflect on the fact that the Greeks did not have available a special language for the construction of a scientific theory, like the Greek language will be for the other civilizations. The later peoples have built the entire scientific terminology taking heavily from Greek

\footnotetext{
${ }^{7}$ Politecnico di Bari, AA 2000-2001 (XI), School of Architecture, via E. Orabona 4, Monday, March 12, 2001.
} 

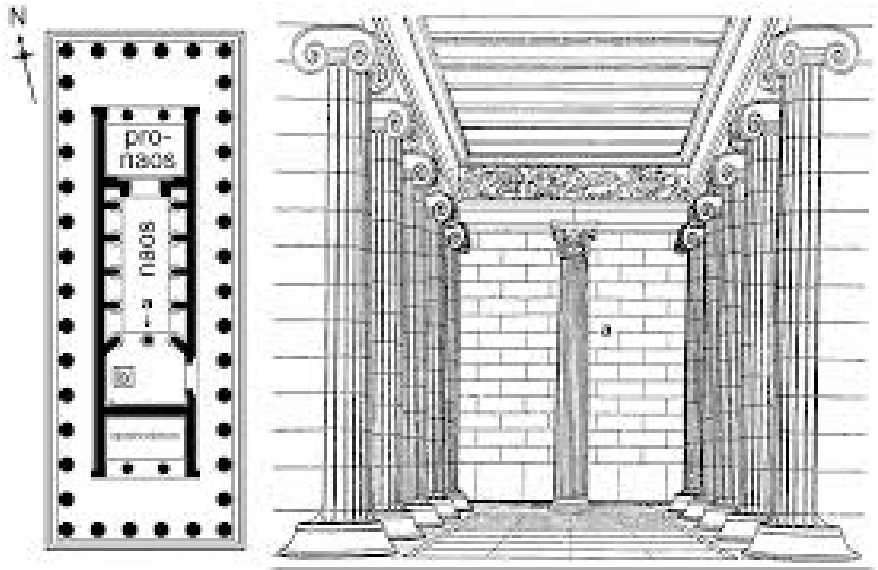

Figure 2. Floor plan and transverse section of the temple.

language, and this provided an additional tool to distinguish the technical term used from the conventional meaning that it used to have in the common language.

The Greeks of course could not do it: using the terms of their daily language, the process of abstraction had to be carried out in a somehow more conscious way. A simple example: when a student speaks, today, of a trapezium (or trapezoid, in North America), or when we talk of a circus trapeze, these words lack more mundane meanings. By contrast, a student in Euclid's time who spoke of a trapezium

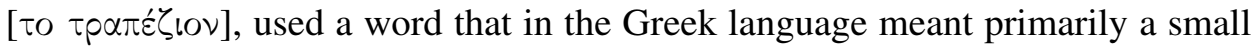
bench, and this required a more conscious level of abstraction.

Then how do you go from a real word to its abstraction? For example, with the word "straight line" it is obvious that we are referring to a geometric entity and not to a real object. So, in which way from a "straight line" that is a real object, which can be traced with a stylus, with a pen on a papyrus or on a sheet of paper, was born the straight line of geometry? A possible answer is that it arises precisely from the strictly deductive structure of the theory.

Deductive theory. A deductive theory consists of a set of propositions, all based on a few, basic assumptions that in the case of the Euclidean geometry are the "postulates". Considering admissible not all the statements but only those deductible rigorously with a deductive method from "postulates", narrows the semantic field of the term of ordinary language.

The "real" straight line can be green or red or can have any possible attribute; however, because none of the five postulates of Euclid speaks of colors, it is clear that no color will ever appear in any theorem. Nothing about colors is deducible from the postulates; colors are used in other contexts. Similarly there is no mention of thickness, and the thickness of a line will never appear in geometry. 


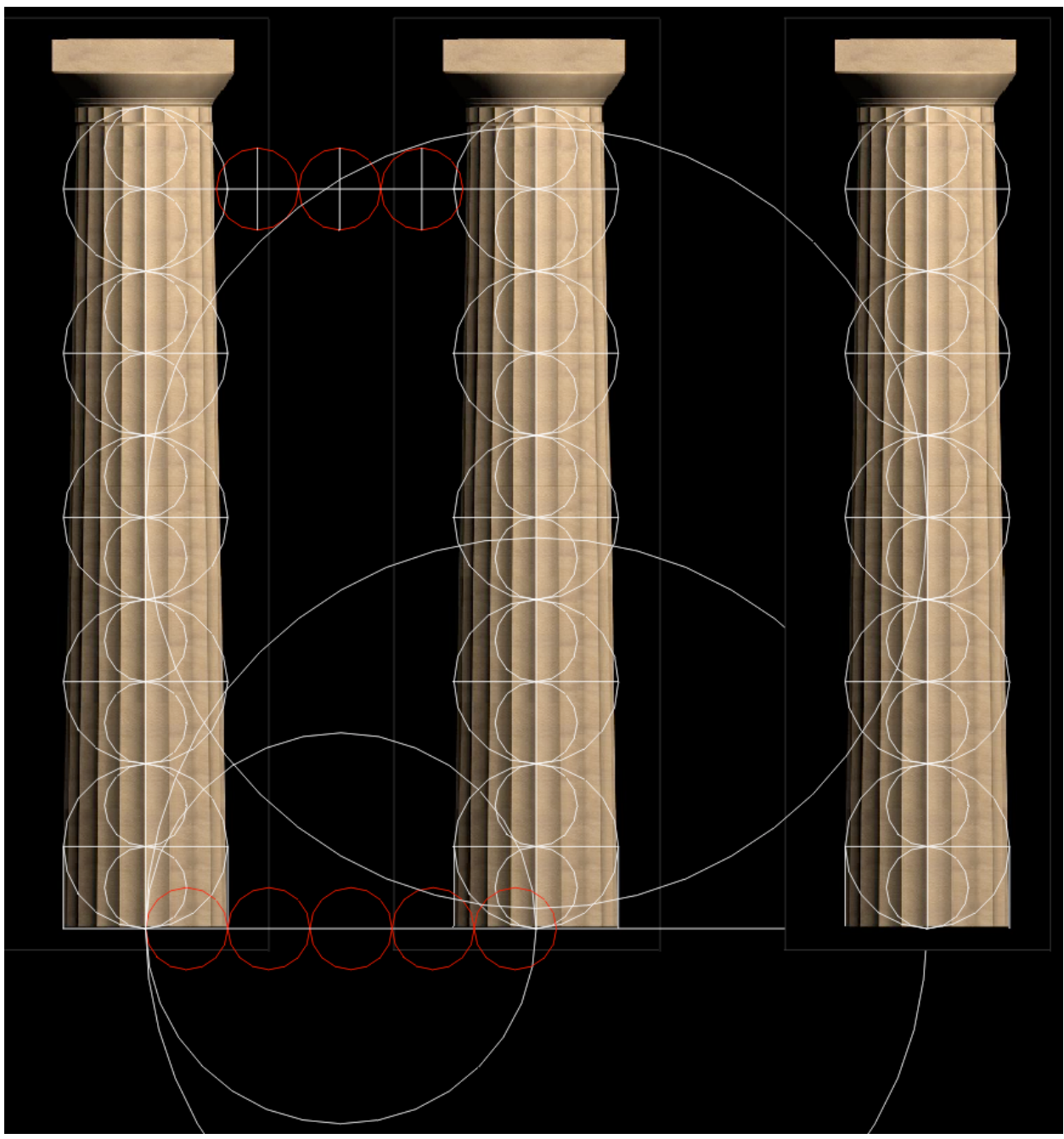

Figure 3. Proportional studies of the Doric order (external peristasis) of the temple of Apollo at Bassae (by G. Fallacara).

Theoretical concepts or postulates, and their constructability. Thus in this way a new concept arises that is the theoretical concept. But it is important to note that the postulates retain a role (that for instance the axioms have not in modern mathematics) in connecting the theory and the practical use of the language. The postulates have indeed a dual nature: from the point of view of the theory they are statements about theoretical entities, born thanks to their function; but in any case, they continue to be phrases with an "ordinary" meaning. For instance we are accustomed to think, thanks to the first postulate of Euclid, that the statement that a straight line passes through two points is seen an internal statement of the 

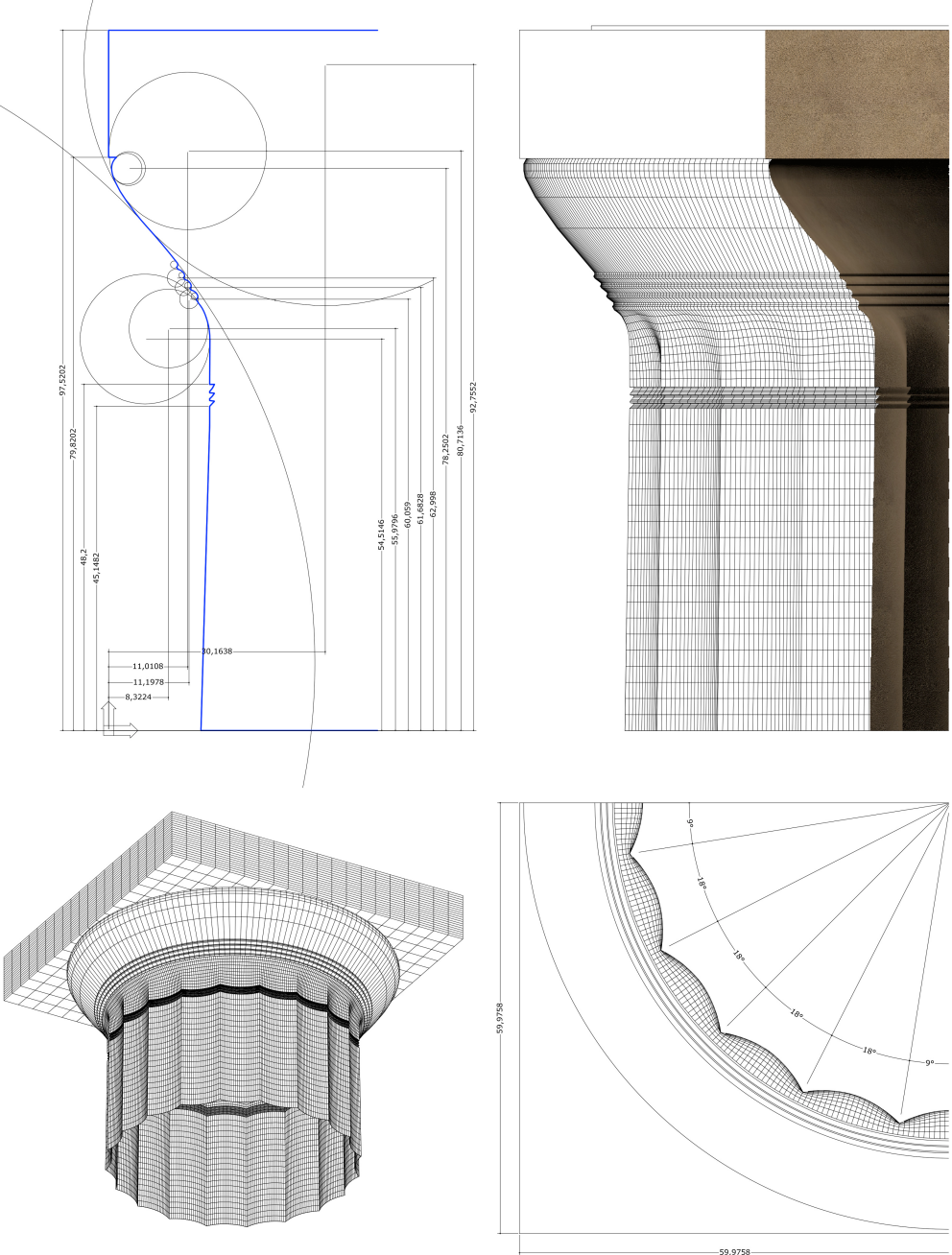

Figure 4. Constructive geometries of capital 9A (by G. Fallacara).

geometric theory which relates those abstract entities which are the points and the straight lines. If we make a literal translation of the first postulate of Euclid's Elements, namely that between two "signs" you can always draw a straight line, this statement has a meaning both from the point of view of the theory, but also refers to a real activity of drawing, and this allows a concrete application of the theory. When this connection is broken, you cannot understand anymore what is the relationship between theory and the real world.

The diffusion of the deductive method and the building of models. Eg. The hidraulycs, the astronomy. Another important aspect is the changes that these concepts experienced in the modern era, escaping to the original meanings. The 
scientific theories we are talking about are not mathematical theories in the current sense of the word mathematics; they have a much broader scope of application: for example, in his mechanics works, Archimedes uses exactly the same demonstration scheme we are talking about, but there he talks about machines and not of geometric drawings. And of course also the drawings are something real that is built like machines; think to the hydrostatic theories of Archimedes, or to the Treaty On Floating Bodies, which is concerned with a number of theorems on bodies floating in a fluid.

But the floating bodies of Archimedes are not the real and concrete bodies that float in the life of every day: with these they have the same ratio that the Euclidean straight line has with the straight line of the drawing. Indeed the floating bodies of Archimedes never undergo gusts of wind, the water does not swell, there is no surface tension, and so on. In other words, only those features of the floating bodies are maintained which are mentioned in the hydrostatic postulates. Everything else is automatically neglected. Ultimately, a model is built that considers only the consequences of a part of the characteristics chosen from the real world. The same thing happens in astronomy.

Hypotheses and postulates. A crucial point of these scientific theories is that they consist of theorems proved rigorously starting from some statements that are often defined "hypotheses" rather than postulates (the word often is regarded as synonymous of "postulates"). The term hypothesis derives from a Greek word that has a different meaning from the modern terminology, namely it is "the basis", what it is placed under, the foundation.

It is important to understand how you choose the hypotheses, how you choose the postulates. There are a number of statements which are very easily verifiable, but generally the postulates are neither simple nor verifiable. For instance, in the way in which Archimedes explains the heliocentric theory in the "Sand Reckoner", it is not at all easy to verify Aristarchus' "hypothesis" that the Earth moves and that the Sun is stationary, because all the experience through the millennia seems to say the opposite. So the postulates are not something whose truth is obvious. This also applies to the postulates of geometry, if only for their being so general: how do you verify that for any pair of signs you can always draw a straight line?

Nor we can assume the criterion of simplicity. The idea to start from the simple is a pre-Euclidean idea, coming from the Pythagorean School: if you want to build a geometry starting from the simplest entities, these should be the points. But it has never succeeded to build a geometry starting from the points, because aporias were always arising, because always there had been some problems. And then Euclid decided to start halfway, directly from the concepts of circles and straight lines, without starting from the concept of point, and this was a tremendous idea. 
The starting hypotheses are neither the easiest nor the most verifiable. But there is another fundamental criterion that must be satisfied, that is: from them it must be possible to deduce what is observed with a deductive method - what Plato called

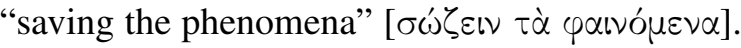

It is important to note that the phenomena are not intended here in the modern meaning of the word. There are two different starting points of the theory: one is the logical starting point that is the postulates of the theory; and then there is the starting point of the phenomena, that is all that you can immediately perceive with the senses.

In the case of astronomy, the starting point of the modern theory is the assumption that the sun is stationary and the earth moves, while the phenomenological starting point is the observation of planetary motions, in particular their retrograde movement. That is, we observe the motion of the planets, and notice that they move some times "forward" and sometimes "backward": here appearances are very complex and we try to save them, deducing the theory from simple hypotheses.

So, if we have some simple hypotheses from which it is possible to deduce what appears, then we have been able to build a valid scientific theory.

Hellenistic science and its applications. Hellenistic science was what it was precisely because, despite it changed the object of investigation compared to the classical philosophy, focusing in a sense "on the parts" rather than "on the entire", it kept the spirit of the old philosophy, the contemplative spirit that the Greeks called theoretical. Greek science was animated by the theoretical and contemplative force, which urges us to consider visible things as chinks through which you can access to the ones that are not directly accessible. This is an approach that the pragmatictechnological modern mentality seems to have lost, or at least marginalized. The explicit description of the process of abstraction that leads to the formation of geometric entities is typical of Greek culture. The idea of solving problems with theorems is a typical Greek cultural product, that no other civilization had conceived.

So, the superiority of the Greeks on other peoples on this particular point, is not purely quantitative but qualitative, because what they created, establishing philosophy, is a novelty in some absolute sense. Indeed, it was the philosophy, because of its rational categories, to make possible the birth of science and, in a sense, to generate it.

Constructible geometric entities. Let us go back to theorems. In order to create theorems you must first have developed the logic, as Greeks did analyzing the forms of reasoning used in speeches; and then you have to try to derive a large number of logical propositions from very few propositions, chosen as postulates. 

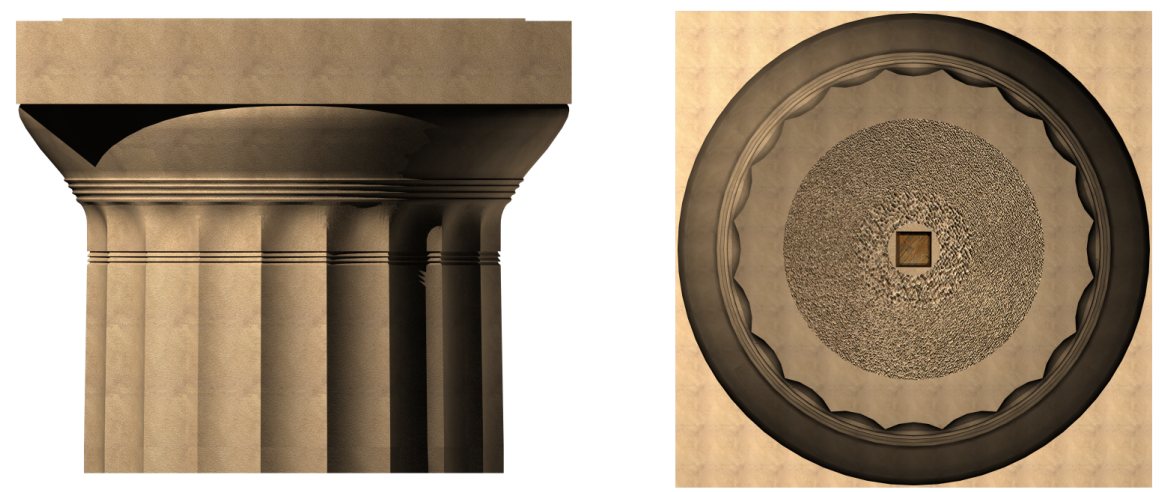

Figure 5. Left: virtual reconstruction of capital $9 \mathrm{~A}$ and its drum (by G. Fallacara). Right: virtual reconstruction of horizontal section of capital 9A with its empolion (from bottom).
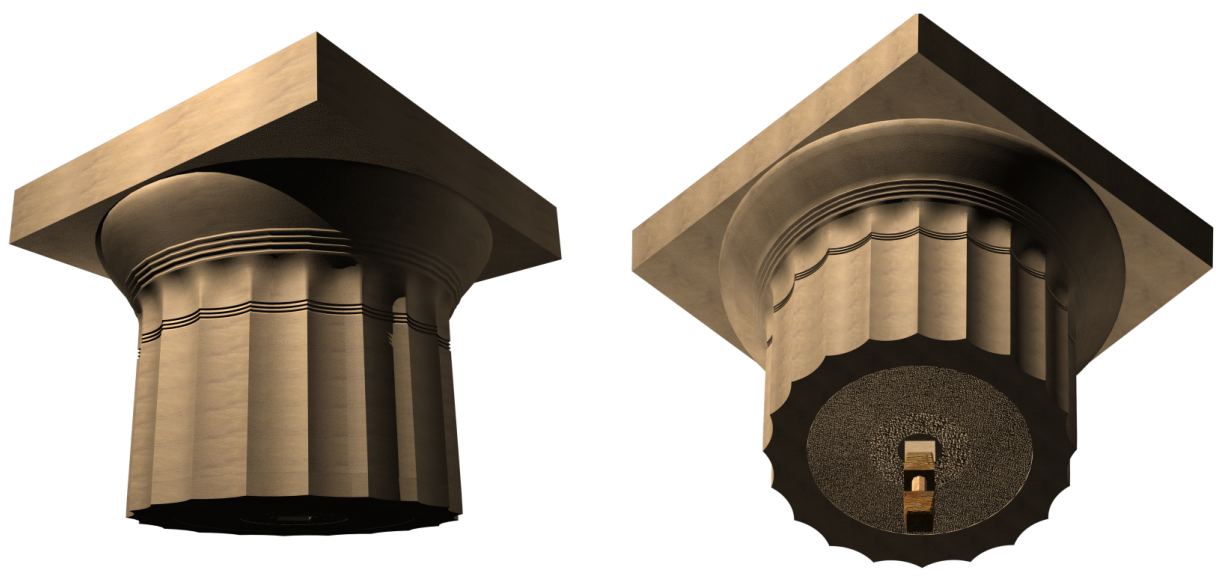

Figure 6. 3D view of capital 9A, from above and below.

It must then be determined which geometric entities are possible subject of mathematical proofs. The choice made by Euclid is to admit only constructible geometric entities on the basis of the postulates, that is, with a ruler and a compass.

\section{Conclusions: a research paradigm was changed due to the meeting of the "two cultures"}

As a conclusion, I want to come back to the impact of Russo's way of thinking on my work by briefly mentioning a few research products of the aforementioned lines of thought. In 2001-2003 the author supervised the PhD Thesis: Serial production 


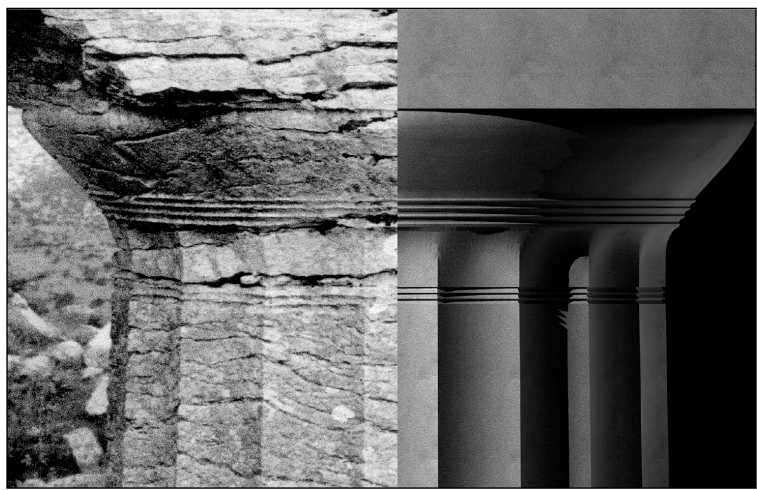

Figure 7. Comparison between the real capital and its virtual reconstruction.

in the architectonic ornamentations: from Hellenistic age to prototyping processes with CAD-CAM techniques ${ }^{8}$. This research object was a result of the investigation on the diversity between the mode of production of the Greek classic architecture and the Hellenistic one, developed also through the comments of Lucio Russo to our observations on the Hellenistic building site, to its methods and to the techniques of cutting the stone. A year later the seminar on the temple of Apollo at Bassae, it was promoted a symposium devoted to architecture in cut stone and to the stereotomy. ${ }^{9}$ The title was Architecture and stereotomy, tradition and innovation. ${ }^{10}$ This was an important moment of transition between the previous research

\footnotetext{
${ }^{8} \mathrm{PhD}$ Student: Annalisa Di Roma.

9"Stereotomy, from the Greek words $\sigma \tau \varepsilon \rho \varepsilon o=$ solid and $\tau o \mu \eta=$ cutting, is the science of cutting the solids (stone and wood) that through projective graphic techniques is able to determine the exact forms of the elements constituting the architectural system, and to realize them.

It combines the set of geometric codified procedures, consistent and repeatable, able to design and to represent whole buildings, or parts thereof, made of stone or wood. ... By extension of meaning, stereotomy stood to indicate during the 16th, 17th and 18th centuries the Art of Building, or Baukunst or Art de Batir." In C. D'Amato, Studiare l'architettura, Roma, Gangemi, 2014, p. 27.

${ }^{10}$ The initiative was taken by the Department of Civil Engineering and Architecture of Politecnico di Bari and the Department of Representation and Survey of the University of Rome "La Sapienza." The sessions were:

1) From survey to model (M. Docci, A. Ambrosi);

2) Mathematical model and numerical model (R. Migliari, Trevisan C.);

3) Idea, design, construction (C. D'Amato, S. Alevridis);

4) The replica of capital 9A of the temple of Apollo at Bassae (CMS);

5) Practical applications (G. Falcone, G. Valenti, V. Cascione, G Fallacara, A. Di Roma);

6) Theories, methods and instrumentation of contemporary stereotomy (L. Nisi, M. Curuni, M. Ciammaichella, E. Guglielmotti);

7) Theories, methods and tools of traditional stereotomy (E. Rabasa Diaz, M. Greco and M. Sganga, C. Bianchini, M. Fasolo, M. Alicino and V. Chieti, A. Di Roma, E. De Nichilo, G. Fallacara, G. Pinto, G. Radicchio, C. Zaccaria, P. Perfido).
} 
on architecture in cut stone, both with traditional methods and CNC machines, and the study of stereotomic architecture that would characterize my research in the following years.

Finally, I want to mention that the mature fruit of this season was the birth of Classic curriculum in the degree course in architecture of the School of architecture of Bari.

Since the academic year 2005-2006 the School of Architecture of Bari offers, together with a General curriculum, a Classical one. This is mostly inspired by the archeologic studies and by the Bauforschung, the consolidated tradition of studies in the Germanic world between 19th and 20th centuries about the knowledge of ancient architecture. Today this curriculum is not offered by other Schools of Architecture all over the world. In this School scientific knowledge, mathematical thought and physical theories are highlighted as fundamental ingredients for the basic education of young architects.

Received 7 Nov 2016. Revised 10 Oct 2017. Accepted 27 Oct 2017.

Claudio D'Amato: claudio.damatoguerrieri@poliba.it 


\section{Guidelines for Authors}

Authors may submit manuscripts in PDF format on-line at the submission page.

Originality. Submission of a manuscript acknowledges that the manuscript is original and and is not, in whole or in part, published or under consideration for publication elsewhere. It is understood also that the manuscript will not be submitted elsewhere while under consideration for publication in this journal.

Language. Articles in MEMOCS are usually in English, but articles written in other languages are welcome.

Required items. A brief abstract of about 150 words or less must be included. It should be selfcontained and not make any reference to the bibliography. If the article is not in English, two versions of the abstract must be included, one in the language of the article and one in English. Also required are keywords and a Mathematics Subject Classification or a Physics and Astronomy Classification Scheme code for the article, and, for each author, postal address, affiliation (if appropriate), and email address if available. A home-page URL is optional.

Format. Authors are encouraged to use $\mathrm{IAT}_{\mathrm{E} X}$ and the standard amsart class, but submissions in other varieties of $\mathrm{T}_{\mathrm{E}} \mathrm{X}$, and exceptionally in other formats, are acceptable. Initial uploads should normally be in PDF format; after the refereeing process we will ask you to submit all source material.

References. Bibliographical references should be complete, including article titles and page ranges. All references in the bibliography should be cited in the text. The use of $\mathrm{BIBT}_{\mathrm{E}} \mathrm{X}$ is preferred but not required. Tags will be converted to the house format, however, for submission you may use the format of your choice. Links will be provided to all literature with known web locations and authors are encouraged to provide their own links in addition to those supplied in the editorial process.

Figures. Figures must be of publication quality. After acceptance, you will need to submit the original source files in vector graphics format for all diagrams in your manuscript: vector EPS or vector PDF files are the most useful.

Most drawing and graphing packages - Mathematica, Adobe Illustrator, Corel Draw, MATLAB, etc. - allow the user to save files in one of these formats. Make sure that what you are saving is vector graphics and not a bitmap. If you need help, please write to graphics@msp.org with as many details as you can about how your graphics were generated.

Bundle your figure files into a single archive (using zip, tar, rar or other format of your choice) and upload on the link you been provided at acceptance time. Each figure should be captioned and numbered so that it can float. Small figures occupying no more than three lines of vertical space can be kept in the text ("the curve looks like this:"). It is acceptable to submit a manuscript with all figures at the end, if their placement is specified in the text by means of comments such as "Place Figure 1 here". The same considerations apply to tables.

White Space. Forced line breaks or page breaks should not be inserted in the document. There is no point in your trying to optimize line and page breaks in the original manuscript. The manuscript will be reformatted to use the journal's preferred fonts and layout.

Proofs. Page proofs will be made available to authors (or to the designated corresponding author) at a Web site in PDF format. Failure to acknowledge the receipt of proofs or to return corrections within the requested deadline may cause publication to be postponed. 
Special issue in honor of

Lucio Russo

Lucio Russo: A multifaceted life

Raffaele Esposito and Francesco dell'Isola

The work of Lucio Russo on percolation Geoffrey R. Grimmett

"Mathematics" and "physics" in the science of harmonics Stefano Isola

From quantum to classical world: emergence of trajectories in a quantum system Rodolfo Figari and Alessandro Teta

Propagation of chaos and effective equations in kinetic theory: a brief survey Mario Pulvirenti and Sergio Simonella

What decides the direction of a current? Christian Maes

A remark on eigenvalue perturbation theory at vanishing isolation distance Fiorella Barone and Sandro Graffi

Some results on the asymptotic behavior of finite connection probabilities in percolation Massimo Campanino and Michele Gianfelice

Correlation inequalities for the Potts model Geoffrey R. Grimmett

Quantum mechanics: some basic techniques for some basic models, I: The models Vincenzo Grecchi

Quantum mechanics: some basic techniques for some basic models, II: The techniques Vincenzo Grecchi

On stochastic distributions and currents

Vincenzo Capasso and Franco Flandoli

A note on Gibbs and Markov random fields with constraints and their moments Alberto Gandolfi and Pietro Lenarda

Quantum mechanics: light and shadows (ontological problems and epistemic solutions) Gianfausto Dell'Antonio

MEMOCS is a journal of the International Research Center for the Mathematics and Mechanics of Complex Systems at the Università dell' Aquila, Italy.

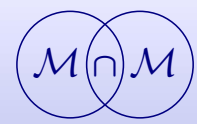

